\title{
Luxemburg-Studien
}

Études luxembourgeoises

6

Peter Gilles / Cristian Kollmann / Claire Muller (Hrsg.)

\section{Familiennamen zwischen Maas und Rhein}

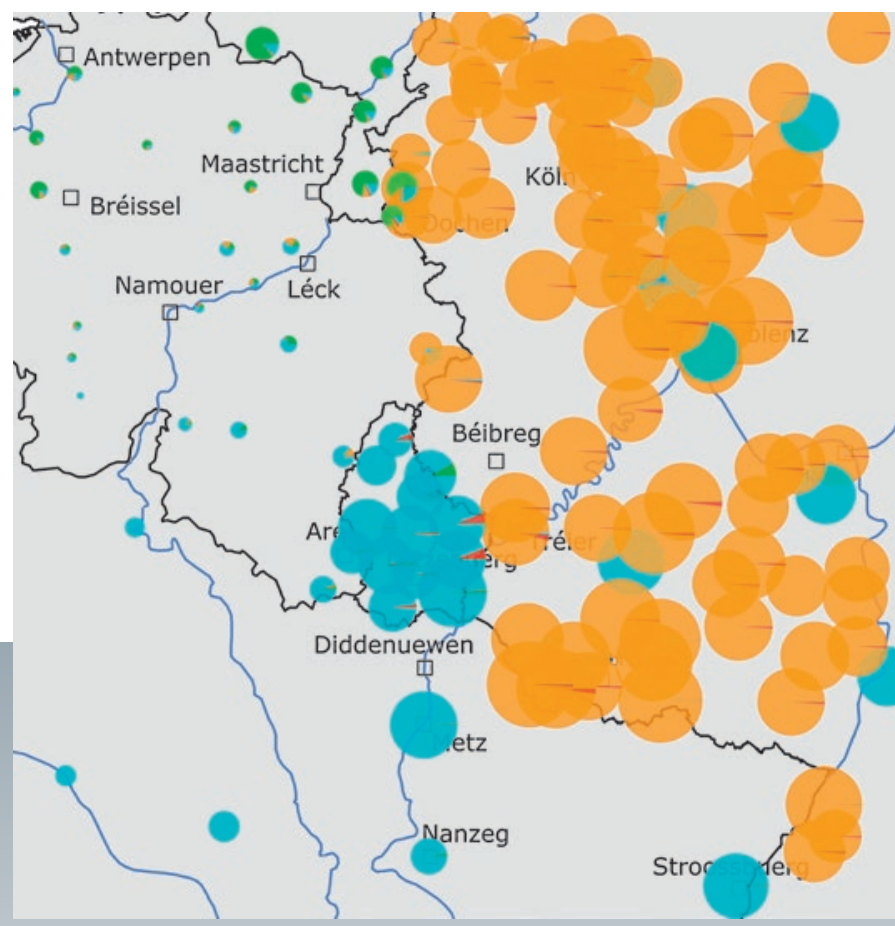


Die Familiennamen im Gebiet zwischen den Flüssen Maas und Rhein stellen infolge der komplexen politisch-historischen Grenzziehungen und durch ihre Lage in der Kontaktzone zwischen Germania und Romania eine besonders vielfältige Quelle für die Namenforschung dar. Der Band umfasst komparative und systematische Beiträge zu den Familiennamenlandschaften in den Grenzregionen von Luxemburg, Belgien, Deutschland und Frankreich, die aus sprachhistorischen, kontaktlinguistischen und kartographischen Perspektiven beleuchtet werden. Diese Artikelsammlung richtet sich damit sowohl an Sprachhistoriker wie auch an Kulturhistoriker.

\section{UNIVERSITÉ DU LUXEMBOURG \\ Unité de Recherche Identités. Politiques, Sociétés, Espaces (IPSE)}

Peter Gilles ist Professor für Sprachwissenschaft an der Universität Luxemburg. Seine Forschungsgebiete sind Phonetik und Phonologie sowie Variationslinguistik und Dialektologie insbesondere des Luxemburgischen.

Cristian Kollmann und Claire Muller sind Wissenschaftliche Mitarbeiter am Forschungsprojekt , Luxemburgischer Familiennamenatlas (LFA)'. 
Familiennamen zwischen Maas und Rhein 


\title{
LUXEMBURG-STUDIEN ÉTUDES LUXEMBURGEOISES
}

\author{
Herausgegeben von \\ Peter Gilles, Markus Hesse, \\ Michel Pauly und Christian Schulz
}

Band 6 
Peter Gilles / Cristian Kollmann / Claire Muller (Hrsg.)

\section{Familiennamen zwischen Maas und Rhein}

\section{PETER LANG}


Bibliografische Information der Deutschen Nationalbibliothek Die Deutsche Nationalbibliothek verzeichnet diese Publikation in der Deutschen Nationalbibliografie; detaillierte bibliografische Daten sind im Internet über http://dnb.d-nb.de abrufbar.

\author{
Umschlagabbildung: \\ Regionale Verteilung der Familiennamen Muller (blau), \\ Mueller (rot), Müller (orange) und (van) Mulder(s)/Smulder(s) (grün) \\ in der Maas-Rhein-Region. \\ c Universität Luxemburg, Institut für luxemburgische Sprach- \\ und Literaturwissenschaft
}

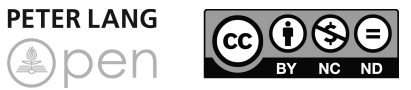

Open Access: Dieses Werk ist lizensiert unter der Creative Commons Lizenz Namensnennung - Nicht kommerziell -

Keine Bearbeitungen 4.0 International (CC BY-NC-ND 4.0).

Den vollständigen Lizenztext finden Sie unter: https://creativecommons.org/licenses/by-nc-nd/4.0/deed.de

Diese Publikation wurde begutachtet.

() Peter Gilles / Cristian Kollmann / Claire Muller, 2014

Peter Lang $\mathrm{GmbH}$

Internationaler Verlag der Wissenschaften

Berlin

www.peterlang.com 


\section{Inhalt}

Vorwort

Grundstrukturen der luxemburgischen Familiennamenlandschaft

Der Luxemburgische Familiennamenatlas (LFA)

Peter Gilles

Regionalismen in den Luxemburger Familiennamen

Cristian Kollmann

Les noms de famille en images: les interférences germaniques dans les

noms français du ,Luxemburgischer Familiennamenatlas“

Claire Muller

Die luxemburgischen Familiennamen entlang der historischen Grenze zu

Deutschland - Projektskizze und vorläufige Ergebnisse

Walter Amaru Flores Flores

Namenvarianten an der Sprachgrenze, genealogisch überprüft.

Jean-Claude Muller

La „loi des deux syllabes“: une réponse structurelle à la fixation des noms

de famille en Wallonie

Jean Germain

Auf der Schnittstelle zwischen Germania und Romania: Die Familiennamen

in Belgien

Ann Marynissen

Deutscher Familiennamenatlas Band 6: Patronyme. Konzept und

linksrheinische Beispiele

Kathrin Dräger, Konrad Kunze

Das ,Digitale Familiennamenwörterbuch Deutschlands ‘

Möglichkeiten und Ziele am Beispiel regionaler Namen

Fabian Fahlbusch, Rita Heuser

Anschriften der Autorinnen und Autoren. 



\section{Vorwort}

Erfreulicherweise kann für die Namenforschung seit einigen Jahren wieder eine rege Forschungsaktivität festgestellt werden. Für die Familiennamen manifestiert sich dies u.a. in zahlreichen europäischen Großprojekten wie dem ,Deutschen Familiennamenatlas' (DFA), dem ,Digitalen Familiennamenwörterbuch Deutschlands‘ (DFD), den ,Familiennamen Österreichs' (FamOs), den ,Family Names of the United Kingdom‘ (FaNUK) oder dem ,Luxemburgischen Familiennamenatlas' (LFA). Nicht zuletzt zeugen zahlreiche Monographien, Sammelbände, Artikel und Qualifikationsarbeiten der letzten Dekade vom neu erwachten linguistischen, dialektologischen sowie sprachund kulturhistorischen Interesse an der Namenforschung, das weit über das vorherrschende genealogische Moment hinaus geht. Grob zusammengefasst lassen sich zwei aktuelle Trends beobachten: Zum einen können Namenverteilungen durch neue Visualisierungsverfahren in einem ungleich größeren Umfang kartographiert werden und mit weiteren sprachlichen, i.e. dialektologischen Isoglossen, und/oder außersprachlichen Informationen korreliert und durch quantitative Auswertungen validiert werden. Zum anderen rücken prononciertere linguistische Fragestellungen in den Fokus, die nicht nur lautgeschichtliche Herleitungen sondern insbesondere morphologische Aspekte betreffen.

Im Kontext dieser beiden Trends sind auch die Beiträge des vorliegenden Bandes angesiedelt, die auf eine Tagung der Universität Luxemburg mit dem Titel „Familiennamen zwischen Maas und Rhein. Etymologien, Sprachkontakt, Kartierung“ (21.-22. September 2011) zurückgehen. Der gemeinsame Ausgangspunkt der folgenden Studien ist demnach ein regionaler: Initiiert durch die Arbeiten am ,Luxemburgischen Sprachatlas' wurde der geographische Raum zwischen den Flüssen Maas und Rhein als Maximalausdehnung eines komplexen Familiennamengeflechts identifiziert. Dieses weist historisch bedingt große Ähnlichkeiten auf, hat sich jedoch aufgrund politischer Grenzziehungen seit dem 17. Jh. mehr und mehr diversifiziert. Das Themenspektrum umfasst die Sprachgeschichte, inklusive des historischen Sprachkontakts zwischen Germania und Romania, ferner die Namenwortbildung sowie insgesamt die Kartierung von Familiennamen.

Die vorliegende Publikation enthält eine Auswahl der auf der Tagung gehaltenen Vorträge in ausgearbeiteter und teilweise modifizierter Form. Alle Beiträge wurden einem Begutachtungsverfahren unterzogen. In fünf Beiträgen liegt der Schwerpunkt auf Luxemburg und der angrenzenden Großregion (Gilles, Kollmann, C Muller, Flores, J-C Muller). In zwei weiteren Aufsätzen richtet sich der Fokus auf Belgien, wobei jeweils Wallonien (Germain) und Gesamtbelgien (Marynissen) ins Auge gefasst werden. Die Beiträge von Dräger/Kunze und Fahlbusch/Heuser schließlich erweitern den Blickwinkel des „Deutschen Familiennamenatlasses“ (DFA) auf das angrenzende Luxemburg und Belgien. Gemeinsam ist damit allen Beiträgen die grenzüberschreitende Perspektive. 
An dieser Stelle möchten wir allen Kolleginnen und Kollegen, insbesondere dem Gutachter, für ihre konstruktive Mitarbeit an der Erstellung dieses Bandes danken. Für die Mitarbeit bei der Gestaltung des Bandes danken wir Mara Bilo, Benoît Junk und Caroline Döhmer.

Luxemburg, im Dezember 2013

Die Herausgeber 


\title{
Grundstrukturen der luxemburgischen Familiennamenlandschaft Der Luxemburgische Familiennamenatlas (LFA)
}

\author{
Peter Gilles
}

\begin{abstract}
Der folgende Beitrag resümiert ausgewählte Ergebnisse des Projekts ,Luxemburgischer Familiennamenatlas (LFA)'. Nicht nur durch die Randlage im historischen germanophonen Sprachgebiet hat sich in Luxemburg eine charakteristische Namenlandschaft herausgebildet, die durch vielfältige Sprachkontakte mit (hoch-)deutschen und romanischen Sprachen/Varietäten gekennzeichnet ist. Die Analyse bezieht sich auf räumliche Verteilungsmuster von Namen und Namentypen sowie auf sprachhistorische Aspekte. Die Projektergebnisse werden in Form eines Namenbuches und eines Namenatlasses publiziert.

Nach einer Vorstellung von Methodik und Datengrundlage (Kap. 1) folgt eine Präsentation ausgewählter Ergebnisse (Kap. 2).
\end{abstract}

\section{Methodik und Datengrundlage des LFA}

Innerhalb der Zielsetzung des LFA $^{1}$ erfolgt die Beschreibung der luxemburgischen Familiennamenlandschaft in kartographischer und sprachhistorischer, i.e. etymologischer und phonologischer Perspektive. Dabei ist der Fokus primär auf Luxemburg gerichtet. Aufgrund der geographischen Ausdehnung, der Randlage an der germanischromanischen Sprachgrenze und vielfältiger historischer Gebietsveränderungen erscheint es jedoch unverzichtbar, die angrenzenden Regionen systematisch in die Analyse mit einzubinden. Vielfältige Sprachkontakte mit deutsch- und romanisch-sprachigen Regionen und natürlich auch die massive Immigration im Verlauf des 20. Jh. verliehen der luxemburgischen Familiennamenlandschaft ihre charakteristischen Züge. In der Sicht dieser grenzüberschreitenden Familiennamenkunde werden also neben Luxemburg, Belgien (besonders Wallonien mit seinen historisch deutschsprachigen Gebieten um Arel, St. Vith und Eupen), Frankreich (besonders die Départements Moselle und Meurthe-et-Moselle) und Deutschland (besonders Saarland, Rheinland-Pfalz, Teile von Nordrhein-Westfalen) in die Untersuchung einbezogen. Als forschungspraktische Maximalausdehnung des Untersuchungsgebietes hat sich die Region zwischen Rhein und Maas als eine brauchbare Grundeinheit herausgestellt, innerhalb derer sich die meisten

1 Das Projekt ,Luxemburgischer Familiennamenatlas (LFA)‘ wurde vom Fonds National de la Recherche (FNR) gefördert und von 2009 bis 2012 am Laboratoire für luxemburgische Sprache- und Literaturwissenschaft der Universität Luxemburg unter meiner Leitung durchgeführt. Mitarbeiter im Projekt waren Claire Muller, Cristian Kollmann und W. Amaru Flores Flores. Kooperationspartner waren Claudine Moulin (Trier), die das Projekt ursprünglich auch initiiert und beantragt hat, sowie der ,Deutsche Familiennamenatlas (DFA)` der Universitäten Freiburg (Konrad Kunze) und Mainz (Damaris Nübling). 
für Luxemburg relevanten Namenstrukturen beschreiben lassen. Methodisch wie inhaltlich schließt sich der LFA damit an vergleichbare Vorhaben zu Deutschland (DFA, Steffens 2013) und Belgien (Herbillon/Germain 1996, Marynissen 1994, Marynissen/ Nübling 2010) an.

Die Hauptdatenbasis stammt aus Telefonbuchdaten, Geburts- und Melderegistern: Für Luxemburg handelt es sich um die Telefonbuchdaten des editus-Verlags von 2009, für Deutschland um die Telefonbuchdaten der Deutschen Telekom von 2009, für Belgien um das Melderegister von 2008 und für Frankreich um die kumulierten Geburtenregister des ,Institut national de la statistique et des études économiques (INSEE) ‘ für die Jahrgänge 1920 bis 1970. Die deutlich geringere Anzahl verschiedener Namen in den französischen Daten erklärt sich daraus, dass alle Namen mit einer Häufigkeit unter 30 nicht zur Verfügung gestellt wurden. Trotz der teilweise unterschiedlichen Struktur der Daten ist aufgrund der sehr hohen Anzahl die Vergleichbarkeit gewährleistet. ${ }^{2}$ Über die quantitative Zusammensetzung gibt Tab. 1 Aufschluss.

Tab. 1: Quantitative Zusammensetzung des Familiennamenbestandes

$\begin{array}{lllll} & \begin{array}{l}\text { Staat } \\ \text { Namen } \\ \text { types }\end{array} & \begin{array}{l}\text { Namenträger } \\ \text { token }\end{array} & \begin{array}{l}\text { Anteil an Rhein-Maas-Region } \\ \text { Namen } \\ \text { types }\end{array} & \begin{array}{l}\text { Namenträger } \\ \text { token }\end{array} \\ \text { Luxemburg } & 36.326 & 163.206 & 36.326 & 163.206 \\ \text { Deutschland } & 1.287 .087 & 23.526 .460 & 340.017 & 3.125 .101 \\ \text { Belgien } & 498.015 & 11.402 .069 & 162.269 & 2.430 .201 \\ \text { Frankreich } & 131.056 & 60.740 .370 & 19.855 & 6.304 .034\end{array}$

Die Type/Token-Ratio beträgt für Luxemburg 4,5, d.h. auf einen Namen (,type') entfallen durchschnittlich 4,5 Namenträger/Telefonbucheinträge (,token'). Im Vergleich dazu beträgt die Type/Token-Ratio für Deutschland 21 und für Belgien 33. In Luxemburg gibt es also deutlich mehr seltener vorkommende Namen als in den Nachbarländern. Genauere Einsichten in die luxemburgischen Namendaten gewährt Abb. 1, wo eine Aufteilung in Häufigkeitsklassen vorgenommen wird. Zur Abschätzung von historischen Veränderungen finden sich rechts die entsprechenden Daten von 1880, die aus Volkszählungsdaten gewonnen wurden (Müller 1887). In Bezug auf die Types ist zu erkennen, dass heute ca. $90 \%$ der Namen mit den geringsten Häufigkeiten von 1 bis 10 vorkommen, während dieser Anteil 1880 nur bei $65 \%$ lag. Bezogen auf die Tokens bedeutet dies, dass heute ca. $38 \%$ aller erfassten Personen einen Namen der untersten Häufigkeitsklasse tragen. Diese Häufigkeitsverteilung illustriert die starke Diversifizierung des Luxemburger Familiennamenbestandes und ist auf den hohen Anteil der nicht-luxemburgischen Wohnbevölkerung (ca. $44 \%$ ) zurückzuführen.

2 Zur Diskussion der Vorteile, aber auch möglicher Unzulänglichkeiten solcher Datentypen, insbesondere der Telefonbuchdaten, vgl. DFA und Cheshire et al. (2011: 582f.). 


\section{Types 2007}

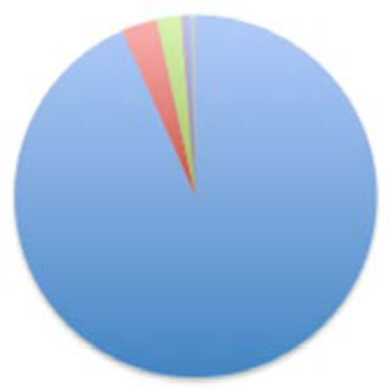

Token 2007

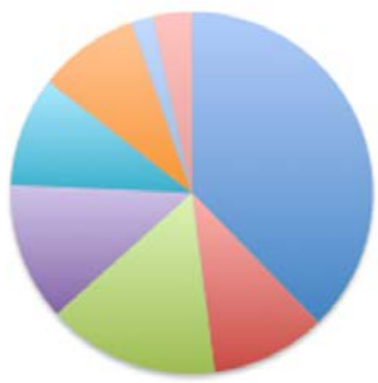

Types 1880

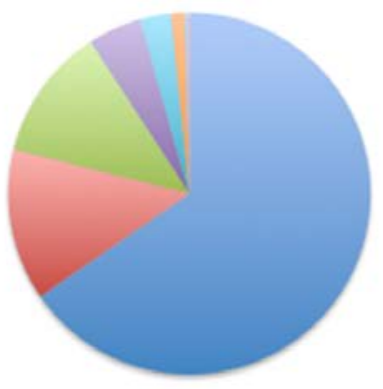

$=1$ bis 10

$=11$ bis 20

$=21$ bis 50

$=51$ bis 100

$=101$ bis 200

$=201$ bis 500

$=501$ bis 1000

\section{Token 1880}

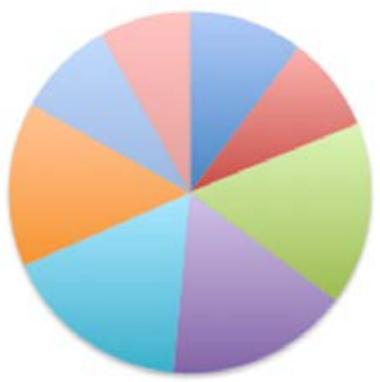

= 1 bis 10

$=11$ bis 20

$=21$ bis 50

=51 bis 100

101 bis 200

$=201$ bis 500

$=501$ bis 1000

Abb. 1: Aufteilung der luxemburgischen Familiennamen von 2009 und 1880 in Häufigkeitsklassen

Als zusätzliche Quellen, insbesondere zur historischen Sondierung, wurden neben den bereits erwähnten Volkszählungsdaten von 1880 (Müller 1887), Feuerstättenverzeichnisse von 1561, 1611, 1656 (Oster 1965) und als älteste greifbare Schicht die Rechnungsbücher (1388-1500) der Stadt Luxemburg (Moulin/Pauly 2007ff.) herangezogen, deren Namenbestand von Gniffke (2010) in einem Namenbuch zusammengestellt wurde. Darüber hinaus wurden auch genealogische Datenbanken ${ }^{3}$ konsultiert, in deren stetig wachsenden Datenbeständen aus Geburts- und Heiratsregistern sich wichtige Hinweise über die Transmission und Veränderung von Familiennamen im generationellen Wechsel aufspüren lassen. Innerhalb dieser historischen Quellendaten nehmen die Volkszählungsdaten von 1880 die wichtige Funktion eines Filters ein: Da es das Ziel des LFA ist, die historische Struktur der Luxemburger Familiennamenlandschaft zu erfassen, bleiben alle Namen, die nach 1880 überwiegend durch Immigration hinzugekommen sind, aus der Untersuchung ausgeblendet. Dies betrifft überwiegend portugiesische und italienische Einwanderernamen wie da Silva (239, Rang 41), dos Santos (178, Rang 66), Ferreira (202, Rang 58) oder Rossi (67, Rang 321). Die Untersuchung konzentriert sich also ausschließlich auf Namen, die bereits 1880 anzutreffen waren.

$3 \quad$ Z.B. http://www.deltgen.com oder http://www.luxroots.com. 
Zur Verwaltung und Bearbeitung des Namenbestandes wurde eine internetbasierte Datenbank entwickelt, ${ }^{4}$ in der alle relevanten Informationen eingegeben werden. Zur Namenklassifikation und -beschreibung wurden folgende Kriterien berücksichtigt:

- Name

- Lemma oder Variante

- wenn Lemma: Angabe des Basisnamens

- wenn Variante: Angabe des zugehörigen Namens

- phonetische Transkription für ausgewählte Namen

- Namentyp

- Morphologische und/oder sprachhistorische Kennzeichen

- statistische Angaben zu Häufigkeitsrang und Anzahl

- Etymologie

- Weiterführende sprachhistorische Diskussion

- Beschreibung der Verbreitung

- Historische Belege

Die Datenbank als wichtigstes Arbeitsinstrument wird über verschiedene Formulare ,gefüttert" (vgl. Abb. 2 im Anhang). Da alle Felder sortierbar sind und individuell oder kollektiv durchsucht werden können, lassen sich die Namen nach vielen gewünschten Kriterien ausgeben (z.B. alle Namen auf -tges, alle Herkunftsnamen, alle mehrdeutigen Namen, Namen mit Häufigkeiten zwischen 20 und 30 etc.). Über ein Exportskript können die Daten in Form eines alphabetisch sortierten Namenbuchs oder als XML-Daten zur weiteren Manuskriptherstellung (z.B. in Adobe InDesign) ausgegeben werden.

Auf diese Art und Weise wurden zwischen 2009 und 2012 ca. 2500 Familiennamen unter Zuhilfenahme von onomastischer Forschungsliteratur, Handbüchern und Wörterbüchern (insbesondere Luxemburger Wörterbuch (LWB) und Rheinisches Wörterbuch (RhWB) etymologisiert und ausführlich kommentiert. Ein illustrativer Ausschnitt aus diesem Namenbuch findet sich im Anhang. ${ }^{5}$

Parallel zur Beschreibungskomponente wurde eine Kartographie-Komponente entwikkelt, die sowohl eine flexible, grenzüberschreitende Kartierung ermöglicht, die Karten für verschiedene Teilräume generiert, als auch ein auf Nachhaltigkeit ausgerichtetes Instrument für eine auszubauende europäische Familiennamengeographie darstellt. ${ }^{6}$ Somit

4 Das Datenbanksystem basiert auf einer Javascript-Lösung mittels ,dhmlx (http://www.dthmlx. com), die auf das Datenbanksystem MySQL als Backend aufsetzt.

5 Die Veröffentlichung der Druckversion des Namenbuches ist für 2013 vorgesehen.

6 Vgl. dazu Cheshire et al. (2009, 2011), die auf der Grundlage der Namendaten von zahlreichen europäischen Ländern (ca. 8 Millionen verschiedene Namen von 152 Millionen Namenträgern) mit Hilfe von Isonymieberechnungen, Räume mit ähnlichen Nameninventaren ermitteln. Auch Luxemburg wurde berücksichtigt, und es wurden interessanterweise Übereinstimmungen des Nameninventars von Südluxemburg mit dem Elsass und der Region Bozen in Südtirol ermittelt (Cheshire et al. 2011: 591). Unter der Adresse http://worldnames.publicprofiler.org ist eine weiträumige Namenkartierung für Europa, Amerika und teilweise auch Asien möglich. 
wurde ein System entwickelt, das aufbauend auf einem Geographischen Informationssystem (GIS) mit realen Geokodierungen arbeitet und das Kartenmaterial für die Grundkarte dynamisch von verschiedenen externen Anbietern oder aus eigenen Quellen beziehen kann. Im Gegensatz zu den Kartierungsprogrammen des Deutschen Familiennamenatlasses, familiennaam.be (http://www.familienaam.be) oder dem Online-Dienst geogen (http://christoph.stoepel.net/geogen), erlaubt das System des LFA prinzipiell eine weltweite Kartierung. Dazu muss für die Namendaten lediglich die Information über Häufigkeit und Ortspunkt vorliegen. Für alle Ortspunkte wird die Geolokalisierung mittels des Längen- und Breitengrades verwendet und anschließend auf einer dynamisch generierten Karte angezeigt. ${ }^{7}$ Abb. 3 (Anhang) zeigt die Bedienungsoberfläche der Kartierung.

Das Kartierungsmodul verfügt über folgende Funktionalitäten: Auf einer Europakarte können die relativen Häufigkeiten für bis zu vier Namen (oder Namengruppen) in größenskalierten Kreisdiagrammen dargestellt werden. Als Grundkarte wird eine Modifikation der ,OpenStreetMap“ verwendet, die lediglich größere Ortschaften/Städte, Flüsse und Landesgrenzen anzeigt. ${ }^{8}$ Verschiedene weitere Grundkarten können ausgewählt werden (z.B. Google Terrain, Google Satellite, OpenStreetMap, Karte der Einteilung deutscher Dialekte, historische Karten der Großregion u.a.). Mit der Maus kann die Karte an beliebigen Stellen vergrößert oder verkleinert werden, sodass die Verbreitungen in verschiedenen Regionen untersucht werden können. Die Grundkarte der Kartierung lässt sich dahingehend einschränken, dass nur die Namen für die Rhein-Maas-Region, für Luxemburg, Belgien, Deutschland oder - in maximaler Ausdehnung - für alle Länder kartiert werden. Über die Suchfelder links werden die zu kartierenden Namen eingegeben. Diese Felder verarbeiten auch Suchmuster mit ,regulären Ausdrücken', sodass beliebige Namenstrukturen oder -gruppen zusammengefasst kartiert werden können. Über eine Schnittstelle zur Namenklassifizierung werden unter ,Namenkommentare ‘ die Etymologien und sprachhistorischen Diskussionen der kartieren Namen eingeblendet. Verschiedene Statistiken informieren über das Vorkommen der Namen in den vier Ländern. Durch Auswählen eines Symbols auf der Karte erhält der Benutzer Informationen über die Zusammensetzung nicht nur der kartierten, sondern aller Namen für diesen Ortspunkt. Hier können Häufigkeitslisten ausgegeben werden, die z.B. alle Namen für diesen Datenpunkt oder aggregiert für ein größeres Gebiet enthalten (z.B. Deutschland: dreistelliges Postleitzahlengebiet, Frankreich: Departement, Luxemburg: Kanton, Belgien: Gebiet eines dreistelligen NIS-Code). Für Druck-Publikationen kann die Ebene der

7 Es basiert im Wesentlichen auf dem Ebenenverwaltungssystem ,OpenLayers` (http://www. openlayers.org), mit dessen Hilfe internetbasiert verschiedene georeferenzierte Informationsquellen (i.e. Karten diverser Anbieter wie ,Google Maps‘ oder ,Open Street Map', Vektordaten wie z.B. Symbole und Flächen etc.) überlagert werden können. Durch die Anbindung an ein Datenbanksystem (MySQL) ist es so möglich, dynamische Karten mit automatisch skalierenden Häufigkeitssymbolen für jeden beliebigen Namen zu erstellen. Die Layoutkontrolle geschieht über die GIS-Bibliothek ,GeoExt' (http://geoext.org), die speziell für die flexible Einbindung von Datenbankabfrage-Elementen, Fenster-Verwaltung und Multilayer-Kartenverwaltung entwickelt wurde.

8 Dazu wurde auf den Internet-Dienst,Cloudmade` zurückgegriffen (http://www.cloudmade.com). 
Häufigkeitssymbole im Vektorformat SVG exportiert werden und maßstabsgetreu über beliebige Grundkarten montiert werden.

Eine öffentliche Version zur Kartierung aller Familiennamen im Rhein-Maas-Raum wurde im Mai 2012 unter der Adresse http://lfa.uni.lu zur Verfügung gestellt. Gegenüber der internen Kartierungssoftware verfügt diese Version des LFA, hauptsächlich aus datenschutztechnischen Gründen, über weniger Funktionen: Es können die relativen Häufigkeiten von bis zu vier Namen kartiert werden. Eine einfache Platzhaltersuche ermöglicht es, auch Namengruppen mit gemeinsamen Zeichenketten darzustellen. Darüber hinaus werden auch die Etymologien der ca. 2200 häufigsten Luxemburger Familiennamen sowie Namenstatistiken angegeben. Auf komplexe Suchmöglichkeiten mittels regulärer Ausdrücke, Zoomfunktion und Informationen zu einzelnen Ortspunkten wurde hingegen verzichtet; diese bleiben der internen Wissenschaftler-Version vorbehalten. Neben der Kartierungsmöglichkeit von Luxemburger Namen lassen sich damit natürlich auch alle Namen im Saarland, Rheinland-Pfalz, Nordrhein-Westfalen, Wallonien oder Lothringen kartieren, und der LFA wird damit auch zu einem Kartierungsinstrument, um regionale Namenstrukturen in den angrenzenden Ländern darzustellen.

\section{Ausgewählte Resultate}

\subsection{Häufigkeitsbasierte Raumbildungen}

Ein erster Überblick über die luxemburgische Namenlandschaft manifestiert sich im Vergleich der 30 häufigsten Namen in Luxemburg und verschiedenen angrenzenden Arealen in Belgien und Deutschland (Tab. 2). Im Vergleich mit der unmittelbar angrenzenden wallonischen Province de Luxembourg und Gesamt-Belgien bilden die luxemburgischen Namen einen starken Kontrast, denn praktisch keiner der überwiegend französischen Namen ist auch in Luxemburg häufig. Die germanisch-romanische Sprachgrenze ist damit in den Familiennamen recht deutlich konturiert. In dieser Übersicht kommt allerdings nicht zum Ausdruck, dass nahezu alle häufigen französischen Namen mit mittlerer oder niedriger Häufigkeit auch in Luxemburg vorkommen: So liegt z.B. Lambert in Luxemburg auf Rang 148, Gérard auf Rang 268, Martin auf Rang 72 und Thiry auf Rang 165. Der Grad der Mischung von romanischen und germanischen Namen lässt sich also erst dann abschätzen, wenn auch mittelfrequente Namen berücksichtigt werden (vgl. Claire Muller im Druck).

Tab. 2: Ranglisten der 30 häufigsten Familiennamen

\begin{tabular}{llllll}
\hline Rang & Belgien & $\begin{array}{l}\text { Provinz } \\
\text { Luxemburg (B) }\end{array}$ & Luxemburg & PLZ 546 (D) & Deutschland \\
\hline 1 & Peeters & Lambert & Schmit & Schmitt & Müller \\
2 & Janssens & Gérard & Muller & Müller & Schmidt \\
3 & Maes & Gillet & Weber & Schneider & Schneider \\
4 & Jacobs & Martin & Hoffmann & Becker & Fischer \\
5 & Mertens & Thiry & Wagner & Wagner & Weber
\end{tabular}




\begin{tabular}{|c|c|c|c|c|c|}
\hline Rang & Belgien & $\begin{array}{l}\text { Provinz } \\
\text { Luxemburg (B) }\end{array}$ & Luxemburg & PLZ 546 (D) & Deutschland \\
\hline 6 & Willems & Guillaume & Thill & Hoffmann & Meyer \\
\hline 7 & Goossens & Georges & Schmitz & Weber & Wagner \\
\hline 8 & Claes & Léonard & Schroeder & Schmitz & Becker \\
\hline 9 & Wouters & Poncelet & Reuter & Meyer & Schulz \\
\hline 10 & De Smet & Simon & Klein & Jakobs & Hoffmann \\
\hline 11 & Pauwels & Collard & Becker & Marx & Schäfer \\
\hline 12 & Vermeulen & Louis & Kieffer & Heinz & Bauer \\
\hline 13 & Hermans & Noël & Kremer & Klein & Koch \\
\hline 14 & Michiels & Mathieu & Faber & Theis & Richter \\
\hline 15 & Dubois & François & Meyer & Roth & Klein \\
\hline 16 & Aerts & Collignon & Schneider & Krämer & Wolf \\
\hline 17 & Lambert & Thomas & Weis & Michels & Schröder \\
\hline 18 & De Vos & Adam & Schiltz & Willems & Neumann \\
\hline 19 & Smets & Antoine & Simon & Schmidt & Schwarz \\
\hline 20 & Martens & Lejeune & Welter & Zimmer & Zimmermann \\
\hline 21 & Van de Velde & Jacques & Hansen & Reuter & Braun \\
\hline 22 & Desmet & Poncin & Majerus & Schuh & Hofmann \\
\hline 23 & De Clercq & Laurent & Ries & Scherf & Schmitt \\
\hline 24 & Hendrickx & Denis & Meyers & Reis & Hartmann \\
\hline 25 & Van Damme & Schmit & Kayser & Franzen & Krüger \\
\hline 26 & Dupont & Toussaint & Steffen & Thiel & Lange \\
\hline 27 & Claeys & Collin & Krier & Philippi & Werner \\
\hline 28 & De Backer & Hubert & Braun & Koster & Schmitz \\
\hline 29 & $\begin{array}{l}\text { Van Den } \\
\text { Broeck }\end{array}$ & Arnould & Wagener & Schröder & Meier \\
\hline 30 & Stevens & Lemaire & Diederich & Lex & Schmid \\
\hline
\end{tabular}

Die Ähnlichkeit mit den Namen im dreistelligen Postleitzahlengebiet 546 (Raum Bitburg in der Eifel) oder auch noch mit Gesamt-Deutschland ist hingegen recht offensichtlich. Viele der häufigen luxemburgischen Namen finden sich ebenfalls häufig im Postleitzahlengebiet 546. Viele Namen erscheinen, teils mit minimaler Abweichung in der Schreibung, beiderseits der Landesgrenze (Schmit/Schmitt, Muller/Müller, Wag(e)ner) Wagner, Rot/Roth). Dennoch lassen sich auch hier zahlreiche Unterschiede feststellen: So sind Simon, Ries, Majerus oder Diederich in PLZ 546 seltener.

Eine exakte Einschätzung, inwieweit sich Regionen in ihrem Nameninventar wirklich unterscheiden, kann durch die bloße Inspektion und punktuelle Vergleiche solcher Ranglisten nicht erreicht werden. Dazu ist es vielmehr nötig, möglichst vollständige Nameninventare mit statistischen Methoden der Bioinformatik zu vergleichen, wie sie 
in der quantitativ orientierten Namenforschung angewandt werden. ${ }^{9}$ Als ein geeignetes Vergleichsmaß hat sich hier die sog. ,Isonymie“ etabliert, die als die Ähnlichkeit von zwei Populationen auf der Grundlage der Häufigkeitsstruktur der Familiennamen definiert ist (Lasker 1985, Liu et al. 2012). Dieses Verfahren wurde im Folgenden auf die zwölf Kantone Luxemburgs sowie auf je drei bzw. vier angrenzende, ähnlich große Gebiete in Belgien und Deutschland angewandt (i.e. in Belgien die Arrondissements Arel, Bastnach und Verviers - in letzterem liegt auch die Deutschsprachige Gemeinschaft mit den Hauptorten St. Vith und Eupen; in Deutschland die dreistelligen Postleitzahlengebiete 542 (Trier), 543 (Mosel), 545 (Wittlich), 546 (Bitburg). Ziel dieser statistischen Prozedur war es erstens, innerluxemburgische Räume mit ähnlichen Nameninventaren zu ermitteln und zweitens, die (Un)ähnlichkeit mit den angrenzenden belgischen und deutschen Gebieten statistisch valide zu bestimmen. Aus dem Gesamt der 94.190 Namen-Types, die 752.483 Namenträger repräsentieren, wurden nur diejenigen Namen berücksichtigt, die in einem der Gebiete mindestens zehn Mal vorkommen. Durch den Ausschluss der selteneren Namen wird der durch rezente Migration insbesondere für Luxemburg sehr hohe Anteil ausländischer Namen reduziert, sodass eine Beschränkung auf die ,autochthonen“ Namen möglich wird. Damit blieben 7.885 Types (503.800 Namenträger) für die eigentliche Analyse übrig. Für jede der 19 Regionen wurde der Isonymie-Wert nach dem Lasker-Verfahren ermittelt, d.h. das Maß der Ähnlichkeit der Nameninventare, wobei natürlich auch die Häufigkeit der einzelnen Namen berücksichtigt wird. Anschließend wurden in einer Matrix alle Regionen untereinander verglichen. In einer Clusteranalyse wurden dann die Distanzen zwischen allen möglichen Paarungen der 19 Regionen ermittelt und schließlich als Dendrogramm dargestellt (Abb. 4). Gut abgrenzbare Cluster sind an den relativ langen vertikalen Linien erkennbar. Demnach lässt sich die Namenmenge - wenig überraschend - in ein klar abgegrenztes romanisches (A) und germanisches (B) Cluster einteilen. Dabei unterscheidet sich das (ehemals) deutsch- und luxemburgischsprachige Arrondissement Arlon etwas von den beiden übrigen belgischen Arrondissements, was auf die teilweise noch hohen Anteile germanischer Namen im Areler Land zurückzuführen ist (i.e. Schmit, Muller, Thill, Wagner, Schmitz, Klein, Schroeder, Feller, Reuter u.a.).

Deutlicher als möglicherweise angenommen, hebt sich aber auch das deutsche, die Eifel und die Moselgegend umfassende Cluster $\mathrm{C}$ vom luxemburgischen ab. Die höchste Ähnlichkeit mit dem deutschen Nameninventar weisen die nördlichen Kantone Vianden, Clerf und Wiltz (D) auf. Insgesamt reflektiert die Cluster-Analyse tatsächlich die bekannte Dialekteinteilung des Luxemburgischen (vgl. Gilles 1999: 49ff.): Hohe Ähnlichkeit untereinander weisen die Kantone der bevölkerungsreichsten Cluster um die Stadt Luxemburg und im Süden auf (F), was dem Zentrum und dem sog. Minett entspricht. Als eigenes Cluster lässt sich auch der Osten mit den Kantonen Echternach und Grevenmacher ausgrenzen (F), mit typischen Familiennamen wie Osweiler (22), Ries (376), Weydert (191), Wohl (70). Schließlich bildet der Norden mit den Kantonen Vianden, Clerf und Wiltz (D) das wohl am besten ausgrenzbare Cluster der 
Luxemburger Familiennamen. Typische Namen wie Arend (158), Bertemes (160), Clesen (58), Lanners (202), Neser (30), Nosbusch (48), Scheer (109), Spaus (48) sind hier besonders häufig zu finden.

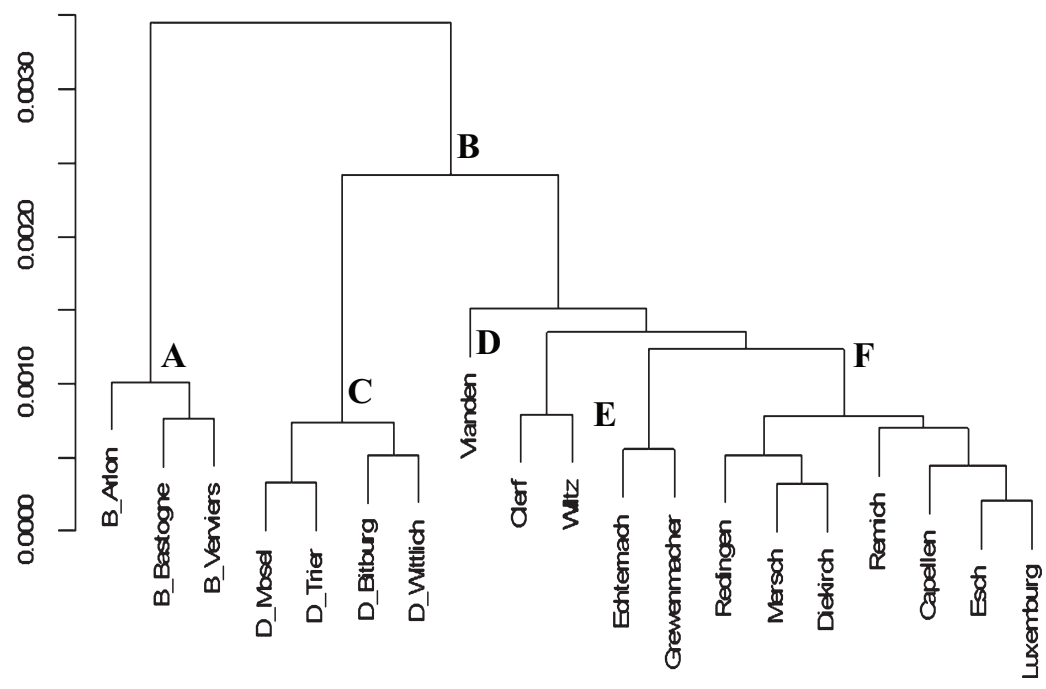

Abb. 4: Dendrogramm einer Clusteranalyse zur Distanzbestimmung zwischen den zwölf luxemburgischen Kantonen und drei angrenzenden Regionen in Belgien und Deutschland mittels Isonymien (zur Beschreibung der Cluster siehe Text)

Der Vorteil der Isonymie-Berechnungen liegt darin, hohe Populationen direkt miteinander vergleichen zu können, was über die bloße Analyse der Ranglisten nicht erreicht werden kann. Nachteile dieser Methode liegen darin, dass auch Varianten von Namen als eigene Namen interpretiert werden, d.h. offensichtliche Varianten wie Muller/Müller, Schroeder/Schröder, Wagener/Wagner oder Gérard/Gerard werden als verschiedene Namen behandelt. Doch könnte durch eine entsprechende Aufbereitung der Daten dieser Nachteil ausgeglichen werden. Weiterhin ist aus der statistischen Berechnung nicht direkt ersichtlich, welche charakteristischen oder häufigen Namen tatsächlich für die jeweiligen Abgrenzungen von Regionen verantwortlich sind. Aber gerade für eine quantitative Untermauerung können solche Isonymie-Berechnungen wertvolle Ergänzungen bei der Bestimmung von Räumen und ihren Grenzen liefern.

\subsection{Verteilung der Namentypen}

Zur Klassifizierung der Familiennamen wird auch im LFA auf die klassische Taxonomie der Benennungsmotive zurückgegriffen (vgl. die kritische Diskussion in Kollmann 2011). Folglich wird differenziert zwischen Familiennamen aus Rufnamen (Martin, Thill, Simon, Clément), nach der Herkunft (Mersch, Metz, Olinger, Erpelding), nach der Wohnstätte (Mousel, Berg, Linden), nach dem Berufsnamen (Sadeler, Kieffer $<$ mhd. küefer, Krier $<$ mhd. krüeger) und aus Übernamen (Klein, Weis, Ries, Wolff). Nicht immer ist das 
Benennungsmotiv eindeutig zu bestimmen; in solchen Fällen von Konkurrenzetymologie werden im Namenbuch alle Alternativen mitangegeben.

Eine Quantifizierung der Namentypen für Luxemburg und angrenzende Länder und Regionen zeigt Abb. 5, die aus Angaben in Flores Flores (2010), Marynissen/Nübling (2010) und Marynissen (in diesem Band) zusammengestellt ist. Das luxemburgische System ist demnach gekennzeichnet durch einen hohen Anteil von Rufnamen (34\%), gefolgt von Berufsnamen (20\%) und Herkunftsnamen (17\%). Übernamen und Wohnstättennamen sind hingegen relativ selten vertreten. Im Vergleich treten nun teils gravierende Unterschiede zutage: Luxemburg zeigt weniger Berufsnamen und Übernamen als Deutschland, während der Anteil an Rufnamen und auch Wohnstättenamen ungefähr identisch ist. Bei den Rufnamen manifestiert sich ein deutlicher Kontrast zur Region Flandern und zur direkt angrenzenden Provinz Luxemburg in Belgien, wo sich hohe bis sehr hohe Anteile für Rufnamen finden. Von allen betrachteten Ländern/Regionen fällt als Charakteristikum für Luxemburg der relativ hohe Anteil an Herkunftsnamen auf. So gehören die letztlich auf Ortsnamen in Luxemburg oder dem grenznahen Umland zurückgehenden Reding (283), Lanners (202) (< Ortsname Lannen), Mersch (193), Erpelding (170), Olinger (163) (< Ortsname Olingen), Biver (164)/Biwer (79), Fischbach (143) oder Berchem (136) zu den häufigen bis sehr häufigen Namen. Insgesamt zeigt sich, dass Luxemburg durch eine eigene Mischung der Namentypen gekennzeichnet ist und sich dadurch von den umgebenden Regionen abgrenzt (vgl. auch Flores Flores in diesem Band).

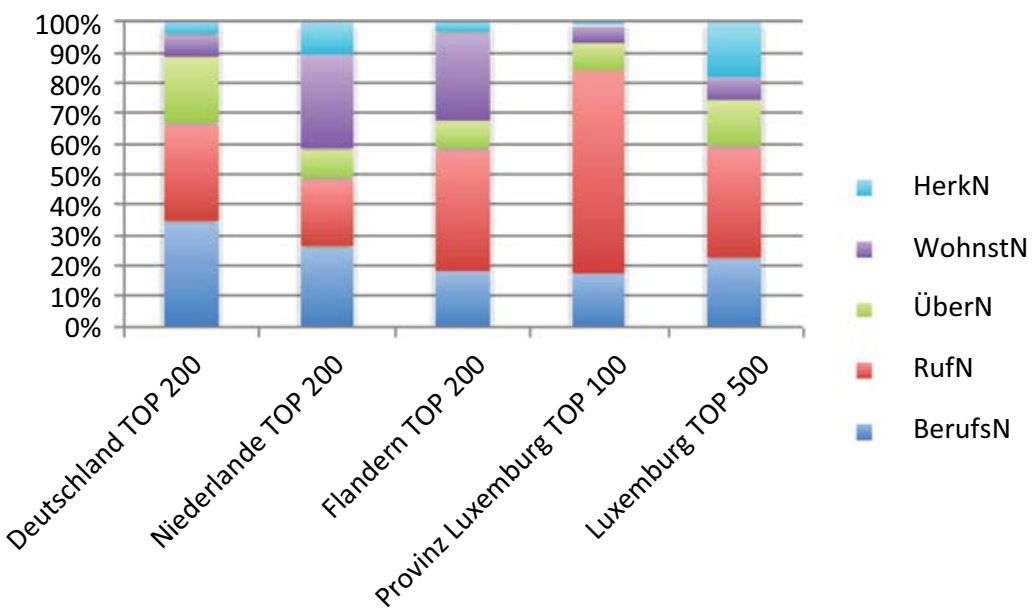

Abb. 5: $\quad$ Einteilung der Familiennamen in fünf Ländern/Regionen gemäß ihrer Benennungsmotive

\subsection{Sprachhistorische Entwicklungslinien}

Die quantitativen Analysen bestätigen die Existenz einer luxemburgischen Familiennamenlandschaft, die bei aller Eigenständigkeit doch immer auch, je nach Phänomen unterschiedlich starke, Gemeinsamkeiten mit dem Umland aufweist. Um dieses erste Ergebnis weiter zu konkretisieren, sollen im Folgenden nun einige sprachhistorische Aspekte 
beleuchtet werden, die in ihrer Gesamtheit die Spezifik des Inventars der luxemburgischen Familiennamen charakterisieren:

1. Kombination bestimmter Familiennamen

2. Spannungsverhältnis zwischen, Verhochdeutschung' und regional-moselfränkischen Lautentwicklungen

3. Präferenz für bestimmte Bildungsweisen

4. Beibehaltung archaischer Schreibweisen

Hinzukommen die vielfältigen Sprachkontaktprozesse infolge von Übernahme und Integration französischer Namen, die in diesem Beitrag jedoch nicht weiter dargestellt werden; vgl. ausführlich dazu C. Muller im Druck und C. Muller in diesem Band. Alle diese Eigenschaften zusammen genommen, bilden dann die Spezifik der luxemburgischen Familiennamenlandschaft.

\subsubsection{Kombination bestimmter Familiennamen}

Auf namen-lexikalischer Basis manifestiert sich die Spezifik des Luxemburger Raumes, indem hier bestimmte Namen auftauchen, die anderswo entweder seltener oder überhaupt nicht vorkommen. Oben bereits wurden die zahlreichen Herkunftsnamen erwähnt, die sich auf Ortsnamen in Luxemburg oder in der Grenzregion beziehen. Dazu gehören weiterhin auch alle auf Luxemburg beschränkte Namen wie z.B. Schaul (113), Stoos (53), Heuertz (59), Jemming (44), Origer (78), Bauler (80) oder Kerger (69). Teilweise ist deren Etymologie nur schwer ermittelbar, teilweise handelt es sich um Herkunftsnamen zu verschollenen und/oder ansatzweise rekonstruierbaren Toponymen. Weiterhin tragen zur spezifisch luxemburgischen Prägung lexikalische Regionalismen bei, die sich aus regionalsprachlichen Appellativa entwickelt haben. Mit Hilfe der Dialektwörterbücher des Moselfränkischen (i.e. Luxemburger Wörterbuch (LWB), Rheinisches Wörterbuch (RhWB)) können die relevanten Bedeutungsaspekte ermittelt werden. Einige Beispiele:

- Geimer (41) zu ,Ingwer' (LWB, RhWB)

- Harles (35) zu ,Hornisse“ (LWB)

- Schmartz (32) zu ,Schramme, Striemen, Narbe‘ (RhWB)

- Tock (28) zu ,Dickkopf, Starrkopf‘ (LWB)

- Spautz (29) zu lb. Spaut,Spucke‘ (LWB)

- Kandel (25) zu lb. Kondel ,Dachrinne‘ (LWB)

- Klensch (26) zu ,Klinke‘ (LWB)

Charakteristisch sind natürlich auch die französischen Familiennamen in Luxemburg, die aufgrund der Jahrhunderte währenden Migration über die germanisch-romanische Sprachgrenze hinweg schon früh in Luxemburg Fuß fassen konnten. Bereits in den Rechnungsbüchern der Stadt Luxemburg (1388-1500) finden sich französische Namen wie Colin, Geoffrey, Gilles, Guillaume, Guy (vgl. Gniffke 2010: 42). Migrationsbedingt kann es dann folglich heute auch zum Nebeneinander von französischem und germanischem 
Äquivalent kommen. In je unterschiedlichen Kombinationen findet sich Becker (496) neben Boulanger (17), Ludwig (117) neben Louis (66), Klein (521) (auch in Komposita/ Ableitungen) neben Petit (64), Schiltz (405) neben Gilles (23) (und Ableitungen) oder Wilhelm (83) neben Guillaume (29). Meist ist dabei das französische Äquivalent seltener als das germanische. Karte 1 (im Anhang) illustriert, wie sich Frantz(en) (120) und François (57) verteilen. Neben der Mischung in Luxemburg ist ebenfalls zu erkennen, dass François auch leicht in der Eifel verbreitet ist und Frantz(en) sich auch in Lothringen und der belgischen Provinz Luxemburg findet.

\subsubsection{Spannungsverhältnis zwischen ,Verhochdeutschung' und regional- moselfränkischen Entwicklungen}

Das luxemburgische Nameninventar ist gekennzeichnet einerseits durch das Vorhandensein von überwiegend standarddeutschen Familiennamen, aber andererseits auch durch regional-moselfränkische Laut- und Graphieentwicklungen. Nach der Entstehungsphase der Familiennamen ab dem 14. Jh., wie sie für Luxemburg etwa in den Rechnungsbüchern der Stadt Luxemburg greifbar wird (vgl. Moulin/Pauly 2007, Gniffke 2010), sollte es noch bis zum Ende des 18. Jh. dauern, bis die Erblichkeit der Familiennamen durch gesetzliche Regelungen fixiert wurde. Erst in dieser Phase der Offizialisierung erlangen Familiennamen auch relative Festigkeit in ihrer schriftlichen Form. Zu dieser Zeit hatte sich im Luxemburger Raum die Diaglossie zwischen der sich flächendeckend etablierenden standarddeutschen Schriftsprache und dem moselfränkischen Dialekt (= emergentes Luxemburgisch) voll etabliert. Aufgrund dieser Entwicklung ist es nicht verwunderlich, dass in dieser Zeit weite Teile der luxemburgischen Familiennamen nach den graphematischen und phonematischen Prinzipien des Standarddeutschen verschriftet wurden. Mit dieser Fixierung der standarddeutschen Schreibung hat sich dann auch nach und nach die Aussprache nach der Schreibung herausgebildet. Es kann gemutmaßt werden, dass durch diese ,Verhochdeutschung' die Herausbildung eines in Schrift und Lautung prononciert eigenständigen luxemburgischen Familiennameninventars gebremst wurde. Erkennbar ist dies heute daran, dass Familiennamen (meist Berufs(über)namen), die von Appellativen abgeleitet wurden, standarddeutsch sind, wohingegen das zugehörige nominale oder verbale Appellativ die luxemburgische Form zeigt.

$\begin{array}{ll}\text { Appellativ } & \text { Familienname } \\ \text { Kach } & \text { Koch }(165) \\ \text { kleng } & \text { Klein }(521) \\ \text { Päiffert } & \text { Peiffer (166), Pfeifer (26) } \\ \text { Schmatt } & \text { Schmit (1515) } \\ \text { Steen } & \text { Stein }(75) \\ \text { Wiewer } & \text { Weber }(1059) \\ \text { Woner } & \text { Wagner }(1004)^{10} \\ \text { Zëmmer, Zammermann } & \text { Zimmer }(172)\end{array}$

10 Ganz selten erscheint Wohner (5), das als regionalsprachliche Entsprechung zu Wagner gelten könnte. 
Dem gegenüber tragen dennoch nicht wenige Namen regionalsprachliche Züge, die kennzeichnend für das Luxemburgische, aber teilweise auch für das weitere Westmitteldeutsche sind. Die daraus entstehende Phänomenverteilung führt zu einem regional gestaffelten Kontinuum, das sich über heutige Ländergrenzen hinweg erstreckt und alte siedlungsgeschichtliche, genealogische und dialektale Verhältnisse aufdeckt. Die im Folgenden vorgestellten regionalsprachlichen Entwicklungen haben jedoch überwiegend in Luxemburg ihre Reflexe in den Familiennamen hinterlassen (vgl. ausführlicher dazu Kollmann in diesem Band).

Im Luxemburgischen ist intervokalisches $g$ weitestgehend ausgefallen (vgl. Won ,Wagen', soen ,sagen', seeёn ,sägen') und dieser Lautwandel wird auch in einigen Namen reflektiert: In diesem Sinne sind also Krier (341) und Plier (31) von Krüger und Pflüger herzuleiten. Häufiger finden sich allerdings standarddeutsch-nähere Formen mit erhaltenem $g$, das entweder als Plosiv [g] oder frikativisch als [z] oder [в] realisiert wird: Jaeger (63), Krieger (14), Krueger (6), Reger (8), Kariger (21), Origer (78), Kugener (95).

Entrundung von gerundeten Palatalvokalen, ein generelles Merkmal des Mittelfränkischen, zeigt folgende Auswahl. Neben diesen existieren auch meist seltenere Parallelformen, in denen die gerundeten Vokale erhalten geblieben sind oder infolge späterer Prozesse verhochdeutscht wurden.

\begin{tabular}{|c|c|c|c|}
\hline Bemtgen (49) & ['be:mcen] & ,Bäumchen“ & neben Baum (99) \\
\hline Kieffer (496) & {$\left[\mathrm{ki}^{\mathrm{k}} \mathrm{fe}^{\mathrm{R}}\right]$} & ,Küfer' & neben Kuffer (62) \\
\hline Krier (341) & {$\left[\mathrm{kRi}: \mathrm{e}^{\mathrm{R}}{ }^{\prime} \mathrm{kR} 3 \mathrm{Ie}^{\mathrm{R}}\right]$} & ,Krüger' & neben Krueger (6) \\
\hline Ney (173), Nei (9) & [naI] & ,neu ${ }^{6}$ & neben Neu (136), Neumann (69) \\
\hline Prim (51) & [prim] & ,Prüm‘ & neben $\operatorname{Pr}(\mathrm{u} \mid \ddot{u}) \mathrm{m}(16)$ [prym] \\
\hline Schlesser (84) & ['flæse ${ }^{\mathrm{R}}$ ] & ,Schlosser ${ }^{6}$ & neben Schloesser (41) \\
\hline
\end{tabular}

Die moselfränkische Senkung von kurzem $i$ zu $a$ (vgl. Finger $>$ Fanger $)$ tritt insgesamt in Familiennamen recht selten zutage:

$\begin{array}{llll}\begin{array}{l}\text { Schlammes }(14) \\ \text { Lampach }(20)\end{array} & \text { neben } & \begin{array}{l}\text { Schli }(m) m(56) \\ \text { neben }\end{array} & \begin{array}{l}\text { zu ,schlimm } \\ \text { (Toponym) }\end{array} \\ \text { Lanners }(202) & & & \text { zu ,Linde }\end{array}$

Auch die Koronalisierung von vormaligem [ç] zu [6, ]] wird nur selten verschriftlicht. Für ein sekundäres Merkmal mit niedrigem Bewusstseinsgrad ist dies nicht weiter verwunderlich. Es finden sich neben häufigem Michel (48), Bichel (27) die selteneren Varianten Mischel (13) und Bischel (6). Eischer existierte noch in der Volkszählung von 1880, ist jedoch heute nur noch als Eicher (66) vorhanden. Auch dies lässt sich als Verhochdeutschung deuten.

Die Assimilation der auslautenden Konsonantenverbindung [Rs] zu [S] ist als ein zentrales Element der Phonologie des Luxemburgischen anzusehen (vgl. Dokter $+s>$ Doktesch ,Doktorin', besonders $>$ besonnesch ,besonders', anders $>$ anesch ,anders'). Sie findet sich recht häufig auch in Familiennamen, wo das $-s$ als vormalige Genitivendung in einer Konstruktion aus genitivischem Beinamen und Rufnamen interpretiert werden kann; vgl. diefferdingers clais (=,Klaus aus Differdingen“) im Rechnungsbuch der Stadt 
Luxemburg von 1464/1465 (Gniffke 2010: 54) oder auch noch heute gebräuchliche Fremdnamennennungen des Typs Contesch Claude (=,Claude Conter $\left.{ }^{6}\right)$. Von der Assimilation betroffen sind also alle Namen, die auf -er $+-s$ enden (häufig Berufsnamen). Wie die folgende Übersicht zeigt, finden sich auch hier häufig(er) Parallelformen mit ausgebliebener Assimilation.

$\begin{array}{lll}\text { Lamesch }(100) & \text { neben } & \text { Lam(m)ers (5) } \\ \text { Meisch }(56)^{11} & \text { neben } & \text { Meyers (357) } \\ \text { Mullesch (5) } & \text { neben } & \text { Mullers (1) } \\ \text { Petesch (42) } & \text { neben } & \text { Peters (210) } \\ \text { Rodesch (58) } & \text { neben } & \text { Roders (1) } \\ \text { Schneidesch (2) } & \text { neben } & \text { Schneiders (22) } \\ \text { Thimmesch (44) } & \text { neben } & \text { T(h)immers (2) }\end{array}$

Gegenüber der Volkszählung von 1880 ist die Assimilation in der Kompositionsfuge rückgängig gemacht und dem Schriftdeutschen angeglichen worden, so dass sich Donneschbach zu heutigem Donnersbach (5) und Weimeschkirch zu Weimerskirch (43) entwickelte.

Nur wenige Familiennamen beachten die sog. $n$-Regel des Luxemburgischen, nach der silbenfinale $-n$ nur dann realisiert werden, wenn das folgende Wort mit $h, d, t, t s, n$ oder Vokal beginnt (vgl. Gilles 2006). Dieses Phänomen betrifft damit nur Komposita, die jedoch bei den luxemburgischen Familiennamen insgesamt relativ selten sind. Folglich spielt auch die $n$-Tilgung hier nur eine untergeordnete Rolle. Beispiele sind u.a. Siebenbour (23), Prommenschenkel (16), Hellenbrand (21), Nurenberg (25), Reifenberg (14), Dillenburg (2). Die $n$-Regel wird beachtet in Dennemeyer (7), Dennewald (11). Variabel sind Steinmetz (153) / Steimetz (8), Brandenbu(o)rg(er) (92) / Brandebo(u)rg(er) (1) oder Leinenweber (1) / Leineweber (6). In der spontanen Aussprache kann der finale Nasal gemäß der luxemburgischen Phonologie hingegen entfallen, z.B. ['gudəka:f] für Gutenkauf, ['diləbu:ə६] für Dillenburg. Allerdings setzt sich auch hier mehr und mehr die Tendenz durch, den Familiennamen (insbesondere bei der Selbstnennung des Namens) auch in der schriftsprachlichen Form, also immer mit [n], auszusprechen (z.B. ['Rokənbro:t] Rokkenbrod (2)). Die Schreibungen der Volkszählung von 1880 zeigen hingegen noch eine stärkere Beachtung der $n$-Regel (z.B. Bohneberger, Dillebourg, Gantebein, Kettemeyer, Decktebrunnen), woraus geschlossen werden kann, dass im Verlauf des 20. Jhs. einige Namen weiter der schriftdeutschen Norm angepasst worden sind.

Im Gegensatz zum Deutschen, wo sich die Aussprache unmittelbar aus der graphischen Form ergibt, existiert im Luxemburgischen für zahlreiche Namen neben der schriftdeutschen Form eine regionalsprachliche Aussprache, die auf der Lautform der luxemburgischen Appellative oder Toponyme basiert, aus denen der Familienname abgeleitet ist. Die Auswahl der schriftdeutschen oder luxemburgischen Aussprache wird durch den pragmatischen Kontext der Namensnennung gesteuert (Nennung des eigenen Namens, Nennung des Namens einer anderen Person, ironische Verwendungen u.a.).

11 Bei der Entstehung dieser Form ist auch eine Krasis mit im Spiel, sodass aus zweisilbigem Meyesch (<Meyer-s) einsilbiges Meisch resultiert. Vgl. auch lb. Määsch, Mäesch (LWB). 


\begin{tabular}{|c|c|c|}
\hline & schriftdeutsch & luxemburgisch \\
\hline Behm (22) & [be:m] & [b3im] \\
\hline Erpelding (170) & ['cəpəldın] & ['iopəlden] \\
\hline Glesener (88) & ['gle:zənə⿱⿱ & 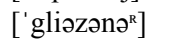 \\
\hline Kremer (493) & 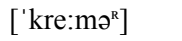 & {$\left[{ }^{\prime} \operatorname{kr} 3 \mathrm{Im}^{\mathrm{R}}{ }^{\mathrm{R}}\right]$} \\
\hline Krier (341) & {$\left[\mathrm{kri}: \partial^{\mathrm{R}}\right]$} & {$\left[{ }^{\prime} \mathrm{kr} 3 \mathrm{I} \partial^{\mathrm{R}}\right]$} \\
\hline Mersch (193) & [meər $\left.\int\right]$ & {$\left[\mathrm{mi} \int\right]$} \\
\hline Nosbusch (48) & ['nosbuf] & ['nosbəf] \\
\hline Schroeder (616) & [' $\left.\mathrm{fr} \varnothing: \mathrm{d} \partial^{\mathrm{R}}\right]$ & [' $\mathrm{r}$ r3Id $\left.{ }^{\mathrm{R}}\right]$ \\
\hline Theves (28) & ['te:vəs] & ['t3ıvəs] \\
\hline Wolff (237) & [volf] & ['voləf] \\
\hline
\end{tabular}

Genauere Studien zu den Verwendungskontexten der beiden Varianten stehen noch aus. Es scheint sich herauszukristallisieren, dass die luxemburgische Variante meist gewählt wird, wenn über eine (oft auch nicht anwesende) Person gesprochen wird. Umgekehrt wird die an der schriftdeutschen Form orientierte Aussprache eher dann verwendet, wenn die Person ihren eigenen Familiennamen ausspricht. Zur Dokumentation der Aussprachen enthält das Namenbuch des LFA phonetische Transkriptionen für diejenigen Namen, die entweder über eine solche luxemburgische Parallelform verfügen oder deren Aussprache aus weiteren Gründen nicht adäquat aus der schriftdeutschen Form abgeleitet werden kann (vgl. Ausschnitt aus dem Namenbuch im Anhang).

Ein weiteres regionalsprachliches Phänomen betrifft die Verteilung von Schwa in unbetonten Silben. Während im Deutschen in von Appellativa abgeleiteten Berufs(über)namen das Schwa eines ursprünglichen Trochäus meist synkopiert wird (vgl. Wagen +er > Wagner; Kessel + er $>$ Keßler), bleiben diese Schwas in den luxemburgischen Familiennamen überwiegend erhalten. Als häufigste phonologische Struktur lässt sich der Wortausgang, Schwa $+l / n+$ Schwa $+r^{6}$ identifizieren. Wie die folgenden Beispiele zeigen, existieren auch hier synkopierte Varianten, die jedoch meist seltener vorkommen.

$\begin{array}{ll}\text { nicht synkopiert } & \text { synkopiert } \\ \text { Beideler (10) } & \text { Beidler (18) } \\ \text { Bosseler (68) } & \text { Bossler (1) } \\ \text { Fideler (1) } & \text { Fiedler (36) } \\ \text { Kesseler (35) } & \text { Kessler (25) } \\ \text { Kugener (95) } & \text { Kugner (1) } \\ \text { Sadeler (16) } & \text { Sadler (51) } \\ \text { Schosseler (41) } & \text { Schoessler (1) } \\ \text { Wagener (305) } & \text { Wagner (1004) } \\ \text { Wiseler (17) } & -\end{array}$

Beispielhaft finden sich auf Karte 2 alle Namen kartiert, die auf - $\beta$ ler/s(s)ler bzw. - $\beta e l e r /-$ es(s)eler enden. Es ist klar zu erkennen, dass in Luxemburg die nicht-synkopierten Formen die eindeutige Mehrheit stellen, während in Deutschland, aber auch in Lothringen und im Elsass die Synkope vorherrscht. Die höchsten Anteile nicht-synkopierter Formen finden sich im nördlichen Rheinland und generell im Niederdeutschen, stellen dort aber meistens die seltenere Variante dar. 
Nicht nur für die Familiennamen ist die Nicht-Durchführung der Synkope charakteristisch, sondern sie ist auch ein wichtiges Merkmal der Phonologie des Appellativwortschatzes (vgl. trëppelen ['trəpələn] ,trippeln', sammmelen ['zamələn] ,sammeln“, Fënsteren ['fənstərən] ,Fenster-P1'). Die Synkoperesistenz wird im Allgemeinem einer stärkeren Silbensprachlichkeit des Luxemburgischen zugeschrieben (vgl. Szczepaniak 2010, Gilles 2013). In der Aussprache hingegen sowohl der Namen als auch der Appellativa kann heute eine Tendenz zur Synkope beobachtet werden (also ['kæslə글 für Kesseler, ['fənstrən] für Fënsteren), deren Systematik zum jetzigen Forschungsstand noch unklar ist.

Das Wirken der Synkope scheint auch die treibende Kraft bei der Entstehung von Marx (<Markus), Lux (< Lucas), Laux ( $<$ diphthongiertem Lucas) gewesen zu sein. In diesen Fällen wurde der Vollvokal der unbetonten Silbe infolge der Nebensilbenabschwächung zunächst zu Schwa reduziert, welches dann in der Folge ganz ausgefallen ist.

\subsubsection{Präferenz für bestimmte Bildungsweisen}

Die Luxemburger Namenlandschaft wird weiterhin durch die Präferenz bestimmter morphologischer Bildungsweisen geprägt, die zwar auch in anderen, meist angrenzenden Region zu finden sind, jedoch in ihrer Kombination typisch und damit konstitutiv für den Luxemburger Raum sind.

Erwähnenswert erscheint hier zunächst, dass Kompositionsbildungen im Vergleich mit den deutschen Familiennamen eher selten sind. Am häufigsten erscheinen Herkunftsnamen auf -weiler (Trierweiler (93), Osweiler (22)) und -scheid (Manderscheid (77), Heiderscheid (70), Wanderscheid (55)), deren Verbreitung sich in den linksrheinischen Gebieten Deutschlands fortsetzt (Karte 3). ${ }^{12}$ In Bezug auf die Berufs- und Standesnamen finden sich unter den TOP 500 lediglich: Hoffmann (1017), Schumacher (310), Steinmetz (153), Kauffmann (114), Feiereisen (74), Neumann (69), Federspiel (69), Weisgerber (69). Die überbordende Kompositionsfreudigkeit, die das Deutsche für alle Benennungsmotive kennt, hat sich im Luxemburgischen, wie übrigens auch in Belgien und in den Niederlanden (vgl. Marynissen/Nübling 2010: 359), nur ansatzweise entwickelt.

Charakteristisch sind weiterhin die häufigen Latinisierungen germanischer Namen, die wahrscheinlich im 16./17. Jahrhundert entstanden sind. Oft existieren die ursprünglichen Namen parallel weiter neben den Latinisierungen. Die Latinisierung kann durch die Anfügung des Nominativ-Suffixes -us, der Genitiv-Suffixe $-y /-i /-i s$ und/oder durch die Übersetzung des ursprünglich germanischen Namens ins Lateinische (Schmit $>$ Faber, Muller > Molitor) erreicht werden.

ursprünglicher Name

Adam (81)

Antoine (15), Anton (16)

Beck (100)
Latinisierung

Adami (4), Adamy (22)

Antoni (2), Antony (127)

Beckius (64)

12 Zur Verbreitung der häufigen Flurnamen auf -scheid im nördlichen Rheinland vgl. Vogelfänger 2010 . 


$\begin{array}{ll}\text { Bissen (63) } & \text { Bisenius (74) } \\ \text { Consthum (3) } & \text { Conzemius (46) } \\ \text { Schmit (1515) } & \text { Faber (454) } \\ \text { Fuchs (36) } & \text { Foxius (5) } \\ \text { Frantz (64) } & \text { Franziskus (6) } \\ \text { Greis (35) } & \text { Grisius (44) } \\ \text { Hubert (87) } & \text { Huberty (218) } \\ \text { Jacob (32) } & \text { Jacobi (1), Jacoby (221) } \\ \text { Lambert (109) } & \text { Lamberty (53) } \\ \text { Meyer (403) } & \text { Majerus (389) } \\ \text { Martin (172) } & \text { Martini (12), Martiny (19) } \\ \text { Michel (48) } & \text { Michaelis (14) } \\ \text { Muller (1097) } & \text { Molitor (291) } \\ \text { Paul (25) } & \text { Paulus (109), Pauly (175) } \\ \text { Peter (38) } & \text { Petri (3), Petry (108) } \\ \text { Steffen (332) } & \text { Stephany (85) } \\ \text { Valentin (11) } & \text { Valentini (17), Valentiny (23) } \\ \text { Wagner (1004) } & \text { Vannerus (3) } \\ \text { Kieffer (496) } & \text { Wietor (26) } \\ \text { Wilhelm (83) } & \text { Wilhelmi (1), Wilhelmy (16) } \\ \text { Wiltz (5) } & \text { Wiltzius (63) }\end{array}$

In Bezug auf die Genitivendung wird die Schreibung mit $<\mathrm{y}>$ derjenigen mit $<\mathrm{i}>$ vorgezogen (vgl. für Belgien auch Marynissen in diesem Band). Karte 4 zeigt die Variantenverteilung der drei im Untersuchungsgebiet häufigsten unflektierten Patronyme Jakob, Hubert, Paul, Winand (orange) und ihre latinisierten Formen auf $-y$ (blau) und $-i$ (rot). Demnach befindet sich das Zentrum der $y$-Genitive klar in Luxemburg, die Formen strahlen aber noch relativ weit in die ehemaligen luxemburgischen Gebiete um Bitburg in Deutschland und in die belgische Provinz Luxemburg hinein. Sie finden sich aber auch noch im Moselgebiet und am Mittelrhein. Genitive auf $-i$ sind in Luxemburg keine anzutreffen, in Konkurrenz zu $-y$ begegnen sie überwiegend im Westmitteldeutschen und Rhein-Maasländischen.

Mit dem Westmitteldeutschen teilt das Luxemburgische das frequente Vorkommen von diminuierten Familiennamen. In diesen Bildungen erscheint das standarddeutsche Suffix - chen fast ausschließlich in der mittelfränkischen Schreibung -gen, das jedoch fast immer als [6ən] ausgesprochen wird. ${ }^{13}$ Das Diminutivsuffix wird an einsilbige Stämme angehangen und erscheint regulär als -gen, wenn der Stamm auf alveolaren Plosiv endet.

$\begin{array}{lll}\text { Ausgangsform } & \text { Diminutiv } & \text { flektierter Diminutiv } \\ \text { Bund } & - & \text { Boentges (24) } \\ \text { Felt (< Valentin) } & \text { Feltgen (69) } & \text { Feltges } \\ \text { Frieden (63) } & \text { Friedgen (13) } & \\ \text { Hild (13) } & \text { Hildgen (19) } & \\ \text { hold } & \text { Hoeltgen (19) } & \\ \text { Hund } & \text { Hintgen (14) } & \end{array}$

13 Der einzige Name auf $<$ chen $>$ ist Hippertchen (15). 


$\begin{array}{lll}\text { Kiepe } & \text { Kipgen (20) } & \\ \text { Lude } & \text { Lutgen (102) } & \\ \text { Mathias (50) } & \text { Mathgen (17) } & \\ \text { Peter (38) } & - & \text { Petges (4) } \\ \text { Schild/Schuld } & \text { Schildgen (5) } & \text { Schiltges (18) } \\ \text { Wirth (71) } & \text { Wirtgen (22) } & \end{array}$

Aus koartikulatorischen Gründen und/oder aus Analogie zu den obigen Formen hat sich bei den übrigen Stammausgängen im Diminutiv ein epenthetisches $t$ herausgebildet, das heute ein fester Bestandteil des Familiennamens geworden ist. Dieser Plosiv entstand im Zuge der Vorverlagerung des ursprünglichen Frikativs [ç] zu prä-palatalem [6] (,Koronalisierung'). Damit wurde der Frikativ des Diminutivsuffixes nahezu homorgan mit den alveolaren Stammauslauten von z.B. Will oder Jenn, wodurch es zur Herausbildung des epenthetischen $t$ 's zwischen Stamm und Suffix kommen konnte.

$\begin{array}{lll}\begin{array}{l}\text { Ausgangsform } \\ \text { Baum (99) }\end{array} & \begin{array}{l}\text { Diminutiv } \\ \text { Bemtgen (49) }\end{array} & \text { Alektierter Diminutiv } \\ \text { Dell(22) } & \text { Deltgen (26) } & \\ \text { Henn (7) } & \text { Hentgen (10) } & \text { Hentges (56) } \\ \text { Jenn (8) } & \text { Jentgen (35) } & \text { Jentges (16) } \\ \text { Koeune (40) } & - & \text { Koentges (1) } \\ \text { Mill(en) (12) } & \text { Miltgen (18) } & \\ \text { Ottilie } & \text { Thilgen (27) } & \text { Thil(1)ges (57) } \\ \text { Schinn } & \text { Schintgen (125) } & \\ \text { Schor } & \text { Schortgen (31) } & \\ \text { Thill (791) } & \text { Thiltgen (14) } & \text { Thiltges (19) } \\ \text { Will (6) } & \text { Wil(d|t)gen (99) } & \end{array}$

Im Falle von Bemtgen scheint hingegen das epenthetische $t$ die Folge einer analogischen Angleichung an die übrigen Namen dieser Gruppe zu sein.

Wie die rechte Spalte in den beiden letzten Übersichten zeigt, sind weitere Familiennamen infolge der Flexion des Diminutivs entstanden. Durch das Anhängen der starken Genitivendung $-s$ an einen diminuierten Namen wurde jedoch nahezu durchgängig der Nasal der Diminutivendung getilgt (vgl. Henn $>$ Hentgen $>*$ Hentgens $>$ Hentges). Die Zwischenstufe mit erhaltenem Nasal hat sich heute nur bei sehr wenigen Familiennamen erhalten (Schiffgens (2), Hensgens (2), Luettgens (1)).

Ebenfalls mit weiten Teilen des Westmitteldeutschen teilt das Luxemburgische das häufige Vorkommen der flektierten Patronyme, zu deren häufigsten Meyers, Peters, Michels und Jacobs gehören. Die kombinierte Kartierung einiger flektierter Patronyme in Karte 5 zeigt die hohen Frequenzen des Phänomens in Luxemburg, im Ruhrgebiet, im Rheinland und auch in Flandern.

Eine ähnliche Verteilung ergibt die Kartierung der flektierten und unflektierten Formen des hochfrequenten Berufsnamens für den Schmied auf Karte 6, die als grenzüberschreitende Ergänzung zu entsprechenden Karten im DFA (Band 1, K. 149, 150) und bei Steffens (2013: 118) verstanden werden kann. Erkennbar ist, wie die flektierten Schmitz-Formen $(<$ Schmit-s) vom Ruhrgebiet und dem Rheinland kommend sich in hoher Häufigkeit auch 
noch in Luxemburg finden, dort aber etwas seltener als unflektiertes Schmit vertreten sind. Als Smets, Smeets, Smits aber auch als Schmitz findet sich der Name etwas weniger, aber weite Flächen abdeckend in Flandern und Wallonien. Im Saarland, aber auch in Frankreich schließlich ist die flektierte Form praktisch nicht mehr vertreten.

Darüber hinaus können häufig Kurzformen von Patronymen beobachtet werden, die meist infolge von Reduktion und anschließendem Ausfall prä- oder posttonischer Silben entstanden sind. So konnte z.B. das Patronym Matthäus nach Ausfall der ersten Silbe (und Frikativierung des Gleitlautes) Theves.

\begin{tabular}{|c|c|c|}
\hline Ausgangsname & Kurzform & flektierte Kurzform \\
\hline Adam (81) & Dahm (122) & Dahmen (1) \\
\hline Agnes (35) & - & Noesen (66), Neser (30) \\
\hline Antonius & Thinnes (92) & \\
\hline Balthasar (27) & Baltes (22) & \\
\hline \multicolumn{3}{|l|}{ Bartholomäus } \\
\hline Barth (18), & Barthel (124) & \\
\hline Blasius (11) & Blees (4) & Blasen (43) \\
\hline Christoffel (2) & Stoffel (109) & \\
\hline Cornelius (18) & Nilles (220) & \\
\hline Elisabeth & Lies (33) & Elsen (121) \\
\hline Friederich (48) & - & Frieden (63) \\
\hline Jacob (32) & Koob (46) & \\
\hline Johannes (1) & Hans, (9) Jans (61), Jenn (8) & Hansen (370) \\
\hline Katharina & - & Treinen (26), Ketter (25) \\
\hline Kilian (1) & Kill (56) & \\
\hline Lorenz (19) & Len(t)z (255) & \\
\hline Marguerite & Margue (22) & Grethen (148) \\
\hline Martin (172) & Marth (29), Thein (99) & \\
\hline Matthäus & Mathay (34) & \\
\hline Matthäus & Theves (28), Thewes (44) & \\
\hline Mathias (50) & Mathes (29) & \\
\hline Mathias (50) & Theis (272) & Theisen (268) \\
\hline Mathias (50) & Thies (49) & \\
\hline Nicolas (52) & Colas (19) & \\
\hline Nikolaus & Clees (103), Cloos (93) & \\
\hline Ottilie & Thilgen (27), & Thilges (49) \\
\hline Robert (57) & Roob (27) & \\
\hline Theresa & Troes (25) & \\
\hline Wilhelm (83) & - & Wiltgen (76) \\
\hline
\end{tabular}

Die Kurzformen ihrerseits können wiederum, wie schon bei den Diminutiven gezeigt, flektiert werden (überwiegend mit schwachem -en). In Bezug auf die Verbreitung dieser Bildungsweise ordnet sich Luxemburg in belgische und westdeutsche Verhältnisse ein (vgl. auch Marynissen/Nübling 2010).

\subsubsection{Beibehaltung archaischer Schreibweisen}

In Bezug auf die Schreibung luxemburgischer Familiennamen sind nicht wenige Unterschiede zu den deutschen Namen festzustellen. Auf ältere, archaische Schreiweisen 
deuten die vielfach erhaltenen Doppelschreibungen von Konsonanten. Dazu gehören v.a. $t z$ - und $c k$-Schreibungen nach Nasal (Heintz (102), Lentz (236), Wantz (73), Frantz (64), Bintz (49), Dentzer (32), Krantz (30); Franck (109), Funck (65), Schanck (60), Juncker (35), Ronck (34), Linckels (33)). Karte 7 zeigt, dass die -ntz-Schreibungen als nahezu exklusiv luxemburgisch angesehen werden können. Ähnlich verhält es sich mit auslautenden -ff-Schreibungen (Wolff $(237)^{14}, \operatorname{Alff}(25)$, Wintersdorff (15)). Auch bei den Zusammensetzungen mit ,-dorf' dominieren in Luxemburg ebenfalls die Doppelschreibungen (Karte 8). Es ist gerade dieses orthographische Detail, das sich interessanterweise nur in Luxemburg findet. ${ }^{15}$

\section{Resümee}

Es war das Ziel dieses Aufsatzes, zentrale Eigenschaften der luxemburgischen Familiennamenlandschaft vorzustellen. Die spezifische Randlage und Größe, der jahrhundertelange Sprachkontakt mit romanischen Varietäten, das spezifische Spannungsverhältnis zwischen regionalsprachlichen und hochdeutschen bzw. standarddeutschen Eigenschaften und nicht zuletzt die Eigenstaatlichkeit haben dazu beigetragen, dass von einer charakteristisch luxemburgischen Familiennamenlandschaft gesprochen werden kann. Im Rahmen des Projektes ,Luxemburgischer Familiennamenatlas“ werden diese Ergebnisse in einem Namenbuch und einem Atlas (in Druckform und online) zur Verfügung gestellt.

In der statistischen Clusteranalyse konnten spezifische Grenzlinien bzw. Zusammengehörigkeiten in Luxemburg und seinen angrenzenden Regionen in Belgien, Deutschland und Frankreich ermittelt werden. In Bezug auf sprachhistorische Entwicklungslinien konnte gezeigt werden, dass sich erstens (wie in anderen Regionen auch) aus genealogischen und migrationellen Gründen spezifische Kombinationen von Familiennamen herausgebildet haben. Zweitens koexistieren hochdeutsch-standarddeutsche Namen, die sich auch im umgebenden Westmitteldeutschen finden, neben regionalsprachlichmoselfränkischen in einem spezifischen Mischungs- und Spannungsverhältnis (vgl. Baum neben Bemtgen). Drittens deutet sich eine Präferenz für bestimmte morphologische Bildungsweisen an (Latinisierungen, Diminutive, Kurzformen von Patronymen), die Luxemburg mit dem angrenzenden Westmitteldeutschen teilt. Viertens schließlich existieren bestimmte graphematische Merkmale (z.B. häufige Doppel-f-Schreibungen), die auf ältere Verschriftlichungstraditionen hinweisen.

In Bezug auf die Kartierung ist deutlich geworden, dass durch eine ausschließliche Fokussierung auf Luxemburg die geographisch-namenkundliche und sprachhistorische Einordnung nicht möglich ist. Innerluxemburgisch lässt sich am ehesten noch ein Kontrast zwischen Nordluxemburg (Ösling) und Zentralluxemburg (Gutland) beobachten, der auch durch entsprechende Dialektunterschiede im Appellativwortschatz gestützt wird. Die weitere Kontextualisierung im Gebiet zwischen Maas und Rhein hingegen

14 Zur Varianz $f / f f$ in Namen mit Wolf siehe DFA 2, S. 168-181.

15 Für weitere graphematische Besonderheiten vgl. Kollmann (in diesem Band). 
belegt die Relevanz von größeren europäischen Arealen für die Verbreitung von Familiennamen, Familiennamentypen und die mit ihnen verbundenen sprachhistorischen Prozessen. Dabei ergibt sich eine Bindung entweder an den westmitteldeutschen oder den wallonisch-nordfranzösischen Raum, wobei sich erstere als die wichtigere erweist, weil die überwiegende Mehrheit der luxemburgischen Namen germanischen Ursprungs ist.

Durch die konsequente Anwendung der grenzüberschreitenden Perspektive erhält die Namenforschung eine vielversprechende europäische Dimension. Da es offensichtlich ist, dass die Verteilung von Familiennamentypen sich nur teilweise an einzelsprachlichen oder nationalen Grenzen orientiert (vgl. Marynissen/Nübling 2010: 331), kann diese neue europäische Perspektive zu einem erweiterten Verständnis zur Entstehung, Ausbreitung und linguistischer Struktur der Familiennamenlandschaft beitragen.

\section{Literatur}

Cheshire, James / Mateos, Pablo / Longley, Paul A.: Family names as indicators of Britain's changing regional geography. CASA Working Paper 149. 2009. URL: www.casa.ucl. ac.uk/publications/ workingpapers.asp.

Cheshire, James / Mateos, Pablo / Longley, Paul A.: Delineating Europe's Cultural Regions: Population Structure and Surname Clustering. In: Human Biology. 2011 83(5), 573-598.

DFA $=$ Deutscher Familiennamenatlas. Herausgegeben von Konrad Kunze und Damaris Nübling. Band

1. Grafematik/Phonologie der Familiennamen. I: Vokalismus von Christian Bochenek und Kathrin Dräger. Berlin/New York 2009.

Flores Flores, Walter Amaru: Zu einer Typologie der luxemburgischen Familiennamen. Das System der luxemburgischen Familiennamen im Zentrum Europas. Magisterarbeit, Universität Mainz. Mainz 2010.

Gilles, Peter (im Druck): Phonological domains in Luxembourgish and their relevance for the phonological system. In: Caro Reina, Javier / Szczepaniak, Renata (eds.): Phonological Typology of Syllable and Word Languages in Theory and Practice. Berlin/New York: de Gruyter.

Gniffke, Andreas: Die Personennamen der Stadt Luxemburg von 1388-1500. Namenbuch und namenkundliche Analyse auf Basis der Rechnungsbücher der Stadt Luxemburg. Unveröffentlichte Dissertationsschrift, eingereicht an der Universität Luxemburg 2010.

Herbillon, Jules / Germain, Jean: Dictionnaire des Noms de Famille en Belgique romane et dans les Régions limitrophes. Bruxelles: Crédit Communal 1996.

Kollmann, Cristian: Methodische Fragen und Überlegungen zur Typologisierung der Familiennamen am Beispiel des Luxemburgischen Familiennamenatlasses. In: Ziegler, Arne / WindbergerHeidenkummer, Erika (Hrsg.): Methoden der Namenforschung. Methodologie, Methodik und Praxis. Berlin 2011, 47-62.

Muller, Claire (im Druck): Les noms de famille français du Luxembourg. In: Nouvelle Revue d'Ononmastique.

LWB = Luxemburger Wörterbuch. Herausgegeben von der Wörterbuchkommission. 5 Bände. Luxemburg 1950-1975. Ergänzungsband 1977. URL: infolux.uni.lu/worterbucher/.

Marynissen, Ann: Limburgse familienamengeografie. In: Naamkunde 26. 1994, 243.-301.

Marynissen, Ann / Nübling, Damaris: Familiennamen in Flandern, den Niederlanden und Deutschland - ein diachroner und synchroner Vergleich. In: Dammel, Antje / Kürschner, Sebastian / Nübling, Damaris (Hrsg.): Kontrastive Germanistische Linguistik, Teilband 1. Hildesheim (Germanistische Linguistik; 206-209). 2010, 311-362.

Klees, Henri: Geographie der Luxemburger Familiennamen (nach der Volkszählung von 1930). Herausgegeben vom Institut Grand-ducal, Section de Linguistique, de Folklore et de Toponymie. Luxemburg 1989 (= Beiträge zur luxemburgischen Sprach- und Volkskunde Nr. 18). 
Lasker, Gabriel Ward: Surnames and Genetic Structure. Cambridge: Cambridge University Press 1985.

Liu, Yan / Chen, Liujun / Yuan, Yida and Jiawei Chen: A study of surnames in china through isonymy. In: American Journal of Physical Anthropology. 2012, 148(3), 341-350.

Moulin, Claudine / Pauly, Michel (Hrsg.): Die Rechnungsbücher der Stadt Luxemburg. Erstes Heft 1388-1399 2007, Zweites Heft 1400-1430 2008, Drittes Heft 1444-1453 2009, Viertes Heft 14531460 2010, Fünftes Heft 1460-1466 2010. Luxemburg 2007-2010 (= Schriftenreihe des Stadtarchivs Luxemburg Bd. 1-5).

Müller, Nik.: Die Familien-Namen des Grossherzogthums Luxemburg. Luxemburg 1887.

Oster, Edouard: Nos noms de familles au 17e siècle. In: Biographie nationale du pays de Luxembourg 1965, Faszikel 13, 17-121.

RhWB $=$ Rheinisches Wörterbuch. Herausgegeben von Josef Müller, Heinrich Dittmaier, Rudolf Schützeichel und Mattias Zender. 9 Bände. Bonn, Berlin 1928-1971. URL: www.woerterbuchnetz.de.

Szczepaniak, Renata: Phonologisch-typologischer Wandel des Deutschen und des Luxemburgischen im Kontrast. In:Dammel, Antje / Kürschner, Sebastian / Nübling, Damaris (Hrsg.): Kontrastive Germanistische Linguistik, Teilband 1. Hildesheim (Germanistische Linguistik; 206-209). 2009, 85-110.

Steffens, Rudolf: Familiennamenatlas Rheinland-Pfalz, Hessen, Saarland. Ubstadt-Weiher u.a.: verlag regionalkultur 2012.

Vogelfänger, Tobias: Nordrheinische Flurnamen und digitale Sprachgeographie: sprachliche Vielfalt in räumlicher Verbreitung. Köln/Weimar: Böhlau 2010. 


\section{Anhang}

\section{A. Auszug auf dem Namenbuch}

Agnes ['agnæs] (Rang: 721, 2007: 35, 1880: 42). Gleiche Basis Neser, Nestgen, Noesges. Etymologie Metronym zum gleichlautenden Rufnamen. $\mathrm{Zu}$ Grunde liegt griech. hagnós ,keusch, rein; hehr, geheiligt', das im Vulgärlateinischen mit lat. agnus ,Lamm‘ in Verbindung gebracht wurde. Verbreitung Agnes findet sich überwiegend in Luxemburg, ebenso die flektierten Kurzformen Nesen (tendenziell in der nördlichen Hälfte) und Noesen (der Nordwesten ausgenommen). Als derivierte Kurzformen erscheinen Neser im Norden Luxemburgs und lose verstreut in der südlichen Hälfte Deutschlands, ferner Nestgen ausschließlich im Süden Luxemburgs. Als entsprechende flektierte Bildungen gelten Noesges im Südwesten Luxemburgs und bei Diekirch sowie in der Eifel, ferner Nösges und Nesges beide überwiegend in der Eifel. Historische Belege Fsv: 1561 Nessen. 1611 Agnessen, Nestges, Noesers. GDB: 1604 Noesen. 1660 Noesers $=>1691$ Noesen. 1668 Noeser. 1670 Agnès. 1682 Nesen $=>1710$ Neesen $=>1713$ Noesen. 1694 Noeser $=>1737$ Noesers. 1735 Neser. 1796 Noesgen. 1882 Noesges. 1890 Noes. MACKel (Rang: 570, 2007: 43, 1880: 38). Etymologie 1. Jüngere Form von $\uparrow$ Maquil. 2. Kurzform von $\uparrow$ Remakel. Verbreitung Mackel erscheint besonders in der südlichen Hälfte Luxemburgs und bildet auch einige Nester in Deutschland, doch nicht in Grenznähe zu Luxemburg. In Deutschland ist mit weiteren Konkurrenzetymologien zu rechnen (z.B. Kurzform zu Markwardt, Markhardt).

NeSEN ['ne:zən] (Rang: 1795, 2007: 14, 1880: 23). $\uparrow$ Noesen.
Neser (Rang: 846, 2007: 30, 1880: 27). Etymologie Deriviertes Metronym zu Nes. Bei diesem Familiennamen, der heute in Luxemburg nicht mehr vorkommt, handelt es sich um eine regionalsprachliche Kurzform von $\uparrow$ Agnes.

Nestgen ['næs't6on] (Rang: 4682, 2007: 5, 1880: 6). Etymologie Metronym zum gleichlautenden Rufnamen oder deriviertes Metronym zu Nes. Bei diesem Familiennamen, der heute in Luxemburg nicht mehr vorkommt, handelt es sich um eine regionalsprachliche Kurzform von $\uparrow$ Agnes. NoEsen ['nø:zən // 'neIzən] (Rang: 327, 2007: 66, 1880: 72). Variante(n) Nesen. Etymologie Flektiertes Metronym zum gleichlautenden Rufnamen. Dieser erscheint als lb. Néis (auch in Hausnamen Néisen) und ist Kurzform von $\uparrow$ Agnes. Die Schreibung mit oe des Familiennamens ist hyperkorrekt und entstand in Anlehnung an Familiennamen wie Schroeder $=1 \mathrm{~b}$. Schréider.

NoesGes ['nø:sjəs] (Rang: 10275, 2007: 3, 1880: 0). Etymologie Flektiertes Metronym zu $\uparrow$ Noesgen. Dieser Name ist Metronym zum gleichlautenden Rufnamen oder deriviertes Metronym zu $\uparrow$ Noes. Die Grundlage ist in beiden Fällen eine regionalsprachliche Kurzform von $\uparrow$ Agnes.

REMACKel (Rang: 2232, 2007: 11, 1880: 11). $\uparrow$ Remakel.

REMACLE ['Rəma:kəl] (Rang: 3737, 2007: 7, 1880: 47). Etymologie Französisches Patronym zum gleichlautenden Rufnamen. Dieser stammt aus Remac(u)lus. $\uparrow$ Remakel.

REMAKEL ['Rəma:kəl] (Rang: 1151, 2007: 22, 1880: 30). Variante(n) Remackel. 
Gleiche Basis Mackel, Remacle. Etymologie Patronym zum gleichlautenden Rufnamen. Dieser stammt aus Remac(u) $l u s$, das als Latinisierung von germ. $R i$ magil angesehen wird. Das erste Element Rim- ist nicht sicher zuordenbar, allenfalls zu an. hrìmr ,Reif* oder ahd. hrīm ,Reim‘, ggf. auch got. rimis ,Ruhe‘. Das zweite Namenelement gehört am ehesten zu germ. *agil- als Weiterbildung von *agja-, Ecke'. Verbreitung Remakel sowie die seltenere Variante Remackel kommen ausschließlich in Luxemburg vor. Die französische Form Remacle ist in Luxemburg niederfrequent, häufig jedoch in Wallonien. Zur Verbreitung der potenziellen
Variante Mackel, siehe dort. Weitere Diskussion Zur regionalen Popularität des Rufnames hat sicher auch der Heilige Remaclus, Bischof im 7. Jh. und Gründer der Klöster Stablo und Malmedy, beigetragen. Im Französischen erscheint der Familienname als Remacle. Die wallonischen Aussprachen lauten Rumâke (Bastnach) und Rumâke (La Gleize). Zu historischen Belegen in Wallonien und weiterführender Literatur, vgl. Germain-Herbillon, S. 855. Historische Belege Fsv: 1561 Maculus. 1611 Mackels, Maclus, Remacle. 1656 Macles. GDB: 1772 Remacle $=>1802$ Remackel. 1811 Maquel $\Rightarrow 1843$ Mackel. 1852 Remackel $=>1890$ Remakel . 


\section{B. Karten und Abbildungen}

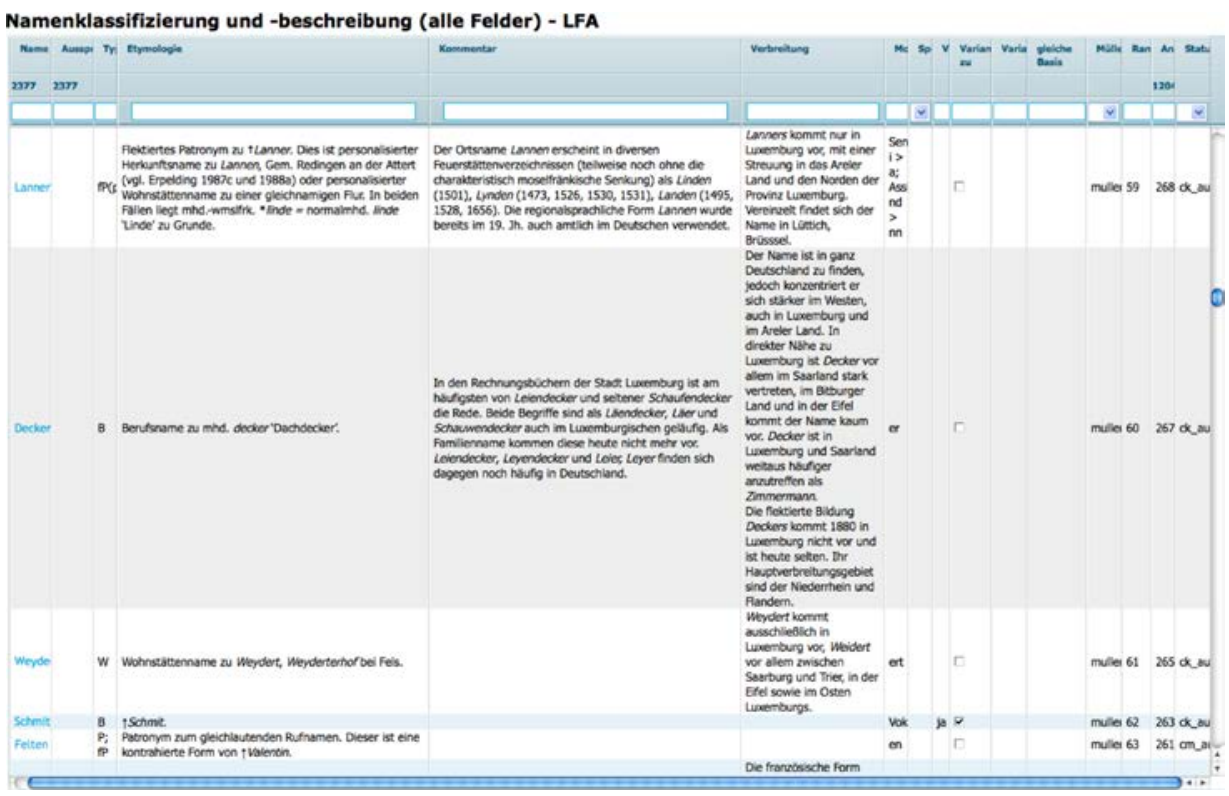

Abb. 2: $\quad$ Zentrale Bedienungsoberfläche der Datenbank des Luxemburgischen Familiennamenatlasses

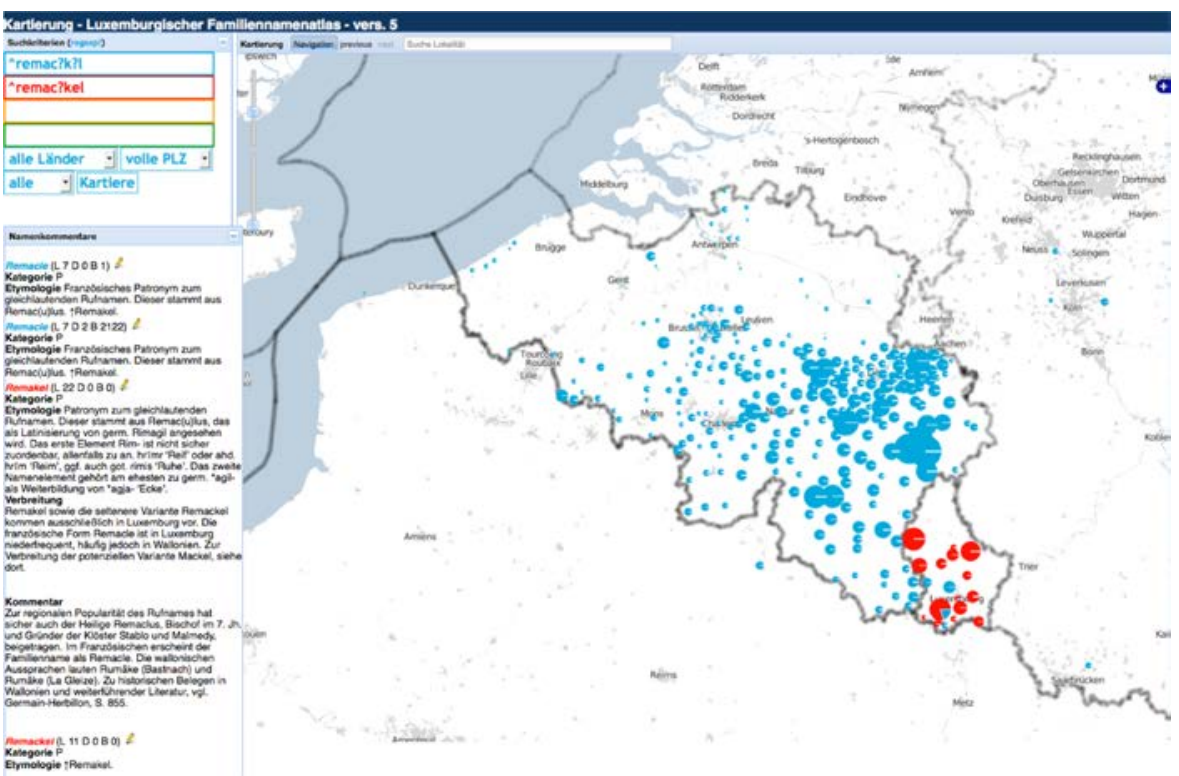

Abb. 3: Bedienungsoberfläche der Kartierungssoftware des Luxemburgischen Familiennamenatlasses 


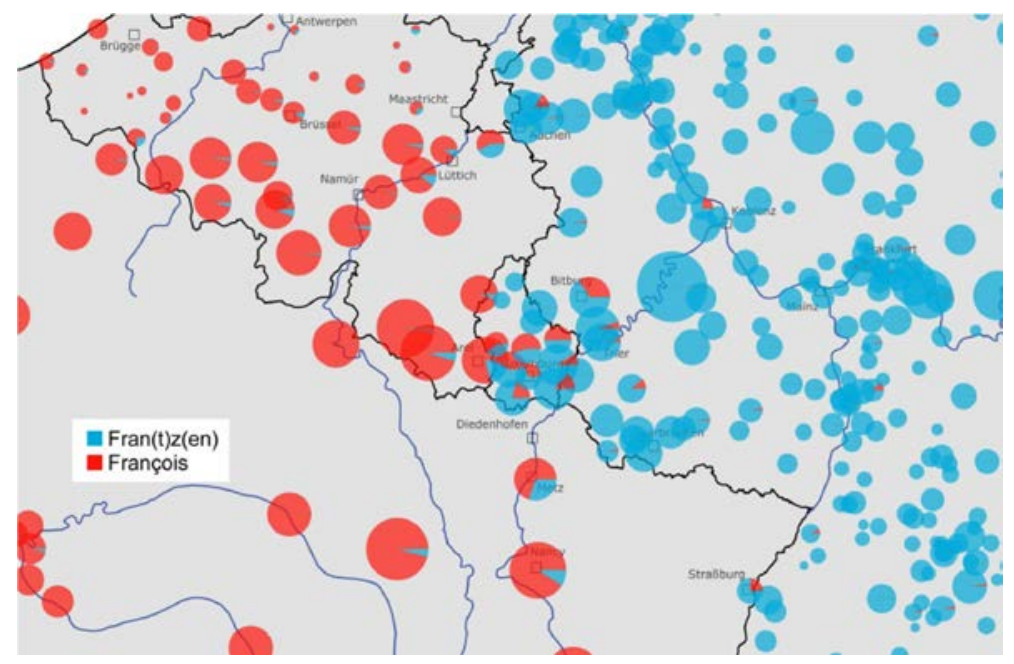

Karte 1: $\quad$ Regionale Verteilung von germanischem Fran(t)z(en) (blau) und romanischem François (rot)

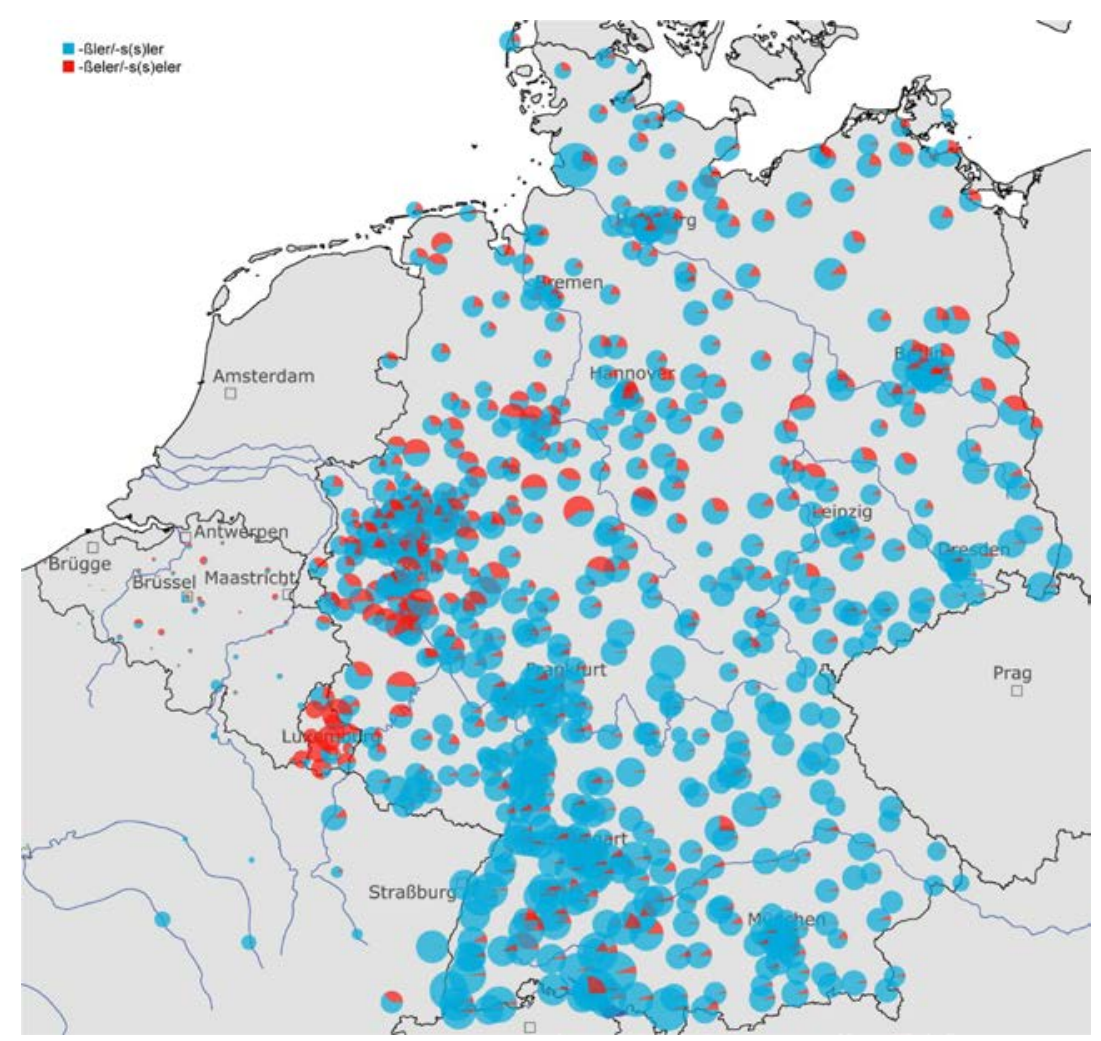

Karte 2: $\quad$ Kombinationskarte zur Verteilung der Namenausgänge -ßler/s(s)ler (= synkopiert; blau) im Vergleich mit -ßeler/-s(s)eler (= nicht synkopiert, rot) 


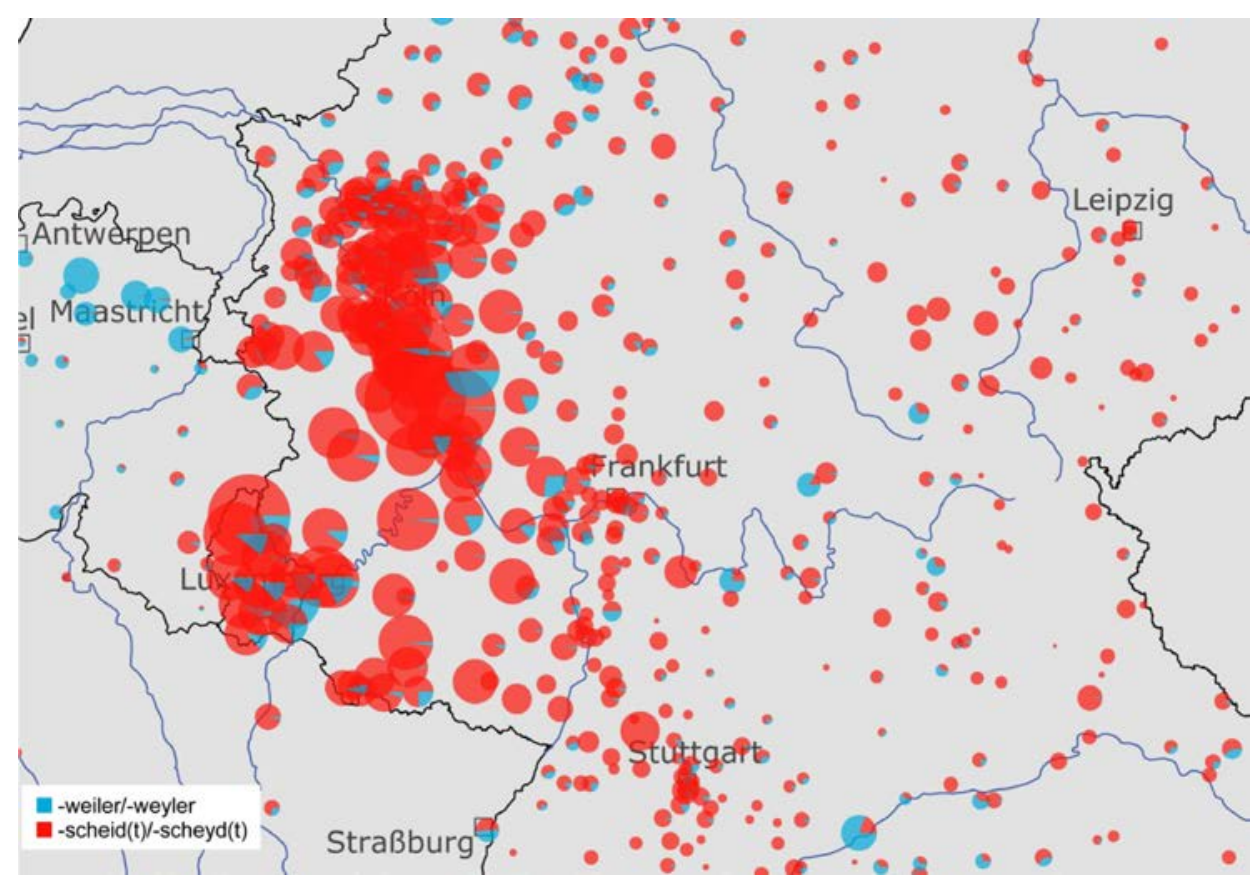

Karte 3: Verteilung der Herkunftsnamen auf-weiler/-weyler (blau) und -scheid(t)/-scheyd(t) (rot)

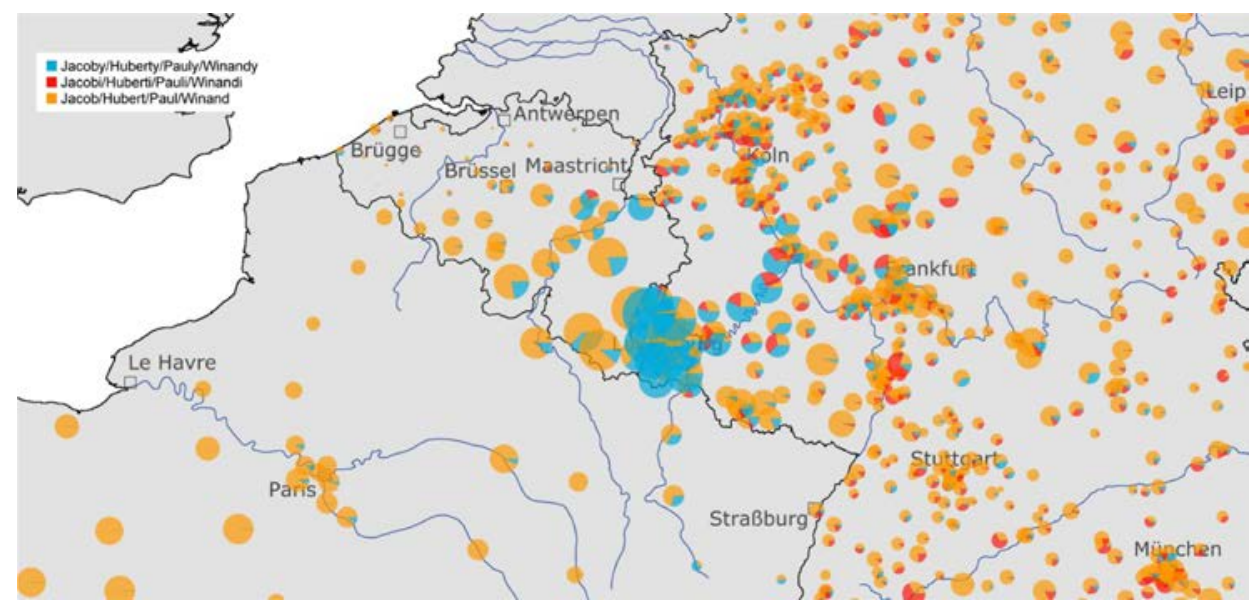

Karte 4: Kombinationskarte der Latinisierungen auf -y (blau: Jacoby, Huberty, Pauly, Winandy), auf -i (rot: Jacobi, Huberti, Pauli, Winandi) neben den Grundformen (orange: Jacob, Hubert, Paul, Winand) 


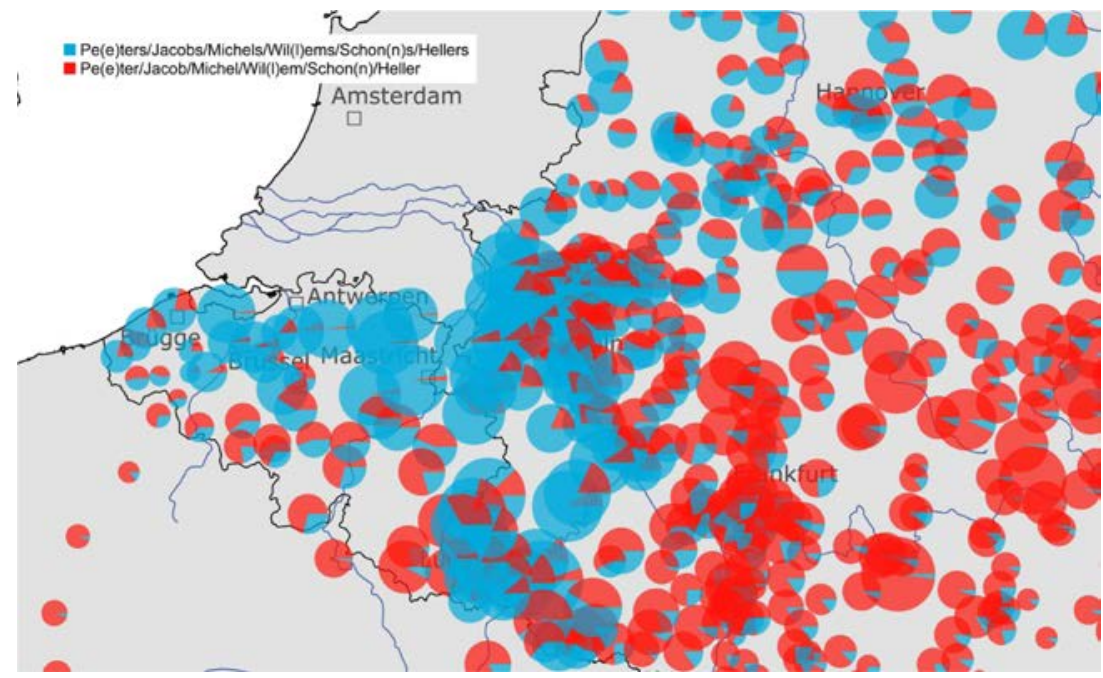

Karte 5: Vorkommen von flektierten Patronymen (blau; Peters, Jacobs, Michels, Willems, Schons, Hellers) im Vergleich zu den unflektierten Formen (rot; Peter, Jacob, Michel, Willem, Schon, Heller)

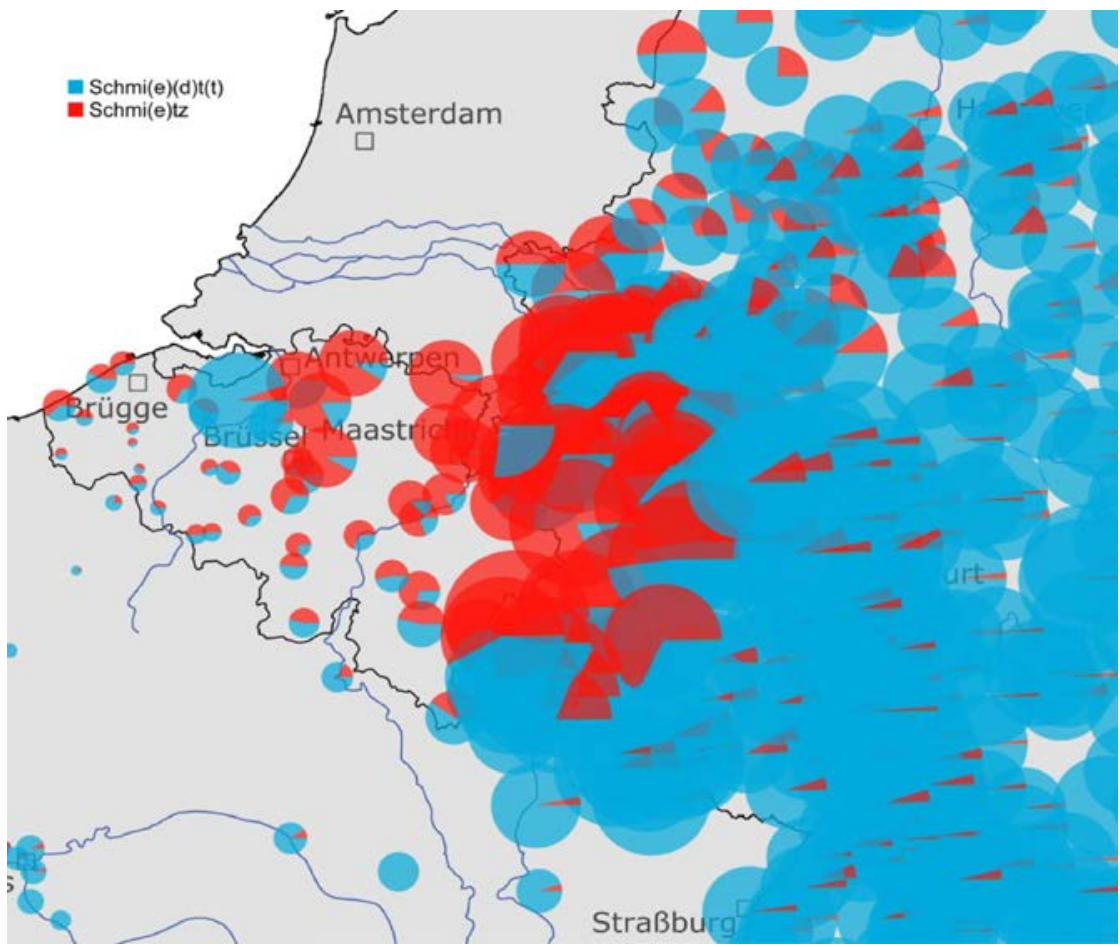

Karte 6: $\quad$ Kombinationskarte für den flektierten Berufsnamen Schmitz, Schmietz, Sme(e)ts, Smits u.a. (blau) im Kontrast zu nicht-flektiertem Schmit(t), Schmidt, Sme(e)t, Smit u.a. 


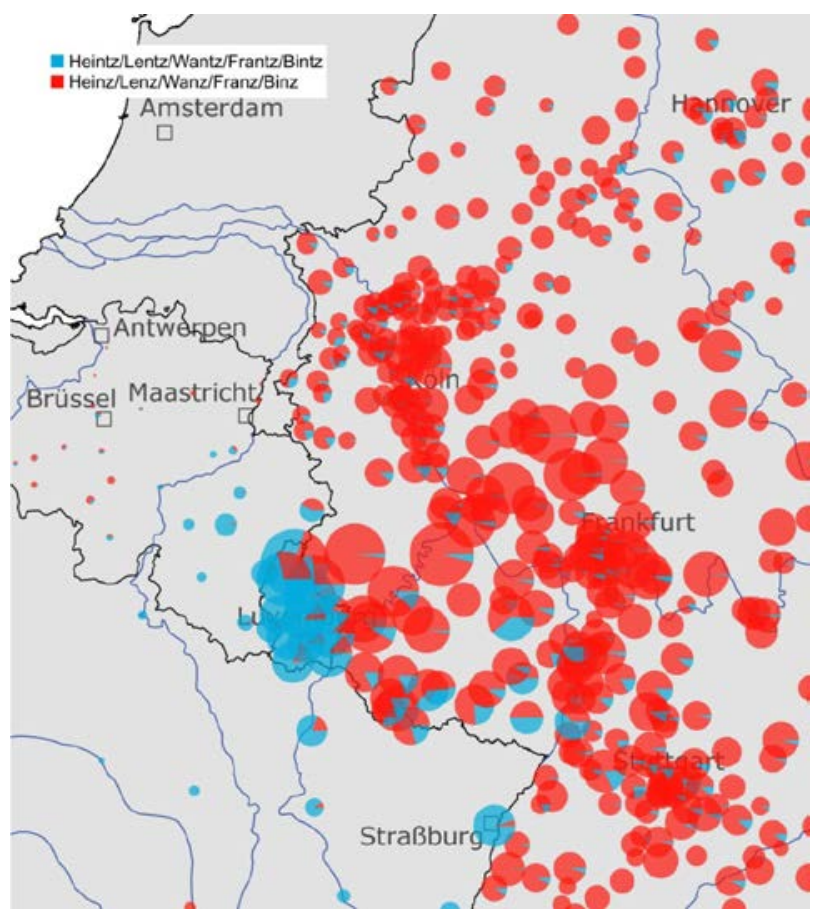

Karte 7: Kombinationskarte von -ntz-Schreibungen in Heintz, Lentz, Wantz, Frantz, Bintz u.a. (blau) im Kontrast zu -nz-Schreibungen (Heinz, Lenz, Wanz, Franz, Binz u.a.) (rot)

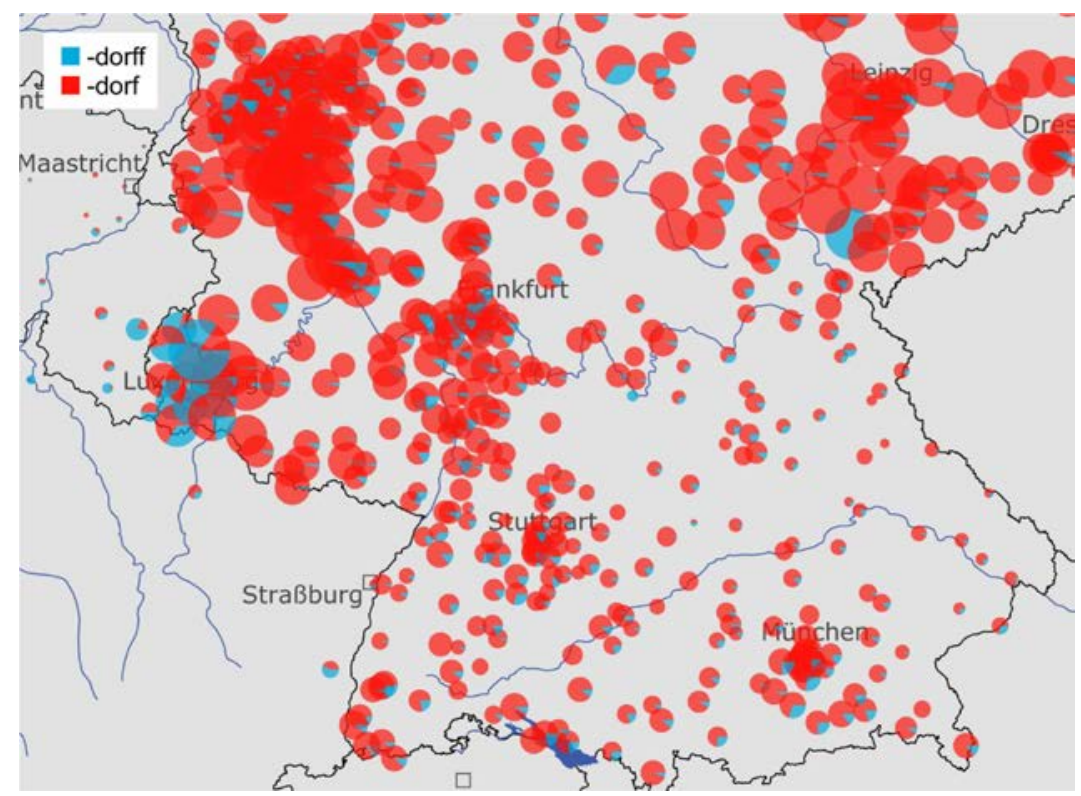

Karte 8: $\quad$ Kombinationskarte der Namen auf-dorff (blau) im Kontrast zu -dorf (rot) 



\title{
Regionalismen in den Luxemburger Familiennamen
}

\author{
Cristian Kollmann
}

\begin{abstract}
Im Raum Luxemburg gibt es, wie in anderen Regionen, eine Reihe von Familiennamen, die regionale Merkmale aufweisen. Diese betreffen u.a. die Graphie, Phonologie, Morphologie, Wortbildung, den Sprachkontakt sowie die Lexik. Ebenso wie in anderen Regionen sind bestimmte Familiennamen in Luxemburg allein schon auf Grund ihrer Verbreitung als regionale Namen einzustufen, ohne dass diese aus linguistischer Sicht besonders hervortreten würden, man denke zum Beispiel an die Herkunftsnamen.

Im vorliegenden Beitrag liegt der Fokus auf jenen Familiennamen, die die häufigsten regionalen Auffälligkeiten speziell in der Graphie und Phonologie zeigen, wobei die zu untersuchenden Namen gleichzeitig die Voraussetzung der etymologischen Eindeutigkeit weitestgehend erfüllen müssen. Ausgewählte Kartierungsbeispiele sollen dabei illustrieren, in welcher Intensität und in welchem Radius ein jeweiliger Regionalismus in der Graphie bzw. Phonologie verbreitet ist.
\end{abstract}

\section{Einleitung}

Das Luxemburger Territorium bildet sprachgeschichtlich mit dem westmitteldeutschen Dialektraum ein Kontinuum. ${ }^{1}$ Besonders gut sichtbar wird dies in der Familiennamenlandschaft der Maas-Rhein-Region ${ }^{2}$ mit Luxemburg im Fokus. Wir haben es mit einer Region zu tun, die sich auch außerhalb des deutschen Staatsgebiets (Luxemburg, Deutschsprachige Gemeinschaft, Areler Land, Deutschlothringen) durch einen Familiennamenschatz auszeichnet, der als Ganzes betrachtet mehrheitlich deutscher Herkunft ist und in dem sich, besonders im nichtdeutschländischen Teil, gleichzeitig eine Reihe von Sprachkontaktphänomenen finden lassen. ${ }^{3}$ Eine Übereinstimmung der Staatsgrenzen mit den Verbreitungsgrenzen eines Familiennamens ist nur selten festzustellen, da die heutigen Staatsgrenzen mit den historischen Sprachgrenzen kaum übereinstimmen. Allein durch die bloße Gegenüberstellung des Luxemburgischen, des Standarddeutschen und Standardfranzösischen wird das sprachgeschichtlich zusammenhängende Territorium mit dessen mundartlichem Unterbau nicht fassbar - dies geschieht erst durch die Betrachtung der Familiennamen.

Zur Entstehungszeit der Familiennamen, dem späten Mittelalter und der frühen Neuzeit, waren das Deutsche und Französische noch nicht in dem Ausmaß standardisiert und vereinheitlicht wie heute. Regionale Spracheinflüsse hatten nicht nur mündlich, sondern auch in den jeweiligen Schriftsprachen noch einen größeren Anteil. Die germanophonen Familiennamen speziell im Raum Luxemburg entstanden auf westmitteldeutscher,

1 Zur Sprachgeschichte des Luxemburgischen vgl. bes. Bruch 1953 und Bruch 1954, wobei manche Thesen überholt sind.

2 Zur genauen Absteckung der Maas-Rhein-Region siehe den Beitrag von Peter Gilles in diesem Band.

3 Der Sprachkontakt wird untersucht von Claire Muller in diesem Band. 
im engeren Sinne mittelfränkischer und vereinzelt sogar moselfränkischer Grundlage. Während zu einem Teil dieser regionalsprachliche Hintergrund in den Familiennamen bis heute durchschimmert, gab es in anderen Fällen, stets im Bestreben, eine möglichst einheitliche, überregionale Sprache zu schaffen, Anpassungen an die jeweils sich herausbildende Standardsprache. Als solche existierte auf germanophoner Seite lange nur das Deutsche. Das Luxemburgische kam erst mit der Staatsgründung 1839 und dem gleichzeitigen Aufkommen eines luxemburgischen Nationalgefühls ins Spiel. Nur allmählich begann der auf Luxemburger Territorium gesprochene moselfränkische Dialekt, sich aus dem deutschen Sprachraum herauszulösen und sich als ,Luxemburgisch ${ }^{6}$ zu begreifen. ${ }^{4}$ Die Familiennamen dagegen, die bis zu diesem Zeitpunkt weitgehend gefestigt waren, nahmen an der Ausgliederung des Luxemburgischen kaum noch teil. Nur vor diesem Hintergrund ist es zu erklären, warum heute die primäre Sprache der Luxemburger Bevölkerung, nämlich das Luxemburgische, als etwas anderes empfunden wird als jene Sprache, das Deutsche, in der ihre Familiennamen verschriftlicht wurden. Zwar zeigt dieses Deutsch durchaus tendenziell regionale Einschläge, doch sie dringen nur vereinzelt bis zum heutigen Luxemburgischen vor.

Anders stellt sich die Situation im mündlichen Gebrauch der Familiennamen dar: Einerseits gilt bei den meisten standarddeutsch geschriebenen Familiennamen de facto auch standarddeutsche Aussprache, wodurch sich der Name vom entsprechenden Appellativ deutlich abhebt: z.B. Schmit vs. Schmadd (Appellativ), Muller / Müller (beidemal mit $\ddot{u}$ zu sprechen) vs. Mëller (Appellativ), Weber vs. Wiewer (Appellativ). Andererseits, und das ist luxemburgspezifisch, gilt bei manchen Namen, trotz standarddeutscher oder zumindest nichtluxemburgischer Schreibung, eine luxemburgische Aussprache, besonders im luxemburgischen Kontext und vor allem dann, wenn der Unterschied zwischen der standarddeutschen und luxemburgischen Aussprache nicht erheblich ist: z.B. Behm $=1 \mathrm{~b}$. Béim (ebenso Appellativ ,Böhme'), Kremer $=1 \mathrm{~b}$. Kréimer (= ebenso Appellativ ,Krämer'), Schroeder $=1 b$. Schréider (ebenso Appellativ für den Verlader von Wein- und Bierfässern; doch auch der Zuschneider für bestimmte Materialien käme in Frage ${ }^{5}$ ). Etwas größer ist der Unterschied zwischen Erpelding $=1 \mathrm{~b}$. Ierpeldeng (Herkunftsname zu Erpeldingen, lb. Ierpeldeng für eine der drei gleichnamigen Ortschaften in Luxemburg), Glesener $=\mathrm{lb}$. Gliesener (für den Glaser), Kieffer $=\mathrm{lb}$. Kéifer (ebenso Appellativ „Küfer“ für den Böttcher), Krier $=1$ b. Kréier $\left(\right.$, Krüger $\left.^{6}\right),{ }^{6}$ Gutenkauf $=1 \mathrm{~b}$. Guddekaf $($ Berufsübername für einen Kaufmann oder Krämer, bei dem man gut oder billig kaufen konnte).

Insgesamt treten derartige Fälle mit spezifisch luxemburgischen Aussprachen seltener auf als jene Regionalismen, die sich über die Graphie äußern und damit eine bestimmte Aussprache vorgeben. Diese kann, außer in jenen Fällen, in denen mit dem Standarddeutschen als Bezugssystem lediglich Schreibvarianz vorliegt, ebenso regional markiert sein und damit Hinweise auf die historische Phonologie der jeweiligen Mundarten liefern. Die Thematik rund um die Graphie und Phonologie soll im Folgenden konkretisiert werden.

$4 \quad$ Vgl. hierzu bes. Gilles 1998 und Gilles 1999.

5 Genaueres siehe 2.1.4.

6 Genaueres siehe 3.1.2. 


\section{Graphie}

Da in Luxemburg neben dem Deutschen die Verwaltungssprache seit alters das Französische war, konnten deutsche Namen nach französischen Orthographieregeln oder zumindest „französisierend“ geschrieben werden, z.B. Schoumacher. Umgekehrt bestand für die französischen Namen, insbesondere nachdem diese auch phonologisch an das regionale Deutsche bzw. Luxemburgische angepasst waren, die Möglichkeit einer deutschen Graphie, z.B. Schiltz < afrz. Giles. Der größere Teil der Auffälligkeiten in der Graphie der Luxemburger Familiennamen ist jedoch weniger dem Sprachkontakt geschuldet, sondern bestimmten regionalen, insbesondere westmitteldeutschen Schreibgepflogenheiten, die sowohl den Vokalismus als auch den Konsonantismus betreffen.

\subsection{Vokalismus}

Das auffälligste Merkmal in der Graphie der Vokale besteht darin, dass statt 〈ä〉 und 〈ö〉 die Digraphe 〈ae〉 und 〈oe〉 verwendet werden, während ein Digraph 〈ue〉 für 〈ü〉 kaum vorkommt. Dies hat erstens historische, zweitens darstellungstechnische Gründe und liegt drittens am Einfluss des Französischen: In der Liste der Volkszählung des „Grossherzogthums Luxemburg“ von $1880^{7}$ erscheinen für den $\ddot{a}$ - und $\ddot{o}$-Laut bis auf wenige Ausnahmen die Ligaturen 〈æ〉 und 〈œ〉. ${ }^{8}$ Der $\ddot{u}$-Laut wird dagegen durchwegs mit 〈ü〉 wiedergegeben. In der Volkszählung von $1930^{9}$ finden sich 〈æ〉 und 〈œ〉 nunmehr aufgelöst in den Digraphen 〈ae〉 und 〈oe〉, und statt 〈ü〉 gilt durchwegs 〈u〉, das sich somit an der französischen Orthographie orientiert. Die Darstellung der Ligaturen $\longleftrightarrow æ$ und 〈œ〉 war möglicherweise technisch nicht möglich und dürfte gleichzeitig als zu archaisch empfunden worden sein, weshalb 〈ae〉 und 〈oe〉 gewählt wurden. Technische Schwierigkeiten dürften ggf. ebenso die Graphien mit Trema 〈ä〉, 〈ö〉, 〈ü〉 bereitet haben, weshalb der Umlaut 〈ü〉 gemäß der französischen Graphie für [y] unmarkiert blieb. Dieselben Graphien wie bereits 1930, nämlich 〈ae〉, 〈oe〉, 〈u〉, zeigen die Familiennamen der luxemburgischen Festnetzabonnenten von 2009. Der pauschalste Grund dafür ist, dass im Telefonverzeichnis alle Namen in Kapitallettern erscheinen und dabei auf Diakritika verzichtet wird. Außerhalb dieser (semi-)offiziellen Listen ist die Graphie 〈ü〉, etwa in Müller, durchaus noch in Gebrauch, wenngleich sie nach 1945 auf Grund ihres deutschen Erscheinungsbildes an Popularität eingebüßt hat. Die genaue Anzahl der jeweiligen aktuellen Graphien mit oder ohne Diakritika kann jedoch nicht eruiert werden, da die offiziellen Volkszählungsdaten aus Datenschutzgründen selbst für Forschungszwecke nicht zugänglich sind. In der Volkszählungsliste von 1880 ist jedenfalls ausschließlich Müller und nie Muller aufgeführt. ${ }^{10}$

7 Ediert von Müller 1887.

8 Die Ausnahmen sind Bäcker (7), Bäker (13), Flöner (25), Flörchinger (11), Flöschinger (11), Frölich (1), Hungershöfer (1), Knörze (1). Die Zahl in Klammern bezieht sich auf die Anzahl der Namenträger.

9 Reproduziert in Klees 1989.

10 Zu den Umlautgraphien in Deutschland, vgl. Dräger / Kunze 2010: 8-39. 
Ein weiteres Merkmal in der Graphie der Luxemburger Familiennamen ist 〈y〉 in den Verbindungen 〈ey〉, 〈ay〉. Die Graphie 〈ey〉 reicht allgemein in ihren Ursprüngen in frühneuhochdeutsche Zeit zurück ${ }^{11}$ und hat sich in der Onymik in manchen Fällen halten können, während sie im Appellativschatz zugunsten von 〈ei〉 durchwegs aufgegeben wurde. Im Bereich der Familiennamen ist sie in Luxemburg im Gegensatz zu der deutschländischen Nachbarregion häufiger anzutreffen. Dabei spielt es keine Rolle, ob mhd. $\bar{l}, e i$ oder $\bar{u}$, das über $e u$ regionalsprachlich zu $e i$ entrundet wurde, zu Grunde liegt.

Untenstehend nun Beispiele, die die genannten Graphien illustrieren.

\subsection{1 〈ae〉 für 〈ä〉, 〈e〉 für den Primär- und Sekundärumlaut}

Am häufigsten erscheint 〈ae〉 in Schaeffer (85) 12 / Schaefer (61), Berufsname zu mhd. schäfer ,Schäfer', Jaeger (63), Berufsname zu mhd. jäger, jeger ,Jäger', und Kraemer (58), Berufsname zu mhd. krämer, mhd.-md. krēmer ,Krämer'. Allerdings ist zu berücksichtigen, dass gelegentlich der Umlaut mehrheitlich sowohl in Luxemburg als auch im übrigen Kartierungsgebiet als einfaches 〈e〉 notiert wird, etwa im Fall von Kremer (493) ${ }^{13}$ Ein ähnlicher Fall wie Kremer vs. Kraemer ist Glesener (88) vs. Glaesener (36), ${ }^{14}$ Berufsname zu mhd.-mslfrk. *gläsener, Glaser', umgelautete Variante von mhd. glaser, wobei das Vorkommen dieses Familiennamens auf Luxemburg beschränkt ist. ${ }^{15}$ In jenen Familiennamen, wo Primärumlaut vorliegt, ist die Notation 〈ae> bzw. in Deutschland 〈ä〉 extrem selten: Becker (496) vs. Baecker (3), Berufsname zu mhd. bècker ,Bäcker'. Der Grund dafür ist, dass 〈ä〉 für den Primärumlaut erst im Neuhochdeutschen, und zudem sporadisch, nach dem Prinzip der Stammschreibung verwendet wird.

\section{$2.1 .2\langle o e\rangle$ für $\langle\ddot{o}\rangle$}

Am häufigsten zu Tage tritt die Graphie 〈oe〉 für 〈ö〉 in Goergen (249), flektiertes Patronym zu Görg als regionalsprachliche Variante von Georg, gefolgt von Goedert (230), Patronym zum gleichlautenden Rufnamen aus ahd.-mfrk. *Godihard mit Kompositionsvokal $i$ wie z.B. in ahd. Godifrid. Am dritthäufigsten ist oe in Koenig (128), Übername zu mhd. künnic, künec ,König'.

11 Vgl. Frühnhd. Gr., § L 27.

12 Die Angaben in Klammern hinter den Namen beziehen sich im Folgenden auf die Anzahl der Festnetzanschlüsse in Luxemburg aus dem Jahr 2009. Diese werden, wie die Volkszählungsdaten von 1880, mit der Datenbank und Kartierungssoftware des Luxemburgischen Familiennamenatlasses (LFA) verwaltet. Auch die Etymologien in diesem Beitrag folgen jenen des LFA, dessen OnlineVersion unter http://lfa.uni.lu zu finden ist.

13 Zum Tonvokal in Kramer, Krämer, Kremer in Deutschland, siehe DFA I, Karte 24.

14 Mit resegmentiertem Suffix -ner nach dem Muster von Berufsbezeichnungen wie Hafener, Wagener.

15 Im angrenzenden deutschen Gebiet gelten dagegen Glaser und Glasmacher (Letzteres überwiegend um Aachen und am Niederrhein). Speziell zum Tonvokal in Glaser, Gläser, Gleser in Deutschland, siehe DFA I, Karte 1. Siehe auch 2.1.3. 


\section{1 .3 〈oe〉 hyperkorrekt für ä oder e}

Im Luxemburgischen sind, wie in übrigen westmitteldeutschen Dialekten, mhd. $\bar{a}$ (Sekundärumlaut von $\bar{a}$ ), mhd. $\bar{o}$ (Umlaut von $\bar{o}$ ) sowie mhd. $\bar{e}$ in éi zusammengefallen: vgl. lb. Stréisselchen ,Sträßchen“, béis ,böse‘, Séil ,Seele“ ${ }^{6}{ }^{16}$ Ferner hat in offener Silbe sowie vor bestimmter Mehrfachkonsonanz (mitunter nur lokal) ein Zusammenfall von mhd. $\dot{e}$ (Primärumlaut von $a$ ), mhd. $\ddot{a}$ (Sekundärumlaut von $a),{ }^{17}$ mhd. $\ddot{o}$ (Umlaut von $o$ ) und mhd. $e$ in ie stattgefunden: vgl. lb. Glieser,Gläser', Niecht ,Nächte', Biedem ,Böden', Fieldchen (stadtlb.) ,kleines Feld'. In einigen Luxemburger Familiennamen steht die Graphie 〈oe〉 demnach nicht für mhd. $\bar{o}, \ddot{o}$, sondern hyperkorrekt für einen jener Vokale, die im Luxemburgischen auf synchroner Ebene von historischem $\bar{o}, \ddot{o}$ nicht unterschieden werden können. Einige der hierfür nicht besonders zahlreichen Beispiele sind: Stroesser (11), Berufsübername zu rhein. Strässer ,Pflasterer' (RhWB), Goerens (71), flektiertes Patronym zu lb. †Géierend < Gērend als zerdehnte Form von Gernd, das aus einer Langform mit ahd. gēer ,Speer' (Gērnand, Gērnōt) kontrahiert ist, Noesen (66), flektiertes Metronym zu lb. Néis als Kurzform von Agnes < Agnēs, Gloesener (23), dem, wie unter 2.1.1 bereits angemerkt, häufigeres Glesener (88), Glaesener (36) entspricht (siehe Karte 1). Kein Beispiel lässt sich für die hyperkorrekte Schreibung 〈oe〉 für mhd. $\ddot{a}$ (kurzer Sekundärumlaut von $a$ ) beibringen; ${ }^{18}$ ein lediglich historisches Beispiel für mhd. $e$ ist dagegen Foeltgen, Wohnstättenname zu stadtlb. Fieldchen ,kleines Feld“ mit im Jahr 1880 insgesamt nur 3 Namenträgern.

\subsection{4 〈oe〉 für ö oder hyperkorrekt für ä, e?}

Insgesamt am häufigsten erscheint 〈oe〉 in Schroeder (616). Jedoch ist 〈oe〉 in diesem Fall historisch zweideutig, denn bei diesem Familiennamen handelt es sich um eine Agensbildung einerseits zu mhd.-md. schrōden , hauen, schneiden, abschneiden' für den $\mathrm{Zu}$ schneider von Kleidern, Steinen, Metall oder Holz, andererseits zu mhd.-mfrk. schrāden ,u.a. rollen, wälzen, besonders Wein- und Bierfässer auf- und abladen oder zu Wagen befördern', demnach für den Verlader von Wein- und Bierfässern; entsprechend gilt das Appellativ lb. Schréider (LWB) oder pfälz. Schräder (PfWB). Im zweiten Fall wäre die Graphie 〈oe〉 hyperkorrekt und erklärt sich wie unter 2.1.3 erwähnt dadurch, dass im Luxemburgischen mhd. $\bar{a}, \bar{e}$ mit mhd. $\bar{o}$ in éi zusammengefallen sind.

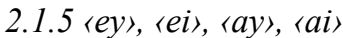

Beispiele mit der häufigsten Graphie 〈ey〉 sind Seyler (101) vs. Seiler (48), Berufsname zu mhd. seiler ,Seilmacher', Weyrich (92) vs. Weirich (42), Patronym zum gleichlautenden Rufnamen aus ahd. Wīhrīh, sowie Ney (173) vs. Nei (9), Übername zu mhd. nü̈we

16 Wobei bereits im Mittelhochdeutschen mitteldeutscher Prägung $\bar{a}$ und $\bar{e}$ nicht mehr unterschieden werden, indem diese meist als 〈e〉 erscheinen (vgl. Mhd. Gr., § L 38).

17 Wobei speziell der Zusammenfall des Primär- und kurzen Sekundärumlauts bereits für das Mittelhochdeutsche mitteldeutscher Prägung gilt (vgl. Mhd. Gr., § L 30).

18 Ein potenzieller Kandidat wäre Schlechter (39), Berufsname zu mhd. *slähter, umgelautete Form von mhd. slahter ,Schlachter', wobei das LWB als Appellativ nur umlautloses Schluechtert anführt. 
,neu' für den Neusiedler. Allerdings rangiert die nicht entrundete Variante Neu (136) nicht besonders weit hinter Ney. ${ }^{19}$ Am allerhäufigsten innerhalb wie außerhalb des Großherzogtums gilt 〈ey〉 in Meyer (403), Standesname zu mhd. meier ,Meier, Oberbauer'. Mit großem Abstand weiter unten in der Rangliste erscheinen Mayer (80), Meier (41) und Maier (11) ${ }^{20}$ Die Graphie 〈ay> tritt dagegen am öftesten in Kayser (340) auf, Übername zu mhd. keiser ,Kaiser'. Weitaus seltener sind Kaiser (89), Keiser (56), Keyser (9). Kayser ist in Luxemburg die vorherrschende Form, gefolgt von Kaiser. Auf deutscher Seite und im Moseldepartement ist es umgekehrt, während sich im Elsass beide Formen die Waage halten. Überall dünn gestreut sind Keiser und Keyser (siehe Karte 2). Der zweithäufigste Name mit 〈ay〉 ist May (99), Übername zu mhd. meie ,Mai‘, z.B. für einen im Mai geborenen Menschen, während sich die Variante Mai (7) im extrem niederfrequenten Bereich befindet. Eine Gegenüberstellung der Hauptvarianten Seyler, Meyer sowie Kayser, May zeigt, dass die Graphie 〈ay〉 nur dann verwendet wird, wenn das entsprechende Appellativ in der deutschen Standardsprache mit ‘ai〉 geschrieben wird (Seiler, Meier vs. Kaiser, Mai). Der Archaismus bei 〈ey〉, 〈ay〉 kommt somit lediglich dem Graphem 〈y〉 zu.

\subsection{Konsonantismus}

Im Bereich des Konsonantismus sind die Graphien 〈ck〉, 〈tz〉 und 〈ff〉 nach Konsonant und Diphthong hervorzuheben. Ähnlich wie bei 〈y〉 in 〈ey〉, 〈ay〉 handelt es sich um eine archaische Schreibweise, die in frühneuhochdeutsche Zeit zurückreicht und in der Orthographie des Appellativschatzes aufgegeben wurde.

\section{2 .1 〈ck〉 nach Konsonant und Diphthong}

Am häufigsten zeigt sich «ck〉 in Franck (109), Patronym zum gleichlautenden Rufnamen oder Herkunftsname Franke, doch ist die Variante Frank (96) nur ungleich seltener. Größer ist der Frequenzabstand zwischen Fonck (98), Funck (65) vs. Funk (46), Fonk (3), Berufsübername zu mhd. vunke ,Funke‘ für den Schmied oder Übername zum selben Appellativ für einen lebhaften Menschen. Die Graphien 〈ck〉 kommen überwiegend in Luxemburg, dem Areler Land und dem Moseldepartement vor (siehe Karte 3). ${ }^{21}$ Ein sehr klares Bild zeigen auch Leick (41), Leuck (19) vs. Leik (1), Herkunfts- oder Wohnstättenname zum Flussnamen Leuk bei Saarburg. Eine Ausnahme, doch mit geringem Häufigkeitsabstand, ist Winckel (26) vs. Winkel (29), Wohnstättenname zu mhd. winkel ,Winkel, Ecke'. Auch die niederfrequenten Formen Starck (3) vs. Stark (8), Übername zu mhd. starc, stark, gewaltig, kräftig, groß`, sind als Ausnahme zu werten, wobei $S t$ arck zumindest in Belgien und dem Moseldepartement mit großem Abstand häufiger als Stark begegnet. ${ }^{22}$

19 Zur Entrundung siehe 3.1.4.4.

20 Zum Tonvokal in Meier und Seiler in Deutschland, siehe DFA I, 464-477.

21 Zur Senkung $u>o$ siehe 3.1.3.3.

22 Zur Verbreitung von $c k, c k h, g k$ nach $n, l, r$ in Deutschland, siehe DFA II, 628-641. 


\section{$2.2 .2\langle t z\rangle$ nach Konsonant und Diphthong}

Am repräsentativsten für diese Graphie sind Schiltz (405) vs. Schilz (21), Patronym zum gleichlautenden Rufnamen aus afrz. Giles, sowie Holtz (34) vs. Holz (13), Wohnstättenname zu mhd. holz ,Wald, Gehölz‘. Eine Ausnahme ist u.a. Schultz (12) vs. Schulz (17), Scholz (9), Amtsname zu mhd. schultheize, der Verpflichtungen befiehlt, Richter, Schultheiß', doch ließe sich diese dadurch begründen, dass der Familienname in Luxemburg überwiegend unsynkopiert als Scholtes (275) begegnet. ${ }^{23}$ Eine speziell für Luxemburg geringe Aussagekraft besitzt dagegen Schweitzer (70) vs. Schweizer (2), Herkunftsname Schweizer, da der Name in der Graphie mit 〈tz〉 auch in Westdeutschland weit verbreitet ist. ${ }^{24}$

\subsection{3 〈ff〉 nach Konsonant und Diphthong}

Einige der zahlreichen Beispiele hierfür sind Kieffer (496) vs. Kiefer (45), Berufsname zu mhd. küefer ,Böttcher' (siehe Karte 4), ${ }^{25}$ Wolff (237) vs. Wolf (53), Übername zu mhd. wolf, Wolf‘ oder Patronym zum gleichlautenden Rufnamen als Kurzform von Rufnamen mit ahd. wolf, Wolf', Peiffer (166), Pfeiffer (26) vs. Peifer (2), Pfeifer (6), Berufsname zu mhd.-wmd. pïfer $=$ normalmhd. pfiffer ,Pfeifer, Spielmann' ${ }^{6}{ }^{26}$ Ebenso repräsentativ sind Familiennamen mit -dorff vs. -dorf wie Bettendorf (59) vs. Bettendorf (44), Herkunftsname zu Bettendorf (lb. Bettenduerf) für eine Luxemburger Ortschaft in der gleichnamigen Gemeinde. ${ }^{27}$

\section{Phonologie}

Archaischere bzw. aus der Perspektive des standardneuhochdeutschen Appellativschatzes

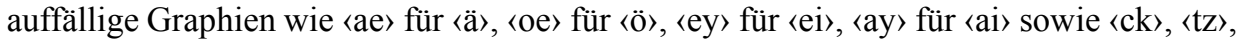
$\langle\mathrm{ff}\rangle$ für jeweils $\langle\mathrm{k}\rangle,\langle\mathrm{z}\rangle,\langle\mathrm{f}\rangle$ nach Diphthong oder Konsonant bringen keine abweichende Aussprache der Familiennamen mit sich. Es handelt sich lediglich um graphische Varianten. Anders dagegen bei den phonologischen Varianten, die regionalsprachlich markiert sind: Die diesen zu Grunde liegende regionale Aussprache hat einen derart großen Einfluss, dass sie zuletzt auch über die Graphie an die Oberfläche tritt. Doch ist dies in Luxemburg, wie in anderen Regionen, eher die Ausnahme als die Regel. Regionalsprachliche Merkmale in der Phonologie konnten, falls überhaupt, nur zu Tage treten, wenn diese möglichst einem größeren Dialektraum eigen waren und sie somit zum Zeitpunkt der Fixierung der betreffenden Familiennamen noch nicht als ,dialektal' im heutigen Sinne wahrgenommen wurden. Dies betrifft sowohl alte, aus dem Westgermanischen ererbte Merkmale, als auch bestimmte Neuerungen, die in spätmittelhochdeutscher und frühneuhochdeutscher Zeit schon weiträumig etabliert waren.

23 Zur Senkung $u>o$ siehe 3.1.3.3.

24 Zur Varianz $t / t z$ in Deutschland, siehe DFA II, 494-507.

25 Zur Entrundung siehe 3.1.4.5.

$26 \mathrm{Zu} p$ vs. $p f$ siehe 3.2.4.

27 Zur Varianz $f / f f$ in Namen mit Wolff, Dorf, Senf u.a. in Deutschland, siehe DFA II, 168-181. 


\subsection{Vokalismus}

Wenn man als Bezugsystem das Westgermanische heranzieht, handelt es sich bei den regionalsprachlichen Merkmalen im Bereich des Vokalismus durchwegs um Neuerungen. Sie können unterschiedlichen Alters sein und sind jedoch in der neuhochdeutschen Standardsprache vielfach nicht eingetreten. Je nach Phänomen reicht die Zeitspanne dieser Neuerungen vom Frühalthochdeutschen bis zum Frühneuhochdeutschen. Die wichtigsten bestehen in folgenden Vorgängen: Monophthongierung, Senkung, Diphthongierung, Hebung, Kürzung, Entrundung, Verdumpfung.

\subsubsection{Monophthongierung}

Die Monophthongierung betrifft im Folgenden mhd. ei, ou und dessen Umlaut öu. Jedoch sind entsprechende Reflexe in den Familiennamen äußerst rar.

\subsubsection{Mhd. ei >lb. ee [e:]}

Im Mitteldeutschen wird ei im 12./13. Jh. monophthongiert. Das Ergebnis u.a. im Mittelfränkischen ist $\bar{a}$ oder $\bar{e} .^{28}$ Im modernen Luxemburgischen gilt $e$, ee [e:], veraltend noch $\ddot{a}$, $\ddot{a} \ddot{a}[\varepsilon$ : $]:$ Seel, Säl ,Seil ${ }^{‘}$, Rees, Räs ,Reise ${ }^{6}{ }^{29}$ Eines der wenigen Beispiele für diesen Vorgang in Familiennamen ist Mesemburg (18), Mesenburg (1), wobei jedoch die Varianten Meysembourg (36), Meisenburg (5), Meysenburg (4) häufiger vorkommen. Zu Grunde liegt ein Herkunftsname zu Meysemburg (lb. Meesebuerg) für eine Ortschaft in der Gemeinde Fels. Nur vereinzelt und historisch belegt sind 1704 Reiser $=>1735$ Reser, ${ }^{30}$ Übername zu mhd. reiser , der eine Reise, einen Feldzug macht, Krieger' oder 1739 Selers $=>1769$ Seiler. ${ }^{31}$

\subsubsection{Mhd. ou > lb. a, aa [a:]}

In Teilen des Mitteldeutschen wird seit dem 12./13. Jh. ou zu $\bar{o}$ monophthongiert, ${ }^{32}$ und dieses erscheint u.a. im heutigen Luxemburgischen weiter gesenkt zu $a$, aa [a:]: Bam ,Baum‘. Ein sicheres Beispiel in Familiennamen ist Schambourg (30), Schamburger (1), wobei die Monophthongierung auch im zu Grunde liegenden Toponym gegriffen hat. Es handelt sich um einen Wohnstättennamen zu Schamburg $<$ Schauenburg (zu mhd. schouwen ,schauen“) für eine Burg im Zentrum von Bartringen.

\subsubsection{Mhd. öu > lb. ee [e:]}

Mhd. $\ddot{o} u$, der Umlaut von ou, wird im Mittelfränkischen zu $\bar{o}$ monophthongiert ${ }^{33}$ und ergibt durch Entrundung u.a. im Luxemburgischen ee [e:], veraltend $\ddot{a}$, ää: Beemchen,

28 Vgl. Mhd. Gr., § L 45.

29 Doch siehe auch 3.2.6.

30 http://deltgen.com. Hierbei handelt es sich um eine genealogische Online-Datenbank. Das Zeichen $\Rightarrow$ drückt einen Generationswechsel aus.

31 http://deltgen.com.

32 Vgl. Mhd. Gr., § L 46.

33 Vgl. Mhd. Gr., § L 47. 
Bäämchen ,kleiner Baum‘. Dasselbe Appellativ hat auch einen Familiennamen hervorgebracht: Bemtgen, Wohnstättenname zu mhd. *böumchìn, Diminutivform von mhd. boum ,Baum'. Bemtgen ist ausschließlich in Luxemburg verbreitet. Im Saarland sowie vereinzelt im Rheinland erscheint der Familienname in der standarddeutschen Version Bäumchen. Bemtgen/Bäumchen ist eines der seltenen Beispiele dafür, wo die heutige Namengrenze mit der Staatsgrenze korreliert (siehe Karte 5).

\subsubsection{Monophthongierung > Senkung > Diphthongierung/Hebung und Kürzung: Mhd.} ie, uo, üe $>m h d .-m d . \overline{1}, \bar{u}, \bar{u}$ > $m h d .-m f r k$. è, ō, ö $>l b$. éi, ou, éi / i, u, i

Im 11./12. Jh. fand im Mitteldeutschen die Monophthongierung $i e>\bar{i}, u o>\bar{u}, \ddot{u} e>\ddot{u}$ statt, und hieraus im 13. Jh. u.a. im Mittelfränkischen die Senkung $\bar{l}>\bar{e}, o u>\bar{o}$, üe $>\bar{o} .{ }^{34} \mathrm{Die}$ Diphthonge mhd.-mfrk. $\bar{e}, \bar{o}, \bar{o}$ haben in Luxemburg je nach Position unterschiedliche Ergebnisse erbracht, erscheinen einerseits diphthongiert als éi [er], ou [ov], éi [er]: léif ,lieb', Fouss ,Fuß', Féiss ,Füße', andererseits, durch Hebung und Kürzung, als $i$ [i], $u$ $[\mathrm{u}], i[\mathrm{i}]$ : richen ,riechen', Brudder ,Bruder', Bridder ,Brüder'. Für die Diphthongierung von mhd.-mfrk. $\bar{e}, \bar{o}, \bar{o}>\mathrm{lb}$. éi, ou, éi in Familiennamen findet sich nur ein historisches Beispiel: 1793 Krier $=>1833$ Kreer, ${ }^{35}$ Berufsname zu mhd. krüeger ,Schankwirt; Töpfer, Geschirrhändler ${ }^{6}{ }^{36}$ In Kreer wird man den Versuch der Wiedergabe der Aussprache lb. Kréier sehen dürfen. ${ }^{37}$ Für die Senkung und Kürzung von mhd.-mfrk. $\bar{e}, \bar{o}, \bar{o}>\mathrm{lb} . i, u, i$ ließe sich, obwohl Letztere nicht durch die folgende Doppelkonsonanz explizit markiert ist, Diderich (22), Diderrich (19), Patronym zum gleichlautenden Rufnamen aus ahd. Diederīch, beibringen, zumal selbst die mit großem Abstand häufigere Hauptvariante Diederich (317) im Luxemburgischen mit kurzem $i$ gesprochen wird. Das Hauptverbreitungsgebiet von Diderich liegt in der südlichen Hälfte Luxemburgs und dem Areler Land, während Diderrich ausschließlich in Luxemburg auftritt (siehe Karte 6). ${ }^{38}$

\subsubsection{Senkung}

Die Senkung bezieht sich im Folgenden auf jene von frühahd.-mfrk. $i$ und deren weitere Reflexe im Luxemburgischen sowie auf jene von mhd. $o$ und $u$.

\subsubsection{Frühahd.-mfrk. $i>l b$. é [e], ë [ə]; frühahd.-mfrk. ï>lb. $a$}

Im Frühalthochdeutschen mittelfränkischer (und teilweise rheinfränkischer) Prägung fand die Spaltung des Phonems $i$ in geschlossener Silbe statt: Stand in der Folgesilbe ein Mittel- oder Tiefzungenvokal oder folgte keine Silbe, wurde $i$ zu einem offenen $i$ gesenkt. In diesem Zusammenhang spricht man von $i_{2}$, das im Folgenden als $i$ notiert wird. In den übrigen Fällen blieb $i$ als geschlossenes $i$, das als $i_{1}$ bezeichnet wird,

34 Vgl. Mhd. Gr., § E 34 und § L 48-50.

35 http://deltgen.com.

36 Zur Entrundung $\ddot{u} e>$ ie siehe 3.1.4.5, zum $g$-Schwund siehe 3.2.

37 Es gilt nämlich zusätzlich zu beachten, dass für éi und ou in der frühen Orthographie 〈e'〉 und 〈o'〉 galten: de Ro'de Le'w , der rote Löwe'.

38 Zur Quantität des Tonvokals in Dietrich in Deutschland, siehe DFA I, 636-641. 
erhalten. Das Ergebnis von $\ddot{i}\left(i_{2}\right)$ in weiten Teilen des Westmoselfränkischen und damit auch im Luxemburgischen ist, außer vor $h, r$ und sch, überwiegend $a$ [a]: ahd.-mfrk. *smï ,Schmied ${ }^{*}>$ lb. Schmadd, jenes von $i\left(i_{1}\right)$ ist vor Velar é [e]: ahd. dicki, dick $>$ lb. déck, in den übrigen Fällen der Zentralvokal $\ddot{e}$ [ə]: frühahd.-mfrk. *smittja ,Schmitte $>$ lb. Schmëtt. ${ }^{39}$ Der Weg von $\ddot{z}$ zu $a$ im Westmoselfränkischen verlief über eine zentrale Zwischenstufe, deren Qualität in etwa derjenigen des heutigen Zentralvokals lb. $\ddot{e}$ entsprochen haben dürfte..$^{40}$

Einen frühen Beleg für die Senkung $i>a$ im Luxemburgischen enthalten die für den Zeitraum zwischen 1388 und 1500 überlieferten Rechnungsbücher der Stadt Luxemburg. ${ }^{41}$ Gleich mehrfach erscheint in den genannten Quellen das Toponym Lampach. ${ }^{42}$ Dieses ist mit heutigem Limpach, lb. Lampech, für eine Ortschaft in der Gemeinde Rekkingen zu identifizieren. Als entsprechende Familiennamen gelten heute sowohl Limpach (38) als auch Lampach (20). Das Erstglied des Namens entspricht ahd.-mfrk. *lïnda (= normalahd. linda) ,Linde', das lb. Lann ergab. Lediglich einen indirekten Beleg für die Senkung $\ddot{i}>a$ in den Rechnungsbüchern liefert zaimerman, tzaymerman, ${ }^{43}$ Berufsname zu mhd.-mslfrk. *zïmmerman ,Zimmermann'. In den Graphien 〈ai〉, 〈ay〉 ist 〈i〉, 〈y〉 Dehnungszeichen, das hyperkorrekt eingesetzt wurde. Als Familienname gilt heute durchwegs Zimmermann (26), als Appellativ lb. Zammermann, wobei jüngere Sprecher die bereits modernere Form Zëmmermann gebrauchen. Ebenso, um bei den Rechnungsbüchern zu bleiben, einen indirekten Hinweis auf die Senkung $\ddot{i}>a$ gibt $s m o t,{ }^{44} \mathrm{im} \mathrm{Ge}-$ gensatz zu den mit großem Abstand häufigeren ,Normalbelegen' mit 〈i〉 oder 〈y〉 wie smit, smyt ${ }^{45}$ Berufsübernamen zu mhd.-mslfrk. *smït ,Schmied'. In der insgesamt nur dreimal vorkommenden Graphie 〈o〉 darf möglicherweise eine Verschreibung für 〈a〉 gesehen werden, desgleichen in goltsmot, das ein einziges Mal auftaucht, neben frequenterem goltsmit, - smyt. ${ }^{46}$ Ein eindeutiger Beleg mit $<a 〉$ in der Berufsbezeichnung für den Schmied taucht erst in einem Feuerstättenverzeichnis aus dem Jahr 1528 auf: der Smatt. ${ }^{47}$ Angesichts der historisch singulären Belege, die einen Reflex der Senkung zeigen, ist es nicht überraschend, dass heute die Formen mit 〈i〉 ausnahmslos gelten. Die frequentesten Varianten sind Schmit (1525), Schmitt (194), Schmidt (99).

39 Das Ergebnis von $i$, vor $h$ und $r$ ist dagegen $i i$ [i:], das speziell vor $r$ bei der jüngeren Generation mit einem Schwa-Nachschlag zu hören ist: ahd.-mfrk. *gisïht, Gesicht" $>$ lb. Gesiicht, ahd.-mfrk. *wïrd ,Wirt ${ }^{\star}>$ lb. Wiirt, Wiert. Das Ergebnis von $i_{2}$ vor $s c h$ ist wie bei $i_{1}$ der Zentralvokal $\ddot{e}$ [ə]: ahd.-mfrk. *dïk, Tisch`> lb. Dësch.

40 Genaueres zur Senkung von frühahd. $i$ sowie von mhd. $o$ im Luxemburgischen, siehe Kollmann 2013 (im Druck).

41 Ediert von Moulin / Pauly 2007-2010. Des Weiteren liegen eine personennamenkundliche Analyse (Gniffke 2010) sowie eine graphematisch-phonologische Analyse (Ravida 2012) vor.

42 Z.B. 1455/56 clais van lampach (Gniffke 2010: 358 ).

43 Z.B. 1448/49, zaimerman 1452/53 tzaymerman (Gniffke 2010: 509-513)

44 Z.B. 1477/78 smot (Gniffke 2010: 477-78).

45 Z.B. 1467/68 smyt, 1478/79 smit (Gniffke 2010: 476).

46 1472/73 goltsmot (Gniffke 2010: 443).

47 http://mambra.lu. 
Keinen Niederschlag in den Rechnungsbüchern findet die Senkung $\ddot{i}>a$ in den hochfrequenten Formen fas-, faß-, vas-, vasßbender, ${ }^{48}$ Berufsnamen zu mhd.-mfrk. vazbinder ,Fassbinder'. Dies hat folgenden Grund: Speziell in Agensableitungen auf ahd. -āri ( $>$ mhd. - $\bar{a} r e$ ) mit ahd.-mfrk. $\ddot{i}\left(i_{2}\right)$ als Stammvokal verhält sich dieses $\ddot{i}$ in seinem weiteren Verlauf wie einfaches $i\left(i_{1}\right)$ und fungiert als analoger Umlaut. Entsprechend lautet das luxemburgische Appellativ Faassbënner (vgl. WLM), demnach mit ,Umlaut‘ im Gegensatz zum zu Grunde liegenden Verb lb. bannen ,binden'. Weitere sich ebenso verhaltende Agensbildungen sind das Simplizium lb. Bënner ,Binder (von Garben)`, ferner Spënner ,Spinner' zu lb. spannen ,spinnen', Sprénger ,Springer‘ zu lb. sprangen ,springen“, Schwëmmer neben seltenerem umlautlosem Schwammer ,Schwimmer' zu lb. schwammen ,schwimmen“ (vgl. LWB). Als Familienname erscheint der Fassbinder heute in Luxemburg sowohl mit dem dem neuhochdeutschen Standard entsprechenden Stammvokal: Fassbinder (17), Fasbinder (13), als auch mit 〈e〉: Fassbender (13), Fasbender (5). ${ }^{49}$

Ein weiteres Beispiel für die Senkung $i>\ddot{e}$ ist Schmoetten (11), Wohnstättenname zu mhd. smitte ,Schmiede ‘ < frühahd. *smittja. Die Graphie 〈oe〉steht hier nicht für historisches $\ddot{o}$, sondern für jenen Zentralvokal lb. $\ddot{e}$, der phonetisch einem $\ddot{o}$ sehr nahekommt. Dabei ist das Vorkommen der Variante Schmoetten auf Luxemburg beschränkt. Auf deutscher Seite entspricht ihr Schmitten (siehe Karte 7).

Ein sicheres Beispiel für die Senkung von $\ddot{i}\left(i_{2}\right)>a$ in einem gegenwärtigen Familiennamen ist Schammel (25), Übername zu mhd.-mslfrk. * schïmmel = normalmhd. $\operatorname{schim}(m)$ el ,Schimmel; weißes Pferd' (< ahd. skimbal) für einen Menschen mit grauer oder weißer Haarfarbe. Schammel kommt ausschließlich in Luxemburg vor, Schimmel ausschließlich in Deutschland, doch kaum in Grenznähe zum Großherzogtum (siehe Karte 8). Ein weiteres Beispiel ist Lanners (202), flektiertes Patronym zu $\dagger$ Lanner als personalisierter Herkunftsname zu Lannen für eine Ortschaft in der Gemeinde Redingen an der Attert. Allerdings begegnet die Senkung bereits im Toponym, das aus Linden stammt. Wie für Zimmermann und Schmit nur noch historisch bezeugt ist dagegen die Senkung $\ddot{i}>a$ im folgenden Familiennamen: 1656 Schlammen $=>1692$ Schlimmen,${ }^{50}$ flektiertes Patronym, und dies Übername zu mhd.-westmslfrk. ${ }^{*} \operatorname{sïm}(p)=$ normalmhd. $\operatorname{slim}(p)$, schief, schräg, verkehrt‘. Der Familienname erscheint heute noch unflektiert als Schlimm (8), das entsprechende Appellativ als lb. schlamm ,hinkend, lahm; schief, krumm“ (LWB).

Ein Beispiel für die Senkung $i>e$ außerhalb des Gebietes der $i$-Spaltung ist Engeldinger (20). Hierbei handelt es sich um einen personalisierten Herkunftsnamen zu einem abgegangenen Toponym rheinfrk. Engeldingen für das spätere Hagendingen, jetzt amtlich nur noch frz. Hagondange, das eine Gemeinde im Moseldepartement bezeichnet. Innerhalb des Gebiets mit $i$-Spaltung musste das Toponym ahd.-mfrk. *Ïngoldingun ,bei den Leuten des Ingold' lauten, woraus die u.a. 1526 bezeugte Form Angeldingen.

48 Z.B. 1427/28 vasbender, 1445/46 vaßbender, 1480/81 faßbender 1499/1500 fasbender (Gniffke 2010: 425-427).

49 Zum Tonvokal in Binder, Binner, Bender, Benner und -binder, -bender in Deutschland, siehe DFA I, Karte 43 und 44.

50 http://deltgen.com. 
Außerhalb des Gebiets mit $i$-Spaltung und somit im angrenzenden Rheinfränkischen lautete das Toponym ahd.-rheinfrk. *Ingoldingun, dem später u.a. 1583 Ingeldingen und 1473 Engeldingen entsprechen. ${ }^{51}$

\subsubsection{Mhd. o > lb. a}

Mhd. kurzes offenes $o$ in geschlossener Silbe wurde in spätmittelhochdeutscher Zeit in Teilen des Westmoselfränkischen zu velarem $a$ [a] gesenkt, wenn der zu senkende Vokal nicht unmittelbar vor $h$, Liquid, Nasal oder sch stand: mhd. kochen ,kochen ' $>1 \mathrm{~b}$. kachen Erste, wenngleich nur sporadische Hinweise auf die Senkung von $o$ zu $a$ tauchen in den Rechnungsbüchern auf, doch findet sie dort nur in einem einzigen Begriff ihren besonderen Niederschlag, und zwar in Apperer, Gehilfe im Baugewerbe ${ }^{.}{ }^{52}$ Die Frequenz der Variante mit $a$ ist dabei bemerkenswerterweise weitaus höher als bei Opperer. Der Begriff lässt sich auf ahd.-md. *opperāri zu lat. *operārius abbilden und begegnet heute im Luxemburgischen als Apperer ,Handlanger' (LWB). Ein entsprechender Familienname kommt heute in Luxemburg nicht mehr vor. In den Rechnungsbüchern indirektes Zeugnis von der Senkung $o>a$ gibt der Einzelbeleg kaich, ${ }^{53}$ Berufsname zu mhd.-mslfrk. koch ,Koch'. Wie bei 〈ai〉 in zaimerman ist 〈i〉 als hyperkorrektes Dehnungszeichen aufzufassen. Als Familienname gilt heute ausschließlich Koch (26), als Appellativ lb. Kach. Ein ebenfalls historischer Beleg für die Senkung mhd. $o>$ lb. $a$ findet sich 1880 in Rackenbrod (1) vs. Rockenbrod (13), heute nur noch Rockenbrot (2), Berufsübername zu mhd. rocke, rogge ,Roggen' und brōt ,Brot' für den auf Roggenbrot spezialisierten Bäcker. Sichere Beispiele für die Senkung in den gegenwärtigen Luxemburger Familiennamen sind dagegen in keinem Fall beizubringen.

\subsubsection{Mhd. u >lb. o}

Ausgehend vom Mittelfränkischen und Hessischen findet im Mitteldeutschen ab dem 12. Jh. die Senkung von kurzem $u$ zu geschlossenem $o$ statt. ${ }^{54}$ Konkret im Luxemburgischen erscheint dieses $o$, außer vor $r$ und germ. $h$, als $o$ : mhd. junc ,jung ${ }^{\text {‘ }}>$ lb. jong. In den Luxemburger Familiennamen lassen sich für diese Senkung zahlreiche Beispiele finden: Scholtes (275), Scholtus (34), Amtsname zu mhd. schultheize ,der Verpflichtungen befiehlt, Richter, Schultheiß`. Scholtes konzentriert sich eindeutig in der Großregion, wobei außerhalb Deutschlands die Varianten mit $o$ gar ausschließlich sind (siehe Karte 9) ${ }^{55}$ Mit wie auch ohne Senkung in Luxemburg erscheint Fonck (98), Fonk (3) vs. Funck (65), Funk (46). ${ }^{56}$

Ein Beispiel, in dem sich die Senkung dagegen kaum durchgesetzt hat, ist Juncker (35), Junker (57) vs. Jonker (4), Standes- oder Übername zu mhd. juncherre, junger Herr, junger (noch nicht Ritter gewordener) Adliger, Junker, Edelknabe‘.

51 Genaueres siehe Kollmann 2011.

52 Z.B. 1461/62 apperer (Gniffke 2010: 412f).

53 1499/1500 kaich (Gniffke 2010: 452f).

54 Vgl. Mhd. Gr., § L 26 und $\S$ L 35.

55 Zum Tonvokal in Schulte vs. Scholten in Deutschland, siehe DFA I, Karte 98.

56 Zur Schreibvarianz 〈k〉 vs. 〈ck〉 siehe 2.2.1. 


\subsubsection{Entrundung}

Erste Anzeichen für die Entrundung in hochdeutschen Mundarten reichen in das $12 .{ }^{57}$ bzw. 13. Jh. ${ }^{58}$ zurück und finden sich zuerst im Bairischen. Das Moselfränkische wurde von der Entrundung erst in frühneuhochdeutscher Zeit erfasst, und in der am nächsten verwandten Dialektgruppe, dem Ripuarischen, ist die Rundung bis heute erhalten geblieben.

Beispiele für die Entrundung in den Luxemburger Familiennamen sind zahlreich. Im Einzelnen sind folgende am häufigsten:

\subsubsection{Mhd. $\ddot{\mathrm{o}}>\mathrm{e}$}

Schlesser (84) vs. Schloesser (41), Berufsname zu mhd. *slözzer, umgelautete Nebenform von slozzer ,Schlosser'; entsprechend das Appellativ lb. Schlässer. Das Vorkommen der entrundeten Form Schlesser konzentriert sich überwiegend auf Luxemburg und Ostlothringen. Mit der Graphie 〈oe〉 erscheint der Name vor allem in Luxemburg. In Deutschland, insbesondere im mittleren Westen, gilt die Entsprechung Schlösser (siehe Karte 10). ${ }^{59}$

\subsubsection{Mhd. $\ddot{\mathrm{u}}>\mathrm{i}$}

Prim (51) vs. Prum / Prüm (16), Herkunftsname zu Prüm (mda. Prim) für eine Ortschaft in der gleichnamigen Gemeinde im Eifelkreis Bitburg-Prüm. Prim ist überwiegend in Luxemburg und dem Moseldepartement anzutreffen, während auf deutscher Seite Prüm dominiert, das in Luxemburg als Prum / Prüm auftritt.

\subsubsection{Mhd. $\overline{\mathrm{o}}>\mathrm{e}$}

Flener (30) vs. Floener (24), Übername zu mhd. vlöhenen, flüchten, durch Flucht entfernen, in Sicherheit bringen' (Lexer), nhd. flöhnen ,bewegliches Gut flüchten, d.h. in Sicherheit bringen' (DRW). Beide Varianten des Namens sind fast nur in Luxemburg zu finden.

\subsubsection{Mhd. $\overline{\mathrm{u}}>\mathrm{eu}>\mathrm{ei}$, ey}

Ney (173), Nei (9) vs. Neu (136), Übername zu mhd. nü̈we ,neu' für den Neusiedler. ${ }^{60}$ Das Hauptverbreitungsgebiet von Ney liegt in Luxemburg, dem Saarland und Moseldepartement. Nei ist überall sehr selten, am häufigsten jedoch in Luxemburg. Neu begegnet überall sehr häufig mit großflächigen Nestern.

Feiereisen (74), Feyereisen (44), Berufsübername zu mhd. vürīsen ,Feuereisen“ für den Hersteller und Verkäufer von Feuereisen, also Spezialeisen zum Feuerschlagen. Feiereisen gilt fast ausschließlich in Luxemburg, Feyereisen außer in Luxemburg häufiger in Belgien, besonders dem Areler Land. Feuereisen ist insgesamt selten und erscheint ausschließlich in Deutschland, besonders im Raum Trier und Saarbrücken (siehe Karte 11).

57 Vgl. Mhd. Gr., § L 25.

58 Vgl. Fnhd. Gr., § L 36.

59 Zur Verteilung Schlosser vs. Schlösser in Deutschland, siehe DFA I, Karte 80.

60 Zur Schreibvarianz 〈ei〉 vs. 〈ey〉 siehe 2.1.5. 
Ein Beispiel, das lediglich Tendenzen aufzeigt, ist u.a. Breyer (32), Breier (4) vs. Breuer (47), Berufsname zu mhd. brüwwer, Brauer` für den Bierbrauer, da die nicht entrundete Form häufiger ist als die entrundete. ${ }^{61}$

\subsubsection{5 üe > ie}

Kieffer (496), Kiefer (45), Berufsname zu mhd. küefer ,Böttcher‘ ${ }^{62}$ Kieffer ist die dominierende Form in Luxemburg, aber auch in Lothringen und dem Elsass. In der deutschen Nachbarregion gilt überwiegend Kiefer.

Krier (341), Krieger (14) vs. Kruger / Krüger (5). Krier zeigt ein klares Hauptverbreitungsgebiet: Luxemburg, allerdings kaum im Norden, Areler Land und übriges Wallonien, Ostlothringen, Saarland. Im übrigen Deutschland bildet diese Variante keine größeren Nester. Die in Deutschland dominierende Form ist überall Krüger. Eine weitere Variante mit Entrundung ist Krieger (14), doch besteht Konkurrenzetymologie zu mhd. krieger ,Streiter, Kämpfer' (siehe Karte 12). ${ }^{63}$

Plier (31), Plyer (4), Berufsname zu mhd.-wmd. *plüeger (normalmhd. phluoger) ,Pflugmacher'. Plier ist überwiegend in Luxemburg (mit dem höchsten Vorkommen im Kanton Redingen) und dem Areler Land verbreitet. Sehr selten und auf den Süden Luxemburgs beschränkt ist die Schreibvariante Plyer. Plieger und Plüger kommen in Luxemburg überhaupt nicht vor und sind auch in Deutschland sehr selten. Die dort mit großem Abstand dominierende Form ist Pflüger. ${ }^{64}$

\subsubsection{Verdumpfung: mhd. $\overline{\mathrm{a}}>$ lb. o, oo [o:]}

Die Verdumpfung von mhd. $\bar{a}$ ist seit dem 12. Jh. in vielen hochdeutschen Mundarten zu beobachten. ${ }^{65}$ Im Luxemburgischen gilt $o$, oo [o:], sowie speziell vor Nasal ou [ou]: mhd. blāwe $>$ lb. blo; mhd. strāze $>$ lb. Strooss; mhd. mānōt $>$ lb. Mount.

Sichere Beispiele für die Verdumpfung in Familiennamen sind sehr rar. Eines davon ist: Schrobiltgen (10), Übername zu lb. Schrobiltgen (Vianden) ,streitsüchtiger Mann“ (LWB). Beim Appellativ handelt es sich um ein Kompositum mit lb. schro, boshaft, bösartig, rücksichtslos; ungezogen, unartig‘ (LWB) < mhd. schrāch, schrōch ,mager, dürr, rauh, grob“ (Lexer) und einer pejorativen Diminutivbildung von mhd. buole ,naher Verwandter, Geliebter, Liebhaber‘. Der Familienname zeigt die größte Verbreitung im Areler Land. In der Schreibvariante Schrobildgen ist er vereinzelt um Saarbrücken zu finden (siehe Karte 13). ${ }^{66}$

61 Zum Tonvokal in Brauer, Bräuer in Deutschland, siehe DFA I, 506-513.

62 Zur Schreibvarianz 〈f〉 vs. 〈ff〉 siehe 2.2.3.

63 Zum Tonvokal in Krüger in Deutschland, siehe DFA I, 274-279.

64 Zum g-Schwund in Krier und Plier/Plyer siehe 3.2.3. Hingegen zur Verteilung Pflüger vs. Plöger in Deutschland, siehe DFA II, Karte 36.

65 Vgl. Mhd. Gr., § L 37.

66 Genaueres siehe Kollmann 2010. 


\subsection{Konsonantismus}

Ein Hauptmerkmal im Konsonantismus des Mittelfränkischen besteht darin, dass westgerm. $\hbar, d, g$ in althochdeutscher Zeit nicht - über die Mediae $b, d, g$ als Zwischenstufe - zu Tenues verschoben wurden. Sogar die frikativische Aussprache von ursprünglich westgerm. $b$ und $g$ blieb im Inlaut außer nach Nasal sowie im Auslaut, wo Verhärtung eintrat, nachweislich lange bewahrt. ${ }^{67}$ Dieser Zustand gilt im Wesentlichen bis heute, nur dass $g$ und in manchen Fällen auch $b$ in intervokalischer Stellung geschwunden ist. Beispiele für das Luxemburgische: westgerm. *graban, graben“ > ahd.-mfrk. *graちan > lb. gruewen; westgerm. *graちa- ,Grab“ > ahd.-mfrk. *graf > lb. Graf; westgerm. *geちan ,geben'> ahd.-mfrk. *geban > lb. ginn; westgerm. *đaga- ,Tag'> ahd.-mfrk. *dach > lb. Dag; westgerm. *wagōn, Wagen'> ahd.-mfrk. *wago $>$ lb. Won.

Ein weiteres, dem Mittel- und Rheinfränkischen gemeinsames Merkmal ist die unterbliebene Verschiebung von westgerm. $p$ im Anlaut sowie im Inlaut nach $m$, vornehmlich im Ripuarischen auch nach Liquid. Auch westgerm. $p p$ bleibt unverschoben. ${ }^{68}$ Beispiele für das Luxemburgische: westgerm. *pīpan ,pfeifen' $>$ ahd.-mfrk. pïffan $>$ lb. päiffen; westgerm. *đorpa- ,Dorf* > ahd.-mfrk. dorp > lb. Duerf; westgerm. *appla-> ahd.-mfrk. appul > lb. Apel.

Ein Vorgang, der jedoch nicht die 2. Lautverschiebung betrifft, ist die althochdeutsche Frikativenschwächung, die Folgendes besagt: Die Reihe der stimmlosen Reibelaute germ. $f, p, x$, $s$ wurde seit etwa 750 in stimmhafter Umgebung lenisiert. ${ }^{69}$ Während im Standardneuhochdeutschen die Lenisierung bis auf jene von $s$ rückgängig gemacht wurde, bleibt sie in einzelnen neuhochdeutschen Mundarten auch in anderen Fällen erhalten. Im Luxemburgischen gilt der Erhalt der Stimmhaftigkeit auch bei $w[\mathrm{v}]<f$ in stimmhafter Umgebung im Inlaut. Beispiel: westgerm. *ofna- ,Ofen' $>$ ahd. ovan $>$ lb. Uewen.

Ein weiteres, jedoch rezenteres und kleinräumig auftretendes Phänomen, das in dieser Form überwiegend auf das Luxemburgische beschränkt ist, betrifft die Velarisierung von mhd. $n$ nach langem Hochzungenvokal oder fallendem Diphthong im Inlaut: mhd. schīnen $>\mathrm{lb}$. schéngen, mhd. meinen $>\mathrm{lb}$. mengen.

Untenstehend nun Beispiele für die genannten regionalsprachlichen Phänomene in den Familiennamen.

\subsection{1 westgerm. $\mathrm{b}>l b . \mathrm{w}[v]$}

Groff (29), Grof (11), Übername zu mhd.-wmd. grof = normalmhd. grop , an Masse groß, dick, stark; unfein, ungebildet'; entsprechend lb. graff, grob“. Das Vorkommen von Groff beschränkt sich auf die südliche Hälfte Luxemburgs und das Elsass, wo jedoch aus lauthistorischen Gründen eine andere Etymologie zu erwägen ist. Seltener und ebenfalls nur in der südlichen Hälfte des Großherzogtums, dagegen etwas häufiger in Deutschland, ist

67 Vgl. Mhd. Gr., § E 39 und $\S$ L 62.

68 Vgl. Ahd. Gr. I, § 131; Mhd. Gr., § E 39 und § L 61.

69 Vgl. Ahd. Gr. I, § L 63. 
Grof zu finden. Insgesamt ist jedoch in Deutschland Grob am häufigsten, das punktuell auch in Lothringen (Moseldepartement, Vogesen) vorkommt (siehe Karte 14).

Ewert (69), vs. Ebert, Patronym zum gleichlautenden Rufnamen als kontrahierte Form von Eberhard. Das Hauptverbreitungsgebiet von Ewert liegt eindeutig in Luxemburg, während auf deutscher Seite Ebert dominiert. Eine sehr klare Verteilung zeigen entsprechende flektierte Bildungen: Ewertz (19) konzentriert sich in Luxemburg, um Prüm und Wittlich und streut vereinzelt u.a. im Saarland. Ebertz ist dagegen im moselfränkischen Sprachgebiet nicht zu finden.

\subsection{2 westgerm. đ $>l b . \mathrm{d}$}

Sadler (51), Sadeler (16) vs. Sattler (3), Berufsname zu mhd.-wmd. *sadeler ,Sattler'. Sadler ist überwiegend in Luxemburg und dem Moseldepartement anzutreffen, die nicht synkopierte Variante Sadeler in Luxemburg und dem Areler Land. Auf deutscher Seite dominiert Sattler (siehe Karte 15).

Sondag (10) vs. Sonntag (28), Sontag (3), Übername zu mhd.-wmd. sundach, sondach = normalmhd. suntac, Sonntag' wohl für einen Menschen, von dem man sich Glück und Segen erhoffte. Anders als beim vorigen Namen liegt der Schwerpunkt der Verbreitung der unverschobenen Formen im Areler Land, das somit in diesem Fall als Rückzugsgebiet zu interpretieren ist (siehe Karte 16).

\subsection{3 westgerm. g > lb. Ø in intervokalischer Stellung}

Beispiele hierfür sind Krier (341) und Plier (31) / Plyer (4), die unter 3.1.4.5 behandelt wurden. Dabei sind die $g$-losen Formen fast nur außerhalb Deutschlands zu finden.

\subsection{4 westgerm. $\mathrm{p}>\mathrm{lb} . \mathrm{p}$}

Peiffer (166), Peifer (2), vs. Pfeiffer (26), Pfeifer (6), Berufsname zu mhd.-wmd. pïfer ,Pfeifer, Spielmann', entsprechend das Appellativ lb. Päifert (mit sekundärem Agens- $t$ ). Die Variante Peiffer mit unverschobenem $p$ dominiert mit großem Abstand überall außerhalb Deutschlands. Außerdem treten in Luxemburg Formen mit einfachem $f$ im Inlaut seltener auf (siehe Karte 17) ${ }^{70}$.

Peffer (87), Peffer (1), Berufsübername oder Übername zu mhd.-wmd. peffer = normalmhd. pfeffer ,Pfeffer' am ehesten für den Pfefferhändler, allgemein den Gewürzkrämer oder -liebhaber; vgl. auch das Appellativ lb. Peffer. Peffer kommt fast ausschließlich in Luxemburg vor, während Pfeffer dort nicht zu finden ist (siehe Karte 18).

Putz / Pütz (181), Wohnstättenname zu mhd.-md. pütze = normalmhd. pfütze ,Brunnen“; vgl. entsprechend lb. Pëtz ,Schöpf-, Ziehbrunnen“. Formen mit verschobenem $p f$ sind in Luxemburg überhaupt nicht anzutreffen. ${ }^{71}$

70 Zur Schreibvarianz 〈f〉 vs. 〈ff〉 siehe 2.2.3. Zur Varianz $p / p f / f(f)$ in Namen mit Pfeifer und Pfeffer in Deutschland, siehe DFA II, 62-71.

71 Hingegen zum Tonvokal in Pütz, Pfitzner in Deutschland, siehe DFA I, Karte 67. 
3.2.5 westgerm. $\mathrm{f}>$ ahd. $\mathrm{v}>l b . \mathrm{w}[v]$

Munhoven (28), Munhowen (19), Herkunftsname zu Monhofen (lothr. Monuewen, frz. Monom) für eine Gemeinde im Moseldepartement. Munhoven und Munhowen kommen ausschließlich in Luxemburg vor, Letzteres besonders im Süden. Munhofen erscheint nur sehr vereinzelt im angrenzenden Saarland (siehe Karte 19).

\subsubsection{Velarisierung von $\mathrm{n}$}

Hengen (109), vs. Heinen (213), Heynen (33), flektiertes Patronym zu Heng, Heine als Kurzform von Heinrich. Die Variante mit velarisiertem $n$ kommt fast ausschließlich in Luxemburg vor, wenngleich auch dort die unvelarisierte Form dominiert (siehe Karte 20). ${ }^{72}$

\section{Konklusion}

Die Regionalismen in den Luxemburger Familiennamen treten auf mehreren grammatischen Ebenen zu Tage. In diesem Beitrag wurde die Untersuchung der Regionalismen auf die Graphie und Phonologie beschränkt, wobei nur jene Namen ausgewählt wurden, deren Etymologie weitestgehend eindeutig ist.

Für die Graphie lässt sich allgemein festhalten, dass in der Maas-Rhein-Region Archaismen auf nichtdeutschem Staatsgebiet öfter anzutreffen sind, was sich am konsequentesten am Digraph 〈ff〉 zeigt. Dies ist gewiss als Ausdruck einer größeren Normierungsresistenz als in Deutschland zu werten. In der Phonologie lässt sich eine Reihe von westmitteldeutschen Merkmalen beobachten. Doch ist die Zahl jener Familiennamen, die diese Merkmale nicht zeigen, weitaus höher. Speziell exklusiv mittelfränkische, moselfränkische oder gar luxemburgische Merkmale lassen sich dagegen äußerst selten feststellen. So finden sich zwar Beispiele für die Entrundung wie mhd. Küefer $>$ Kiefer, mhd. Krüeger $>$ Krier. Doch auf der Stufe Kiefer, Krier sind diese Namen stehen geblieben, und die luxemburgische Weiterentwicklung zu Kéifer, Kréier wurde nicht mehr mitgemacht. Kéifer und Kréier gelten nur im überwiegend mündlichen luxemburgischen Kontext. Im Konsonantismus hingegen zeigt sich die größte Normierungsresistenz beim unverschobenen $p$. Dies könnte daran liegen, dass die Affrikate $p f$ dem Westmitteldeutschen völlig fremd ist.

Eine regelrechte Systematik bei den Regionalismen lässt sich nicht erkennen, oft sind diese einzelwortbasiert und zeigen lediglich Tendenzen auf. Zudem sind die Regionalismen in den seltensten Fällen auf das Territorium des heutigen Luxemburgs beschränkt. Vielmehr weisen sie eine von Osten herreichende Kontinuität auf, die zum Teil auch noch im Areler Land und in Deutsch-Lothringen anzutreffen ist, allerdings in höherer Konzentration im nichtdeutschen Teil der Maas-Rhein-Region.

72 Zur Velarisierung von $n$ siehe auch den Beitrag von Ann Marynissen in diesem Band, wo das Thema ausführlich und grenzübergreifend behandelt wird. 


\section{Literatur}

Ahd. Gr. I = Braune, Wilhelm: Althochdeutsche Grammatik I, Laut- und Formenlehre, 15. Auflage, bearbeitet von Ingo Reiffenstein, Tübingen, 2004.

Bruch, Robert: Das Luxemburgische im westfränkischen Kreis, Luxemburg, 1954 (= Publications Littéraires et Scientifiques du Ministère de l'Éductaion Nationale 2).

Bruch, Robert: Grundlegung einer Geschichte des Luxemburgischen, Luxemburg, 1953 (= Publications Littéraires et Scientifiques du Ministère de l'Éductaion Nationale 1).

DFA I = Deutscher Familiennamenatlas, Herausgegeben von Konrad Kunze und Damaris Nübling, Band 1, Grafematik/Phonologie der Familiennamen, I: Vokalismus von Christian Bochenek und Kathrin Dräger, Berlin / New York, 2009.

DFA II = Deutscher Familiennamenatlas, Herausgegeben von Konrad Kunze und Damaris Nübling, Band 2, Grafematik / Phonologie der Familiennamen, II: Konsonantismus von Antje Dammel, Kathrin Dräger, Rita Häuser und Mirjam Schmuck, Berlin / New York, 2011.

Dräger, Kathrin / Kunze, Konrad: Umlautzeichen in deutschen Familiennamen. In: Zunamen. Zeitschrift für Namenforschung 5, 2010, 8-39.

DRW $=$ Deutsches Rechtwörterbuch, Herausgegeben von der Heidelberger Akademie der Wissenschaften, Heidelberg, 1914ff. URL: http://woerterbuchnetz.de

Frühnhd. Gr. = Ebert, Robert P.: Reichmann, Oskar / Solms, Hans-Joachim / Wegera, Klaus-Peter, 1993: Frühneuhochdeutsche Grammatik.

Gilles, Peter: Dialektausgleich im Lëtzebuergeschen, Zur phonetisch-phonologischen Fokussierung einer Nationalsprache, Tübingen, 1999.

Gilles, Peter: Die Emanzipation des Lëtzebuergeschen aus dem Gefüge der deutschen Mundarten. In: Zeitschrift für Deutsche Philologie, Nr. 117, 1998, 20-35.

Gniffke, Andreas: Die Personennamen der Stadt Luxemburg von 1388-1500, Namenbuch und namenkundliche Analyse auf Basis der Rechnungsbücher der Stadt Luxemburg, Unveröffentlichte Dissertationsschrift, eingereicht an der Universität Luxemburg, 2010.

Les Annuaires du Luxembourg, Édition, 2009.

Lexer = Lexer, Matthias 1872-1778: Mittelhochdeutsches Handwörterbuch, 3 Bände, Leipzig, Nachdruck Stuttgart, 1970. URL: http://woerterbuchnetz.de

LWB = Luxemburger Wörterbuch, Herausgegeben von der Wörterbuchkommission, 5 Bände, Luxemburg, 1950-1975, Ergänzungsband, 1977. URL: http://infolux.uni.lu/worterbucher/

Klees, Henri: Geographie der Luxemburger Familiennamen (nach der Volkszählung von 1930), Herausgegeben vom Institut grand-ducal, Section de Linguistique, de Folklore et de Toponymie, Luxemburg, 1989 (= Beiträge zur luxemburgischen Sprach- und Volkskunde Nr. 18).

Kollmann, Cristian: Schrobiltgen Biltgen (und Schreibvarianten), Rubrik „Wuert vum Mount“ (September 2010) auf http://infolux.uni.lu

Kollmann, Cristian: Vergessener Ortsname: Wo liegt Engeldingen? Rubrik „Wuert vum Mount“ (August 2011) aufhttp://infolux.uni.lu

Kollmann, Cristian: Neues zu Engeldingen, Rubrik „Wuert vum Mount“ (September 2011) auf http:// infolux.uni.lu

Kollmann, Cristian: Schmadd - Schmëdd - Schmidd-s, Haff-Huef: Senkung und unterbliebene Senkung von $i$ und $o$ zu $a$ im Luxemburgischen. In: Deutsche Dialekte, Konzepte, Probleme, Handlungsfelder, Tagungsband des 4. Kongresses der Internationalen Gesellschaft für Dialektologie des Deutschen vom 13. bis 15. September 2012 an der Universität Kiel, Herausgegeben von Michael Elmentaler, Markus Hundt, Jürgen Erich Schmidt, Stuttgart, 2013. Im Druck.

Mhd. Gr. = Mittelhochdeutsche Grammatik, Von Hermann Paul, 25. Auflage, neu bearbeitet von Thomas Klein, Hans-Joachim Solms, Klaus-Peter Wegera und Heinz-Peter Prell, Tübingen, 2007.

Moulin, Claudine / Pauly, Michel (Hgg.): Die Rechnungsbücher der Stadt Luxemburg, Erstes Heft 1388 1399 2007, Zweites Heft 1400-1430 2008, Drittes Heft 1444-1453 2009, Viertes Heft 1453-1460 2010, Fünftes Heft 1460-1466 2010, Luxemburg, 2007-2010 (= Schriftenreihe des Stadtarchivs Luxemburg Bd, 1-5). 
Müller, Nik: Die Familien-Namen des Grossherzogthums Luxemburg, Luxemburg, 1887.

PfWB = Pfälzisches Wörterbuch, Begründet von Ernst Christmann, fortgeführt von Julius Krämer, bearbeitet von Rudolf Post unter Mitarbeit von Josef Schwing und Sigrid Bingenheimer, 6 Bde, Wiesbaden / Stuttgart, 1965-1997. URL: http://woerterbuchnetz.de

Ravida, Fausto: Graphematisch-phonologische Analyse der Luxemburger Rechnungsbücher 1388-1500, Ein Beitrag zur Historischen Stadtsprachenforschung, Heidelberg, 2012.

RhWB $=$ Rheinisches Wörterbuch, Herausgegeben von Josef Müller, Heinrich Dittmaier, Rudolf Schützeichel und Mattias Zender, 9 Bände, Bonn / Berlin 1928-1971. URL: http://woerterbuchnetz.de

WLM = Wörterbuch der luxemburgischen Mundart, Luxemburg, 1906. URL: http://infolux.uni.lu/ worterbucher/

\section{Internet}

http://deltgen.com

http://infolux.uni.lu

http://lfa.uni.lu

http://mambra.lu

http://woerterbuchnetz.de

\section{Kartenanhang}

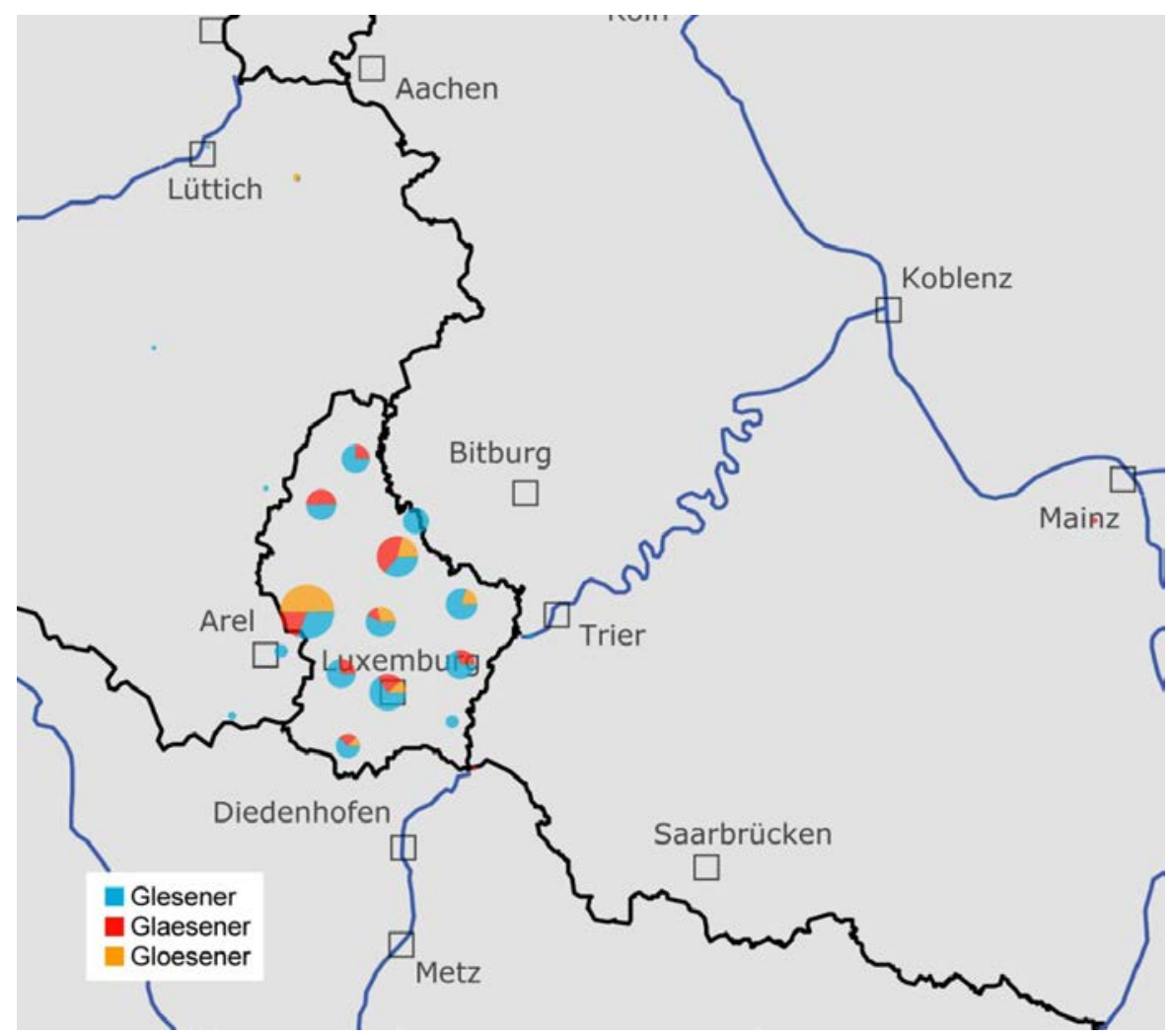

Karte 1: Glesener, Glaesener, Gloesener (s. 2.1.1 und 2.1.3) 


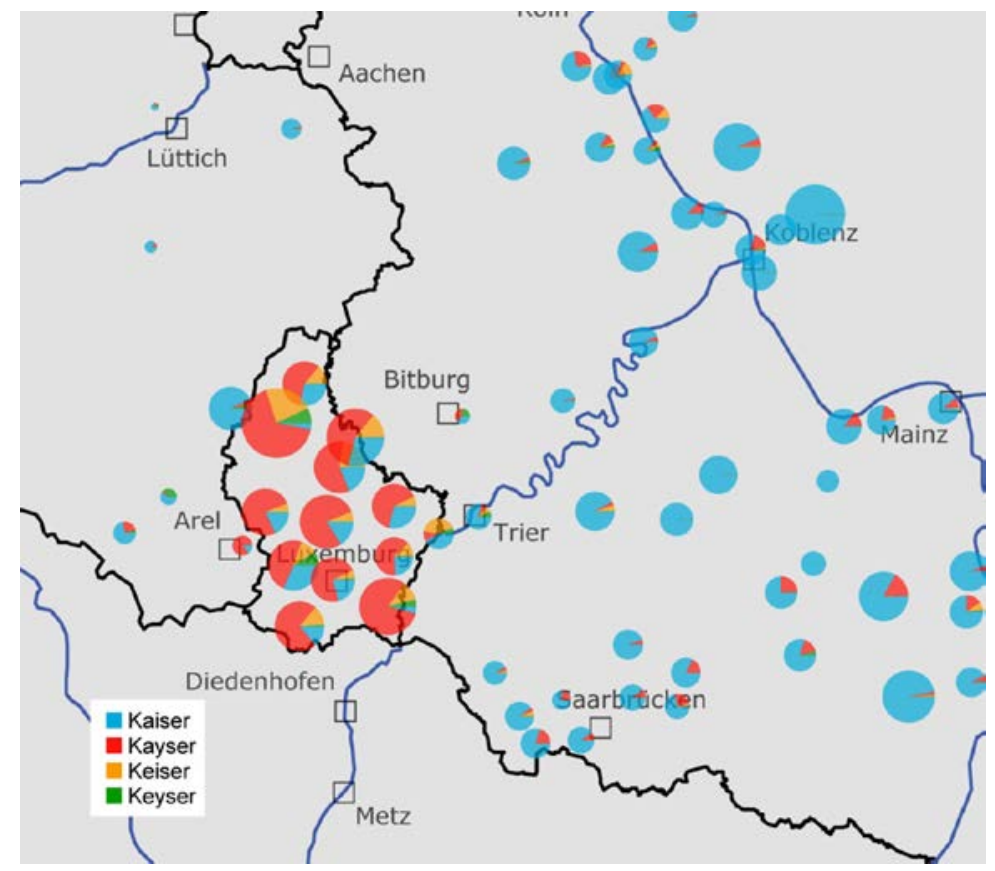

Karte 2: $\quad$ Kaiser, Kayser, Keiser, Keyser (s. 2.1.5)

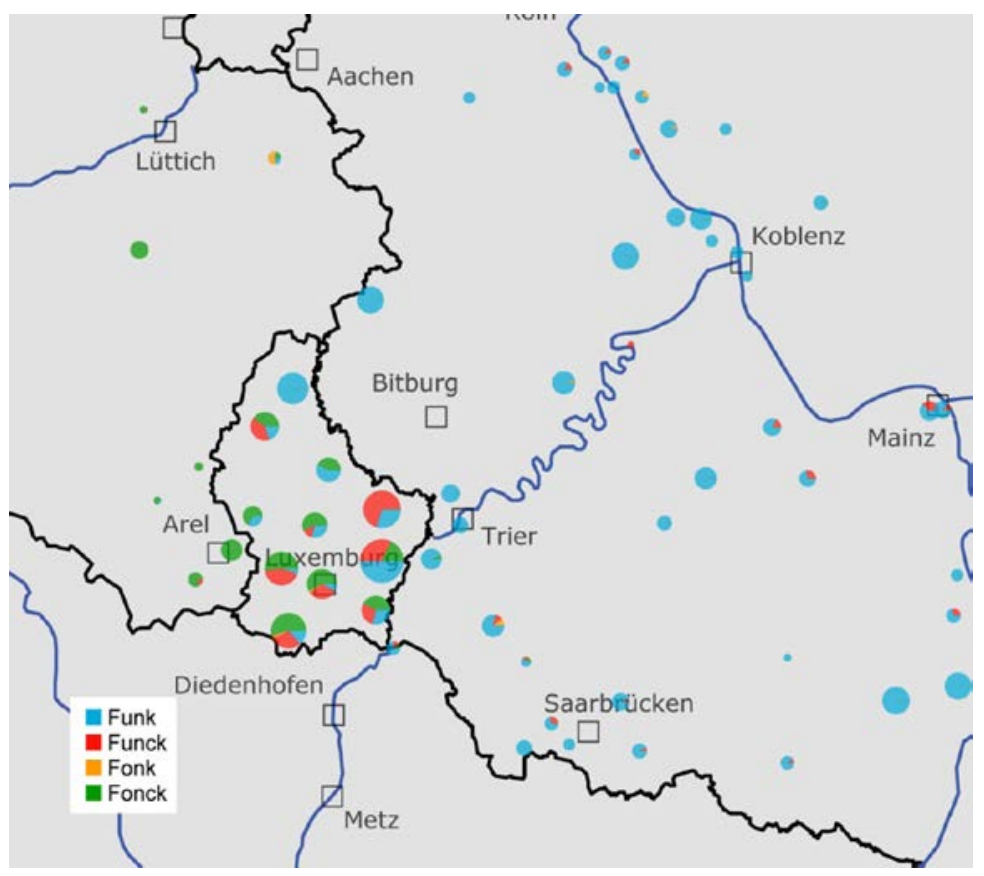

Karte 3: $\quad$ Funk, Funck, Fonk, Fonck (s. 2.2.1 und 3.1.3.3) 


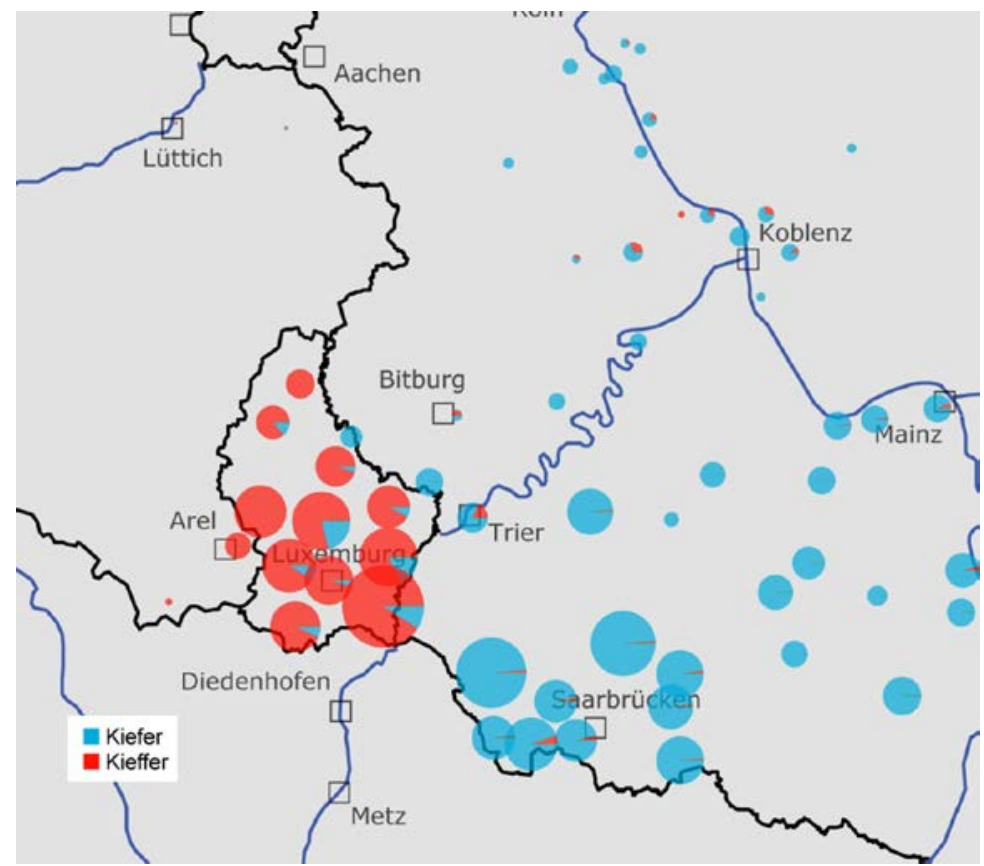

Karte 4: $\quad$ Kiefer, Kieffer (s. 2.2.3 und 3.1.4.5)

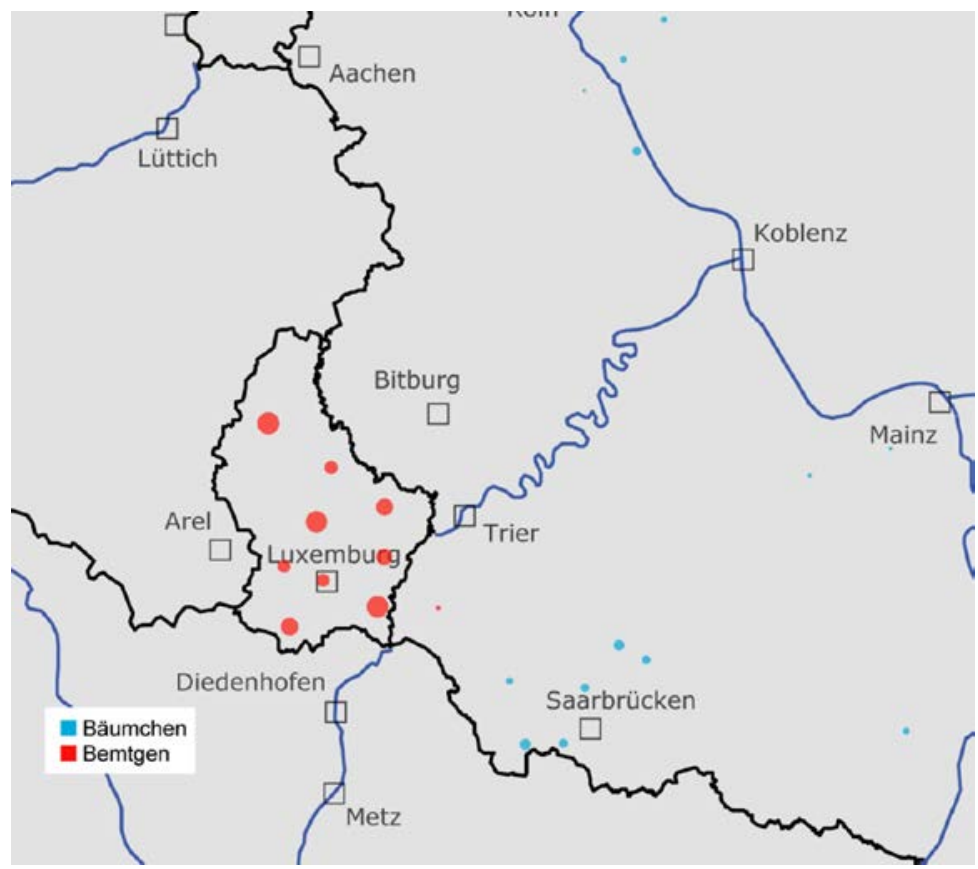

Karte 5: $\quad$ Bäumchen, Bemtgen (s. 3.1.1.3) 


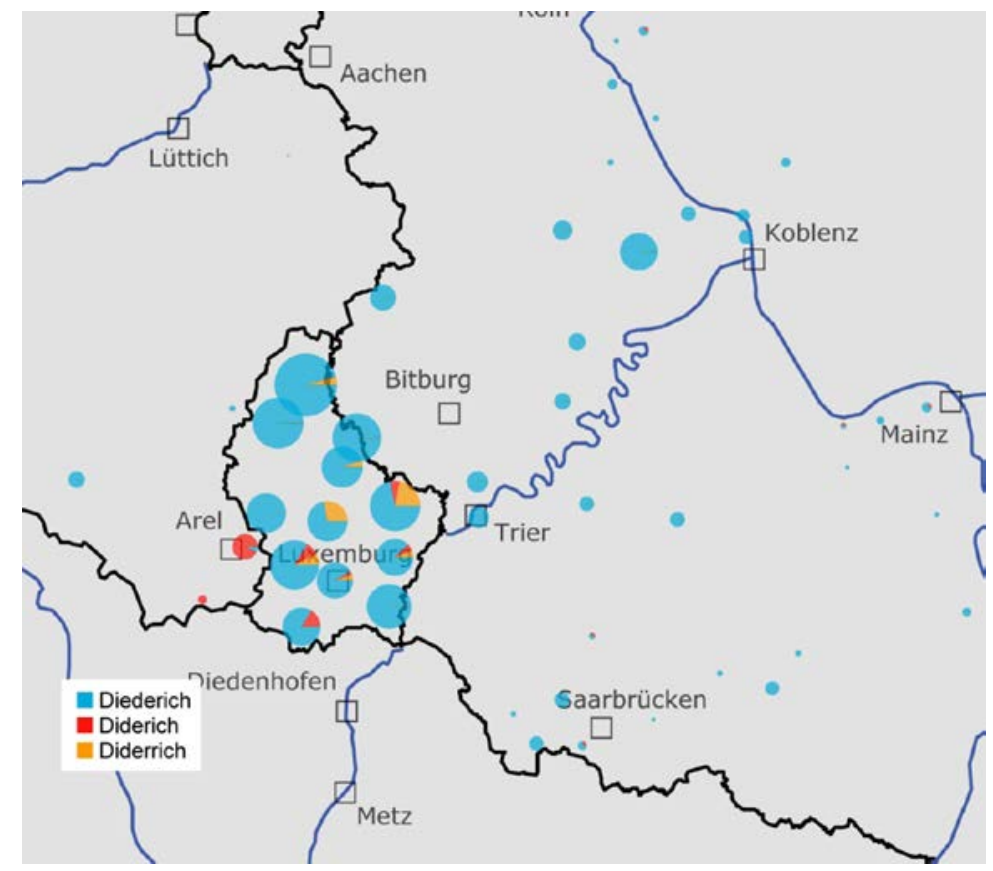

Karte 6: $\quad$ Diederich, Diderich, Diderrich (s. 3.1.2)

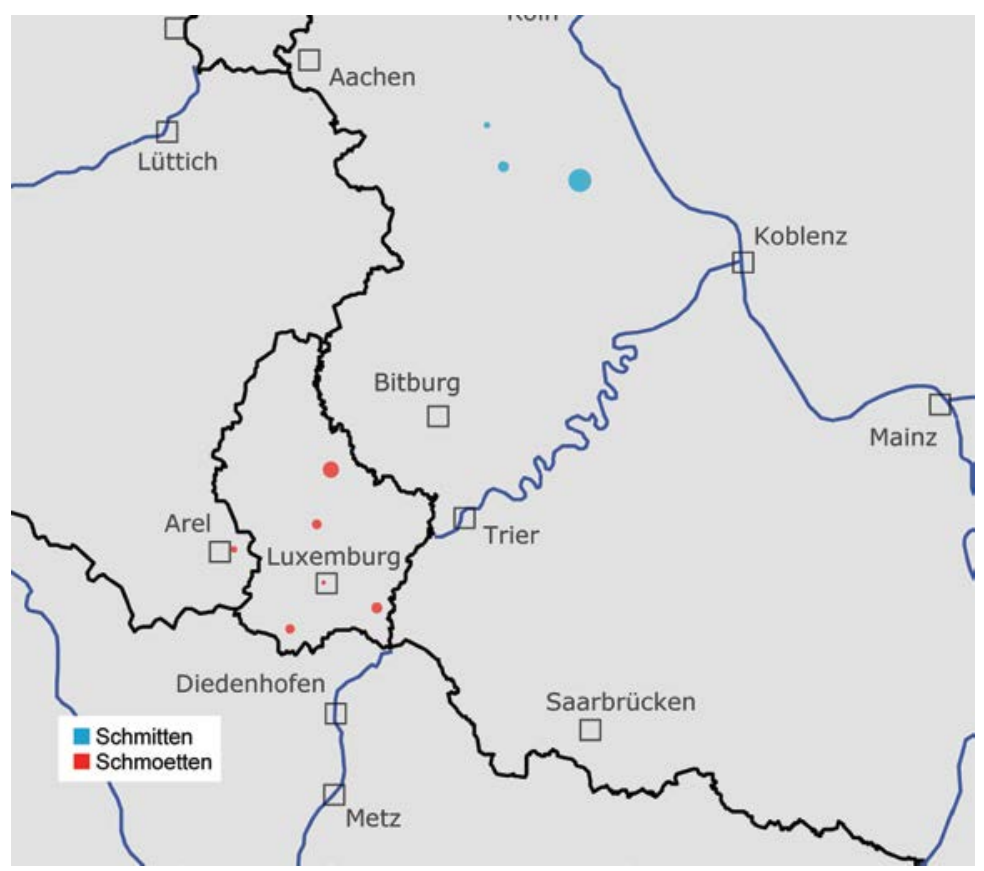

Karte 7: $\quad$ Schmitten, Schmoetten (s. 3.1.3.1) 


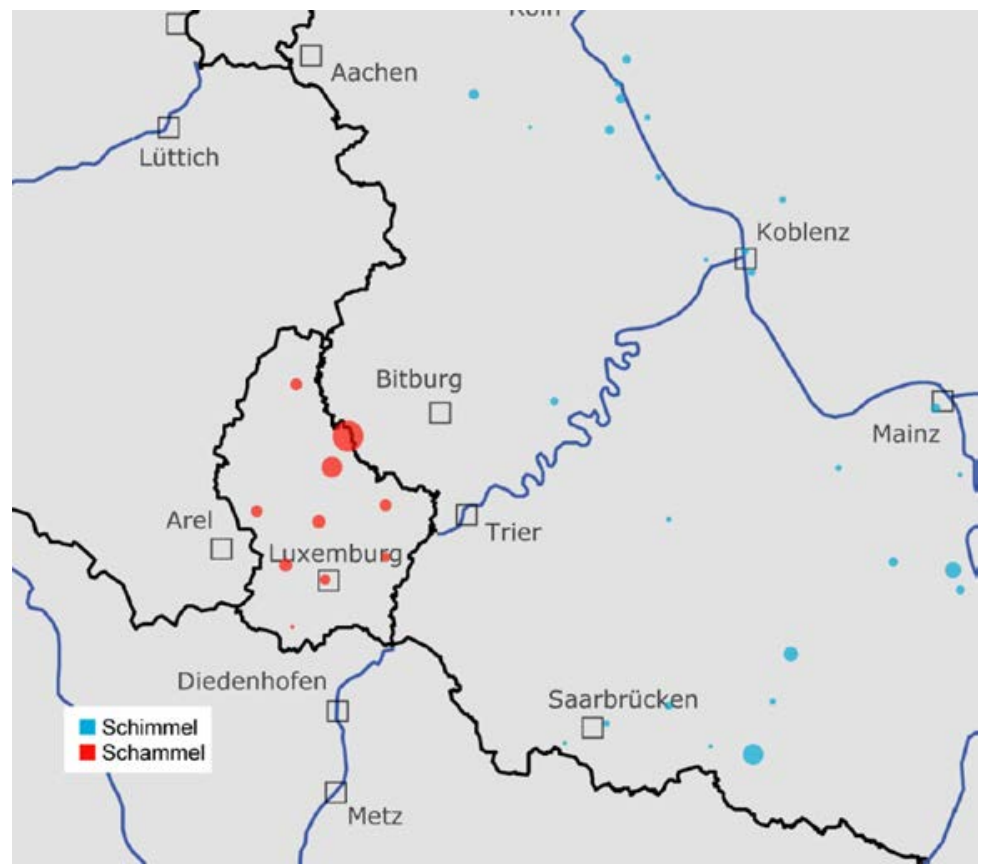

Karte 8: $\quad$ Schimmel, Schammel (s. 3.1.3.1)

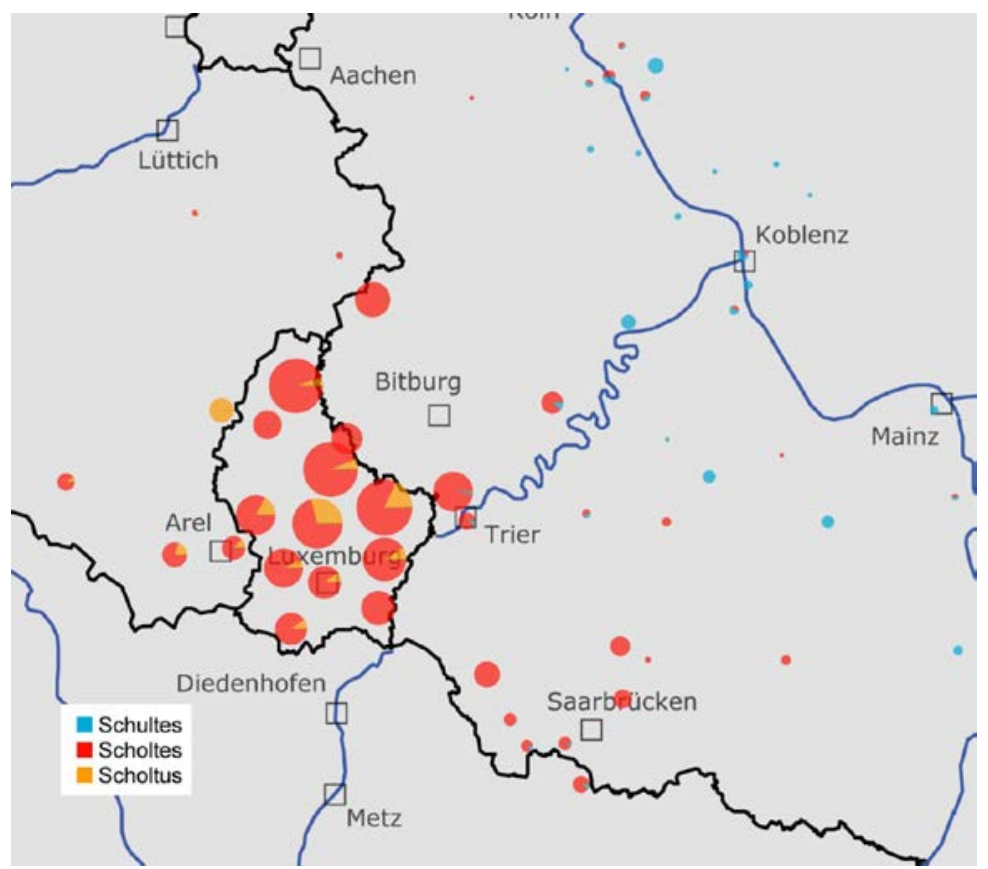

Karte 9: $\quad$ Schultes, Scholtes, Scholtus (s. 3.1.3.3) 


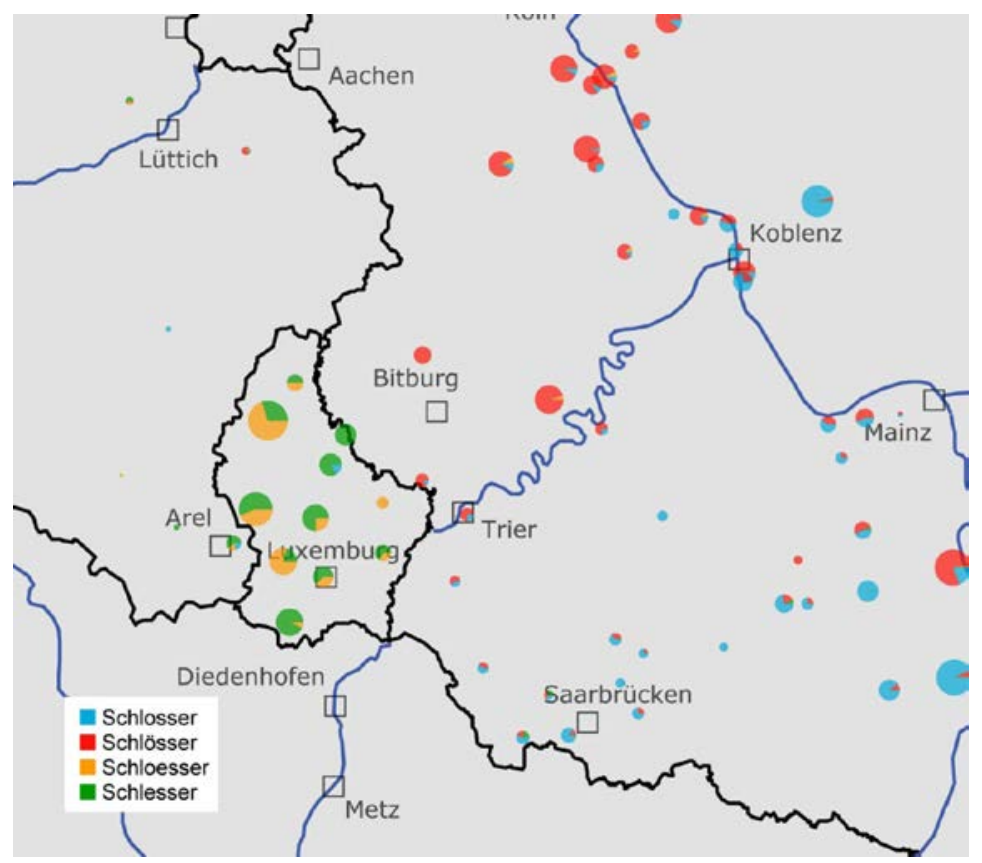

Karte 10: $\quad$ Schlosser, Schlösser, Schloesser, Schlesser (s. 3.1.4.1)

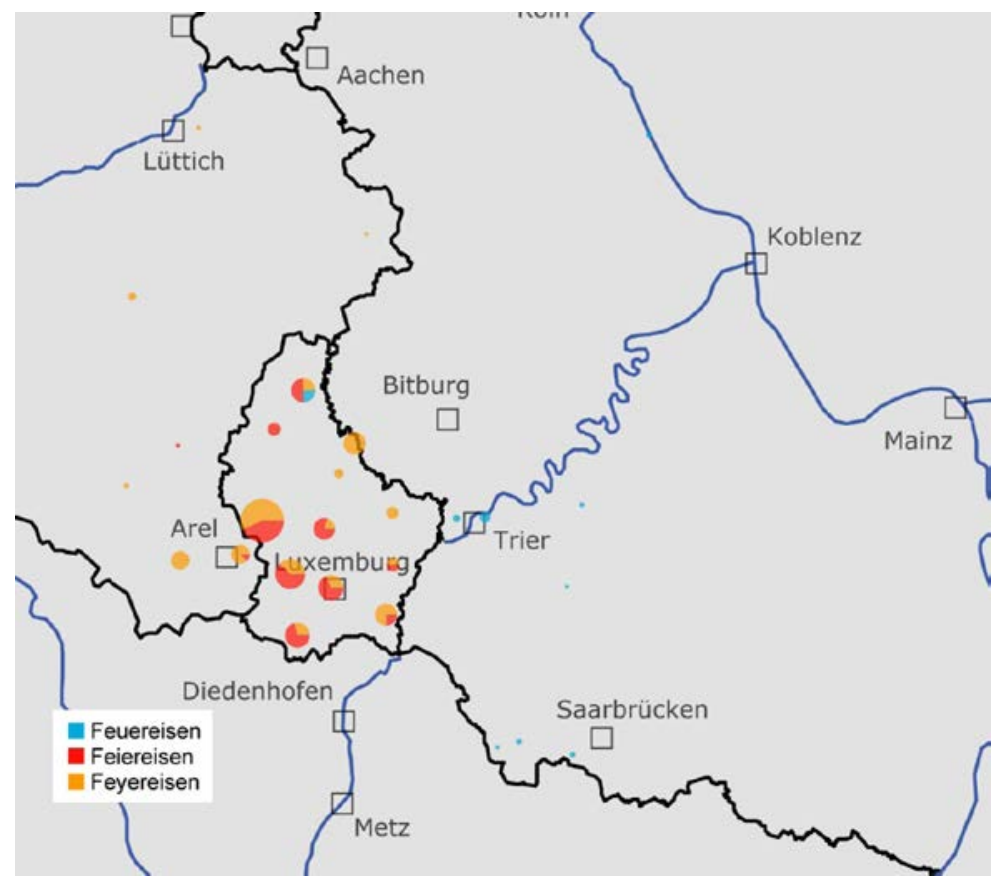

Karte 11: Feuereisen, Feiereisen, Feyereisen (s. 3.1.4.4) 


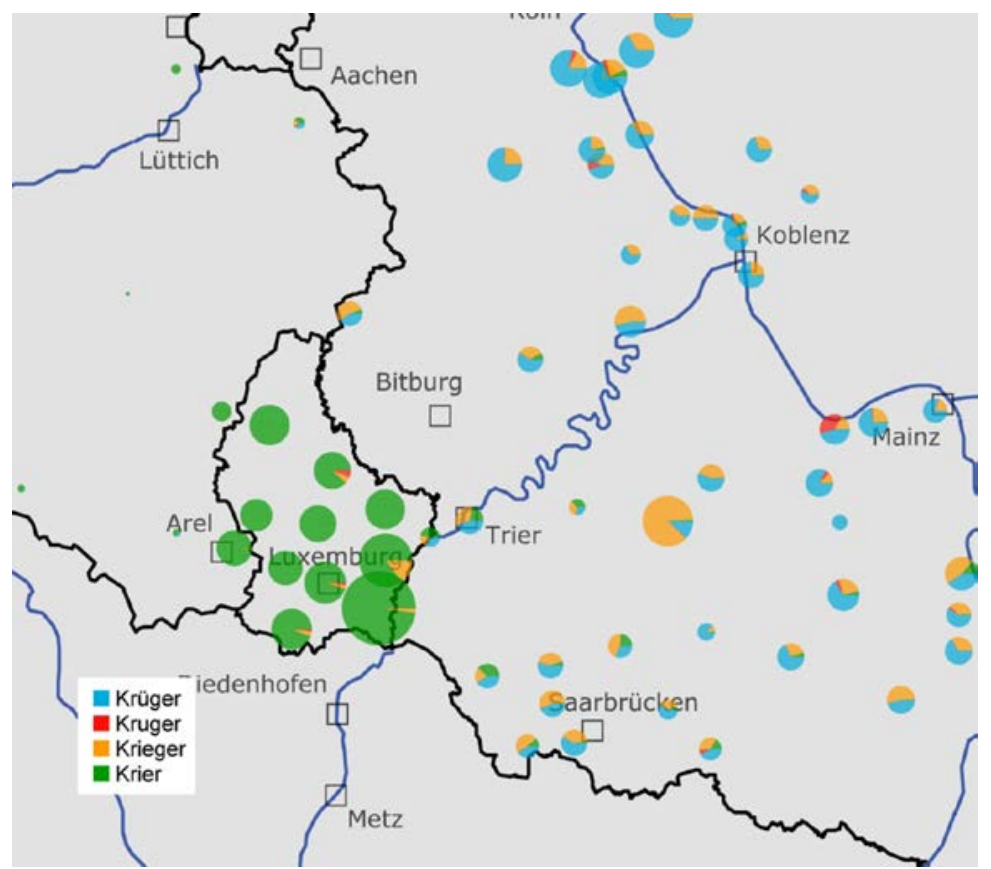

Karte 12: $\quad$ Krüger, Kruger, Krieger, Krier (3.1.4.5 und 3.2.3)

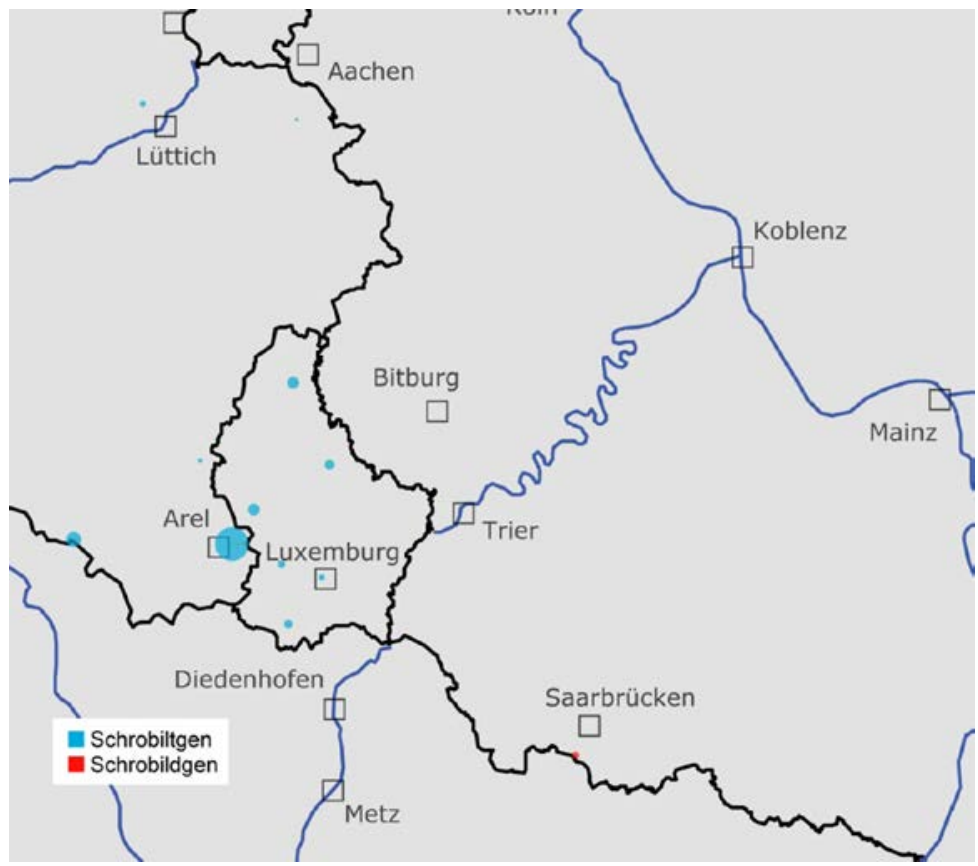

Karte 13: $\quad$ Schrobiltgen, Schrobildgen (s. 3.1.5) 


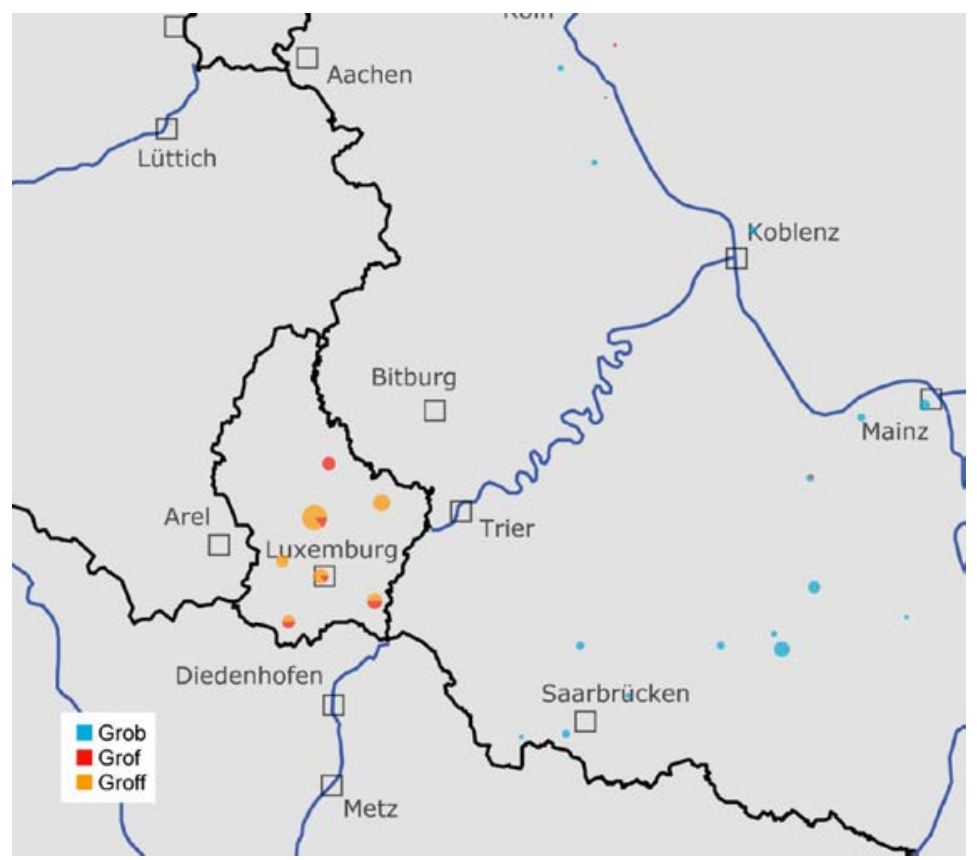

Karte 14: Grob, Grof, Groff (s. 3.2.1)

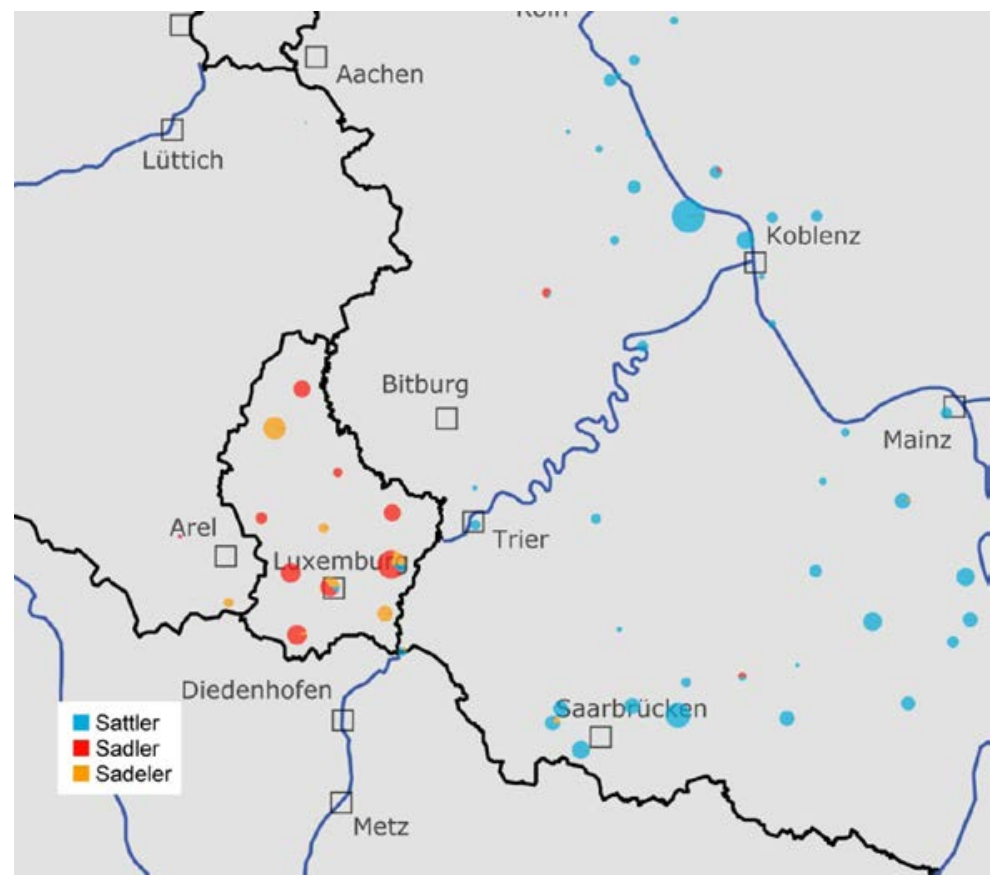

Karte 15: $\quad$ Sattler, Sadler, Sadeler (s. 3.2.2) 


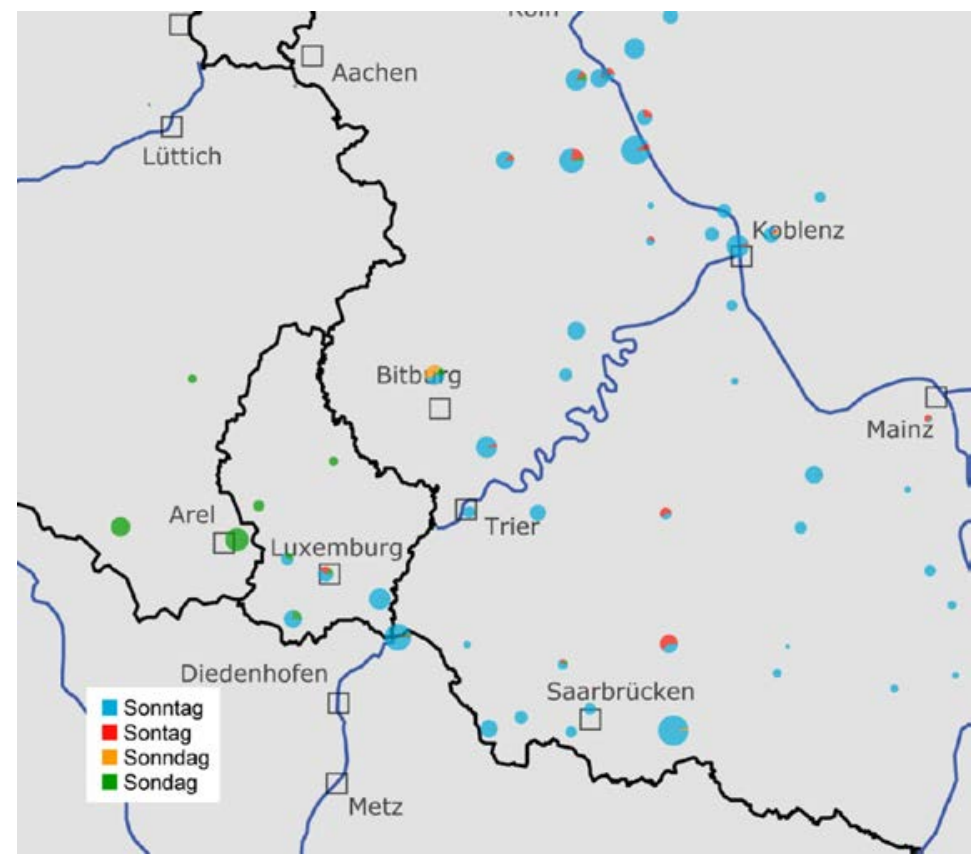

Karte 16: Sonntag, Sontag, Sonndag, Sondag (s. 3.2.2)

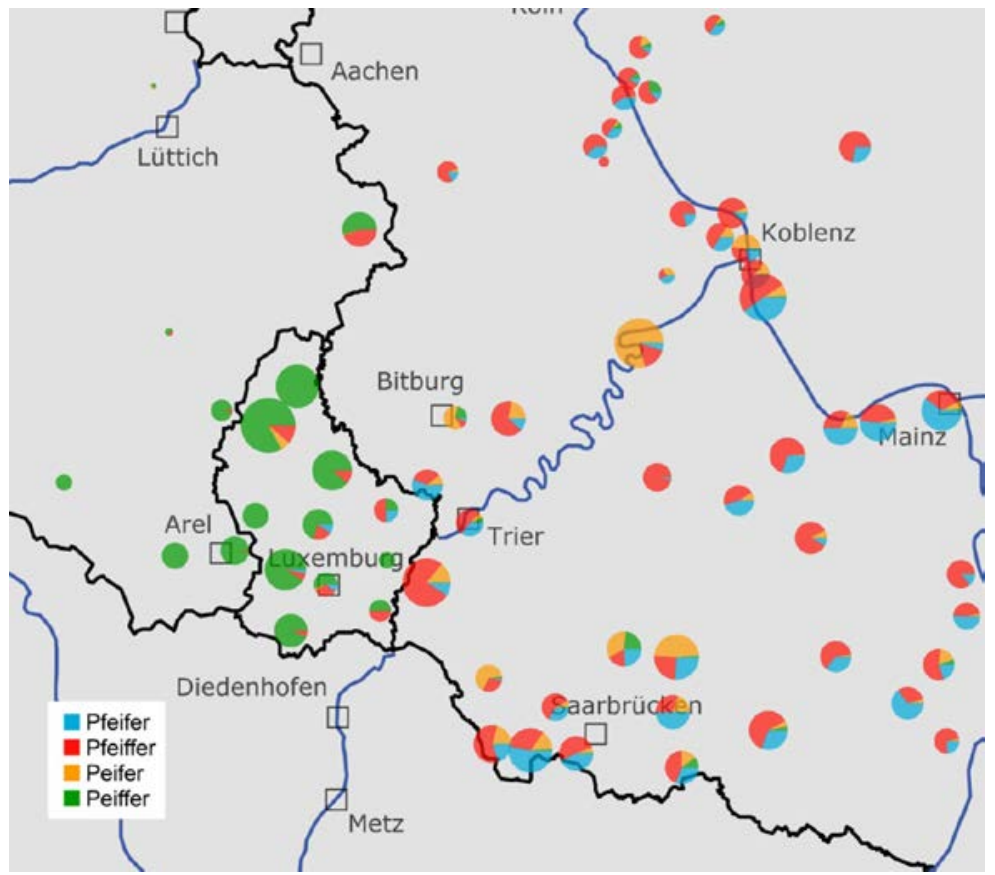

Karte 17: $\quad$ Pfeifer, Pfeiffer, Peifer, Peiffer (s. 2.2.3 und 3.2.4) 


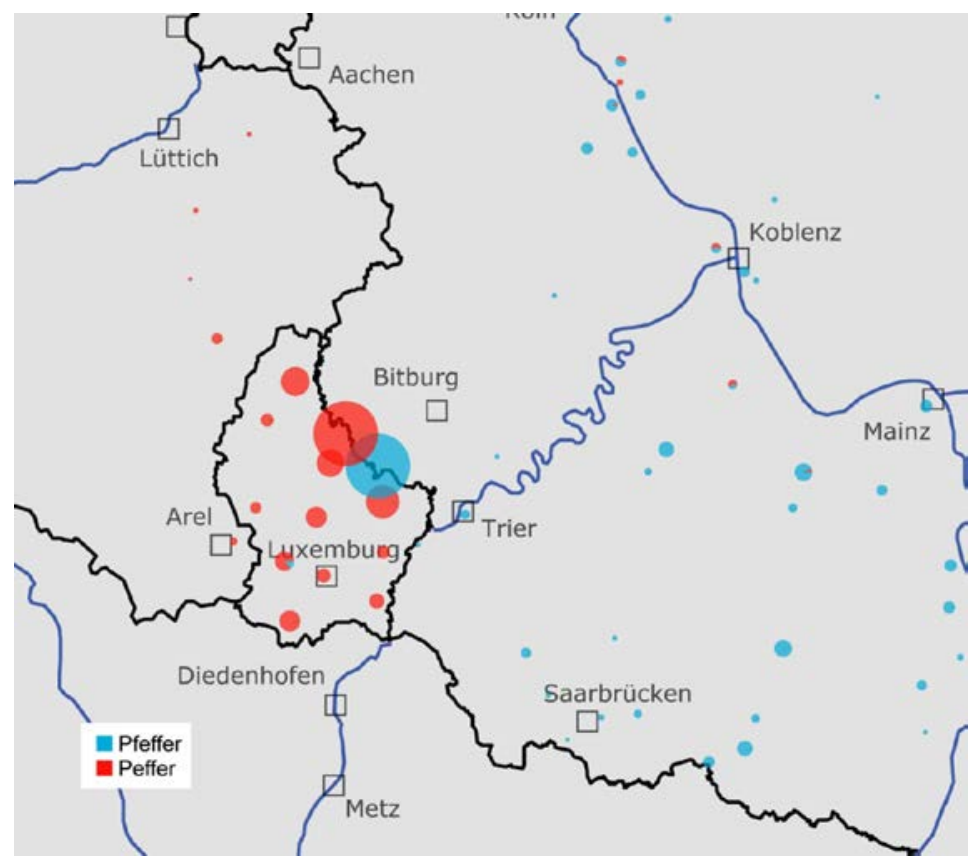

Karte 18: $\quad$ Pfeffer, Peffer (s. 3.2.4)

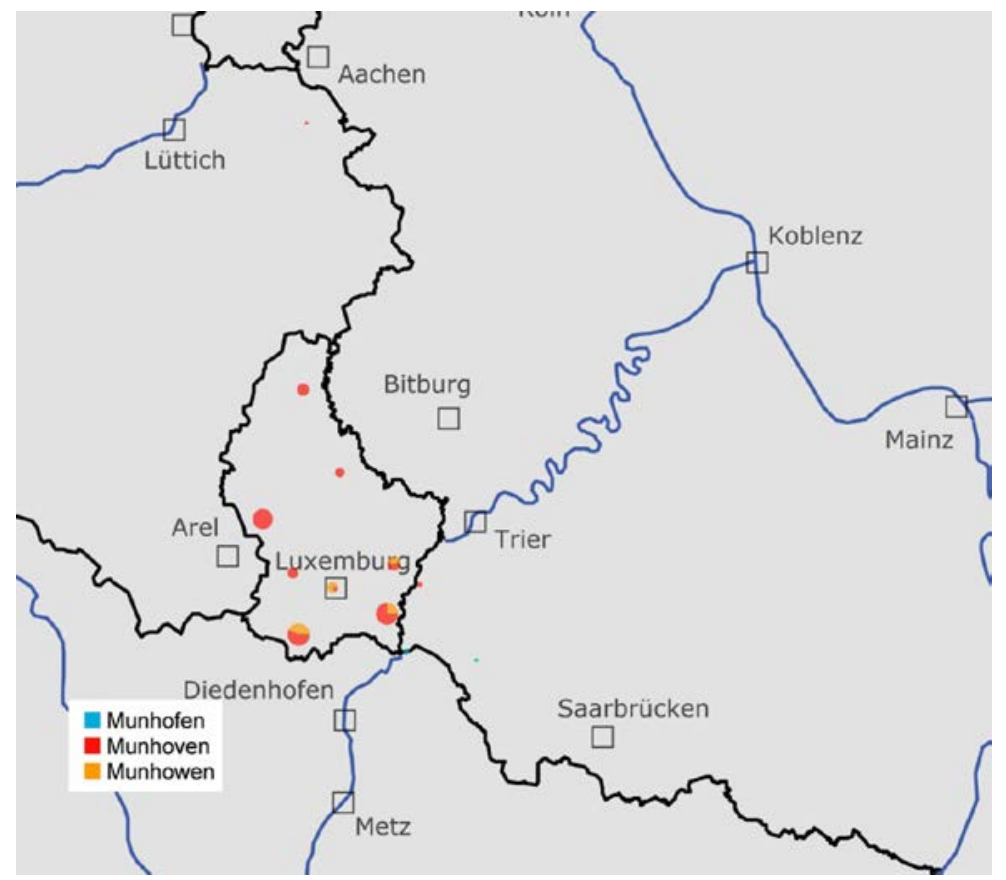

Karte 19: $\quad$ Munhofen, Munhoven, Munhowen (s. 3.2.5) 


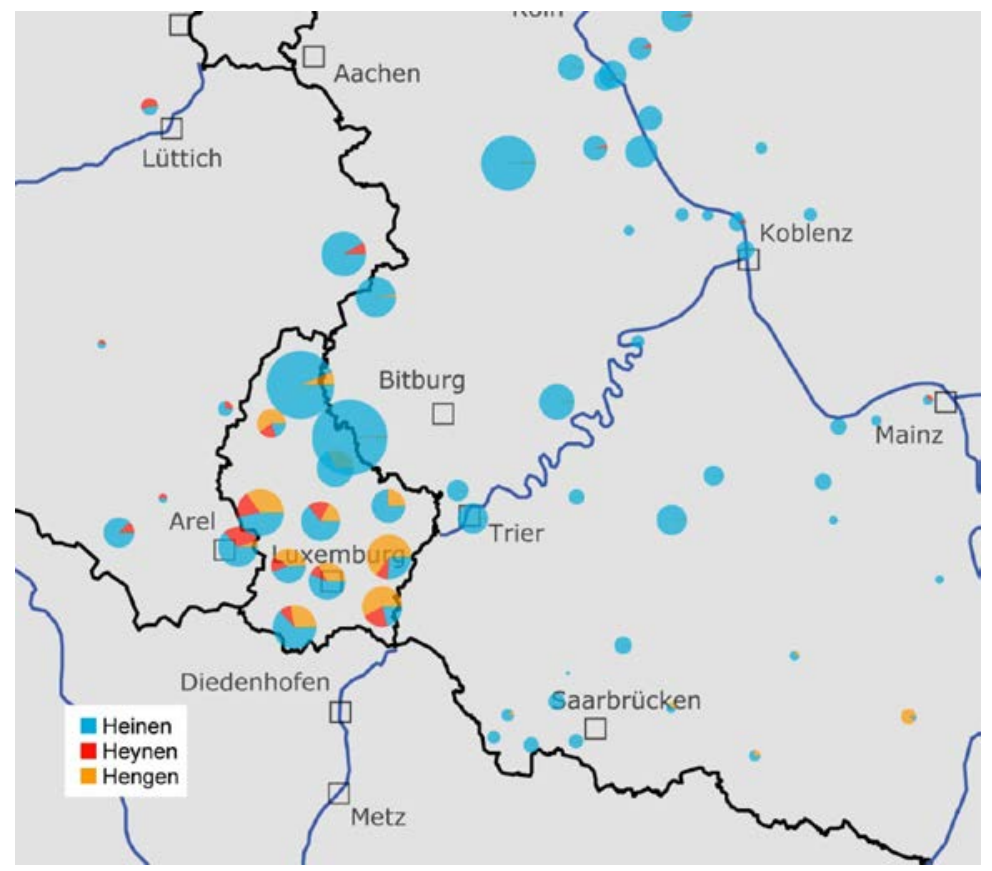

Karte 20: Heinen, Heynen, Hengen (s. 3.2.6) 



\title{
Les noms de famille en images: les interférences germaniques dans les noms français du, Luxemburgischer Familiennamenatlas
}

\author{
Claire Muller
}

\begin{abstract}
An den Familiennamen zwischen Maas und Rhein lassen sich Sprachkontaktphänomene besonders gut und häufig beobachten. In Luxemburg ist der Kontakt zwischen der französischen und der hochdeutschen Sprache umso intensiver, da im historisch germanophonen Luxemburg sowohl das Französische als auch das Hochdeutsche als Verwaltungssprache dien(t)en. Der folgende Beitrag untersucht die Germanisierung französischer Namen in Luxemburg und versucht mit Hilfe von Karten deren Regionalität aufzuzeigen.
\end{abstract}

\section{Aperçu quantitatif}

Les noms de famille dans les territoires entre Meuse et Rhin constituent un fonds particulièrement riche pour l'étude des phénomènes de contacts linguistiques. Le contact entre le français et le haut-allemand a été d'autant plus fréquent au Luxembourg que ces deux langues ont servi et servent encore de langues administratives, le luxembourgeois développant encore le statut d'une langue écrite et normée. Les deux corpus principaux du projet $\mathrm{LFA}^{1}$, l'un historique (recensement de la population en 1880), l'autre contemporain (annuaire téléphonique de 2009), livrent ainsi une série intéressante de noms de famille français transformés au contact de l'allemand. En outre de la présentation quantitative et étymologique des noms de famille analysés, cette contribution proposera pour chaque nom attesté dans le corpus de 2009 une carte, afin de vérifier le caractère régional de ces noms et de confronter leur distribution géographique à l'étymologie retenue.

La base de données ,2009 contient $0,14 \%$ de noms de famille français, germanisés ou non, sur le total des noms de famille. La plupart de ces noms de famille sont arrivés au Luxembourg à une époque récente; ainsi, un tiers de ces noms se trouvaient déjà dans le corpus , $1880^{\circ}$. Des sources complémentaires remontant au XVII ${ }^{e}$ siècle, comme le recensement des feux de 1656, montrent toutefois que les noms français ont une longue histoire sur le territoire luxembourgeois. Ainsi, parmi les 65 noms français recensés en 1656, sept figurent encore aujourd'hui parmi les dix noms français les plus fréquents du Luxembourg: Bernard, Clément, Colin (< Nicolas), Georges, Lambert, Martin et Mathieu.

En ce qui concerne les ressemblances et différences du paysage anthroponymique luxembourgeois avec les territoires voisins (aujourd'hui) francophones, nous avons pu montrer dans Muller (2013) qu'il faut différencier les noms à haute fréquence des noms à basse fréquence. Le pays d'Arlon en Belgique, se rapprochant du Luxembourg par

1 Pour une présentation globale du projet et des corpus, ainsi que des outils et des conventions de cartographie, voir la contribution de Peter Gilles dans cet ouvrage. 
une histoire et une langue communes, partage surtout des noms moyennement et peu fréquents avec le Luxembourg (notamment les noms marqués dialectalement). Les noms de famille à haute fréquence sont presque tous français, témoins de l'arrivée massive d'une population francophone au XIX ${ }^{\mathrm{e}}$ siècle dans le Areler Land. Le département de la Moselle a en commun avec le Luxembourg les noms de famille allemands qui représentent, comme au Grand-Duché, l'écrasante majorité des anthroponymes. Il ne compte par contre qu'une quantité infime de noms issus de parlers dialectaux, romans ou germaniques. Cependant, le Luxembourg, le pays d'Arlon et le département de la Moselle se réunissent par leurs deux noms les plus fréquents qui sont, comme en Allemagne, Schmi(d)t et Müller. Le Luxembourg, quant à lui, se différencie de ses voisins par la présence de noms français germanisés.

\section{Les différents types d'adaptations}

Le modèle de communication structuraliste est à la base de la définition de l'interférence linguistique. En effet, comme chaque énoncé appartient à une langue naturelle, il permet de considérer ,qu'un énoncé peut contenir plusieurs éléments appartenant à une autre langue que le reste de l'énoncé" (Weinreich 1977:23). L'interférence linguistique, „accident de bilinguisme entraîn[é] par un contact entre les langues“ (Debyser 1970:34), peut ainsi être définie très simplement comme „l'influence réciproque de structures appartenant à deux systèmes linguistiques différents"33 (Czochralski 1971:5). L'interférence peut être positive ${ }^{4}$ quand la reprise d'éléments étrangers apporte un enrichissement de la langue de départ, un phénomène qui concerne surtout les transferts lexicaux. Dans le cas spécial de l'anthroponymie, l'intégration de noms de famille français au luxembourgeois pourrait globalement constituer un tel transfert positif. L'interférence est négative lorsque le contact interlangue a comme conséquence des erreurs dans la langue de départ, phénomène auquel sont confrontés tous les apprenants d'une langue étrangère. Les interférences phonétiques appartiennent à ce deuxième cas de figure. En effet, l'étude de l'interférence phonétique décrit et analyse

„la manière dont un locuteur perçoit et reproduit les phonèmes d'une langue selon le modèle d'une autre. [...] L'interférence intervient lorsqu'un locuteur bilingue identifie un phonème du système secondaire avec un phonème du système primaire et qu'il soumet lors de la production ce phonème aux règles du système primaire“, (Weinreich 1977:30). ${ }^{5}$

„[...] eine Äußerung einige Elemente enthält, die zu einer anderen Sprache gehören als der Rest der Äußerung“.

3 „[...] die gegenseitige Einwirkung und Beeinflussung von Strukturen, die zwei verschiedenen Sprachsystemen angehören“.

4 C'est ce que Weinreich appelle le transfer.

5 ,[...] die Art und Weise, wie ein Sprecher die Laute der einen Sprache [...] nach dem Muster der anderen wahrnimmt und reproduziert [...]. Interferenz tritt ein, wenn ein Zweisprachiger ein Phonem des Sekundärsystems mit einem des Primärsystems identifiziert und es bei seiner Hervorbringung als Laut den phonetischen Regeln der Primär-sprache unterwirft.“ 
Ainsi, un nom de famille français modifié phonétiquement au contact du luxembourgeois, présente le cas d'une interférence négative. Les noms de famille français du Luxembourg montrent des interférences à différents niveaux. La germanisation de la prononciation du nom dans tout énoncé oral en luxembourgeois est l'interférence la plus systématique; l'accentuation sera déplacée vers l'avant: fr. Meunier [møn'je] < lb. Meunier ['mønje], la neutralisation germanique (Weijnen 1964) s'appliquant systématiquement (fr. Georges [30R3] < lb. Georsch [30RS]). À l'écrit, certaines modifications peuvent intervenir afin de rapprocher un nom des usages d'écriture allemands, cependant nous n'avons trouvé que de rares exemples où cette adaptation graphique intervient sur un phonème non modifié phonétiquement: fr. Vercollier [venkol'je] $<\mathrm{lb}$. Wercollier [ver'kolje]. Dans les noms de famille fr. Vidon $<1 \mathrm{~b}$. Widong ou fr. Collin $<1 \mathrm{lb}$. Kolling, la prononciation de la première lettre ne change pas par le changement de graphie, mais la prononciation du nom est de toute façon modifiée par la germanisation du suffixe nasal.

Certaines interférences phonétiques du luxembourgeois dans les noms de famille d'origine française peuvent se traduire au niveau graphique. Ces phénomènes de contact d'abord oraux ont donc dû être particulièrement fréquents pour devenir visible à l'écrit. Les noms de famille relevés dans les corpus du projet LFA témoignent des phénomènes consonnantiques et vocaliques suivants:

- la spirantisation de [j],

- la neutralisation germanique

- par assourdissement sonore de [d] final,

- par assourdissement de [v] final,

- par assourdissement de la fricative post-alvéolaire [3] finale,

- l'occlusion de [s],

- l'allongement de la diphtongue [wa] $<$ [ua:],

- la perte de la nasale, remplacée par une voyelle courte suivie de la vélaire [ $\mathrm{y}]$.

A cette liste peut s'ajouter éventuellement la fricatisation intensifiée de la chuintante [J], un phénomène qui s'observe à l'écrit (Laroche > Larosch), mais dont l'emploi à l'oral reste à prouver.

\section{La spirantisation de $[j]$}

En français moderne, une légère spirantisation peut être constatée auprès de consonnes sourdes suivis d'un [j] suivi d'une voyelle. Cette spirantisation a été renforcée lors de l'adaptation du nom de famille français en luxembourgeois, surtout pour les noms à suffixe [je] (graphie ,ier') ${ }^{6}$ :

6 Comparer aussi: fr. n.m. quartier > lb. n.m. Quärtchen, fr. n.m. cloutier > lb. n.m. Kläutchen (LWB). Pour une analyse plus détaillée de la spirantisation, voir Muller (2012). 
Scharpantgen (1880:0, 2009:19), Scharpentier (1880:8, 2009:0), Charpentier (1880:62, 2009:39), Charpantier (1880:4, 2009:6)

Scharpantgen [JaR'panteən] est la germanisation de Charpentier, nom de métier français très fréquent. Scharpantgen témoigne non seulement de la substitution du suffixe français [je] par le suffixe luxembourgeois [(t)6ən], mais également de la dénasalisation de la voyelle accentuée. Charpentier est plus fréquent au Luxembourg que sa variante germanisée qui n'est relevée qu'une première fois lors d'un recensement en 1930 et semble être une forme très jeune. Scharpantgen (en bleu) se concentre dans le sud du Luxembourg, là où se trouve également la majorité des formes Charpentier (en rouge), ainsi que la variante très rare Charpantier (non représentée). La forme picarde Carpentier (en jaune) est dans le nord de la France et en Wallonie plus répandue que Charpentier, elle est répandue jusque dans le Pays d'Arlon et l'ouest du Grand-Duché du Luxembourg. Cependant, c'est bien la forme française standard qui est à l'origine du nom luxembourgeois Scharpantgen.

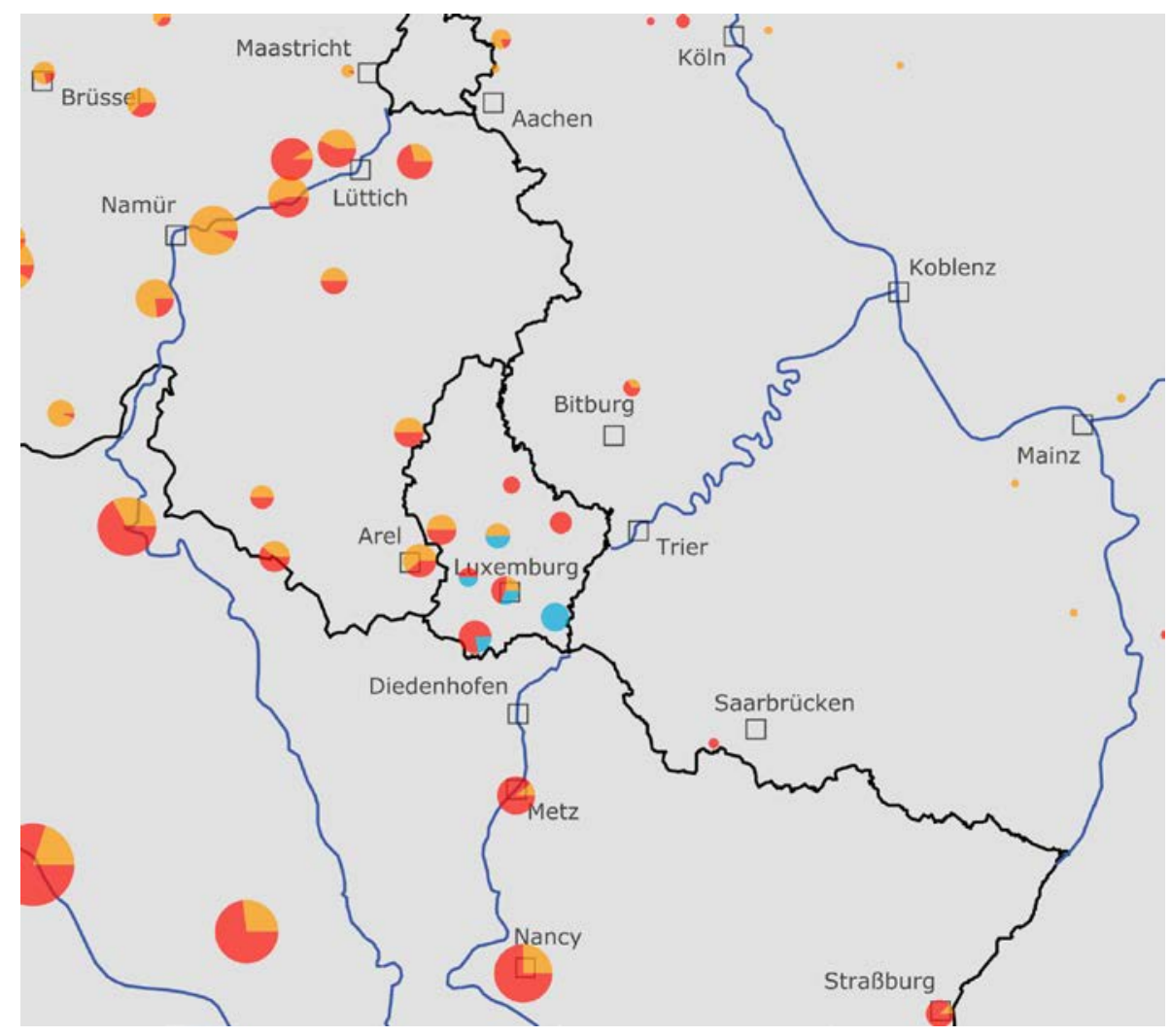

Carte 1: $\quad$ Scharpantgen (bleu), Charpentier (rouge), Carpentier (jaune)

7 Nous indiquerons à côté du nom le nombre d'occurrences du nom dans les deux corpus principaux du LFA (1880 et 2009). 
Kapgen (1880:8, 2009:19), Capgen (1880:2, 2009:0)

L'étymologie de Kapgen, ['kapsən] renvoie le plus probablement au diminutif du n.f. all. Kappe ,bonnet' (lb. n. f. Kap ${ }^{8}$ ) qui désigne le fabricant ou le porteur de celui-ci; l'absence de Umlaut demande toutefois à être analysée davantage. La germanisation du nom de famille Capier, issu ou bien nom de profession pour le fabricant ou le porteur de capes, ou bien de la forme picarde de Chapier, qui provient du nom de profession pour le fabricant de chapes, auraient également pu être à l'origine de Kapgen, même si la rareté du nom $\mathrm{Ca}$ pier ne plaide pas en faveur d'une origine française. Kapgen (en bleu) et Kap (en rouge) se concentrent au Luxembourg. Capier (en jaune) se trouve en Belgique uniquement à Liège, et compte-tenu des intenses migrations entre Liège et le Luxembourg, Capier ne peut pas être exclu avec certitude comme origine de Kapgen. Les rares occurrences de Chapier sur le territoire allemand ne peuvent malheureusement pas être visualisées.

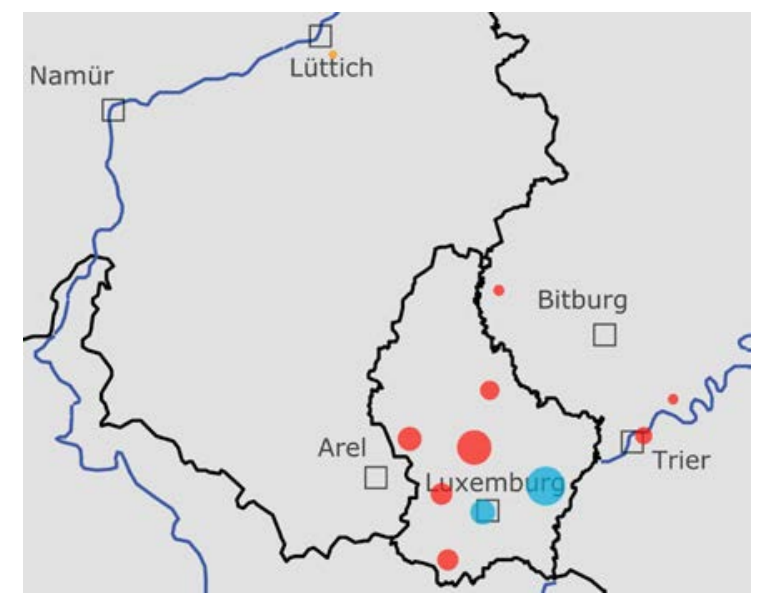

Carte 2: $\quad$ Kapgen (bleu), Kap (rouge), Capier (jaune)

\section{Haustgen (1880:12 occurrences, 2009:3 occurrences)}

Si phonétiquement, le nom Haustgen, prononcé ['haustcən ou 'hæ:ustcen], pourrait être expliqué par une étymologie française, et si la géolocalisation du nom germanisé et de son original français est proche, les relevés dans des sources historiques du XVIII et du $\mathrm{XV}^{\mathrm{e}}$ siècle créent un doute. En effet, le nom de métier Houssier, [hus'je], qui désigne le marchand ou le fabricant de housses (pour chevaux), se transformerait par adaptation $\mathrm{du}$ suffixe français [je] en lb. [tcən] et par la diphtongaison de [u] en [au] (comparer lb. Kläutgen > fr. cloutier) en ['haustcən]. Cette explication élargirait aussi l'adaptation par spirantisation à des suffixes [je], qui ne sont pas introduits par consonne occlusive sourde ([p], $[\mathrm{t}],[\mathrm{g}]$ ), mais par consonne fricative [s].

8 Les exemples du lexique luxembourgeois sont repris, dans leur orthographe originale, du dictionnaire LWB, consultable en ligne sur infolux.uni.lu. 
Houssier n'est pas localisable au Luxembourg; en Belgique, le nom occupe un territoire à l'ouest de Roubaix jusqu'à Bertrix et Liège. En France, le nom est confiné à l'ouest sans continuation avec la présence du nom en Belgique. Houssier est ainsi proche du Luxembourg, sans être présent ni dans le pays d'Arlon ni au Luxembourg même. Haustgen quant à lui, se retrouve uniquement au centre du Grand-Duché.

Finalement, ce sont les occurrences du nom Haustgen dans des sources plus anciennes qui invitent à prendre en considération deux autres étymologies, germaniques cette-fois. Hauschtges relevé en 1722 (Deltgen 2012) serait un argument en faveur de l'étymologie proposée par Debrabandere 1993:651 qui explique le nom par un diminutif du francique Haust, lb. Hauscht ,tas de foin'; ce serait donc un nom caractérisant l'habitation. Le nom Haust ne peut toutefois pas être situé à proximité du Luxembourg. Les occurrences relevées dans les livres de compte (Gniffke 2011) du XV'e siècle ouvrent l'étymologie à une troisième possibilité: huschin, husgin, husgün, hußges pourraient représenter des formes encore non diphtonguées de Haustgen et provenir ainsi d'un diminutif de moyen-haut-allemand hüze (hiüze) ,gai, insolent‘ pour former un surnom se référant au caractère léger du porteur.

\section{Etgen (1880:24, 2009:10), Étienne (1880:22, 2009:22)}

Ce nom de famille se reconstruit également à partir d'une forme française spirantisée: fr. Etienne [et'jen] < lb. Etgen ['ətcən]. La particularité de cette adaptation est qu'il ne s'agit pas d'un [e] fermé dans le suffixe comme dans [je], mais bien d'un [ع] ouvert, suivi d'une consonne. Cependant, la spirantisation transforme ici également [jen] $>[(\mathrm{t}) 6 ə n]$. Etienne, et sa variante Estienne (en rouge), se retrouvent partout dans les aires francophones, ainsi qu'au nord et au sud-ouest du Luxembourg. La forme germanisée Etgen (en bleu) est ancrée au Luxembourg et, du côté allemand, le long de la Moselle.

\section{Malget (1880:85, 2009:65), Maillet (1880:23, 2009:17), Mailliet (1880:54, 2009:53)}

Transmis par le wallon et germanisé au Luxembourg, ce nom, dont une explication plus détaillée peut être trouvé dans Muller (2012), est un témoin particulier du contact linguistique entre le luxembourgeois et ses langues voisines. En effet, pour que le nom français Maillet [ma'je] soit adapté en Malget ['malze], il a fallu appliquer la prononcia-

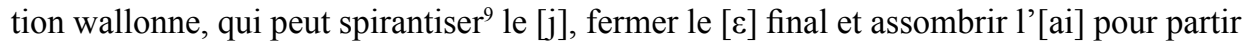
d'une forme [mal'je], qui suit alors la même adaptation en luxembourgeois déjà observée devant des consonnes occlusives sourdes. Le mécanisme d'adaptation fonctionne donc aussi devant consonne liquide. La fricative originale étant sonore, elle est voisée: [je] > [3e]: [mal'je] < ['malze]. Si la forme standard Maillet (en jaune) connaît une distribution géographique étendue, sa version germanisée Malget (en bleu) ne peut être trouvée qu'au Luxembourg. Une forme Mailliet (en rouge), dont la graphie témoigne éventuellement d'une étape intermédiaire de la spirantisation, est également confinée au Luxembourg.

9 La lexicographie wallonne ne nous a malheureusement pas encore permis de découvrir d'attestations convaincantes. Alors qu'en fin de mot, [1] peut être non spirantisé dans certaines variantes du wallon: fr. famille = w. famil [famil]; mais fr. bouteille = w. botaye [botzj], en milieu de mot par contre, le [1] semble plutôt s'amenuiser: fr. paille =w. paye [paj]; fr. village = w. viyaedje [vijatf]. 


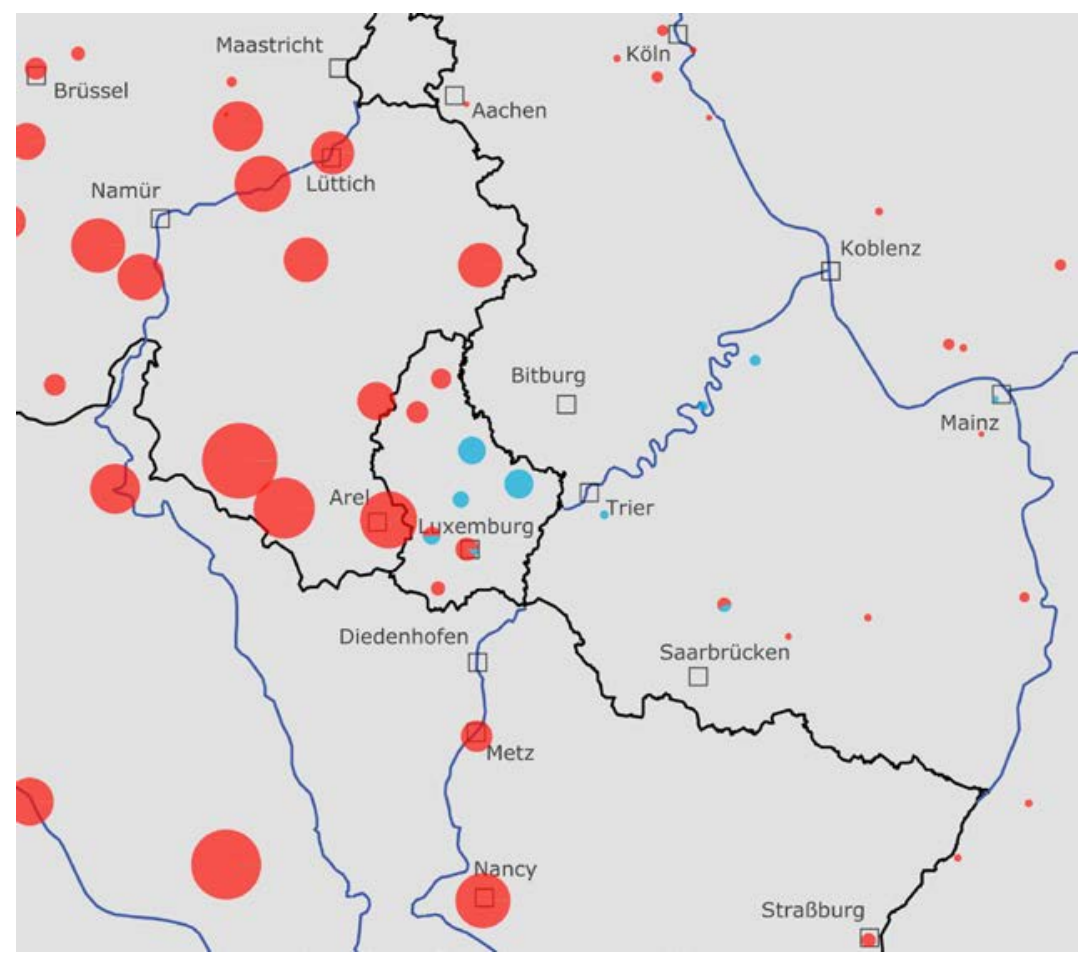

Carte 3: $\quad$ E(s)tienne (rouge), Etgen (bleu)

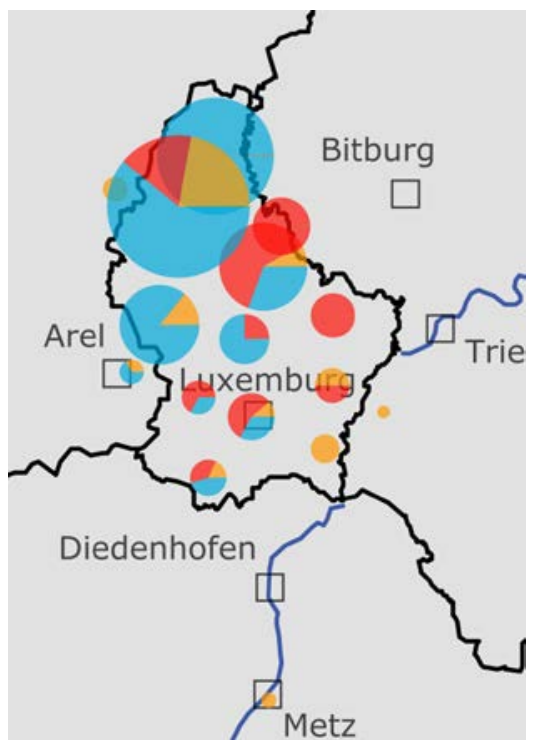

Carte 4: $\quad$ Maillet (jaune), Malget (bleu), Mailliet (rouge) 
Rosseljong (1880:18, 2009:6), Rossillion (1880:6, 2009:0)

En prenant en considération les mécanismes d'adaptation par spirantisation, le nom luxembourgeois Rosseljong (en bleu), prononcé ['Rosəlzon], situé dans le sud-ouest et le centre du Luxembourg, ainsi que dans le Pays d'Arlon, a certainement une étymologie française. Une base étymologique valide constitue wall.-lorr. rossillion $<$ a.fr. part. passé rossi ,rougi ${ }^{6}+$ diminutif [õ] , le petit roux'. Rossillion (en rouge) existe, bien que rarement en Lorraine (6 naissances en 1891-1915 dans les départements d'Ardennes, Meuse et Meurthe-et-Moselle). Le nom d'origine française Roussillon, qui se concentre autour du canton du même nom (sud-est de la France), pourrait s'adapter également en Rosseljong, en ajoutant l'abaissement du [u] vers [o] opéré pendant la période du moyen-haut-allemand (voir ci-dessous), mais aucune continuité géographique n'est observée entre Roussillon et Rosseljong.

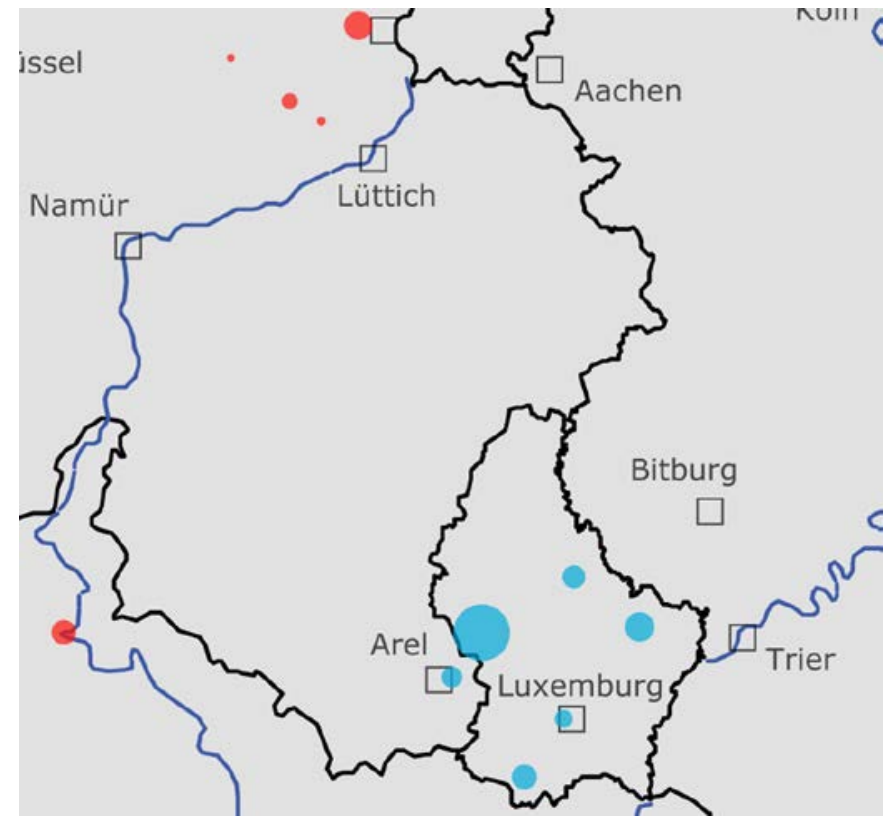

Carte 5: $\quad$ Rosseljong (bleu), Rossillion (rouge)

Jopa (1880:10, 2009:14), Duhautpas (1880:0, 2009:7)

Afin de remonter à l'étymologie de ce nom de famille, il faut inverser plusieurs mécanismes de transformation. En effet, la base généalogique Deltgen 2012 montre les différentes étapes de l'adaptation du nom Duhautpas [dyho'pa] vers Jopa ['zopa:], notamment l'apocope de la préposition $d u$ : Duhautpas $(1672)=>$ Hiaupas $(1751)$, et la palatalisation de [ho] vers [hio]: Hiaupas (1751). La spirantisation de [hio] < [30] donne enfin Jopa (1730). La palatalisation [ho] vers [hio] semble néanmoins être un phénomène unique. L'influence du wallon permet de considérer une autre explication pour la forme Hiaupas: en partant d'une forme wallone wa. *Dihautpas, la 
contraction et ensuite l'apocope de l'article permet de passer à * Dhiaupas > Hiaupas. La localisation ne montre aucune continuité entre les occurrences du nom Duhautpas (en rouge) dans le nord de la France (15 porteurs de 1966-1990) et ceux du nordouest du Luxembourg. La forme germanisée Jopa (en bleu) est localisée uniquement au Luxembourg.

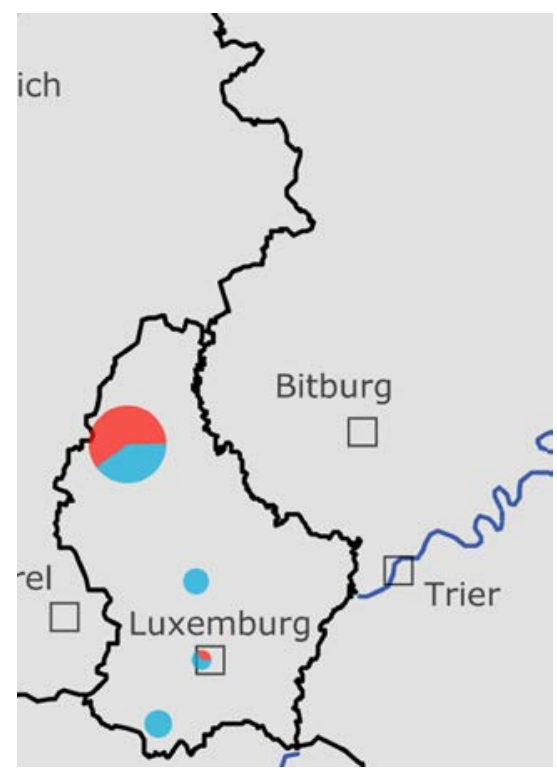

Carte 6: Jopa (bleu), Duhautpas (rouge)

\section{La neutralisation germanique par assourdissement du [d] final}

En français, la différenciation entre les consonnes constrictives sourdes et sonores est phonologiquement significative (p. ex. fr. cage vs. fr. cache). En moyen-haut-allemand par contre, et donc en luxembourgeois également, cette opposition est neutralisée en position finale en faveur de la constrictive sourde avec une telle systématicité que „la neutralisation généralisée de toutes constrictive et fricative sonores finales s'est traduite dans une règle de la langue allemande standard. “10 (Wängler 1960:67). Cette règle de la neutralisation germanique concerne également les fricatives sonores finales, voir Meibauer 2002 pour un aperçu général.

Si dans le lexique luxembourgeois, cette neutralisation est observée souvent à l'oral mais rarement à l'écrit (par exemple fr. étude [e'tyd] < lb. Etüd [e'tyt]), il en est de même pour les noms de famille, où un seul cas relevé dans le corpus est susceptible de reproduire ce trait d'interférence.

$10,[\ldots]$ die im Deutschen allgemeine Auslautverhärtung aller stimmhaften Verschluß- und Reibelaute gar in einer Regel der deutschen Hochsprache niedergeschlagen [hat]“. 
De la Hamette (1880:0, 2009:18), Delahamette (1880:0, 2009:5)

Ce nom d'origine n'existe avec le suffixe «-ette` qu'au Grand-Duché du Luxembourg (en bleu: Delahamette, en rouge: De la Hamette). Le nom est une forme germanisée de de la Hama(i|y)de, qui remonte à Lahamaide (La Hamaide), un village dans le Hainaut belge, fief des seigneurs de La Hamaide, anoblis au XIII ${ }^{\mathrm{e}}$ siècle. En Belgique, les variantes De la Hamayde (en jaune), de la Hamaide (non représentée) et de l'Hamaide (non représentée) sont très rares et aucun porteur n'est recensé près du lieu d'origine du nom. Seul le nom Hamaide est assez fréquent et répandu à la fois en Belgique, mais également en France. L'interprétation du nom est cependant problématique à cause d'un nom marocain très semblable (Hamaïde).

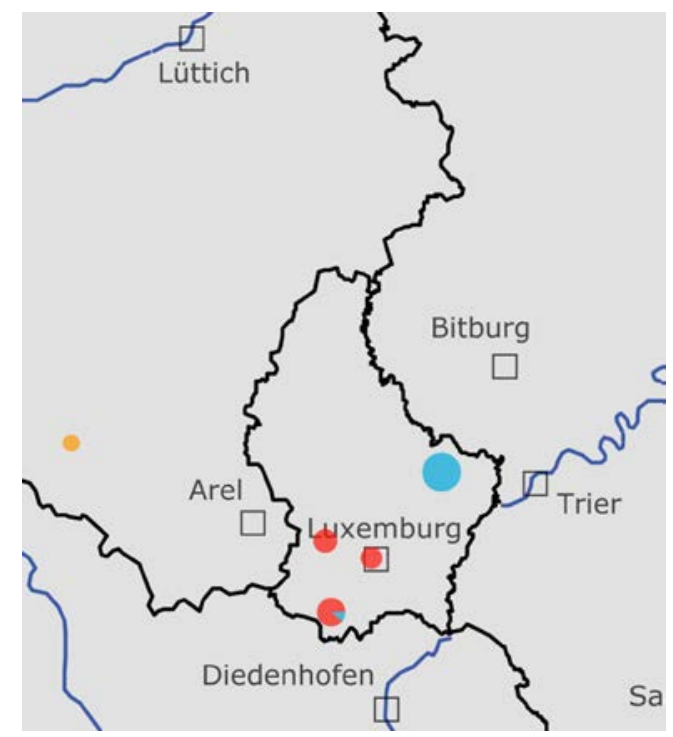

Carte 7: $\quad$ Delahamette (bleu), De la Hamette (rouge), De la Hamayde (jaune)

\section{La neutralisation germanique par assourdissement du [v] final}

Pour ce phénomène d'interférence, un exemple seulement a pu être relevé dans le corpus. Dans le lexique, les exemples trouvés ne sont également pas nombreux et ils ont tous conservé la graphie 〈V〉, par exemple fr. betterave $>1 \mathrm{lb}$. Betterav, fr. grave $>\mathrm{lb}$. grav, fr. octave $>$ lb. Octav (LWB).

\section{Lakaff (1880:54, 2009:8), Lakaf (1880:4, 2009:2), Lacave (1880:33, 2009:7)}

Les deux variantes proviennent de la germanisation du nom Lacave, nom qui désigne un lieu d'habitation. Il faut comprendre cave au sens topographique comme un endroit creux, un fossé. Les variantes germanisées (Lakaff en bleu, Lakaf non visible à cause de seulement deux occurrences) montrent par rapport au modèle français en outre de l'assourdissement 
de [v], l'allongement du deuxième [a] ainsi que l'avancement de l'accent: ['laka:f]. Elles apparaissent, à l'exception de Lakaf dans la Sarre, uniquement au Luxembourg. Lacave (en jaune) montre une distribution en deux grands ensembles indépendants: le plus grand se trouve dans le sud-ouest de la France, le deuxième recouvre le nord-est de la France. Quelques porteurs se retrouvent également au Luxembourg; nous avons ainsi indiqué la présence sur le même territoire à la fois de la forme française et de la forme germanisée correspondante.

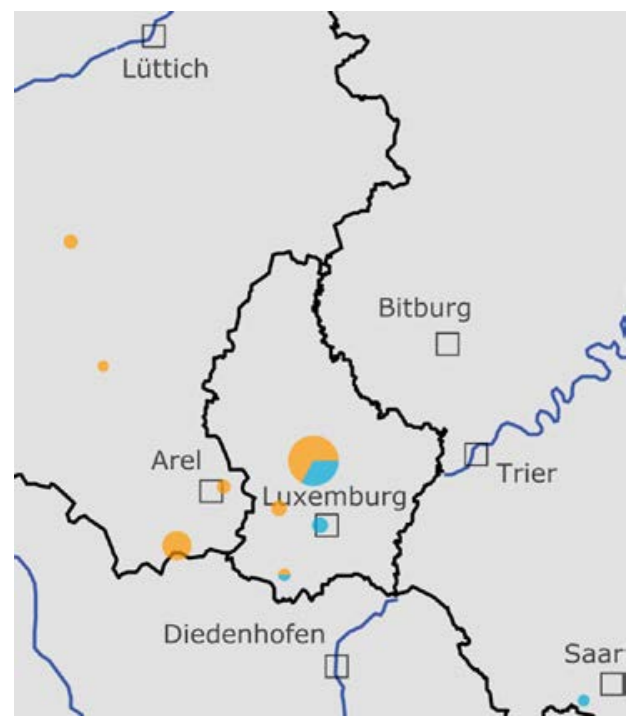

Carte 8: Lakaff (bleu), Lacave (jaune)

\section{La neutralisation germanique par désonorisation de la fricative sonore finale}

$\mathrm{Au}$ contact des locuteurs germaniques, les fricatives finales des noms français sont désonorisées. Ce type d'interférence se traduit dans le lexique dans de nombreux exemples à l'écrit, comme: fr. bagage $>\mathrm{lb}$. Bagaasch, fr. colportage $<\mathrm{lb}$. Kolportaasch, fr. contre-plaquage $>$ lb. Konterplackaasch, fr. ménage > lb. Mënaasch, fr. ravage > lb. Rawaasch, e.a. (LWB).

\section{Parrasch (1880:12, 2009:8), Parrache (1880:2; 2009:0), Parage (1880:4; 2009:15)}

Le nom de famille français Parage est à l'origine des formes germanisées Parrasch et Parrache. Ces deux noms montrent des degrés différents d'interférence; si le suffixe français 〈-age〉 est assimilé phonétiquement et graphiquement dans Parrasch, dans Parrache, la fricative est reproduite à l'écrit par un suffixe français. Cette forme n'est pas attestée dans le corpus le plus récent. Le dédoublement du 〈r〉 pose question; il semble cependant exprimer une prononciation avec une voyelle finale brève ['para:f], contrairement aux exemples du lexique se terminant en [a:]. Le nom de famille appartient à la catégorie des noms d'habitation, comme en témoignent les noms de divers hameaux 
surtout dans le sud-ouest de la France. L'origine du toponyme Parage remonte à l'ancien français et occitan parage, paraige n.m. ,haute naissance'. C'est surtout un terme juridique dans le système féodal, qui désigne un mode de légation où les biens du père sont partagés entre tous les enfants, contrairement au droit d'aînesse. ${ }^{11}$ Le toponyme Parage désigne ensuite des biens obtenus par parage. L'explication de l'origine de Parrasch et Parrache est cependant quelque peu remise en doute par la géolocalisation ${ }^{12}$ des noms: Parrasch (en bleu) n'existe qu'au Grand-Duché du Luxembourg, tandis que Parage (en rouge) n'existe que rarement (4 porteurs) dans la Province de Luxembourg très près de la frontière luxembourgeoise. Même si le nom est présent dans les Ardennes, c'est dans le sud-ouest de la France que le nom est de loin le plus fréquent. La carte semble ainsi suggérer que la Province de Luxembourg soit à l'origine de l'importation du nom, car c'est dans cette zone de contact que le nom est aujourd'hui le mieux attesté. Toutefois, seule une étude généalogique pourrait éclaircir les circonstances exactes de ce contact.

\section{Delosch (1880:9; 2009:10), Deloge (1880:3; 2009:9)}

Deloge est un des deux noms de notre inventaire ne se terminant pas sur une syllabe finale en [a], mais en [o]. Le nom désigne l'origine géographique du porteur et se réfère au nom de lieu Loge, fréquent dans le nord de la France. Le toponyme est également fréquemment attesté comme nom de hameaux et de lieux-dits. Le toponyme et le nom de famille existent également au pluriel: top. Les Loges, anthrop. Deslosges. L'étymologie du toponyme remonte à a.fr. loge < germ. laubja, hutte, cabane'. Au contact des locuteurs allemands, le

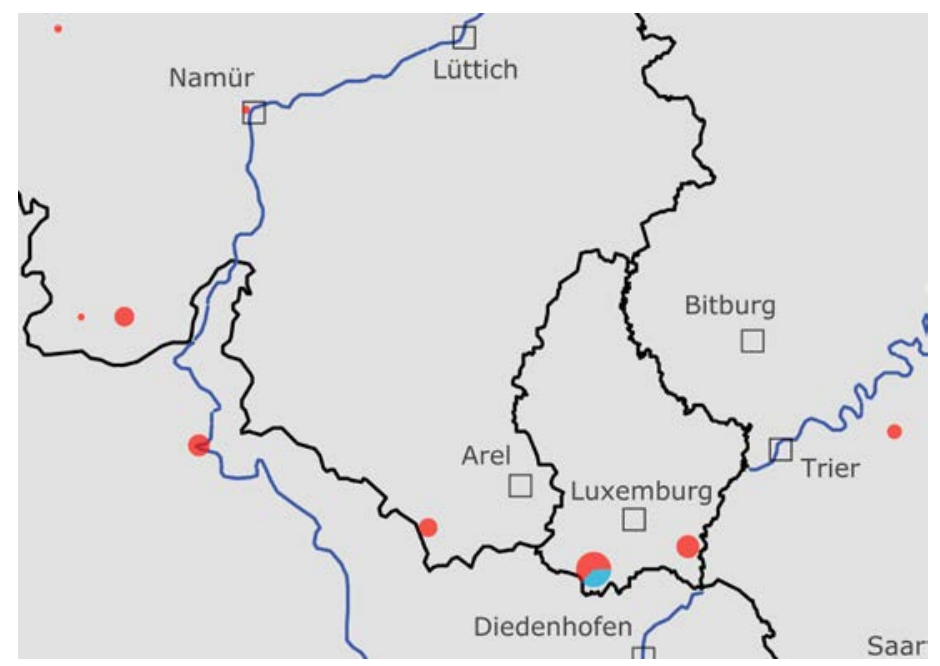

Carte 9: $\quad$ Parrasch (bleu), Parage (rouge)

11 Parage, n.m. ,Mode de détention particulier d'un fief entre frères afin de pallier les inconvénients du partage tout en présentant l'indivisibilité au fief, l'aîné seul prêtant hommage au suzerain et répondant des services au fief", TLFi.

12 Pour une meilleure lisibilité, les données sont exceptionnellement représentées par communes. 
nom s'est adapté en Delosch ['dəlo:f] (en bleu); cette forme germanisée n'est attestée qu'au Luxembourg. Deloge (en rouge) est également présent au Luxembourg, bien que moins fréquemment; ce nom est surtout répandu en Belgique (259 porteurs) et dans le nord de la France (66 des 326 naissances enregistrées entre 1966-1990). Les départements de la Meuse et de la Meurthe-et-Moselle entrent également dans la zone de diffusion du nom Deloge; ainsi, toute la zone romane limitrophe au Luxembourg a pu fournir ce nom au Luxembourg.

\section{Lepasch (1880:0; 2009:13), Lepage (1880:22, 2009:58)}

Lepasch est la forme germanisée du nom français Lepage, ou Le page. Elle est absente du recensement de 1880, mais nous avons pu la trouver dans le recensement de la population du doyenné de Mersch de 1766. La prononciation ['ləpa:f] présente une voyelle finale longue, comme dans les exemples du lexique. Le nom Lepage appartient à la catégorie des noms désignant un état ou une fonction: il est composé de l'article défini le agglutiné à a.fr. n.m. page, ,jeune garçon, valet'. Lepage (en rouge) est un nom très répandu en Belgique (1296 occurrences) et en France (3135 naissances sur l'ensemble du territoire entre 1966 et 1990), surtout dans la moitié nord de l'hexagone. Le Page est une variante régionale, surtout de la Bretagne. La forme germanisée Lepasch (en bleu) n'est attestée que sur le territoire du Luxembourg.

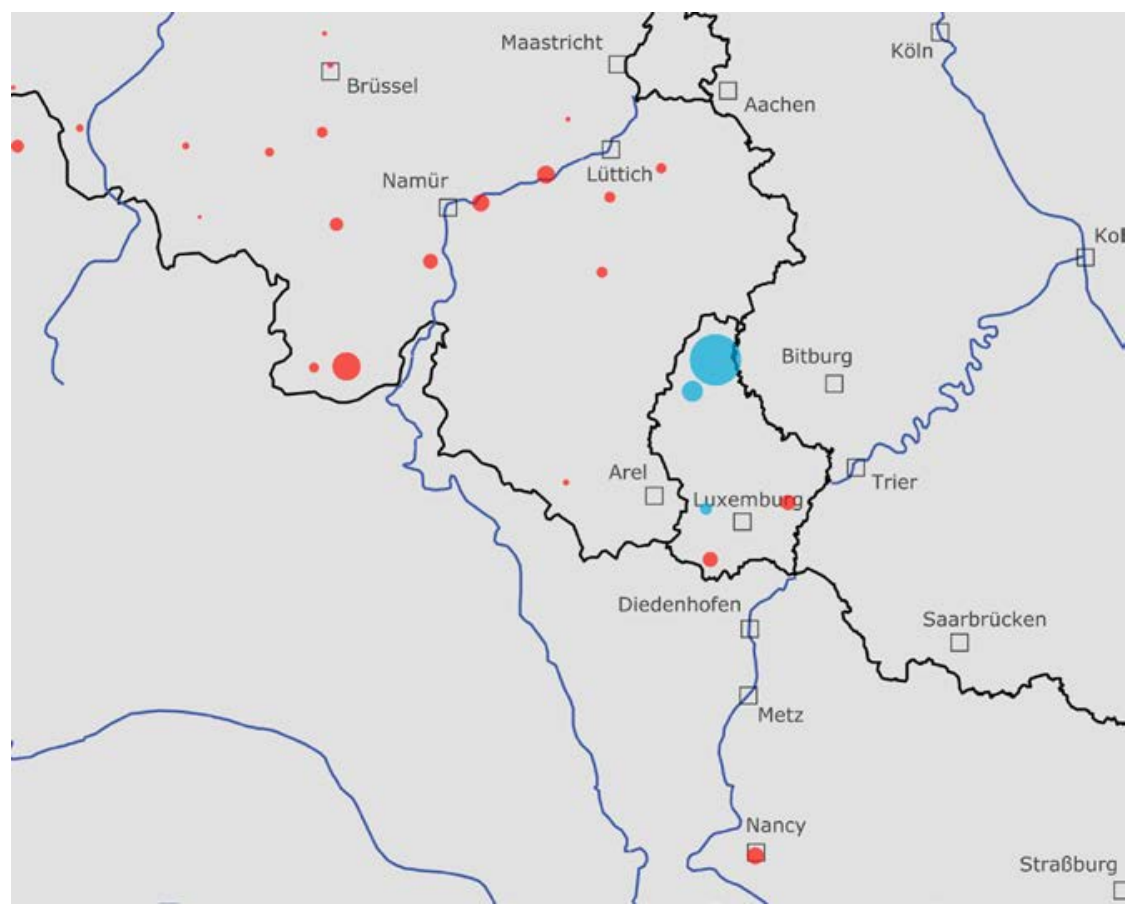

Carte 10: Delosch (bleu), Deloge (rouge) 


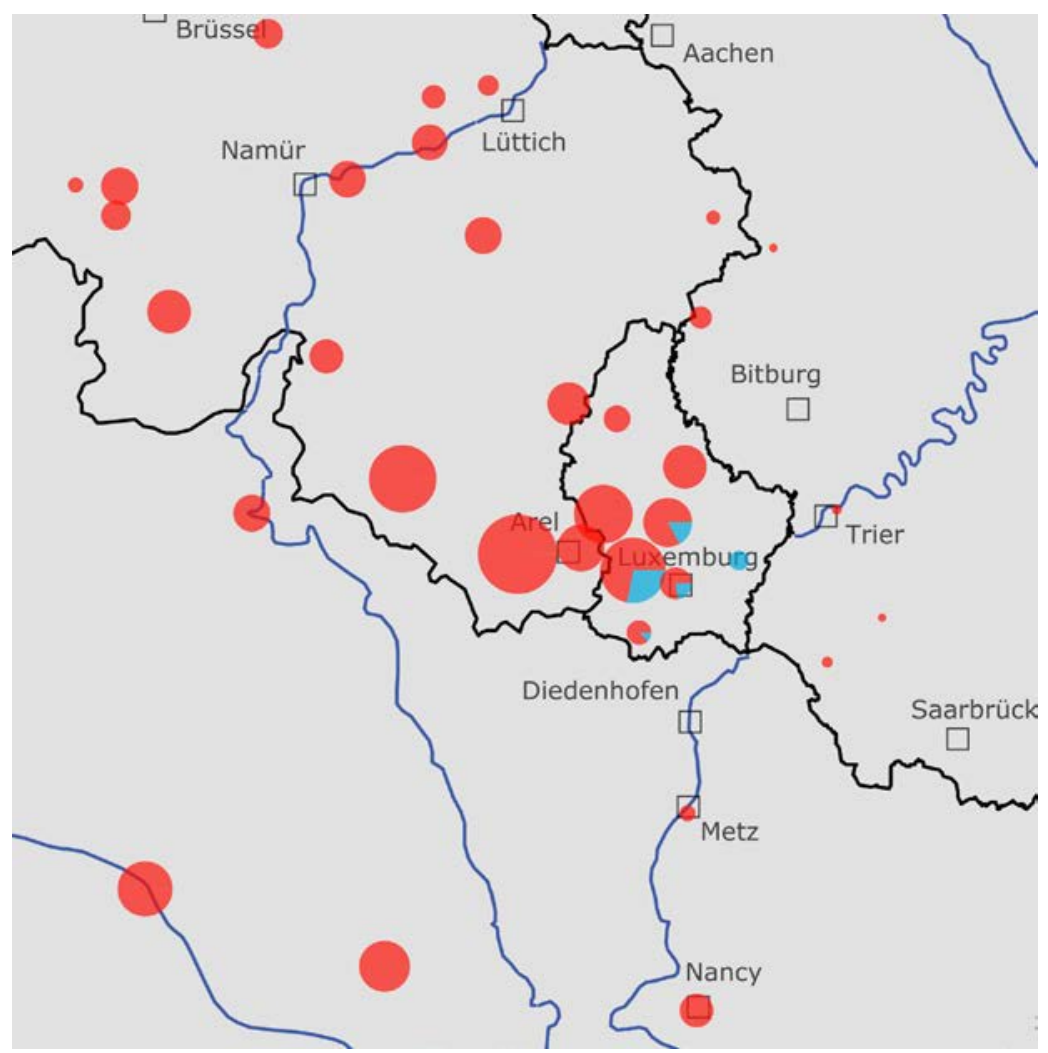

Carte 11: $\quad$ Lepasch (bleu), Lepage (rouge)

\section{La fricatisation de la chuintante $\int$}

Il faut se poser la question si la substitution graphique de «ch〉 pour 〈sch〉 dans les mots $\mathrm{du}$ lexique (fr. retouche $>\mathrm{lb}$. Retusch, fr. sacoche $>\mathrm{lb}$. Zakosch, LWB) et dans certains noms de famille ne traduit pas une tendance des locuteurs germanophones à reproduire la chuintante française avec une fricatisation plus large, plus forte. L'adaptation serait cependant tellement subtile que seule une étude phonético-acoustique permettrait de définitivement affirmer que l'adaptation est ici uniquement graphique.

Larosch (1880:33; 2009:11), Larosche (1880:16, 2009:9), Laroche (1880:15, 2009:30)

Le nom de famille français Laroche se réfère ou bien au lieu d'origine du porteur, comme Laroche-en-Ardenne dans la Province de Luxembourg, ou bien au lieu d'habitation du porteur, comme les nombreux noms de hameaux et lieux-dits Laroche / La Roche en France et en Belgique. Le nom se laisse décomposer en la, article défini féminin, et fr. n.f. roche. La forme germanisée Larosch se distingue de son modèle français Laroche uniquement par la spirantisation plus prononcée de [J]. L'avancement de l'accent n'a pas 
eu lieu; le nom est prononcé [la' $\left.\mathrm{R} \iint\right]$. En France, Laroche (en jaune) est la forme standard, elle est très fréquente (plus de 4000 naissances, 1966-1990). En Belgique et dans le nord de la France, c'est également Laroche qui domine à côté des formes régionales Larock et Laroque (< pic. roque, roche'). Les formes germanisées (Larosch, en bleu; Larosche, en rouge) n'existent qu'au Luxembourg; quelques rares porteurs sont attestés dans le département de la Moselle.

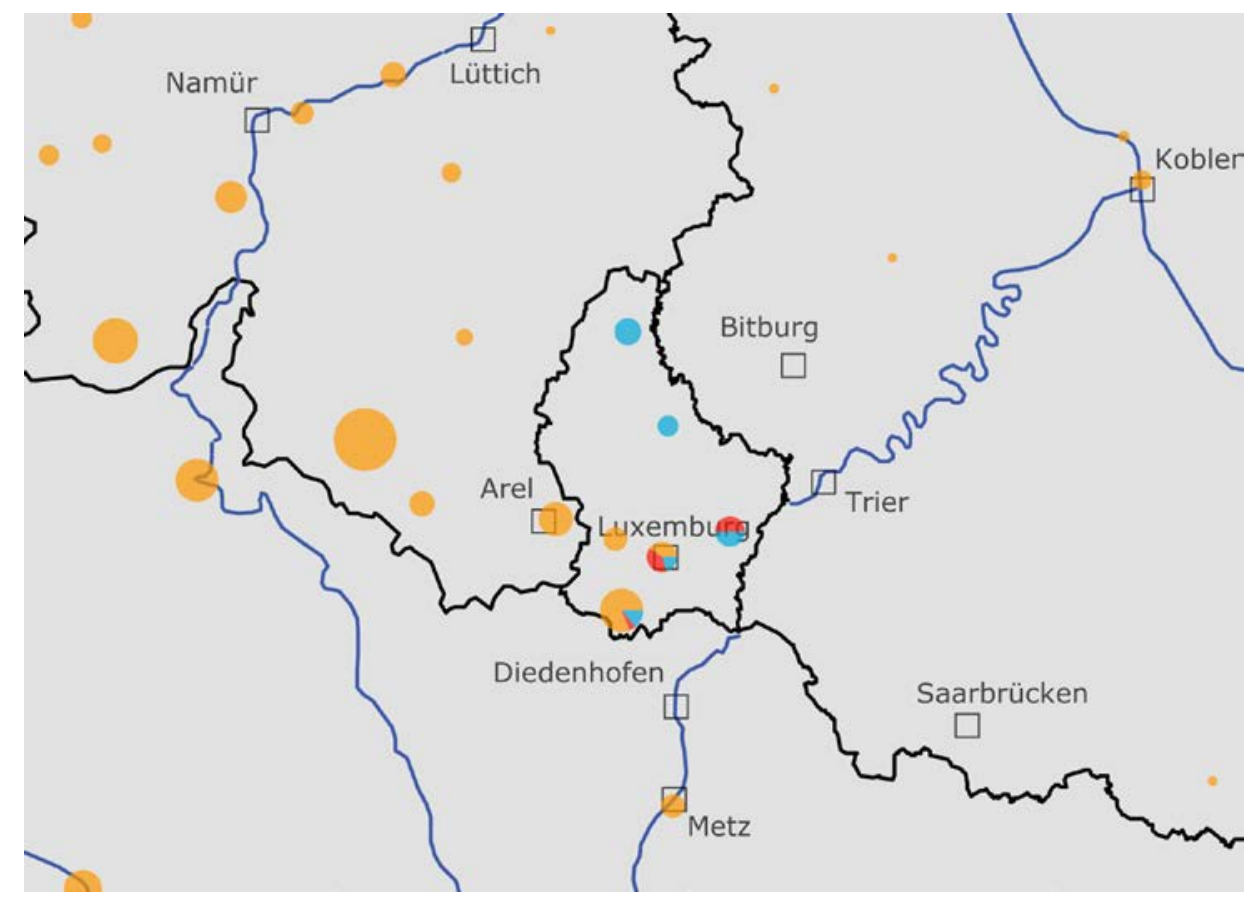

Carte 12: $\quad$ Larosch (bleu), Larosche (rouge), Laroche (jaune)

Duschaing (1880:4, 2009:0), Duschène (1880:3, 2009:8), Duchêne (1880:16, 2009:19), Duchaine (1880:0, 2009:1)

L'augmentation de la spirantisation se produit également à l'intérieur du nom, comme en témoignent Duschaing et Duschène, deux formes germanisées à différents degrés du nom Duchêne, qui caractérise un lieu d'habitation. Duschaing montre, en plus de la spirantisation, le remplacement de la nasale finale, alors que l'écriture Duschène rend uniquement compte de la spirantisation. La prononciation du 〈u〉 dans Duschaing et Duschène semble être plutôt $[\mathrm{y}]$, donc française, mais le nombre très restreint de porteurs laisse ce problème en suspens. Duschene (en bleu) présente un foyer limité sur le territoire grand-ducal, alors que Duchêne (en rouge) est très répandu sur l'ensemble des territoires francophones, même si les différents foyers sont trop petits pour être représentés sur la carte ci-dessous. En Wallonie, ainsi que dans le nord de la France, la forme Duchesne (en jaune) domine, mais elle est inconnue au Luxembourg. 


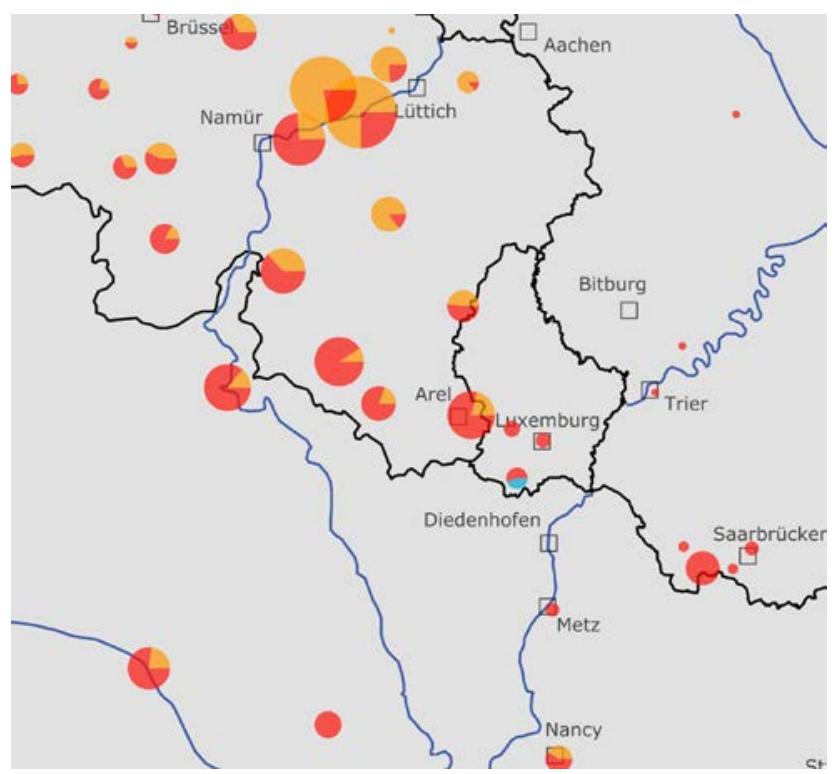

Carte 13: Duschene (bleu), Duchêne (rouge), Duchesne (jaune)

Duschang (1880:4, 2009:5), Duchamp (1880:8, 2009:8), Duchamps (1880:0, 2009:2)

Duschang est la forme spirantisée et dénasalisée de Duchamp(s), nom caractérisant le lieu d'habitation. Avec moins de 100 porteurs, Duchamp (en rouge) et Duchamps (en jaune) ne sont pas des noms fréquents en France; Duchamps est plus fréquent en Belgique avec 131 porteurs. Sur la carte, Duschang (en bleu) apparaît seulement au Luxembourg. La prononciation du 〈u〉 est également hésitante, comme pour Duschaing décrit ci-dessus.

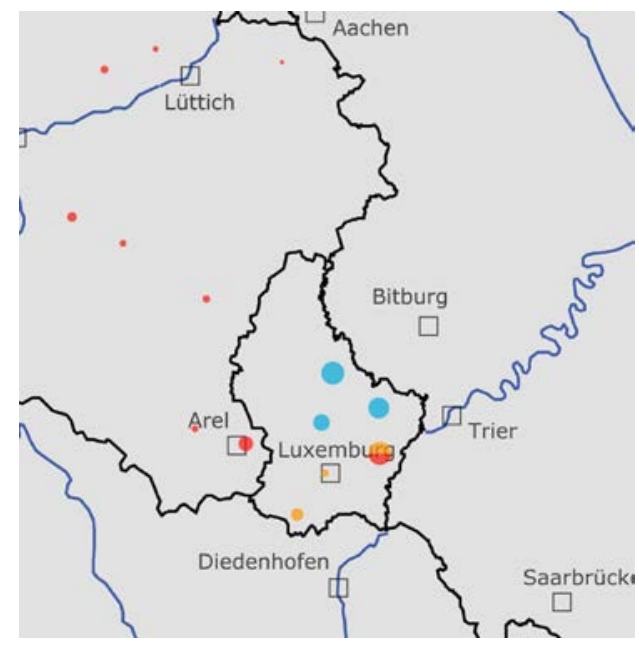

Carte 14: Duschang (bleu), Duchamps (jaune), Duchamp (rouge) 
Lavasch (1880:1, 2009:0), Lavache (1880:0, 2009:0)

L'origine du nom rare et aujourd'hui disparu Lavasch ['lava]] semble résider dans la germanisation du matronyme français Lavache. Ce nom, composé de l'article la agglutiné au n.f. vache, appartient à la catégorie des surnoms et se référait très probablement à la corpulence de sa porteuse. Comment cependant le nom français, présentant seulement deux foyers très régionaux en France (le premier recouvre les départements du Calvados et de la Manche avec 41 naissances entre 1996-1990, tandis que le deuxième recouvre le département de la Nièvre avec 12 naissances entre 1966-1990), a pu donner la forme Lavasch, dont l'attestation de 1880 au Luxembourg est l'unique relevé dans le corpus, seule une étude généalogique pourrait le mettre en évidence.

\section{La neutralisation germanique par désonorisation de la fricative sonore initiale}

Il existe également des cas où la fricative sonore initiale est désonorisée lors du processus d'adaptation en langue luxembourgeoise, ou, le cas échéant, en langue allemande.

Schaack (1880:257, 2009:222), Schaak (1880:59, 2009:0), Jacques (1880:38, 2009:24)

Le nom de famille Schaak, qui appartient aux 100 noms les plus fréquents au Luxembourg, s'explique par la germanisation du nom français Jacques. Cette désonorisation ne concerne toutefois que le nom de famille; le prénom luxembourgeois correspondant à Jacques, Jak, se prononce avec une fricative sonore en position initiale: [3a:k].

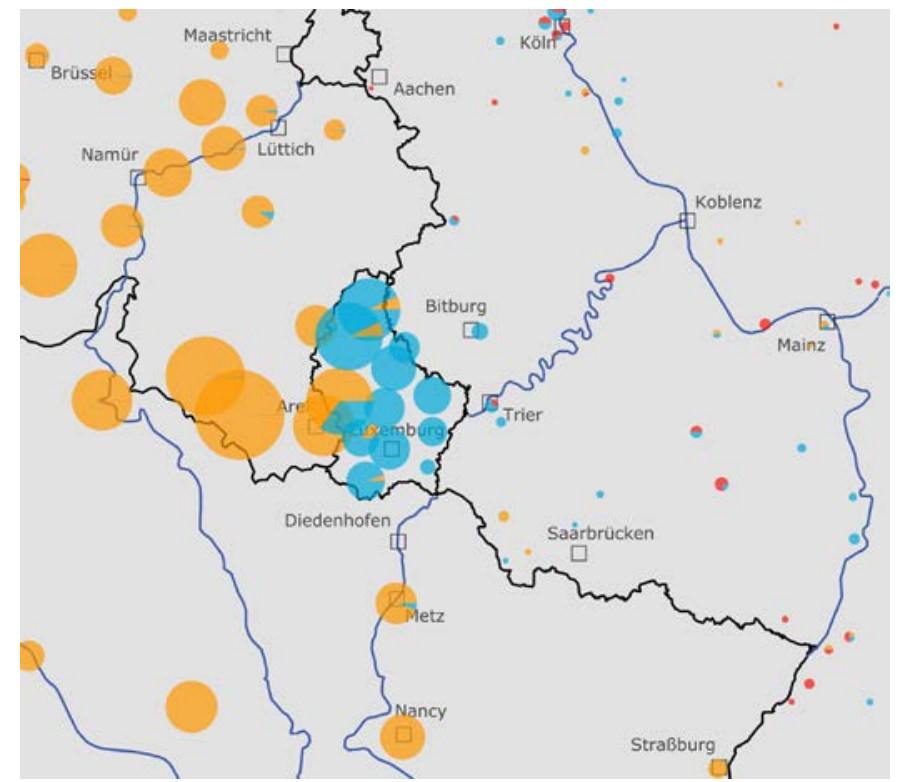

Carte 15: Jacques (jaune), Schaack (bleu), Schaak (rouge) 
Le nom français Jacques (en jaune) est un nom très répandu et très fréquent en France et en Belgique. Les variantes désonorisées Schaack (en bleu) et $S c h a a k^{13}$ (en rouge) sont éparpillées sur tout le territoire de l'Allemagne (195 porteurs pour Schaack, 311 pour Schaak). Au Luxembourg, le nom Schaak connaît une densité particulière, alors que Schaak n'est plus répertorié en 2009.

\section{Schanen (1880:71, 2009:139), Jean (1880:3, 2009:16)}

Déjà les livres de compte de la ville de Luxembourg du XVe siècle analysés par Gniffke 2011 attestent de nombreuses formes présentant la fricative sourde en position initiale à partir du nom français Jean: Schain, Schan; et à partir du nom féminin Jeanne: Schanne, Schannen, Schentgen. En ce qui concerne le nom de famille Schanen, il s'agit d'une forme obtenue par flexion de Schan. Jean (en rouge) est un nom de famille largement répandu aussi bien en France qu'en Wallonie, alors que Schanen (en bleu) connaît une répartition limitée presqu'exclusivement au territoire luxembourgeois, seuls quelques porteurs sont attestés dans l'Eifel et la Sarre côté allemand. En Allemagne est également attestée la présence du nom Schan, qui pourrait, surtout pour les occurrences au Palatinat, représenter la forme germanisée de Jean, sans marque de flexion. La signification du nom Schan, surtout celle des attestations éparpillées sur le reste du territoire est toutefois ambigüe.

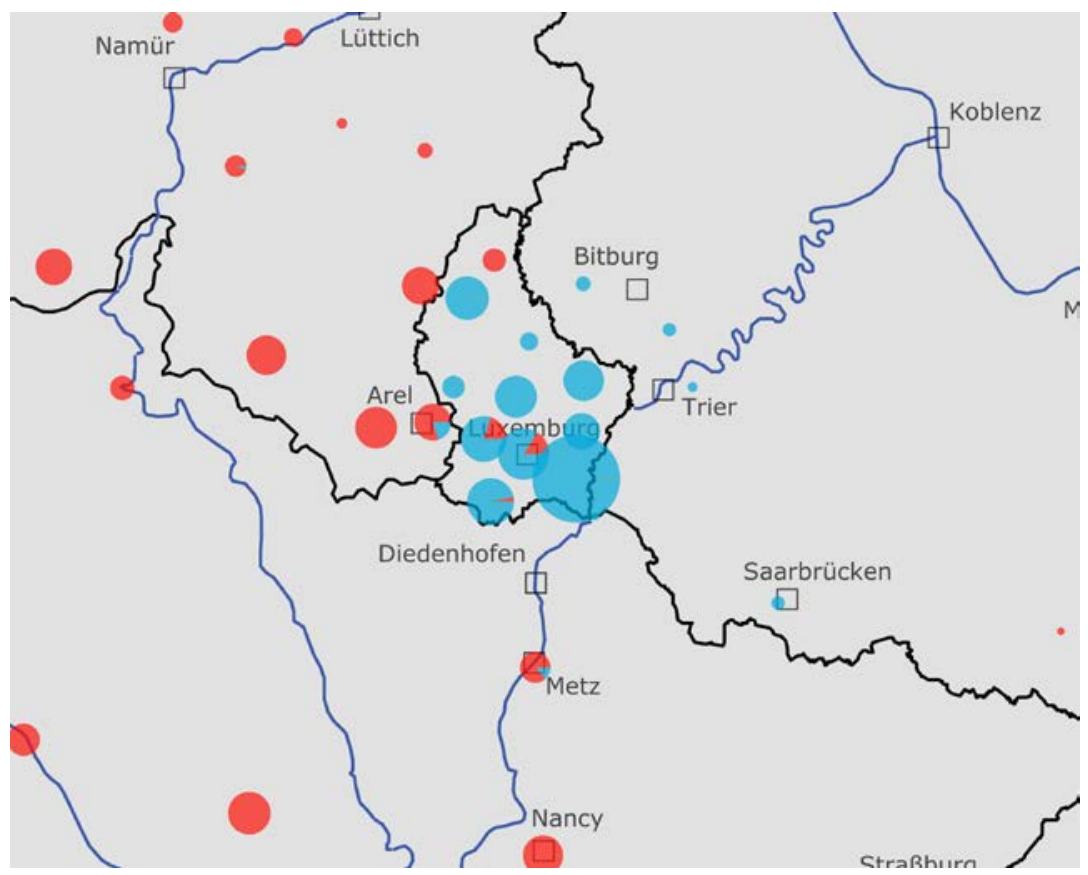

Carte 16: Jean (rouge), Schanen (bleu)

13 Schaak est cependant ambigu, surtout les occurrences dans l'Est de l'Allemagne pourraient s'expliquer par une autre origine, voir p.ex. Gottschald (1932: 334). 


\section{L'occlusion de [s]}

L'occlusion du $[\mathrm{s}]$ initial lors de la germanisation d'emprunts français est un phénomène bien attesté par la lexicographie, notamment dans des cas comme: fr. serre $>1 \mathrm{lb}$. Zär, fr. saisie $>\mathrm{lb}$. Zäsi, fr. sciatique $>\mathrm{lb}$. Ziatick, fr. saucisse $>\mathrm{lb}$. Zoossiss, fr. subside $>\mathrm{lb}$. Zubsid, etc. (LWB). Parmi les noms de famille français accueillis au Luxembourg, certains montrent également ce trait d'interférence.

Zigrang (1880:40, 2009:0), Zigrand (1880:47, 2009:63), Cigrang (1880:27, 2009:35), Cigrand (1880:20, 2009:1)

La perméabilité de la frontière linguistique entre Romania et Germania se manifeste particulièrement dans cet anthroponyme. L'oscillation entre les zones de contact a d'abord conduit à la francisation du nom germanique (germ. sigrant, bouclier victorieux ' $>$ a.fr. Sigrand), ensuite à la germanisation de l'emprunt français (fr. Sigrand $>1$ b. Zigrang). Dauzat 1951 suggère que Sigrand pourrait appartenir à la catégorie des sobriquets et dé signerait une expression familière d'une personne parlant de quelqu'un d'autre. Il appuie son affirmation par l'existence du nom Sigros. La géolocalisation des porteurs sur une

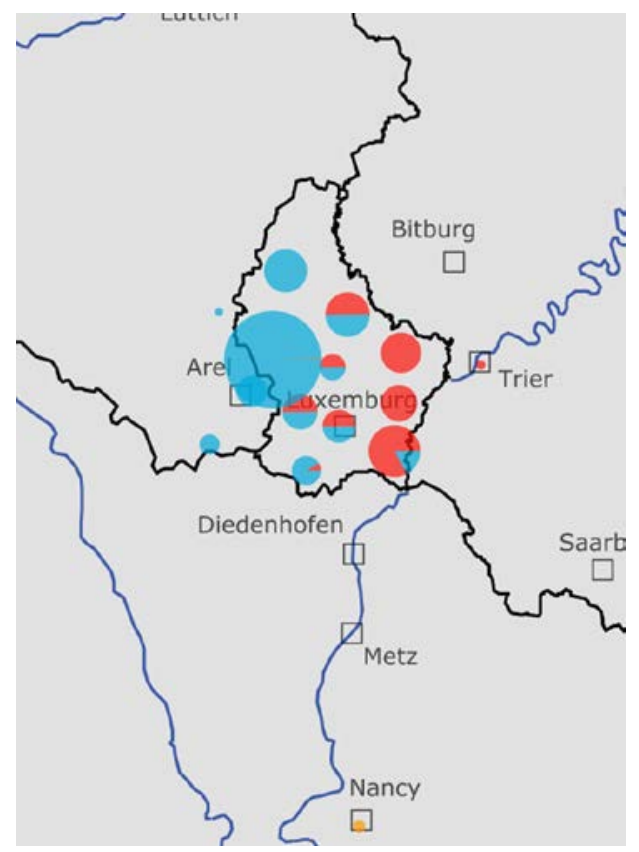

Carte 17: $\quad$ Cigrang (rouge), Zigrand (bleu), Sigrand (jaune)

carte montre toutefois que l'aire de dissémination de Sigrand et celle de Sigros ne s'entrecoupent pas: Sigrand apparait surtout dans les départements de Meurthe-et-Moselle (12 porteurs entre 1966-1990), Moselle (5 porteurs) et Jura (10 porteurs), alors que Sigros est concentré dans le département du Puy de Dôme. De plus, les locutions 
adverbiales sont inconnues comme générateurs d'anthroponymes; il faut donc chercher une autre explication pour Sigros. Le nom se laisserait décomposer en l'adjectif numéral six et le nom masculin gros, ce qui pourrait convenir si l'on se réfère à l'une des trois explications proposées par le TLFi: 'a) Droit dû aux fermiers des aides pour chaque muid de vin que l'on vendait en gros. b) Ancienne mesure de poids, égale à la huitième partie de l'once. c) Ancienne monnaie, de valeur variable'. A proximité du foyer Sigros, le nom de famille Sidenier (décomposable en six et denier, également une unité monétaire) est en fait attesté. Sidenier et Sigros s'expliqueraient par exemple par la désignation par un procédé métonymique du précepteur d'une taxe valant six deniers ou six gros.

Sigrand a produit plusieurs formes montrant des degrés divers d'interférence: $Z i$ grang (non représenté) et Cigrang (en rouge), prononcés ['tsigRay], illustrent à la fois l'occlusion du [s] initial et la substitution de la voyelle nasale. Zigrand (en bleu) et Cigrand (non représenté) montrent uniquement l'occlusion. Les variantes germanisées se concentrent au Luxembourg, avec quelques exceptions dans le pays d'Arlon à l'ouest et à Trèves à l'est. Sigrand (en jaune) survient dans les départements français limitrophes du Luxembourg, la proximité géographique facilitant le contact linguistique. Le nom Cigrand que nous avons interprété comme produit d'une germanisation existe cependant dans un foyer limité dans le sud-ouest de la France. La distance exclut un contact direct; toutefois, un certain lien entre le sud-ouest de la France et le Luxembourg semble se dessiner (v. Parrasch, Lakaf, ci-dessus); les multiples expéditions militaires et échanges commerciaux dans l'histoire du territoire luxembourgeois y jouent sans doute un rôle.

\section{Zossong (1880:0, 2009:10), Sossong (1880:10, 2009:19), Sosson (1880:20, 2009:22)}

Le nom de famille Zossong est le produit d'une germanisation de Sosson, issu de l'ancien français soçon (< lat. socius), compagnon, associé; associé pour labourer'. Zossong a été adapté par l'occlusion du [s] initial et le remplacement de la nasale finale, alors que Sossong ne présente que le remplacement de la nasale finale. La variante doublement germanisée Zossong (en bleu) n'est localisable que au Luxembourg, alors que Sossong (en rouge) apparaît au Luxembourg mais également dans le Pays de la Sarre et en Rhénanie-Palatinat, ainsi que dans le sud de la Province de Luxembourg. Le contact s'est très probablement produit dans la zone de contact belgo-luxembourgeoise, car Sosson (en jaune) y forme le plus grand foyer en Belgique avec environ la moitié des 202 porteurs. Un autre foyer, plus restreint en terme de nombre de porteurs, peut être observé en Basse-Normandie où ont été enregistrées 65 des 74 naissances recensées entre 1891 et 1915 en France. Le nom est également représenté en Lorraine avec une dizaine de porteurs dans le département de la Meurthe-et-Moselle. ${ }^{14}$

14 Signalons encore que quelques cas de confusion entre Sosson et Soisson, nom d'origine, peuvent être relevés dans Deltgen 2012, par exemple: 1721 Sosson $=>1754$ Soisson. 


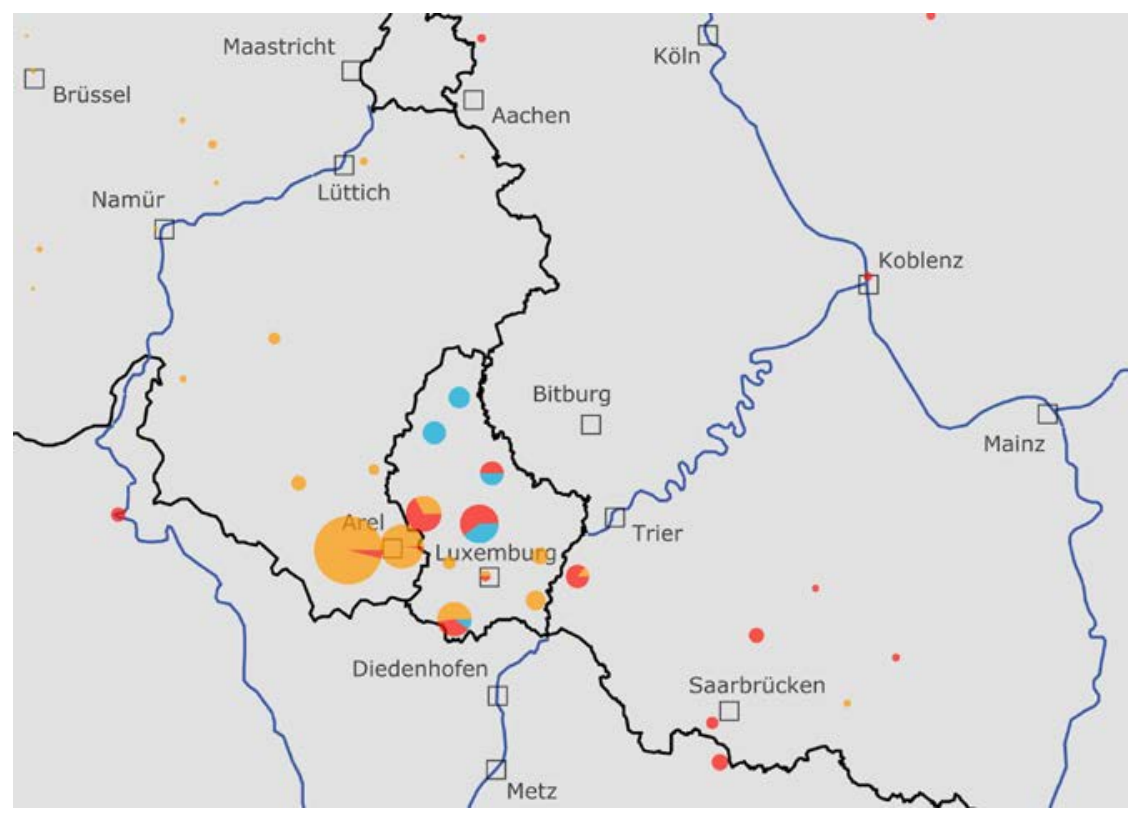

Carte 18: $\quad$ Zossong (bleu), Sossong (rouge), Sosson (jaune)

Zieser (1880:39, 2009:26), Sizaire (1880:0, 2009:2), Cisaire (1880:1, 2009:0)

Le cas de Zieser ['tsi:zəR] est particulier, car il est un rare témoin du contact linguistique entre le wallon et le luxembourgeois. En effet, Zieser est la forme germanisée de wa. Sizaire, une variante rare de fr. César (> lat. Caesar, d'origine inconnue). Sizaire (en rouge) est répandu surtout au sud de la Province de Luxembourg; en effet, plus de la moitié des 171 porteurs enregistrés en Belgique sont représentés à cet endroit sur la carte. L'autre moitié des porteurs est éparpillée dans toute la Wallonie. En France, une cinquantaine de porteurs de Sizaire est localisable dans le département du Nord. Zieser (en bleu) montre la plus grande concentration au Luxembourg, mais également 26 attestations éparpillées dans toute l'Allemagne.

\section{L'allongement de la diphtonge [wa] > [ua:]}

L'allongement de la diphtongue [wa] finale est un trait d'interférence répandu à l'oral, mais rarement exprimé par écrit. Ainsi, la lexicographie du luxembourgeois (LWB) ne nous fournit qu'un seul exemple: fr. pied du roi, unité de mesure dans la production des gants $^{\text {‘ }}>$ lb. Piduar, Pidua. La banque de données du projet LFA ne contient également qu'un unique exemple représentatif pour ce trait de germanisation. ${ }^{15}$

15 Ajoutons cependant les deux exemples relevés dans la base généalogique Deltgen 2012, Borjua et Dalua. Borjua ne peut être que la forme germanisée du nom très répandu Bourgeois ( $<$ a.fr. borgeis 


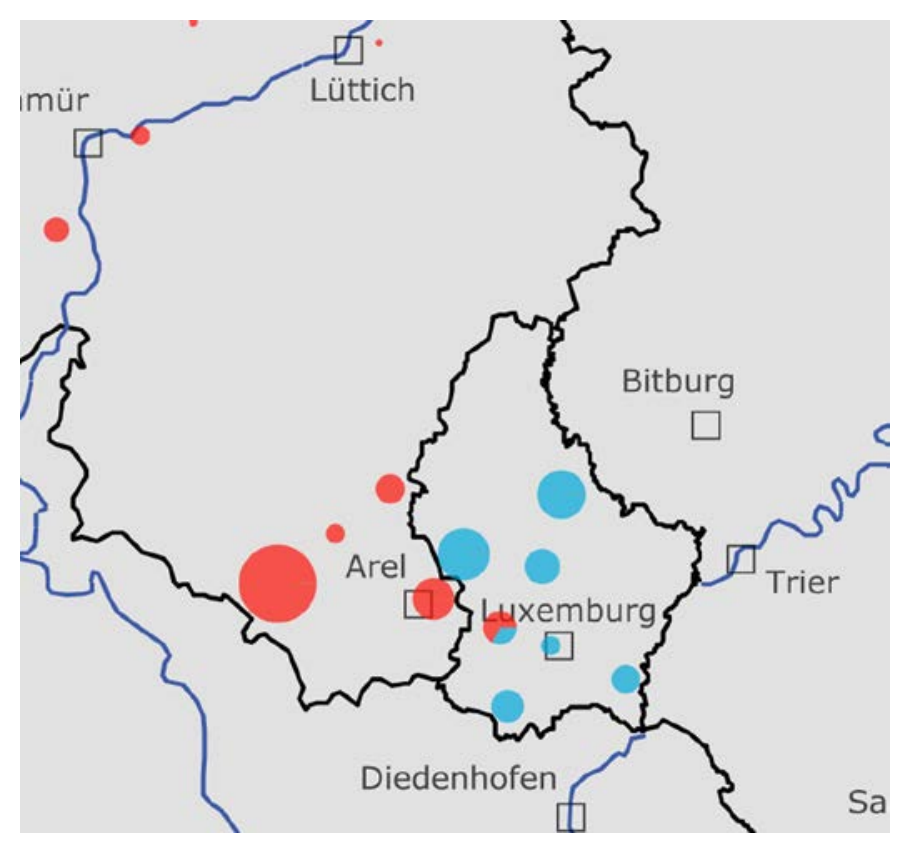

Carte 19: $\quad$ Zieser (bleu), Sizaire (rouge)

Hottua (1880:21, 2009:25), Hottois (1880:1, 2009:0), Hottoi (1880:1, 2009, 0)

Hottua est la forme germanisée du nom d'origine Hottois. Ce dernier se réfère aux habitants de Hotte (wa. Houte) dans la Province de Luxembourg. Les différentes variantes issues du nom, traduisant les hésitations de la mise par écrit d'un nom d'origine alloglotte, peuvent être consultées dans un relevé agnatique en ligne. ${ }^{16}$ Citons parmi les nombreux exemples: 1711 Houtoy $=>1769$ Hottoau $=>1803$ Hottua. Hottua (en bleu) est un nom de famille qui ne se rencontre qu'au Luxembourg; en revanche, sa variante graphique Hotua (en rouge) n'existe que dans la Province de Luxembourg. Hottois (en jaune) est répandu en Wallonie ainsi que dans le département du Nord; toutefois, il fait possiblement référence à d'autres lieux du même nom (p.ex. 1399 au hottois, Limal, Brabant-Wallon, proposé par Germain-Herbillon 1996:541).

,libre habitant d'une ville, jouissant de certains privilèges'), comme le démontre également le relevé généalogique qui atteste pour une même ligne agnatique: 1766 Borjua, 1795 Bourgeois. Nous retrouvons le même type d'attestation pour Dalua: 1852 Dalois, 1853 Dalua. Dalois est un nom rarement rencontré en France (20 naissances seulement entre 1891 et 1990); il se décompose en Alois et la préposition $d^{\prime}$. La présence de la préposition plaide en faveur d'un nom marquant le lieu d'origine du porteur, par exemple les lieux-dits Alloy, département de la Meuse (attesté dans Gocel 201:3), et Les Allois (p.ex. département de la Haute Vienne), Buire-en-Alloy, département de la Somme. L'étymologie du toponyme se rapporte sans doute à a.fr. aloe, domaine héréditaire possédé en pleine propriété, exempt de toute redevance', TLFi.

https://sites.google.com/site/hottua/lenomhottua, consulté le 14.09.2011. 


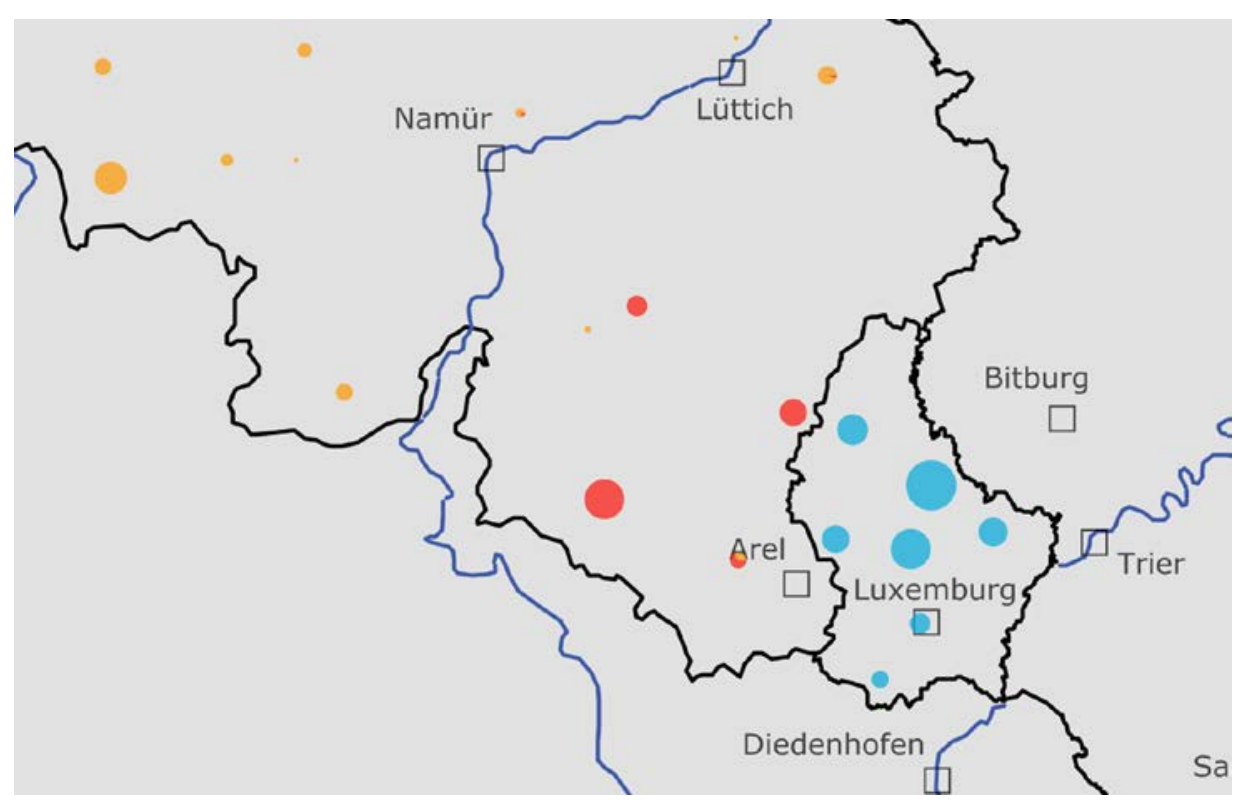

Carte 20: $\quad$ Hottua (bleu), Hotua (rouge), Hottois (jaune)

\section{La perte de la nasale, remplacée par une voyelle courte suivie de la vélaire [y]}

Si l'inventaire des voyelles en allemand est composé uniquement de voyelles orales, le fran-

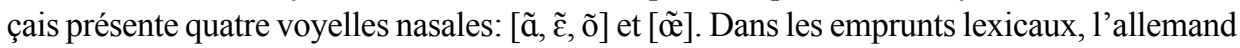
du sud remplace la voyelle nasale par une voyelle orale suivie d'une consonne nasale, ainsi fr. [bal'kõ] < all. [bal'ko:n], alors que dans le nord de l'Allemagne, l'emprunt est prononcé [bal'kon] (Meisenburg/Selig 1998:65). Des études sur la prononciation du français par des locuteurs allemands ont également montré des problèmes dans l'articulation distincte de [ã] et de [õ] (Haberl 2004). Or nous avons pu constater dans Muller 2012 que l'adaptation des nasales finales françaises dans un parler moyen allemand occidental suit une étonnante systématicité: les locuteurs de la langue réceptrice interprètent toujours de la même manière les phonèmes inconnus de la langue source. La voyelle nasale française est traduite par une voyelle orale suivie par la consonne nasale [y]. En cela le luxembourgeois montre la même réalisation de l'interférence que celle constatée par Meisenburg/Selig 1998 pour l'Allemagne du Nord. Cependant, aucune confusion dans la distinction de [ã] et de [õ] n'a pu être relevée dans le corpus, ce qui s'explique probablement par le fait que le locuteur luxembourgeois, à défaut d'être bilingue, est exposé à un contact très étroit avec le français. ${ }^{17}$

17 Ainsi, Bieritz 1971:98 a constaté dans son étude sur l'apprentissage du français par des étudiants de l'université de Bochum qu'après cinq ans d'études, les erreurs de prononciation ne concernaient plus les voyelles nasales. 
Tab. 1: Nasales françaises et leur adaptation en luxembourgeois / moyen allemand

\begin{tabular}{|l|l|}
\hline français & $\begin{array}{l}\text { adaptation en luxembourgeois / } \\
\text { moyen allemand occidental }\end{array}$ \\
\hline$\tilde{\mathrm{a}}$ & ay \\
\hline$\tilde{\mathrm{o}}$ & oy $/$ un \\
\hline$\tilde{\varepsilon}$ & ey / in \\
\hline$\tilde{\mathfrak{e}}$ & ey / in \\
\hline
\end{tabular}

Une carte représentant la localisation des noms français adaptés par perte de la nasale montre que le phénomène n'est pas exclusif au Luxembourg, mais s'y concentre:

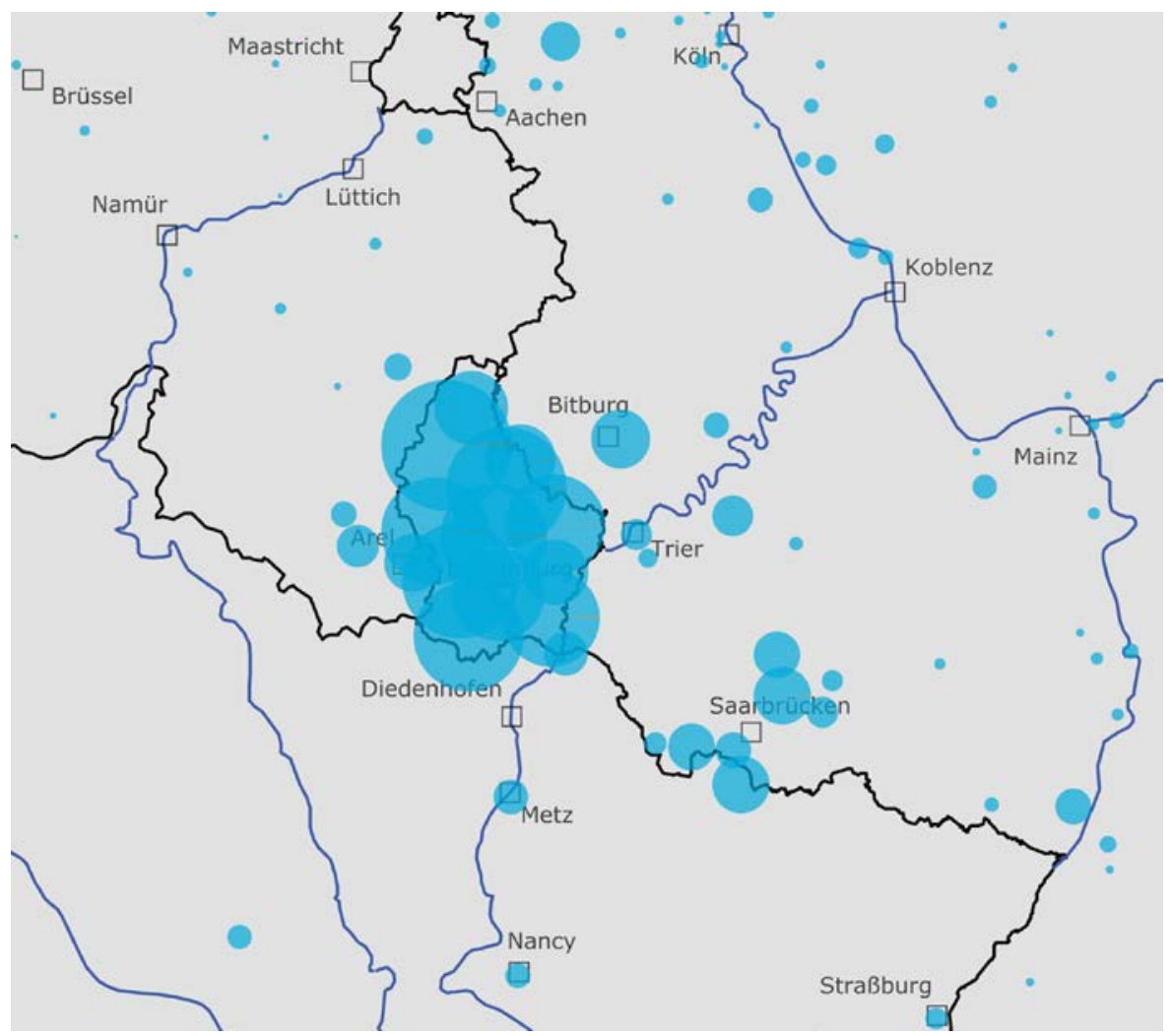

Carte 21: $\quad$ Perte de la nasale: Lallemang, Flammang, Lorang, Colling, Widong

En effet, ces interférences se retrouvent également en Allemagne, surtout dans la Sarre, ce qui contredit la division entre nord et sud faite par Meisenburg/Selig 1998. En France, ce sont certaines formes (surtout Lorang, Flammang) qui se rencontrent dans l'Est. Cependant, c'est la fréquence beaucoup plus élevée au Luxembourg de ce type de germanisation qui la rend caractéristique du paysage anthroponymique du Grand-Duché. 


\section{L'abaissement de [u] vers [o]}

Toute une catégorie de germanisations, bien que concernant des noms particulièrement fréquents, n'entre pas tout à fait dans le cadre de la présente contribution, qui décrit des mécanismes de contact entre le français et le haut-allemand, car ces germanisations ne s'expliquent que par un contact entre le français et le moyen-allemand. Il s'agit de l'abaissement de [u] vers le [o], un changement phonétique s'étant opéré lors du passage du moyen-hautallemand (Mittelhochdeutsch) au nouveau-haut-allemand-précoce (Frühneuhochdeutsch). Les noms français en contact avec le moyen-haut-allemand ont ainsi également été transformés: fr. Bourgeois > lb. Borjua, fr. Louche > lb. Losch, fr. Moulin $>\mathrm{lb}$. Molling, fr. Mousson $>$ lb. Mossong, fr. Toussaint > lb. Tossing, fr. Roussillon > lb. Rosseljong. ${ }^{18}$

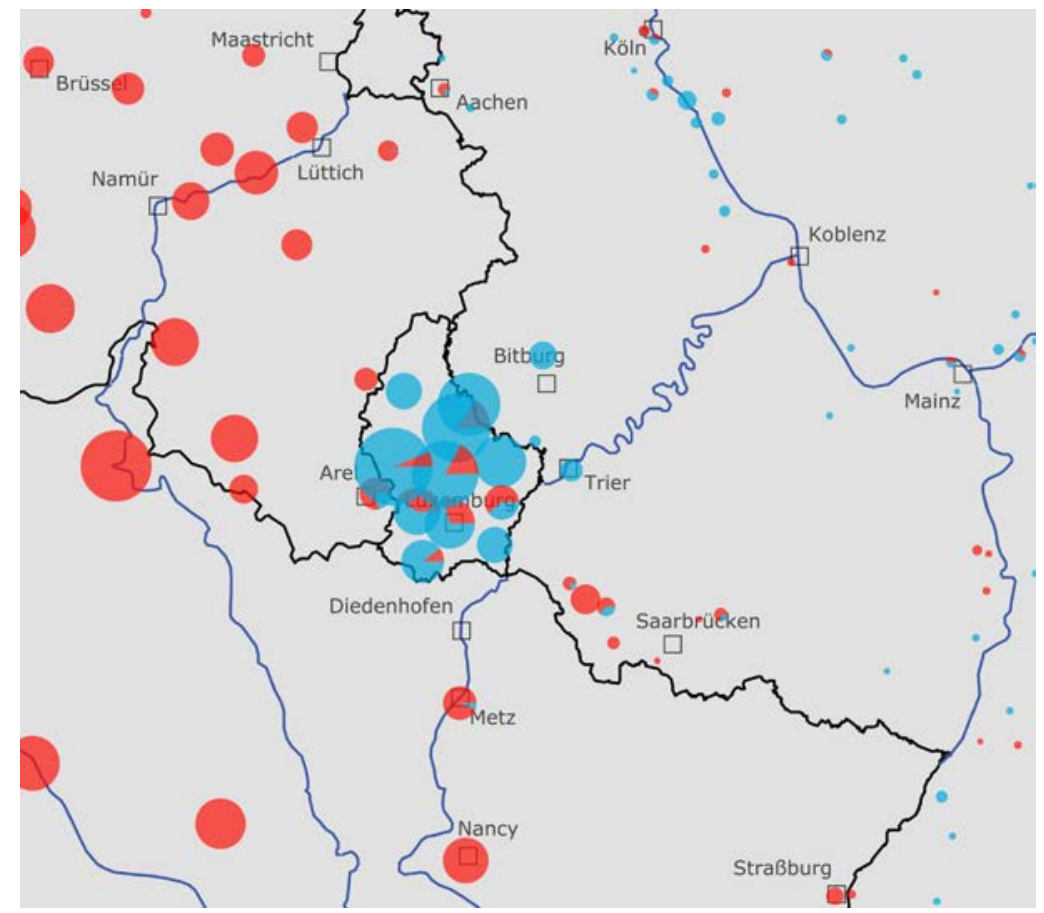

Carte 22: En bleu: Borjua, Losch, Molling, Mossong, Tossing, Rosseljong. En rouge: Bourgeois, Louche, Moulin, Moussson, Toussaint, Ro(u)ssillion

La représentation cartographique de ces quelques noms démontre la régionalité de ce phénomène pour les noms d'origine française. Cette image est attendue si l'on prend en considération la fréquence de l'abaissement $[u]>[0]$ dans les noms d'origine germanique. En projetant les 12 noms les plus fréquents au Luxembourg montrant l'abaissement sur

18 Il existe une autre possibilité, mais que nous ne pouvons pas prouver, faute d'attestations. Le $o$ dans la série des noms présentés a pu être transmis en luxembourgeois directement de l'ancien français (a.fr. borgeis, molin) via le français régional, par exemple le wallon. 
une carte (carte 23), la fréquence spécialement élevée au Luxembourg est saillante, même si le phénomène phonétique en soi est très présent surtout sur l'aire du bas-allemand.

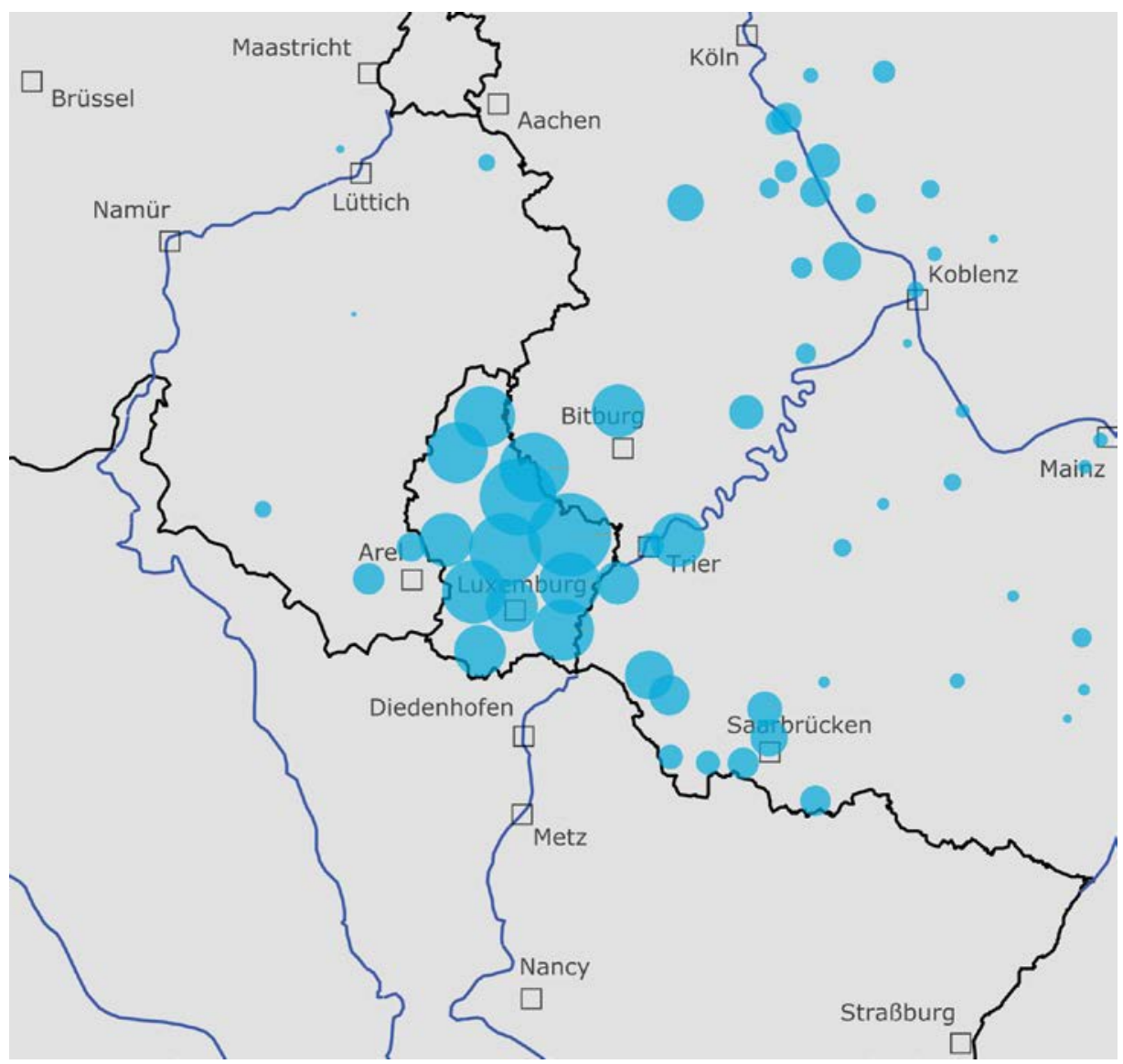

Carte 23: Scholtes, Wolter, Tholl, Hoss, Polfer, Schosseler, Schommer, Konz, Scholler, Strock, Stronck, Grotz

La transcription de l'abaissement dans les noms de famille à la fois d'origine germanique et romane est une caractéristique forte du paysage anthroponymique luxembourgeois. ${ }^{19}$

\section{Conclusions}

La localisation des noms de famille sur une carte permet d'affirmer le caractère régional des phénomènes de germanisation décrits; elle livre des pistes sur la localisation des

19 Voir également la contribution de Cristian Kollmann dans ce volume. 
zones de contact, elle met en relation des foyers de noms géographiquement éloignés, elle permet par la superposition de différents noms cartographiés d'affirmer, de rapprocher ou de mettre en doute des étymologies.

En rassemblant sur une même carte l'ensemble des noms germanisés présentés dans cette contribution, la distribution géographique très régionalisée est saillante. Le Luxembourg apparaît comme haut-lieu de la mise par écrit des adaptations phonétiques effectuées sur les noms de famille français; la carte ci-dessous regroupant tous les porteurs des noms germanisés du corpus renforce cette idée. Ce sont en effet moins les processus phonétiques d'adaptation qui sont spécifiques au Luxembourg, mais la fréquence de la fixation écrite de ceux-ci. Cette tendance à retranscrire des variations linguistiques non standard se montre également dans le traitement des régionalismes phonétiques.

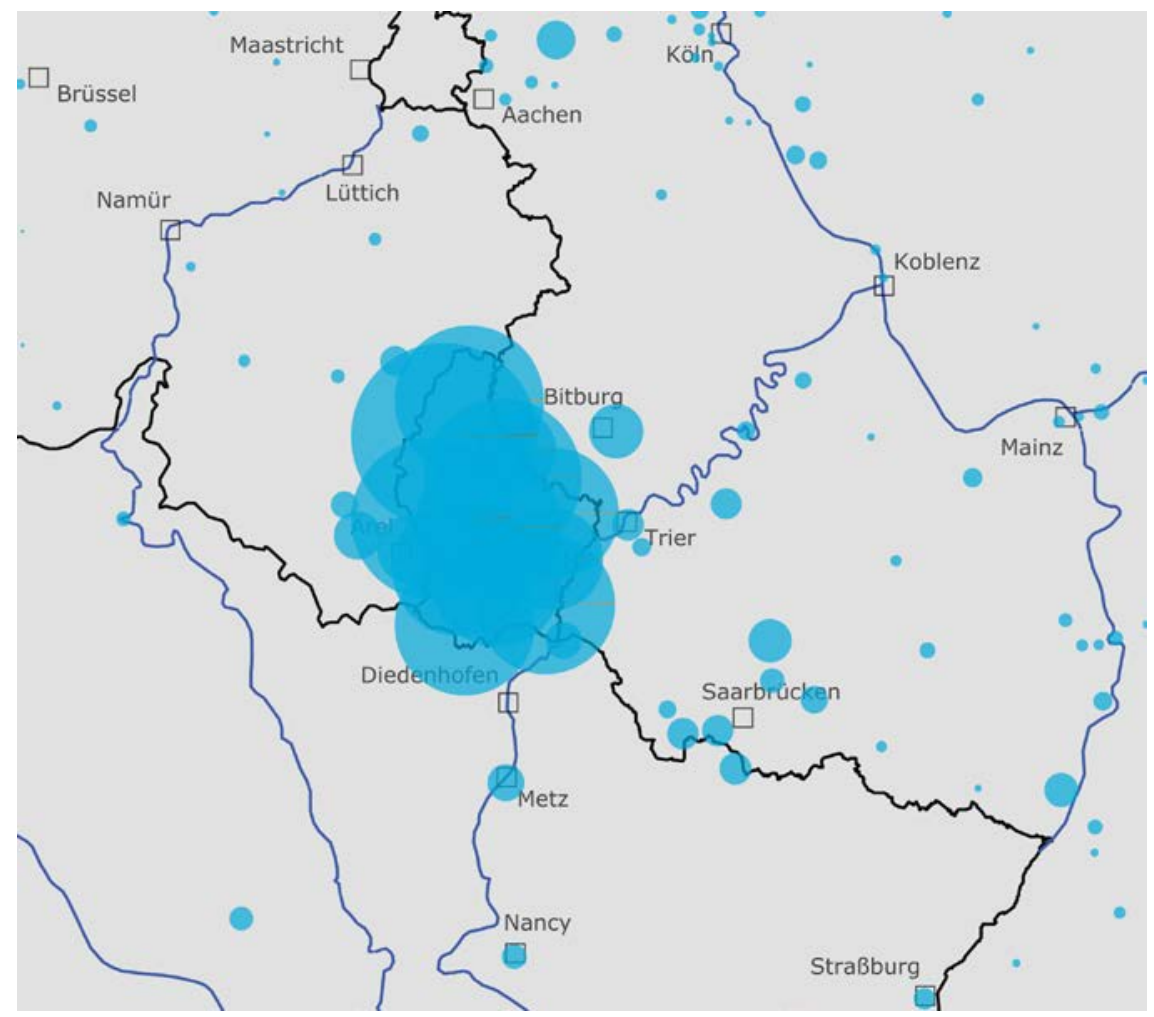

Carte 24: $\quad$ Représentation de l'ensemble des noms germanisés

Les raisons de cette perméabilité du standard écrit au Luxembourg est sans doute à chercher dans la tension entre la langue parlée et la langue standard de la mise par écrit des noms, à savoir l'allemand standard. Leur proximité linguistique crée facilement des interférences négatives en allemand qui semblent profiter d'une grande acceptabilité auprès des locuteurs et des administrations, ce qui explique le grand nombre de noms de famille reproduisant des régionalismes phonétiques. Les interférences dans les noms de famille 
d'origine française rencontrent la même acceptabilité, favorisée par le côtoiement quotidien du français et éventuellement une habitude des interférences linguistiques en général.

Une interrogation à poursuivre est celle de l'avancement de l'accent qui ne semble pas s'opérer systématiquement sur les noms lors de la germanisation, puisque certaines formes échappent à ce processus, comme notamment Larosch et Lavasch. ${ }^{20} \mathrm{Si} \mathrm{l'on} \mathrm{consi-}$ dère les autres noms de famille qui, en luxembourgeois, se terminent par une chuintante, nous y constatons l'avancement de l'accent: Parrasch, Delosch et Lepasch. Nous remarquons ainsi que les noms présentant l'avancement se terminent sur une chuintante issue d'une fricative sonore $\left(3>\int\right)$, alors que les noms dont le modèle français se terminait déjà sur une fricative sourde n'ont pas avancé l'accent lors du processus de germanisation. Cette règle ne se laisse toutefois pas généraliser à défaut d'un corpus plus grand, car déjà le lexique livre des contre-exemples comme fr. barrage < lb. Baraasch [ba' teur chronologique ne peut ainsi pas être exclu; l'âge des emprunts pourrait jouer un rôle.

La question qui accompagne la visualisation cartographique des noms est l'identification des zones d'emprunts, c'est-à-dire de l'endroit d'origine d'un nom de famille germanisé. Les cartes ne peuvent que donner quelques indications générales, surtout quand le modèle français est très répandu. Les noms-modèles à distribution géographique limitée sont les seuls dont l'origine du nom importé est bien sûr plus facile à cerner. Il semble évident que seule l'établissement des lignes agnatiques des différents porteurs pourrait livrer des indications plus spécifiques sur le lieu exact de l'éclosion du nom. La consultation des cartes confirme d'un côté que les noms proviennent des zones romanes limitrophes au Luxembourg, de l'autre côté elle révèle une relation entre le Grand-Duché et le sud-ouest de la France qui nécessite une investigation plus poussée. La cartographie des noms peut cependant mettre en relation des noms géographiquement proches, comme le démontrent le cas de Sigros et Sideniers, ou au contraire écarter des étymologies à cause de leur trop grand éloignement.

La visualisation de l'ancrage géographique des noms de famille par des cartes se confirme donc comme un outil complémentaire très utile pour l'analyse de l'étymologie.

\section{Bibliographie}

Bieritz, Wulf-Dieter: Phonologische Interferenzen, Französische Aussprachefehler deutscher Studenten. Dans: International review of applied linguistics 12, 1974, p. 193-23.

Czochralski, J. A.: Zur Sprachlichen Interferenz. Dans: Linguistics 67, Le Hague, 1971, p. 5-25.

Dauzat, Albert: Dictionnaire étymologique des noms de famille et prénoms de France, Paris, 1951.

Debrabandere, Frans: Verklarend woordenboek van de familienamen in België en Noord-Frankrijk, Bruxelles, 1993.

20 Ajoutons Lagasche, nom de famille attesté uniquement en 1877 dans la base généalogique publique Deltgen 2012 et qui est la forme légèrement germanisée de Lagache. Ce nom, dont l'origine remonte à la forme picarde et normande l'agache < germ. agaza ,pie', appartient à la catégorie anthroponymique des surnoms. Lagache est attesté dans le Nord de la France (606 naissances dans les départements du Nord et de Calais, entre 1966 et 1990); Lagace est plus rare mais également limité au Nord (7 naissances au niveau national entre 1966 et 1990). 
Debyser, Francis: La linguistique contrastive et les interférences. Dans: Langue française 8, 1970, p. $31-61$.

Deltgen 2012 = Base généalogique publique luxembourgeoise, www.deltgen.com.

Gniffke, Andreas: Die Personennamen der Stadt Luxemburg von 1388-1500, Namenbuch und namenkundliche Analyse auf Basis der Rechnungsbücher der Stadt Luxemburg, Thèse, Université de Trèves / Université du Luxembourg, 2011.

Gocel, Marc: La télégraphie aérienne de ,A à Z‘, Association du Mont Saint Quentin, 2001.

Gottschald, Max: Deutsche Namenkunde, Unsere Familiennamen nach ihrer Entstehung u. Bedeutung, Munich, 1932 [1931].

Haberl, Kathrin: Probleme Deutscher mit der französischen Aussprache, Munich, 2004.

Herbillon, Jules/Germain, Jean: Dictionnaire des Noms de Famille en Belgique romane et dans les Régions limitrophes, Bruxelles, 1996.

LWB = Luxemburger Wörterbuch, 5 volumes, Luxembourg, 1950-1975. Complément 1977.

Meibauer, Jörg, et coll.: Einführung in die germanistische Linguistik, Stuttgart/Weimar, 2002.

Meisenburg, Trudel/Selig, Maria: Phonetik und Phonologie des Französischen, Stuttgart, 1988.

Muller, Claire: Les noms de famille français du Luxembourg. Dans: Nouvelle Revue d'Ono-mastique, 2013/2, [à paraître].

TLFi $=$ Trésor de la langue française informatisé, atilf.atilf.fr.

Wängler, Hans-Heinrich: Phonetik des Deutschen mit einer allgemeinen Einführung in die Phonetik, Marburg, 1960.

Weijnen, Antoon: Fonetische en grammaticale parallellen aan weerszijden van de taalgrens. TNTL 80, 1964, p. 1-25.

Weinreich, Uriel: Sprachen in Kontakt, Ergebnisse und Probleme der Zweisprachigkeits-forschung, München, 1977. 



\title{
Die luxemburgischen Familiennamen entlang der historischen Grenze zu Deutschland - Projektskizze und vorläufige Ergebnisse
}

\author{
Walter Amaru Flores Flores
}

\begin{abstract}
This article examines the differences and similarities of the family names in the historical and recent Luxembourgish/German border region. Special attention is paid to the role of the historical border before 1815. The motifs of the most prototypic (i.e. most frequent) family names - based on telephone registers - are investigated. An additional focus is put on occupational names and names of origin. The results can be described in three ways: 1) Some features show a distribution across geographical and linguistic borders. 2) Others seem to follow the historical borders of Luxembourg. 3) The distribution of a last group equals the recent Luxembourgish border. The latter is not easy to explain as the nowadays Luxembourg can't be described as a historical core area. Thus its borders shouldn't have influenced the emergence of the family names.
\end{abstract}

\section{Einleitung - das Projekt „Die luxemburgischen Familiennamen entlang der historischen Grenzen zu Deutschland (LFAG)“}

Der vorliegende Beitrag ist im Rahmen der Arbeit am Promotionsprojekt „Die luxemburgischen Familiennamen entlang der historischen Grenzen zu Deutschland“" entstanden, das die Erfassung und Beschreibung des Nameninventars jener Region zum Ziel hat, die bis 1815 ein Teil Luxemburgs war, dann an Preußen gefallen ist und heute größtenteils zu Deutschland gehört. Die lediglich kleinen Teile, die heute in Belgien bzw. Frankreich liegen, werden in der Untersuchung ausgeklammert, um eine möglichst große Homogenität der zugrundeliegenden Daten zu gewährleisten. ${ }^{2}$

Das fortlaufende Dissertationsprojekt ist dem gerade abgeschlossenen, mehrjährigen Projekt „Luxemburgischer Familiennamenatlas (LFA)“3 angegliedert. Hier wurden über 2000 der häufigsten heutigen luxemburgischen Familiennamen unter Berücksichtigung konkreter historischer, regionaler Daten sowie der spezifischen luxemburgischen Lautgeschichte etymologisiert; eine Reihe der behandelten Namen wurden dabei zum ersten Mal

1 Das skizzierte Promotionsprojekt wird vom Fonds National de la Recherche, Luxemburg (ProjektCode 1029084) unterstützt.

2 Die Namendatenbanken für Luxemburg und Deutschland basieren auf Telefonbuchdaten von Editus (2009) bzw. der Deutschen Telekom (2009) - entlang der historischen Grenze ergänzt um Daten des Onlinetelefonbuchs www.dasoertliche.de (2011). Für Belgien und Frankreich liegen vergleichbare Telefonbuchdaten nicht vor.

3 Informationen zum 2009-2012 an der Universität Luxemburg durchgeführten Projekt „Luxemburgischer Familiennamenatlas (LFA)“ finden sich online unter http://infolux.uni.lu/familiennamen/. Hier gibt es auch die Möglichkeit, Namen grenzüberschreitend zu kartieren. Im Laufe des Jahres werden die Ergebnisse auch in Buchform erscheinen. Vgl. zum LFA auch Gilles (in diesem Band). 
überhaupt bearbeitet, für zahlreiche weitere mussten die bislang geläufigen Etymologien modifiziert oder verworfen werden. Zudem wurde die räumliche Verbreitung der Familiennamen erfasst und für (sprach)historische Zusammenhänge kartographisch fruchtbar gemacht. Da „Namenareale Sprach- und Ländergrenzen überwinden“ (Marynissen/Nübling 2010:360), erfolgte die Kartierung in aller Regel über die heutigen Landesgrenzen hinweg für die gesamte Region zwischen Maas und Rhein oder auch für die kompletten Länder Luxemburg, Belgien, Deutschland und Frankreich.

Zugleich lassen sich aber auch für Luxemburg markante Differenzen im Nameninventar gegenüber den Nachbarländern feststellen. Erstaunlich ist etwa die große Zahl solcher Namen, die in ihrer heutigen Verbreitung fast oder vollständig auf das heutige Staatsgebiet Luxemburgs beschränkt sind. So findet sich in der Datenbank des LFA im Feld „Verbreitung“, in dem die areale Verteilung des jeweiligen Namens beschrieben wird, in immerhin 773 Fällen die Formulierung ,,(fast) nur in Luxemburg“. Dass lediglich rund 1000 als Lemma oder Variante erfasste Namen einen Eintrag im Feld ,Verbreitung“ besitzen, und zudem ohnehin nur die häufigsten Familiennamen Luxemburgs berücksichtigt wurden, macht die Zahl umso interessanter.

Indem der LFA also sowohl die (sprach)grenzüberschreitenden Gemeinsamkeiten als auch Differenzen mehrerer benachbarter Länder bzw. Großregionen in den Blick nimmt, steht er in der Tradition der meisten bisher erschienenen kontrastiven onomastischen Arbeiten wie etwa Marynissen/Nübling (2010), Farø/Kürschner (2007), Nübling (1997, 2004), Lüderitz (2002).

Durch das Disserationsprojekt soll diese Makroperspektive des LFA um einen mikroperspektivischen Blick auf die kleinräumigen Zusammenhänge einer sich wandelnden Grenzregion ergänzt werden und insbesondere mögliche Einflüsse politischer Grenzen auf Familiennamenlandschaften untersucht werden. Der deutsch-luxemburgische Grenzraum ist hierfür insofern besonders gut geeignet, als sich hier die historischen und rezenten Grenzen klar unterscheiden, und somit der mögliche Grenzeinfluss diachron beleuchtet werden kann. Um diesen ermitteln zu können, werden die erhobenen Daten des ehemals luxemburgischen Gebietes mit den Nameninventaren einerseits des heutigen Luxemburg und andererseits eines schon immer „deutschen“ ${ }^{4}$ Vergleichsgebietes, das sich hinter der ehemaligen luxemburgischen Grenze von vor 1815 anschließt, verglichen. Dabei stellt sich speziell die Frage, wie etwa die heute nur in Luxemburg vorkommenden Familiennamen erklärt werden können, da ihre Verteilung einen Grenzverlauf spiegelt, der zum Zeitpunkt der Entstehung und der angenommenen Festwerdung der Familiennamen keineswegs bestanden hat.

In diesem Zusammenhang sollen nun einige vorläufige Ergebnisse vorgestellt werden: Diese betreffen einerseits die Verteilung der verschiedenen Benennungsmotive auf die häufigsten Familiennamen sowie andererseits erste Detailbeobachtungen zu den

4 Der Begriff „,deutsch“ ist für die östliche Seite der historischen Grenze problematisch, insofern zu dieser Zeit kein einheitliches Territorium existierte. Korrekter wäre es, u.a. von einem kurtrierischen, einem preußischen etc. Gebiet zu sprechen. Der Einfachheit halber werden hier dennoch die Begriffe „deutsch“ bzw. ,nicht-luxemburgisch“ verwendet. 
Berufs- und Herkunftsnamen des Untersuchungsgebiets. Über den Vergleich zwischen dem heutigen und dem historischen Luxemburg sowie der angrenzenden deutschen Gebiete soll dabei, wo es sich anbietet, der kontrastive Blick auch auf die Namenlandschaften angrenzender Länder (Belgien, Niederlande und Deutschland) ausgeweitet werden. Zuvor soll aber das skizzierte Untersuchungsgebiet näher bestimmt werden.

\section{Das Untersuchungsgebiet}

Ziel der Arbeit ist die Untersuchung des Einflusses der historischen Grenzen, wie sie zum Zeitpunkt der Entstehung und Festwerdung der Familiennamen bestanden haben. Tatsächlich sind jedoch die Grenzen Luxemburgs häufigen Änderungen unterworfen gewesen: ${ }^{5}$ Seit der ersten Erwähnung der „kleinen Burg“ lucilinburhuc in einer Tauschurkunde zwischen Graf Siegfried und dem Kloster St. Maximin in Trier im Jahre 963 wächst das Territorium Luxemburgs zunächst stetig an, bis es im Jahre 1364 seine größte Ausdehnung erreicht, um anschließend im Wesentlichen durch drei Teilungen bis 1839 seine heutige Form zu erlangen (vgl. Abb. 1).

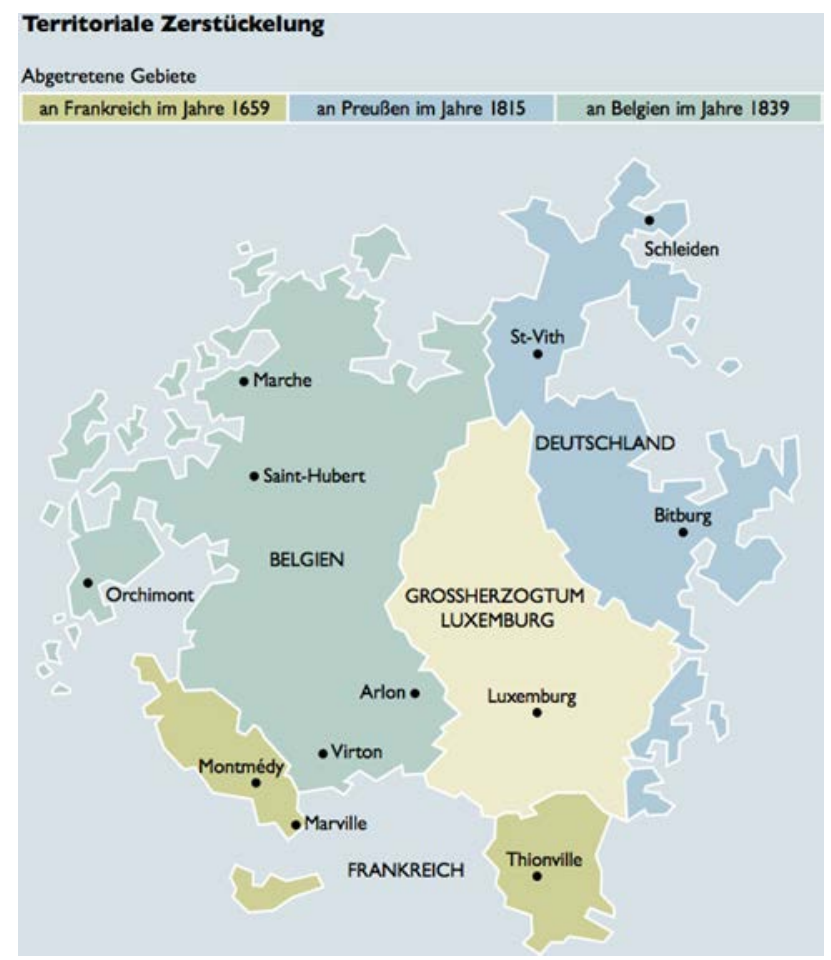

Abb. 1: $\quad$ Die Entwicklung des luxemburgischen Staatsgebiets seit 1364 (aus Thewes 2008:9)

5 Einen kurzen Überblick über die Geschichte Luxemburgs, auch mit Blick auf die Mehrsprachigkeit und wechselnden politischen Verhältnisse liefert etwa Thewes (2008); spezieller mit der Sprachhistorie des Luxemburgischen befasst sich Bruch (1953). 
Die Phase der Ausdehnung des luxemburgischen Territoriums liegt dabei noch vor der Entstehungszeit der Familiennamen und spielt somit hier keine Rolle. Lediglich die Teilung von 1659 fällt in den Zeitraum vor der endgültigen Festwerdung der luxemburgischen Familiennamen, die nach Erpelding (in LFN:19) ab dem 17. Jahrhundert einsetzt, sich aber bis um 1800 zieht. ${ }^{6}$ Wie bei der Abspaltung des überwiegend wallonischsprachigen (Nord-)Westens im Zuge der belgischen Unabhängigkeitsbestrebungen (1839) war die hier relevante luxemburgische Ostgrenze nicht betroffen. Abgesehen von kleineren Verschiebungen und Streitigkeiten im überwiegend kleinsträumigen Bereich kann diese im relevanten Zeitraum als stabil betrachtet werden.

Um die Referenzgebiete der Untersuchung im Detail festzulegen, wurde auf eine neue Edition des Atlas Ferraris zurückgegriffen. Hierbei handelt es sich um ein topographisches Kartenwerk, das ursprünglich von 1771 bis 1777 im kaiserlichen Auftrag vom österreichischen Generalleutnant von Ferraris erstellt wurde, und auf 275 handkolorierten Blättern die gesamten Österreichischen Niederlande, zu denen Luxemburg damals gehörte, erfasste. Anhand des enorm detailreichen und hochauflösenden Kartenmaterials konnten die historischen Grenzverläufe, wie sie direkt vor der Teilung Luxemburgs 1815 bestanden haben, auf Orts- bzw. sogar Einzelhofebene erfasst und auf die heutigen Orte und Siedlungen übertragen werden. Die so ermittelten Ortspunkte wurden anschließend entsprechend der heutigen fünfstelligen PLZ-Gebiete in Gruppen zusammengefasst. ${ }^{7}$ Das Untersuchungsgebiet lässt sich insgesamt in folgende Teile gliedern:

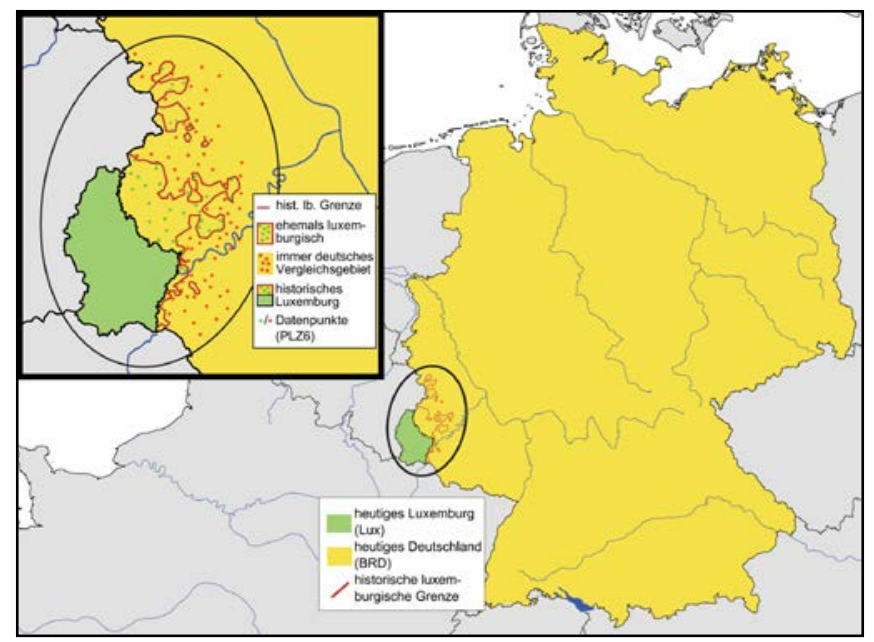

Abb. 2: $\quad$ Das Untersuchungsgebiet mit historischer Grenze und Datenpunkten (entsprechend der PLZ-Gebiete)

6 Dies deckt sich mit der Datierung der Festwerdung in Belgien (1795), Deutschland und den Niederlanden (jeweils 1811) durch Marynissen/Nübling (2010: 318).

7 Da die heutigen deutschen PLZ-Gebiete nicht dem historischen Grenzverlauf folgen, mussten die PLZ-Gebiete entlang der historischen luxemburgischen Grenze für die Untersuchung in ihren jeweils ehemals luxemburgischen und ,immer deutschen“ Anteil, die hier sog. PLZ6, geteilt werden. 
- Im Zentrum steht das eigentliche Untersuchungsgebiet, d.h. das ehemals luxemburgische Territorium auf heute deutschem Gebiet.

- Als ,immer deutsches“ Vergleichsgebiet wurde ein etwa ein PLZ-Gebiet breiter Streifen östlich der historischen Grenze ausgewählt.

- Zusätzlich wird auch das heutige Luxemburg als Vergleichsgebiet berücksichtigt.

Um eine klare Unterscheidung zu gewährleisten wird im Folgenden mit einfachem „Luxemburg“ (auch kurz „Lux“) bzw. „Deutschland“ (auch kurz „BRD“) jeweils auf die heutigen Staatsgrenzen referiert. Das eigentliche Untersuchungsgebiet hingegen wird als „ehemaliges Luxemburg“ (auch kurz „ehem. Lux.“) und das Vergleichgebiet östlich der historischen Grenze als „,deutsches Vergleichsgebiet“" (auch kurz „dt. Vgg.“) bezeichnet. Ehemaliges und heutiges Luxemburg gemeinsam ergeben das „historische Luxemburg“ (auch kurz ,hist. Lux.“).

\section{Die häufigsten Familiennamen und ihre Benennungsmotive}

Der Vergleich der Anteile der verschiedenen Benennungsmotive an den jeweils häufigsten Familiennamen mehrerer Nameninventare gehört zu den geläufigsten Methoden der kontrastiven Onomastik, auch wenn Farø/Kürschner (2007) zurecht auf die damit verbundene Problematik hingewiesen haben: Es kann nicht ohne weiteres davon ausgegangen werden, dass die häufigsten etwa 100 Familiennamen und ihre Benennungsmotive repräsentativ für das jeweilige Nameninventar insgesamt sind. Vielmehr konnten sie zeigen, dass bei einem Ansatz, der statt der „Spitze des Eisbergs“ (Nübling/Marynissen 2010:313) eine Zufallsauswahl aus allen Häufigkeitsbereichen zugrundelegt, deutlich andere Ergebnisse entstehen. So relativieren sich die von Lüderitz (2002) anhand der jeweils 100 häufigsten Familiennamen ermittelten Differenzen zwischen Deutschland und Dänemark nach ihrem Ansatz deutlich.

Ein weiteres, bislang nicht beachtetes Problem der Gegenüberstellung gleich großer TOP-Listen zweier Länder kann in der unterschiedlichen Gesamtanzahl der Types (d.h. verschiedenen Familiennamen) und Tokens (d.h. Namenträger) beider Länder und somit in einem entsprechend abweichenden relativen Anteil an den zugrundeliegenden Nameninventaren liegen: Die TOP 100 kann im Extremfall etwa für ein kleines Inventar bereits bis in die mittleren Häufigkeitsränge vordringen und bei einem wesentlich größeren Vergleichsinventar lediglich die absolute Spitze der Verteilungspyramide abdekken. In der Tat ist auch für den historischen luxemburgischen Grenzraum dieses Problem nicht außer Acht zu lassen, da sich zwar das dt. Vgg. und das heutige Luxemburg in der Größe ihrer Nameninventare recht nahe kommen (30169 bzw. 36326 Types mit 163206 bzw. 163039 Telefonanschlüssen), das ehem. Lux. aber kleiner ist (9381 Typen; 39698 Telefonanschlüsse).

Dennoch wird auch hier auf den Ansatz der TOP-Listen zurückgegriffen, da dieser interessante Ergebnisse zu liefern vermag. Zudem fehlen bislang statistisch ausgereifte Alternativen, die klare Aussagen über die Verteilung der unterschiedlich häufigen Familiennamen von der Spitze bis zum Ende treffen könnten. Der Vergleich der häufigsten 
Familiennamen erscheint somit durchaus gerechtfertigt, wenn man die damit verbundenen Probleme nicht vergisst. Um die Problematik einzudämmen, wird hier der von Marynissen/Nübling (2010) eingeschlagene Weg weitergeführt und die Zahl der untersuchten Namen nochmals von 200 auf 500 erhöht. Die Listen der jeweils 500 häufigsten Familiennamen im ehem. Lux., im dt. Vgg. und im heutigen Luxemburg finden sich im Einzelnen im Anhang.

Anhand dieser Daten soll nun ein Blick auf den Anteil der Benennungsmotive geworfen werden. Generell sind die Quellen der Familiennamenschöpfung in Deutschland und Luxemburg, das ja zur Zeit der Familiennamenentstehung noch Teil des deutschen Sprachgebiets war, dieselben. Problemfälle wie etwa die jüngeren schwedischen Naturnamen gibt es nicht. Dabei kann der in Deutschland weitgehend üblichen Unterteilung in fünf Kategorien gefolgt werden:

- Familiennamen nach dem Beruf (BerufsN) ${ }^{8}$ : Müller/Muller, Schmitz, Weber etc.

- Familiennamen aus Rufnamen (RufN): Peter(s), Thiel(en), Theis, Grethen etc.

- Familiennamen nach der Herkunft (bei Zugezogenen; HerkN): Weiler, Neuerburg, Schweitzer etc.

- Familiennamen nach der Wohnstätte (bei Ortsansässigen; WohnstN): Pütz/Putz/Pitz, Bach, Backes, Dum(m)ong/Dumont etc.

- Familiennamen aus Übernamen (ÜberN): Klein, Braun, Haas, Kayser etc.

Diese klassische Einteilung hat hier gegenüber der im LFA verwendeten, deutlich differenzierteren Klassifizierung, die Kollmann (2011) in einer frühen Version vorstellt, den Vorteil der besseren Vergleichbarkeit mit anderen Arbeiten sowie der besseren statistischen Erfassbarkeit.

Die Klassifizierung folgt im Wesentlichen den Etymologien des LFA, die 96,6 \% der luxemburgischen TOP 500 abdecken. Um auch fürs hist. Lux. und D auf eine ähnlich hohe Abdeckung zu gelangen, wurden zusätzlich für alle nicht vom LFA berücksichtigten Namen, soweit vorhanden, die Etymologien nach dem Duden Familiennamen aufgenommen, sowie ein Abgleich mit möglichen in Frage kommenden Ortsnamen anhand von Thorey/Geis (22003) durchgeführt. Bei Namen mit mehreren konkurrierenden Etymologien wurden diese jeweils anteilig berücksichtigt, d.h. dass etwa bei einem Namen mit je einer Etymologie als Herkunfts-, Berufs- und Wohnstättenname jeder der Kategorien der Wert $1 / 3$ zugeordnet wurde.

Die folgende Tabelle und Grafik zeigen den Anteil der verschiedenen Benennungsmotive an den jeweils häufigsten 500 Familiennamen der drei Gebiete sowie an den 200 häufigsten Familiennamen Deutschlands nach Marynissen/Nübling (2010:323):

8 Die Klasse der „Berufsübernamen“, d.h. solcher Namen, die statt von der Tätigkeit als solcher von einem typischen Werkstück oder -zeug abgeleitet sind, werden hier zu den Berufsnamen gezählt, da die zugrundeliegende Motivation der Benennung auch hier in der Tätigkeit des ersten Namenträgers liegt. Hierzu zählen bspw. Klinkhammer, Ambos, Axt. 
Tab. 1: Die Benennungsmotive der häufigsten Familiennamen in Luxemburg, ehem. Lux., dt. Vgg. sowie in Deutschland insgesamt (BRD)

\begin{tabular}{|l|c|c|c|c|c|}
\hline & RufN & BerufsN & HerkN & WohnstN & ÜberN \\
\hline Luxemburg & $39,7 \%$ & $20,0 \%$ & $19,0 \%$ & $8,1 \%$ & $13,2 \%$ \\
\hline ehem. Lux. & $39,4 \%$ & $20,9 \%$ & $13,9 \%$ & $10,3 \%$ & $15,5 \%$ \\
\hline dt. Vgg. & $40,3 \%$ & $21,4 \%$ & $14,0 \%$ & $8,7 \%$ & $15,6 \%$ \\
\hline BRD & $32,3 \%$ & $34,2 \%$ & $4,3 \%$ & $7,0 \%$ & $22,3 \%$ \\
\hline
\end{tabular}

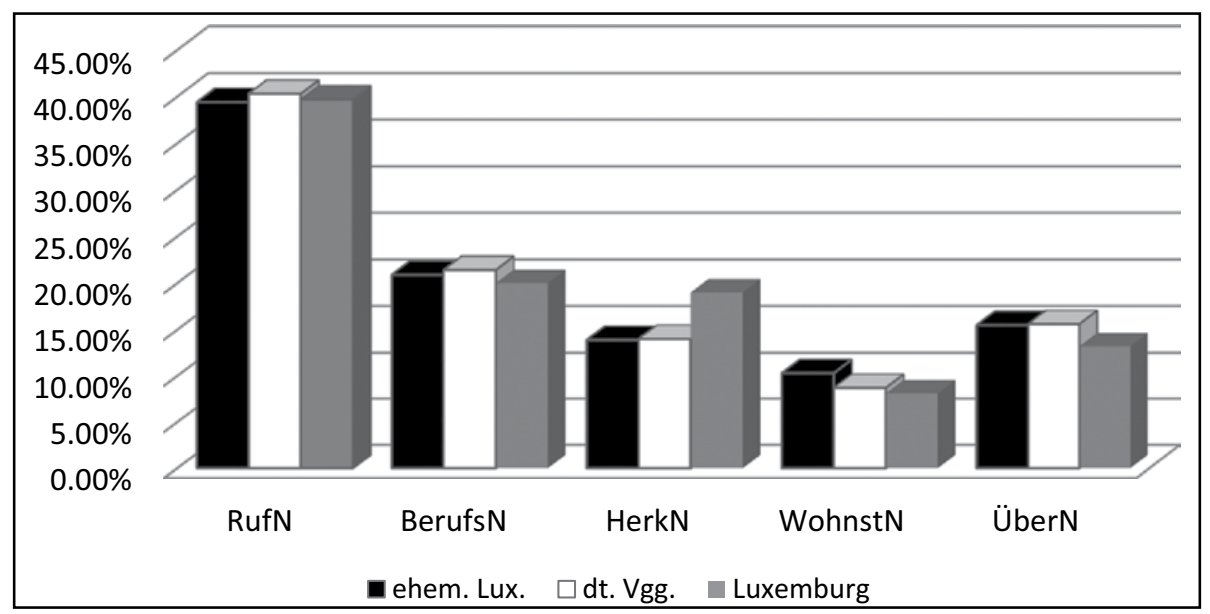

Abb. 3: Die Benennungsmotive der häufigsten Familiennamen in Luxemburg, ehem. Lux. und dt. Vgg.

Auf den ersten Blick fällt auf, dass die Verteilung der Benennungsmotive in Luxemburg, ehem. Lux. und dt. Vgg. relativ ähnlich ist: In allen Gebieten dominieren deutlich die Familiennamen aus Rufnamen mit etwa 40 \% vor den Berufsnamen mit rund $20 \%$. Im hist. Lux. und D folgen die Übernamen vor den Herkunftsnamen, in Luxemburg hingegen ist die Reihenfolge umgedreht. Überall am seltensten sind die Wohnstättennamen. ${ }^{9}$

Die Verteilung in der TOP 200 für Gesamtdeutschland hingegen weicht deutlich ab: Hier führen nicht die Patronyme sondern die Berufsnamen die Liste mit 34,2 \% gegenüber 32,3 \% knapp an. Es folgen mit deutlichem Abstand die Übernamen vor den Wohnstättenund Herkunftsnamen. Diese Unterschiede gerade auch gegenüber dem dt. Vgg. lassen sich als Ausdruck der Regionalität der Familiennamenlandschaften deuten: Die große Anzahl an Patronymen ist demnach typisch für den (Süd-)Westen des deutschen Sprachraums und

9 Dabei ist zu berücksichtigen, dass v.a. Namen aus Tierbezeichnungen (wie Haas, Wolff(f), Bock, Hahn/Haan usw.) ebenso wie solche aus einem insbes. im Genitiv stehenden Rufnamen (wie Peters, Thielen, Jacoby) gerade in Luxemburg stets auch zunächst Hausnamen und daraus abgeleitet erst Familiennamen (und somit Wohnstättennamen) sein können. Da dies jedoch im Einzelfall kaum sicher ermittelbar ist und um eine „Schwemme“ an Wohnstättennamen zu verhindern, wurden solche Namen nie als Wohnstättennamen gewertet. 
findet in Luxemburg seine Fortsetzung. Der wesentlich niedrigere Anteil in der TOP 200 Gesamtdeutschlands wäre dann durch die restlichen Gebiete Deutschlands beeinflusst. Daneben muss aber auch die unterschiedliche Verteilung der Benennungsmotive in den verschiedenen Häufigkeitsstufen berücksichtigt werden: Vergleicht man die Werte der TOP 200 nach Marynissen/Nübling (2010) mit denen für die TOP 100 nach Lüderitz (2002) sowie denen der Zufallsauswahl nach Farø/Kürschner (2007), so zeigt sich, dass der Anteil der Patronyme mit der Anzahl berücksichtigter weniger frequenter Namen stetig steigt: Liegt er in der TOP 100 noch bei $22 \%$, so sind es in der TOP 200 bereits 32,3\% um bei der Zufallsauswahl von Farø/Kürschner (2007) mit 33 \% schließlich die Rangliste anzuführen. Ähnliches gilt auch für die anderen Benennungsmuster: bei zunehmender Größe der Namenauswahl nähern sich die Werte den Zahlen für D an:

Tab. 2: Die Verteilung der Benennungsmotive in Deutschland für verschiedene große Ausschnitte (Farø/Kürschner (2007) haben bei der Auswertung ihrer Zufallsauswahl die Herkunfts- und Wohnstättennamen zu einer Gruppe zusammengefasst)

\begin{tabular}{|l|c|c|c|c|c|}
\hline & RufN & BerufsN & HerkN & WohnstN & ÜberN \\
\hline TOP 100 & $22 \%$ & $44 \%$ & $4 \%$ & $6 \%$ & $24 \%$ \\
\hline TOP 200 & $32,3 \%$ & $34,2 \%$ & $4,3 \%$ & $7,0 \%$ & $22,3 \%$ \\
\hline Zufallsauswahl & $33 \%$ & $25 \%$ & $29 \%$ & & $18 \%$ \\
\hline
\end{tabular}

Es bleibt jedoch festzuhalten, dass auch bei einer Berücksichtigung aller Häufigkeiten wie durch Farø/Kürschner (2007) die Werte für Gesamtdeutschland nicht bis zu denen fürs dt. Vgg. (bzw. ehem. Lux. oder Luxemburg) heranreichen. Dies scheint - bei aller Vorsicht, denn schließlich kann in den noch selteneren Bereichen unterhalb der TOP 500 das Verhältnis wieder umgekehrt sein - doch für eine Regionalität der Werte zu sprechen. Sie spiegeln somit zunächst weniger eine harte Abstufung entlang der (historischen) Grenze als vielmehr einen allmählichen Verlauf im größeren Kontext im Sinne grenzüberschreitender, europäischer Namenareale.

Vergleicht man hingegen nur die regionalen Daten, so zeigen sich drei unterschiedlich deutliche Ausreißer (in Tab. 1 grau hinterlegt). Bei den Herkunfts- und bei den Übernamen weicht der Wert für Luxemburg von denen im dt. Vgg. und ehem. Lux. ab. Gerade der Abstand bei den Herkunftsnamen scheint für eine zufällige Abweichung zu groß und passt auch nicht ins Bild eines langsamen großräumigen Übergangs. Vielmehr findet hier die aktuelle Staatsgrenze ihren Niederschlag in den Familiennamen. Ist einerseits ein Grenzeinfluss gerade bei den Herkunftsnamen, die an Zugezogene vergeben wurden und somit ein gewisses Abbild von Migration darstellen, durchaus plausibel, so wäre ein solcher Einfluss andererseits eigentlich für die historische Grenze zwischen dt. Vgg. und ehem. Lux. zu erwarten. Insofern die Herkunftsnamen die historischen Migrationsbewegungen zur Zeit ihrer Entstehung reflektieren, kann die heutige Grenze, die damals eben noch in keiner Weise Bedeutung hatte, keinen Einfluss gehabt haben. Noch erstaunlicher ist die (wenngleich weniger deutliche) Differenz bei den Wohnstättennamen zwischen Luxemburg und dt. Vgg. auf der einen und ehem. Lux. auf der anderen Seite, stellt doch ehem. Lux. zu keiner Zeit ein eigenständiges, in sich abgeschlossenes Gebiet dar. 
Die Verteilung der Benennungsmotive unter den jeweils 500 häufigsten Familiennamen weist somit auf einen grenzüberschreitenden, größeren Namenraum mit fließenden Übergängen hin. Zugleich lassen sich aber auch die Beobachtungen aus der Arbeit am LFA bestätigen, die auf eine gewisse Eigenständigkeit des Nameninventars des heutigen Luxemburg hinweisen. So fällt neben der recht großen Zahl relativ häufiger Familiennamen, die nur dort vorkommen, die Differenz in der Häufigkeit der Herkunfts- und Übernamen in Luxemburg verglichen mit dt. Vgg. und ehem. Lux. auf. Dieser Grenzverlauf in der Namenlandschaft lässt sich aber im Zusammenhang mit der Entstehung der Familiennamen historisch nicht erklären.

Vielmehr müssen die Ursachen wohl eher in der Zeit der Grenzverschiebung, d.h. um bzw. nach 1815 gesucht werden. Tatsächlich lassen sich Hinweise dafür finden, dass vor allem Schreibvarianten für die heutigen Unterschiede auf beiden Seiten der Grenze verantwortlich sein könnten. So findet sich der häufigste luxemburgische Familienname Schmit in Deutschland in dieser Schreibung ganze 175 Mal, während die in Deutschland häufigste graphische Variante Schmidt in Luxemburg wiederum kaum vorkommt. Daneben lassen sich zahlreiche weitere, auch systematische graphische Besonderheiten der heutigen luxemburgischen Familiennamen finden, so die häufigen Schreibungen mit -ff, -nck oder -ou-. ${ }^{10}$

In diese Richtung weisen auch erste Versuche, den Abstand zwischen verschiedenen Familiennameninventaren über die Differenz der relativen Häufigkeit der einzelnen Typen in ihnen zu ermitteln. Auch hier ist das Problem der unterschiedlichen Größen der Areale noch nicht gelöst, es fällt aber auf, dass der Wert sich für Luxemburg und das ehem. Lux. ebenso wie für Luxemburg und das dt. Vgg. merklich ändert, wenn man alleine die verschiedenen Umlautschreibungen zusammenfasst. Die Differenz zwischen ehem. Lux. und dt. Vgg. hingegen ändert sich hierdurch kaum.

\section{Einige Details zu Berufs- und Herkunftsnamen}

Im Folgenden soll nun ein genauerer Blick auf die Familiennamen der einzelnen Benennungsmotive geworfen werden, um auch an ihnen einige Gemeinsamkeiten und Unterschiede der verschiedenen Gebiete herauszuarbeiten. Im Rahmen dieses Artikels bleibt der Vergleich auf die Berufs- und Herkunftsnamen begrenzt, da für diese zum jetzigen Zeitpunkt die salientesten Ergebnisse vorliegen. Die hier vorgestellten Ergebnisse bleiben dabei eine erste Auswahl und erheben keinerlei Anspruch auf Vollständigkeit, sondern zeigen vielmehr erste Tendenzen auf.

Für dieses Kapitel wurden jeweils nur eindeutig einem Benennungsmotiv zuzuordnende Namen berücksichtigt, d.h. alle Familiennamen, für die mehrere, auf verschiedene Bennenungsmotive weisende Etymologien in Frage kommen, wurden nicht berücksichtigt. Die Auswahl bleibt dabei nicht auf die häufigsten 500 Familiennamen der einzelnen

10 So etwa in den Familiennamen Kieffer, Graffl-graff, Schaeffer/Schäffer; Junck(er), Franck, Funck; zahlreichen Namen mit -bourg/Bourg- (darunter auch Bourggraff), Bourkel/Bourquel/Bourckel (zu Burkel) uvm. Vgl. hierzu auch Gilles (in diesem Band). 
Areale beschränkt, vielmehr sind alle Familiennamen, für die bislang eine Etymologie ermittelt werden konnte, aufgenommen worden.

\section{Berufsnamen}

Wie aufgrund der unterschiedlichen Gesamtzahl der Familiennamen in den einzelnen Gebieten zu erwarten, variiert auch die Zahl der eindeutig als Berufsnamen identifizierten Familiennamen. Stimmen die Verhältnisse im Großen überein, so gibt es trotz ähnlicher Anteile zumindest unter den 500 häufigsten Familiennamen doch eine gewisse Abweichung: So ist die Anzahl der eindeutigen Berufsnamen in Luxemburg, das insgesamt mehr Namen hat als das dt. Vgg., geringer als dort. Auffällig ist auch, dass im ehem. Lux. trotz insgesamt nur eines Drittels an Familiennamen etwa halb so viele Berufsnamen vorkommen wie im dt. Vgg. oder in Luxemburg:

Tab. 3: Anzahl der eindeutigen Berufsnamen

\begin{tabular}{|l|c|c|}
\hline ehem. Lux. & Lux & dt. Vgg. \\
\hline 299 & 547 & 651 \\
\hline
\end{tabular}

Zurückführen lassen sich diese Abweichungen wohl auf zwei Ursachen. Einerseits ist der prozentuale Anteil der etymologisierten Familiennamen im hist. Lux. insgesamt etwa doppelt so hoch wie in Luxemburg und im dt. Vgg. (20\% vs. $10 \%$ ), was auch die absolute Anzahl der identifizierten Berufsnamen erhöht. Auf der anderen Seite sind die Berufsnamen im dt. Vgg. (wie im gesamten Deutschland; vgl. Marynissen/Nübling 2010:326ff.) sehr kompositionsfreudig, was die Anzahl der verschiedenen Types deutlich in die Höhe treibt. Demgegenüber gibt es unter den Berufsnamen im gesamten historischen Luxemburg (d.h. sowohl in Lux als auch im ehem. Lux.) deutlich weniger Komposita. Tab. 4 zeigt stellvertretend die absolute Zahl der Kompositia für die häufigsten Berufsnamen Müller, Meier, Schmi(d)t und Weber inklusive ihrer jeweiligen Schreibvarianten. Mit Abstand die meisten Komposita gibt es jeweils im dt. Vgg. Eine größere Zahl an Komposita gibt es im ehem. Lux. und Luxemburg lediglich mit dem Zweitglied -meier, wobei auch hier die Zahl im dt. Vgg. deutlich höher ist. Ein gestufter Übergang zwischen dt. Vgg., ehem. Lux. und Luxemburg lässt sich insgesamt nicht feststellen.

Tab. 4: Anzahl der Komposita auf-müller, -meier, -schmi(d)t und -weber

\begin{tabular}{|l|c|c|c|}
\hline & ehem. Lux. & Lux & dt. Vgg. \\
\hline -müller & 12 & 8 & 54 \\
\hline -meier & 33 & 51 & 200 \\
\hline -schmit & 6 & 9 & 35 \\
\hline -weber & 3 & 2 & 7 \\
\hline
\end{tabular}

Neben der deutlichen Abweichung in der Kompositionsfreudigkeit, zeigen sich Unterschiede auch bei der (Schreib-)Varianz, die sich bei den Simplizia und Komposita 
gleichermaßen zeigt. Während Weber in allen Gebieten ohne Schreibvariante auftritt, divergiert die Zahl der Varianten bei Müller, Meyer und Schmi(d)t. Insgesamt treten im dt. Vgg. jeweils die meisten Varianten auf, es folgt Luxemburg, die wenigsten Varianten gibt es im ehem. Lux.

Etwas aus der Reihe fällt hier lediglich Müller, das in Luxemburg genauso viele Varianten aufweist, wie im dt. Vgg. Dies erklärt sich zum einen über allgemeine Besonderheiten in der Umlautschreibung des [y] im Luxemburgischen: Umlautschreibung mit Trema $<\ddot{\mathrm{u}}>$ ist hier sehr selten ${ }^{11}$, neben $<$ ue $>$ findet sich durch französischen Einfluss häufig auch $<\mathrm{u}>$ in der Lautung [y] - so ist Muller auch die mit Abstand häufigste Variante in Luxemburg. Zum anderen führt die im Lb. regelmäßige Assimilation von ers > esch hier zu Dubletten der Genitivformen: Neben Mullers etc. tritt auch Mullesch etc. auf.

Tab. 5: Anzahl der Schreibvarianten bei Müller, Meier und Schmi(d)t

\begin{tabular}{|l|l|l|l|}
\hline Davon & ehem. Lux. & Lux & dt. Vgg. \\
\hline Müller $^{12}$ & 2 & 7 & 7 \\
\hline Meyer $^{13}$ & 8 & 9 & 14 \\
\hline Schmit $^{14}$ & 6 & 9 & 13 \\
\hline
\end{tabular}

Die deutlich geringere Anzahl der Schreibvarianten im ehem. Lux. lässt sich wohl in erster Linie über die geringere Gesamtgröße das Nameninventars erklären, ob sie darüber hinaus auch auf eine größere Nähe zu Luxemburg als zum dt. Vgg. hinweist, lässt sich nicht klar beantworten. Auffällig ist hier die verhältnismäßig hohe Anzahl an Varianten bei Meyer, die sich aber ebenfalls schwer deuten lässt.

Interessant ist daneben die Anzahl von Berufsnamen im Genitiv. Diese sind laut Marynissen/Nübling (2010:328ff.) in Deutschland insgesamt selten und haben ihr Zentrum in den Niederlanden und Flandern, von wo sie sich bis in den Nordwesten Deutschlands ziehen. Ihre Zahl ist indes auch im gesamten Untersuchungsgebiet recht hoch: Das beschriebene Areal findet hier also seine Fortsetzung.

11 Tatsächlich verzeichnet die Editus-Telefondatenbank keine Umlautschreibungen, so dass selbst die seltenen Familiennamen mit $<\ddot{\mathrm{u}}>$ nicht in dieser Form notiert sind. Statt $<\ddot{\mathrm{u}}>$ findet sich $<\mathrm{u}>$, statt $<\ddot{a}, \ddot{o}><$ ae, oe $>$.

12 Die Varianten umfassen neben Mueller/Muller u.a. verdumpftes Möller/Moeller, entrundetes Miller sowie die latinisierte Variante Molitor.

13 Die Varianz beruht hier vorwiegend auf den unterschiedlichen Schreibungen -ey-, -ei-, -ay-, -ai-. Dazu kommen Genitiva sowie wiederum die latinisierte Form Majerus, auch in abgeschwächten Varianten wie Majeres.

14 Hier liegen zahlreiche Varianten des Auslauts vor: Schmidt,-tt, $-t,-d$, auch im Genitiv $-t z,-t s$. Hinzu kommen Diminutiva wie Schmidtke und neben latinisierten Formen Faber, Fabry auch französische Varianten wie Lefevre. Keine direkte Variante hingegen ist etymologisch verwandtes Schmoetten, das fast nur im heutigen Luxemburg auftritt und statt des Schmieds die Schmiede bezeichnet. 
Tab. 6: Anzahl der Genitive bei den Berufsnamen

\begin{tabular}{|l|c|c|c|}
\hline & hist. Lux. & Lux & D \\
\hline Anzahl gesamt & 19 & 38 & 35 \\
\hline davon starke Genitive (davon auf -sch) & $18(0)$ & $35(4)$ & $31(1)$ \\
\hline davon schwache Genitive & 1 & 1 & 3 \\
\hline davon lat. Genitive & & 2 & 1 \\
\hline
\end{tabular}

Ebenfalls an die Niederlande und Flandern schließen die in Luxemburg deutlich häufigeren Bildungen mit Artikel und/oder Präposition an (vgl. Marynissen/Nübling 2010:328ff.). So finden sich hier insgesamt 22 Berufsnamen mit germanischem (de etc.) und französischem (le etc.) Artikel (und teilweise Präposition). Deutlich seltener sind solche Bildungen hingegen in D (sechs, nämlich: De Meyer, Lefebre, Lefebvre, Lepage, Leclere, Lefevre) und hist. Lux. (drei, nämlich: Lepage, Lefebre, Lemaire) ebenso wie insgesamt in Deutschland.

\section{Herkunftsnamen}

Herkunftsnamen sind wie alle Familiennamen aus zunächst unfesten Beinamen entstanden, um den Namenträger von anderen Personen mit demselben Rufnamen zu unterscheiden. Dabei wurden Herkunftsnamen solchen Personen gegeben, die aus einem anderen Ort zugezogen waren. Insofern tradieren die Herkunftsnamen historische Migrationsbewegungen. Hierbei können sowohl Mikromigrationen von einem Ort in den nächsten (Densborn, Dondelinger etc.) als auch Makromigrationen aus einem Ort in einen weiter entfernten Ort (dann meist eine größere Stadt, so etwa Luxemb(o)urger) bis hin zur Migration über Landschafts- und auch Landesgrenzen (Böhm, Saxler, Schweitzer) gespiegelt werden.

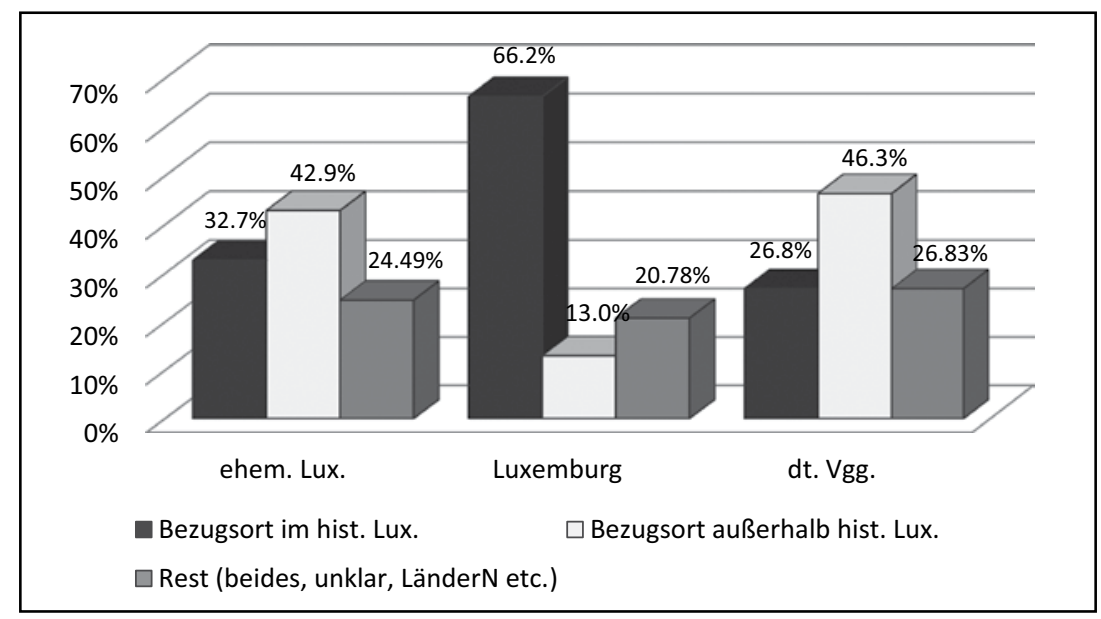

Abb. 4: $\quad$ Die Verteilung der Bezugsorte der Herkunftsnamen ${ }^{15}$

15 Die sog. Ländernamen, d.h. Namen, die nicht auf eine Ortschaft, sondern auf das Land oder Gebiet der Herkunft verweisen, werden hier mit solchen Namen, die auf mehrere Orte auf beiden Seiten 
Um den Einfluss der historischen Grenze Luxemburgs auf Migrationsbewegungen in der Mikroebene zu untersuchen, wurde für alle eindeutigen Herkunftsnamen mit Hilfe sowohl der mit dem Atlas Ferraris ermittelten Ortsliste als auch von Thorey/Geis ( $\left.{ }^{2} 2003\right)$ ermittelt, ob ihr Bezugsort im historisch luxemburgischen Gebiet liegt oder nicht. In der überwiegenden Zahl der Fälle liegen dabei Bezugsorte, die außerhalb des historischen Luxemburgs liegen, heute in Deutschland - auf eine Unterscheidung zwischen solchen historisch nicht-luxemburgischen Orten, die im heutigen Belgien, Frankreich und Deutschland liegen, wurde daher verzichtet.

Die in Abb. 4 wiedergegebenen Werte weisen eine deutliche Differenz zwischen Luxemburg auf der einen und dem dt. Vgg. auf der anderen Seite auf. So liegen die Bezugsorte von etwa zwei Drittel der Herkunftsnamen in Luxemburg auch im historisch luxemburgischen Gebiet, lediglich 13 Prozent hingegen außerhalb des historischen Luxemburg. Dies scheint für einen Einfluss der Grenzen auf die Verteilung der Herkunftsnamen zu sprechen. Hierzu passt auch, dass im dt. Vgg. das Verhältnis gerade umgekehrt ist: Hier liegt mit 46,3 Prozent die Mehrzahl der Bezugsorte außerhalb und lediglich 26,8 Prozent innerhalb des historischen Luxemburg. Erstaunlich ist jedoch, dass auch im ehem. Lux. mit 42,9 Prozent die Mehrzahl der Bezugsorte außerhalb des historisch luxemburgischen Gebietes liegt. Dem stehen lediglich 32,7 Prozent historisch luxemburgische Bezugsorte gegenüber. Das Verhältnis ist zwar im ehem. Lux. etwas ausgeglichener als im dt. Vgg., dennoch überrascht das im Vergleich mit Luxemburg umgekehrte Verhältnis der Werte.

Weisen die Werte im heutigen Luxemburg auf einen deutlichen Einfluss der Grenzen auf die Herkunftsnamen hin, so sprechen die Werte im ehem. Lux. eher dagegen. Dies ist insofern bemerkenswert, als ein Einfluss der heutigen Grenzen kaum plausibel zu machen ist. Erklärungsversuche wären in der Zeit nach der Grenzziehung von 1815 und keinesfalls im Zusammenhang mit der Entstehung der Herkunftsnamen anzusetzen. Somit kämen allenfalls spätere Migrationsbewegungen in Frage, d.h. einerseits ein verstärkter Zuzug aus dem Rest Deutschlands ins ehem. Lux. oder aber eine Abwanderung ehemaliger Luxemburger aus dem ehem. Lux. in den Rest Luxemburgs nach 1815 (wobei jeweils die ererbten Herkunftsnamen mitgewandert wären). Allerdings sind solche Migrationsbewegungen - zumal in dem wohl für einen Niederschlag in den Herkunftsnamen nötigen Ausmaß - nicht bekannt.

Eine größere Nähe des ehem. Lux. zu Luxemburg als zum dt. Vgg. lässt sich hingegen aus einem anderen Vergleich ableiten. Abb. 5 und 6 zeigen die relative Häufigkeit aller eindeutigen Herkunftsnamen aus dem ehem. Lux. in Luxemburg bzw. im dt. Vgg. Dabei wird jeweils wieder zwischen Namen mit einem Bezugsort innerhalb bzw. außerhalb des historischen Luxemburg unterschieden. Der jeweils linke Balken gibt dabei die Anzahl der Namen an, die in Luxemburg bzw. im dt. Vgg. jeweils überdurchschnittlich häufig vorkommen, der rechte Balken hingegen die Anzahl der Namen die unterdurchschnittlich (oder gar nicht) vorkommen.

der Grenze verweisen, oder deren Bezugsort nicht eindeutig ermittelbar ist, zu einer Restgruppe zusammengefasst. 
Während in Luxemburg (Abb. 5) nun jene Herkunftsnamen aus ehem. Lux. mehrheitlich überdurchschnittlich häufig vorkommen, deren Bezugsorte im historischen Luxemburg liegen, so sind es im dt. Vgg. (Abb. 6) gerade jene, deren Bezugsorte außerhalb des historischen Luxemburg liegen.

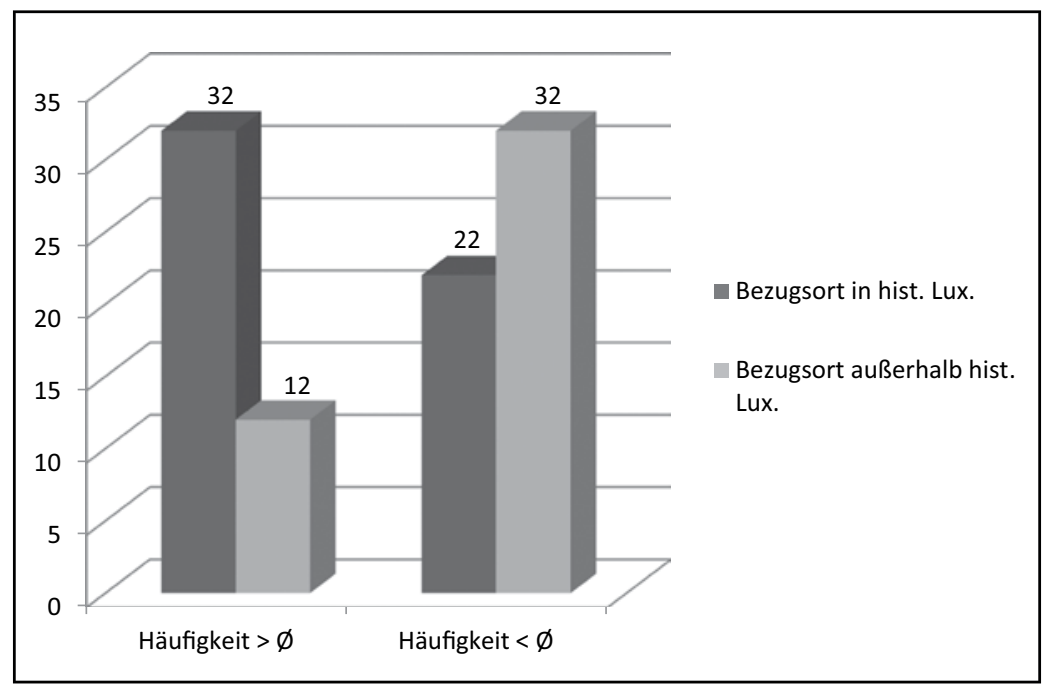

Abb. 5: $\quad$ Bezugsorte der über- und unterdurchschnittlich häufig in Luxemburg vorkommenden Herkunftsnamen (Anzahl absolut)

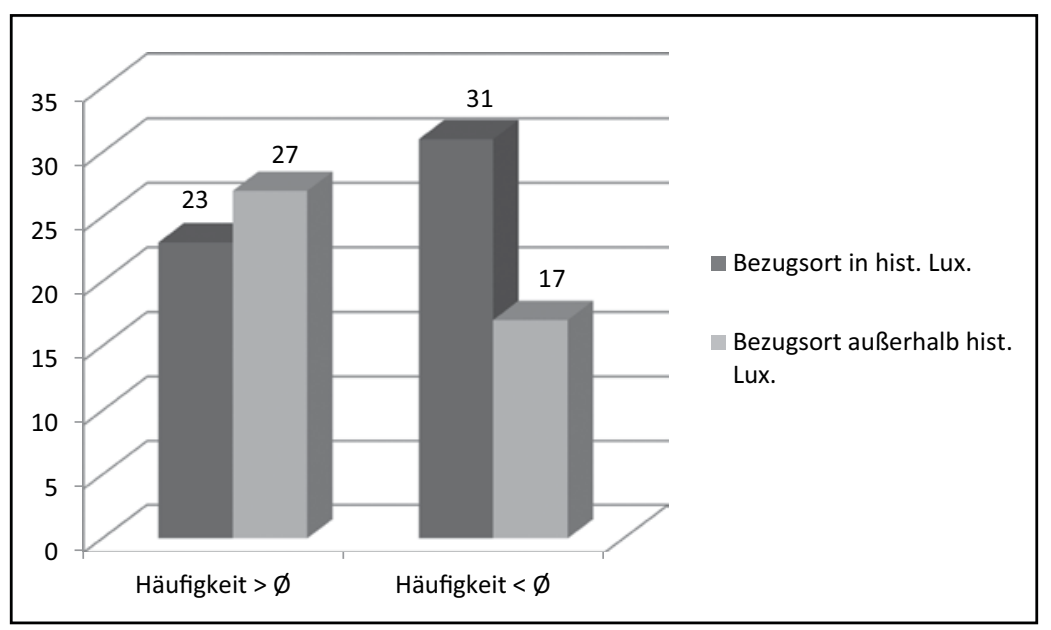

Abb. 6: $\quad$ Bezugsorte der über- und unterdurchschnittlich häufig im dt. Vgg. vorkommenden Herkunftsnamen (Anzahl absolut)

Vergleicht man nur die Anzahl der Namen, die in Luxemburg und D jeweils überhaupt nicht vorkommen, so zeigt sich dieser Gegensatz noch klarer: 


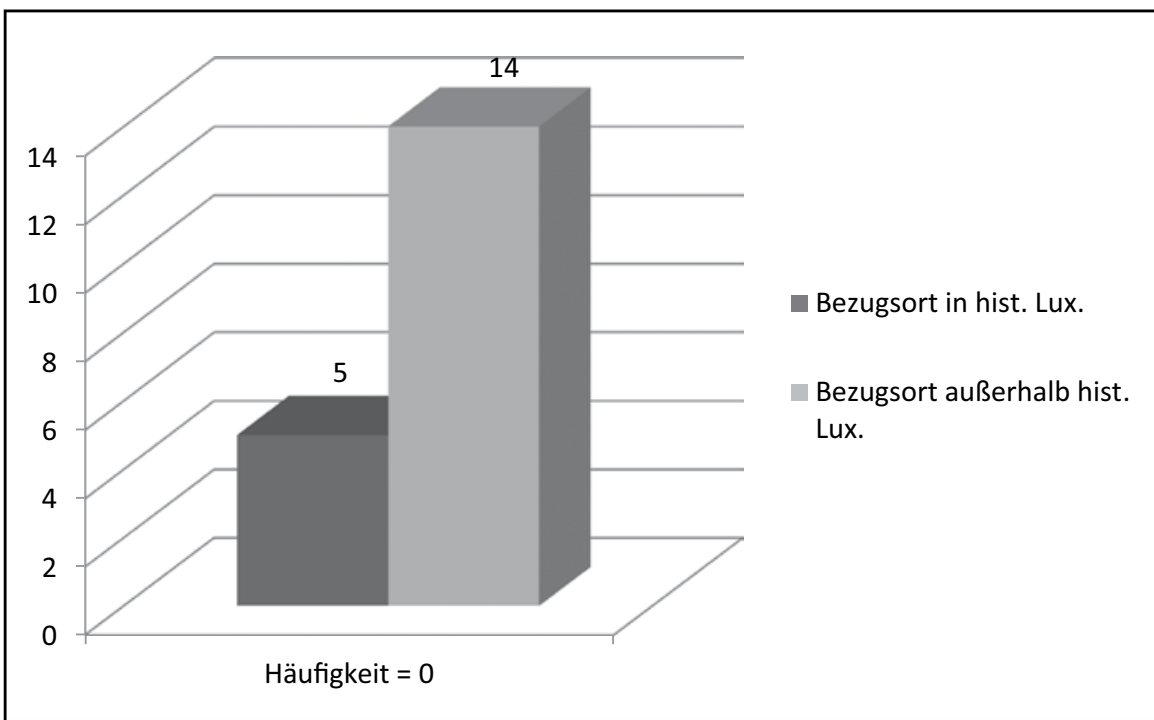

Abb. 7: Bezugsorte der in Luxemburg nicht vorkommenden Herkunftsnamen (Anzahl absolut)

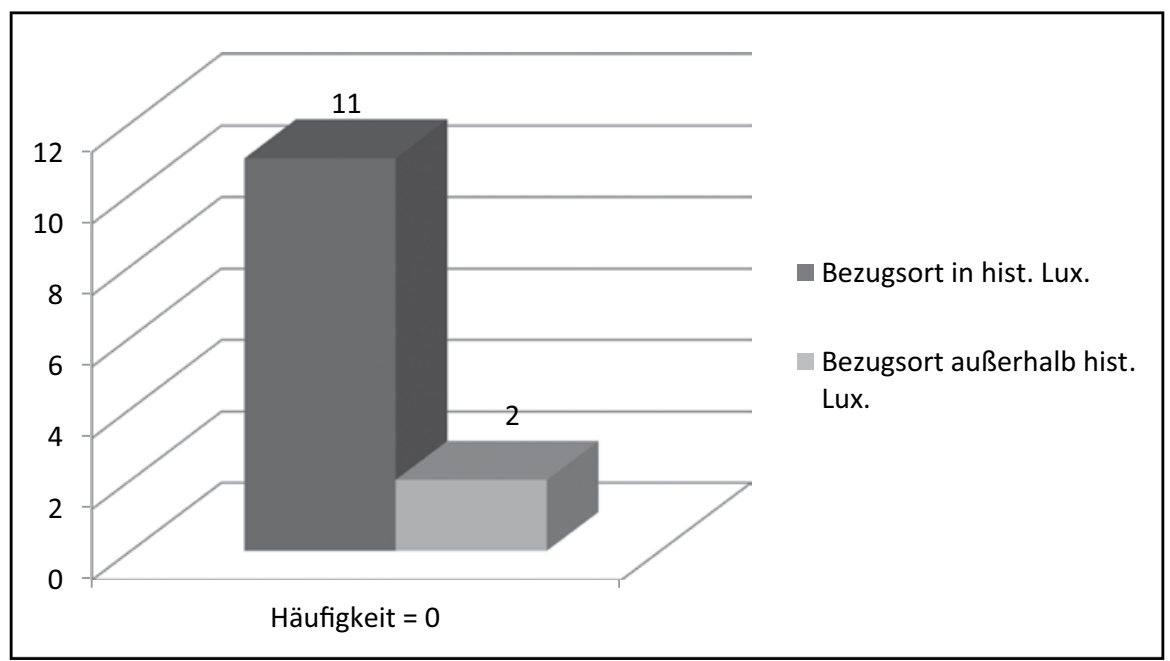

Abb.8: $\quad$ Bezugsorte der im dt. Vgg. nicht vorkommenden Herkunftsnamen (Anzahl absolut)

\section{Fazit und Ausblick}

Insgesamt stützen die hier vorgestellten Daten das von Marynissen/Nübling (2010) skizzierte Bild grenzüberschreitender, europäischer Namenareale. Dabei konnten für das Untersuchungsgebiet sowohl Gemeinsamkeiten als auch (oft gestaffelte) Unterschiede zwischen den einzelnen Teilgebieten gezeigt werden. 
Ein relativ einheitliches Bild für das Untersuchungsgebiet ergaben die Verteilung der Benennungsmotive auf die jeweils häufigsten Familiennamen sowie die durchgehend recht hohe Anzahl der im Genitiv stehenden Berufsnamen. Interessant ist, wie sich diese Gemeinsamkeiten in der Makroperspektive jeweils in größere Areale integrieren lassen. So konnte für die Verteilung der Benennungsmotive gezeigt werden, dass die im Untersuchungsgebiet sehr ähnlichen Werte deutlich von denen der gesamten Bundesrepublik abweichen, und es sich hier folglich um ein im Westen Deutschlands bis nach Luxemburg und an die germanisch-romanische Sprachgrenze reichendes Areal handelt. Es ist davon auszugehen, dass dieses Gebiet keine klare Ostgrenze hat, sondern die Übergänge hier fließend sind. Bei den Berufsnamen im Genitiv wiederum handelt es sich ebenfalls um ein Areal, das nur im Westen Deutschlands auftritt und außer in Luxemburg auch in Flandern und den Niederlanden seine (noch gesteigerte) Fortsetzung findet.

Deutliche Unterschiede innerhalb des Untersuchungsgebietes hingegen lassen sich zwischen den Teilgebieten etwa in Bezug auf die Anzahl der Komposita bei den Berufsnamen aufzeigen. Während in der Makroperspektive Deutschland insgesamt als kompositionsfreudig erscheint, konnte in der Mikroperspektive gezeigt werden, dass es hier deutliche regionale Unterschiede gibt, die der historischen luxemburgischen Grenze zu folgen scheinen: So ist das dt. Vgg. noch durch eine große Zahl an Komposita gekennzeichnet, während bereits im ehem. Lux., ebenso wie in Luxemburg, kaum Kompositionsbildungen $\mathrm{zu}$ verzeichnen sind. Eine rein auf das historische Luxemburg bezogenes Phänomen liegt hier jedoch nicht vor, denn seine Fortsetzung findet dieses kompositionsarme Gebiet wiederum in Flandern und den Niederlanden.

Unterschiede lassen sich ebenfalls bei der Bildung der Berufsnamen mit Artikel/ Präposition sowie bei der Verteilung der Bezugsorte der Herkunftsnamen verzeichnen. Hier allerdings verlaufen die Differenzen nicht entlang der historischen, sondern der heutigen luxemburgischen Grenzen. In Bezug auf die Artikel/Präpositions-Bildungen der Berufsnamen ergibt sich auch hier wieder ein gemeinsames Areal LuxemburgFlandern-Niederlande. Bei der Verteilung der Bezugsorte der Herkunftsnamen wiederum konnte mit dem Vergleich der Häufigkeiten der Herkunftsnamen aus dem ehem. Lux. im dt. Vgg. und in Luxemburg ein Hinweis auf eine doch vorhandene Staffelung erbracht werden.

Dennoch verwundert gerade bei den Herkunftsnamen als Spiegel historischer Migration der Bezug auf die heutigen Grenzen. Zugleich erscheinen die Werte aber zu eindeutig, um als rein zufällig betrachtet zu werden. Um hier mögliche Ursachen nach der Grenzverschiebung von $1815 \mathrm{zu}$ ermitteln, sollen im Rahmen der Dissertation historische Namendaten vom Beginn des 19. Jahrhunderts untersucht werden. Gerade auch in Hinblick auf die Schreibvarianten können diese Daten wohl neue Einsichten liefern. Ebenso soll im Rahmen der Dissertation das erwähnte Problem der TOPListen durch eingehende statistische Vergleiche einzelner Häufigkeitsbereiche sowie eine Vollanalyse einzelner Belegorte abgeschwächt werden. Dies soll durch weitere statistische Vergleichsmöglichkeiten zwischen benachbarten Namenarealen flankiert werden. 


\section{Literatur}

Bruch, Robert (1953): Grundlegung einer Geschichte des Luxemburgischen. Luxemburg (Publications littéraires es scientifiques du Ministère de l'Éducation Nationale; 1 ).

Duden Familiennamen=Kohlheim, Rosa/Kohlheim, Volker (2005): Duden Familiennamen. Herkunft und Bedeutung. Mannheim u.a.

Farø, Ken/Kürschner, Sebastian (2007): Et databasemøde mellem Jensen og Müller. Om kontrastiv antroponomastik metodik. In: TijdSchrift voor Skandinavistiek 28/2, 105-126.

Ferraris, Graf Josef Johann von (2011): De grote atlas van Ferraris. De eerste atlas van België, 1777. Kabinetskaart van de Oostenrijkse Nederlanden en het Prinsbisdom Luik [= Le grand Atlas de Ferraris]. Mit einer Einleitung v. Sara Lammens neu herausgegeben. Tielt.

Kollmann, Cristian (2011): Methodische Fragen und Überlegungen zur Typologisierung der Familiennamen am Beispiel des Luxemburgischen Familiennamenatlasses. In: Arne Ziegler/Erika WindbergerHeidenkummer (Hrsg.): Methoden der Namenforschung. Methodologie, Methodik und Praxis. Berlin, $47-62$.

LFA=Luxemburgischer Familiennamenatlas. Derzeit online unter: http://infolux.uni.lu/familiennamen (ges. 04.02.2013).

LFN=Die Luxemburger und ihre Familiennamen, Circa 17500 Familiennamen, welche von Einwohnern Luxemburger Nationalität getragen wurden oder noch getragen werden und 1880 (8.584) sowie 1984 (13.388) registriert worden sind. Hrsg. Von STATEC. Luxemburg 1984.

Lüderitz, Sandra (2002): Kontrastive Onomastik dänischer und deutscher Familiennamen. Freiburg (Magisterarbeit).

Marynissen, Ann/Nübling, Damaris (2010): Familiennamen in Flandern, den Niederlanden und Deutschlandein diachroner und synchroner Vergleich. In: Antje Dammel/Sebastian Kürschner/Damaris Nübling (Hrsg.): Kontrastive Germanistische Linguistik, Teilband 1. Hildesheim (Germanistische Linguistik; 206-209), 311-362.

Nübling, Damaris (1997): Deutsch-schwedische Divergenzen in Entstehung und Struktur der Familiennamen. Ein Beitrag zur kontrastiven Onomastik. In: Beiträge zur Namenforschung 32/2, 141-173.

Nübling, Damaris (2004): Prinzipien der Proprialitätsmarkierung. Familiennamenindikatoren in den nordeuropäischen Sprachen. In: Astrid van Nahl u.a. (Hrsg.): Namenwelten. Orts- und Personennamen in historischer Sicht. Ergänzungsbände zum Reallexikon der Germanischen Altertumskunde, Bd. 44. Berlin/New York, 466-482.

Thewes, Guy (2008): A propos... Geschichte des Großherzogtums Luxemburg. Übers. v. Patrick Wilwert, hrsg. v. Presse- und Informationsamt der Luxemburger Regierung. Luxemburg.

Thorey, Volker/Geis, Claus (22003): Nachweise genealogischer Quellen im Gebiet der ehemaligen PreuBischen Rheinprovinz. Band 1: Wohnplatzverzeichnis; Band 2: Kirchenbücher, Familenbücher und Verkartungen und Zivilstandsregister im südlichen Teil der preußischen Rheinprovinz. Köln (Westdeutsche Gesellschaft für Familienkunde; 182 u. 183). 


\section{Anhang}

A Die 500 häufigsten Familiennamen im ehemals luxemburgischen Gebiet ehem. Lux.

\begin{tabular}{|c|c|c|c|c|c|c|c|c|c|}
\hline 1 & Müller & 36 & Faber & 71 & Jakobs & 106 & Zimmermann & 141 & Keilen \\
\hline 2 & Schmitz & 37 & Braun & 72 & Schönhofen & 107 & Bermes & 142 & Densborn \\
\hline 3 & Weber & 38 & Michels & 73 & Franzen & 108 & Thelen & 143 & Linden \\
\hline 4 & Becker & 39 & Bauer & 74 & Hecker & 109 & Friedrich & 144 & Thome \\
\hline 5 & Schmitt & 40 & Lichter & 75 & Arens & 110 & Kiefer & 145 & Fisch \\
\hline 6 & Hoffmann & 41 & Fischer & 76 & Moos & 111 & Hammes & 146 & Hau \\
\hline 7 & Schneider & 42 & Haas & 77 & May & 112 & Schreiber & 147 & Niederprün \\
\hline 8 & Wagner & 43 & Wirtz & 78 & Kaufmann & 113 & Lutz & 148 & Lehnen \\
\hline 9 & Peters & 44 & Ewen & 79 & Steffen & 114 & Stolz & 149 & Eppers \\
\hline 10 & Meyer & 45 & Fandel & 80 & Neumann & 115 & Thiex & 150 & Hermanns \\
\hline 11 & Thielen & 46 & Ludwig & 81 & Neises & 116 & Pauls & 151 & Thies \\
\hline 12 & Mayer & 47 & Esch & 82 & Plein & 117 & Gansen & 152 & Engel \\
\hline 13 & Heinz & 48 & Lenz & 83 & Maas & 118 & Meyers & 153 & Schilling \\
\hline 14 & Thiel & 49 & Dederichs & 84 & Goebel & 119 & Dimmer & 154 & Pick \\
\hline 15 & Pütz & 50 & Schuh & 85 & Richter & 120 & Leinen & 155 & Hack \\
\hline 16 & Klein & 51 & Schwarz & 86 & Bach & 121 & Billen & 156 & Haus \\
\hline 17 & Heck & 52 & Klinkhammer & 87 & Willems & 122 & Ballmann & 157 & Gehlen \\
\hline 18 & Krämer & 53 & Schares & 88 & Göbel & 123 & Scholtes & 158 & Steinmetz \\
\hline 19 & Schmidt & 54 & Koch & 89 & Heyen & 124 & Reiter & 159 & Lamberty \\
\hline 20 & Schröder & 55 & Mertes & 90 & Kremer & 125 & Wallenborn & 160 & Jegen \\
\hline 21 & Schumacher & 56 & Hein & 91 & Gasper & 126 & Blum & 161 & Kranz \\
\hline 22 & Theis & 57 & Dahm & 92 & Görgen & 127 & Trierweiler & 162 & Schuster \\
\hline 23 & Schäfer & 58 & Junk & 93 & Hahn & 128 & Streit & 163 & Bormann \\
\hline 24 & Hermes & 59 & Bretz & 94 & Koster & 129 & Zender & 164 & Reichert \\
\hline 25 & Reuter & 60 & Kockelmann & 95 & Kohl & 130 & Gillen & 165 & Strauch \\
\hline 26 & Elsen & 61 & Winter & 96 & Groß & 131 & Theisen & 166 & Wolter \\
\hline 27 & Heinen & 62 & Thommes & 97 & Jansen & 132 & Begon & 167 & Reinert \\
\hline 28 & Zimmer & 63 & Hansen & 98 & Sonnen & 133 & Follmann & 168 & Dichter \\
\hline 29 & Breuer & 64 & Fuchs & 99 & Kauth & 134 & Mohr & 169 & Nickels \\
\hline 30 & Roth & 65 & Endres & 100 & Beck & 135 & Welter & 170 & Weiland \\
\hline 31 & Berg & 66 & Meier & 101 & Leisen & 136 & Meiers & 171 & Metzen \\
\hline 32 & Schilz & 67 & Clemens & 102 & Klasen & 137 & Kirsch & 172 & Maus \\
\hline 33 & Weiler & 68 & Marx & 103 & Kandels & 138 & Antony & 173 & Knauf \\
\hline 34 & Simon & 69 & Molitor & 104 & Brück & 139 & Lux & 174 & Hörnchen \\
\hline 35 & Grün & 70 & Berens & 105 & Steinbach & 140 & Lehnertz & 175 & Ziwes \\
\hline
\end{tabular}




\begin{tabular}{|c|c|c|c|c|c|c|c|c|c|}
\hline 176 & Röder & 214 & Stein & 252 & Metzdorf & 290 & Steil & 328 & Thomas \\
\hline 177 & Steffes & 215 & Hartmann & 253 & Dunkel & 291 & Schaal & 329 & Gruben \\
\hline 178 & Faust & 216 & Habscheid & 254 & Kickertz & 292 & Wawer & 330 & Jacobs \\
\hline 179 & Weimann & 217 & Schoden & 255 & Cremer & 293 & Assmann & 331 & Wilhelm \\
\hline 30 & Adams & 218 & Hoor & 256 & Hilgers & 294 & Kasel & 332 & Classen \\
\hline 181 & Kessler & 219 & Weis & 257 & Peifer & 295 & Tholl & 333 & Allar \\
\hline 182 & Schramer & 220 & Fink & 258 & Jüngels & 296 & Groben & 334 & Spang \\
\hline 183 & Kinnen & 221 & Reichertz & 259 & Pauly & 297 & Gerten & 335 & Reifer \\
\hline 184 & Jung & 222 & Graf & 260 & Colling & 298 & Kootz & 336 & Mai \\
\hline 185 & Backes & 223 & Hauer & 261 & Schlöder & 299 & Berger & 337 & Greif \\
\hline 186 & Steins & 224 & Schommer & 262 & Metz & 300 & Krones & 338 & Juchmes \\
\hline 187 & Otten & 225 & Bohr & 263 & Schon & 301 & Garcon & 339 & Lauer \\
\hline 188 & Wonner & 226 & Feltes & 264 & Schreiner & 302 & Esser & 340 & Wengler \\
\hline 189 & Ewertz & 227 & Krütten & 265 & Gödert & 303 & Holbach & 341 & Reis \\
\hline 190 & Kohn & 228 & Hettinger & 266 & Funk & 304 & Barg & 342 & Burbach \\
\hline 191 & Zunker & 229 & Willmes & 267 & Loscheider & 305 & Barthel & 343 & Stark \\
\hline 192 & Scheuer & 230 & Spartz & 268 & Weires & 306 & Pint & 344 & Burelbach \\
\hline 193 & Welsch & 231 & Jutz & 269 & Enders & 307 & Marbach & 345 & Walter \\
\hline 194 & Bares & 232 & Lempges & 270 & Hubert & 308 & Valerius & 346 & Mutsch \\
\hline 195 & Orth & 233 & Jakoby & 271 & Scheer & 309 & Boesen & 347 & Zeimentz \\
\hline 196 & Wolf & 234 & Poss & 272 & Keil & 310 & Conrad & 348 & Lutgen \\
\hline 197 & Strupp & 235 & Hontheim & 273 & Wenzel & 311 & Sauber & 349 & Pohl \\
\hline 198 & Weides & 236 & Johanns & 274 & Horn & 312 & Weiß & 350 & Geimer \\
\hline 199 & Ademes & 237 & Kreutz & 275 & Schabio & 313 & Epper & 351 & Kramp \\
\hline 200 & Diederich & 238 & Hilger & 276 & Burg & 314 & Berners & 352 & Kotz \\
\hline 201 & Geisen & 239 & Pesch & 277 & Kimmlingen & 315 & Schmalen & 353 & Zens \\
\hline 202 & Ries & 240 & Kolf & 278 & Niesen & 316 & Palzer & 354 & Konz \\
\hline 203 & Rausch & 241 & Crames & 279 & Banz & 317 & Schackmann & 355 & Geschwind \\
\hline 204 & Schulz & 242 & Frank & 280 & Schleder & 318 & Fiedler & 356 & Fries \\
\hline 205 & Klassen & 243 & Zahren & 281 & Mick & 319 & Rings & 357 & Bartz \\
\hline 206 & Nosbüsch & 244 & Pitsch & 282 & Arnoldy & 320 & Neyses & 358 & Karen \\
\hline 207 & Mertens & 245 & König & 283 & Frisch & 321 & Zahnen & 359 & Francois \\
\hline 208 & Lang & 246 & Herrmann & 284 & Hotz & 322 & Bungartz & 360 & Mies \\
\hline 209 & Zwank & 247 & Weinand & 285 & Bisenius & 323 & Baumann & 361 & Mossal \\
\hline 210 & Ferring & 248 & Dahmen & 286 & Ehlenz & 324 & Blasen & 362 & Thull \\
\hline 211 & Schwickerath & 249 & Schier & 287 & Nonnweiler & 325 & Wolff & 363 & Rossler \\
\hline 212 & Neuerburg & 250 & Kraus & 288 & Spoden & 326 & Adam & 364 & Feilen \\
\hline 213 & Olk & 251 & Gerhards & 289 & Rauen & 327 & Pützer & 365 & Kirch \\
\hline
\end{tabular}




$\begin{array}{llllll}366 \text { Fuchsen } & 393 \text { Brenner } & 420 \text { Nesges } & 447 \text { Gierden } & 474 \text { Scholer } \\ 367 \text { Werner } & 394 \text { Paas } & 421 \text { Juchems } & 448 \text { Reger } & 475 \text { Breiling } \\ 368 \text { Junker } & 395 \text { Heinzen } & 422 \text { Nilles } & 449 \text { Pfeiffer } & 476 \text { Stockemer } \\ 369 \text { Alff } & 396 \text { Heinrichs } & 423 \text { Heiles } & 450 \text { Gabler } & 477 \text { Haubrich } \\ 370 \text { Ritter } & 397 \text { Lenzen } & 424 \text { Felten } & 451 \text { Merz } & 478 \text { Platz } \\ 371 \text { Dostert } & 398 \text { Weinandy } & 425 \text { Kleis } & 452 \text { Penning } & 479 \text { Geib } \\ 372 \text { Maier } & 399 \text { Flesch } & 426 \text { Schu } & 453 \text { Gentges } & 480 \text { Conrady } \\ 373 \text { Poensgen } & 400 \text { Köhler } & 427 \text { Fischbach } & 454 \text { Lorth } & 481 \text { Lescher } \\ 374 \text { Beyer } & 401 \text { Diederichs } & 428 \text { Hoff } & 455 \text { Bamberg } & 482 \text { Reinart } \\ 375 \text { Hennes } & 402 \text { Trapp } & 429 \text { Weyandt } & 456 \text { Born } & 483 \text { Weiers } \\ 376 \text { Karges } & 403 \text { Link } & 430 \text { Schütz } & 457 \text { Schroeder } & 484 \text { Denter } \\ 377 \text { Kaiser } & 404 \text { Aubart } & 431 \text { Kuhn } & 458 \text { Melchior } & 485 \text { Heintz } \\ 378 \text { Ernst } & 405 \text { Scherer } & 432 \text { Engels } & 459 \text { Steffens } & 486 \text { Schomer } \\ 379 \text { Zehren } & 406 \text { Gierens } & 433 \text { Winkelmann } & 460 \text { Rodens } & 487 \text { Carl } \\ 380 \text { Goergen } & 407 \text { Huber } & 434 \text { Schweyen } & 461 \text { Seiwert } & 488 \text { Moser } \\ 381 \text { Mirkes } & 408 \text { Back } & 435 \text { Heid } & 462 \text { Kirchen } & 489 \text { Fusenig } \\ 382 \text { Neu } & 409 \text { Pallien } & 436 \text { Schorn } & 463 \text { Klink } & 490 \text { Irsch } \\ 383 \text { Cillien } & 410 \text { Stoff } & 437 \text { Grossmann } & 464 \text { Thurmann } & 491 \text { Palgen } \\ 384 \text { Karpen } & 411 \text { Betzen } & 438 \text { Deutsch } & 465 \text { Lexen } & 492 \text { Valentin } \\ 385 \text { Theisges } & 412 \text { Fabry } & 439 \text { Vogel } & 466 \text { Bläsius } & 493 \text { Leick } \\ 386 \text { Schabo } & 413 \text { Masselter } & 440 \text { Schlösser } & 467 \text { Krebs } & 494 \text { Hupp } \\ 387 \text { Disch } & 414 \text { Candels } & 441 \text { Tossing } & 468 \text { Roderich } & 495 \text { Schuckart } \\ 388 \text { Petry } & 415 \text { Koos } & 442 \text { Ziewers } & 469 \text { Kalle } & 496 \text { Dick } \\ 389 \text { Götz } & 416 \text { Lehmann } & 443 \text { Poth } & 470 \text { Turmann } & 497 \text { Altringer } \\ 390 \text { Igelmund } & 417 \text { Leifgen } & 444 \text { Schons } & 471 \text { Gompelmann } & 498 \text { Harings } \\ 391 \text { Propson } & 418 \text { Stoffel } & 445 \text { Tautges } & 472 \text { Mayers } & 499 \text { Lorig } \\ 392 \text { Mees } & 419 \text { Lambert } & 446 \text { Dingels } & 473 \text { Birringer } & 500 \text { Zirbes }\end{array}$

\section{B Die 500 häufigsten Familiennamen in Luxemburg}

$\begin{array}{lllllll}1 & \text { SCHMIT } & 10 & \text { KLEIN } & 19 & \text { SCHILTZ } & 28 \text { DIEDERICH } \\ 2 \text { MULLER } & 11 \text { KIEFFER } & 20 \text { MEYER } & 29 \text { SCHUMACHER } \\ 3 \text { WEBER } & 12 \text { BECKER } & 21 \text { MAJERUS } & 30 \text { BRAUN } \\ 4 \text { HOFFMANN } & 13 \text { KREMER } & 22 \text { RIES } & 31 \text { WAGENER } \\ 5 \text { WAGNER } & 14 \text { FABER } & 23 \text { HANSEN } & 32 \text { KIRSCH } \\ 6 \text { THILL } & 15 \text { WEIS } & 24 \text { MEYERS } & 33 \text { MOLITOR } \\ 7 \text { SCHMITZ } & 16 \text { SIMON } & 25 \text { KRIER } & 34 \text { REDING } \\ 8 \text { SCHROEDER } & 17 \text { SCHNEIDER } & 26 \text { KAYSER } & 35 \text { SCHOLTES } \\ 9 & \text { REUTER } & 18 \text { WELTER } & 27 \text { STEFFEN } & 36 \text { THEIS }\end{array}$




\begin{tabular}{|c|c|c|c|c|c|c|c|}
\hline 37 & THEISEN & 75 & GENGLER & 113 & MANGEN & 151 & HENGEN \\
\hline 38 & FLAMMANG & 76 & ERPELDING & 114 & THOMA & 152 & KNEIP \\
\hline 39 & MARX & 77 & HERMES & 115 & HILGER & 153 & LAMBERT \\
\hline 40 & GOERGEN & 78 & SCHAACK & 116 & MERGEN & 154 & SCHEER \\
\hline 41 & WEILER & 79 & PEIFFER & 117 & MARTINS & 155 & FRANCK \\
\hline 42 & DA SILVA & 80 & $\mathrm{KOCH}$ & 118 & WINANDY & 156 & PETRY \\
\hline 43 & WOLFF & 81 & BIVER & 119 & ARENDT & 157 & HILBERT \\
\hline 44 & LENTZ & 82 & OLINGER & 120 & MARQUES & 158 & WIES \\
\hline 45 & GOEDERT & 83 & LORANG & 121 & WIRTZ & 159 & CONTER \\
\hline 46 & JACOBY & 84 & LUCAS & 122 & ANTONY & 160 & SCHANEN \\
\hline 47 & NILLES & 85 & BERTEMES & 123 & SCHINTGEN & 161 & JACOBS \\
\hline 48 & JUNG & 86 & GILLEN & 124 & BARTHEL & 162 & ROLLINGER \\
\hline 49 & HUBERTY & 87 & AREND & 125 & DAHM & 163 & SCHOLER \\
\hline 50 & HEINEN & 88 & BERNARD & 126 & $\mathrm{KOHN}$ & 164 & ZENNER \\
\hline 51 & BACK & 89 & LINDEN & 127 & SCHWARTZ & 165 & DEMUTH \\
\hline 52 & PETERS & 90 & STEINMETZ & 128 & ELSEN & 166 & LINSTER \\
\hline 53 & FISCHER & 91 & DOSTERT & 129 & BERG & 167 & THIRY \\
\hline 54 & FELTEN & 92 & LUX & 130 & REULAND & 168 & WAMPACH \\
\hline 55 & ENGEL & 93 & WOLTER & 131 & REINERT & 169 & CLEES \\
\hline 56 & RODRIGUES & 94 & BACKES & 132 & FISCH & 170 & EWEN \\
\hline 57 & HAAS & 95 & MOUSEL & 133 & LUDWIG & 171 & HEINTZ \\
\hline 58 & LANNERS & 96 & MOES & 134 & KRAUS & 172 & LOPES \\
\hline 59 & FERREIRA & 97 & GRETHEN & 135 & SAUBER & 173 & LUTGEN \\
\hline 60 & DECKER & 98 & MATHIEU & 136 & KOHNEN & 174 & SCHREINER \\
\hline 61 & SCHMITT & 99 & KOHL & 137 & MERTENS & 175 & SCHULLER \\
\hline 62 & MERSCH & 100 & BAUSCH & 138 & GOMES & 176 & RECKINGER \\
\hline 63 & WEYDERT & 101 & FISCHBACH & 139 & KAUFFMANN & 177 & KASS \\
\hline 64 & MICHELS & 102 & COLLING & 140 & POOS & 178 & RISCH \\
\hline 65 & PUTZ & 103 & FRISCH & 141 & LANG & 179 & SEYLER \\
\hline 66 & DOS SANTOS & 104 & FERNANDES & 142 & SCHAUL & 180 & BECK \\
\hline 67 & WEYLAND & 105 & SCHARTZ & 143 & RUPPERT & 181 & THOMMES \\
\hline 68 & PAULY & 106 & GONCALVES & 144 & REITER & 182 & TOUSSAINT \\
\hline 69 & PEREIRA & 107 & WILMES & 145 & NICKELS & 183 & LAMESCH \\
\hline 70 & NEY & 108 & SCHAUS & 146 & BOCK & 184 & SCHLEICH \\
\hline 71 & CLEMENT & 109 & BERCHEM & 147 & GEORGES & 185 & SCHMIDT \\
\hline 72 & STEICHEN & 110 & FELLER & 148 & PAULUS & 186 & BAUM \\
\hline 73 & MARTIN & 111 & NEU & 149 & STOFFEL & 187 & EVEN \\
\hline 74 & ZIMMER & 112 & RAUSCH & 150 & DONDELINGER & 188 & THEIN \\
\hline
\end{tabular}




\begin{tabular}{|c|c|c|c|c|c|c|c|}
\hline 189 & MAY & 228 & RIBEIRO & 267 & WILTGEN & 306 & FELTGEN \\
\hline 190 & THILMANY & 229 & LEY & 268 & HEMMER & 307 & KERGER \\
\hline 191 & FONCK & 230 & SCHLESSER & 269 & METZ & 308 & MORES \\
\hline 192 & HECK & 231 & ROTH & 270 & QUINTUS & 309 & FEDERSPIEL \\
\hline 193 & SCHEUER & 232 & DA COSTA & 271 & WEYER & 310 & WEISGERBER \\
\hline 194 & HAMES & 233 & FRISING & 272 & GERARD & 311 & ERNSTER \\
\hline 195 & FRANK & 234 & JOHANNS & 273 & MORIS & 312 & EWERT \\
\hline 196 & BAUSTERT & 235 & PLETSCHETTE & 274 & STEIN & 313 & KASEL \\
\hline 197 & BRUCK & 236 & WILHELM & 275 & TEIXEIRA & 314 & NEUMANN \\
\hline 198 & DUPONT & 237 & LEMMER & 276 & BISENIUS & 315 & FEYDER \\
\hline 199 & KUGENER & 238 & HUSS & 277 & FEIEREISEN & 316 & BERSCHEID \\
\hline 200 & SINNER & 239 & BOURG & 278 & REILAND & 317 & BINTENER \\
\hline 201 & SEIL & 240 & ADAM & 279 & THULL & 318 & GALES \\
\hline 202 & KEMP & 241 & BAULER & 280 & DUARTE & 319 & BOSSELER \\
\hline 203 & PHILIPPE & 242 & KINNEN & 281 & RIPPINGER & 320 & DUHR \\
\hline 204 & GLODT & 243 & MAYER & 282 & STAUDT & 321 & SUNNEN \\
\hline 205 & CLOOS & 244 & MILLER & 283 & WANTZ & 322 & THOME \\
\hline 206 & GLOD & 245 & PINTO & 284 & ALVES & 323 & ROSSI \\
\hline 207 & KOENIG & 246 & SCHILLING & 285 & $\mathrm{BACH}$ & 324 & GODART \\
\hline 208 & REISER & 247 & ANEN & 286 & GOEBEL & 325 & $\mathrm{COLBACH}$ \\
\hline 209 & TRIERWEILER & 248 & BIWER & 287 & GOERENS & 326 & LIPPERT \\
\hline 210 & WEYRICH & 249 & CLEMENS & 288 & WILLEMS & 327 & FAUTSCH \\
\hline 211 & THINNES & 250 & GILSON & 289 & DALEIDEN & 328 & NOESEN \\
\hline 212 & GREISCH & 251 & ZEIMES & 290 & WIRTH & 329 & FELTES \\
\hline 213 & BAUER & 252 & ZEIMET & 291 & ANDRE & 330 & HAHN \\
\hline 214 & JOST & 253 & ENTRINGER & 292 & MARNACH & 331 & KRECKE \\
\hline 215 & KAISER & 254 & ORIGER & 293 & OLIVEIRA & 332 & LOUIS \\
\hline 216 & GLESENER & 255 & REICHLING & 294 & SCHWEITZER & 333 & STRASSER \\
\hline 217 & TRAUSCH & 256 & ROMMES & 295 & HEIDERSCHEID & 334 & EICHER \\
\hline 218 & THIELEN & 257 & BRANDENBURGER & 296 & SCHON & 335 & MENDES \\
\hline 219 & PEFFER & 258 & DUMONT & 297 & KRACK & 336 & FUNCK \\
\hline 220 & HUBERT & 259 & SCHOCKMEL & 298 & GRAAS & 337 & MONTEIRO \\
\hline 221 & KERSCHEN & 260 & BOEVER & 299 & MAAS & 338 & SIEBENALER \\
\hline 222 & HAAG & 261 & EISCHEN & 300 & THIEL & 339 & JUNGERS \\
\hline 223 & SCHUSTER & 262 & MANDERSCHEID & 301 & WOHL & 340 & MEIERS \\
\hline 224 & GRAF & 263 & GLODEN & 302 & DIAS & 341 & BECKIUS \\
\hline 225 & STEPHANY & 264 & DIDIER & 303 & GELHAUSEN & 342 & FRANTZ \\
\hline 226 & SCHAEFFER & 265 & BESCH & 304 & BLEY & 343 & RAACH \\
\hline 227 & KONSBRUCK & 266 & LUDOVICY & 305 & DE SOUSA & 344 & DE OLIVEIRA \\
\hline
\end{tabular}




\begin{tabular}{|c|c|c|c|c|c|c|c|}
\hline 345 & GAASCH & 384 & STOLTZ & 423 & WANDERSCHEID & 462 & REIFFERS \\
\hline 346 & HELLERS & 385 & HEUERTZ & 424 & BLUM & 463 & SADLER \\
\hline 347 & PETIT & 386 & KELLER & 425 & $\mathrm{RECH}$ & 464 & BOHLER \\
\hline 348 & FRIEDEN & 387 & KOSTER & 426 & ALTMANN & 465 & ECKER \\
\hline 349 & WILTZIUS & 388 & LEYDER & 427 & WAHL & 466 & PRIM \\
\hline 350 & SOARES & 389 & SCHONS & 428 & BINTNER & 467 & SCHUMMER \\
\hline 351 & BEFFORT & 390 & NICOLAY & 429 & GEIBEN & 468 & GASPAR \\
\hline 352 & BISSEN & 391 & BOUR & 430 & BASTIAN & 469 & KARIER \\
\hline 353 & HALSDORF & 392 & SCHULER & 431 & BROSIUS & 470 & KIRCHEN \\
\hline 354 & JAEGER & 393 & CLESEN & 432 & SCHAAF & 471 & FRERES \\
\hline 355 & STREFF & 394 & KRAEMER & 433 & SCHANK & 472 & BURG \\
\hline 356 & FEIDT & 395 & MACK & 434 & STRAUS & 473 & GRUN \\
\hline 357 & HAUPERT & 396 & RISCHARD & 435 & PROBST & 474 & MALGET \\
\hline 358 & LOSCH & 397 & RODESCH & 436 & WERNER & 475 & MATHIAS \\
\hline 359 & BINSFELD & 398 & FRANCOIS & 437 & LAMBERTY & 476 & ZIGRAND \\
\hline 360 & HARY & 399 & JUNKER & 438 & PESCH & 477 & BEMTGEN \\
\hline 361 & KUFFER & 400 & KAUFMANN & 439 & HERMANN & 478 & FANDEL \\
\hline 362 & REIFF & 401 & ROEDER & 440 & KREINS & 479 & SCHUH \\
\hline 363 & SANTOS & 402 & BERTRAND & 441 & STOOS & 480 & BINTZ \\
\hline 364 & WESTER & 403 & HURT & 442 & WALDBILLIG & 481 & CONRAD \\
\hline 365 & SCHWEICH & 404 & ROBERT & 443 & WOLF & 482 & LEIDER \\
\hline 366 & THOLL & 405 & HAMEN & 444 & OBERWEIS & 483 & MANNES \\
\hline 367 & JANS & 406 & THOSS & 445 & SASSEL & 484 & THIES \\
\hline 368 & KALMES & 407 & CORREIA & 446 & KUHN & 485 & THILGES \\
\hline 369 & MELCHIOR & 408 & FRANTZEN & 447 & KNEPPER & 486 & FLICK \\
\hline 370 & SCHAEFER & 409 & HENTGES & 448 & NICOLAS & 487 & KLOPP \\
\hline 371 & THOMAS & 410 & KEISER & 449 & SCHUTZ & 488 & RASSEL \\
\hline 372 & WEILAND & 411 & KILL & 450 & WITRY & 489 & HEMMEN \\
\hline 373 & TURMES & 412 & DIMMER & 451 & BIREN & 490 & PUNDEL \\
\hline 374 & SCHANCK & 413 & $\mathrm{ESCH}$ & 452 & GILBERTZ & 491 & BREMER \\
\hline 375 & CONRARDY & 414 & MEDINGER & 453 & HAAN & 492 & KAYL \\
\hline 376 & NOEL & 415 & MEISCH & 454 & HOSS & 493 & NOTHUM \\
\hline 377 & JEITZ & 416 & SCHREIBER & 455 & LAUX & 494 & BERENS \\
\hline 378 & MEIS & 417 & GOETZINGER & 456 & METZLER & 495 & BUCHLER \\
\hline 379 & SILVA & 418 & STEFFES & 457 & OESTREICHER & 496 & FRIEDRICH \\
\hline 380 & ADROVIC & 419 & HEIN & 458 & SCHEUREN & 497 & GINDT \\
\hline 381 & BETTENDORFF & 420 & HEINZ & 459 & STEMPER & 498 & MAJERES \\
\hline 382 & BIEVER & 421 & OSWALD & 460 & WENGLER & 499 & MEDERNACH \\
\hline 383 & LAUER & 422 & REIS & 461 & EIFFES & 500 & MICHEL \\
\hline
\end{tabular}




\section{Die 500 häufigsten Familiennamen im nie luxemburgischen Vergleichsgebiet dt. Vgg.}

\begin{tabular}{|c|c|c|c|c|c|c|c|c|c|}
\hline 1 & Müller & 37 & Hansen & 73 & Bach & 109 & Faber & 145 & Streit \\
\hline 2 & Schmitz & 38 & Schwarz & 74 & Mertens & 110 & Orth & 146 & Hammes \\
\hline 3 & Schneider & 39 & Hein & 75 & Huppertz & 111 & Herrmann & 147 & Kirchen \\
\hline 4 & Becker & 40 & Lauer & 76 & Heck & 112 & Thomas & 148 & Paulus \\
\hline 5 & Schmitt & 41 & Backes & 77 & Kirsch & 113 & Dederichs & 149 & Offermann \\
\hline 6 & Weber & 42 & Mayer & 78 & Friedrich & 114 & Scherer & 150 & Rausch \\
\hline 7 & Hoffmann & 43 & Mertes & 79 & Fuchs & 115 & Reichert & 151 & Theissen \\
\hline 8 & Wagner & 44 & Thielen & 80 & Kuhn & 116 & Theisen & 152 & Adam \\
\hline 9 & Klein & 45 & Haas & 81 & Mohr & 117 & Dahm & 153 & Steinbach \\
\hline 10 & Meyer & 46 & Esser & 82 & Schuster & 118 & Lorenz & 154 & Schommer \\
\hline 11 & Schmidt & 47 & Theis & 83 & Wolf & 119 & Hennen & 155 & Gorges \\
\hline 12 & Braun & 48 & Reuter & 84 & Stein & 120 & Zender & 156 & Küpper \\
\hline 13 & Zimmer & 49 & Pütz & 85 & Bohr & 121 & Jacobs & 157 & Hartmann \\
\hline 14 & Schäfer & 50 & Engel & 86 & Hilgers & 122 & Kirch & 158 & Brausch \\
\hline 15 & Krämer & 51 & Schuh & 87 & Steffes & 123 & Stolz & 159 & Kaufmann \\
\hline 16 & Michels & 52 & Jung & 88 & Barth & 124 & Berens & 160 & Palm \\
\hline 17 & Jansen & 53 & Görgen & 89 & Jäger & 125 & Schuler & 161 & Wollscheid \\
\hline 18 & Peters & 54 & Esch & 90 & Thome & 126 & $\mathrm{He} ß$ & 162 & Steinmetz \\
\hline 19 & Kiefer & 55 & Franzen & 91 & Philippi & 127 & Kohl & 163 & Kreutz \\
\hline 20 & Schröder & 56 & Neumann & 92 & Adams & 128 & Winter & 164 & Hermanns \\
\hline 21 & Schumacher & 57 & Wirtz & 93 & Nießen & 129 & Meiers & 165 & Lehnertz \\
\hline 22 & Koch & 58 & Steffens & 94 & May & 130 & Otto & 166 & Klasen \\
\hline 23 & Marx & 59 & Groß & 95 & Stollenwerk & 131 & Metzen & 167 & Frings \\
\hline 24 & Jakobs & 60 & Ludwig & 96 & Lenz & 132 & Cremer & 168 & Lieser \\
\hline 25 & Heinz & 61 & Zimmermann & 97 & Förster & 133 & Hoff & 169 & Mauel \\
\hline 26 & Breuer & 62 & Werner & 98 & Welter & 134 & Thul & 170 & Schramm \\
\hline 27 & Bauer & 63 & Linden & 99 & Krings & 135 & Spang & 171 & Benz \\
\hline 28 & Fischer & 64 & Maas & 100 & Steffen & 136 & Petry & 172 & Elsen \\
\hline 29 & Thiel & 65 & Meier & 101 & Blum & 137 & Kranz & 173 & Thieltges \\
\hline 30 & Simon & 66 & Kremer & 102 & Lehnen & 138 & Weiler & 174 & Teusch \\
\hline 31 & Roth & 67 & Follmann & 103 & Schreiner & 139 & Fries & 175 & Bollig \\
\hline 32 & Willems & 68 & Hermes & 104 & Molitor & 140 & Pauly & 176 & Keller \\
\hline 33 & Berg & 69 & Schulz & 105 & Thelen & 141 & Richter & 177 & Prinz \\
\hline 34 & Heinen & 70 & Clemens & 106 & Kaiser & 142 & Frank & 178 & Walter \\
\hline 35 & Junk & 71 & Hahn & 107 & Lang & 143 & Endres & 179 & Burg \\
\hline 36 & Reinert & 72 & Klinkhammer & 108 & Schreiber & 144 & Klaßen & 180 & Martin \\
\hline
\end{tabular}




\begin{tabular}{|c|c|c|c|c|c|c|c|c|}
\hline 181 Kurth & 219 & Funk & 257 & Oswald & 295 & Lichter & 333 & Valerius \\
\hline Thömmes & 220 & Lenzen & 258 & Stoffel & 296 & Schaaf & 334 & Scholl \\
\hline Hau & 221 & Keßler & 259 & Weinand & 297 & Rommelfanger & 335 & Berners \\
\hline Lehnert & 222 & Ernst & 260 & Thieser & 298 & Schönhofen & 336 & Nelles \\
\hline Scherf & 223 & Maus & 261 & Bernardy & 299 & Scheuer & 337 & Kohn \\
\hline Henn & 224 & Mahlberg & 262 & Roos & 300 & Haubrich & 338 & Schulte \\
\hline 187 Otten & 225 & Wolff & 263 & Scheid & 301 & Johannes & 339 & Winkel \\
\hline Scholtes & 226 & Schlösser & 264 & Baumann & 302 & Johnen & 340 & Probst \\
\hline Ternes & 227 & Lambertz & 265 & Emmerich & 303 & Metzdorf & 341 & Selzer \\
\hline Gehlen & 228 & Flesch & 266 & Krause & 304 & Vogel & 342 & Scholz \\
\hline Loch & 229 & Zenner & 267 & Krüger & 305 & Rau & 343 & Ewen \\
\hline König & 230 & Beck & 268 & Merten & 306 & Schüller & 344 & Pauls \\
\hline Laux & 231 & Jager & 269 & Schnichels & 307 & Günther & 345 & Keil \\
\hline Reis & 232 & Lennartz & 270 & Weiß & 308 & Lehmann & 346 & Marxen \\
\hline Ehlen & 233 & Graf & 271 & Bock & 309 & Lautwein & 347 & Plein \\
\hline Jakoby & 234 & Eiden & 272 & Busch & 310 & Stoffels & 348 & Pfeiffer \\
\hline Maier & 235 & Herrig & 273 & Philipps & 311 & Poth & 349 & Schon \\
\hline Jost & 236 & Schütz & 274 & Lamberti & 312 & Wilhelm & 350 & Porten \\
\hline Schu & 237 & Wilden & 275 & Alten & 313 & Schilz & 351 & Biewer \\
\hline Berger & 238 & Koll & 276 & Engels & 314 & Bongard & 352 & Greuel \\
\hline Leinen & 239 & Ballmann & 277 & Kraus & 315 & Hardt & 353 & Lange \\
\hline Gerhards & 240 & Dahmen & 278 & Hilger & 316 & Alt & 354 & Sommer \\
\hline Bender & 241 & Haag & 279 & Kramp & 317 & Dewald & 355 & Schulze \\
\hline Lex & 242 & Feilen & 280 & Neukirch & 318 & Carl & 356 & Dietzen \\
\hline Lamberty & 243 & Leyendecker & 281 & Krewer & 319 & Borsch & 357 & Könen \\
\hline Konz & 244 & Reiter & 282 & Pohl & 320 & Lauterbach & 358 & Oberbillis \\
\hline Koster & 245 & Gasper & 283 & Feltes & 321 & Meyers & 359 & Köhler \\
\hline Strauch & 246 & Hack & 284 & Blau & 322 & Horn & 360 & Hermann \\
\hline Brück & 247 & Lutz & 285 & Greif & 323 & Nilles & 361 & Holz \\
\hline Lorig & 248 & Krebs & 286 & Annen & 324 & Schnitzler & 362 & Petri \\
\hline 211 Weis & 249 & Herres & 287 & Knauf & 325 & Reinartz & 363 & Lentes \\
\hline Thull & 250 & Sauer & 288 & Waßong & 326 & Wolter & 364 & Schwind \\
\hline Claßen & 251 & Röder & 289 & Welsch & 327 & Geib & 365 & Dietz \\
\hline Grün & 252 & Trierweiler & 290 & Bies & 328 & Britz & 366 & Eich \\
\hline Steuer & 253 & Boesen & 291 & Conrad & 329 & Birkel & 367 & Hamm \\
\hline Ackermann & 254 & Lauscher & 292 & Brandt & 330 & Göbel & 368 & Monzel \\
\hline Baltes & 255 & Kratz & 293 & Arens & 331 & Jäckels & 369 & Münch \\
\hline Jonas & 256 & Heinrichs & 294 & Pesch & 332 & Milz & 370 & Römer \\
\hline
\end{tabular}




\begin{tabular}{|c|c|c|c|c|c|c|c|c|c|}
\hline 371 & Bell & 397 & Wollgarten & 423 & Ollinger & 449 & Scheer & 475 & Brand \\
\hline 372 & Fisch & 398 & Anton & 424 & Kettern & 450 & Heinrich & 476 & Caspers \\
\hline 373 & Felten & 399 & Born & 425 & Kerber & 451 & Bretz & 477 & Justinger \\
\hline 374 & Binz & 400 & Neises & 426 & Reetz & 452 & Ensch & 478 & Peifer \\
\hline 375 & Huwer & 401 & Hubert & 427 & Helfen & 453 & Ewertz & 479 & Mies \\
\hline 376 & Pick & 402 & Mattes & 428 & Böhm & 454 & Kohr & 480 & Hofmann \\
\hline 377 & Koltes & 403 & Strupp & 429 & Basten & 455 & Pitsch & 481 & Steil \\
\hline 378 & Weiland & 404 & Scholer & 430 & Kopp & 456 & Kinzig & 482 & Ferber \\
\hline 379 & Quint & 405 & Schütt & 431 & Kraemer & 457 & Mauer & 483 & Andres \\
\hline 380 & Christmann & 406 & Seiwert & 432 & Witt & 458 & Kaster & 484 & Decker \\
\hline 381 & Blasius & 407 & Breit & 433 & Rader & 459 & Weyand & 485 & Beyer \\
\hline 382 & Diederichs & 408 & Fritz & 434 & Remmel & 460 & Regnery & 486 & Conrads \\
\hline 383 & Albrecht & 409 & Ostermann & 435 & Zehren & 461 & Wald & 487 & Pelzer \\
\hline 384 & Lutterbach & 410 & Olk & 436 & Hauser & 462 & Schwickerath & 488 & Nettersheim \\
\hline 385 & Ney & 411 & Hübner & 437 & Barbian & 463 & Steines & 489 & Weirich \\
\hline 386 & Theobald & 412 & Konrad & 438 & Büchel & 464 & Reitz & 490 & Schlöder \\
\hline 387 & Biehl & 413 & Thesen & 439 & Maximini & 465 & Schirra & 491 & Schleimer \\
\hline 388 & Hayer & 414 & Schons & 440 & Möller & 466 & Hens & 492 & Rauen \\
\hline 389 & Behr & 415 & Wiesen & 441 & Holbach & 467 & Kautenburger & 493 & Bettendorf \\
\hline 390 & Lux & 416 & Stark & 442 & Horsch & 468 & Poensgen & 494 & Götten \\
\hline 391 & Kasel & 417 & Fusenig & 443 & Kunz & 469 & Reiland & 495 & Brandenburg \\
\hline 392 & Peter & 418 & Deutsch & 444 & Rohles & 470 & Ritter & 496 & Hagen \\
\hline 393 & Kirsten & 419 & Diewald & 445 & Völl & 471 & Thommes & 497 & Bidinger \\
\hline 394 & Stadtfeld & 420 & Kappes & 446 & Trampert & 472 & Thoma & 498 & Mai \\
\hline 395 & Spanier & 421 & Herz & 447 & Saxler & 473 & Gerten & 499 & Kugel \\
\hline 396 & Radermacher & 422 & Hill & 448 & Roder & 474 & Diedrich & 500 & Kläs \\
\hline
\end{tabular}




\title{
Namenvarianten an der Sprachgrenze, genealogisch überprüft
}

\author{
Jean-Claude Muller
}

\begin{abstract}
Der Beitrag ist in drei Kapitel gegliedert und argumentiert anhand von genealogisch überprüften Namenbeispielen sowie mit onomastischen Verbreitungskarten, welche mit Hilfe des an der Universität Luxemburg entwickelten Familiennamenmoduls erstellt wurden. Im ersten Kapitel werden die Dubletten im Bereich der Hausnamen und Familiennamen als luxemburgische Sondererscheinung definiert; diese bleiben danach aber aus der Diskussion ausgeklammert. Im zweiten Kapitel werden sechs konkrete Fallbeispiele aus der genealogischen Praxis von Namensdubletten über die Sprachgrenzen zwischen Germania und Romania hinweg erörtert. Im abschließenden dritten Kapitel werden drei Beispiele von Familiennamen als regionale Herkunftsangaben diskutiert, um dann anhand von ausgewählten Namenstypen alte territoriale Zugehörigkeitsbezeichnungen als Quelle von Familiennamen wahrscheinlich zu machen.
\end{abstract}

\section{Die Variation Hausname versus Familienname}

Seit dem Ende des 19. Jahrhunderts gehen die Luxemburger Namenforscher davon aus, dass rund ein Viertel der Luxemburger Familiennamen aus Ortschafts-, bzw. Gehöfts- oder Siedlungsnamen, d.h. Herkunftsbezeichnungen herzuleiten sind. ${ }^{1}$ Seit die Luxemburger Gesellschaft für Genealogie und Heraldik (A.L.G.H.) im Jahre 1984 mit Sitz im Schloss von Mersch im Landeszentrum gegründet wurde, geben sich deren Forscher immer wieder mit der Problematik der Hausnamen ab, die bis etwa 1750 in den Quellen parallel mit den Familiennamen überliefert werden. Dass eine solche Praxis den mit diesen Spezialnamen nicht Vertrauten schwerwiegende Hindernisse in den Weg legt, leuchtet ein. ${ }^{2}$ Der Autor und der belgisch-luxemburgische Namenforscher Paul Mathieu haben sich terminologisch darauf geeinigt, diese anscheinend spezifisch luxemburgischen Dubletten der Familiennamen, die Hausnamen, als Oikonyme (hergeleitet aus griechisch oĩkos) oder Domonyme (hergeleitet aus lateinisch domus) zu bezeichnen. Das Gebiet ihrer Verbreitung, vom Einsetzen der schriftlichen Quellen vor dem Dreißigjährigen Krieg bis nach dem Zweiten Weltkrieg, scheint sich auf das luxemburgisch-sprechende Gebiet im

1 Vgl. van Werveke 1900. Anhand des Familiennamens Mahowald führt Kollmann 2011 ein Beispiel vor, wie für einen lange kontrovers diskutierten Familiennamen letztendlich ein Herkunftsortsname gefunden werden kann.

2 So trägt z.B. der Pfarrer von Redingen an der Attert von 1733 bis 1756 alle Täuflinge mit den Hausnamen ein, während sein Neffe und Nachfolger ab 1756 nur Familiennamen benutzt. Ein schlüssiger genealogischer Beweis zur Erstellung einer Übersicht konnte nur anhand der Vornamen erbracht werden; vgl. Muller/Muller 1987, 33-107. 
Großherzogtum, im Bitburger Land, im Umland von Arlon und in den belgischen Ostkantonen zu beschränken, während die ehemals territorialgeschichtlich luxemburgischen, aber wallonischsprachigen Gebiete der Provinz Luxemburg von Belgien nicht von diesem Phänomen betroffen zu sein scheinen. Der Sprachwissenschaftler Elmar Neuss ${ }^{3}$ stellte seinerseits diesbezüglich eine klare Isoglosse südlich von Monschau in der Eifel fest; nördlich davon ist der Gebrauch von Hausnamen völlig unbekannt. Viele dieser Haus- oder Vogteinamen in den Luxemburger Dörfern sind von meist weiblichen Vornamen abgeleitet, an welche die Endung -en tritt, was einem alten schwachen Genitiv entspricht: Anen ( $<$ Anna), Even $(<$ Eva), Grethen ( $<$ Margaretha), Joken ( $<$ Jacobus), Plunen ( $<$ Apollonia), Zären/Zehren $(<*$ des Herren, Bezeichnung des Pfarrhauses). Andere wiederum, überwiegend aus Handwerksbezeichnungen hervorgegangen, enden in -esch, das aus -ers assimiliert ist: Schneidesch ( $<$ Schneider), Schouschtesch $(<$ Schuster), Schräinesch $(<$ Schreiner), Koschtesch ( $<$ Küster), Kréimesch ( $<$ Krämer), Millesch ( $<$ Müller), Schréidesch $(<$ (Wein-)Schröter), Woonesch $(<$ Wagener), Zammesch $(<$ Zimmerer $=$ Zimmermann), Péitesch $\left(<\right.$ Peter). ${ }^{4}$ Referentiell funktionieren diese althergebrachten und mittlerweile vom Aussterben bedrohten Hausnamensysteme in den einzelnen Dörfern autark, d.h. es trifft äußerst selten zu, dass bei einer Einheirat in ein anderes Dorf ein solcher Hausname vom einheiratenden Ehepartner mitgebracht wurde. Die Agnatentafel der bekannten Historiker Christian Calmes und Albert Calmes ${ }^{5}$ führt in die Ortschaft Beckerich an der belgisch-luxemburgischen Grenze. Der früheste Ahne, der schriftliche Spuren hinterlassen hat, ist Gabriel Meyers aus der $12 \mathrm{~km}$ entfernten Ortschaft Calmus, welcher am 30.06.1720 in Beckerich Catharina Bengen heiratete und danach nunmehr als Calmus oder Calmes aufgeschrieben wurde. Dessen eigentlicher Familienname konnte bisher nicht festgestellt werden, da die Bezeichnung Meyers in den Pfarrbüchern von Septfontaines/Simmern einen Hausnamen darstellt. Eine andere Person namens Calmes, die auf ähnlichem Wege der Auswanderung aus dem Dorf Calmus in den benachbarten Pfarreisitz Septfontaines zu ihrem Namen gekommen sein mag, hat Émile Arendt ermittelt: Wolfgangus Calmes, um 1580 in Simmern geboren, der 1605 an der Universität Trier eine gedruckt vorliegende Dissertation verteidigte (vgl. Arendt 1993).

Angedacht ist im Rahmen der A.L.G.H. ein Forschungs- und Dokumentationsprojekt, welches alle diese Hausnamen Luxemburgs erfassen soll, und zwar auf der Grundlage der Kartenaufnahmen von etwa 1824 (dem so genannten Urkataster) und einer sprachlichen Erhebung vor Ort. Zu ergänzen sein wird diese Materialsammlung durch das Studium von Notariatsinstrumenten aus dem 17. und 18. Jahrhundert sowie von Zivilstandsurkunden von etwa 1802 bis etwa 1840, in denen sehr oft in der vorgedruckten Kategorie

3 Persönliche Mitteilung aus dem Jahr 2010; Vgl. noch Neuss 2001.

4 Etliche Lokaluntersuchungen liegen seit Kurzem vor: Mathieu 1996, Mathieu 1999, sowie Muller 1985, 1993.

5 Eine Agnatentafel führt alle männlichen Vorfahren eines Probanden auf, die dessen Familiennamen inklusive Varianten aufweisen. Vgl. Calmes 1979; zu Christian Calmes, vgl. Schaffner 1988. 
„Straße innerhalb der Ortschaft“ der Hausname eingetragen wurde (,gestorben, geboren im Haus XY"). Weil diese spezielle Luxemburger Art der Doppelbenennung von Personen sowohl nach ihrem Familiennamen als auch nach dem Hausnamen noch in der Mitte des 19. Jahrhunderts weithin verwurzelt war, können als weitere Quellen innerhalb des Projekts, allerdings mit Vorbehalten, ${ }^{6}$ die Volkszählungen von 1854, 1857 und 1862 herangezogen werden, bei denen eine spezielle Kategorie „Hausname“ auf den Vordrucken steht.

Dieses einleitende Kapitel stellte lediglich eine besondere Kategorie von luxemburgischen Namensvarianten vor, welche im weiteren Verlauf der Erörterungen ausgeklammert bleiben wird. Der nun folgende erste Flügel dieses namenkundlichen Diptychons behandelt Dubletten von Familiennamen über die Sprachgrenze hinweg, während das abschließende dritte Kapitel sich mit territorialen Zugehörigkeitsbezeichnungen beschäftigt.

\section{Fallbeispiele zur Problematik der sprachlichen Dubletten bei eigentlichen Familiennamen}

\subsection{Aus Holtzmacher mach Dubois}

Genealogische Nachforschungen belegen, dass die Familie Holtzmacher ihren Ursprung in Useldingen am Fluss Attert hat. Im Jahr 1749, am 7. Januar, findet ,inter missarum solemnia“ in der Pfarrkirche Ruette bei Virton die Hochzeit zwischen „Jacobum Holsmacher et Joannam Fort" statt, wie der Ortspfarrer Donon in der Pfarrmatrikel festhält. Der Name der Ehefrau begegnet später auch als Le Fort. Der Useldinger Auswanderer, der am 15.02.1776 eine zweite Ehe mit Jeanne Gerard einging, starb am 13.03.1791 in Ruette: „est décédé à Ruette la grand a huit heures et demie après midi Jacques Dubois manoeuvre résident audit lieu agé d'environ Soixante-Sept ans, administré des Sacrements de pénitence...". Soweit die Niederschrift des Pfarrers J.-B. Didier von Ruette. Welcher Forscher würde diesen Todesakt von Jakob Holtzmacher in einem Namenverzeichnis unter Dubois aufgesucht haben?

Im Hochzeitsakt von 1749, niedergeschrieben im künftigen Wohnort Ruette, steht noch die ererbte, germanische Namensform Holtzmacher für den Bräutigam, während der Name des Neuankönmmlings gleich bei den Taufen seiner Kinder romanisiert wurde: z.B. Jacques Dubois, getauft am 04.01.1758 in Ruette, ,fils légitime de Jacque Dubois et de Jeanne Fort". Die Identität des Vaters war demnach nur in einer Familienrekonstruktion mit peinlich genauer Angabe der Namensformen und -varianten zu beweisen. Der Anstoß zur Lösung des genealogischen und namenkundlichen Problems kam unserem Informanten Fernand Limpach, dessen Verwandte Dubois bis 1920 in der Gegend von Ruette wohnten, allerdings durch eine schriftliche Archivnotiz, wo zum Ursprung der

6 Vgl. zu neuentstandenen Hausnamen im 19. Jh. Ensch 1994. Die Originaldokumente dieser Volkszählungen sind als Mikrofilm im Luxemburger Staatsarchiv zugänglich. 
Familie Dubois festgehalten wurde: „Holtz dit Dubois, venu d'Useldange (G.D.) à Ruette vers 1750. Il eut pour fils Martin Dubois...".?

Man kann sich ausmalen, welche phonetischen Schwierigkeiten im wallonischromanischen Umfeld von Ruette die Aussprache des deutsch-luxemburgischen Familiennamens Holtzmacher für den zugewanderten Ehemann der Jeanne (le) Fort bedeutete. Die Umsetzung zu Dubois war im eigentlichen Sinne keine genaue Übersetzung, sie lag dennoch auf der Hand, weil der Familienname Dubois bereits in Ruette im Jahr 1653 belegt ist. Ein Modell war also gegeben, so dass man im Allgemeinen formulieren kann, dass bei personengeschichtlichen Nachforschungen über die Sprachgrenze hinweg stets die sprachliche Adaptation bzw. Übersetzung von Familiennamen bedacht werden sollte. So wird z.B. im Fall der Eisenarbeiterfamilie Klein auf der Bonnerter Platinerie, heute genau auf der belgisch-luxemburgischen Grenze bei Oberpallen gelegen, der Genealoge im romanischen Umfeld sowohl nach Klein als auch nach Petit in den Namenindices suchen müssen (vgl. ausführlicher Bourguignon 1999, 401-402).

\subsection{Die Namensdublette Lejeune - Jung}

Die Exemplifizierung dieser Namensdublette findet sich in einem recht späten Schriftstück im Luxemburger Stadtarchiv, welches darüber hinaus lehrt, dass der Forscher unbedingt die eigenhändigen Unterschriften unter den Dokumenten beachten sollte: „Liberté fraternité Egalité à la Municipalité de Luxembourg. Le soussigné Jean Lejeune cordonnier en cette ville vous invite, citoyens, à avoir le plus favorable égard à Sa pauvreté et à La longue maladie dont il est encore actuellement attaqué et au nombre de cinq enfants qui lui demandent du pain qu'il ne lui est guerres possible de leur donner sans le secours des personnes charitables, et en conséquence à le comprendre dans la liste de ceux qui sont dans le cas de profiter de L'excédent de La répartition de la contribution, dans laquelle il est taxé à 21 pièces. Salut et fraternité. Luxbg le 7 brumaire 4e année“ [Donnerstag, 20.10.1795]. Unterschrift: Johannes Jung (vgl. Muller 1996, 155).

Festzuhalten ist bei diesem Beispiel, dass der Haupttext offensichtlich von der Hand eines französischsprachigen, öffentlichen Schreibers zu Papier gebracht wurde, kaum vier Monate nach der Eroberung der Festung Luxemburg durch das revolutionäre Frankreich. Die Unterschrift stammt allerdings vom Bittsteller selbst, der also wenigstens seinen Namen schreiben konnte. Über das sozialgeschichtliche Interesse des Textes hinaus lehrt dieses Fallbeispiel, dass in zweisprachigem Kontext, wie hier unter der neuen französischen Verwaltung des Wälderdepartments (département des Forêts), die Obrigkeit manchmal Sprachformen niederschreibt, welche nicht mit der zweifellos gesprochenen Sprache übereinstimmen. Für den Forscher ergeben sich bei solch evidenten Übersetzungsvarianten ähnliche Vorsichtsregeln wie bei Beispiel 2.1.

7 Nähere Details sind nachzulesen in der Erstpublikation bei Muller 1996, 154. Man vgl. auch in diesem Band den Beitrag / angeführt werden (Dubois/Denbosch). 


\subsection{Der romanische Ursprung des Familiennamens Hottua}

Eine genealogische Nachforschung des Autors in Zusammenarbeit mit Georges Lepère (Arlon) aus dem Jahr 1988 hat folgende Agnatentafel ergeben: ${ }^{8}$

11. Marguerite Hottua, *22.06.1847 Molinfaing (B), $\infty$ 05.06.1874 Tournai/Neufchâteau mit 10. Victor Klepper.

22. Jacques Hottua, Schmied, * 16.12.1808 Niedermertzig, Gemeinde Feulen (L), $\infty$ (2) 02.07.1849 Saint-Pierre/Neufchâteau (B) mit Catherine Peusche. 44. Michael Hottua, Schmied, * 22.11.1769 Oberfeulen, $†$ 09.06.1812 Niedermertzig, $\infty$ mit Elisabeth Straus (1777-1837); 7 Kinder.

88. Michael Hottua oder Houtois, * 08.03.1744 Oberpallen, $\dagger$ 16.12.1819 Feulen, $\infty$ mit Catherine Klein.

176. Michel Houtois, * 19.03.1720 Oberpallen, $\infty$ mit Catherine Jungers.

352. Michel Houton, * ca. 1700 in Oberpallen, $\infty$ mit Catherine Ludig.

704. Maternus Houton, $\infty$ mit Catherine NN.

Die Schreibvariante -ua in Hottua erklärt sich in diesem Beispiel als typische Germanisierung von rom. -ois. Houtois ließe sich dabei eventuell als derivierter Herkunftsname zu frz. Hotte (wa. Hote) für eine kleine Ortschaft der Gemeinde Feitler/Feitweiler/Fauvillers in der Provinz Luxemburg interpretieren, während beim einmaligen Beleg Houton aus dem Jahr 1667 der Schreiber offensichtlich volksetymologisch an den weiter entfernten Ort wa. Houton, frz. Hotton, bei Marche in den Ardennen dachte. ${ }^{9}$

Etymologische Untersuchungen zu Familiennamen würden wesentlich erleichtert, wenn mehr solcher, auf ernsthaften genealogischen Nachforschungen beruhenden Agnatentafeln, die die genaue Schreibweise der Namen festhalten, vorlägen. So stammt der heutige luxemburgische Familienname Milmeister zum Beispiel nachweislich von der Berufsbezeichnung Mi(e)nnenmeister aus dem Stadtluxemburger Festungsbereich (vgl. Milmeister 1985).

\subsection{Katapom aus Quatrepoint}

Ein einleuchtendes Beispiel für eine nicht nur graphische, sondern auch phonetische Verformung eines ursprünglich romanischen Familiennamens bei der Niederschrift in germanischem Umfeld findet sich in den Pfarrakten der Pfarrei Sankt-Michael, Luxemburg. Dort heißt es in einem Hochzeitsakt vom 01.02.1779: „...matrimonium contraxerunt Dominicus Simon, filius Frederici Simon, linitextoris [Leinenweber] et Mariae Josephae Mackel, conjugum ex Paffendall et virtuosa Joanna Quatrepoint, scribere nesciens,

8 Die hier abgedruckte Agnatentafel ist aufgrund der numerischen Generationenzählung von SosaStradonitz aufgebaut. Erstpublikation in Muller 1996, 155-156 mit detaillierteren Angaben; ein weiterer Träger desselben Familiennamens wird behandelt von Malget 2010.

9 Claire Muller (in diesem Band) vertritt ihrerseits diese Etymologie. Zum Ortsnamen Hotton und der Geschichte dieser Ortschaft vgl. Marquet 1930. Trotz phonologischer Schwierigkeiten $(<\ddot{u}>$ versus $<\mathrm{u}>$ ) möchte der Autor die Herleitung aus dem Gentilnamen Hutois(es), der Bezeichnung der Einwohner der Stadt Huy an der Maas nicht völlig ausschließen. 
Alphonsi Quatrepoint, hortulani [Gärtner] et Anne-Margarithae Peyal conjugum filia legitima“. Die junge Ehefrau verstarb im Alter von nur 39 Jahren, und ihr Begräbnisakt hält fest: ,mortua est et in cemeterio extra portam Eich [auf dem so genannten Siechenfriedhof] sepulta fuit Joanna Katapom, uxor Dominici Simon, linitextoris ex Paffendall“". Die Identität der genannten Ehefrau in beiden Akten steht außer Zweifel wegen des identischen Gattennamens. Der Name Quatrepoint ist auf Französisch irgendwie deutbar, die luxemburgisch-germanisierte Aussprache als Katapom hingegen nicht. Man kann sich konkret die Schwierigkeiten einer genealogischen Nachforschung ausmalen, welche bloß anhand von Namenindices weiterkommen wollte. Ein langer und weitsichtiger Umgang mit der schriftlichen Dokumentation ist hingegen nötig - der Sprachwissenschaftler seinerseits sollte sich vor voreiligen Erklärungen hüten!

Ein anderes konkretes Beispiel einer - diesmal verbessernden - Aussprache in fremdsprachigem Kontext bietet die Entwicklung des Familiennamens Sacmarie aus der Gegend von Redingen/Attert, welcher von der zweiten Auswanderergeneration in den Vereinigten Staaten (Wisconsin) in der zweiten Hälfte des 19. Jh. zu Sainte-Marie „,aufgewertet“ wurde. Ähnlich spielte in der Neuen Welt ein Sohn aus der „Thillsmühle“von Redingen mit dem Sozialprestige einer französischen Aussprache seines Namens, indem er seinen banalen Namen Thill zu einem noch unerklärten Thilly de Fontenay umgestaltete.

\subsection{Strekfus $z u$ Longepied, Hartenfels $z u$ Felsenhart}

Ein interessantes Fallbeispiel einer Lehnübersetzung vom Deutschen ins Französische wurde um das Jahr 1995 von einer französischen Genealogin namens Longepied beigesteuert (Muller 1996, 157). Diese wollte bei der A.L.G.H. Informationen über Geburtsort und -datum ihres Vorfahren François Streffus oder Strickfus erhalten. Der Betreffende sei um 1760-1765 im Luxemburgischen geboren und habe sich nach seiner Heirat in Frankreich am 17.07.1794 unter dem Familiennamen Longepied „naturalisieren“ lassen. Seine Eltern hätten Ulrich Strekfus und Eva Barbara geheißen. Leider konnten in den überlieferten Quellen keine derartigen Namensträger im Luxemburger Land nachgewiesen werden. Die Dublette ist aber an sich unverdächtig, begegnet doch in Luxemburg der Familienname Lichtfus(s), der ursprünglich als Bahuvrīhikompositum wohl jemanden bezeichnete, der eine Wegstrecke oder das Leben „leichten Fußes“ meisterte.

Die Umstellung von Elementen in einem Namenkompositum - wohlbekannt seit der altindischen Kompositionslehre - stellt einen Sonderfall von Namensdubletten dar: A.L.G.H.-Nachforschungen im Bereich der Goldschmiedefamilie Felsenhart in Trier und Arlon ergaben einen wahrscheinlichen Ursprung in Vianden am Ende des 17. Jahrhunderts. Dort war allerdings der Familienname als Hartenfels statt Felsenhart eingetragen, was den Fragesteller zu unbeweisbaren Spekulationen über einen möglichen Konfessionswechsel (protestantisch zu katholisch) mit gleichzeitiger Namensänderung veranlasst hatte.

\subsection{Die Amtmännerfamilien Wolf und Lupus - Dubletten durch Latinisierung}

Loutsch $(1974,534)$ beschreibt in seinem Luxemburger Wappenbuch das Siegel eines Jean Lupus, apostolischer Notar und Pfarrer zu Mersch vom 02.07.1694 mit der lakonischen Bemerkung: „d'une famille de Saint-Vith, Lupus étant évidemment la latinisation 
$d u$ nom Wolf‘. Des Weiteren ist in den Beforter Pfarrmatrikeln im Jahr 1750 von einer Hochzeit die Rede, bei welcher sich der Ehemann Wolff, die Ehefrau hingegen Lupus nennt: „1750 19a Maii contraxerunt matrimonium Joannes georgius Wolff ex Befort, relictus filius petri et barbarae Wolf cum virtuosa anna clara elisabetha Lupus, joannis wilhelmi et mariae judit Lupus filia ex Reulant, eadem parochia“. Die Gattin stammte demnach aus Burg-Reuland im Umland von Sankt-Vith, heute im Dreiländereck zwischen Belgien und Deutschland im hohen Luxemburger Norden gelegen. Diese bizarr anmutende Doppelbenennung Wolff/Lupus könnte ihre Erklärung in der Tatsache finden, dass innerhalb der weitverzweigten Juristen- und Amtmännerfamilie Wolf(f) in SanktVith eine Namensspezialisierung für einzelne Zweige vollzogen wurde: die lateinische Form Lupus blieb dem Sankt-Vither und späteren Burg-Reulander Zweig vorbehalten, während der später in Befort nachzuweisende Zweig exklusiv die deutsche Form Wolf(f) gebrauchte. Das Zusammentreffen beider Varianten in einem Akt beruht demnach wohl auf einer arrangierten Eheverbindung, um eine einzig überlebende Erbtochter des LupusZweiges in den entfernt verwandten Wolff-Zweig einzuheiraten.

Solche latinisierte Namensdubletten sind im Bereich der Luxemburger Familien recht häufig anzutreffen; man denke an den Arloner Humanisten Bartholomäus Latomus (1495-1570), der als Steinmetz geboren wurde; oder noch an die Diekircher Notablenfamilie Vannerus, mit aus Wag(e)ner latinisiertem Familiennamen.

Insbesondere die Latinisierungen von Herkunftsbezeichnungen, die dann zu Familiennamen wurden, scheinen im Bereich des deutschsprachigen Herzogtums Luxemburg, sowie der gesamten Moselgegend gehäuft aufzutreten. Es handelt sich im Idealfall um Familiennamen mit der Endung -ius, die frei an den Herkunftsnamen angefügt wurde (Karte 1). So ergibt der Ortsname Bissen + ius den Familiennamen Bisenius; Wiltz $>$ Witzius; der Familienname Conzemius ist allerdings etwas schwieriger zu erklären, gehört jedoch letztlich wohl zum Luxemburger Toponym Consthum. Eine Kartierung aller auf -ius endenden Familiennamen mit Hilfe des an der Universität Luxemburg entwickelten Moduls eines Familiennamen-Atlasses zeigt sehr deutlich die räumliche Einengung des Phänomens auf den moselländisch-niederrheinischen Bereich. ${ }^{10}$ Die Auflistung luxemburgischer Belegbeispiele aus den Publikationen der Volkszählungen von 1880, 1930 und 1980 ergibt folgendes Inventar: Beckius, Bernius, Besenius, Bisenius, Bonarius, Britzius, Brixius, Brosius, Capesius, Cavelius, Chelius, Conzemius, Cricius, Dixius, Fabricius, Foxius, Gricius, Grisius, Grissius, Grutzius, Hamelius, Hamilius, Jubelius, Karius, Korzilius, Krutzius, Milius, Neisius, Pixius, Rusius, Sartorius, Wiltzius, Wilzius, Wiwinius, Zeutzius. ${ }^{11}$

In diesem Kapitel wurden anhand von Beispielen aus der genealogischen Praxis Dubletten von Familiennamen über die romanisch-germanische Sprachgrenze hinweg

10 Vgl. http://lfa.uni.lu.

11 Detailliert behandelt in Muller 2002. Aus der obigen Liste wurden alle auf lateinische Vornamen zurückgehenden Formen ausgeschlossen. Es sind diese im einzelnen: Ambrosius, Anthonius, Blasius, Confucius, Cornelius, Dionysius, Gregorius, Hortensius, Junius, Lucius, Luzius, Marius, Tullius, Valerius, Walerius, Wallerius. 
dargestellt und erläutert. Der zweite Flügel dieses namenkundlichen Diptychons bespricht abschließend Familiennamenvarianten, welche auf eine Herkunft aus entfernten Landstrichen hinweisen, oder aber sehr alte Territorialverhältnisse und heute verschwundene Landesgrenzen widerspiegeln.

\section{Regionale Herkunftsangaben und territoriale Zugehörigkeits-bezeichnungen als Quelle von Familiennamen}

\subsection{Munkler hergeleitet von der Burg Montclair}

Hoch über einer Saarschleife bei Mettlach im Saarland liegen die Ruinen der bedeutenden spätmittelalterlichen Burganlage Montclair. Diese wurde um 1428-1439 vom Ritter Arnold von Sierck an der Stelle der bis ins 10. Jh. zurückreichenden Burg Skiva auf diesem strategischen Bergrücken erbaut. Zum ersten Mal ins Licht der Geschichte tritt der Burgname Montclair in einer Papsturkunde vom 04.06.1190, in welcher Papst Clemens III. im Lateran dem Erzbischof Johann I. von Trier u.a. den Besitz des „,Castrum de Muncler" bestätigt. Die 1218 ebenfalls benutzte lateinische Benennung „Mons clarus" (Simon, dominus de Monteclaro) kann sowohl , heller, lichter Berg' als auch ,berühmter Berg‘ bedeuten. Als ein Guy de Clermont aus Frankreich im späten 13. Jh. durch Einheirat in den Besitz der Burg Montclair kam, scheint er mit dem etymologischen Spiel der Umstellung der Kompositionselemente, - wie oben im Fall Felsenhart = Hartenfels erläutert, - den Namen Montclair als Name eines neuen Rittergeschlechts begründet zu haben.

Interessant ist es nun festzustellen, dass von diesem Burg- und Geschlechtsnamen anscheinend der sehr viel später belegte Familienname Munkler (und Varianten) herzuleiten ist. ${ }^{12}$ Im Jahr 1496 taucht in den Quellen von Rossdorf bei Darmstadt ein Peter Monckeller auf, der wahrscheinliche Urahn der heutigen Familien Münkler, die in der Gegend von Dieburg wohnen. Wenn man die derzeitigen 59 deutschen Telefonbucheinträge zum Familiennamen Munkler abfragt, fällt die Konzentration von fast der Hälfte (24) im Landkreis Bitburg-Prüm ins Auge. Diese leiten sich von den ersten Belegen in der bis 1815 zu Luxemburg gehörenden Eifelpfarrei Eschfeld her, wo 1696 die Namensform Monkeller $\beta$ zuerst auftaucht. In Eschfeld trägt das Stammhaus das Domonym (siehe oben Kapitel 1) Munkelesch, obwohl das Haus eine Familie mit dem Namen Müller seit Generationen bewohnt. Das luxemburgische Feuerstättenverzeichnis von 1624 nennt in Eschfeld den Namen Monckler, während in jenem von 1611 in der Ortschaft Eschdorf bei Wiltz derselbe Name auch als Monckler überliefert ist. Weitere frühe Belege finden sich 1702 als Moncler in Binsfeld im Luxemburger Norden und 1784 als Munckler in Föhren bei Schweich an der Mosel. Somit kann dieser recht seltene Familienname wohl eindeutig als Germanisierung des ursprünglich romanischen Burg- und Geschlechternamens Montclair gedeutet werden.

\footnotetext{
12 Vgl. Munkler 1988. Alle folgenden Namenbeispiele entstammen dieser 400-seitigen Studie.
} 


\subsection{Croat-Cravatte-Horvath}

Unsere Nachbarn zuhause trugen den Familiennamen Croat, und ihre Enkelkinder gerieten in der Folge der Kriege im auseinanderbrechenden Jugoslawien in den 1990er Jahren auf dem Schulhof arg in Bedrängnis: Bekanntlich ist die Grausamkeit kleiner Kinder grenzenlos, und demzufolge wurden unsere Nachbarskinder wegen ihres angestammten Familiennamens mit den Kriegsverbrechen der Kroaten drangsaliert und gehänselt. Nun gibt es effektiv einen Zusammenhang zwischen diesem seit 1678 im Herzogtum Luxemburg belegten Ethnikon, dem Namen der Einwohner von Kroatien und der Krawatte, im 17. Jh. aus dem gleichbedeutenden französischen cravate entlehnt, „dieses aus Krawat, ,die kroatische Halsbinde', einer mundartlichen Nebenform von Kroate, nach einer bestimmten Halsbinde von kroatischen Reitern“ im Dreißigjährigen Krieg (vgl. Seebold 1989: 411). Bei der Kartierung mit dem Modul des Familiennamen-atlasses der Universität Luxemburg wurden neben der aus dem Ungarischen entlehnten Benennung Horvath die vielfältigen Schreibweisen eingegeben, welche in der Luxemburger Hochzeitskartei der A.L.G.H. vor 1802 vorkommen (siehe Karte 2): der anlautende gutturale Verschlusslaut mit $<\mathrm{C}>$ oder $\langle\mathrm{K}>$, der labiale Glide zwischen den mittleren tiefen Vokalen $<\mathrm{OA}>$, $<$ AVA $>$ sowie die einfache oder verdoppelte Dentalendung $<\mathrm{T}>,<\mathrm{TTE}>$. Am Rande sei festgehalten, dass Angehörige der Familie aus dem Kanton Redingen im 19. Jh. nach Nordamerika auswanderten und dort bis heute ihren Namen als Croatt, mit Betonung auf dem ersten Vokal, schreiben. Neben diesem Familienverband, der seinen Ursprung in der kleinen Ortschaft Lannen bei Redingen hat und Ende des 18. Jh. eine Nebenlinie in Eschdorf hervorbrachte, begegnet ein zweiter Brennpunkt dieses Ethnonyms in Düdelingen im Luxemburger Süden. Dort ging im Jahr 1678 der erste bekannte Namensträger Joannes Cravat die Heirat mit Maria Lux aus Hayange ein. Die Schreibweisen variieren wild durcheinander in beiden nicht miteinander verwandten Zweigen, so dass von einer Beeinflussung der Graphemik durch die Phonetik des fremdartigen Namens auszugehen ist; in Düdelingen überwiegt allerdings die Graphie Cravat. Einmal begegnet sogar die Scheibweise Kravaten, nämlich bei der Taufe einer Anna K. am 02.01.1684 in Everlingen, Pfarrei Ospern. Ursprünglich wurden in Luxemburg mit diesem Namen wohl zwei aus dem Balkan stammende Söldnersoldaten bezeichnet, die sich nach den Gräueln des Dreißigjährigen Krieges unabhängig voneinander im Luxemburger Land niederließen und die zwei nachweisbaren Stammlinien begründeten.

\subsection{Behm - Böhm - Bem}

Behm/Böhm, ein anderer Familiennamenkomplex mit variablen Schreibungen, lässt zweifelsfrei auf einen Ursprung aus dem Land Böhmen schließen, mit dem Luxemburg im Lauf seiner Geschichte seit König Johann (dem Blinden) und dem Bischof und Kanzler Peter von Aspelt zu Beginn des 14. Jahrhunderts vielfältige Kontakte pflegte (Muller 2003b). Am 27.09.1625 erwarb Hieremias Böhem „,aus dem Landt Meissen“ das Bürgerrecht der Stadt Luxemburg und legte den gewöhnlichen Bürgereid ab. Interessant ist die durch diese Archivnotiz belegte Tatsache, dass der erste Namensträger nicht direkt aus dem Land herkam, dessen Name in seinem Familiennamen steckt, sondern seine Vorfahren bereits früher aus Böhmen nach dem sächsischen Meißen an der Elbe migriert waren. Von diesem 
eingewanderten „Urahn“ stammen nachweislich alle Luxemburger Namensträger Behm $\mathrm{ab}$, und die Genealogie erlaubt es, eine Agnatentafel über 12 Generationen zusammenzustellen, was bei der bekannten kargen Quellenlage außergewöhnlich ist (Ensch \& Muller 1990). Nach der Schreibung Böhem von 1625 begegnet in dieser Familie einzig und allein die Namensform Behm, vom Eremiten Theodor Behm (1708-1784) auf der Rittersdorfer Klause und dem Notar Jean-Nicolas Behm $(* 1740)$ in Luxemburg-Stadt bis hin zum Dechanten Michel Behm und seinem Neffen, dem Arzt Jean-Marie Behm in Mersch. Die Kartierung mittels des Luxemburgischen Familiennamenatlasmoduls zeigt eindeutig, dass diese Schreibung Behm vorwiegend in Luxemburg zu finden ist, während Namensformen mit -e am Ende (Behme, Böhme, Boehme, Beme) - z.B. der in Görlitz an der Neiße geborene Mystiker Jakob Böhme - eher auf den deutschen Osten und Norden beschränkt und somit näher an der Ursprungsregion dieses Ethnikons verbreitet sind. Diese Verteilung Behme/Böhme versus Behm/Böhm scheint auch sehr deutlich im entsprechenden Kartenkomplex des DFA Bd. 4 Herkunftsnamen $(2013,86 \mathrm{ff}$.) auf.

\subsection{Bourguignon - Burgund(er)}

Abschließend sollen nun jene Namenstripletten diskutiert werden, die oberflächlich so aussehen, als ob sie unterschiedliche Herkunftsbenennungen je nach Sprachgebiet wären. So lässt die Familiennamenkartierung der romanischen Form Bourguignon vor allem das heutige Königreich Belgien (weniger Flandern als Wallonien) als Hochburg dieses Familiennamens erscheinen (siehe Karte 3). Auch in der belgischen Provinz Luxemburg ist der Name häufig belegt, während er im gleichnamigen Großherzogtum nur im Süden vereinzelt aufscheint, dort in Konkurrenz mit der germanischen Form Burgund und noch seltener Burgunder, das sich an der Mosel konzentriert. In Deutschland gibt es neben verstreuten Einzelbelegen der drei Formen nur für Bourguignon eine Massierung in Köln und für Burgunder eine in Bonn.

Doch der zeitgenössische kartographische Befund lässt die Hypothese als sehr unwahrscheinlich erscheinen, dass in früheren Zeiten eine massive Auswanderung aus Burgund (französisches Herzogtum / duché de Bourgogne sowie Freigrafschaft Burgund / Franche Comté) in die frankophonen belgischen Provinzen stattgefunden hätte, und eine viel weniger zahlreiche in die deutschsprachigen Gebiete. In diesem Fall liegt weniger eine Einwanderung vor als eine Benennung von ortsfesten Untertanen nach ihrem jeweiligen Landesherrn.

Im Laufe des 15. Jahrhunderts erreichten die Herzöge von Burgund mit Sitz in Dijon den Zenit ihrer Macht. Aus dem zwischen französischem Königtum und deutschem Reich eingekeilten Territorium bauten sie eine europäische Großmacht auf, die bald nach Norden expandierte und sich unter Herzog Philipp dem Schönen durch Eroberung der reichen belgischen Provinzen dort dauerhaft etablierte. Bruges/Brügge und Malines/Mechelen wurden die Zentren burgundischer Prachtentfaltung und Machtausübung. Die Stadt und Festung Luxemburg wurde 1443 erobert, nachdem mit Kaiser Sigismund 1437 der letzte Repräsentant der mittelalterlichen Luxemburger Dynastie ohne männlichen Erben verstorben war. Der burgundische Expansionsdrang wurde erst am Ende des 15. Jh. durch militärische Niederlagen Karls des Kühnen in der Schweiz und vor Nancy gebremst. 
Durch die Heirat zwischen Kaiser Maximilian aus dem österreichischen Hause Habsburg und der burgundischen Erbin Maria kamen diese Besitzungen, Luxemburg inbegriffen, im 16. Jh. an die spanischen Habsburger.

Setzt man das allmähliche Festwerden von Familiennamen, vor allem in den Städten, um 1500 an, so leuchtet ein, dass der Familienname Bourguignon und seine Varianten Burgund und Burgunder als Zugehörigkeitsbezeichnungen in dem eben erläuterten, sehr spezifischen historischen Kontext entstanden sind. Sie sind demnach keine Zeugnisse irgendwelcher Migrationen, sondern territoralgeschichtlich begründet. Vorstellen kann man sich etwa, dass solche Benennungen an der Grenze mit dem Königreich Frankreich ihren Ursprung nahmen, indem die burgundischen Untertanen jenseits der Landesgrenze spöttisch als Bourguignons bezeichnet wurden (Muller 2003a).

\subsection{Spanier - Lespagnol - Spaniol}

Spinnt man den Faden ins 16. und 17. Jahrhundert weiter, so kann man eine ganz ähnliche territorialgeschichtliche Begründung für die Namenstripletten Spanier - Lespagnol - Spaniol finden, die als Familiennamen wiederum viel zu häufig belegt sind, als dass sie von einer Einwanderung aus Spanien, wie massiv auch immer diese gewesen sein mag, herrühren könnten. Mit Kaiser Karl V. und seinem Sohn, dem spanischen König Philipp II. und dessen Nachfolgern, regierten die spanischen Habsburger die südlichen, katholisch verbliebenen niederländischen Provinzen (Pays-Bas espagnols) bis zum Spanischen Erbfolgekrieg im Jahr 1700. Ab 1715 wurden sie dann für 80 Jahre von den österreichischen Habsburgern in der Herrscherfunktion ersetzt.

Somit ergibt sich für den Familiennamentypus Spanier - Lespagnol eine mit Bourguignon - Burgund(er) vergleichbare Motivation und Entstehungsgeschichte. Analog ist der Name der Gastwirtschaft „Im spanschen Eck“ zu interpretieren, die sich im Dorf Igel an der Mosel befand, das bis 1815 zum Herzogtum Luxemburg gehörte. Diese oberflächlich bizarre Benennung verdeutlicht aber unzweifelhaft die territorialgeschichtliche Motivation solcher Namengebungen, auch im Bereich der Familiennamen. Die Einwohner aus dem Trierischen nämlich, jene von der anderen Seite der Grenze, bezeichneten so die Dörfer und Untertanen, die von „Spanien“, d.h. den spanischen Habsburgern, von 1520 bis 1700 regiert wurden. Im Dorf Itzig, südlich der Stadt Luxemburg, gibt es heute noch „eng spuenisch“ und „eng franséisch Säit“. Diese heute unverständlich gewordenen, altertümlichen Bezeichnungen reichen in eine Zeit zurück, als das Königreich Frankreich im Rahmen seiner expansiven Reunionspolitik unter Ludwig XIV. Teile des Luxemburger Südens besetzt hielt unter dem Vorwand, diese Dörfer und Dorfteile gehörten zur Herrschaft Roussy/Rüttgen, die französisch war. Das Luxemburger Wörterbuch (IV, 253) führt ebenfalls aus, dass die Substantivierung „am Spueneschen“ „im deutschen Grenzgebiet“ für diejenigen Luxemburger Gebiete gebraucht wird, „die bis $1714 \mathrm{zu}$ den spanischen Niederlanden gehörten“. Man darf daher wohl davon ausgehen, dass die Familiennamen des Typus Spanier usw. zuerst an den Territorialgrenzen, vielleicht sogar als spöttische Bezeichnung für die Nachbarn auf der andern Seite, entstanden sind und sich dann innerhalb der betreffenden Territorien durch Heirat und Binnenwanderung ausgebreitet haben. Solch eine Dynamik lässt ebenfalls die Familiennamenkarte 
mit den Tripletten Spanier - Lespagnol - Spaniol erkennen (siehe Karte 4), wobei die romanische Form mit agglutiniertem bestimmtem Artikel Lespagnol vor allem südlich von Brüssel erscheint, während die schriftdeutsche Form Spanier in Brüssel selbst, jedoch vor allem im Luxemburger Gutland und entlang der Mosel, wie auch entlang der Rheinschiene zu finden ist. Die ältere Namensform für ,Spanier', Spaniol (< español) hat dagegen interessanterweise eine Hochburg im Saarland. Im Großherzogtum Luxemburg rangierte der Familienname Spanier im Jahr 1980 sogar unter den 500 häufigsten Familiennamen.

Am Ende dieser Analyse dürfte deutlich geworden sein, dass Namensdubletten an den Sprachgrenzen, welche sich von Länder- oder Regionennamen herleiten lassen, nicht zwingend Bevölkerungsbewegungen oder Einwanderungen widerspiegeln. Vielmehr können Familiennamen dieser Kategorie auch die Erinnerung an sehr alte territorialgeschichtliche Zusammenhänge, wie tiefgefroren, bewahren.

Halten wir also als Fazit fest, dass Namenetymologien in Grenzgebieten zwischen Territorien oder Sprachen sich oftmals erst durch zusätzliche genealogische Forschungen erschließen lassen. So stößt man im zweisprachigen Kontext nicht nur auf aus Übersetzungen resultierende Namendubletten (Holtsmacher/Dubois, Klein/Petit) sondern auch auf durch lautliche Verformungen entstandene Namen (Katapom < Quatrepoint, Munkler $<$ Montclair) und Latinisierungen (Lupus $<$ Wolff).

Evidente Herkunftsnamen wie etwa Behm oder Croat sollten auf Grundlage genealogischer Daten bewiesen werden, während andere scheinbare Herkunftsnamen nicht zwingend durch Zuwanderung erklärbar sind (Spanier, Lespagnol), sondern auf alte territoriale Zusammenhänge rekurrieren können (hier die historischen niederländischen Provinzen der Pays Bas espagnols).

\section{Literatur}

Arendt, Emile: Ein bisher unbekannter Frühdruck aus der Luxemburger Offizin Birthon. In: de Familjefuerscher, Nr. 10, Luxemburg, 1993, 32.

Bourguignon, Marcel: L`Ėre du Fer en Luxembourg (XVe-XIXe siècles) - Études relatives à l'ancienne sidérurgie et à d'autres industries au Luxembourg, Luxembourg, 1999.

Calmes, Albert: Note biographique. In: Au Fil de 1'Histoire, Luxembourg, 1979, 279-282.

Ensch, Jean: Die neuentstandenen Hausnamen im 19. Jh. In: Livre d'Or de l'Harmonie grand-ducale municipale, Wiltz, 1994, 707-776.

Ensch, Jean / Muller, Jean-Claude: Ligne agnatique Behm. In: de Familjefuerscher Nr. 6, Luxemburg, 1990, 73.

Kollmann, Cristian: Auf der Suche nach Mahowald. In: Hémecht Jg. 63, Luxemburg, 2011, 351-362.

Deutscher Familiennamenatlas (DFA), Herausgegeben von Kunze, Konrad und Nübling, Damaris. Band 4: Familiennamen nach Herkunft und Wohnstätte, Berlin, 2013.

Loutsch, Jean-Claude: Armorial du Pays de Luxembourg, Luxembourg, 1974.

Luxemburger Wörterbuch, Herausgegeben von der Wörterbuchkommission, 5 Bände, Luxemburg, 1950-1975, Ergänzungsband, 1977. URL: http://infolux.uni.lu/worterbucher.

Malget, Jean: 28.04.1710 - Das Testament des Pfarrers vom Helperknapp, Étienne / Stephan: HAUTOIS oder HOTTUA und weitere Dokumente zum Wirken des Stephanus HU(T)TOY, Pastor in HeilbergBuschdorf (1665-1710). In: de Familjefuerscher Nr. 26, Luxemburg, 2010, 53-59.

Marquet, Édouard: Hotton à travers les âges, Tournai/Paris, 1930. 
Mathieu, Paul: Les „baptiseurs“ de maisons - Quelques remarques sur les „Hausnimm“ ou „noms de maisons“. In: Annuaire/Jahrbuch A.L.G.H., 1996, 161-181.

Mathieu, Paul: Les „noms de maisons („Häusnimm“) dans le Pays d'Arlon et plus spécialement à Tintange. In: Annuaire/Jahrbuch A.L.G.H., 1999, 25-77.

Milmeister, Jean: Ligne agnatique Milmeister. In: de Familjefuerscher Nr.2, Luxemburg, 1985, 13.

Muller, Jean-Claude: Hausnamen und Familiennamen von Redingen/Attert, Useldingen und Rippweiler in zwei Seelenverzeichnissen von 1793. In: de Familjefuerscher Nr. 2, Luxemburg, 1985, 11-15.

Muller, Jean-Claude: Démographie et structures familiales dans la paroisse d'Attert en 1745. In: Annuaire/Jahrbuch A.L.G.H., 1993, 161-190.

Muller, Jean-Claude: WOLF/LUPUS oder ähnlich: Zur Problematik der Dubletten bei Familiennamen einige Fallbeispiele. In: Annuaire/Jahrbuch A.L.G.H., 1996, 153-160.

Muller, Jean-Claude: Histoire(s) de... Bissen/Besenius, Konz/Conzemius, Wiltz/Wiltzius - Latinisations: les noms de famille luxembourgeois se terminant en -ius. In: Le Quotidien Nr. 2, Esch-sur-Alzette, 2002, 12.

Muller, Jean-Claude: Onomastique et migrations: Des noms, fossiles de l'histoire territoriale - Bourguignon, Spanier, Liégeois, Oestreicher - patronymes qui n'indiquent pas la provenance mais l'appartenance territoriale. In: Le Quotidien, Esch-sur-Alzette, 28.08.2003. (=Muller 2003a)

Muller, Jean-Claude: Onomastique et migrations: Les Behm venus de Bohême. In: Le Quotidien, Eschsur-Alzette, 18.09.2003. (=Muller 2003b)

Muller-Wirth, Henriette / Muller, Jean-Claude: Die Bevölkerung von Redingen/Attert im Spiegel der Pfarrbücher und der Volkszählung von 1766. In: Festbroschüre Cercle philatélique Les Timbrophiles de 1'Attert, Redingen/ Attert, 1987, 33-107.

Munkler, Bruder Niketius A.: Der Familienname Munkler in seiner 800jährigen Geschichte, Vorkommen in den Geschlechtern v. Walecourt, v. Joinville, c. Clefmont, v. Montclair, v. Sirck, v. Sayn bis in die Gegenwart, Bonn, 1988.

Neuss, Elmar: Rheinische Personennamen aus Mittelalter und früher Neuzeit. In: Rheinische Vierteljahrsblätter 65, Bonn, 2001, 1-33.

Schaffner, Hugues: Bibliographie Christian Calmes. In: Hémecht Jg. 40, 1988 und ergänzend ibidem 41, 1989, 101.

Seebold, Elmar (Hrsg.): Kluge - Etymologisches Wörterbuch der deutschen Sprache, Berlin, 1989.

Van Werveke, Nicolas: Die Familiennamen des Luxemburger Landes, Diekirch, 1900. 


\section{Kartenanhang}

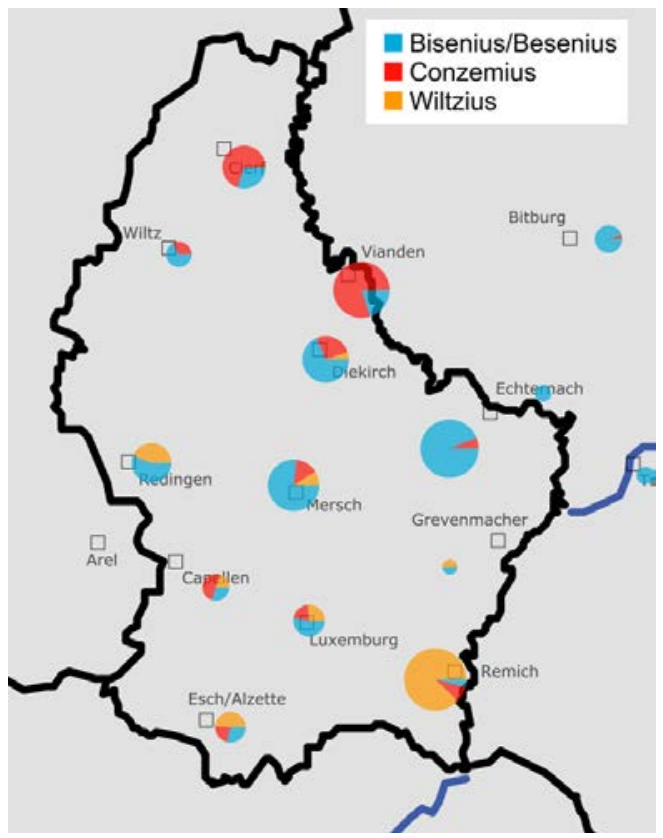

Karte 1: Verteilungskarte einiger Latinisierungen auf-ius, abgeleitet aus den Ortsnamen Bissen, Consthum und Wiltz

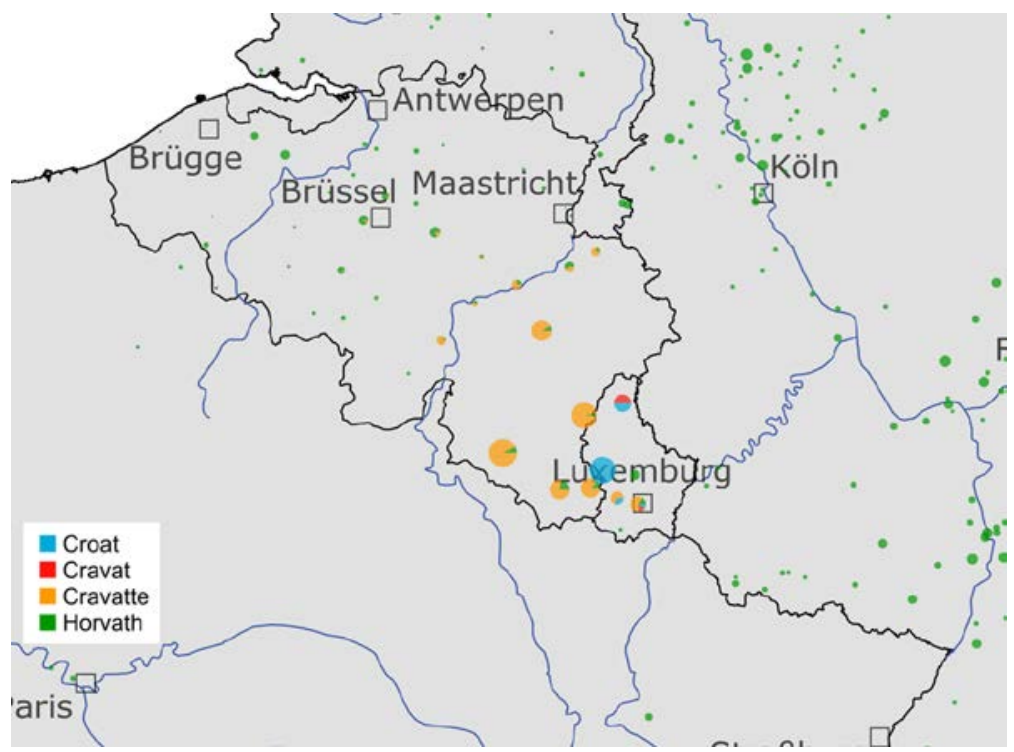

Karte 2: Verteilungskarte des aus dem Ungarischen entlehnten Landesnamens für Kroatien in der Rhein-Maas-Region 


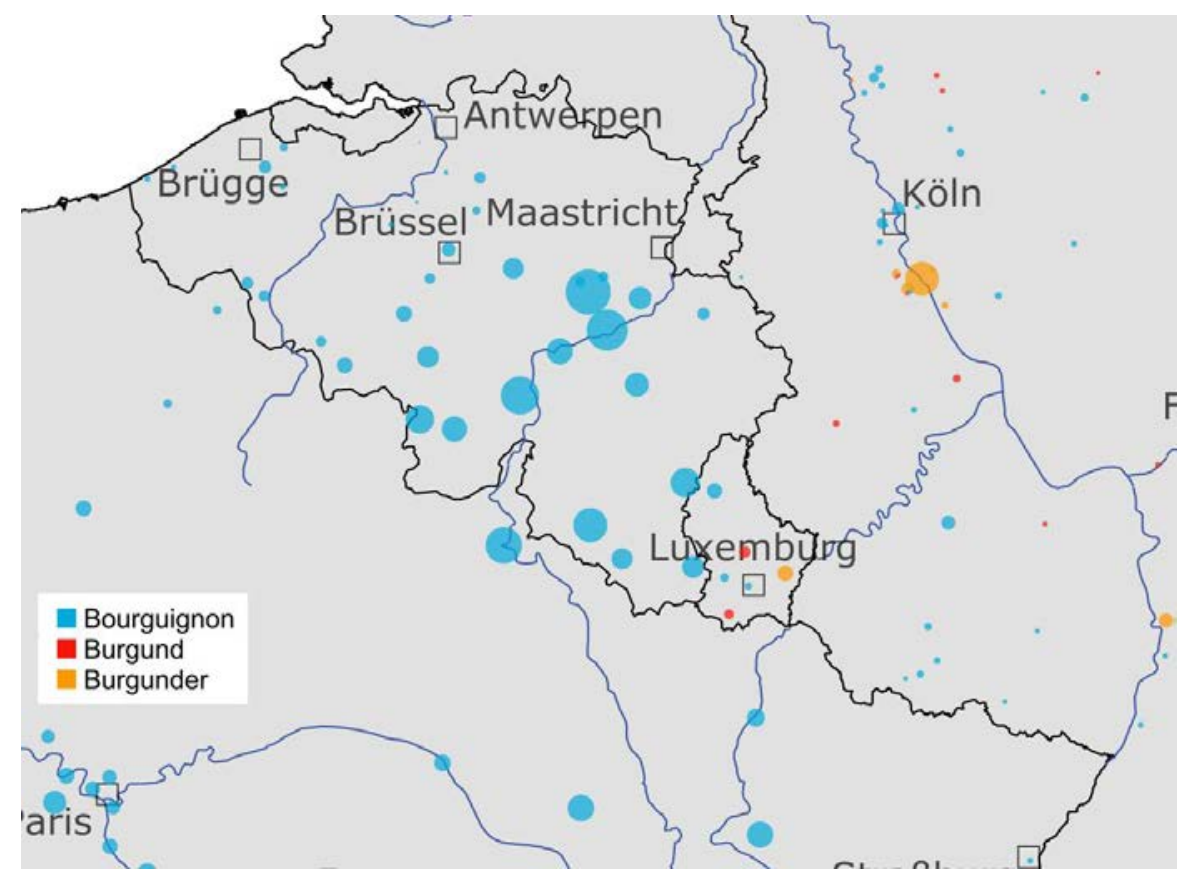

Karte 3: Verteilungskarte der vom Landesnamen Burgund abgeleiteten Familiennamen

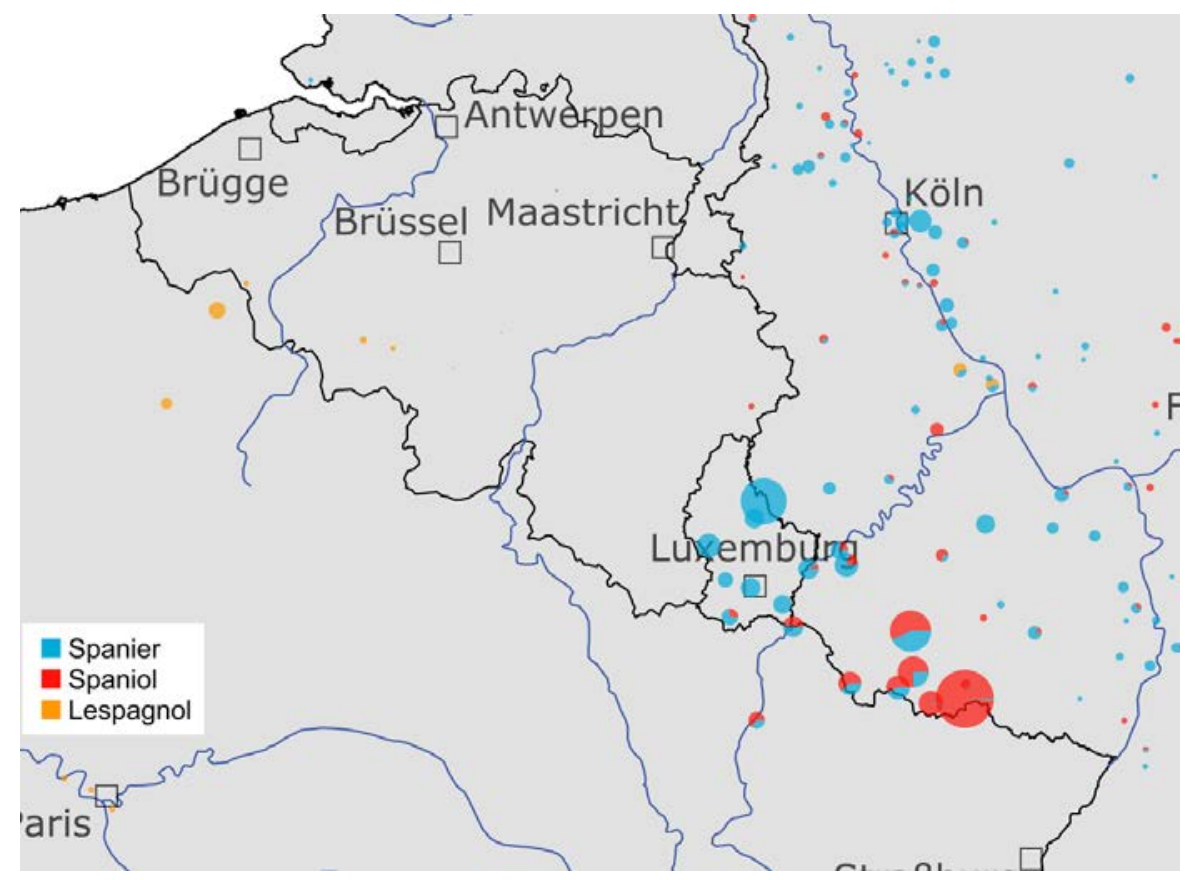

Karte 4: Verteilungskarte der vom Landesnamen Spanien abgeleiteten Familiennamen 



\title{
La „loi des deux syllabes“: une réponse structurelle à la fixation des noms de famille en Wallonie
}

\author{
Jean Germain
}

\section{Résumé}

Dans cet article de synthèse, qui intègre l'essentiel de deux articles précédents peu connus (Germain 2002 et 2005), on examine le rôle joué par un phénomène d'économie morphologique que l'on peut appeler „la loi des deux syllabes", loi implicite qui, selon nous, régit la majeure partie des noms de famille français de Wallonie.

Une première partie concerne l'aphérèse qui a frappé nombre d'anciens noms de baptême sous leur forme hypocoristique, souvent à finalité patronymique, gonflant le corpus wallon des noms de famille modernes issus de noms de baptême. Des exemples liégeois permettent de mettre en perspective ce phénomène.

La seconde partie s'intéresse au phénomène de l'agglutination orthographique de l'article et/ou de la préposition qui s'inscrit dans la même dynamique. Comment et quand s'est fixée cette forme patronymique des anciens surnoms, telle est la question à laquelle on tâche de répondre sur la base des registres de bourgeoisie de la ville de Namur.

Ces diverses recherches ont été menées en marge du projet ,Patronymica Romanica‘, mieux connu sous son nom abrégé PatRom.

\section{Entre affectivité et aphérèse, le destin des hypocoristiques}

Selon la définition des dictionnaires, un „hypocoristique“, tiré d'un mot grec signifiant ,caressant", est un terme qui exprime une intention caressante, affectueuse, notamment dans le langage des enfants ou ses imitations. L'anthroponymie en est un grand consommateur.

En effet, dans la création des futurs noms de famille au Moyen Âge et dans les siècles qui ont suivi, se sont opposées généralement deux forces contradictoires qui ont encore cours aujourd'hui: d'une part l'affectivité qui permet de personnaliser la relation entre le dénommé et le dénommant, d'autre part - comme en contrepoint - la paresse qui permet de gommer la longueur du nom ainsi créé. L'affectivité qui touche les noms de baptême se matérialise par la naissance de nombreux hypocoristiques, généralement concrétisés par des changements de suffixes ou par des adjonctions d'un ou de plusieurs suffixes, voire par des redoublements; nombre d'entre eux ont joué un rôle du point de vue patronymique. Par ailleurs, c'est par une forme de paresse - d'économie du langage - que l'être humain a réduit à deux syllabes la plupart des noms; il a ainsi résolu l'équation par la suppression de syllabes, généralement à l'initiale (aphérèse), plus rarement à la finale (l'apocope).

\subsection{La suffixation hypocoristique: le jeu de l'affectivité}

Au Moyen Âge, en Wallonie comme dans le reste du domaine oïlique français, les créations hypocoristiques ont été d'une très grande vitalité en affectant de nombreux noms de baptême; ce fut surtout le cas avec les noms individuels les plus populaires, ceux des 
apôtres et ceux des saints vénérés localement. Pour le domaine gallo-roman, on peut se référer à ce qu'en disent Dauzat 1977: 307-315 et Mulon 2002. Le procédé perdra de sa vitalité, sans doute au cours du XVI ${ }^{\mathrm{e}}$ siècle. À Namur en tout cas, les hypocoristiques ont disparu complètement comme noms individuels à la fin du XVI $\mathrm{XI}^{\mathrm{e}}$ siècle, mais nombre d'entre eux ont survécu comme noms de famille sous des formes très diverses (cf. Germain 1987).

\section{Chinı.}

Le XXIIe jour de may l'an LXXII.

Declaracion des feuz et mesnaiges ou chastel de Chini et de la franchise d'icelle au dehors dudit chastel.

Jehan Colart.

Jehan de Gouvy.

Jehan Rebenne.

Jakemyn le waingnier.

Ernoult le filz Maistre Piere.
Gérart de Hotte.

Colignon Billa.

Poncin, son gendre.

Gillet de Labby.

Alis vefve, fille Billa.

\section{Sussy (I).}

Pittit Jehan filz Pirlot. Hanrion Macquet.

Jehan Pirlot.

Hennyn le filz Maistre Piere.

Fig. 1: Dans ce dénombrement du comté de Chiny en 1472, presque tous les noms de baptême se présentent sous la forme d'hypocoristiques
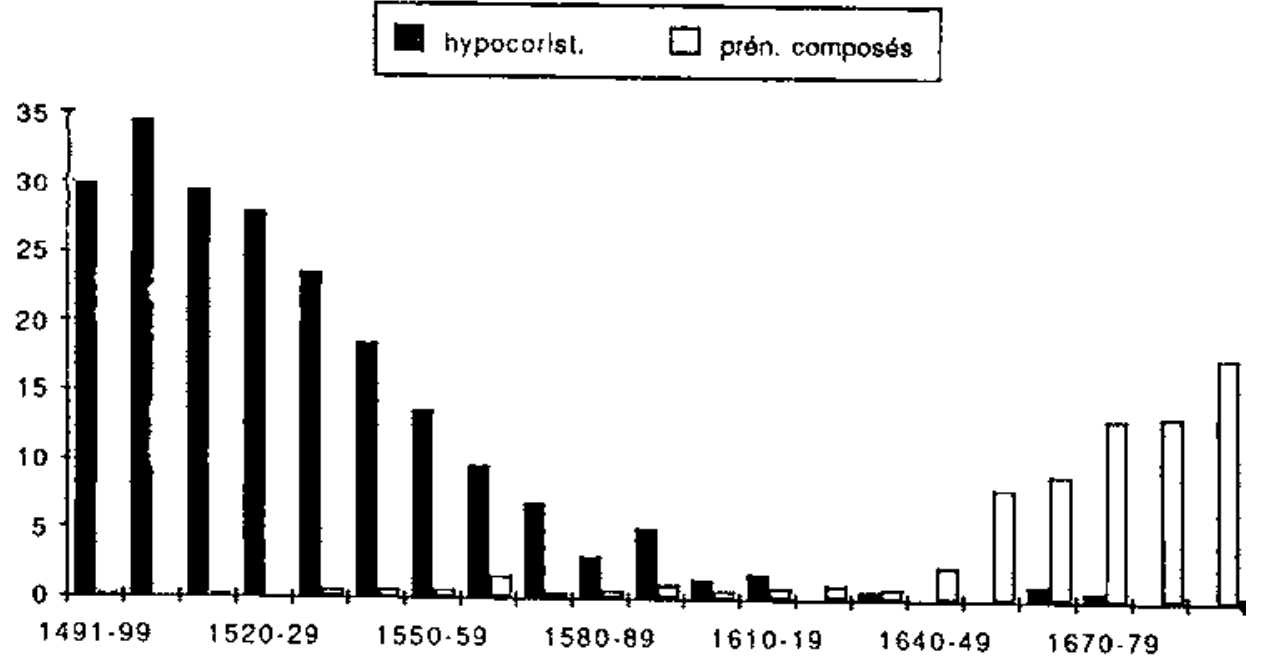

Déclin des hypocoristiques et essor des prénoms multiples

Fig. 2: D'après l'étude des registres de bourgeoisie de Namur, vers 1600, la production naturelle d'hypocoristiques cesse au profit, sans doute, de noms de baptême doubles sous leur forme standard (Collin vs Jean-Nicolas) 
On ne s'étonnera pas dès lors de constater qu'en Wallonie, particulièrement dans les zones proprement wallonnes, ,entre Meuse et Rhin“, ce sont bien ces anciens noms personnels, ces noms de baptême, qui représentent la base de l'anthroponymie actuelle (voir Germain 1989). Et si, parmi les 100 noms de famille les plus fréquents en Wallonie, on note une très forte proportion d'anciens prénoms (63 sur $100 \mathrm{NF}$ ), sous leur forme originelle (48) ou sous une forme „hypocoristique“ (15), ces derniers sont bien présents comme Collard, Bastin, Gillet, Michaux, Collin, Poncelet, Bodart, Gilson, Massart, etc. C'est encore plus vrai dans la province de Luxembourg où les 20 premiers noms de famille les plus répandus sont tous d'anciens noms de baptême (Lambert, Gérard, Martin, Gillet, Thiry, etc.). Ces proportions ne sont pas nécessairement valables pour l'ensemble du corpus, même si les anciens noms individuels, notamment d'origine germanique, munis de suffixes simples (et, on, ot, otte, in, oul, art) ou complexes (eç-on, eç-oul, in-et, in-on, ot-et, on-et), en restent la base d'un point de vue statistique.

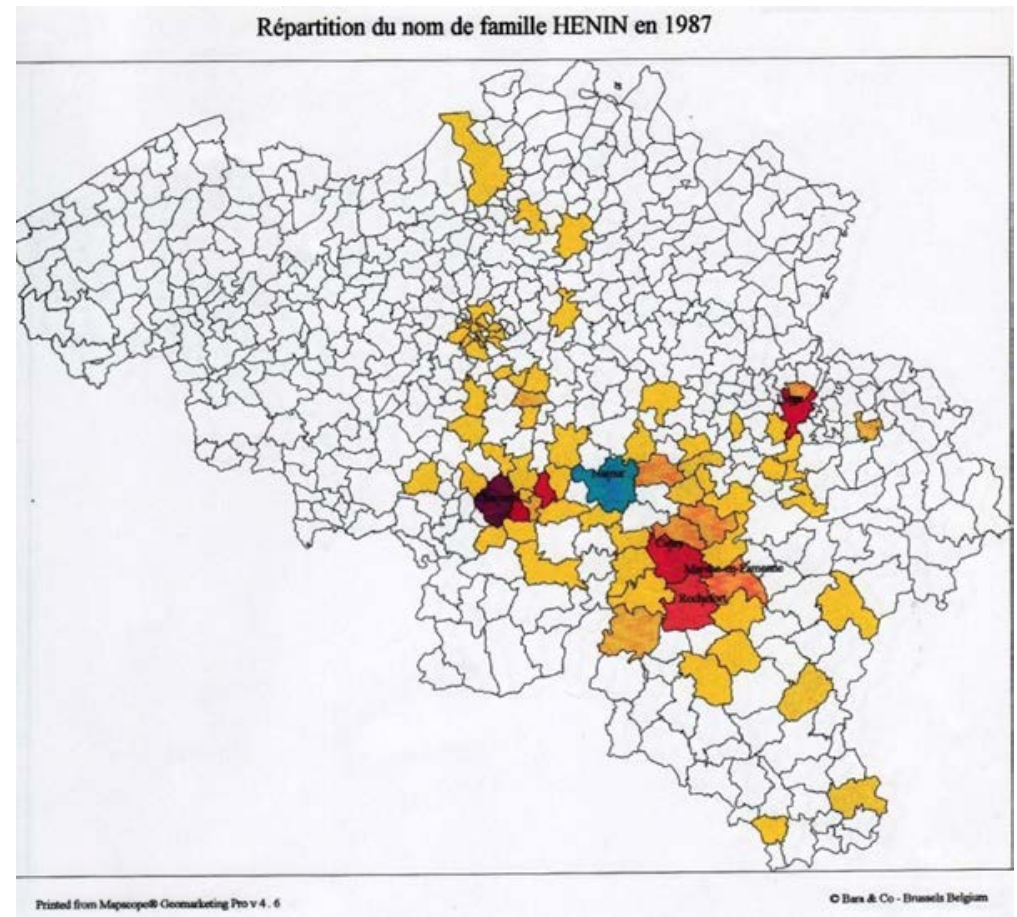

Fig. 3: Henin, un nom de famille célèbre héritier d'un hypocoristique de Je(h)an, est bien concentré au sud de la zone namuroise, aux confins du Condroz et de la Famenne.

\subsection{La suffixation hypocoristique: un morphème patronymique?}

On le sait, la plupart des langues disposent d'un morphème permettant d'exprimer la filiation patronymique. En se limitant aux frontières de l'Europe, on pense bien entendu aux diverses langues germaniques, scandinaves et slaves. C'est le cas aussi de l'espagnol avec son suffixe -ez (Fernández, Sanchez, González, Pérez) ou -iz (Hórtiz); pour l'italien, il y a toujours débat 
à propos des noms se terminant en $-i$ (Rossi, Bianchi, De Benedetti), certains y voyant un génitif, d'autres un pluriel. Tel n'est pas le cas du français qui ne dispose pas dans son arsenal morphologique d'un tel suffixe. Ceci n'est qu'une hypothèse, mais nous estimons que cette absence en français d'un suffixe patronymique spécifique ou d'une forme génitive exprimant l'idée de „fils de“ a eu des conséquences sur la création anthroponymique. Comme nous le verrons, certains textes permettent de saisir sur le vif le procédé de la dérivation: père et fils portent le même nom individuel, sans doute latinisé au moment du baptême, mais l'usage populaire les a distingués dans la vie quotidienne au moyen de dérivés, d'hypocoristiques.

Dans le cadre de cet article, nous profitons d'une belle série d'exemples wallons, particulièrement liégeois (Body 1879 et Renard 1952: 234-6), pour illustrer le propos et tâcher de comprendre le système ou une partie du système.

Pour rappel, la Wallonie liégeoise fait partie du domaine gallo-roman, dont elle représente la variante la plus septentrionale aux confins du domaine germanique. Cette série intéressante de dérivés anthroponymiques de la ville et de la région de Liège date principalement des XIII ${ }^{\circ}$ et XIV siècles, c'est-à-dire de la période de création anthroponymique. Il s'agit principalement de noms attestés dans des cartulaires, dans des ,échevinages“ ainsi que chez le chroniqueur liégeois Jacques de Hemricourt; pour plus de détails, voir Renard 1952: 230-231.

Nous nous attachons surtout à comprendre comment s'est opérée la distinction - dans l'usage oral et/ou écrit - entre père et fils au Moyen Âge lorsque ceux-ci portaient le même nom individuel, ce qui constituait une coutume très fréquente (voir aussi Herbillon 1952). Les dérivés ou hypocoristiques de toutes sortes ont bien sûr été mis à contribution... Mais de quelle manière?

Cette création d'hypocoristiques à valeur affective et/ou diminutive est illustrée par les quelques exemples liégeois ci-dessous:

- 1289 Thomas Massars

- 1311 Thomas dictus Masson

- 1314 Thomas dit Massotte

- 1345 Antonio dicto Tonard

- 1345 Lambertus dictus Lambot

- 1345 Nicolaï [génitif de Nicolaus] dicti Colegnon

- 1351 Wautier dit Wautoulle

- 1570 Denixhe dit Nisson

- s.d. Nicolas dit Colignon de Marchin

- s.d. Jacque dit Jamin

- s.d. Renard dit Rennekin de Strée.

Dans les exemples ci-dessous, il apparaît très nettement que l'hypocoristique représenté par la forme dérivée du nom (réduite parfois par aphérèse) remplit une fonction distinctive, permettant de distinguer aisément le fils du père:

- 1288 Renardon, filh Renar

- 1289 Rennechon, li fil Renard

- 1314 Wathelet fis Waltier 
- 1317 Gerardon ki fut fils ledit Gerar

- 1320 Hanes fils Johan

- 1321 Radelettus filius quondam Radulphi

- 1323 Rassequinus filius domini Rassonis

- 1325 Hanettus, filius quondam Petit Johan

- 1330 Thomasins fis adit Thomas de Hemericurt

- 1330 Bodechon filz Bauduin

- 1338 Henrotea fil Henri

- 1341 Sallemotte fil jadis Salemon

- 1345 Johannes dictus Hanicote, filius quondam Hanicar.

Est-ce toujours aussi simple? Non, puisque le système ne peut fonctionner que sur deux générations. La distinction entre père et fils portant le même nom individuel peut se faire aussi sous une autre forme hypocoristique, si le père est déjà connu par une forme dérivée du nom de baptême, soit avec une forme surdérivée, soit avec une substitution de suffixe. C'est le cas dans les exemples qui suivent:

- 1314 Anthonius dictus Tonette, filius quondam Tonardi

- 1314 Thomassinus filius Massoie d'Astenoir

- 1325 Thoneto, filio quondam Thonardi

- 1303 Mones li fis Monar

- 1325 Pirecote filz jadis Pirekin = ( ?) 1348 Pierekotte Pïerekin de Huy

- 1329 Gilotias filz Gilet

- 1314 Colins, fis maistre Colar

- 1349 Colchon fil Colinet

- 1341 Collegnon fil Colin (= Nicolas)

- 1303 Hankines fils Hankien.

Il peut arriver que le fils reprenne la forme simple du nom individuel, alors que pour le père et le grand-père on usait déjà de ce nom sous une forme hypocoristique; ainsi dans les exemples ci-dessous:

- 1317 Gerar, fil Girardin

- 1318 Bastianus filius quondam Bastoule

- 1338 Pietre, fils Pirot Pireal [= Pirot, fils de Pireal ?]

- 1343 Jehan fils Haneton

- 1348 Egidii Giloie

- 1349 Eustasse, fil Stasselot, fil jadis Willaume Stassar.

Ajoutons qu'un stade ultérieur est atteint lorsque le surnom lui-même est dérivé, comme c'est le cas dans ces exemples étonnants, particulièrement dans celui affectant le composé phrastique Boileau (= qui boit de l'eau):

- 1350 Boylottes, fil do dit Boyleawe

- Id. Boyloteau fils dedit Boyleawe 
- \pm 1400 Everar dit Burlurea [fils d'] Everar Burlur

- \pm 1400 Johan ly Polleray [fils de] Johan le Polen.

Ceci nous amène à conclure, certainement pour le nord du domaine d'oïl dont fait partie la Wallonie, que les formes dérivées ou hypocoristiques des noms individuels ont pallié implicitement, de façon structurelle, l'absence d'un suffixe patronymique. Cela mériterait certainement d'être approfondi pour le reste du domaine français et de la Romania, mais il conviendrait de disposer pour cela de séries fournies, analogues à celles de Liège.

\subsection{Un contrexemple: les génitifs latins}

Nous avons dit qu'il n'y a pas de marque morphologique pour le génitif marquant la filiation en domaine wallon. Une exception de taille est toutefois constituée par les génitifs latins. Ces anciens noms de baptême au génitif sont extrêmement fréquents dans les pays germaniques, tout particulièrement en Allemagne et au Grand-Duché de Luxembourg; comme il s'agit d'un phénomène transfrontalier, il n'est donc nullement étonnant de les trouver en Belgique dans la frange orientale qui avoisine ces deux pays. Étant savants par définition, ils échappent à la règle des deux syllabes; pas de contraction ni d'aphérèse ne sont observées. Il peut y avoir une similitude avec le génitif faible en -en et le génitif fort en $-s$ dans les domaines néerlandais et flamand voisins (voir le DFA III, p. 68 sv.)

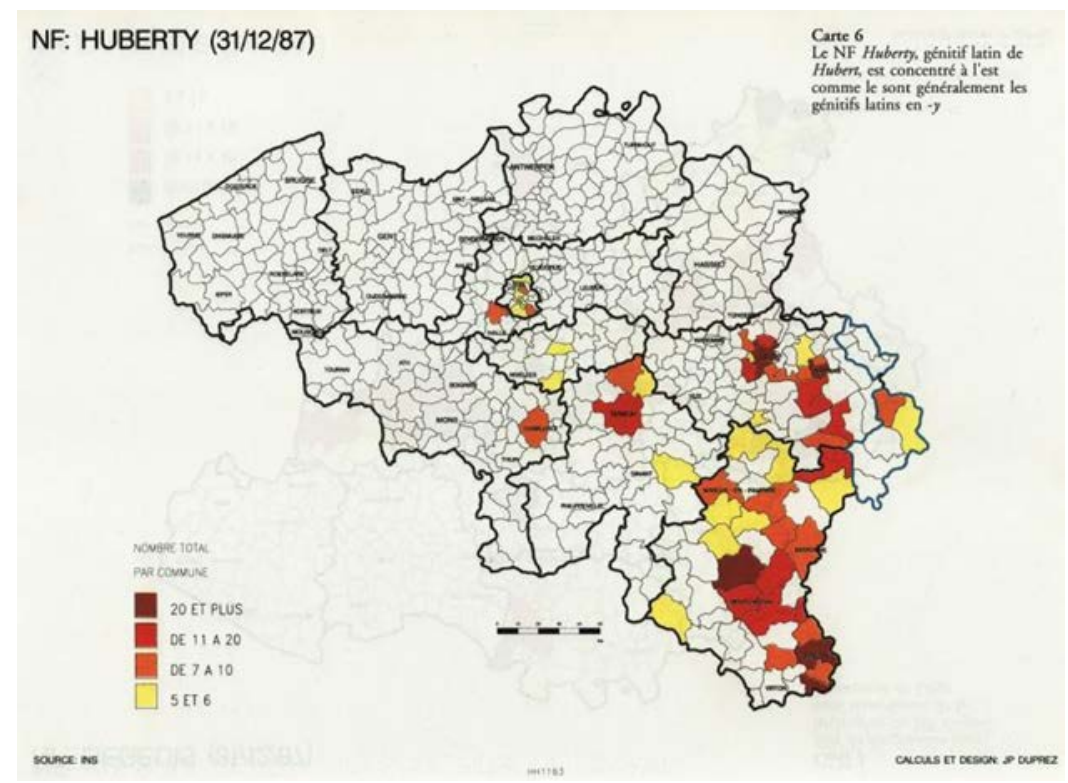

Fig. 4: La répartition géographique du nom de famille Huberty, le long de la frontière grand-ducale, est caractéristique des noms résultant de génitifs patronymiques.

Par ordre alphabétique, les génitifs latins plus fréquents sont: Alberty, Bernardy, Conrardy, Dominicy, Egedy (<Aegidius), Gérardy/Gerardy, Huberti/Huberty, Jacobi/Jacoby, 
Lamberti/Lamberty, Mathei, Michaelis/Michaëlis, Nicolay qui garde sa prononciation traditionnelle [aï], Pasquasy (< Pascasius), Pauli/Paulis, Petri/Pétry, Philippi, Quiriny, Remacly, Renaldi (de Renaud), Renardy, Roberti/Roberty, Servaty, Simonis, Stephany/ Stéphany, Urbany, Valentiny, Walthery/Walthéry, Winandy... Même si la plus grande partie de ces noms sont d'anciens prénoms, on rencontre également quelques noms de métier ou de fonction sous une forme génitive savante comme Fabry, Mercatoris, Militis, etc.

Généralement, c'est le $-y$ final qui marque cette forme génitive, mais le $-i$ fournit régulièrement une alternative. Il convient toutefois de ne pas confondre cette forme avec les noms équivalents d'origine italienne (Alberti, Bernardi, Damiani, Donati, Valentini, etc.); en cas de doute, la répartition aréologique du nom de famille est déterminante, selon qu'elle épouse le bassin industriel mosan (de Charleroi à Liège) ou qu'elle se confonde avec les régions orientales en périphérie du domaine germanique, voir Germain 2005.

\subsection{Les grandes heures de l'aphérèse}

Pour rappel, en linguistique, l'aphérèse se définit comme la disparition d'un ou de plusieurs sons à l'initiale d'un mot, tandis que l'apocope résulte de l'amuïssement d'un ou de plusieurs sons à la fin d'un mot.

Ce n'est un secret pour personne, la paresse - ou plutôt la loi du moindre effort - gouverne la plupart de nos actes. La langue n'échappe pas à la règle. Pour des raisons d'économie, l'homme est ainsi fait qu'il calcule inconsciemment ses efforts. Pourquoi faire usage de trois ou quatre syllabes, quand on peut n'en utiliser qu'une ou deux ?

Il en est de même aujourd'hui avec les mots savants de la langue française, qui ont été construits à partir des étymons latins ou grecs, que ce soit sous la forme de composés ou avec des suffixes standards. Bien vite, l'usager de la langue a simplifié de tels termes en les tronquant: automobile est devenu auto, vélocipède s'est réduit à vélo, télévision a hésité entre télé et $T V$ (tévé), etc. Les exemples abondent dans les créations récentes et les innovations technologiques: ordi pour ordinateur, $P C$ pour personal computer, bolo pour bolognaise, etc.

Il en va ainsi dans le langage des jeunes, qui recourent davantage encore à cette forme économique du langage, en usant et abusant de l'apocope. Les prénoms de jeunes se voient ainsi tronqués presque systématiquement de leur(s) dernière(s) syllabe(s): Nicolas s'appellera Nico, Sébastien sera Séba, Jean-Sébastien généralement Jean-Seb ou J.S., etc. L'apocope a donc succédé à l'aphérèse; elle l'a même complètement remplacée, peut-être sous l'influence de l'anglais.

Bien avant l'apocope, c'est toutefois l'aphérèse qui a connu de longs siècles d'existence et de prépondérance. Dès le Moyen Âge en effet, elle a résolu le problème de l'allongement des noms personnels (noms de baptême à l'époque), dont les formes populaires avaient été affectées de multiples suffixes, patronymiques ou non, comme nous l'avons vu précédemment. L'aphérèse a donc agi au moment où les hypocoristiques étaient encore des noms individuels, et non pas quand ils se fixent en tant que seconds noms, comme futurs noms de famille. En Wallonie, le processus de l'aphérèse a du reste persisté pratiquement jusqu'à la Seconde Guerre mondiale dans l'usage oral et populaire des prénoms dialectaux, avant d'être suppléé par l'apocope. 
Les exemples sont nombreux, les familles particulièrement représentées étant celles de Je(h)an, Jacques, Nicolas, Thomas, Antoine et Eustache/Istasse. En voici un bref inventaire partiel extrait de notre dictionnaire (Germain/Herbillon 2007):

- Alexandre $>$ Sandre (Delsandre), Sante, Sandron, Santkin, etc.

- Antoine (w. Antône) > Toinet, Thonet, Tonin, Tonard, T(h)onon (avec ou sans $h$ )...

- Denis > Nizet, Nisart, Nisette, Nison; Nihon, Nihart, etc.;

- Eustache (Istasse en ancien français de Wallonie) > Stasse, Stassart, Stassin, etc.;

- Innocent > Nocent, Nossain, Nossent, Nossin;

- Jacques (Jakemes en ancien français) $>$ Jacquemin $>$ Jacqueminet $>$ Minet;

- Jean, Jehan $>$ Jehanin $>$ Hanin; Hanart, Hano, -otte, etc.; > Jehenin $>$ Henin, etc.

- Nicolas $>$ Colet, Colot, Colard, Colaux, Colin (d'où Colinet, Coligneau, Colignon), Colasse, Colson, Colsoul, etc.;

- Sébastien > Bastin, Bastien;

- Thomas $>$ Thomasson > Masson (d'où Massonnet, devenu Sonnet), Massart, Massau(x), Masseau, Massot, Massotte, Massenet, Massenaux, Masset, Massillon, Massin (d'où Massinon et Massinet, devenu Sinet).

Comme l'indiquent ces différents exemples, nombre de noms de famille résultent du processus de l'aphérèse et, si l'on excepte l'initiale (ultime substrat du radical), ces noms ne consistent plus qu'en un double suffixe. Binet peut ainsi résulter de l'aphérèse de Lambinet (Lambert) ou de Robinet (Robert); Sonnet peut être un Gilsonet, un Massonnet ou un Rensonnet; Kinet/Quinet peut hésiter entre Gilkinet et Raskinet; Minet sera généralement un Jacqueminet; etc.

À cela s'ajoutent des formes contractées comme Jacquemin $>$ Jamin, Gérardot $>J a$ dot, Gérardin ou Girardin > Jadin, Jehennin > Genin, Perrotin (dérivé de Pierre) > Protain, Protin. Elles sont toujours guidées par le souci de limiter à deux syllabes la forme du nom. Parallèlement, Thomassin et Thomasson peuvent devenir Massin, Masson ou Thomsin, Thom(p)son. Quant à Philippot, il s'est mué en un acrobatique Phlypo...

Enfin, les noms composés de l'adjectif grand, petit, beau, etc. + le prénom Je(h)an qui représentait un quart de la population masculine sont particulièrement fréquents au sud du sillon Sambre-et-Meuse et en Ardenne; y foisonnent les NF (noms de famille) Beaujean, Bonjean, Maljean, Grandjean, Petitjean, Vieuxjean, Jeunejean, etc., sans oublier la forme redoublée Jeanjean. Il s'agit là de formations tardives $\left(\mathrm{XV}^{\mathrm{e}}-\mathrm{XVI}^{\mathrm{e}}\right.$ siècles), dans des régions où la fixation s'est faite plus tard que dans les régions tôt urbanisées. Bien que certaines formes présentent plus de deux syllabes, il est probable que dans la version orale en wallon ce n'était pas le cas: p'tit Djan, djon.ne Djan, etc.

\section{Du surnom au nom de famille: l'agglutination de l'article}

Un autre phénomène - distinct dans le temps et dans les noms affectés - marqué par la „loi des deux syllabes“ a affecté de façon très significative la forme et la structure des noms de famille, à savoir l'intégration ou non de l'article et/ou de la préposition au 
moment de leur fixation définitive (voir aussi la contribution suivante de notre collègue Ann Marynissen, Auf der Schnittstelle zwischen Germania und Romania: die Familiennamen in Belgien). Pourquoi s'appelle-t-on Legrand et Petit, et non Grand et Lepetit?

Pour l'étude de cette évolution déterminante dans l'histoire de nos noms, nous avons eu recours à un corpus historique de noms de personnes namurois, intéressant et homogène, en l'occurrence les noms des „,bourgeois“ enregistrés dans le registre de la bourgeoisie namuroise de 1491 à 1796, soit sur une longue durée de plus de trois siècles (voir Van Damme-Mairesse 1982 et Van Damme-Mairesse/Delvigne 1986). Il ne s'agit pas là d'un registre officiel, mais on est sans doute plus près de la réalité quotidienne des noms, puisque ce sont des bourgeois qui se sont déclarés eux-mêmes. Nous avons sélectionné différents types de surnoms au début du corpus avec l'article défini (qui datent de la fin du XV siècle): des noms de métier, des sobriquets (physiques ou moraux), ainsi que quelques noms ethniques; le test s'est prolongé avec des noms d'origine, précédés encore à cette époque de la préposition de.

Dans son traité d'anthroponymie française, Dauzat 1977: 303 évoque de façon assez générale le problème: „Pour cette série, on a vu que l'article était toujours employé dans les anciens textes: il ne s'agit donc pas d'une addition de l'article là où l'on trouve la soudure, mais d'une suppression, là où l'article est absent; soudure ou élimination sont connexes au passage du nom commun au nom propre“. Pour le reste, Albert Dauzat se limite à évoquer les différenciations régionales, sans parler de la date de cette agglutination, ni de la longueur respective des divers noms communs affectés par cette agglutination.

\subsection{La fixation des surnoms d'origine lexicale}

Dans les registres de bourgeoisie de Namur, les dix noms les plus courants de chaque catégorie ont été retenus, à savoir ceux qui avaient le meilleur rendement; ce n'est donc pas un critère neutre qui a été utilisé pour la sélection, afin de pouvoir assurer une validité statistique de base.

Ont été prises en compte les variantes graphiques mineures (le saige pour le sage, blancq pour blanc, etc.). Par contre, nous avons évité les noms commençant par une voyelle ou par un $h$ - parce que les notations sont moins assurées: dès 1491, on trouve toujours Lallemand, Lardinois, Lartillier, Lamedeu, Lescrinier, Lhost, presque toujours sans apostrophe, ce qui peut paraître suspect.

Une des hypothèses de départ est que l'accrétion de l'article ou de la préposition est fonction du nombre de syllabes du nom lui-même; nous avons dès lors constitué plusieurs séries de surnoms selon le nombre de syllabes que comporte le surnom lui-même (1, 2 ou 3$)$.

La période 1491-1796 a été répartie en 31 décennies (la dernière étant incomplète puisque les registres s'arrêtent en 1796). Pour chacun des noms ou surnoms, a été comptabilisé dans chacune des décennies le nombre de porteurs du nom enregistrés au moment de leur admission à la bourgeoisie, selon qu'ils sont désignés par la forme simple du nom (Brun), la forme avec article non agglutiné (le Brun ou Le Brun) ou la forme avec article agglutiné (Lebrun). N'ont pas été pris en compte les dérivés du nom simple, ce qui aurait pourtant été intéressant dans l'équilibre général du système, pour évaluer l'importance des deux syllabes. En effet, généralement, le surnom d'un brun donne comme noms de famille soit Lebrun soit Bruneau, Brunet, etc.; pour un blanc (de cheveux), ce sera Leblanc ou Blanchard, Blanchot, etc. 
Les différents types de surnoms délexicaux (ethniques, physiques ou moraux, noms de métier ou de dignité) ne présentent pas de différence significative dans le traitement. Par contre, le nombre de syllabes de chacun d'entre eux a une importance considérable.

Pour les surnoms déterminés par un article à l'origine, nous avons veillé à déterminer l'évolution dans le temps selon que le lexème servant de surnom est composé d'une, deux ou trois syllabes.

$1^{\circ}$ Surnoms monosyllabes

Surnoms à 1 syllabe

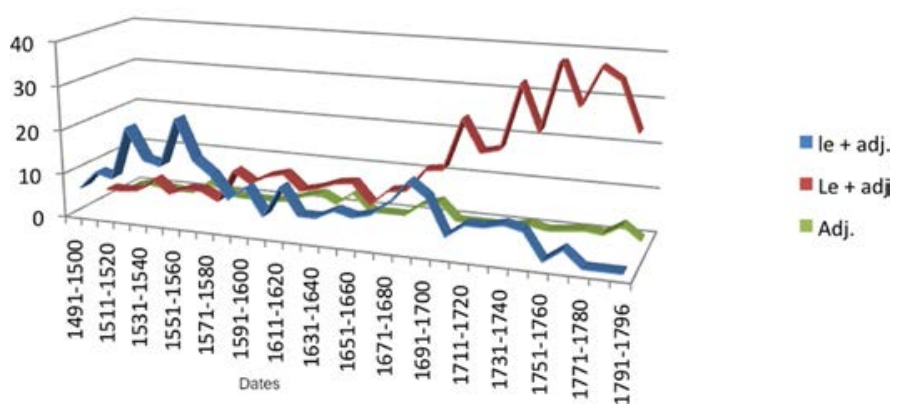

Fig. 5: $\quad$ Le surnom qui ne comporte qu'une seule syllabe bénéficie très rapidement de l'accrétion de l'article, type Legrand.

- L'accrétion de l'article devient rapidement la règle à laquelle n'échappent que peu de noms, uniquement des surnoms comme Coq qui présentent une finale consonantique forte, et en l'occurrence gutturale (Grand dans une moindre mesure);

- Ce phénomène d'accrétion s'amorce dès $1521 / 30$, l'accrétion est aussi courante que la non-accrétion entre $1571 / 80$ et 1691/1700 et s'impose pratiquement comme la règle absolue (à quelques exceptions près) vers 1700/10 et surtout 1751/60.

$2^{\circ}$ Surnoms à deux syllabes

Surnoms à 2 syllabes

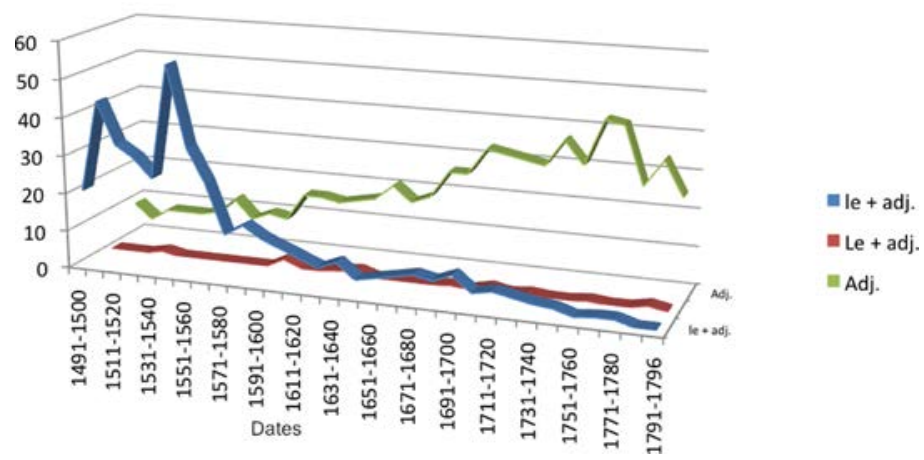

Fig. 6: $\quad$ Le surnom qui comporte deux syllabes perd assez vite l'accompagnement de l'article, type Petit. 
- L'accrétion de l'article (type Lepetit) n'atteint le score de $10 \%$ qu'une seule fois dans la décennie 1631/40 et disparait pratiquement dès 1711/20;

- La disparition de l'article, quant à elle, ne s'amorce vraiment (à quelques exceptions près) que vers $1561 / 780$ pour devenir la norme dès $1611 / 20$ - 1641/50 et atteindre plus de $90 \%$ des cas en 1721/30.

$3^{\circ}$ Surnoms à trois syllabes et plus

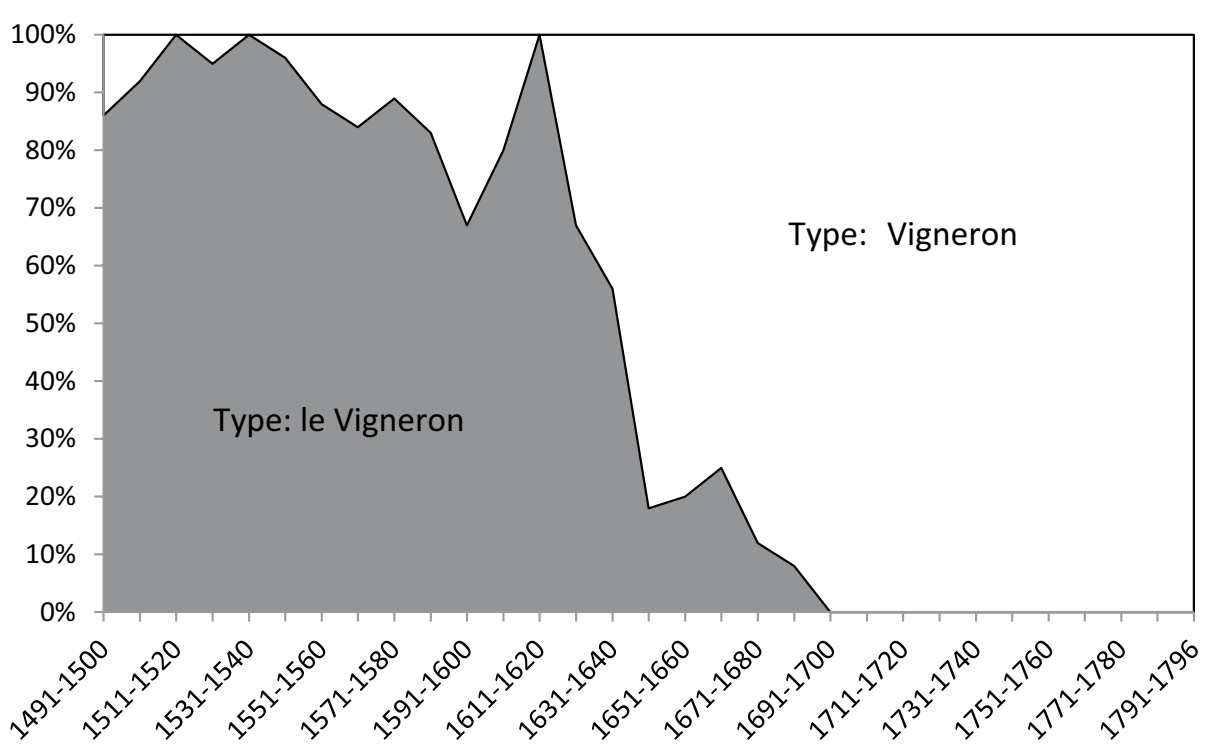

Fig. 7: $\quad$ Si le surnom comporte plus de deux syllabes, type Vigneron, l'article disparaît au plus tard à la fin du XVII siècle.

- Ce n'est qu'exceptionnellement qu'on trouve des cas de noms avec accrétion de l'article, du type Lechanteur;

- La disparition de l'article dépasse les $10 \%$ des cas dès 1551/60 (donc un peu plus tôt) pour devenir nettement prédominante dans la décennie 1641/50 et être définitive dès $1681 / 1690$.

On est frappé tout de suite par la similitude et le parallélisme chronologique des processus d'évolution des différents cas, ce que confirment partiellement les noms détoponymiques.

\subsection{Le sort de la préposition dans les noms d'origine toponymique}

Pour les noms d'origine introduits par la préposition de, la situation est en effet parallèle même si l'on constate un certain décalage. Il est du reste plus difficile de les appréhender car les noms à base de noms de lieu ou „détoponymiques“ se renouvellent plus rapidement et empêchent une comparaison significative, particulièrement les noms à une syllabe. 
Pour les NF détoponymiques à deux syllabes, le processus de disparition de la préposition de (type Velaine) s'amorce vraiment vers 1600 - après quelques essais en 1531/40 pour devenir prédominant à partir de 1621/30 et surtout depuis 1671/80.

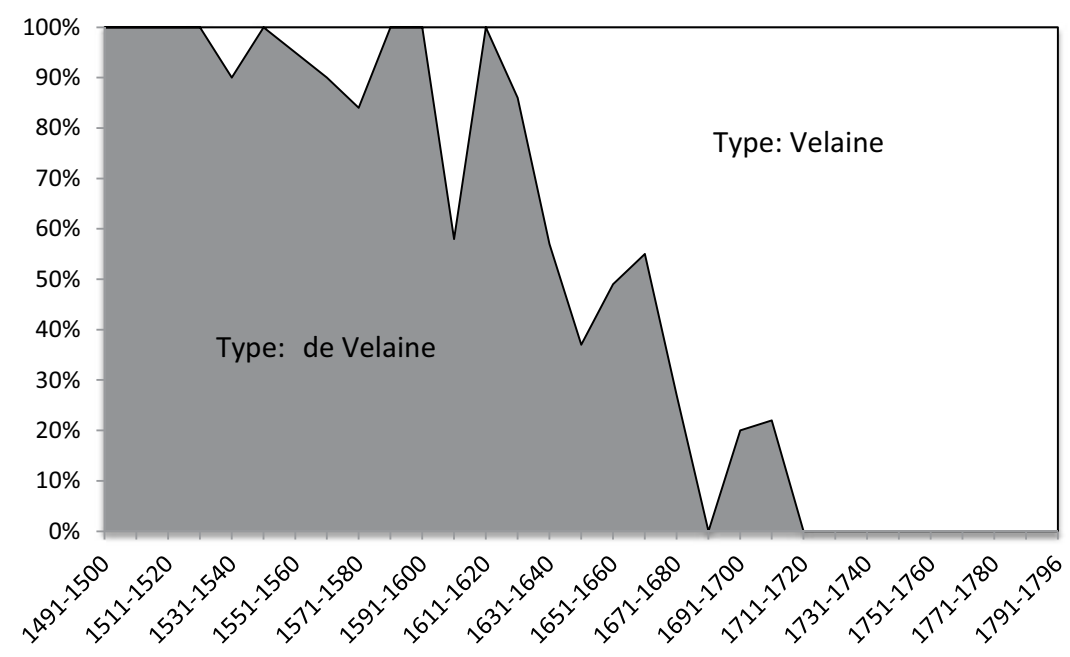

Fig. 8: $\quad$ Comme les surnoms, les noms d'origine toponymique comportant deux syllabes (type Velaine) ne requièrent pas l'accrétion de la préposition de.

Quant aux NF détoponymiques à trois syllabes, le même processus commence également vers 1600 , pour être à égalité vers $1641 / 50$ et dominant vers $1681 / 90$. Grande similitude donc avec les NF détoponymiques à deux syllabes.

On notera, soit dit en passant, que dans le domaine linguistique wallon, certains de ces noms détoponymiques peuvent avoir intégré la préposition sous sa forme dialectale wallonne $d i$ au lieu de $d e$. Quelques exemples significatifs parmi d'autres dans l'anthroponymie contemporaine: Dispa(s), Dispaux, Duspeaux $=$ de Spa; Dister $\sim$ Dester $=$ de Ster, nom de lieu très courant dans l'est de la Wallonie; Distèche $\sim$ Destexhe $=$ de Stexhe, hameau de Horion-Hozémont (arr. de Liège); Distate, Distatte $\sim$ Destate, Destatte $=$ de Statte, à Huy; Discry = de Scry, à Abée (arr. de Huy); Digneff, Dignef, Digneffe = de Jeneffe, w. Gn'gnèfe (arr. de Waremme ou de Dinant); Diskeuve $\sim$ Deskeuvre = de Skeuv(r)e, hameau de Natoye (arr. de Dinant); Distave = de Stave (arr. de Philippeville), etc.

Bien qu'il existe des exceptions comme Dissewiscourt, Disséwiscourt = de Séviscourt, hameau de Bras (arr. de Neufchâteau), les noms détoponymiques obéissent de manière générale à la loi des deux syllabes. Il est vrai que l'aphérèse n'est pas possible sans la défiguration du nom de lieu éponyme (qui possède déjà de toute façon trois syllabes). Il en va de même pour quelques noms qui servent de contre-exemples à notre démonstration, comme par exemple Dedessuslemoustier ou Doutrelepont.

Nous pouvons dès lors conclure qu'en Wallonie et dans le nord du domaine gallo-roman, les noms de famille ont de préférence une consistance d'une ou de deux syllabes; la longueur moyenne des mots en français doit du reste être similaire. Le système anthroponymique a structuré implicitement les NF pour arriver à ce nombre de syllabes. Il y a bien 
entendu de nombreuses exceptions, mais il s'agit certainement d'une tendance profonde et réelle que l'on peut dater ainsi:

- milieu du XVI ${ }^{\mathrm{e}}$ siècle pour l'amorce de l'évolution;

- $1^{\text {re }}$ moitié du XVII ${ }^{\mathrm{e}}$ siècle pour la phase d'équilibre;

- fin XVII ${ }^{\mathrm{e}}$ siècle / début XVIII ${ }^{\mathrm{e}}$ siècle pour la généralisation des divers processus.

Accrétion de l'article dans les SN à 1 syllabe

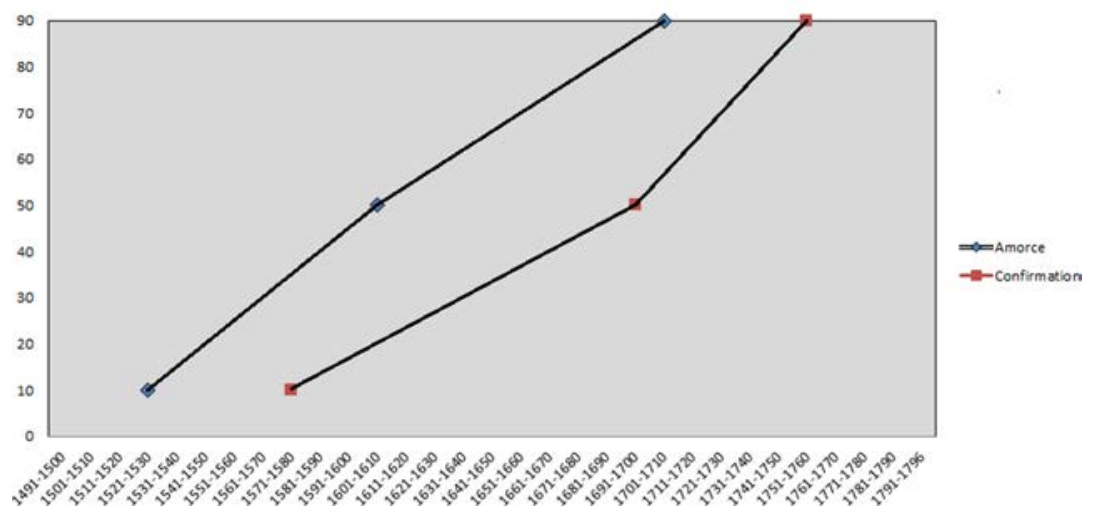

Fig. 9: L'accrétion de l'article, pour les surnoms à une seule syllabe, s'amorce dès le XVIe siècle et est terminée au début du XVIII siècle

Il va de soi que Namur n'est pas pour autant exemplatif pour l'ensemble du domaine d'oïl. Il faudrait des points de comparaison avec d'autres régions de ce vaste domaine linguistique, particulièrement avec la Normandie qui est la région où subsistent le plus grand nombre de noms de famille avec accrétion de l'article, comme le montre un graphique donnant le score respectif des NF Le Chevalier/Lechevalier dans les différentes zones. La Wallonie est très nettement la région où se vérifie notre hypothèse de départ de manière évidente.

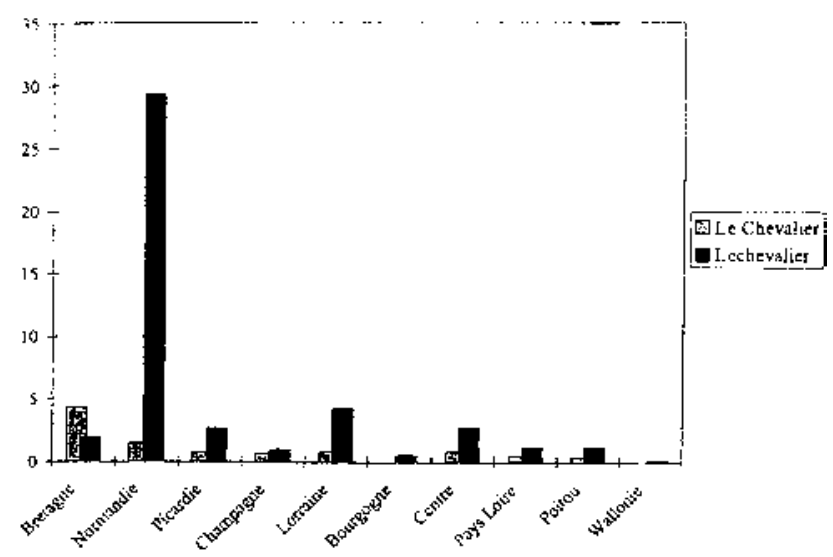

Fig. 10: $\quad$ À l'inverse de la Normandie, la Wallonie n'apprécie guère les noms de famille trop longs avec accrétion de l'article, type Lechevalier $\sim$ Chevalier. 


\section{Pour conclure}

La „loi des deux syllabes“, telle est la théorie que nous avons jugé bon de développer dans cet article consacré d'une part à l'aphérèse des dérivés hypocoristiques et d'autre part à la fixation des noms de famille en Wallonie. D'aucuns pourront trouver la démarche subjective, orientée, voire partiale. Que l'on ne se trompe pas, il ne s'agit pas d'une loi comme en proposent la physique, la chimie ou les mathématiques. C'est une loi qui supporte de nombreuses exceptions, mais qui reste une tendance lourde du domaine linguistique particulier que constitue la réalité onymique, située au cœur de l'humain et de l'expressif.

\section{Bibliographie}

Body, Albin: Étude sur les noms de famille du pays de Liège: origine, étymologie, classification. Bulletin de la Société liégeoise de littérature wallonne 17, 1879, 1-227.

Dauzat, Albert: Traité d'anthroponymie française. Les noms de famille de France. $3^{e}$ éd., Paris 1977.

Familienamen in België (site Internet): http://www.familienamen.be/

Germain, Jean: Les prénoms à Namur de 1491 à 1699. Bulletin de la Commission royale de Toponymie et Dialectologie 60, 1987, 23-81 = Les prénoms à Namur aux $\mathrm{XVI}^{\mathrm{e}}$ et XVII ${ }^{\mathrm{e}}$ siècles. Dans: Actes du XVI ${ }^{e}$ Congrès international des Sciences onomastiques (Québec, 1987). Québec 1990, $273-286$.

Germain, Jean: La fréquence des noms de famille contemporains en Belgique. Bulletin de la Commission royale de Toponymie et Dialectologie 62, 1989, 139-162.

Germain, Jean: Du surnom au nom de famille. La fixation formelle des noms de famille en domaine d'oïl à partir de l'exemple de Namur (Wallonie). Dans: Actas do XX Congreso internacional de Ciencias onomásticas (Santiago de Compostela, 20-25 setembro 1999). A Coruña 2002, $1409-1414$.

Germain, Jean: Des langues romanes inégales face à la productivité anthroponymique: l'expérience du projet PatRom. Dans: Brozović-Rončević, Dunja / Caffarelli, Enzo (eds), Denominando il mondo. Dal nome comune al nome proprio (Atti del simposio internazionale, Zara, 1-4 settembre 2004). Roma (QuadRIOn 1) 2005, 215-226.

Germain, Jean: Les noms de famille italiens en Belgique. Dans: Rivista italiana di onomastica 13, 2007, $503-530$.

Germain, Jean: Se dénommer autrefois, s'appeler aujourd'hui, en Gaume et ailleurs. Dans: Les Cahiers Brunehaut (Chiny), $\mathrm{n}^{\circ} 22,2011,19-22$.

Germain, Jean / Herbillon, Jules: Dictionnaire des noms de famille en Wallonie et à Bruxelles. $2^{\mathrm{e}}$ éd., Bruxelles 2007.

Hanton, Catherine / Schmidt, Béatrice: Dérivation lexicale et dérivation anthroponymique. Dans: Klein, Jean-René et al. (éds): Théorie linguistique et applications informatiques. Actes du $16^{\mathrm{e}}$ Colloque européen sur la grammaire et le lexique comparés (24-27 septembre 1997). Louvain-la-Neuve 1999, vol. 2 (= CILL 25, 1999, 12), 31-47.

Herbillon, Jules: Prénoms archaïques, Bulletin de la Société royale Le Vieux-Liège, t. 3 1946-1950, 123-124, 148-149, 163-165, 187-188, 201-203, 233-236, 249-253, 321-324, 385-389, 407-409, $462-463$.

Herbillon, Jules: Hypocoristiques masculins à suffixe féminin au Pays de Liège. Dans: Mélanges de philologie romane offerts à M. Karl Michaëlsson. Göteborg 1952, 256-263.

Mulon, Marianne: Les suffixes dans les noms de famille français. Dans: Origine et histoire des noms de famille. Essais d'anthroponymie. Paris 2002, 19-31.

PatRom (site Internet): http://patrom.fltr.ucl.ac.be/historique/ 
Pierret, Jean-Marie: Les noms de personne en Wallonie (Notes pour le cours ROM 2480). Louvain-laNeuve: UCL 2001.

Pierret, Jean-Marie: L'origine du nom de famille Massart. Petite récréation anthroponymique. Dans: Aventures et voyages au pays de la Romane. Pour Pierre Massart, Cortil-Wodon. 2002, 239-245.

Renard, Edgard: Notes d'anthroponymie liégeoise. Bulletin de la Commission royale de Toponymie et Dialectologie 26, 1952, 229-283.

Van Damme-Mairesse, Michèle: Répertoire des bourgeois de Namur 1516-1699. Bruxelles: AGR 1982.

Van Damme-Mairesse, Michèle/Delvigne, Guy: Répertoire des bourgeois de Namur 1700-1796.

Bruxelles: AGR 1986. 



\title{
Auf der Schnittstelle zwischen Germania und Romania: Die Familiennamen in Belgien
}

\author{
Ann Marynissen
}

\begin{abstract}
In diesem Beitrag werden die Bildung und Verbreitung der flämischen und wallonischen Familiennamen miteinander verglichen. Obwohl sie zu verschiedenen Sprachräumen gehören (germanisch bzw. romanisch), weisen die Familiennamen auf beiden Seiten der Sprachgrenze in Belgien sowohl in lexikalischer als auch in grammatikalischer Hinsicht beträchtliche Parallelen auf. In Flandern kommt der alte Kontrast zwischen dem fränkischen und dem ingwäonischen Gebiet zum Ausdruck. In Wallonien setzt sich das Wallonische im Osten vom Pikardischen im Westen ab.

Was die Produktivität der Namengebungsmotive betrifft, kontrastieren im Osten der beiden belgischen Landsteile die Rufnamen als frequenteste Kategorie mit den frequenteren Wohnstättennamen und Übernamen im Westen.

Auch das grammatische Erscheinungsbild der germanischen bzw. romanischen Familiennamen in Belgien ist geprägt von einem Ost-West-Gegensatz, der die Sprachgrenze überschreitet. Die germanischen und die romanischen Wohnstättennamen in Belgien sind nach ähnlichen Mustern gebildet. Die Genitivierung von Ruf- und Berufsnamen, im Germanischen entweder mit den Endungen -s oder -en, im Romanischen mit der lateinischen Endung $-i /-y$, markiert onomastisch ein großes rhein-maasländisches Areal, das sich über Belgien, die Niederlande und Deutschland erstreckt.

Auch die rheinische Velarisierung $n>n g$ hat in den rhein-maasländischen Familiennamen ihre Spuren hinterlassen.
\end{abstract}

\section{Einleitung}

Belgien liegt auf der Bruchlinie der germanischen und romanischen Kultur. Die quer durch Belgien verlaufende germanisch-romanische Sprachgrenze teilt das kleine Land in eine flämische und eine französische Sprachgemeinschaft und sorgt somit für linguistische Diversität. In der zweisprachigen Region der Hauptstadt Brüssel sind sowohl das Niederländische als auch das Französische die offiziellen Sprachen. Das Deutsche ist, als dritte offizielle Sprache Belgiens, in der Deutschsprachigen Gemeinschaft im Osten Belgiens anerkannt. Im Areler Land, das offiziell Französischsprachig ist, wird neben dem Französischen ein mit dem Luxemburgischen eng verwandter moselfränkischer Dialekt gesprochen. Die germanophone Gemeinschaft im Areler Land, das historisch zum deutschen moselfränkischen Sprachraum gehört, ist im Gegensatz zur Deutschsprachigen Gemeinschaft von Belgien nicht offiziell anerkannt.

In diesem Beitrag wird die Bedeutung der Sprachgrenze für die Bildung der Familiennamen aus dem germanischen Norden und dem romanischen Süden Belgiens bewertet. Zudem wird die sprach- und ggf. staatsgrenzüberschreitende Verbreitung einiger Namentypen, die typisch Rhein-Maasländisch sind, behandelt. 


\section{Die Produktivität der Namengebungsmotive}

Familiennamen haben eine historisch-etymologische Bedeutung, anhand welcher sie verschiedenen Benennungsmotiven zugeordnet werden. Hinsichtlich ihrer Namensgebungsmotive werden die Familiennamen traditionell in fünf lexikalische Motivgruppen eingeteilt (siehe Goossens 2011a für eine Taxonomie der niederländischen Familiennamen):

1. Familiennamen aus Rufnamen (Patronyme, Metronyme), z.B. Janssens, Peeters, Gérard, Léonard.

2. Berufs-, Amts- und Standesnamen, z.B. Smets, Lefèvre, De Koninck, Leroy.

3. Wohnstättennamen, z.B. Vandenbosch, Dubois.

4. Herkunftsnamen, z.B. Van Brussel, Halleux, Liégeois, Lallemand.

5. Übernamen, z.B. De Jong, Dewitte, Lejeune, Leblanc.

Die unterschiedlichen Namengebungsmotive sind nicht gleichmäßig über den Sprachraum verteilt: Es gibt erhebliche geographische Unterschiede bezüglich ihrer Produktivität, die im Folgenden erst anhand von Familiennamendaten aus dem Jahr 1987 (Belgien) bzw. 1993 (Niederlande) für den niederländischen Sprachraum und dann anhand von Daten aus dem Jahr 2007 für Belgien illustriert werden (siehe Anhang).

\subsection{Die Produktivität der Motive im niederländischen}

Sprachraum (Material: 1987 / 1993)

Die geographische Verteilung der fünf lexikalischen Motivgruppen im niederländischen Sprachraum wurde in einem früheren Aufsatz untersucht (siehe Marynissen 2010, 17-24). Methodisch wurde dabei wie folgt vorgegangen: Den 100 häufigsten Namen pro flämischer und niederländischer Provinz wurde das jeweilige Benennungsmotiv zugeordnet. Konnte ein Name auf zwei Motive zurückgeführt werden (z.B. Moors als Patronym oder als Übername), so wurden beide Motive je zur Hälfte gezählt, im Falle dreifacher Bedeutungskonkurrenz zu je einem Drittel. Anschließend wurde der prozentuale Anteil der fünf Motivgruppen an den 100 häufigsten Namen berechnet. Für Flandern wurde die Namensliste des belgischen Melderegisters von 1987 benutzt (Eintrag ab 5 Namensträgern pro Gemeinde), für die Niederlande stand mir die Liste mit den Namen der Telefonanschlüsse von 1993 zur Verfügung (Eintrag ab 2 Namensträgern pro Gemeinde).

Auf Karte 1 wird die frequenteste Motivgruppe pro Provinz abgebildet. Im niederländischen Sprachraum kommen erhebliche regionale Unterschiede zum Vorschein.

In Flandern ist die Bezeichnung der familiären Verwandtschaft das frequenteste Namensgebungsmotiv. In gleich vier der fünf flämischen Provinzen (Limburg, Antwerpen, Brabant und Ost-Flandern) bilden die Patronyme die größte Motivgruppe. Absolute Spitzenreiter sind die Provinzen Limburg (56\%) und Brabant (50\%), in denen (mehr als) die Hälfte der 100 frequentesten Namen auf den Vornamen des Vaters (ggf. auch der Mutter, insbes. in Limburg) zurückgehen. Auch die Provinz Antwerpen weist einen besonders großen Anteil an Rufnamen auf (43\%). In Ost-Flandern ist der patronymische Typus ebenfalls stark vertreten, jedoch ist er mit $32 \%$ weniger dominant als in der östlichen Hälfte Flanderns. Der Anteil an Rufnamen nimmt in Flandern von Ost nach West ab: In 
West-Flandern gewinnt eine andere Motivgruppe die Oberhand, nämlich jene der Wohnstättennamen $(35 \%)$.

In den Niederlanden ist das Bild um einiges variierter. Die südöstlichen Provinzen Limburg und Gelderland schließen sich dem ausgedehnten östlichen flämischen Gebiet mit einer starken Präsenz an Rufnamen an (Limburg: 56 \%, Gelderland, 35 \%). Auffallend ist, dass die niederländische Provinz Limburg eine ebenso hohe Prozentzahl an Rufnamen aufweist wie das belgische Limburg (56 \%): Dieses Ergebnis verdeutlicht die historische Einheit dieser Region innerhalb des niederländischen Sprachgebietes.

Nord-Brabant hat einen speziellen namenkundlichen Charakter: Es ist die einzige Provinz, in der Herkunftsnamen als das frequenteste Namensgebungsmotiv auftreten (30\%). Da diese Provinz dialektgeographisch deutlich in einen westlichen und einen östlichen Teil auseinanderfällt, kann vermutet werden, dass bei einer entsprechenden Zweiteilung der Provinz die Dominanz der Herkunftsnamen im Osten noch schärfer ausgeprägt wäre, während der Westen sich vermutlich in das größere niederländische Ganze einfügen würde (Goossens 2011b, 178).

In allen anderen niederländischen Provinzen führen entweder die Berufsnamen oder aber die Wohnstättennamen die Rangordnung an: Beide Motivgruppen erreichen überall Werte von mindestens $30 \%$. In Süd-Holland (33\%), Utrecht (34 \%), Flevoland (33\%), Overijssel (34\%) und Friesland (39\%) dominiert der Verweis auf die Wohnstätte des ersten Namensträgers als Benennungsmotiv; in Groningen (36\%), Drenthe (30\%), Nord-Holland (36\%) und Zeeland (30\%) ist ein Familienname am häufigsten durch die Referenz an den Beruf, das Amt oder den Stand des Namenträgers entstanden.

\subsection{Die Produktivität der Motivgruppen in Belgien (Material: 2007)}

Meine Untersuchung zur Häufigkeit der lexikalischen Motivgruppen im niederländischen Sprachraum habe ich für ganz Belgien anhand von neuerem Material - die Familiennamen des vollständigen belgischen Melderegisters vom 31.12.2007 - wiederholt. Die Liste des Melderegisters, die mir von der Generaldirektion Statistik und Wirtschaftsinformation Belgiens zur Verfügung gestellt wurde, enthält die Familiennamen aller Personen, die ihren Wohnsitz in Belgien haben, und die Frequenz jedes Namens pro Gemeinde. Das Material von 2007 wird der gegenwärtigen belgischen föderalen Staatsstruktur gerecht: Die ehemalige Provinz Brabant wurde in Flämisch- und Wallonisch-Brabant aufgeteilt, und die Familiennamen, die in der Region Brüssel-Hauptstadt vertreten sind, sind separat aufgelistet. Für jede belgische Provinz und für Brüssel wurden der prozentuale Anteil der fünf Motivgruppen an den 100 frequentesten Familiennamen berechnet und die am häufigsten vertretene Motivgruppe abgebildet (Karte 2).

\subsubsection{Rufnamen}

Im Vergleich zur Karte mit den Zahlen zum niederländischen Sprachraum von 1987 (Belgien) bzw. 1993 (Niederlande) hat der Anteil der Rufnamen an den 100 häufigsten Familiennamen in den flämischen Provinzen 20 Jahre später noch etwas zugenommen: von $56 \%$ auf $61 \%$ in Limburg, von $43 \%$ auf $45 \%$ in Antwerpen, von $32 \%$ auf $34 \%$ in Ost-Flandern. West-Flandern bleibt die einzige Provinz, in der ein anderes Motiv die 
Oberhand hat: Hier dominieren die Wohnstättennamen (32\%). Die Rufnamen schneiden in West-Flandern mit $23 \%$ an zweiter Stelle ab.

Die Dominanz der Rufnamen im Osten von Flandern setzt sich bis nach Wallonien fort. Der Anteil der Rufnamen bei den 100 frequentesten Namen liegt in Lüttich (mit $62 \%$ ), Namür (mit $63 \%$ ) und in Luxemburg (mit $66 \%$ ) sogar noch höher als in Limburg. Auch in Hennegau bildete die Namensgebung nach dem Vater das produktivste Motiv (41\%), jedoch nähert sich der Prozentanteil der Rufnamen mehr an den ostflämischen Prozentsatz (34 \%) an als an die Zahlen für die östlichen wallonischen Provinzen.

Die lexikalische Motivierung der germanischen bzw. romanischen Rufnamen in Belgien wird von einem Ost-West-Gegensatz geprägt. In Flandern zeichnet sich eine Grenze zwischen dem ingwäonischen und dem fränkischen Gebiet ab; in Wallonien zeigt sich eine Trennlinie zwischen dem pikardischen und dem wallonischen Gebiet.

\subsubsection{Wohnstättennamen und Übernamen}

Den Rufnamen folgen anteilsmäßig die Wohnstättennamen und die Berufsnamen. Erstere nehmen sowohl in Flandern als auch in Wallonien von Ost nach West zu (Karte 3).

Auch die Übernamen weisen einen Ost-West-Gegensatz auf: In den drei westlichsten belgischen Provinzen erreichen sie Werte von $14 \%$ (Hennegau), $15 \%$ (Ost-Flandern) bis $18 \%$ (West-Flandern). In allen anderen östlicher gelegenen Provinzen wird ein Anteil von $10 \%$ nicht überschritten (Karte 4 ).

\subsubsection{Berufsnamen}

Die Berufsnamen sind mit Prozentzahlen, die in allen Provinzen zwischen 12 und 22 liegen, geographisch ziemlich gleichmäßig über Belgien verteilt (Karte 5).

\subsubsection{Herkunftsnamen}

Die Kategorie der Herkunftsnamen ist am wenigsten vertreten: mit Ausnahme der Provinz Antwerpen ( $9 \%$ ), wo Herkunftsnamen häufiger sind als Übernamen, steht sie in der Rangordnung ganz unten. In Brüssel und Wallonisch-Brabant fehlen in der Liste der 100 häufigsten Namen die Herkunftsnamen völlig (Karte 6).

\subsection{Die Familiennamen in der Hauptstadt Brüssel}

Die zweisprachige Satzung der Hauptstadt Brüssel spiegelt sich in den Familiennamen wider.

Top-20 der häufigsten FN in Brüssel, 31.12.2007:

\begin{tabular}{|c|c|}
\hline Peeters & (ndl.) \\
\hline Janssens & (ndl.) \\
\hline Dubois & (fra.) \\
\hline Jacobs & (ndl.) \\
\hline Mertens & (ndl.) \\
\hline Nguyen & (ausl.) \\
\hline Diallo & (ausl.) \\
\hline
\end{tabular}




$\begin{array}{ll}\text { Maes } & \text { (ndl.) } \\ \text { Michiels } & \text { (ndl.) } \\ \text { Martin } & \text { (fra.) } \\ \text { Lambert } & \text { (ndl.) } \\ \text { Dupont } & \text { (fra.) } \\ \text { Simon } & \text { (fra.) } \\ \text { Dumont } & \text { (fra.) } \\ \text { Goossens } & \text { (ndl.) } \\ \text { Leroy } & \text { (fra.) } \\ \text { Willems } & \text { (ndl.) } \\ \text { Benali } & \text { (ausl.) } \\ \text { Laurent } & \text { (fra.) } \\ \text { Leclercq } & \text { (fra.) }\end{array}$

Von den 20 häufigsten Brüsseler Namen sind acht niederländisch (alle Patronyme), neun französisch (4 Patronyme, 3 Wohnstättennamen, 2 Berufsnamen) und drei sind ausländischer, nicht-europäischer Herkunft: Nguyen (vietnamesisch), Diallo (guineisch), Benali (arabisch). Die gleichen Verhältnisse trifft man bei den 100 frequentesten Brüsseler Namen aus dem Jahr 2007 an: Davon sind 45 französisch, 39 niederländisch und 16 sind ausländische, außereuropäische Namen.

Die Vielfalt der Namen spiegelt den multikulturellen Charakter der Hauptstadt Brüssel wider. In den letzten Jahrzehnten haben sich große Gruppen nicht-westlicher Migranten in Brüssel niedergelassen. Zum Vergleich: 1987 gab es noch keine ausländischen Familiennamen in der Top-100-Liste von Brüssel; die 100 häufigsten Familiennamen verteilten sich dafür jedoch ziemlich gleichmäßig über 53 flämische und 47 romanische Familiennamen (Herbillon / Germain 1996: 878).

Außerdem geben die Familiennamen die weitgehende Französierung von Brüssel wieder. Auf Grund der Brüsseler Namensliste könnte man vermuten, dass Brüssel derzeit eben so viel niederländischsprachige wie französischsprachige Einwohner zählt, jedoch ist dies überhaupt nicht der Fall. Nur eine kleine Minderheit der Brüsseler Bevölkerung (9,3 \%) ist in einer rein niederländischsprachigen Familie aufgewachsen, 51,5\% dagegen wurden ausschließlich auf Französisch erzogen (Janssens 2001, 34). Viele ursprünglich niederländischsprachige Brüsseler tragen zwar noch einen niederländischen Familiennamen - den sie gesetzlich nicht wechseln konnten -, aber haben im Laufe des 19. und 20. Jahrhunderts die niederländische Sprache gegen das Französische getauscht (cfr. Janssens 2001, 43).

\section{Die grammatikalische Bildung der Familiennamen}

Appellative und Rufnamen konnten auf verschiedene Weisen zu Eigennamen umgeformt werden. Zur grammatikalischen Bildung der Familiennamen gibt es beiderseits der germanisch-romanischen Sprachgrenze ähnliche Möglichkeiten.

1. Juxtaposition: Der Name wird in einer Beiordnung hinter den Vornamen gestellt, z.B. Dekker, Jong, Lambrecht, Put, Amsterdam; Marchal, Petit, Pierre, Fontaine. 
2. Voranstellung des Artikels, z.B. De(n) Dekker, De Jong, Den Uyl; Lejeune, Lafontaine.

3. Genitivierung mit der Genitivendung $-s,-i$ oder -en, z.B. Deckers, Jongen, Lambrechts, Puts; Petri, Fabry. In manchen Fällen wird im Niederländischen außerdem ein Genitivmorphem vorgefügt, z.B. Smeyers, Slangen.

4. Bildung von Derivationen bzw. Kompositionen durch Suffigierung bzw. Zusammensetzung, z.B. Gysseling, Bekaert, Haanstra; Willemsen, Broekmans, Aertgeerts, Langenberg; Collin, Collard, Pierret, Grandjean, Petiffrere.

5. Voranstellung einer Präposition, ggf. mit dem Artikel kombiniert, z.B. Van Acker, Vandeputte, Ter Horst, Aendekerk; Dumont, Deprez, Delafontaine, Delcroix, Desruelles.

Wie die Beispiele zeigen, treten die unterschiedlichen Bildungsmöglichkeiten in mehreren lexikalischen Motivgruppen auf (cfr. Goossens 1995).

Nicht nur die lexikalische Motivierung, sondern auch der morphosyntaktische Aufbau der Familiennamen weist geographische Unterschiede auf. Im Folgenden wird die Verteilung der grammatikalischen Namentypen bei den Rufnamen, den Berufs- und Übernamen und den Wohnstättennamen dargestellt, sowohl im niederländischen Sprachraum, zu dem Flandern gehört, als auch in Wallonien.

\subsection{Die Bildung der Rufnamen: Nominativ oder Genitiv}

\subsubsection{Der niederländische Sprachraum}

Karte 7 stellt die Verteilung der Patronymtypen im niederländischen Sprachraum dar (cfr. Marynissen / Nübling 2010, 331-337). Die Karte wird von Genitivnamen dominiert. Bei der großen Mehrheit der niederländischen Patronyme setzt sich der Rufname im Genitiv durch das Suffix -s fort: z.B. Peeters, Janssens, Willems, Jacobs, Hendrickx, Mertens. Bei Metronymen oder bei Patronymen, die aus schwach flektierten männlichen Rufnamen entstanden sind, tritt - vor allem in Limburg - die schwache Genitivendung -en hervor, z.B. Baeten, Heylen, Rutten, Vrancken.

Darüber hinaus gibt es im Zentrum des Sprachraums ein Gebiet, wo neben den Namen mit -s-Endung die volle Verbindung -sone ,Sohn' tradiert wird, im Niederländischen geschwächt zu -sen: Namenspaare wie Willems / Willemsen, Hendrickx / Hendriksen, Jacobs / Jacobsen treten hier als Varianten nebeneinander hervor.

Familiennamen aus juxtaponierten Patronymika beschränken sich in Flandern auf den Südwesten. Sie treten vorwiegend bei alten, zweisilbigen Rufnamen germanischer Herkunft auf, z.B. Lambrecht, Geeraert, Rombout. Auch im Westen von Flandern sind Genitivpatronyme wie Pieters, Willems, Martens, Roels usw. frequent vertreten.

\subsubsection{Die romanischen Patronyme in Belgien}

\subsubsection{Nominativ}

Die Rufnamen in Wallonien erscheinen prototypisch im Nominativ, siehe z.B. die Verbreitung des Namens Gérard in Belgien (Karte 8).

Die romanische Form des ursprünglich germanischen Namens Gerard ist in ganz Wallonien weit verbreitet. Bei Namen wie Simon, Michel, Guillaume, Louis, Lambert, 
Mathieu, Georges, Bernard, Bertrand und vielen anderen Patronymen sind ähnliche Verbreitungsbilder anzutreffen. Viele Wallonen haben einen Familiennamen, der formal identisch mit einem Vornamen ist, z.B. Louis Michel oder Michel Louis.

\subsubsection{Genitiv}

Nur bei den lateinischen Formen von christlichen Heiligennamen ist eine Genitivierung der Rufnamen möglich. Im Osten von Wallonien kommen neben den üblichen Nominativbildungen auch Varianten mit der Genitivendung $-i$ / -y vor, z.B. Hubert / Huberty, Jacob / Jacobi, Nicolas / Nicolay, Paul(us) / Pauly, Petrus / Petri, Robert / Roberti, Gérard / Gerardy, Bernard / Bernardy, Bartholomé / Barthélemy.

Der Umfang der Verbreitung der Namen auf $-i$ ist zwar pro Rufname verschieden, aber die lateinischen Genitivformen situieren sich in jedem Fall im Osten von Wallonien, in den Provinzen Lüttich, Luxemburg und Namür.

Auf Karte 9, mit der Verbreitung der Varianten von Jacob in Belgien, zeichnen sich drei Gebiete ab. Die niederländische Form mit dem $-s$-Genitiv (Jacobs) ist in ganz Flandern belegt, sie ist jedoch in der östlichen Hälfte Flanderns wesentlich frequenter als im Westen. Die niederländische Variante Jacobs ist außerdem im ganzen Norden von Wallonien ziemlich weit verbreitet. Hier wird die beträchtliche Migration von Flamen nach Wallonien im 19. und 20. Jahrhundert ersichtlich. In Wallonien ist der Nominativ Jacob die prototypische Form: Er wird in vielen wallonischen Gemeinden vorgefunden, aber bekommt im Osten von Wallonien Konkurrenz vom lateinischen -iGenitiv: Jacobi/ Jacoby, dessen Formen sich in den Provinzen Lüttich und Luxemburg konzentrieren. Der lateinische Nominativ Jacobus ist einige Male an der Nordseeküste anzutreffen. Die Genitive Huberty, Nicolay / Nicolai / Nicolaij haben eine ähnliche Verbreitung wie Jacobi/ Jacoby. Die Varianten von Bartholomy sind in Lüttich und im Süden von Luxemburg, aber auch in der Provinz Namür, gut vertreten.

Auf Karte 10, die die Morphemvarianten von Paul darstellt, konkurrieren die Nominativvarianten Paul und Paulus mit dem Genitiv Pauli / Pauly, der sich von der Provinz Lüttich bis zum Süd-Limburg erstreckt. Auch im Osten der Provinz Namür gibt es einige Träger des Namens Pauli / Pauly. Bei den Namen Petri / Petry, Roberti / Roberty und Gerardi / Gerardy liegt der Schwerpunkt der Genitivformen in der Provinz Lüttich; Sie erstrecken sich aber sowohl bis nach Süd-Limburg als auch bis in den Norden von Luxemburg.

Obwohl in Flandern und Wallonien ein unterschiedlicher grammatischer Typ vorherrscht, nämlich Genitiv- bzw. Nominativformen, stimmen die beiden Landesteile geographisch in dem Sinne überein, dass die seltenen niederländischen Nominativpatronyme, die nur in der westlichen Hälfte von Flandern bei alten zweistämmigen germanischen Namen wie Rombout, Geeraert, Lambrecht usw. vorkommen, sich in südlicher Richtung an die zahlreichen romanischen Nominativformen anschließen. Umgekehrt setzt sich die Verbreitung der seltenen romanischen Genitivpatronyme, die nur bei lateinischen Formen von christlichen Heiligennamen möglich sind und nur im Osten von Wallonien vorkommen, im östlichen flämischen Gebiet mit einer zahlenmäßigen Überlegenheit der $-s$-Genitivpatronyme fort. 
Die vielen französischen Rufnamen, die in Wallonien Familiennamen geworden sind, wie z.B. Georges, André, Mathieu, François, Etienne, Pierre, Jacques, Antoine usw. eignen sich nicht zur Genitivierung.

\subsubsection{Hypokoristika und Diminutiva}

Der wallonische Rufnamenschatz wird von einer Vielzahl an hypokoristischen und diminutivierten Patronymen ergänzt, z.B. Collard, Collin, Collignon, Collinet $(<\mathrm{Ni}-$ colas), Pierlot, Piron, Pirard, Pierson (Hypokoristika von Pierre) und Pirkin, Piret, Pierret, Pierrot (Diminutive von Pierre). Von dem französischen Rufnamen Jacques sind Hypokoristika wie Jacquard, Jacquemin, Jacquemard, Jacquemotte, Jacquerez, Jacquot und Diminutiva wie Jacquet und Jacquemet abgeleitet (Debrabandere 1993, 262-263; 968-969; 649). Die Verbreitung einige dieser Suffixe ist schon von wallonischen Namenkundlern kartiert und kommentiert worden (Herbillon / Germain 1996: 899-904). Derartige, mit hypokoristischen Suffixen oder Diminutivsuffixen gebildete Patronyme kommen zwar auch in Flandern vor, aber in viel beschränkterem Maße als in Wallonien. Einige Beispiele sind Wuytack, Wuytens, Ghyssels, Reyntiens, Drieskens.

\subsection{Die Bildung der Berufs- und Übernamen: Artikel oder Genitiv}

\subsubsection{Der niederländische Sprachraum}

Karte 11 stellt die Morphemstruktur der Berufsnamen im niederländischen Sprachraum dar. Es lässt sich eine globale Dreiteilung des Sprachraums erkennen. In der nördlichen Hälfte des Sprachraums herrscht der artikellose Nominativtyp vor, z.B. Dekker, Bakker, Kuiper, Visser, Mulder usw. Im Südwesten zeichnet sich ein Gebiet mit vorangestelltem Artikel ab, z.B. De Backer(e), De Cuyper(e), De Meyer(e), De Ridder(e), De Poorter(e). Der Artikel erscheint nördlich des de-Gebiets bei manchen Namen, konditioniert durch den Anlaut, im Akkusativ: z.B. Den Bakker, Den Dekker, Den Hartog, Den Ridder (siehe Marynissen 2005, 110-111). Im Südosten des Sprachraums stehen Berufsnamen in der Regel im Genitiv: z.B. Deckers, Cuypers, Beckers, Slegers, Winnen. Eine auffällige Subkategorie des Genitivtyps bilden Namen mit sowohl präfigiertem als auch suffigiertem Genitivmorphem wie Smulders ( $<$,des Müllers'), Smeyers $(<$,des Meiers'), Spaepen $(<$,des Papen'). Das anlautende $s$, das vor $m, p, w, l, g$ und vor Vokalen bis heute erhalten blieb, ist ein Rest des Genitivartikels, wie er auch in den niederländischen Zeitangaben 's avonds, 's ochtends konserviert ist (siehe auch Marynissen/Nübling 2010, 329-331).

Die niederländischen und flämischen Übernamen zeigen die gleichen Morphemtypen wie die mehrsilbigen Berufsnamen (Karte 12).

Auch die Verbreitung der Morphemtypen stimmt weitgehend mit der Verbreitung der mehrsilbigen Berufsnamen überein. Der einzige Unterschied zu den Berufsnamen ist, dass die einsilbigen Übernamen im Nominativareal in der Regel einen Artikel erhalten haben: z.B. De Lange, De Bruin, De Groot usw. Nur im Nordwesten, in der Randstad 
Holland, zeichnet sich ein Mischgebiet ab, in dem Übernamen mit und ohne Artikel alternieren: De Jong / Jong, De Rooij / Rooij (siehe auch Marynissen / Nübling 2010, 338-340).

\subsubsection{Die romanischen Berufs- und Übernamen in Belgien}

\subsubsection{Genitivierung}

Ebenso wie bei den Rufnamen eignen sich nur lateinische Formen von romanischen Berufsnamen zur Genitivierung mit $-i /-y$. Das einzige Beispiel für einen lateinischen Berufsnamen mit einer guten Verbreitung ist der Name Faber und seine Varianten (Karte 13).

Der Typ mit dem französischen Artikel Le (Lefè(b)vre) kommt in ganz Wallonien und in der westlichen Hälfte von Flandern, dem Gebiet mit vorangestelltem Artikel, vor. In West-Flandern erscheint der Artikel auch in niederländischer Form: Defever, Defevere. Die Genitivform Fabri / Fabry und ihre Varianten kontrastieren im Osten von Wallonien mit den Le-Formen: In den Provinzen Lüttich, Wallonisch-Brabant und Namür gibt es eine erhebliche Dichte des Namens Fabri. Ebenso wie bei den genitivierten Rufnamen Pauli, Jacobi und anderen erstreckt sich der Genitiv bis zum Norden von Luxemburg und bis nach Limburg. In dem pikardischen Teil von Wallonien fehlen die Genitivformen erwartungsgemäß. Der Ost-West-Gegensatz in Flandern zwischen Nominativformen mit vorangestelltem De im Westen (z.B. De Smet) und Genitivformen auf $-s$ im Osten (z.B. Smets) setzt sich in Wallonien fort, mit dem Unterschied, dass die Formen mit dem französischen Artikel Le in ganz Wallonien vertreten sind.

\subsubsection{Mit oder ohne Artikel}

Bei den Berufsnamen und Übernamen, die auf ein französisches Etymon zurückgehen und dementsprechend nicht genitiviert werden können, ist die Silbenstruktur ausschlaggebend für die An- oder Abwesenheit eines Artikels. Einsilbigen Namen wie Lemaire, Lecomte, Leroy; Lejeune, Lebrun, Legrand, Lemoine, Legros, Leblanc, Lenoir usw. wird in der Regel ein Artikel vorangestellt. Bei mehrsilbigen Berufs- und Übernamen ist das in der Regel nicht der Fall, z.B. Mar(é)chal, Meunier, C(h)arlier, Parmentier, Brasseur, Barbier, Boulanger; Petit, Bruneau, Rousseau, Rosset, Noiret, Joly.

Familiennamen, die mit den Antonymen grand und petit gebildet wurden, sind in fast allen wallonischen Gemeinden präsent (Karte 14).

In grammatischer Hinsicht unterscheiden sich die großen Leute aber von den Kleinen. Bei dem einsilbigen Etymon grand ist so gut wie immer der Artikel Le vorangestellt worden, also Legrand, parallel zum niederländischen De Groot. Dahingegen fehlt bei den Familiennamen Petit der Artikel völlig. In West-Flandern erscheint der sprachlich hybride Name Degrand(e), mit niederlandisiertem Artikel de, ähnlich wie die ebenfalls westflämischen Berufsnamen Defever / Defevere.

Die Karte mit den Varianten von marchal und comte illustriert romanische Berufsnamen, die mit oder ohne Artikel gebildet werden können (Karte 15). 
Dem einsilbigen Etymon comte wird fast immer der Artikel Le vorangestellt, parallel zu Niederländisch De Graaf; beim zweisilbigen Etymon marchal fehlt dagegen der Artikel fast immer.

\subsection{Die Bildung der Wohnstättennamen beiderseits der Sprachgrenze}

In der Kategorie der Wohnstättennamen findet man an beiden Seiten der romanischgermanischen Sprachgrenze eine große Menge von Namen, die sowohl in lexikalischer als auch in grammatischer Hinsicht auf analoge Weise gebildet wurden. Beispiele für flämische und wallonische Familiennamen mit demselben Grundwort und denselben grammatischen Elementen sind Vandenbosch / Dubois, Verbrugge / Dupont, Duprez / Vandermeersch, Verplaetse / Delplace, Verstraete / Delarue, Vandercruyssen / Delacroix, Vandecapelle / Delachapelle und viele andere.

\subsubsection{Präposition}

Bei Wohnstättennamen und Herkunftsnamen, die entweder den Wohnsitz oder die Herkunft des Namensträgers zum Ausdruck bringen, ist der Lokativ der prototypische Kasus, z.B. Vande(n)berg(h)(e)(n) / Dumont, Van Aken, De Roubaix. Sowohl in Flandern als auch in Wallonien werden Wohnstättennamen in der Regel mit vorangestellter Präposition und einem Artikel geformt. Die gegenseitigen Entsprechungen Vandenberghe bzw. Dumont kommen in fast ganz Flandern bzw. Wallonien vor, auch in flachen Gegenden (Karte 16).

\subsubsection{Mit oder ohne Suffix / Endung}

Weniger frequent, aber ebenso in den beiden belgischen Landesteilen vertreten, sind juxtaponierte Wohnstättennamen, z.B. Fontaine (in Flandern Fonteyne), Ruelle; Berg, Put, Beke. Im Niederländischen können Wohnstättennamen darüber hinaus im östlichen Genitivgebiet eine Genitivendung bekommen, z.B. Berg(h)s, Puts, Beeckx, eine Möglichkeit, die es im Französischen nicht gibt. Suffigierung ist in beiden Sprachgruppen möglich, z.B. Bergman(s), Broekaert; Bosquet, Croiset.

\subsubsection{Mit oder ohne Artikel}

Wohnstättennamen mit vorangestelltem Artikel ohne Präposition sind nur im Romanischen bekannt, z.B. Laruelle, Lacroix, Lafontaine. Eine Auswertung der vielfältigen Formen des Artikels und der Präposition in den wallonischen Familiennamen findet man in Herbillon / Germain 1996, 37-40. Als Beispiel für die mögliche Variation gelten die Varianten von Ruelle (Karte 17). Ruelle, Laruelle und Delaruelle sind die wichtigsten Varianten; Nebenformen sind Deruelle, Druelle, Desruelles und Delruelle.

Die germanischen und die romanischen Wohnstättennamen in Belgien sind größtenteils nach ähnlichen Mustern gebildet worden. Ein Ost-West-Gegensatz lässt sich in dieser Kategorie in Wallonien nicht nachweisen, da sich die französischen Etyma dieser Namen nicht zur Genitivierung mit einer Endung eignen. In Flandern ist der Gegensatz zwischen östlichen Genitivformen und westlichen Nominativformen zwar vorhanden, aber schwerer sichtbar als bei den Ruf-, Berufs- und Übernamen, da der Lokativtyp in dieser Kategorie allgemein vorherrscht, sowohl im Norden als auch im Süden von Belgien. 


\section{Staats- und Sprachgrenzen überschreitende rhein-maasländische Merkmale}

\subsection{Die Genitivierung mit -s, -en oder - $\mathrm{i}$ / -y}

Die Genitivierung von Familiennamen überschreitet sowohl die romanisch-germanische Sprachgrenze als auch die Staatsgrenzen zwischen Belgien, den Niederlanden und Deutschland. In einem großen rhein-maasländischen Areal haben zahlreiche Namen eine Genitivendung bekommen: entweder eine der germanischen Endungen $-s$ oder -en oder die lateinische Endung $-i /-y$.

\subsubsection{Die starke Genitivendung -s}

Das starke Genitiv-s tritt im Südosten des niederländischen Sprachgebiets in allen Namenkategorien hervor, z.B. Peeters, Mertens; Cuypers, Custers; Molemans, Puts; Sterckx, Donckers; Vugts.

In Deutschland sind mit starkem Genitiv gebildete Patronyme (z.B. Derks, Peters, Wolters, Melchers) und Berufsnamen (z.B. Küppers, Kösters, Deckers, Beckers) vornehmlich im Westen des Bundeslandes anzutreffen. Patronyme im starken Genitiv dominieren im Nordwesten Deutschlands bis zum Emsland, während südlich davon, im Münsterland, am Niederrhein und im Rheinland bis zur Mosel, vor allem entsprechende Berufsnamen überwiegen (siehe Karte 6 in Heuser / Nübling 2010, 45).

In Belgien konzentrieren sich genitivierte Berufsnamen germanischer Herkunft wie Beckers und Deckers im Osten von Flandern, in Wallonien erstrecken sie sich bis zur angrenzenden Provinz Lüttich (Karte18).

Sie sind einerseits Zeugen der historischen Migration von Flamen in die wallonischen Industriegebiete ab dem 19. Jahrhundert, andererseits weisen sie auf die historischen Verbindungen zwischen der Grafschaft Loon und dem Fürstbistum Lüttich hin.

\subsubsection{Die schwache Genitivendung -en}

Die Genitivendung -en, mit der im Niederländischen vor allem Ruf- und Übernamen der schwachen Flexion (z.B. Rutten, Otten, Heynen; Haenen) und Übernamen aus substantivierten Adjektiven (z.B. Jongen, Langen, Kleinen, Grooten) flektiert werden, erreichen im niederländischen Sprachgebiet ihre höchste Frequenz in den beiden Provinzen Limburg (siehe Marynissen 2010, 27-29, Karten 10-11).

Dieser Namentyp setzt sich im deutschen Rheinland fort: Die stärkste Verdichtung zeigen die mit schwachem Genitiv gebildeten Familiennamen des Typs Rufname + -en (z.B. Otten) am Niederrhein im Raum Heinsberg, Erkelenz und Viersen. Ebenso ballen sich Berufsnamen mit schwachem Genitiv am Niederrhein, z.B. Scholten (Heuser / Nübling 2010, Karten 8 und 9, 47-48).

Auch in Wallonien ist bei bestimmten Familiennamen, die auf ein germanisches Etymon zurückgehen, die schwache Genitivendung vertreten.

Auf Karte 19 wird die Verbreitung der Patronyme Otten und Heinen / Heynen und der Übernamen Jongen und Ha(e)nen dargestellt. Ebenso wie bei den Berufsnamen auf $-s$ setzen sich diese Namen von Limburg in der Provinz Lüttich fort. Der germanische 
Rufname Heinen ist außerdem in den zwei germanophonen Gebieten Walloniens, den Ostkantonen und in der Gegend von Arel, verbreitet.

\subsubsection{Die lateinische Genitivendung -i / -y}

Genitivierung mit der lateinischen Endung $-i /-y$ ergibt sich nur bei lateinischen Formen von Berufsnamen und christlichen Heiligennamen als Möglichkeit: z.B. Fabri, Huberty, Jacobi, Nicolay, Pauly, Petri, Roberti, Gerardy, Bernardy, Barthélemy, Corneli, Philippy, Conradi. Die Verbreitung der einzelnen Genitivnamen auf $-i$ in Belgien wurde unter 3.1.2 und 3.1.3. beschrieben. Lateinische Genitive kommen in Lüttich, Luxemburg und Namür, den drei östlichen Provinzen Walloniens, vor. Manche Namen erstrecken sich außerdem bis in den Süden von Limburg. Erwartungsgemäß ist dieser Namentyp auch im mittleren Westen von Deutschland und im Großherzogtum Luxemburg vertreten.

\subsection{Die rheinische Velarisierung $\mathrm{n}>\mathrm{ng}$}

Die rheinische Velarisierung $n>n g$, die vor allem im Ripuarischen und in den angrenzenden Gebieten verbreitet ist (Besch / Knoop 1983, 1132), hat im Rhein-Maas-Gebiet bei den Familiennamen ihre Spuren hinterlassen.

Die Widerspiegelung dieses Dialektmerkmals in deutschen Familiennamen ist vor kurzem im Band Konsonantismus des Deutschen Familiennamenatlas dokumentiert worden (Kunze / Nübling 2011, 786-797). Karte 354 stellt die Verbreitung der velarisierten Varianten Frings, Krings, Brings und Rings dar. Frings und Brings sind velarisierte Formen der patronymischen Kurzformen Fri(e)ns, Bri(e)ns, die auf den lateinischen Rufnamen Severinus zurückgehen. Krings, aus den Kurzform Kri(e)ns, geht auf den Rufnamen Quirinus zurück. Rings, aus Ri(e)ns, kann von Severinus oder Quirinus abgeleitet sein. Die vier kartierten Familiennamen mit velarem Nasal im Auslaut konzentrieren sich im ripuarischen Gebiet, das von Theodor Frings als Kernzone dieses Phänomens betrachtet wird (Besch / Knoop 1983, 1132). Belege für diese Velarisierung reichen ins 16. Jahrhundert zurück: in Köln Frings ,Severinus' und Krings ,Quirinus (Besch / Knoop 1983, 1133).

Diese Velarisierung überschreitet die Staatsgrenzen: Velarisierte Kurzformen von diesen lateinischen Heiligennamen sind gleichfalls im Südosten der niederländischen Provinz Limburg, im Osten der belgischen Provinz Lüttich und im nördlichen Teil der Deutschsprachigen Gemeinschaft Belgiens, die allesamt zum ripuarischen Dialektgebiet gehören, zu finden.

Die Karte mit der Verbreitung aller Voll- und Kurzvarianten von Severinus (Karte 20) ortet den Namen Frings im Süden von Niederländisch-Limburg, wo er mit den Varianten der Kurzformen Frijns / Fryns und den Varianten der Vollformen Severeyns / Severijns / Severens konkurriert.

Außerhalb des ripuarischen Gebiets haben sich nur die Vollformen als Familiennamen durchsetzen können. Die vollen Varianten mit der starken Genitivendung $-s$ sind in Belgisch-Limburg und Antwerpen verbreitet, die Varianten Sever(e)ijnen, mit der schwachen Genitiv -en, sind in Nord-Brabant anzutreffen. 
Die Verteilung der Varianten des lateinischen Namens Quirinus unterscheidet sich in einigen Aspekten von den Severinus-Formen (Karte 21).

Die velarisierte Kurzform Krings erstreckt sich diesmal vom Südosten der belgischen Provinz Limburg bis in den Osten von Lüttich. Die Kurzformen Krijns, Krijnen und Varianten sind zahlreicher vertreten als die Vollformen Quirijns und Quirijnen, die sich im Großen und Ganzen auf dem Nordwesten der Provinz Antwerpen beschränken. Die Kurzformen mit schwacher Genitivendung (Crijnen, Krijnen, Crynen, Krynen) haben sich von ihrem Ursprungsgebiet Nord-Brabant bis in die Randstad Holland verbreitet.

Der aufgrund der rheinischen Velarisierung entstandene Konsonant -ng ist auch in Wohnstättennamen mit proklitischer Präposition in oder an + Artikel belegt: An den $>$ Angen, an der $>$ Anger, In den > Ingen, In der > Inger. Namen wie Angenendt und Ingenhaag sind nur am deutschen Niederrhein vertreten (siehe Karten 3-4 in Heuser / Nübling 2010, 41-43 und Karte 357 in Kunze / Nübling 2011, 791). Im Niederländischen sind die entsprechenden Wohnstättennamen Aangeveld, Angenent, Ingenbleek, Ingenhut, Ingenhoven, Ingendael, Ingenpas, Ingebos und ihre Varianten in einem südöstlichen Streifen vom Achterhoek bis zum Süden Limburgs beheimatet (siehe Marynissen 2010, Karte 14, 32-33).

Im belgischen Familiennameninventar gibt es eine weitere Reihe von Familiennamen, die in ihrer Orthographie Spuren einer velarisierten Aussprache aufweisen, aber von den germanischen Familiennamen mit rheinischer Velarisierung zu unterscheiden sind. Hierbei handelt es sich um ursprünglich romanische Familiennamen, die mit Suffixen mit einem nasalierten Vokal wie -in, -on, -an, gebildet sind und bei denen der Nasalkonsonant des Auslauts mit $n g$ geschrieben wird. Sie weisen auf Velarisierung dieser romanischen Elemente bei Entlehnung ins Germanische hin, cfr. Limburgisch persóng (prison), sjampeljóng (champignon), charlatang (charlatan) (siehe Belemans 2001, 256-258).

Beispiele für Familiennamen mit -ng sind: Colling $<$ Collin, Bastings $<$ Bastin, Goffings $<$ Goffin, Rasking $<$ Raskin, Verding $(h)<$ Verdin, Tossings $<$ Toussaint, Marting $<$ Martin, Allarding $<$ Allardin, Lordong $<$ Lourdon, Dussong $<$ Duson, Cottong $<$ Cotton, Bertrang $<$ Bertrand, Flammang $<$ Flammand, Bemong $<$ Beaumont. Viele dieser Familiennamen kommen in Süd-Limburg (Goffings, Rasking, Verding(h), Bastings, Bemong) und / oder der Gegend von Lüttich (Collings, Bastings, Tossings) vor. Andere sind nur im germanophonen Teil der Provinz Luxemburg (Gegend von Arel) verbreitet: Allarding, Lordong, Cottong.

\section{Schlussfolgerung}

Die germanisch-romanische Sprachgrenze in Belgien bildet für die Familiennamen insofern eine Bruchlinie, als die Familiennamen in Flandern hauptsächlich mit niederländischen Appellativen, die wallonischen Familiennamen aber hauptsächlich mit französischen Appellativen gebildet worden sind.

Die Bildungsweise der Familiennamen selbst weist dagegen in beiden belgischen Landsteilen beträchtliche Parallelen auf. Die Ost-West-Gegensätze, die die flämischen 
Namen kennzeichnen, setzen sich in Wallonien fort. Sie überschreiten die Sprachgrenze. In Flandern kommt der alte Kontrast zwischen dem fränkischen und dem ingwäonischen Gebiet zum Ausdruck. In Wallonien setzt sich das Wallonische im Osten vom Pikardischen im Westen ab.

Im lexikalischen Bereich kontrastieren auf beiden Seiten der Sprachgrenze mit einer jeweils zahlenmäßigen Überlegenheit die Rufnamen im Osten zu den Wohnstättennamen und Übernamen im Westen.

In grammatischer Hinsicht setzt sich sowohl in Flandern als auch in Wallonien ein westliches Gebiet mit einer überwiegenden Anzahl von Nominativnamen - entweder juxtaponiert oder mit vorangestelltem Artikel - vom östlichen Areal mit einer Dominanz von Genitivnamen ab. Dieser grammatische Ost-West-Gegensatz ist in Wallonien weniger ausgeprägt als in Flandern, da sich nur ein beschränkter Teil des wallonischen Namenpotentials zur Genitivierung eignet. Im Osten von Wallonien konkurrieren die vorhandenen Genitivformen mit Nominativbildungen.

Das grammatische Merkmal der Genitivierung überschreitet nicht nur die germanischromanische Sprachgrenze in Belgien, sondern auch die Staatsgrenzen zwischen Belgien, den Niederlanden und Deutschland. In einem großen rhein-maasländischen Areal haben verschiedene Familiennamen entweder eine germanische Genitivendung (-s/-en) oder eine lateinische Genitivendung $(-i /-y)$ bekommen.

Auch die rheinische Velarisierung $n>n g$, ein Dialektmerkmal, das vor allem im Ripuarischen und in den angrenzenden Gebieten vertreten ist, überschreitet die Staatsgrenzen: Sie ist sowohl in niederländischen, belgischen, als auch in deutschen Familiennamen, die im rhein-maasländischem Gebiet beheimatet sind, zu finden.

\section{Literatur}

Belemans, R.: Hoe schrijft Jang van Zjier van Zjef de Smeed zich? Over Limburgers die niet naar Jan, Pier of Klaas genoemd zijn. In: De Tier, V. / Marynissen, A. m.m.v. Brok, H.: (red.) Het dialectenboek 6. Van de streek. De weerspiegeling van dialecten in familienamen, Groesbeek: Stichting Nederlandse Dialecten, 2001, 253-271.

Besch, W. / Knoop, U.: Dialektologie: Ein Handbuch zur deutschen und allgemeinen Dialektforschung, Band II, Berlin: De Gruyter, 1983.

Debrabandere, F.: Woordenboek van de familienamen in België en Noord-Frankrijk, L.J. Veen Amsterdam / Antwerpen, 2003.

Kunze, K. / Nübling, D. (Hrsg.): Deutscher Familiennamenatlas, Band 2: Graphematik / Phonologie der Familiennamen II: Konsonantismus, Verlag Walter de Gruyter Berlin / New York, 2011.

Herbillon, J. / Germain, J.: Dictionnaire des noms de famille en Belgique romane, Band I und II, Bruxelles, 1996.

Goossens, J.: Motiefgeografie van Nederlandse familienamen. In: Naamkunde 27, 1995, 1-31.

Goossens, J.: Namenklassen und ihre Spiegelung in der niederländischen Familiennamengeographie. In: Heuser, R. / Nübling, D. / Schmuck, M. (Hrsg.): Familiennamengeographie: Ergebnisse und Perspektiven europäischer Forschung, Berlin / New York: De Gruyter, 2011a, 43-60.

Goossens, J.: Buchbesprechung von Cornelissen, G. / Eickmans, H. (Hrsg): Familiennamen an Niederrhein und Maas. Von Angenendt bis Seegers / Zeegers. Schriftenreihe der Niederrhein-Akademie / Academie Nederrijn, Band 9, Bottrop: Peter Pomp Verlag, 2010. In: Niederdeutsches Jahrbuch 134, 2011b, 177-179. 
Heuser, R. / Nübling, D.: Von Angenendt über Derix, Janssen und Terlinden bis Elspaß. Niederrheinische Familiennamen im Rahmen des Deutschen Familiennamenatlasses. In: Cornelissen, G. / Eickmans, H. (Hrsg): Familiennamen an Niederrhein und Maas. Von Angenendt bis Seegers / Zeegers. Schriftenreihe der Niederrhein-Akademie / Academie Nederrijn, Band 9, Bottrop: Peter Pomp Verlag, 2010, $37-66$.

Janssens, R.: Taalgebruik in Brussel. Taalverhoudingen, taalverschuivingen en taalidentiteit in een meertalige stad. Brusselse Thema's / Thèmes Bruxellois / Brussels Themes 8, Brüssel, 2001.

Marynissen, A.: Die geographische Streuung der Familiennamentypen im niederländischen Sprachgebiet. In: Niederdeutsches Wort, Band 45, 2005, 105-120.

Marynissen, A.: Ursprung, Motivierung und Bildung von Familiennamen am Beispiel des niederländischen Sprachgebiets. In: Cornelissen, G. / Eickmans, H. (Hrsg): Familiennnamen an Niederrhein und Maas. Von Angenendt bis Seegers / Zeegers. Schriftenreihe der Niederrhein-Akademie / Academie Nederrijn, Band 9, Bottrop: Peter Pomp Verlag, 2010, 11-35.

Marynissen, A. / Nübling, D.: Familiennamen in Flandern, den Niederlanden und Deutschland - ein diachroner und synchroner Vergleich. In: Dammel, A. / Kürschner, S. / Nübling, D. (Hrsg.): Kontrastive Germanistische Linguistik. Themenband der Reihe ,Germanistische Linguistik', Teilband 1, Hildesheim: Olms Verlag, 2010, 311-362.

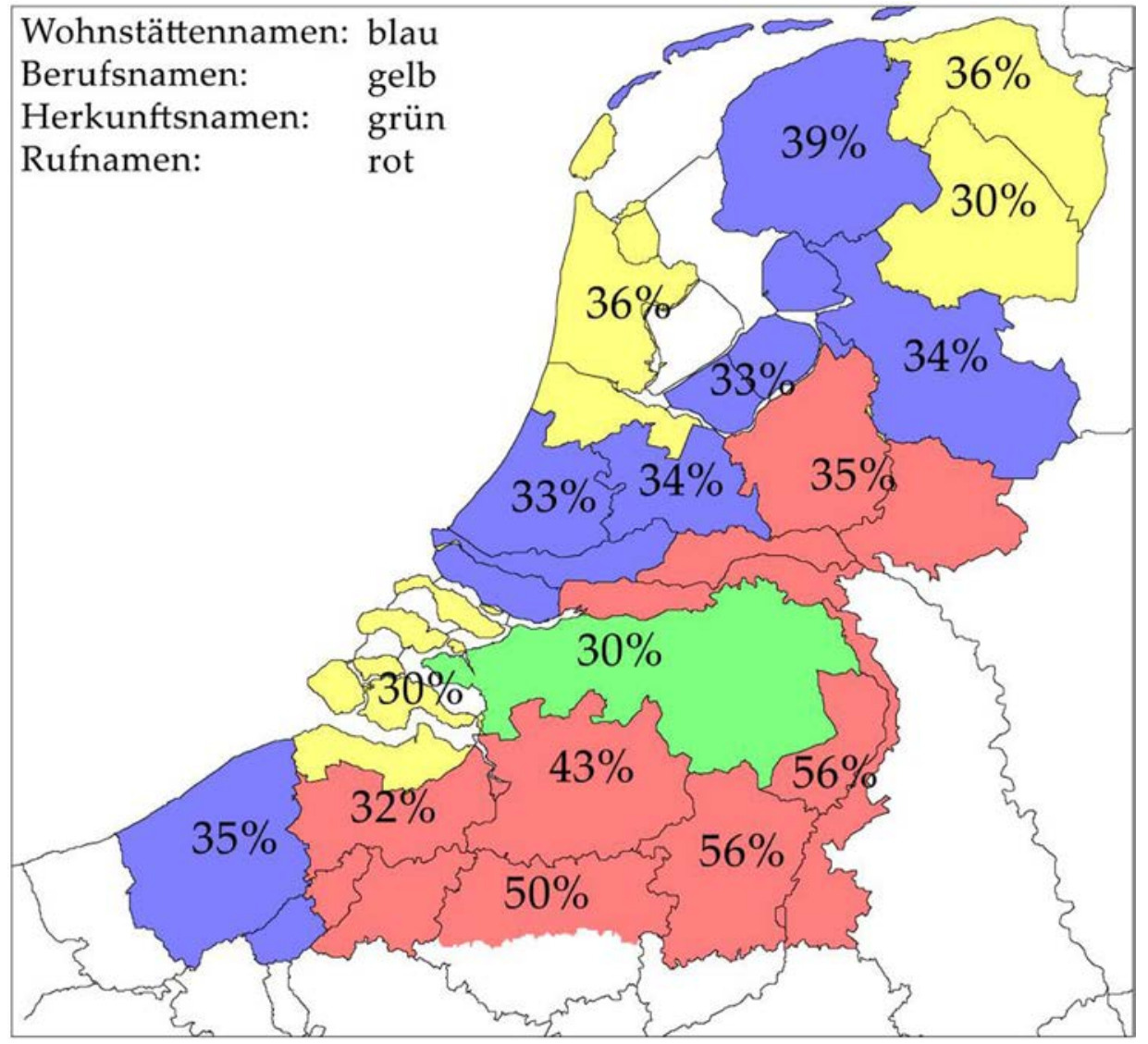

Karte 1: Die frequenteste Motivgruppe pro flämischer (1987) bzw. niederländischer (1993) Provinz 


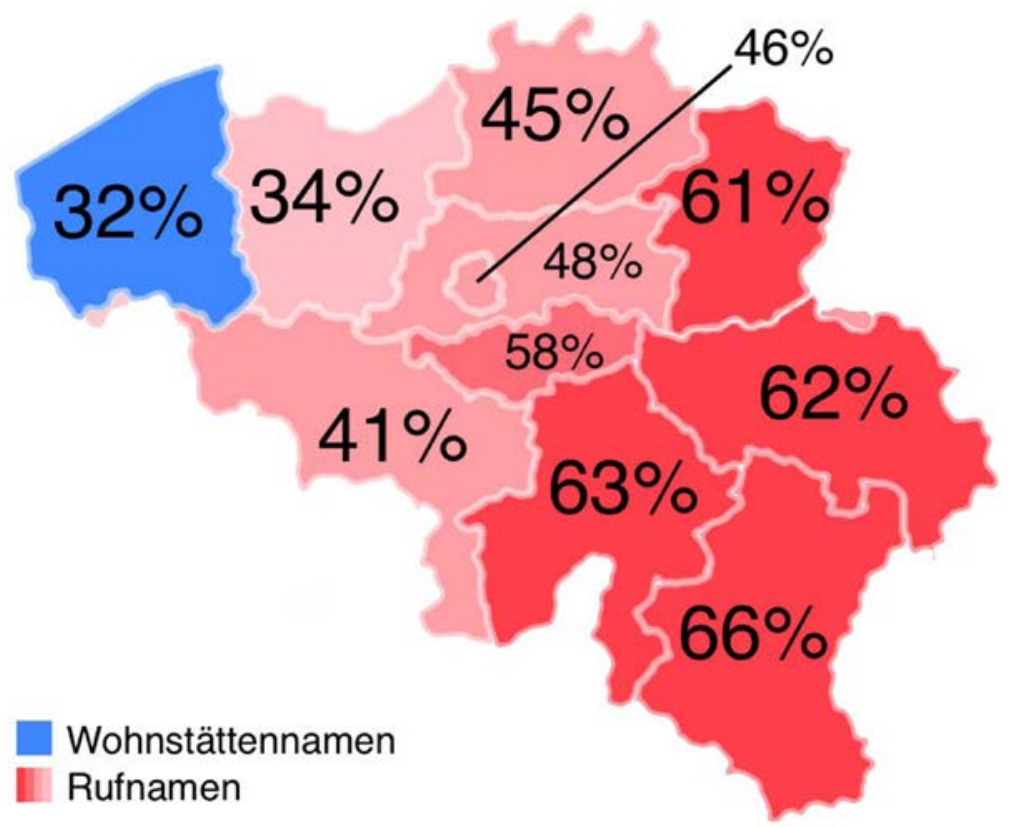

Karte 2: $\quad$ Die frequenteste Motivgruppe pro belgischer Provinz (2007)

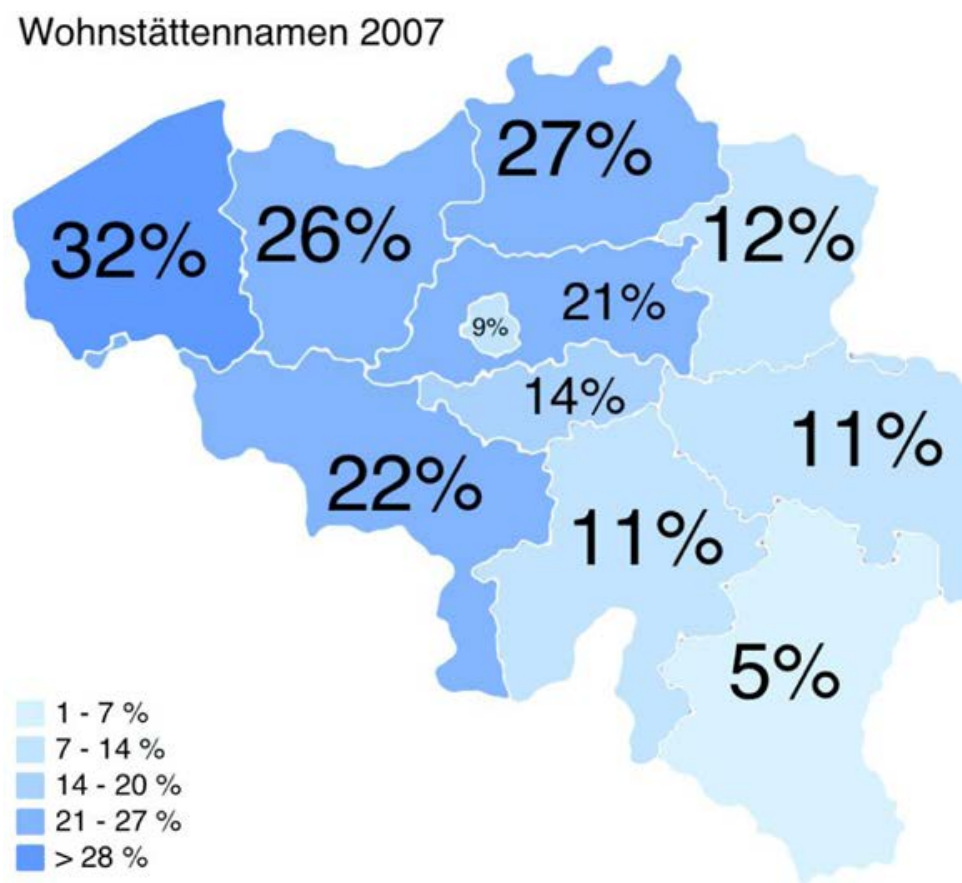

Karte 3: $\quad$ Prozentanteil der Wohnstättennamen (Belgien) 
Übernamen 2007

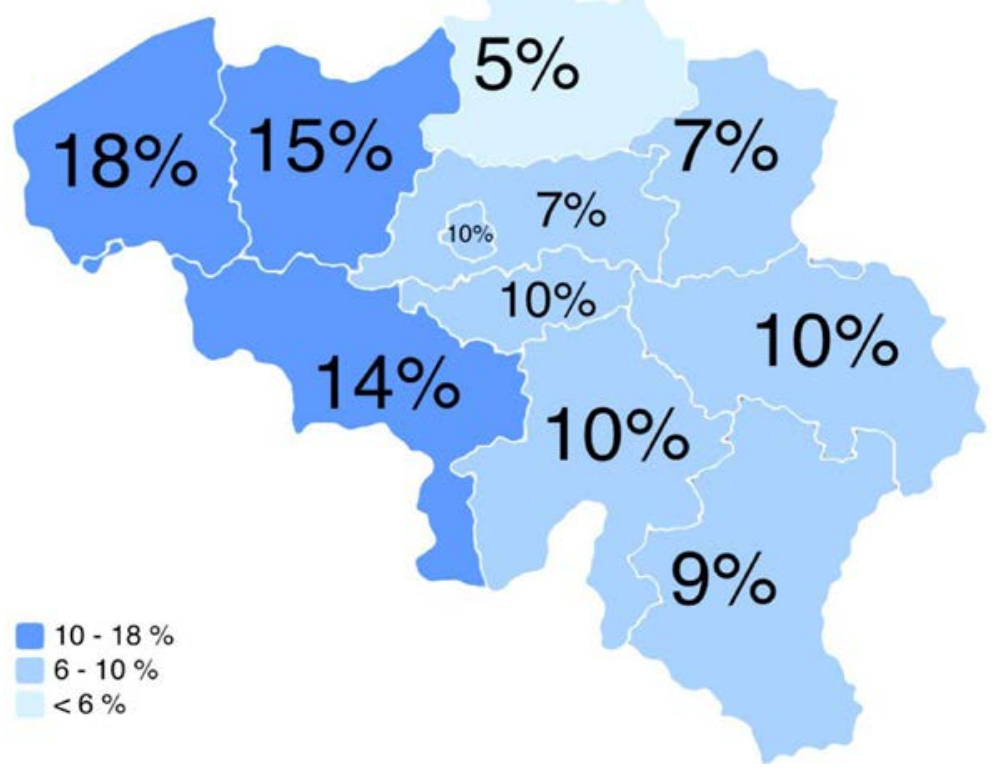

Karte 4: $\quad$ Prozentanteil der Übernamen (Belgien)

Berufsnamen 2007

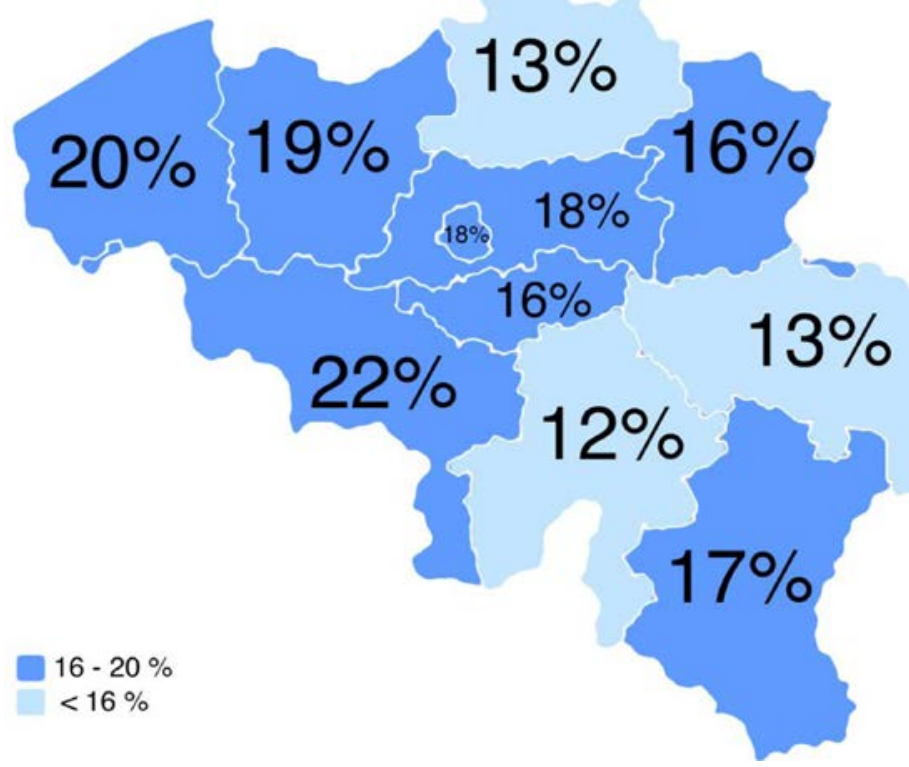

Karte 5: $\quad$ Prozentanteil der Berufsnamen (Belgien) 
Herkunftsnamen 2007

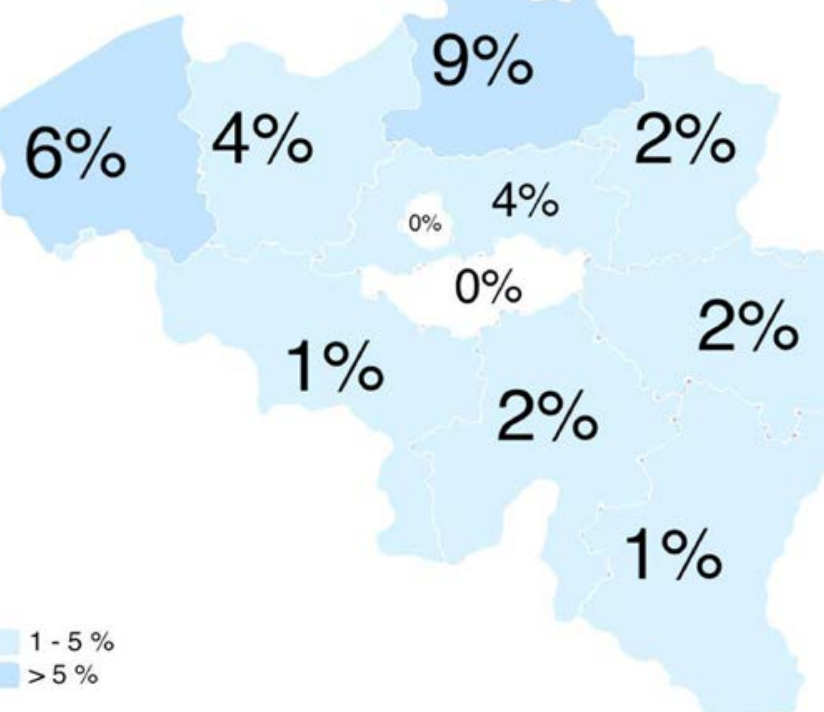

Karte 6: $\quad$ Prozentanteil der Herkunftsnamen (Belgien)

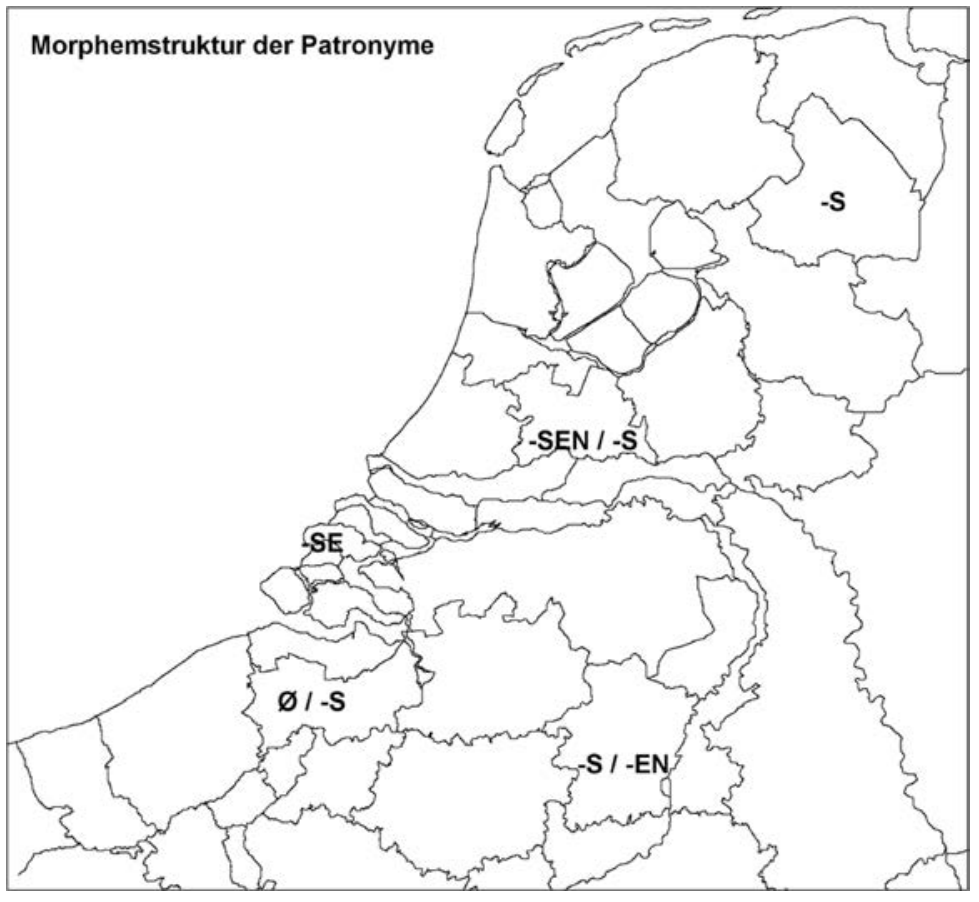

Karte 7: $\quad$ Morphemstruktur der Patronyme (niederländischer Sprachraum) 


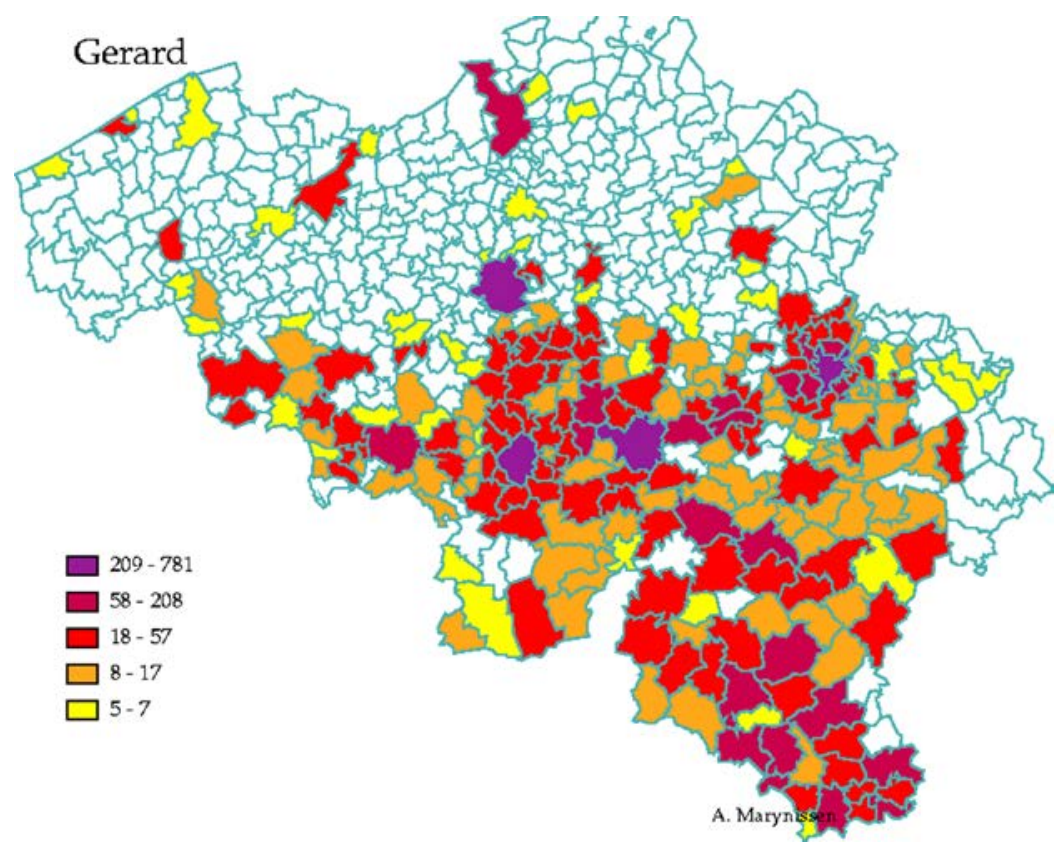

Karte 8: Der Familienname Gérard in Belgien

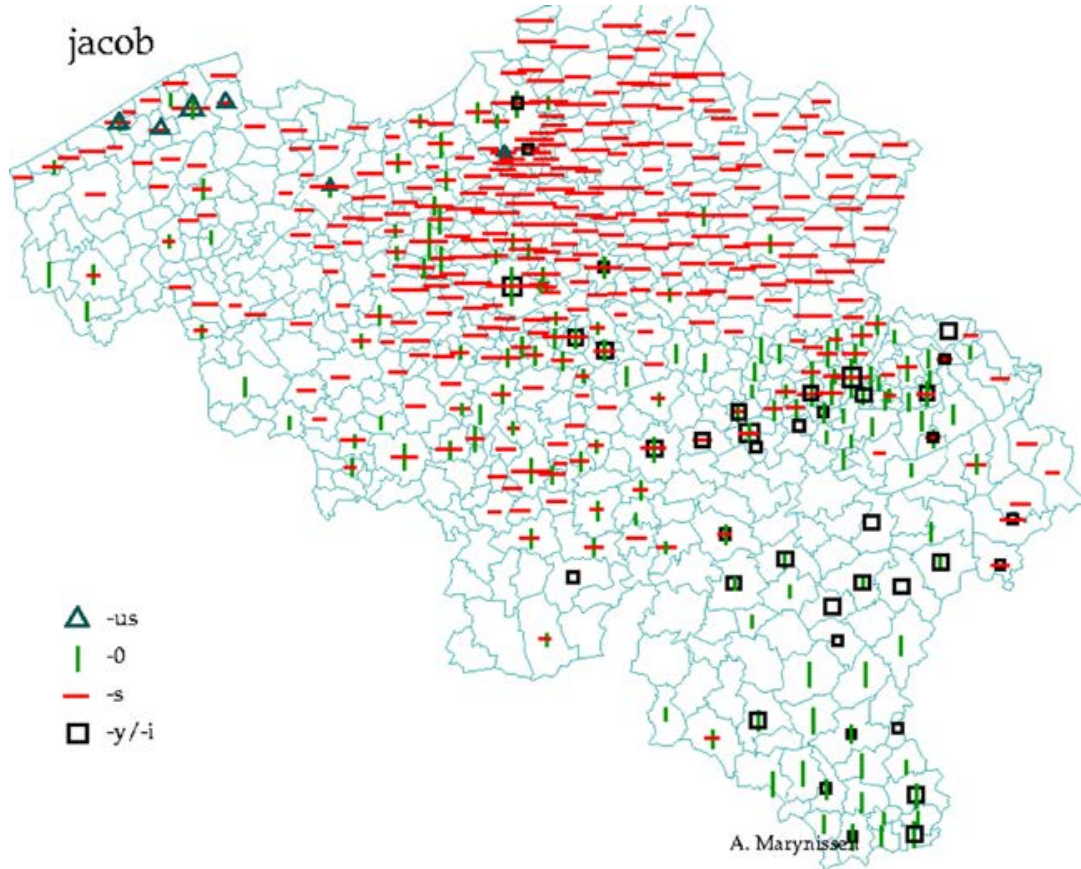

Karte 9: $\quad$ Die Verbreitung der Varianten von Jacob (Belgien) 


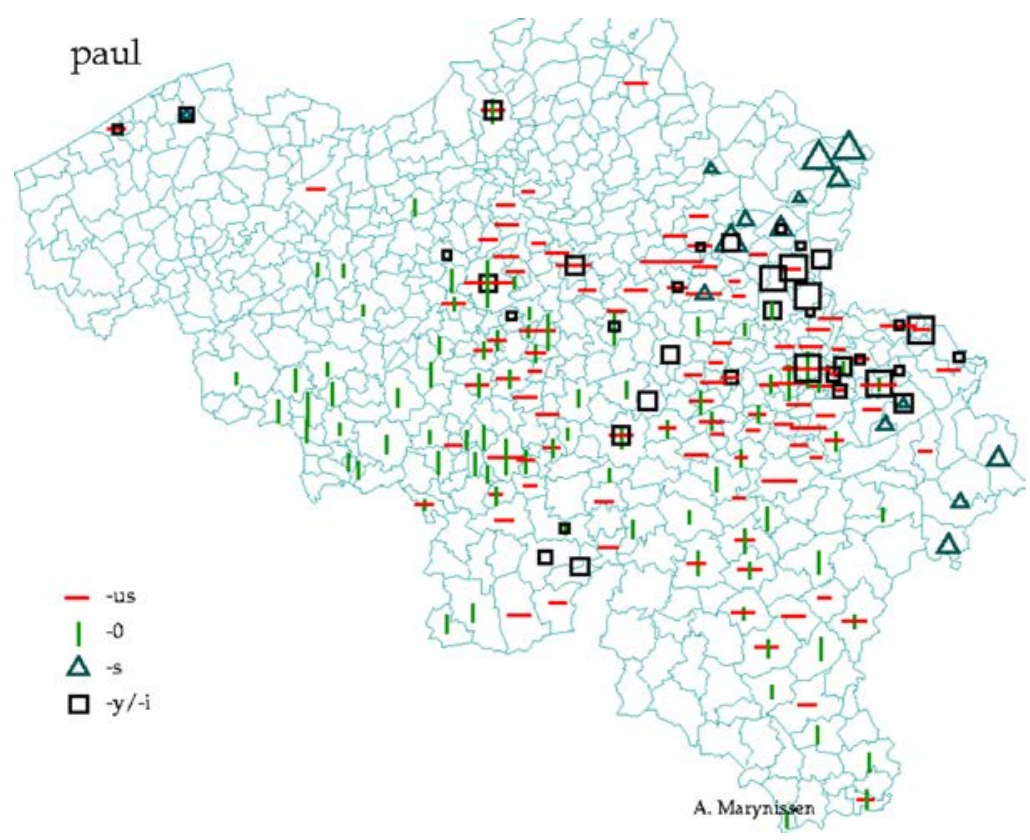

Karte 10: $\quad$ Die Verbreitung der Varianten von Paul (Belgien)

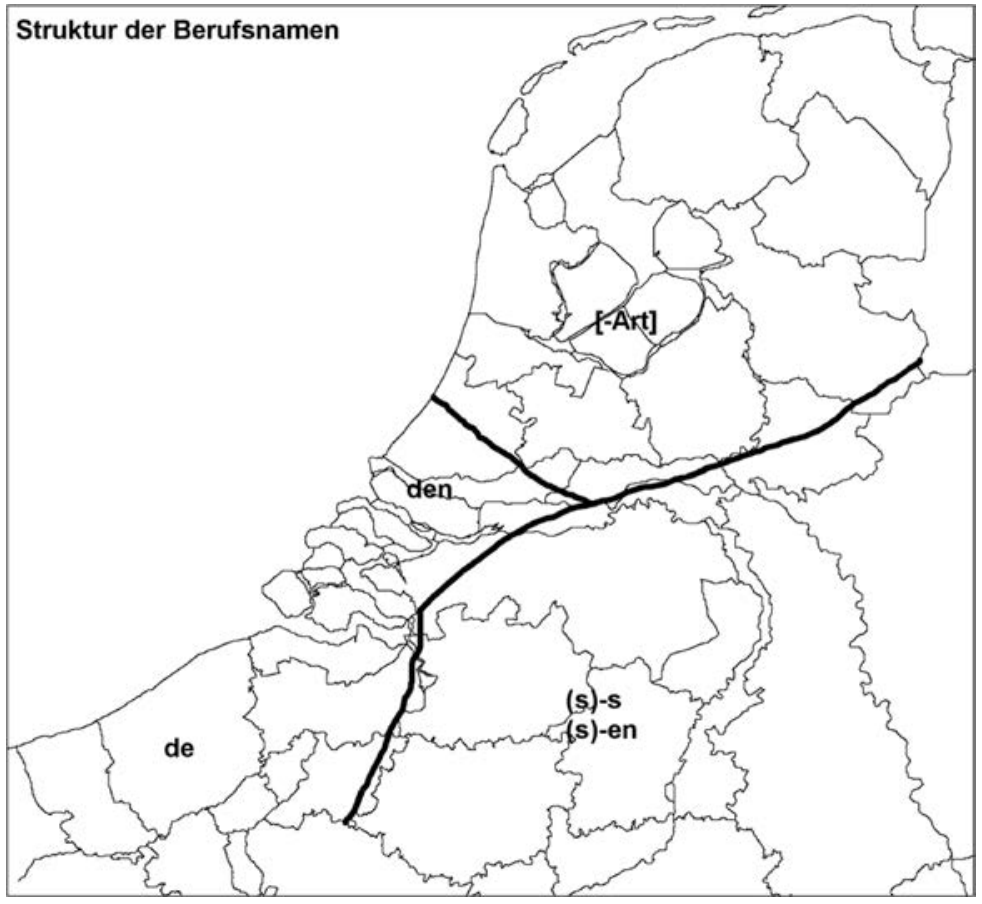

Karte 11: $\quad$ Morphemstruktur der Berufsnamen (niederländischer Sprachraum) 


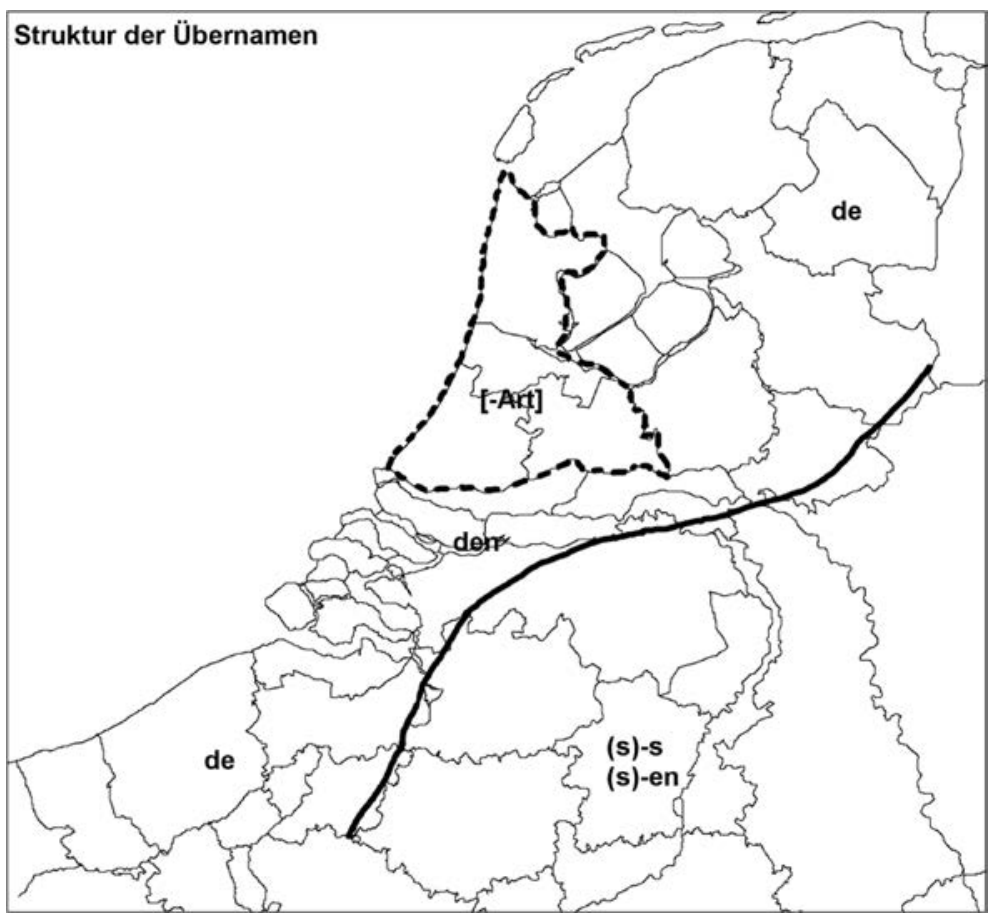

Karte 12: $\quad$ Morphemstruktur der Übernamen (niederländischer Sprachraum)

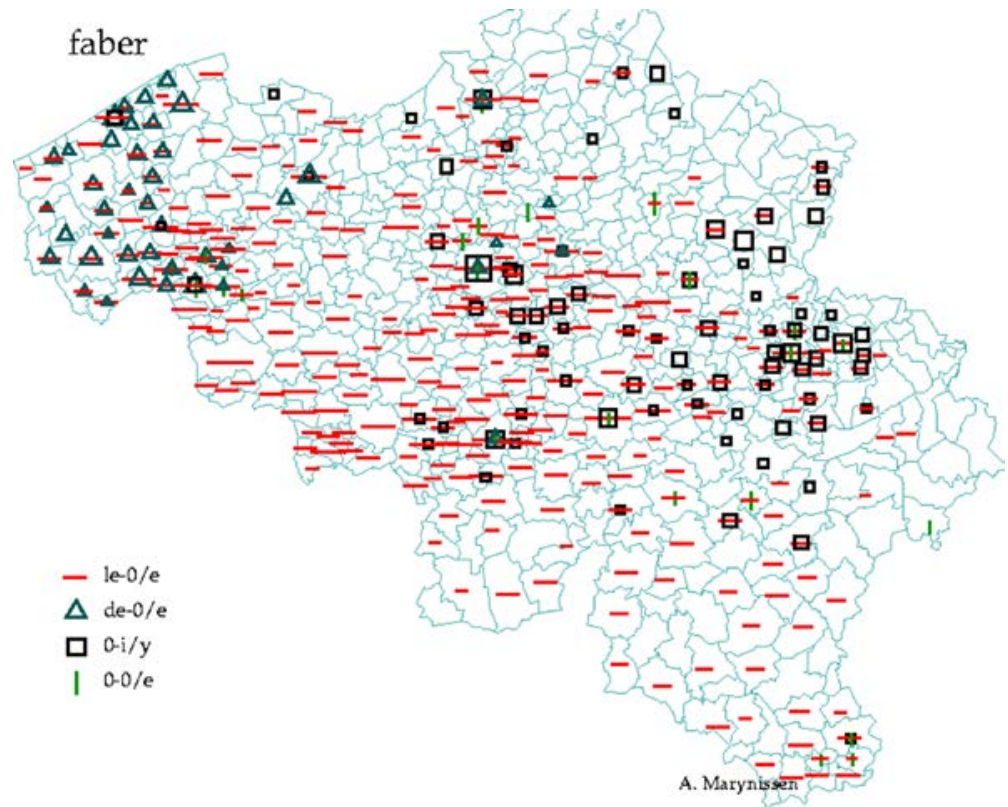

Karte 13: $\quad$ Varianten von lat. faber (Belgien) 


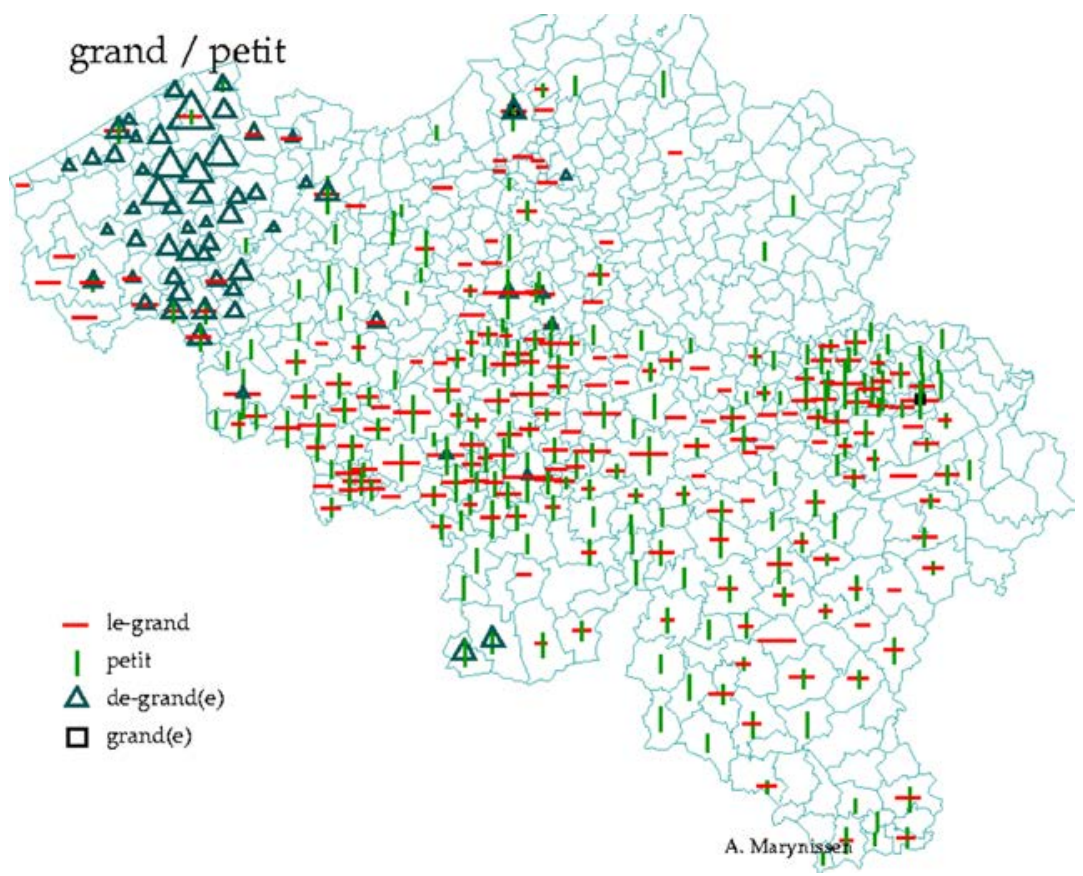

Karte 14: $\quad$ Familiennamen mit grand und petit mit oder ohne Artikel (Belgien)

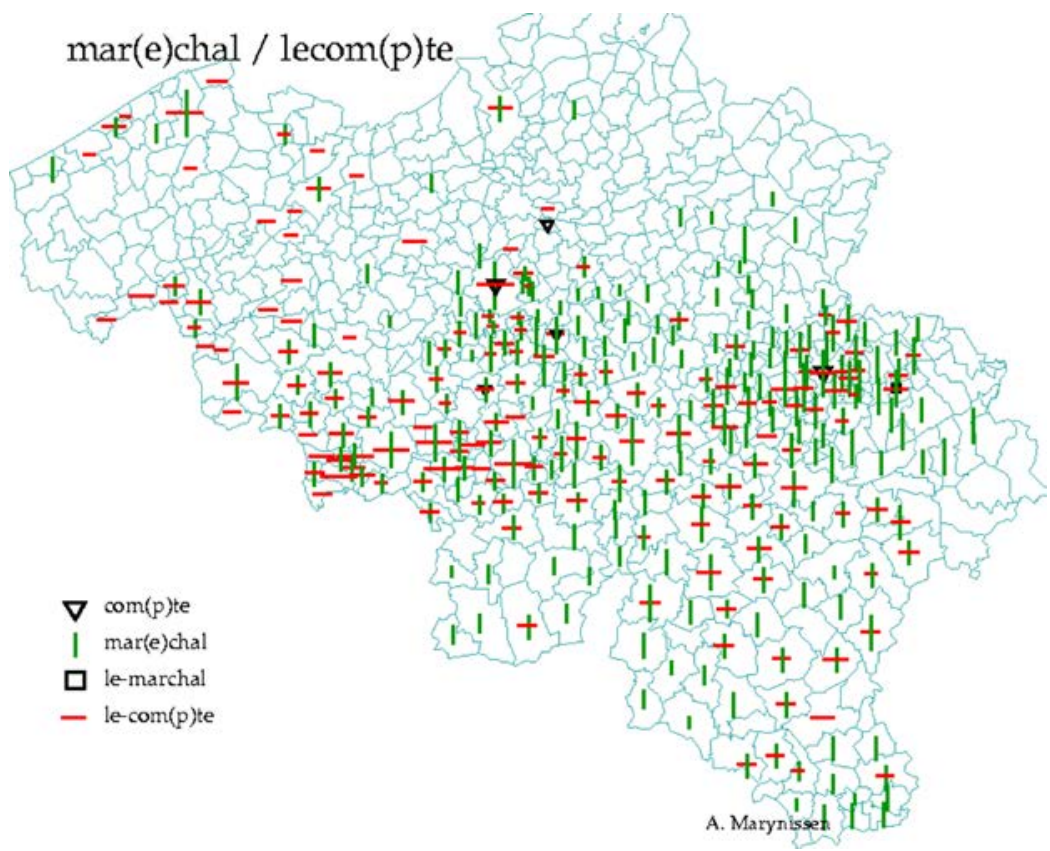

Karte 15: Die Varianten von marchal und comte mit oder ohne Artikel (Belgien) 


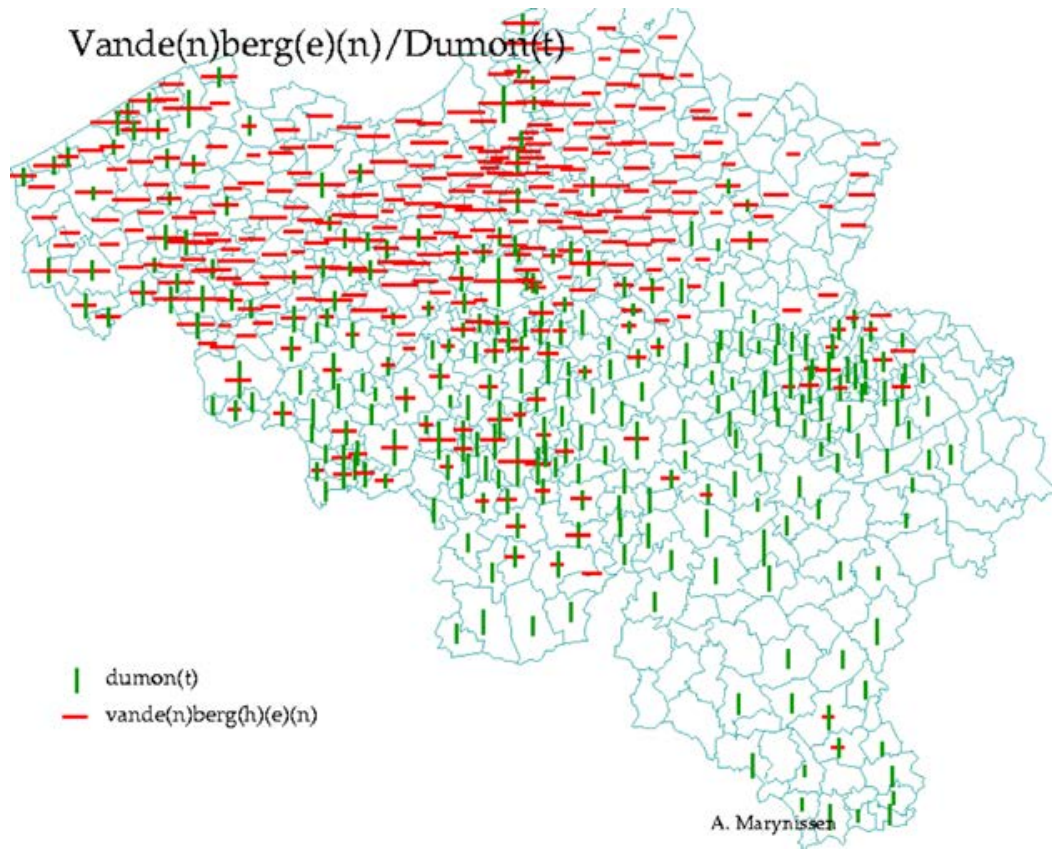

Karte 16: $\quad$ Die Verbreitung der Namensvarianten von berg bzw. mont (Belgien)

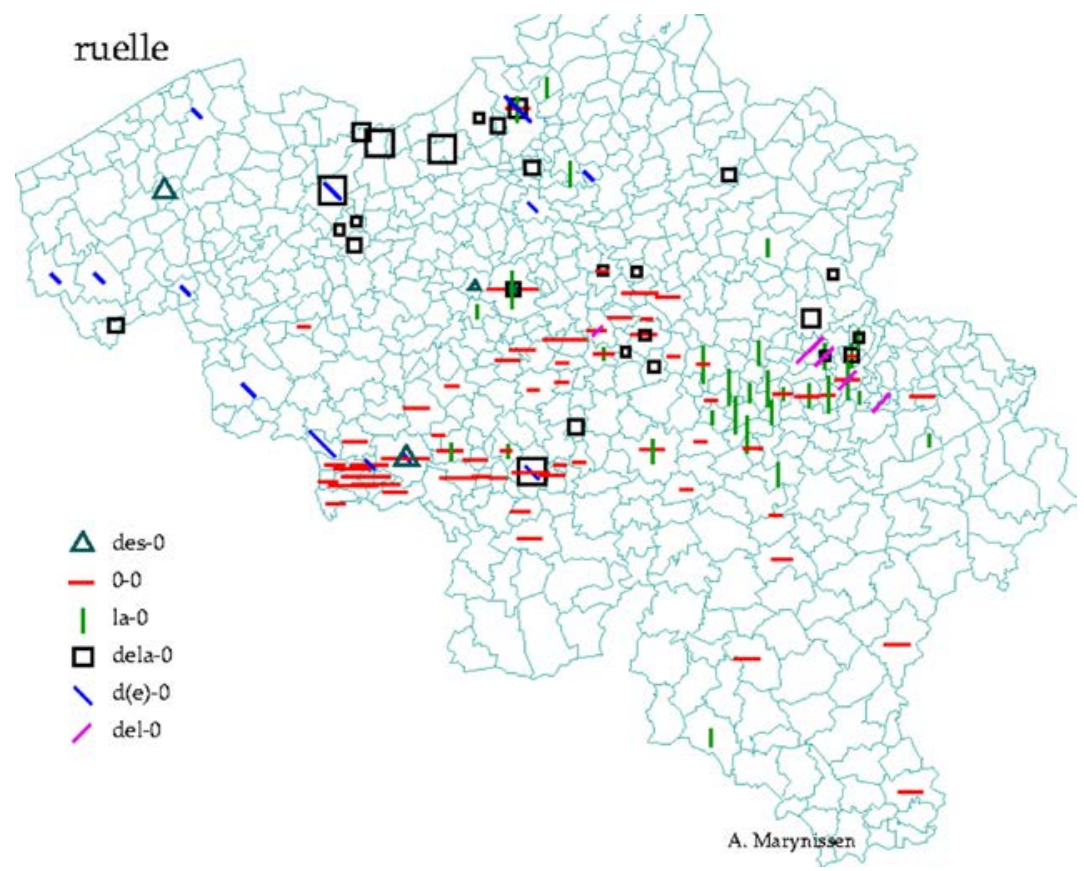

Karte 17: Die Varianten von Ruelle (Belgien) 


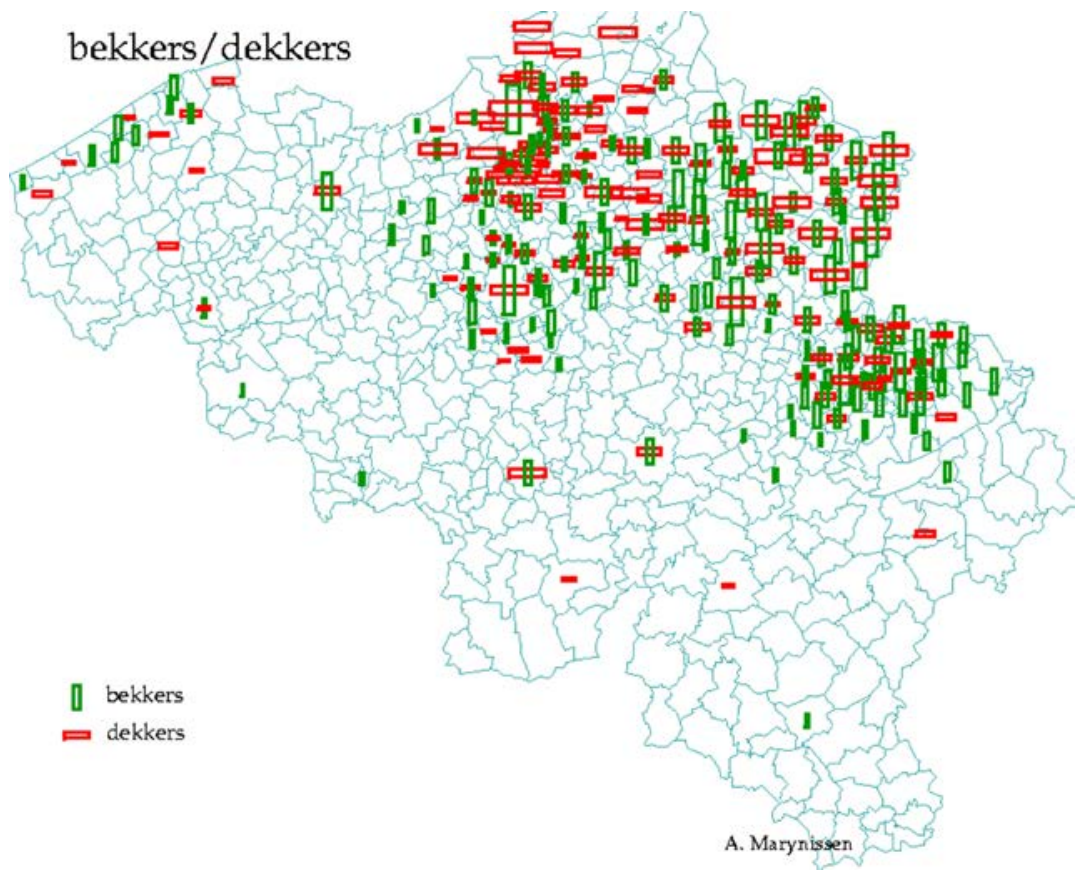

Karte 18: $\quad$ Die genitivierten Berufsnamen Beckers, Deckers und ihre Varianten (Belgien)

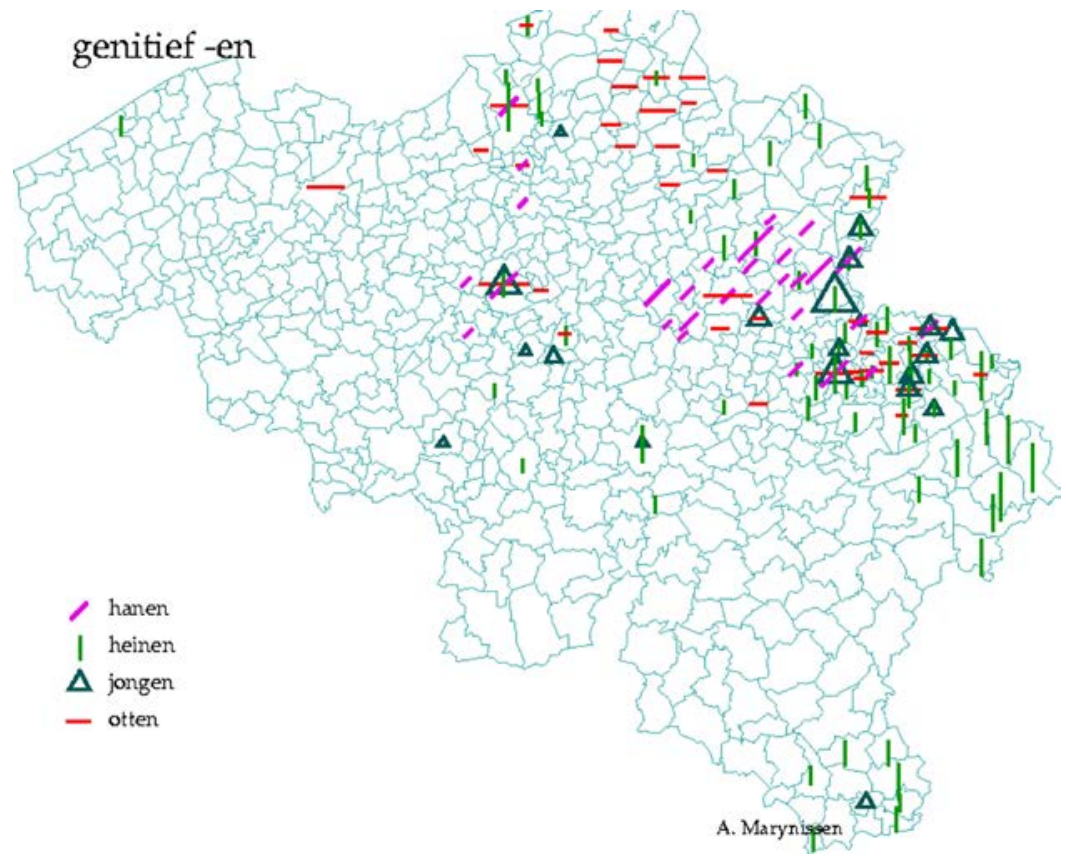

Karte 19: Familiennamen mit schwacher Genitivendung -en (Belgien) 


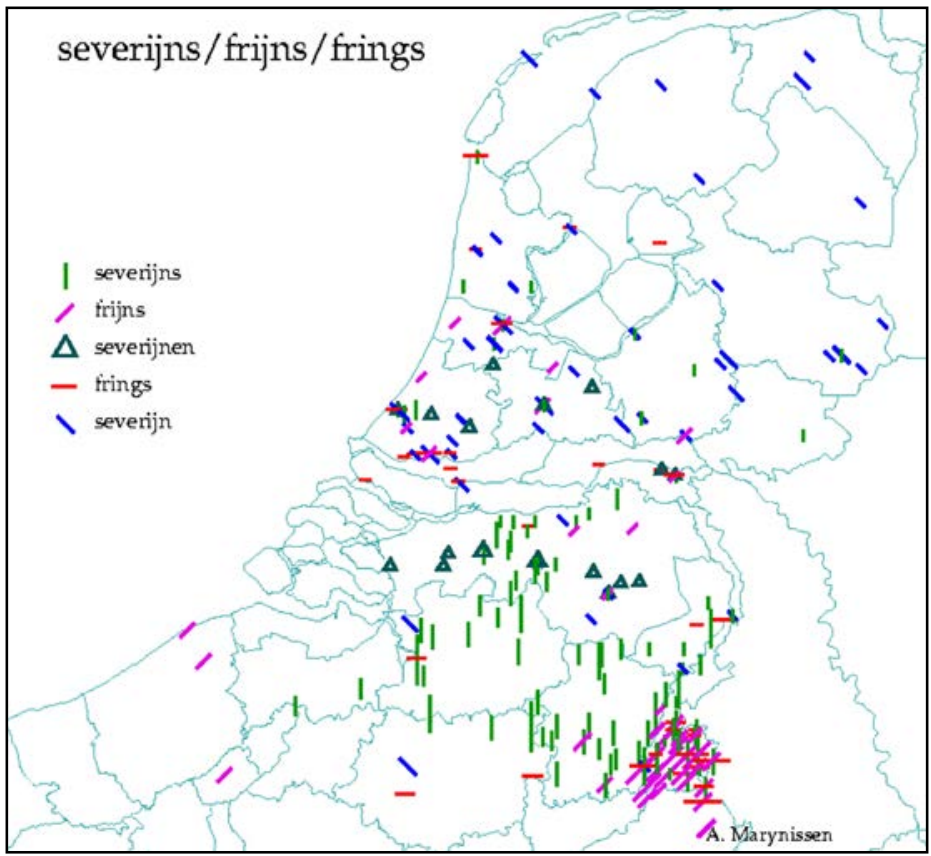

Karte 20: $\quad$ Voll- und Kurzformen von Severinus (niederländischer Sprachraum)

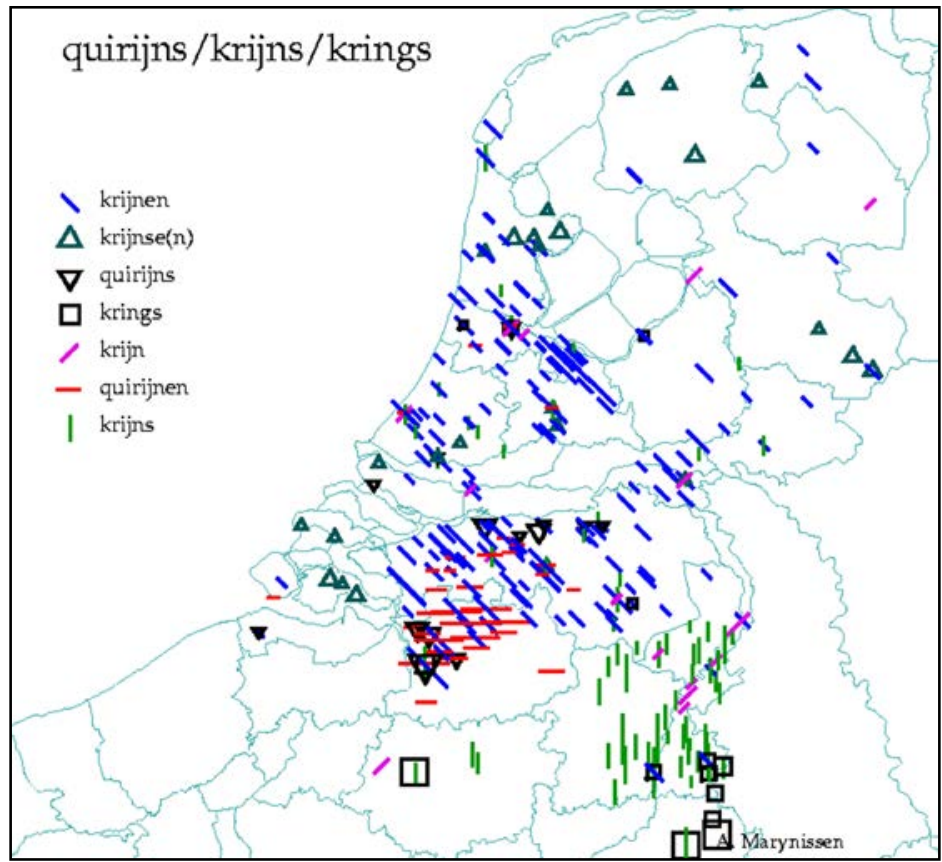

Karte 21: Voll- und Kurzformen von Quirinus (niederländischer Sprachraum) 


\section{Anhang}

Prozentualer Anteil der lexikalischen Motivgruppen an den 100 häufigsten Familiennamen pro belgischer Provinz und Brüssel.

Material: Vollständig belgisches Melderegister, Stand 31.12.2007. Die Daten wurden mir von der Generaldirektion Statistik und Wirtschaftsinformation Belgiens zur Verfügung gestellt.

Es wurden fünf Motivgruppen unterschieden: Rufnamen, Berufsnamen, Wohnstättennamen, Herkunftsnamen und Übernamen. Die Kategorie ,Rufnamen' enthält sowohl Vaternamen (z.B. Peeters, Janssens, Claes, Louis, Michel, Martin) als auch (viel seltenere) Mutternamen (z.B. Baeten, Achten, Luyten).

Die Kategorie ,ausländischer Name', die nur in der Hauptstadt Brüssel belegt ist, enthält Namen, die weder flämischer noch wallonischer, sondern ausländischer Herkunft sind (z.B. N'Guyen, Diallo, Benali, Yilmaz, Bah, Hajji).

Herzlichen Dank an Andrea Theissing und Christina Weise für die Hilfe bei der Auswertung der Daten.

Provinz Antwerpen

$\begin{array}{lr}\text { RufN } & 45.50 \% \\ \text { BerufsN } & 13.00 \% \\ \text { WohnstättenN } & 27.50 \% \\ \text { HerkunftsN } & 9.00 \% \\ \text { ÜberN } & 5.00 \%\end{array}$

Provinz Flämisch-Brabant

RufN

$48.33 \%$

BerufsN

$18.33 \%$

WohnstättenN

$21.00 \%$

HerkunftsN

$4.50 \%$

ÜberN

$7.83 \%$

Provinz Limburg

RufN

$61.17 \%$

BerufsN

$16.67 \%$

WohnstättenN

$12.00 \%$

HerkunftsN

$2.50 \%$

ÜberN

$7.67 \%$

Provinz Ost-Flandern

RufN

BerufsN

WohnstättenN 
HerkunftsN

$4.00 \%$

ÜberN

$15.50 \%$

Provinz West-Flandern

RufN

$23.00 \%$

BerufsN

$20.83 \%$

WohnstättenN

$32.17 \%$

HerkunftsN

$6.00 \%$

ÜberN

$18.00 \%$

Region der Hauptstadt Brüssel

RufN

$46.50 \%$

BerufsN

$18.00 \%$

WohnstättenN

$9.00 \%$

ÜberN

$10.50 \%$

ausländischer $\mathrm{N}$

$16.00 \%$

Provinz Wallonisch-Brabant

RufN

$58.67 \%$

BerufsN

$16.50 \%$

WohnstättenN

$14.50 \%$

ÜberN

$10.33 \%$

Provinz Namür

RufN

$63.67 \%$

BerufsN

$12.83 \%$

WohnstättenN

$11.00 \%$

HerkunftsN

$2.00 \%$

ÜberN

$10.50 \%$

Provinz Hennegau

RufN

$41.00 \%$

BerufsN

$22.00 \%$

WohnstättenN

$22.00 \%$

HerkunftsN

$1.00 \%$

ÜberN

$14.00 \%$

Provinz Lüttich

RufN

BerufsN 


$\begin{array}{lr}\text { WohnstättenN } & 11.00 \% \\ \text { HerkunftsN } & 2.00 \% \\ \text { ÜberN } & 10.50 \%\end{array}$

Provinz Luxemburg

RufN

BerufsN

WohnstättenN

HerkunftsN

ÜberN
$66.50 \%$

$17.50 \%$

$5.50 \%$

$1.50 \%$

$9.00 \%$ 


\title{
Deutscher Familiennamenatlas Band 6: Patronyme. Konzept und linksrheinische Beispiele
}

\author{
Kathrin Dräger, Konrad Kunze
}

\begin{abstract}
Der sechste Band des Deutschen Familiennamenatlasses (DFA) ist für die Familiennamen aus Rufnamen (Patronyme) vorgesehen. In ihm soll der Patronymenschatz erstmals umfassend unter arealem Aspekt dokumentiert werden. Im folgenden Beitrag werden Probleme und Konzept dieses Vorhabens anhand linksrheinischer Beispiele erörtert, wobei über die Westgrenzen Deutschlands in die Nachbarländer ausgegriffen wird. Dabei zeichnet sich die Region zwischen Rhein und Maas auch bei der Verbreitung von Patronymen in sprachlicher wie in außersprachlicher Hinsicht als einheitlicher Kulturraum ab.
\end{abstract}

\section{Der grammatische und der lexikalische Teil des DFA}

Familiennamen sind einerseits sprachliche Zeichen und als solche Untersuchungsgegenstand der Sprachwissenschaft. Andererseits unterliegen Namen innerhalb der sprachlichen Zeichen spezifischen Sonderbedingungen; einmal aufgrund ihrer Funktion, vor allem indem sie auf Individuen rekurrieren und in ihrer Existenz an diese gebunden sind. Zum anderen aufgrund ihrer Entstehungsbedingungen, denn Namen sind nicht nur an Individuen fixierte Sprache, sondern bei ihrer Entstehung wurden auch bestimmte Sachverhalte an Sprache fixiert, in der sie präsent bleiben können, auch wenn die Sachverhalte längst vergangen sind. Diese doppelte Fixierung in außersprachlichen Herkunfts- und Verwendungszusammenhängen qualifiziert Namen zu einer erstrangigen Quelle auch vieler nichtlinguistischer Disziplinen.

Diese Sachlage erfordert eine Aufteilung des Deutschen Familiennamenatlasses $(D F A)^{1}$ in einen ersten Teil, der nur ausdrucksseitige Phänomene behandelt und linguistische Zielsetzungen verfolgt, und einen zweiten Teil, der Aspekte der inhaltlichen Motivation und der Fixierung der Namen an Personen und Sachverhalte aufgreift und somit den hier ansetzenden interdisziplinären Interessen gerecht zu werden versucht.

Der erste, „grammatische“ Teil ist bereits erschienen. Er umfasst drei Bände zu den grammatischen Ebenen der Graphematik, Phonematik, Morphematik und Syntagmatik. Der zweite, ,lexikalische“ Teil ist in Arbeit. Er untersucht die Familiennamen unter dem Gesichtspunkt, inwieweit sich in ihnen Verbreitung und Varianz lexikalischer Einheiten spiegeln, etwa der appellativische Wortschatz des Mittelalters in Berufs- und Übernamen, der Ortsnamenschatz (Makro- und Mikrotoponyme) in Herkunfts- und Wohnstättennamen oder der historische Rufnamenschatz in Patronymen. Der lexikalische Teil wird nach der Entstehungsmotivation der Familiennamen in die fünf üblichen Namengruppen gegliedert. Band IV des DFA behandelt die Namen nach Herkunft und Wohnstätte, Band V

1 Zum Gesamtkonzept des Atlasses s. DFA I, S. XXV-XXXVII und Kunze/Nübling 2007. 
die Namen nach Berufen sowie nach körperlichen, charakterlichen oder biographischen Merkmalen, Band VI die Familiennamen, die aus Rufnamen entstanden sind. Sie werden der Kürze halber Patronyme genannt, auch wenn es nicht immer der Rufname des Vaters war, aus dem die betreffenden Bei- und späteren Familiennamen entstanden sind.

\section{Die Ausgangslage für den Patronymenband}

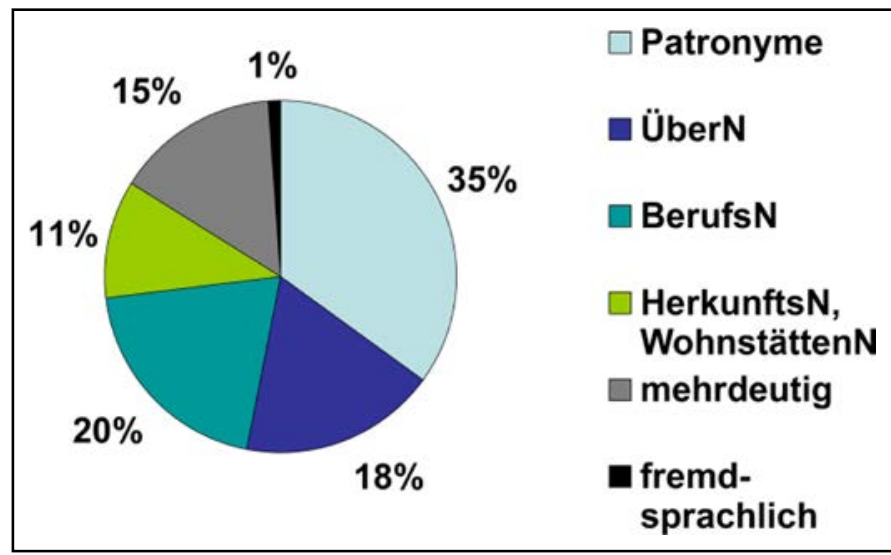

Abb. 1: Zusammensetzung des Inventars der 1000 häufigsten Familiennamen in Deutschland nach Telefonanschlüssen 1996 (nach Kohlheim/Kohlheim 2001, 285, Abb. 1)

Der Anteil der Patronyme am heutigen Familiennamenschatz Deutschlands ist zurzeit noch nicht exakt zu bestimmen. Kohlheim/Kohlheim 2001 haben die 1000 häufigsten Familiennamen in Deutschland daraufhin untersucht: Die Patronyme erweisen sich als die mit Abstand größte Gruppe; ihr Anteil am Namenschatz, gerechnet an der Zahl der verschiedenen Namen (nicht der Namenträger) beträgt über ein Drittel. ${ }^{2}$ Dies darf man allerdings nicht auf den gesamten Namenschatz übertragen, der in Bereichen der weniger frequenten Namenvorkommen möglicherweise, ja wahrscheinlich völlig anders proportioniert ist. ${ }^{3}$ Doch steht außer Frage, dass bei den Patronymen eine in Types- und Tokenzahl gewichtige Materialmenge zu bewältigen sein wird.

Die Patronyme heben sich von den anderen Namengruppen vor allem durch folgende Besonderheiten ab: Sie sind die einzige Namengruppe, die ausnahmslos selbst wieder von Namen abgeleitet (deonymisch) ist. Dagegen beruhen die Namen nach Berufen auf Appellativen (deappellativisch), die nach persönlichen Merkmalen oft auf Adjektiven

2 Kohlheim/Kohlheim 2001, 285. - Bei den 200 häufigsten Familiennamen in den Provinzen des niederländischen Sprachgebiets schwankt der Anteil der Patronyme zwischen maximal 56 \% (Belgisch und Niederländisch Limburg) und minimal 5 \% (Südholland), Marynissen 2010.

3 Dabei dürfte der Anteil der Patronyme aufgrund ihrer Variantenvielfalt bei den weniger frequenten Namen zunehmen, vgl. Farø/Kürschner 2007 und den Beitrag „Die luxemburgischen Familiennamen entlang der historischen Grenze zu Deutschland - Projektskizze und vorläufige Ergebnisse“ in diesem Band. 
(deadjektivisch), die Namen nach Herkunft und Wohnstätte können teils deonymisch, teils deappellativisch sein. Eine Erfassung der aus Rufnamen entstandenen Familiennamen trägt damit wesentlich auch zur Erforschung des spätmittelalterlichen Rufnamenschatzes bei, der in den Familiennamen konserviert ist. Hier eröffnet sich ein erster und zugleich der rationellste Zugang, um großräumig die Verbreitung spätmittelalterlicher Rufnamen überhaupt zu erschließen, und die einzige Möglichkeit, ihre Verwendung in der damals gesprochenen Sprache (und nicht nur in mehr oder weniger offiziellen urkundlichen Aufzeichnungen, also dialektal Haupert statt Hubert, Wörz statt Werner usw.) zu rekonstruieren.

Während die übrigen Familiennamengruppen in der Hauptsache auf Sprachmaterial germanischer (im Osten auch slawischer) Herkunft beruhen, geht die hier zu untersuchende Gruppe in Süddeutschland zu durchschnittlich $25 \%$, in Nord- und Ostdeutschland zu über $50 \%$ auf durch die christliche Kultur aus fremden Sprachen adaptierte Rufnamen zurück (Kunze 2004a, 75). Nicht zuletzt deswegen ist diese Gruppe gegenüber den anderen durch die größte Vielfalt an Varianten gekennzeichnet. Die Vielfalt wird einerseits, wie auch bei den anderen Gruppen, durch unterschiedliche sprach- und schreibdialektale Realisierungen (Herrmann/Hermann/Hörmann) verursacht, zudem durch verschiedene Möglichkeiten der Namenbildung (Jacob/Jacobs/Jacoby/Jacobsen); andererseits kommen durch Umakzentuierung bei der Adaption fremder Rufnamen bedingte Aufspaltungen hinzu (Matthias: Matt/Thies/Hias), Kontraktionen (Mattes) usw., sodann die Herausbildung verschiedener Typen von Kurz- und Lallformen bei ererbten und fremden Rufnamen (Aegidius: Egid/Gilg/Gille/Gill/Ilg) und schließlich die enorme Potenzierung der Varianz durch Überkreuzung all dieser Prozesse. In nicht wenigen Fällen haben sich aus einem einzigen Rufnamen hunderte von Familiennamen entwickelt. Kathrin Dräger hat soeben den wohl extremsten Fall Nikolaus untersucht, aus dem allein in Deutschland nicht weniger als $4000(!)$ verschiedene Familiennamen entstanden sind oder wenigstens in dieser Hinsicht diskutiert wurden oder werden können (Dräger 2013).

Damit wird verständlich, dass die Patronyme unter dem Aspekt ihrer großräumigen Verbreitung die am wenigsten untersuchte Gruppe sind, obwohl hier Grundlegendes über die Familiennamenforschung hinaus auch für die Rufnamen zu erarbeiten wäre und damit auch zur Kulturgeschichte, insbesondere zur Geschichte der gesprochenen Sprache, zur mittelalterlichen Heiligenverehrung, zur Aneignung dynastischer Leitnamen usw.

\section{Kriterien der Namenauswahl}

Aus dieser Lage ergibt sich als zentrales Problem, wie man die großen Materialmengen, die Grundstrukturen ihrer Entfaltung und die wichtigsten Perspektiven ihrer Auswertbarkeit unter den Gesichtspunkten möglichster Repräsentativität und Exemplarizität in einem Atlasband von maximal 1000 Seiten auffängt.

Konkret heißt das, es müssen Beispiele ermittelt werden, die es ermöglichen, einerseits die Rezeptionsräume einer repräsentativen Anzahl ererbter und fremder Rufnamen im Familiennamenschatz anhand des Gesamtfelds ihrer zahlen- und typmäßig relevanten Varianten abzustecken. Andererseits soll es möglich werden, durch Vergleich solcher Beispiele regionale Präferenzen in den Formen der Aneignung und des Gebrauchs der 
Ausgangsnamen aufzuweisen, beispielsweise die Bevorzugung von Vollformen (Eberhard), zweistämmigen (Ebert) oder einstämmigen Kurzformen (Eber), Lallformen (Eppe), Flexion (Eberts, Evertz), Suffigierung (Eberl, Ebbing, Eppmann) usw., das heißt, generelle Prozesse der vielfältigen Namenaufspaltung in ihren räumlichen Konturen zu erfassen.

Das bisher einzige auf die Gruppe der Familiennamen aus Rufnamen konzentrierte Lexikon, Linnartz 1958, setzt auf dem Stand der damaligen Forschung als Lemmata 324 „deutsche“ und 268 ,fremde“ Rufnamen an, aus denen sich in Deutschland Familiennamen entwickelt haben. Aus einigen, etwa Heinrich, Nikolaus oder Johannes, ging eine enorme Menge von Familiennamen hervor, bei anderen, etwa Ewald, Urban oder Adam, ist deren Anzahl überschaubar. ${ }^{4}$ Für eine umfassende Dokumentation dieser im Einzelnen sehr unterschiedlich ausgeprägten Namenfülle wären, würde man etwa den 592 Lemmata bei Linnartz 1958 jeweils ein Kapitel (im DFA = einen Kartenkomplex) widmen, über 5000 Druckseiten erforderlich. Es ist also eine rigorose Auswahl zu treffen. Rechnet man pro Kartenkomplex durchschnittlich fünf Karten, mit Kommentartext insgesamt zehn Seiten, können maximal 100 Ausgangsnamen behandelt werden, also etwa ein Sechstel der bei Linnartz 1958 angesetzten Ausgangsnamen.

Die Auswahl dieses Sechstels erfolgt hauptsächlich nach folgenden Kriterien: Das erste Auswahlkriterium ist die Namenfrequenz. Von den tausend häufigsten Familiennamen in Deutschland beruhen 347 auf Rufnamen, an erster Stelle Hartmann mit 50.690 Telefonanschlüssen/ca. 140.000 Personen, an letzter Janzen mit 3.602 Telefonanschlüssen/ ca. 10.000 Personen. Sie lassen sich 100 Ausgangsnamen bzw. Namengliedern germanischer Herkunft und 42 Ausgangsnamen nichtgermanischer Herkunft zuordnen. ${ }^{5}$ Diese häufigsten Namen sollen daraufhin geprüft werden, wieweit sie als „Kerne“ geeignet sind, um die herum unter Berücksichtigung weiterer Kriterien Kartenkomplexe angelegt werden könnten. So treten beispielsweise Eberhardt mit den Ableitungen Ebert, Evers, Eberle sowie die wenigstens teilweise dazugehörigen Namen Ebel und Ebeling unter den frequentesten Namen auf. Daher werden sie als „Kernnamen“ für einen Komplex „Eberhart" aufgegriffen - sie sind in folgender Aufstellung fett gedruckt - und dann ins Umfeld weiterer Varianten wie Eber, Eberlein, Epp, Ebbing usw. (zusammen mit dem als Ausgangsnamen konkurrierenden Eberwien) eingebettet. ${ }^{6}$

Titel

Hauptkarte

1. Nebenkarte

2. Nebenkarte

3. Nebenkarte
Eber[hart, -wien]

Typen Eberhardt, -hard, -hart

Typen Eberwein, -wien

Typen Ebert, Eber

Typen Eberts, Ebertz, Ebers, Evers, Ewers

4 Als Beispiel für eine durchschnittliche Namenmenge kann Hubert gelten, bei dem Linnartz 1958, 82f. 95 Familiennamen anführt.

5 Laut Kohlheim/Kohlheim 2001, 285-298, nach Telefonanschlüssen, Stand 1996.

6 Von Familiennamen aus Eber[hart, -wien] sind in Luxemburg nach Daten von 1930 vertreten: E(v/w)en 270+211, Ewert(s) 164+1, Eberhard(t) 42+18, E(v/w)erling 42+11, Evera(r)d 20+36, Ewera $(r) d 7+23$, Evra(r)d 4+22, E(w/v)ers 17+12, Evert(z) 17+3, Ewra(r)d 1+15, Ewertz 14, Ebel 9, Eppe 3, Everhard(t) 2+1, Ewerhard 2 (Institut Grand-Ducal 1989, 85, 93f.). 
4. Nebenkarte

5. Nebenkarte

6. Nebenkarte
Typen Eberle, Eberlein, Eberl

Typen Epp, Epe, Ebben, Ebbes, Ebsen, Ebi, Ebbing, Eppmann

Typen Ebel, Ebeling, Eble, Epple, Eppler

Die Kartenkomplexe werden so angelegt, dass jeweils der Ausgangsname (manchmal mehrere Ausgangsnamen: Eberhart, Eberwien) den Titel bildet. Auf der Hauptkarte werden Varianten der unverkürzten Vollform dokumentiert (Eberhardt, -hard, -hart), in den Nebenkarten die Ausfaltung im Familiennamenschatz anhand verkürzter $(\operatorname{Eber}(t)$ usw.), flektierter (Eberts, Ebben usw.), suffigierter (Eberle, Ebbing usw.) Varianten. Vorangestelltes „Typen“ signalisiert, dass hier mehrere, etwa auf graphematischer Ebene variierende Formen zusammengefasst sind (Typ Eberwien: Eberwien, Ewerwien, Everwien, Everwin).

Als zweites Auswahlkriterium dient die Vielfalt der Namenrezeption. Nicht berücksichtigt werden Fälle, bei denen Rufnamen in ihrer Rezeption als Familiennamen nicht oder kaum diversifiziert wurden, etwa Ewald, Helfrich oder Salomon und/oder keine kartierenswerte Arealbildung aufweisen, etwa Erdmann, Jonas oder Jordan. Die Verbreitung solcher Einzelnamen kann ein Interessent unschwer über das Internet einsehen.

Als drittes Auswahlkriterium kommt hinzu, wieweit Patronyme schon an anderer Stelle in ihrer räumlichen Verbreitung und Ausfaltung behandelt sind. So brauchen etwa die Fälle Jacobus, Matthias/Matthäus, Nikolaus und Wenzel nicht mehr aufgegriffen zu werden. ${ }^{7}$ Das gilt auch für die zahlreichen Patronyme, die aufgrund ihrer graphematischen, phonologischen oder morphologischen Varianz schon in den grammatischen Bänden des DFA dokumentiert sind. So ist, um ein links des Rheins konzentriertes Beispiel zu wählen, der Fall Wienand/Weinand schon in DFA I (Vokalismus) im Kapitel „MonophthongDiphthong-Varianz i(e),ei“ dokumentiert. ${ }^{8}$

Diese Familiennamen gehen auf den Rufnamen Wignand (zu ahd. wigg ,Kampf ${ }^{\star}+$ nand ,kühn') mit Ausfall des -g- zurück. Die Grenze der hier kartierten Typen stimmt nach Ausweis fünfstelliger PLZ fast genau mit der Landesgrenze zwischen Nordrhein-Westfalen und Rheinland-Pfalz überein. ${ }^{9}$ Typ Wienand setzt sich vor allem nach Belgien hin fort, mit zahlreichen Belegen für Winan $(d / t)(s)$. Noch häufiger ist die Form Wynan $(d / t)(s)$, die auf der deutschen Seite im Raum Aachen konzentriert ist (Anm. 9) und deren lautliche Realisierung in Deutschland unklar ist; im niederländischen Sprachgebiet jedenfalls ist $y$ in Eigennamen meist Ligatur für $i j$, ein Diphthong mit dem Lautwert [عi]. Während Wynan $(d / t)(s)$ in Belgien dominiert, ist in den Niederlanden Wijnan $(d / t)(s)$ am häufigsten. ${ }^{10}$ In Luxemburg treten im Jahr 1930 diphthongische Formen mit ei, ey insgesamt

7 Debus 2011; Dräger 2013; Kunze 2004b; Kunze 2005.

8 DFA I, K. 171 mit Karte für ganz Deutschland, in Abb. 2 Ausschnitt Westdeutschland.

9 Die Lage der häufigsten unter den Typen Wienand und Weinand subsumierten Familiennamen ist im Kommentartext angegeben: "Im einzelnen finden sich Wienand fast nur östl. des Rheins, Wienands in PLZ 40 Düsseldorf, 41 Mönchengladbach und 47 Duisburg, Wynands in PLZ 52 Aachen, Winands in PLZ 50 Köln, Winand in PLZ 53 Bonn, Weinandt im Raum Gießen, Weynand im Raum Siegen“(DFA I, 390).

10 Belgien: Wynant(s) 606+2032, Wynand(s) 3+171, Wynandt 18; Winand(s) 744+11, Winant(s) 214+71, Winandts 4, Wienand 1; Wijnant(s) 268+1119, Wijnand(s) 4+77, Wijnandts 38; Weynant(s) 9+261, Weynand(s) 179+19, Weinand 51, Weynandt 4 (Einwohner 1998, www.familienaam.be, 28.06.12). 
126-mal auf, monophthongisch ist hauptsächlich Winandy belegt. ${ }^{11}$ Die Ausgangsform Wignand ist in Deutschland nur noch in Weigenand 27 erhalten, das vor allem im Raum Saarbrücken - Mannheim vorkommt; auf der Karte sind alle sonstigen Varianten erfasst, und somit ist der Fall in DFA I (Vokalismus) schon erschöpfend dokumentiert.

\section{Typ Wienand 1677:}

Wienand(s) 776+238, Winand(s)

230+159, Wynand(s) 11+165,

Wienandt(s) 44+25, Wynant(s) 7+15,

Wienant(s) 1+3, Winandt(s) 1+1,

Wynends 1.

Typ Weinand 896:

Weinand(s) 806+8, Weinandt 36, Weynand(s) 35+9, Weynandt 1 ,

Weynant 1 .

Kartentyp: relativ; Ausschnitt Westdeutschland; Kreise pro dreistellige PLZ, entspricht 0,02-3,93\%; Flächen pro zweistellige PLZ, Anzeigeschwelle 0,50\%; Tokenangaben beziehen sich auf Gesamtdeutschland.

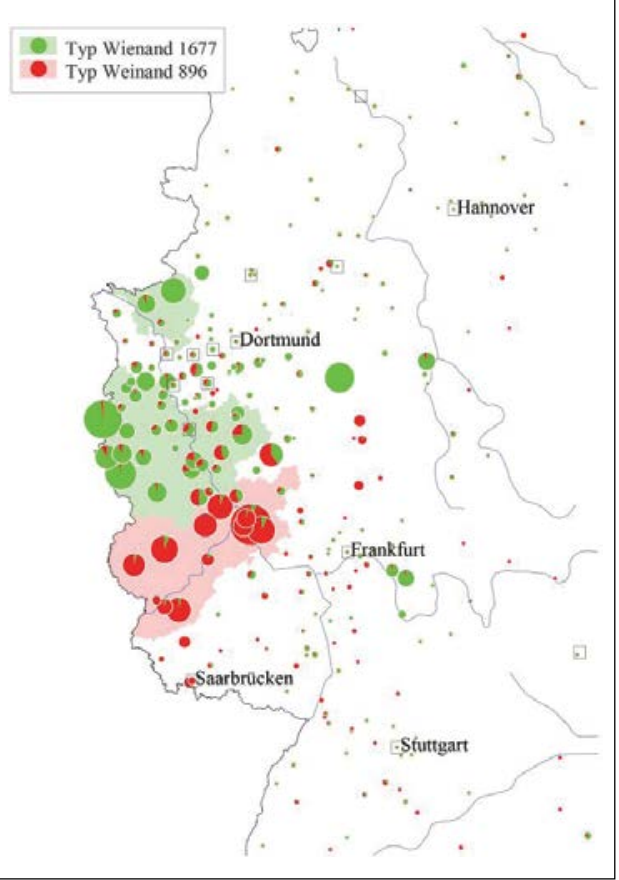

Abb. 2: $\quad$ Wienand/Weinand in Westdeutschland (nach DFA I, K. 171)

Ähnlich steht es mit Patronymen aus Walter. Die räumlich klar ausgeprägte Opposition Walter/Walther (th überwiegt in Thüringen und Sachsen außer der Lausitz, sonst $t$ ) ist in DFA II (Konsonantismus), K. 166 behandelt und die Opposition Walter/Wolter im Kapitel ,Monophthongvarianz $a, o$ “ in DFA I (Vokalismus), auch für genitivisches Walters/ Wolters, kontrahiertes Wahler(s)/Wohler(s) sowie für die Kurzformen Walz/Wolz (K. 33, $34,36,37)$. Hier wurden auch Varianten mit Umlaut des Stammvokals (Welter/Wälter) in den Kartenkomplex mit einbezogen, weil die umgelauteten Fälle eine Lücke im westmd. Raum schließen, die sich zwischen den Wolter-Vorkommen im Norden und den WalterVorkommen im Süden auftut. Im DFA ist Welter/Wälter eine eigene Karte gewidmet (DFA I, K. 35), auf der die Konzentration dieser Namen links des Rheins eindrucksvoll

Niederlande: Wijnand(t)s 3442+98, Wijnant(s) 38+429, Wijnand 280; Wynand(t)s 49+7, Wynant(s) $<5+28$; Winand(t)s 151+7, Winant(s/z) 83+5, Wienand(s) 6+5, Wienandts $<5$; Weinand(s) 100+16, Weynants $<5$ (Einwohner 2007, www.meertens.knaw.nl, 28.06.12).

11 Winand(y/i) 240+3, Winands 1, Wynandi 1; Weinand(t) 32+22, Weynand(t) 6+43, Weinandy 23 (Einwohner 1930, Institut Grand-Ducal 1989, 400, 407, 411, 417). 
zutage tritt; auf Abb. 3 werden die umgelauteten Fälle ${ }^{12}$ in ihrer Relation zu den nicht umgelauteten dokumentiert.

Typ Walter 46123:

Walt(h)er 33659+276, Walt(h)ers $12185+3$.

Typ Wolter 12856 :

Wolt(h)er $9115+3708$, Wolt(h)ers $30+3$.

Typ Welter 2810:

Welt(h)er 2324+172, Welters 314.

Typ Wälter 153:

Wält(h)er 136+13, Waelt(h)er 3+1.

Kartentyp: relativ; Ausschnitt Westdeutschland; Kreise pro dreistellige PLZ, entspricht 0,53-8,07\%; Tokenangaben beziehen sich auf Gesamtdeutschland.

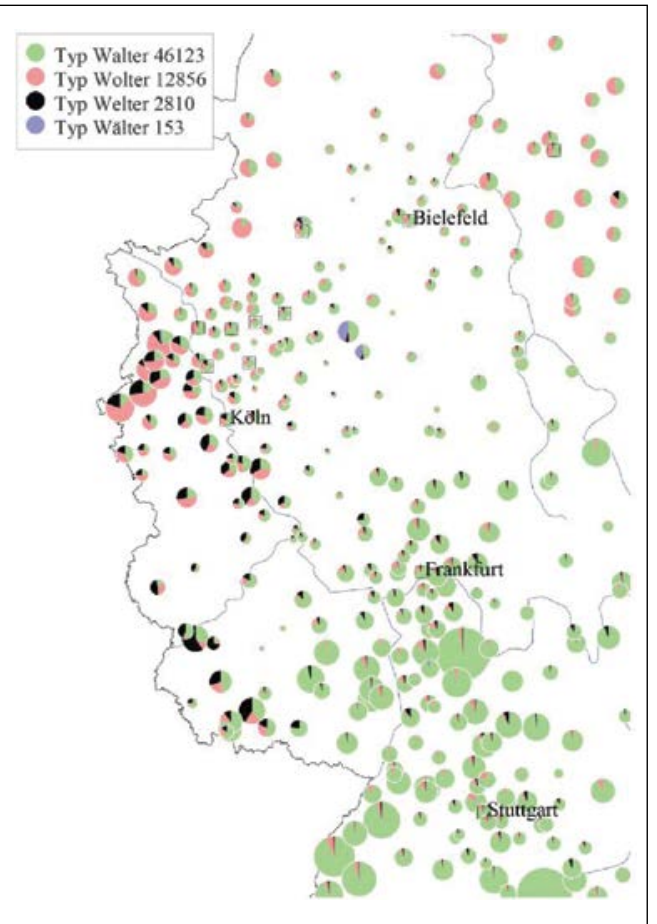

Abb. 3: $\quad$ Walter/Wolter/Welter/Wälter in Westdeutschland (nach DFA I, K. 33 und 35)

In Luxemburg stand Welter im Jahr 1930 an 15. Stelle der häufigsten Familiennamen. Walt(h)er und Wolter(s) erreichten hier zusammen nicht ein Drittel seines Vorkommens. ${ }^{13}$ In Deutschland stehen dagegen Walter, Walther und Wolter auf den Rängen 37, 146 und 219 der frequentesten Familiennamen. Mit den zitierten Karten sind die häufigsten Ausfaltungen des Namens Walter ausreichend im Grammatikteil des DFA dokumentiert. Der Fall Walter braucht also nicht mehr im Patronymenband behandelt zu werden.

Anders steht es, um ein weiteres für den linksrheinischen Raum relevantes Beispiel aufzugreifen, im Fall Lambert. Der heilige Lambert war an der Wende vom 7. zum 8. Jahrhundert Bischof von Maastricht und ist Stadtpatron von Lüttich. Die mittelalterlichen und frühneuzeitlichen Kultstätten des Heiligen konzentrieren sich in den heutigen südlichen Niederlanden, in Belgien, Luxemburg und im linksrheinischen Deutschland,

12 Wölter 22 ist verstreut im mittel- und niederdeutschen Gebiet verbreitet und wird aufgrund seiner geringen Frequenz nicht auf der Karte erfasst.

13 Welter 1097, Wolter(s) 304+1, Walt(h)er 36+15 (Einwohner 1930, Institut Grand-Ducal 1989, 394, 403, 416, 424). 
s. Abb. 4. Entsprechend oft dürfte er hier als Namenspatron gewählt worden sein, was sich wiederum in den Patronymen niederschlagen musste.

Einige Patronyme aus der Vollform Lambert sind in DFA II (Konsonantismus) aufgegriffen, zunächst unter dem Gesichtspunkt der Assimilation von $m b>m m$ (K. 9: Lambert, Lammert, Lamert). Dabei erscheinen die betreffenden Patronyme insgesamt erwartungsgemäß vor allem am Nordwestrand von Deutschland (Abb. 5).

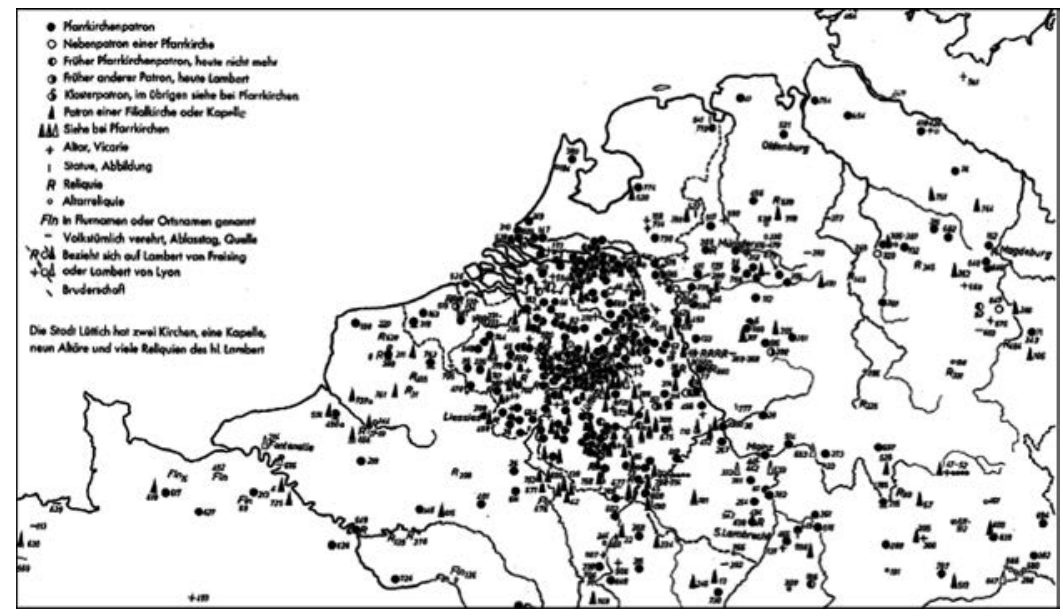

Abb. 4: Verbreitung der Verehrung des heiligen Lambert von Lüttich (Ausschnitt aus Zender 1959, Karte 1)

Typ Lambert 4755:

Lambert 1509, Lamber $(t) z$ 88+1216, Lampert 991, Lamber $(t) \mathrm{s} 403+115$, Lempert 278, Lembert 93, Lempertz 49, Lampertz 13.

Typ Lammert 3826: Lammer $(t) s$ 2422+20, Lammert 909 , Lammer $(t)$ z 40+203, Lemmert 124, Lemmerz 86, Lämmert 22.

Typ Lamert 1080:

Lamers 887, Lamer $(t) z 50+79$, Lamert 64.

Kartentyp: relativ; Kreise pro dreistellige PLZ, entspricht $0,02-4,34 \%$.

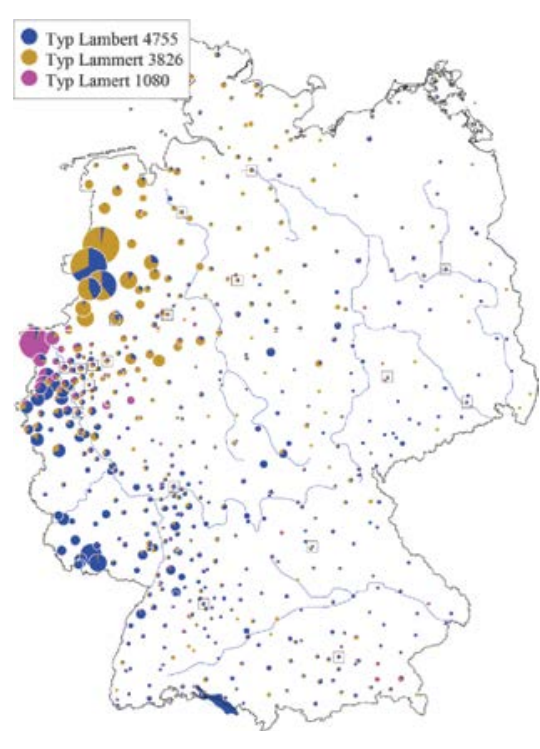

Abb. 5: $\quad$ Lambert/Lammert/Lamert in Deutschland (nach DFA II, K. 9) 
Die Vollformen ohne Assimilation setzen sich zahlreich nach Westen hin in Belgien und Luxemburg fort.

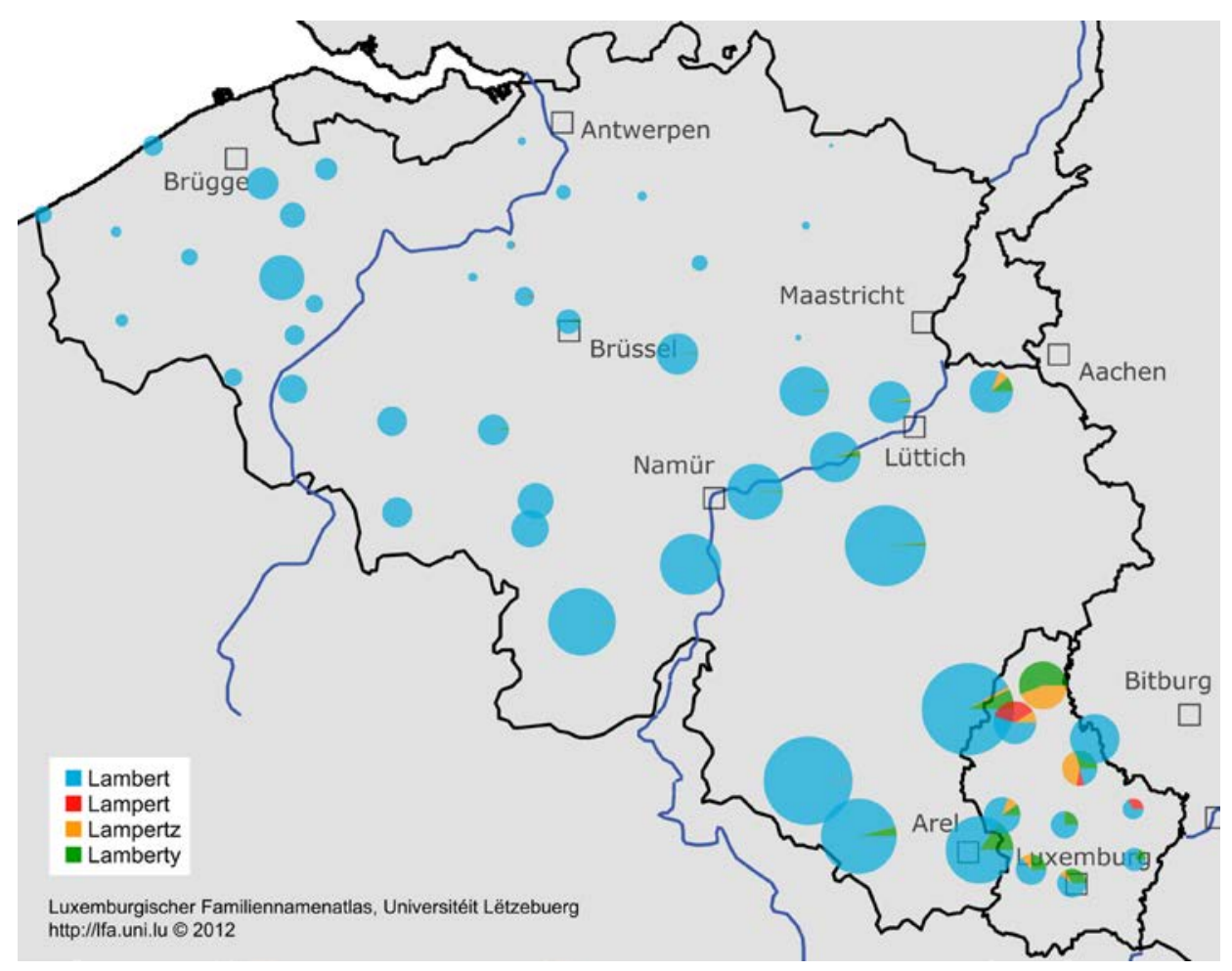

Abb. 6: Lambert, Lampert, Lampertz, Lamberty in Belgien (Kartierung: Luxemburgischer Familiennamenatlas)

Völlig anders sind die mit $k$-Diminutivsuffix versehenen Kurzformen vom Typ Lembke, Lemke gelagert (DFA II, K. 10, hier Abb. 7). Sie finden sich weit entfernt von den Vollformen und markant im Nordosten konzentriert.

Damit stellt sich die Frage, ob die so unterschiedlichen Verbreitungsgebiete sprachlich bedingt sind, etwa durch das $k$-Suffix, oder ob sie auf außersprachliche Faktoren zurückgehen. Eine weitere Lambert-Karte, die sich im Kapitel „Position von $r$ in Patronymen mit -bert und -brecht" im DFA findet (II, K. 394: Lambert, Lambrecht, hier Abb. 8), könnte zu folgender Hypothese führen: Typ Lambrecht ist im Unterschied zu Typ Lambert vor allem im östlichen Deutschland verbreitet. Die Diminutiva Lem(b)ke dürften also wohl eher auf Lambrecht als auf Lambert zurückgehen. Die Frage wäre, ob der heilige Bischof eventuell nur für den Namentypus Lambert Pate stand, nicht aber für den Typus Lambrecht, und in diesem, vom Kult des Heiligen ganz unberührt, der germanische Rufname weiterlebt, und ebenso in Lem(b)ke. 
Um solche Fragen zu verfolgen, wären noch andere Karten zu Patronymen aus Lambert nötig, etwa zu den zahlreichen latinisierten Namen vom Typ Lambertus und Lamberti oder zu den Kurzformen der Typen Lampe oder Lande, zu suffigierten KurzformenTypen wie Lamping, Lämpel oder Lanz. Das heißt, dass der Fall Lambert (im Unterschied zu den vorigen Fällen) nicht in den Grammatikbänden erschöpft ist und im Patronymenband aufgegriffen werden muss. ${ }^{14}$

Typ Lembke 1815:

Lemb(c)ke 1176+398,

Lempke(n) 151+24,

Lampke 66.

Typ Lemke 8761:

Lem (c)ke 7777+661,

Lemken 127, Lamke(n)

58+64, Lemkens 44,

Lemmke 16, Lämke 14.

Kartentyp: relativ; Ausschnitt Norddeutschland; Kreise pro dreistellige PLZ, entspricht 0,02-3,73\% ; Tokenangaben beziehen sich auf Gesamtdeutschland.

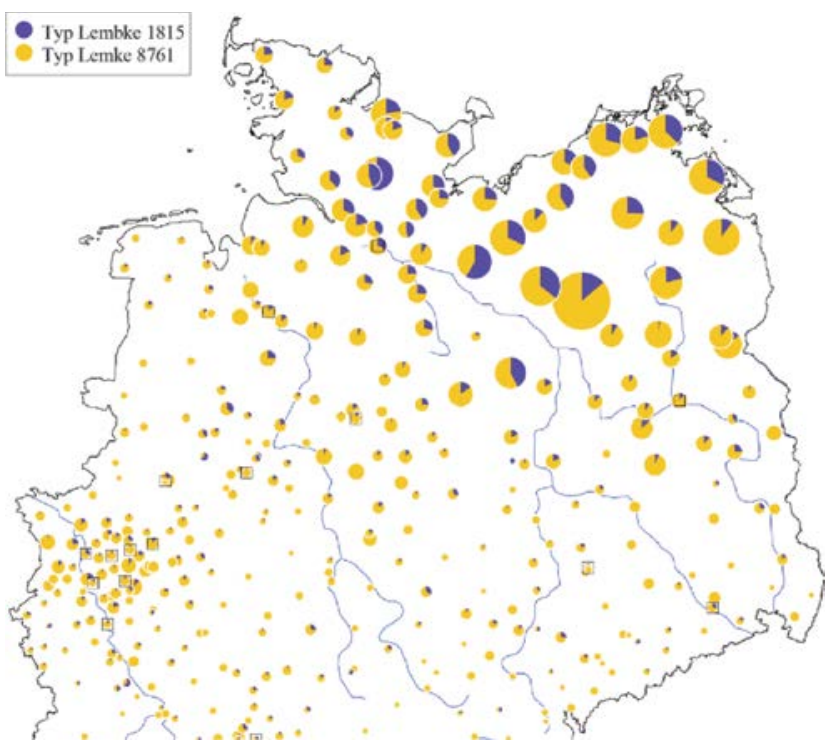

Abb. 7: $\quad$ Lembke/Lemke im nördlichen Deutschland (nach DFA II, K. 10)

14 Der entsprechende Kartenkomplex könnte folgendermaßen aufgebaut werden:

Titel

Hauptkarte

1. Nebenkarte

2. Nebenkarte

3. Nebenkarte

4. Nebenkarte

5. Nebenkarte

6. Nebenkarte

7. Nebenkarte

8. Nebenkarte
Lam[bert]

Typen Lambert, Lambertus, Lamberty, Lambertz

Typen Lambrecht, Lamprecht

Typen Lammert, Lammertz, Lemmert, Lemmertz

Typen Lammer, Lammers, Lemmer, Lemmers

Typen Lampe, Lamp, Lampen, Lamping, Lampmann

Typen Lemme, Lemm, Lemmen

Typen Lembke, Lemke, Lämpel, Lampl

Typen Lande, Land, Landes

Typen Lanz, Lantzsch 
Typ Lambert 4397 :

Lambert 1509, Lambertz 1216,

Lampert 991, Lambers 403,

Lempert 278.

Typ Lambrecht 3952:

Lamprecht 2372, Lambrecht 1580.

Kartentyp: relativ; Kreise pro dreistellige PLZ, entspricht 0,02-2,90\%o; Flächen pro zwei-

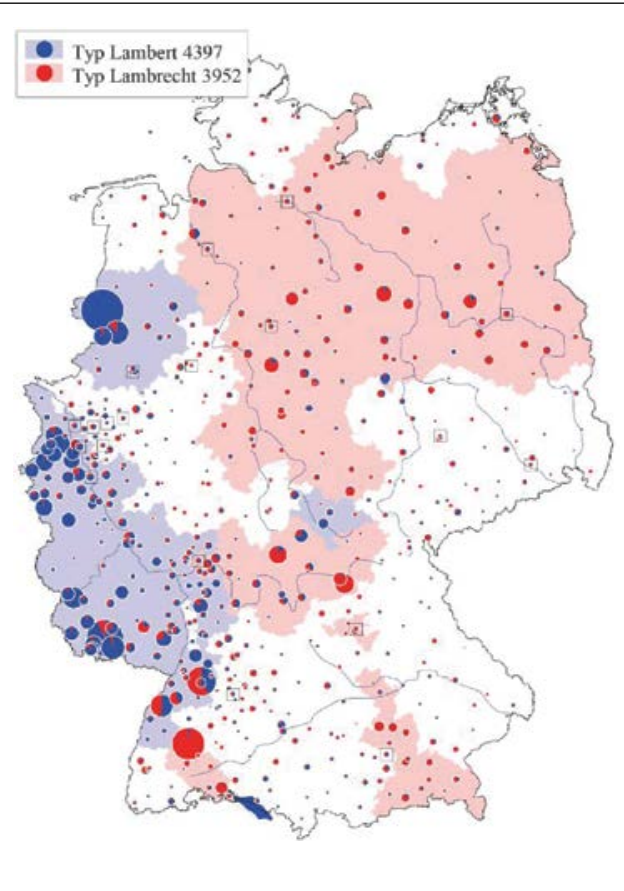

Abb. 8: $\quad$ Lambert/Lambrecht in Deutschland (nach DFA II, K. 394)

Mit den hier genannten Auswahlkriterien lässt sich folgender Entwurf eines Inhaltsverzeichnisses für den Patronymenband erstellen: Teil I enthält (vorläufig) 36 dithematische männliche Ausgangslemmata germanischer Herkunft, alphabetisch von Adel[bert] bis Wil[helm] geordnet. Die eckige Klammer besagt, dass auch andere Rufnamen mit Erstglied Adel- einbezogen werden müssen, weil bei Kurzformen wie App nicht mehr zu klären ist, inwieweit sie aus Adelbert, -bold oder -brand usw. entstanden sind. Ein zusätzlicher Kartenkomplex gilt den wenigen monothematischen Ausgangsnamen germanischer Herkunft wie Ernst oder Karl. Teil II enthält (vorläufig) 20 männliche Ausgangslemmata fremdsprachiger Herkunft von Adam bis Urban. Teil III gilt weiblichen Ausgangsnamen wie Otilie oder Margareta.

\section{Patronyme aus germanischen Rufnamen}
1 Adel[bert]
2 Arn[old]
9 Engel[bert]
16 Heidenreich
3 Bern[hart]
10 Fried[rich]
17 Heil[wig]
4 Bert[old]
11 Geb[hart]
18 Hein[rich]
5 [Rein]bot
12 Ger[hart]
19 Helm [mut]
6 [Hilde]brand
13 Gis[brecht],
$20 \mathrm{Her}$ [bert]
7 Diet[rich]
Giesel[her]
$21 \mathrm{Kon}[\mathrm{rad}]$
8 Eber[hart]
14 Gott[fried]
22 Lam[bert]
15 Hart[mann]
23 Leon[hart] 


$\begin{array}{lcc}24 \text { Lieb[hart] } & 30 \text { Rein[hart] } & 35 \text { Wig[bert] } \\ 25 \text { Liut[bald] } & 31 \text { Rüdi[ger], } & 36 \text { Wil[helm] } \\ 26 \text { Mark[wart] } & \text { Ru[precht] } & 37 \text { Monothematische Ruf- } \\ 27 \text { Mein[hart] } & 32 \text { Sieg[fried] } & \text { namen (Ernst, Karl) } \\ 28 \text { Rade[bold] } & 33 \text { Stein[hart] } & \\ 29 \text { Reich[hart] } & 34 \text { Traut[wein] } & \end{array}$

\section{Patronyme aus fremsprachigen Rufnamen}

$\begin{array}{lll}1 \text { Adam } & \text { 8 Johannes } & 15 \text { Petrus } \\ 2 \text { Ägidius } & 9 \text { Kilian } & 16 \text { Philippus } \\ 3 \text { Augustinus } & 10 \text { Laurentius } & 17 \text { Sebastianus } \\ 4 \text { Balthasar } & 11 \text { Marcus } & 18 \text { Stanislaw } \\ 5 \text { Cornelius } & 12 \text { Mauritius } & 19 \text { Thomas } \\ 6 \text { Franziscus } & 13 \text { Michael } & 20 \text { Urbanus } \\ 7 \text { Jodocus } & 14 \text { Paulus } & \end{array}$

\section{Metronyme}
1 Aus germanischen
Rufnamen
1.1 Her[gart]
1.2 Otilie
usw.
2 Aus fremdsprachigen
Rufnamen
2.1 Katharina
2.2 Margareta
usw.

Dieser noch sehr vorläufige Entwurf wird nach Inangriffnahme des Bandes durch intensive Recherchen im Einzelnen korrigiert und ergänzt werden müssen. Doch wird aus ihm ersichtlich, dass es möglich sein wird, die Fülle der Patronyme und Metronyme in einer durch klare Kriterien begründbaren Auswahl in einem einzigen Band des DFA exemplarisch zu dokumentieren.

\section{Materialbasis der Kartenkomplexe}

Nach der Festlegung der einzelnen Ausgangsnamen, denen jeweils ein Kartenkomplex gewidmet wird, ergibt sich als weitere Frage, welche aus einem bestimmten Ausgangsnamen entwickelten Patronyme für die Kartierung ausgewählt werden. Wie anfangs erwähnt, können aus manchem Ausgangsnamen hunderte von Familiennamen entstanden sein. Es gilt nun, um die oben unter „Kriterien der Namenauswahl“ beschriebenen „Kernnamen“ herum sinnvoll weitere Varianten zu gruppieren. Da es im Zusammenhang eines Atlasses, der die arealen Grundstrukturen des Namenschatzes aufdecken will, kaum auf vereinzelte Familiennamen oder auf Belege mit nur geringer Häufigkeit ankommt, kann man sich auf die Darstellung frequenter Varianten konzentrieren. Zur Reduzierung der Materialfülle sollen, wie schon in den bisherigen Bänden des DFA praktiziert, vor allem zwei Verfahren dienen: das Operieren mit Frequenzschwellen und die Typenbildung. 
Als Beispiel sollen die vor allem im westmitteldeutschen Raum konzentrierten Patronyme aus dem Rufnamen Valentin dienen. Sie sind mit K. 88 in DFA II (Konsonantismus) und dem zugehörigen Kommentartext schon hinreichend dokumentiert und werden deshalb im Patronymenband nicht mehr behandelt werden. Doch das Beispiel wird hier aufgegriffen, weil es einerseits vor allem den linksrheinischen Raum betrifft und weil sich andererseits mit einer einzigen Suchformel nahezu alle Patronyme aus Valentin in Deutschland aus der DFA-Datenbank ermitteln und sich damit die beiden eben genannten Verfahren gut illustrieren lassen.

Zur Ermittlung der Varianten wird als Suchauftrag der reguläre Ausdruck $(V \mid F)$

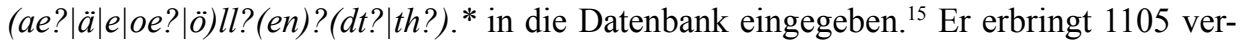
schiedene Namen, darunter viele, die nicht auf Valentin zurückgehen, sondern beispielsweise Herkunfts-/Wohnstättennnamen zu ,Feld“ sind. Von den 1105 ermittelten Namen kommen fast die Hälfte, 520 Namen, nur ein einziges Mal vor und 1006 Namen weniger als 50-mal. Es verbleiben 99 Namen; zieht man davon ausländische Fälle wie italienisch Valentino, Herkunfts-/Wohnstättennnamen zu ,Feld‘ und weitere nicht zu Valentin gehörige und etymologisch unklare Fälle ab, verbleiben nach Einsatz dieses Frequenzfilters von $\geq 50$ Tokens (= Telefonanschlüsse) noch folgende 20 Namen (nach Häufigkeit, absteigend): Valentin 1616, Velten 1354, Felten 872, Velte 577, Faltin 540, Feltes 298, Foltin 279, Felden 241, Vallentin 167, Valtin 136, Faltus 127, Velden 123, Feldges 108, Foltyn 102, Völtl 86, Feltgen 73, Foltys 67, Valentiner 64, Valtinke 63, Feldl $61 .{ }^{16}$

Nun wäre es aber zum einen immer noch zu aufwändig, zum anderen im Sinne der Erfassung von arealen Strukturen des Namenschatzes auch nicht sinnvoll, all diese Namen einzeln zu kartieren. Daher kommt das zweite Verfahren für die Reduzierung des zu kartierenden Materials zur Anwendung, die Typenbildung. Als hauptsächliche Varianzen finden sich in dem eben erstellten Namenkorpus: die Varianz von initialem $V / F$, von a/e als Stammvokal, von $l / l l$, von Erhalt/Schwund der Mittelsilbe -en-, von $t / d$, von $i / e$ in der Endsilbe, von flektierten/unflektierten und von suffigierten/unsuffigierten Namen. Diese Varianzen hängen natürlich teilweise miteinander zusammen. Sie werden daraufhin überprüft, welche von ihnen deutlich voneinander abgesetzte Areale bilden. Dabei ergibt sich, dass Oppositionen wie a/e, Erhalt/Schwund der Mittelsilbe usw. ziemlich willkürlich verteilt und damit nicht kartierenswert sind. Solche nicht kartierenswerten Befunde sowie die Verbreitung nicht kartierter niederfrequenter und/oder konkurrenzbehafteter Namen können im Kommentar der Kartenkomplexe kurz beschrieben werden.

Die deutlichste areale Opposition tritt bei initialem $V / F$ innerhalb des eingedeutschten Typs Velten auf; entsprechend sind in DFA II (Konsonantismus), K. 88 die Einzelnamen zu zwei Typen Velten mit $V$ - und Felten mit $F$-, hier mit einer Tokenschwelle von 10, zusammengefasst und auf der Karte einander gegenübergestellt. ${ }^{17}$ Insgesamt sind diese Namen vor

15 In diesem Suchauftrag bedeuten $(. . \mid \ldots)=$ alternativ; ? = fakultativ; .* = alles, was nachfolgt.

16 Bei den hier aufgezählten Familiennamen sind zum Teil Konkurrenzen möglich, etwa bei Feldl mit Herkunfts- und Wohnstättennamen zu ,Feld', die jeweils im Kommentartext diskutiert werden.

17 Datenbasis von DFA II, K. 88:

Typ Felten 11 Types/2130 Tokens: Fel(t/d)en 872+241, Faltin(s) 540+13, Folt(i/e)n 279+21, Fel(t/d)ens 48+26, Falten 36, Feltin 28, Feldten 26. 
allem links des Rheins konzentriert, darüber hinaus auch in Hessen und im nördlichen Baden anzutreffen. Die Schreibweise mit $F$ - dominiert nördlich der Mosel bei den linksrheinischen Vorkommen, auch am Westrand von Rheinland-Pfalz und im Saarland. In der übrigen Pfalz und bei den rechtsrheinischen Namenvorkommen überwiegt Schreibung mit $V$-. Ein Blick über die deutsche Grenze nach Westen anhand der Beispiele Felten, Faltin, Velten, Valtin ergibt, dass sich dort vor allem die Schreibweise $F$ - durchgesetzt hat (Abb. 9). ${ }^{18}$

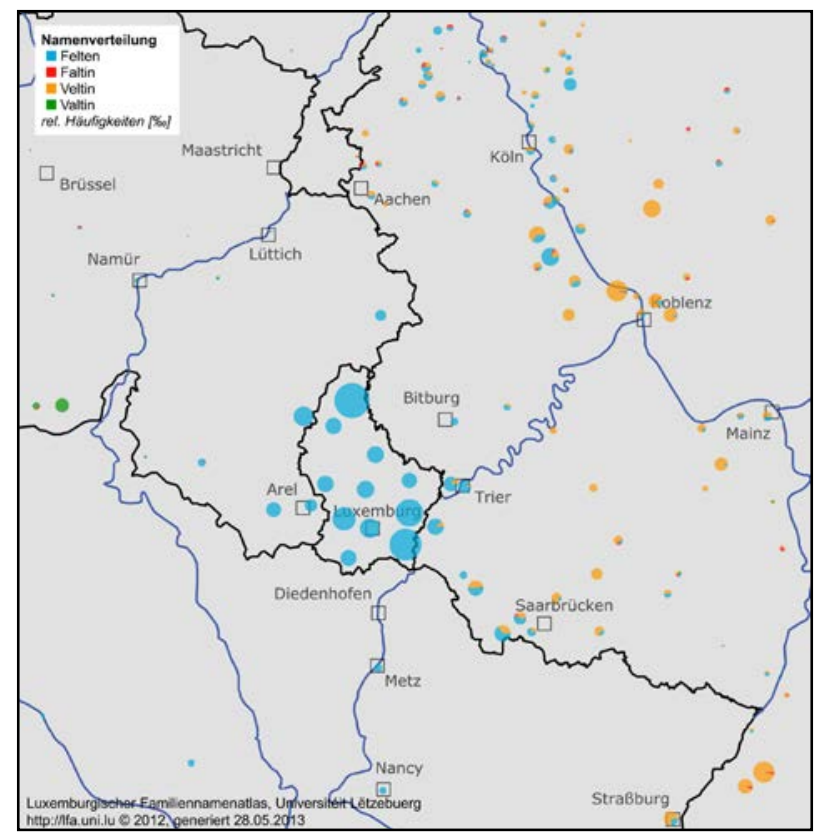

Abb. 9: Patronyme aus Valentin: Felten, Faltin, Velten, Valtin (Luxemburgischer Familiennamenatlas, Online-Kartierung, http://lfa.uni.lu/, 06.07.12)

Typ Velten 8 Types/1704 Tokens: Velten(s) 1354+31,Valt(e/i)n 19+136, Veld(t)en 123+10, Veltin 18, Voltin 13.

Die Verbreitungsgebiete von Velten mit $t$ und Felden mit $d$ decken sich weitgehend, was dafür spricht, beide als Patronyme von Valentin anzusehen und Felden mit $d$ nicht wie bisher üblich als Herkunfts- oder Wohnstättennamen zu ,Feld‘ einzustufen.

18 Felten belegte in Luxemburg im Jahr 1930 Rang 75 der häufigsten Familiennamen (Institut GrandDucal 1989, 425). Valtin wird von Morlet 1991, 950 in Frankreich als Kontraktionsform aus Valetin (zu Valet ,kleines Tal') angesetzt, nur in Lothringen als Patronym zu Valentin. Laut Debrabandere 2003, 1242 ist Valtin in Belgien und Nordfrankreich Patronym zu Valentin. Den lateinnahen Patronymen Val(l)entin 1616+167, Val(l)entien 30+2, Vallenthin 13, Valenthin 11, Valentins 2, die im westlichen Hessen besonders konzentriert, aber auch sonst in ganz Deutschland verbreitet sind, entsprechen mit $F$ - nur Fal(l)entin 13+7 (Nordrhein-Westfalen). Sie sind nicht kartiert, sondern im Kommentartext (ebd., S. 195) aufgeführt. Fragen wie die, warum sich diese Patronyme (innerhalb Deutschlands) insbesondere im Westmitteldeutschen konzentrieren, obwohl der namengebende Heilige Bischof von Passau war und hauptsächlich in Bayern, Österreich und Südtirol verehrt wurde und wird, können im Absatz "Hinweise", der jeden Kartenkomplex abschließt, angesprochen werden. 


\section{Anlage der Kartenkomplexe}

Generell sollen die Namen in den einzelnen Kartenkomplexen so weit wie möglich in der Reihenfolge Vollformen - Reduktionsformen - Kurzformen dokumentiert werden; die Einordnung flektierter und suffigierter Formen erfolgt je nach Materiallage innerhalb dieser Gruppen oder in weiteren, eigenen Gruppen.

Als Beispiel für die Anlage eines Kartenkomplexes sollen im Folgenden die Patronyme aus dem Rufnamen Cornelius dienen. Seine Verbreitung wurde dadurch bestimmt, dass durch Übertragungen von Reliquien des heiligen Papstes (251-253) Cornelius im Frankenreich sein Kult vor allem in dem aus Abb. 10 ersichtlichen Raum populär wurde. Wichtigstes Kultzentrum in Deutschland ist Kornelimünster bei Aachen.

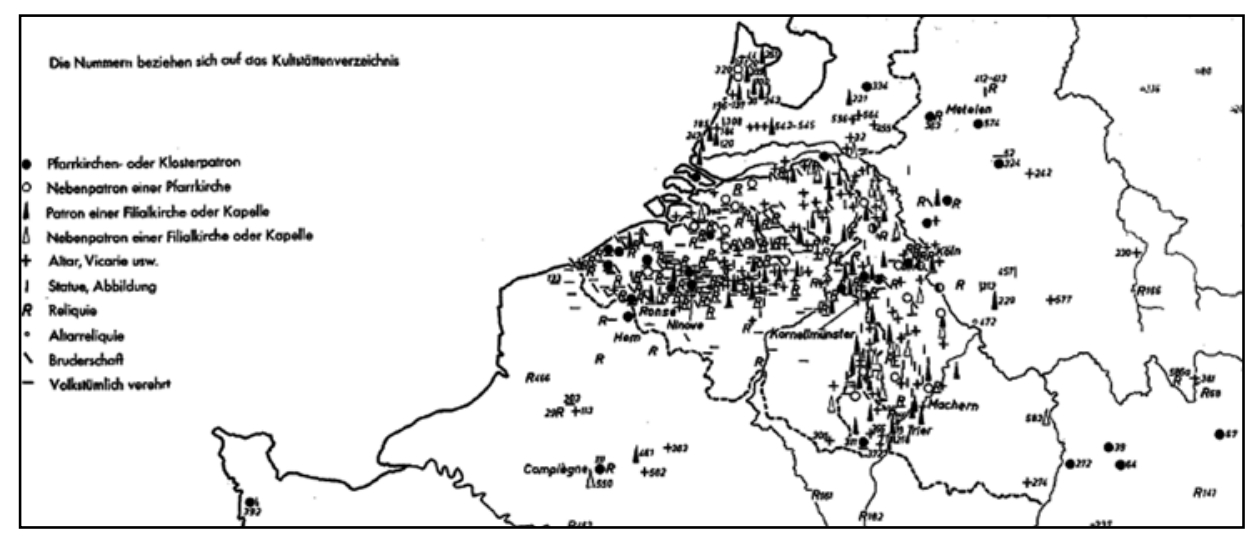

Abb. 10: $\quad$ Verbreitung der Verehrung des heiligen Cornelius (Ausschnitt aus Zender 1959, Karte 8)

Die Hauptkarte des vorgesehenen Kartenkomplexes (Abb. 11) dokumentiert die Verbreitung der Vollformen und bezieht deren flektierte und suffigierte Varianten mit ein. Es ergeben sich folgende räumlich klar voneinander getrennte Typen: Typ Cornelius in Ostfriesland und am Mittelrhein, Typ Cornelissen am Niederrhein, Typ Cornely an der Mosel usw. Mittelrheinisches Cornelius und Cornely setzt sich z.B. auch nach Luxemburg hin fort, ${ }^{19}$ nach Belgien hinein vor allem Cornelis und Cornelissen. ${ }^{20}$

Die nächsten Karten gelten den Patronymen aus Kurzformen. Bei ihnen zeichnen sich zwei weit voneinander getrennte Verbreitungszentren ab, wenn man die Kurzformen in Typen mit kurzem bzw. mit langem Stammvokal sortiert. Links des Rheins konzentrieren sich die kurzvokalischen Typen (Abb. 12): Nelles im Raum Linnich - Köln - Bad Neuenahr-Ahrweiler - Üxheim, dann der genitivische Typ Nellessen, der am Westrand von Deutschland von Kleve bis Monschau bzw. Bonn herrscht. Im Dreieck Duisburg - Mönchengladbach - Bonn findet sich Nellen, welches patronymischer schwacher Genitiv zu

19 Corneli(u)s 1+47, Cornel(y/ie) 20+2 (Einwohner 1930, Institut Grand-Ducal 1989, 61).

20 Cornelis 6743, Cornelisse(n) 9+1458, Cornely 89, Cornel(l) 50+4, (C/K)ornelius 16+1, Carniel 8, Cornielje 1 (Einwohner 1998, www.familienaam.be, 02.07.2012). 
Nell 698 sein dürfte. Nell tritt im Westmitteldeutschen, aber auch nördlich des Bodensees auf und kann Patronym aus Cornelius, aber auch Übername zu mhd. nël(le) ,Spitze, Scheitel, Kopf ${ }^{*}$ sein (Duden Familiennamen 2005, 475). Im Westmitteldeutschen dürfte Ersteres, im oberdeutschen Raum Letzteres gelten. An der Mosel und südlich davon dominiert Nilles, in Westfalen Nillies, in denen der Stammvokal durch ehemaliges -ius von $e$ zu $i$ erhöht wurde. Vor allem Nilles setzt sich nach Luxemburg hinein fort; es belegte hier im Jahr 1930 Rang 41 der häufigsten Familiennamen. ${ }^{21}$ In Belgien finden sich dagegen die auf Abb. 12 kartierten Familiennamen kaum. ${ }^{22}$

Typ Cornelius 1654:

(C/K)ornelius $1550+104$

Typ Cornely 443:

(C/K)ornely 217+57, (C/K)orneli 51+106,

Kornelli 12,

Typ Cornelis 48:

Cornelis.

Typ Cornelissen 349:

Cornelisse(n) 14+241, Corneli( $\beta / s)$ en $78+16$.

\section{Typ Cornelissen 432:}

Cornelsen 276, Kornels(e/o)n 86+2,

Cornel( $\beta /$ ss)en $25+22$.

Typ Cornehl 392:

(C/K)ornehl 175+11, (C/K)ornel 70+29,

(C/K)ornell 44+41, Carnehl 12, Corneel 10.

Typ cornils 296:

cornils.

Kartentyp: relativ; Kreise pro dreistellige PLZ, entspricht $0,02-2,17 \%$.

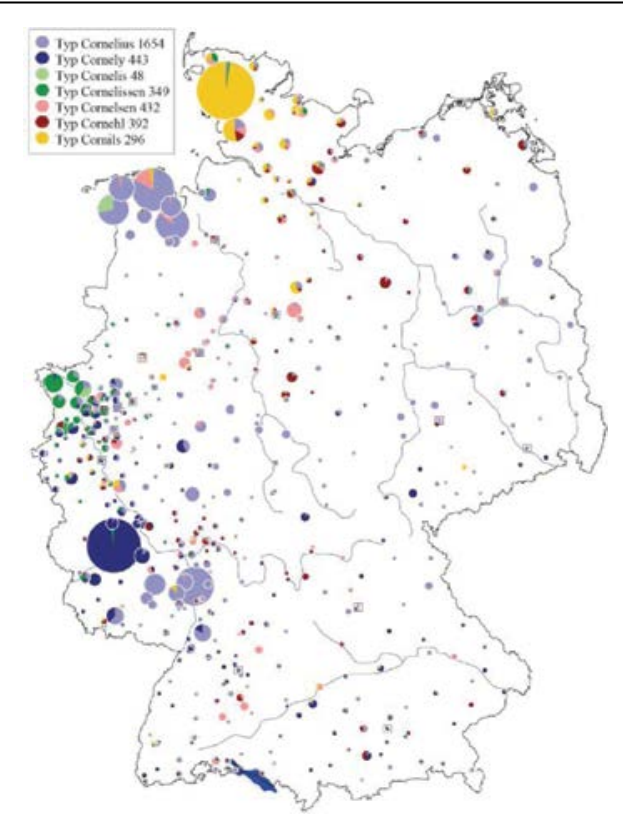

Abb. 11: Cornelius/Cornely/Cornelis/Cornelissen/Cornelsen/Cornehl/Cornils in Deutschland (Kartierung: DFA)

Völlig anders gelagert erscheinen dagegen die langvokalischen Typen Nehls und Nehlsen (Abb. 13). Es besteht kein Zweifel, dass es sich auch bei ihnen um Kurzformen von Cornelius handelt. ${ }^{23}$

21 Nilles 561, Nell(en) 12+5, Nelles(sen) 1+1 (Einwohner 1930, Institut Grand-Ducal 1989, 267, 272).

22 Von den kartierten Namen nur Nilles 57 (Einwohner 1998, www.familienaam.be, 04.10.2010).

23 Hinzu kämen eventuell noch Anteile der Vorkommen von Nie(h)ls 79+12, Nils 15 sowie Ni(e)lsen 30+1905, Nils(s)on 118+276, Niels(s)on 59+30. Erstere sind hauptsächlich im Raum Hamburg und in Holstein konzentriert, Letztere massiv in Schleswig(-Holstein) und vor allem in Skandinavien. Diese Namen werden gewöhnlich als Patronyme zu Nikolaus gedeutet, was auch durch Gleichungen wie Nicolaus $=$ Niels 1598 in Flensburg (Bahlow 2005, 357) belegt ist. In Dänemark urkundet 
Typ Nelles 1027:

Nelles 994, Nelli(e)s 18+15.

Typ Nellessen 633:

Nelles(s)en 91+331, Nelleßen 81,

Nellissen 82, Nellißen 48.

Typ Nellen 363:

Nellen.

Typ Nilles 507:

Nilles 370, Nillies 137.

Kartentyp: relativ; Kreise pro dreistellige PLZ, entspricht $0,01-2,01 \%$.

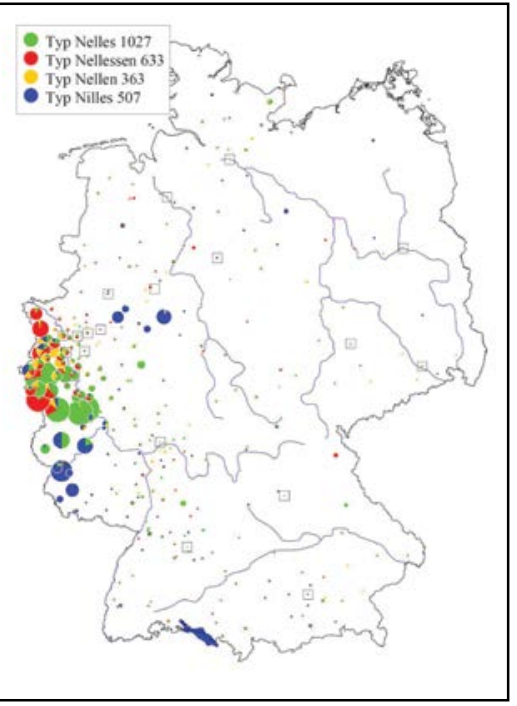

Abb. 12: Nelles/Nellessen/Nellen/Nilles in Deutschland (Kartierung: DFA)

Typ Nehls 1495:

Nehl(e)s 1274+22, Neels 199 .

Typ Nehlsen 374:

Nehlsen 249, Neelsen 125.

Kartentyp: relativ; Kreise pro dreistellige PLZ, entspricht $0,01-1,37 \%$

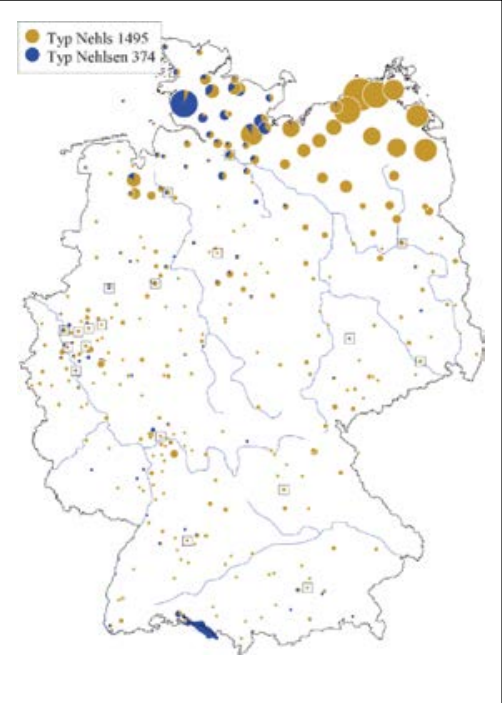

Abb. 13: Nehls/Nehlsen in Deutschland (Kartierung: DFA)

Bischof Nikolaus von Aarhus auch als Niels von Aarhus. Andererseits legt die Verbreitung von Cornils 296 (s. Abb. 11) und Cornilsen 11 in Schleswig-Holstein und Hamburg die Möglichkeit nahe, dass Familiennamen wie Niels und Nielsen in manchen Fällen durchaus auf Kurzformen von Cornelius zurückgehen können (vgl. Dräger 2013, 246-249). Die Frage nach den Hintergründen der Raumbildungen auf Abb. 11, 12 und 13 ist noch offen. 
Weitere Karten könnten wie Abb. 14 mit Nilius, Nelius, Nilges, Nilgen, Nelgen, Nielen und Nie(h)l die Verbreitung weiterer, weniger frequenter Patronyme aus Kurzformen von Cornelius dokumentieren.

Nilius 231

Nelius 176

Nilges 305

Nilgen 110

Nelgen 20

Nielen 134

Nie(h)l $59+35$

Kartentyp: absolut; Kreise pro dreistellige PLZ,

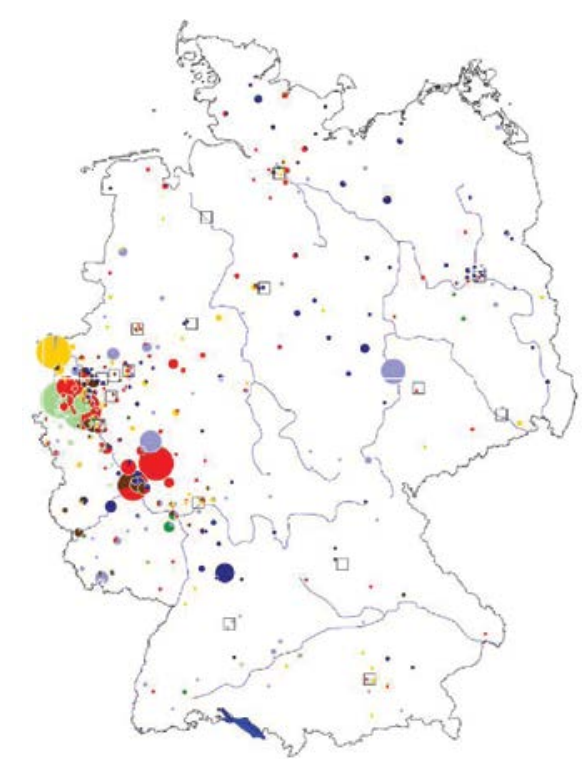
entspricht 1-38 Tokens.

Abb. 14: Nilius/Nelius/Nilges/Nilgen/Nelgen/Nielen/Nie(h)1 in Deutschland (Kartierung: DFA)

\section{Zwei Merkmale linksrheinischer Patronyme}

Abschließend sollen noch zwei Phänomene angesprochen werden, die bei den vorangehenden Beispielen wiederholt als Charakteristika des hier behandelten Raums auftraten. Zum einen hat sich in den Familiennamen besonders häufig der patronymische Genitiv verfestigt. Das gilt zwar auch für deappellativische Familiennamen wie Schulten, Meyers oder Langen, doch Patronyme sind davon in einem besonderen Maß betroffen. Sie treten etwa bei den bisher besprochenen Beispielen im Fall Lambert als Lamber $(t)(s / z)$, Lampen und Lemmen auf, im Fall Cornelius als Cornelissen, Nellessen und Nellen. Der schwache Genitiv auf -en in Patronymen zeigt in Deutschland nach Ausweis von DFA III, K. 20 ein klar umrissenes Hauptverbreitungsgebiet nördlich des Hunsrückkamms und westlich des Rheins sowie ein zweites, kleineres in Ostfriesland und den angrenzenden Regionen. Das deutsche -en-Gebiet setzt sich dabei in den angrenzenden niederländischen und belgischen Regionen fort. ${ }^{24}$ In Luxemburg

24 Marynissen 1994, 270-293; Marynissen 2010, 28f.; Marynissen/Nübling 2010, 331-337. - In den Niederlanden finden sich beispielsweise Heijnen 4973, Heinen 2051, Heynen 255, Hijnen 184 
finden sich im Jahr 1930 unter den häufigsten 100 Familiennamen sechs Patronyme im schwachen Genitiv: Position 20 Hansen ( $<$ Johannes), 31 Theisen $(<$ Matthias/ Matthäus), 40 Goergen (< Georg), 44 Heinen (< Hein[rich]), 96 Grethen (Metronym $<$ Margaretha) und 100 Gillen ( $<$ Aegidius). ${ }^{25}$

Hansen 15258

Theisen 1960

G(ö/oe)rgen $1296+243$

Heinen 3833

Grethen 50

Gillen 206

Kartentyp: relativ; Ausschnitt Westdeutschland; Kreise pro fünfstellige PLZ, entspricht 0,05-30,09\%; Tokenangaben beziehen sich auf Gesamtdeutschland.

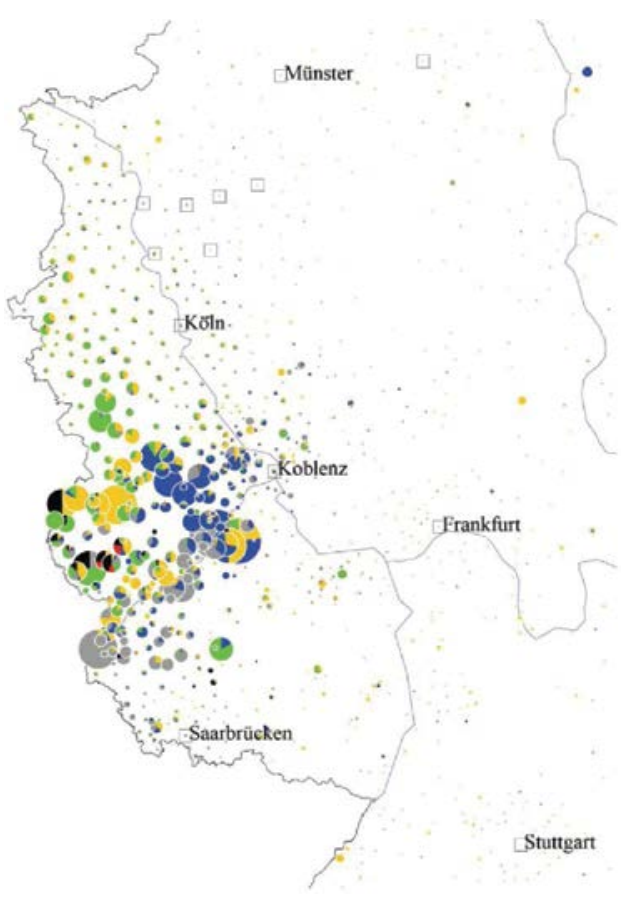

Abb. 15: Hansen/Theisen/G(ö/oe)rgen/Heinen/Grethen/Gillen in Deutschland (Kartierung: DFA)

Abb. 15 zeigt, wie weit sich das Verbreitungsgebiet dieser Familiennamen in Deutschland fortsetzt. ${ }^{26}$ Die Patronyme im schwachen Genitiv stellen eines der markantesten Kennzeichen des hier zur Debatte stehenden Raums dar. Ihr Verbreitungsradius bedarf noch einer Erklärung. ${ }^{27}$

(Einwohner 2007, www.meertens.knaw.nl, 05.07.12), in Belgien Heinen 1079, Heynen 685, Hynen 6 (Einwohner 1998, www.familienaam.be, 05.07.12).

25 Institut Grand-Ducal 1989, 424-426.

26 Während das Hauptverbreitungsgebiet von Theisen, G(oe/ö)rgen, Heinen, Grethen und Gillen im auf der Karte gezeigten Raum liegt, finden sich bei Hansen in diesem Raum nur ca. $40 \%$, aber rund $50 \%$ weit entfernt in Schleswig-Holstein, wo es sich aber nicht um den schwachen Genitiv auf -en, sondern um das Suffix -sen handelt, s. DFA III, K. 84. Die restlichen Vorkommen sind verstreut und auf Bevölkerungsmobilität zurückzuführen. Vgl. auch Heuser/Nübling 2010, K. 8 (Otten), K. 10 (Cornelissen, Gillissen u.a. am Niederrhein); Steffens 2008, K. 2 (Thelen).

27 Besonders zu beachten wäre hier neben patronymischer Motivation des Genitivs (, des Heinen Nachkomme') die Rolle von Hofnamen (,der aus/in des Heinen Hof'). 
Der starke Genitiv auf $-s$ hat in Familiennamen ein weniger eng begrenztes Verbreitungsgebiet als das des Genitivs auf -en. In Deutschland ist dieser Namentyp im gesamten Norden sowie im äußersten Westen nördlich der Mosel konzentriert, s. DFA III, K. 6-19, 88-92. Er setzt sich ebenfalls in den angrenzenden niederländischen und belgischen Gebieten fort und hat seine südwestlichsten Ausläufer in Luxemburg. Die frequentesten Patronyme mit Genitiv-s in Luxemburg sind Peters (vgl. Abb. 17), Michels und Wilmes, im Jahr 1930 auf Rang 62, 68 und 97 der häufigsten Familiennamen. ${ }^{28}$

Charakteristisch für westmitteldeutsche Patronyme ist zum anderen die Latinisierung, vor allem durch die Versetzung deutscher Familiennamen in den lateinischen Genitiv. ${ }^{29}$ So tritt in den hier bisher erwähnten Beispielen der Genitiv Lamberti, Lamberty einerseits in Ostfriesland, andererseits vor allem im Raum Trier und in der westlichen Eifel konzentriert auf. ${ }^{30}$ Aus Winand (Abb. 2) latinisiert ist Winandy, das 1930 Rang 163 unter den häufigsten Namen in Luxemburg einnahm; und unter den 100 häufigsten Familiennamen dieses Landes finden sich die lateinischen Genitive $\mathrm{Ja}$ coby, Huberty und Pauli auf den Rängen 51, 52 und 73. ${ }^{31}$ Abb. 16 fasst die Verbreitung lateinischer Genitive in sieben häufigen Fällen (mit ihren Schreibvarianten) zusammen: Jacobi, Petri, Pauli, Nikolai, Caspari, Conradi und Wilhelmi. Sie konzentrieren sich in den Regionen am mittleren Rhein und nehmen nach Westen hin kontinuierlich zu. ${ }^{32}$ Diese Zunahme gilt nicht nur für den Typus insgesamt, sondern auch speziell für die Vorliebe, den lateinischen Genitiv nicht mit $-i$, sondern mit $-y$ zu schreiben, wie aus Abb. 16 deutlich hervorgeht. Bei den drei oben genannten häufigsten luxemburgischen Fällen beträgt im Jahr 1930 das Verhältnis $-y$ : $-i 1371$ : 10, hier erreicht also $-y$ einen Anteil von über $99 \%$.

28 Institut Grand-Ducal 1989, 424f.

29 Zur Latinisierung s. DFA III, K. 338-342 (ins Lateinische übersetzte Berufsnamen), K. 343-348 (lateinische Nominativendung -us in Patronymen), K. 349-355 (lateinische Genitivendung $-i$ in Patronymen), K. 356-360 (lateinische Genitivendungen -is und -ae in Patronymen), K. 361-365 (lateinisches Suffix -ius).

30 Diese Lage und die häufige Schreibung mit $-y$ spricht dafür, dass hier Familiennamen italienischer Herkunft nur zu geringem Anteil zu veranschlagen sind, anders als bei Valentini, das in Deutschland verstreut vorkommt und zweifellos meist auf Einwanderung aus Italien beruht. Lamberti wie Valentini treten in Italien tausendfach auf.

31 Institut Grand-Ducal 1989, 425-427.

32 Dasselbe Latinisierungsareal wie auf Abb. 16 zeichnet sich in Deutschland auch bei lateinischen Nominativendungen ab, etwa bei germanischen Namen im Fall Hubertus, bei lateinischen Namen im Fall Paulus, DFA III, K. 343 und 347. Auffällig ist dabei, dass in Luxemburg Paulus reichlich, Hubertus aber 1930 kein einziges Mal auftritt. Der ungrammatische Genitiv Michaely findet sich in Luxemburg 46-mal, Michaeli einmal, häufiger ist Mich(a)elis 19+86 (Institut Grand-Ducal 1989, 254). In Deutschland ist Michael(y/i) 144+127 im Saarland konzentriert und darüber hinaus verstreut bis zum Rhein (DFA III, K. 356). Im westmitteldeutschen Raum gibt es außerdem zahlreiche latinisierte Familiennamen, die nicht auf Rufnamen zurückgehen; vgl. in Luxemburg unter den 100 häufigsten Familiennamen im Jahr 1930: Rang 18 Faber (< Schmidt u.ä.), 22 Majerus (< Maier u.ä.), 28 Molitor (< Müller, Möller; Institut Grand-Ducal 1989, 424). 


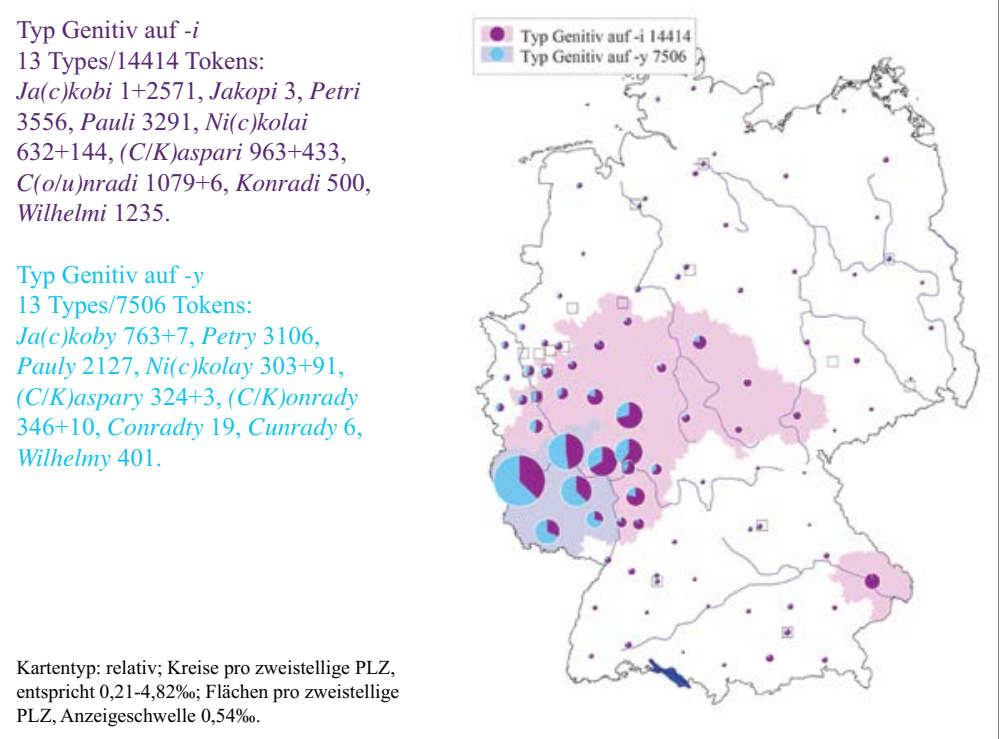

Abb. 16: Genitiv auf-i/Genitiv auf -y in Deutschland (Kartierung: DFA)

Wieder bleibt die Frage nach den Hintergründen der Verbreitung dieser Phänomene gerade in dieser Region noch zu klären. Die räumliche Verbreitung lateinischer Genitive kann nicht durch die Verbreitung deutscher Genitive bedingt sein, vor allem deswegen nicht, weil die Areale deutscher und lateinischer Genitive sich nur peripher überschneiden, wie etwa aus dem Beispiel Peters versus Petr(i/y) in Abb. 17 ersichtlich ist.

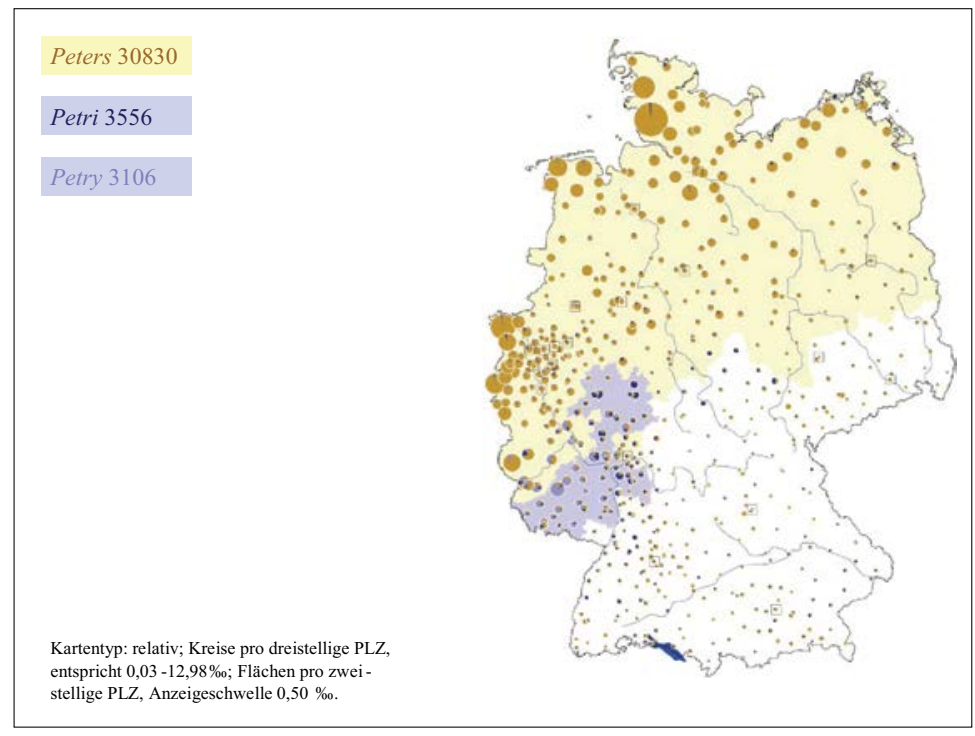

Abb. 17: Peters/Petri/Petry in Deutschland (Kartierung: DFA) 
Trotz der wenigen Beispiele, die hier angeführt werden konnten, dürfte dreierlei deutlich geworden sein: erstens, wie unerlässlich und ergiebig es ist, familiennamengeographische Forschung über heutige Landesgrenzen hinweg zu betreiben; zweitens, wie deutlich dabei die Region zwischen Rhein und Maas auch im Bereich der Patronyme in sprachlicher wie in außersprachlicher Hinsicht als einheitlicher Kulturraum hervortritt; drittens, dass und wie es möglich sein könnte, die Strukturen des überreichen Patronymenschatzes im beschränkten Rahmen nur eines einzigen DFA-Bandes zu konturieren und repräsentativ zu dokumentieren.

\section{Literatur}

Bahlow, Hans: Deutsches Namenlexikon, Familien- und Vornamen nach Ursprung und Sinn erklärt, 16. Auflage, Frankfurt / Main, 2005.

Debrabandere, Frans: Woordenboek van de familienamen in België en Noord-Frankrijk, Grondig herziene en vermeerderde uitgave, Amsterdam, Antwerpen, 2003.

Debus, Friedhelm: Matthäus und Matthias in deutschen Familiennamen, Varianten und Verbreitung. In: Heuser, Rita / Nübling, Damaris / Schmuck, Mirjam (Hgg.): Familiennamengeographie, Ergebnisse und Perspektiven europäischer Forschung, Berlin / New York, 2011, 255-268, 373-377.

DFA I = Kunze, Konrad / Nübling, Damaris (Hgg.): Deutscher Familiennamenatlas, Band 1: Graphematik / Phonologie der Familiennamen I: Vokalismus, Von Christian Bochenek / Kathrin Dräger, Berlin / New York, 2009.

DFA II = Kunze, Konrad / Nübling, Damaris (Hgg.): Deutscher Familiennamenatlas, Band 2: Graphematik / Phonologie der Familiennamen II: Konsonantismus, Von Antje Dammel / Kathrin Dräger / Rita Heuser / Mirjam Schmuck, Berlin / New York, 2011.

DFA III = Kunze, Konrad / Nübling, Damaris (Hgg.): Deutscher Familiennamenatlas, Band 3: Morphologie, Von Fabian Fahlbusch / Rita Heuser / Jessica Nowak / Mirjam Schmuck, Berlin, New York, 2012.

Dräger, Kathrin: Familiennamen aus dem Rufnamen Nikolaus in Deutschland, Regensburg, 2013.

Duden Familiennamen, Herkunft und Bedeutung. Bearbeitet von Rosa Kohlheim und Volker Kohlheim, 2., völlig neu bearbeitete Auflage, Mannheim u.a., 2005.

Farø, Ken / Kürschner, Sebastian: Et databasemøde mellem Jensen og Müller, Om kontrastiv antroponomastik metodik. In: Tijdschrift voor Skandinavistiek 28, 2007, 105-126.

Heuser, Rita / Nübling, Damaris: Von Angenendt über Derrix, Janssen und Terlinden bis Elspaß, Niederrheinische Familiennamen im Rahmen des Deutschen Familiennamenatlasses (DFA). In: Cornelissen, Georg / Eickmans, Heinz (Hgg.): Familiennamen an Niederrhein und Maas, Bottrop, 2010, 37-66.

Institut Grand-Ducal. Section de linguistique, de folklore et de toponymie (Hg.): Geographie der Luxemburger Familiennamen, Nach der Volkszählung von 1930, Luxemburg, 1989.

Kohlheim, Rosa / Kohlheim, Volker: Von Hartmann bis Janzen, Die Patronymika unter den 1000 häufigsten Familiennamen in Deutschland. In: Braun, Angelika (Hg.): Beiträge zu Linguistik und Phonetik, Festschrift für Joachim Göschel zum 70. Geburtstag, Stuttgart, 2001, 283-307.

Kunze, Konrad: dtv-Atlas Namenkunde, Vor- und Familiennamen im deutschen Sprachgebiet, 5. überarbeitete und erweiterte Auflage, München, 2004a.

Kunze, Konrad: Václav / Wenzel, Böhmens Patron in Deutschlands Familiennamen. In: Behr, HansJoachim / Lisový, Igor / Williams-Krapp, Werner (Hgg.): Deutsch-böhmische Literaturbeziehungen, Germano-Bohemica, Festschrift für Václav Bok zum 65. Geburtstag, Hamburg, 2004b, 96-118.

Kunze, Konrad: Jakobus in (nieder)deutschen Familiennamen. In: Röckelein, Hedwig (Hg.): Der Kult des Apostels Jakobus d.Ä. in norddeutschen Hansestädten, Tübingen, 2005, 181-213.

Kunze, Konrad / Nübling, Damaris: Der Deutsche Familiennamenatlas (DFA), Konzept, Konturen, Kartenbeispiele. In: Beiträge zur Namenforschung, Neue Folge 42, 2007, 125-172. 
Linnartz, Kaspar: Unsere Familiennamen, Band 2: Aus deutschen und fremden Vornamen im Abc erklärt, 3. Auflage, Bonn / Hannover / Hamburg, 1958.

Marynissen, Ann: Limburgse familienamengeografie. In: Naamkunde 26, 1994, 243-301.

Marynissen, Ann: Ursprung, Motivierung und Bildung von Familiennamen am Beispiel des niederländischen Sprachgebiets. In: Cornelissen, Georg / Eickmans, Heinz (Hgg.): Familiennamen an Niederrhein und Maas, Bottrop, 2010, 11-35.

Marynissen, Ann / Nübling, Damaris (2010): Familiennamen in Flandern, den Niederlanden und Deutschland - ein diachroner und synchroner Vergleich. In: Dammel, Antje / Kürschner, Sebastian / Nübling, Damaris (Hgg.): Kontrastive germanistische Linguistik. Hildesheim u.a., 2010, 311-362.

Morlet, Marie-Thérèse: Dictionnaire étymologique des noms de famille, Paris, 1991.

Steffens, Rudolf: Der „Kleine Atlas westmitteldeutscher Familiennamen“. In: Ernst, Peter / Patocka, Franz (Hgg.): Dialektgeographie der Zukunft, Akten des 2. Kongresses der Internationalen Gesellschaft für Dialektologie des Deutschen (IGDD) am Institut für Germanistik der Universität Wien, 20. bis 23. September 2006, Stuttgart, 2008, 269-292.

Zender, Matthias: Räume und Schichten mittelalterlicher Heiligenverehrung in ihrer Bedeutung für die Volkskunde, Die Heiligen des mittleren Maaslandes und der Rheinlande in Kultgeschichte und Kultverbreitung, Düsseldorf, 1959.

\section{Internet}

http://www.familienaam.be

http://lfa.uni.lu

http://www.meertens.knaw.nl/nfd 



\title{
Das ,Digitale Familiennamenwörterbuch Deutschlands ${ }^{6}$ Möglichkeiten und Ziele am Beispiel regionaler Namen
}

\author{
Fabian Fahlbusch, Rita Heuser
}

\begin{abstract}
Viele Familiennamen aus Deutschland sind in keinem Namenbuch erfasst. Vor allem seltene, regionale Namen sucht man meist vergebens. Für deren Deutung müssen häufig viele Nachschlagewerke und Einzelstudien konsultiert werden, bis aus den widersprüchlichen Angaben ein plausibles Bild entsteht. Diese Mängel machen ein neues Familiennamenwörterbuch dringend erforderlich, welches die bisherigen Erkenntnisse vereint, teilweise korrigiert und auch revidiert. Zudem sind viele bislang unerforschte Namen erstmals in ein Nachschlagewerk aufzunehmen. Der Beitrag stellt die Notwendigkeit eines modernen digitalen Familiennamenwörterbuchs heraus und präsentiert das Projekt ,Digitales Familiennamenwörterbuch Deutschlands‘. Die Möglichkeiten und Ziele eines solchen Lexikons sollen am Beispiel von regionalen Namen mit besonderer Berücksichtigung der Grenzregion Niederlande - Belgien - Luxemburg - Frankreich - Deutschland ausführlich erläutert werden.
\end{abstract}

\section{Die verfügbaren Namenbücher für Deutschland}

Der größte Teil der Familiennamen in Deutschland ist lexikographisch nicht erfasst. Aus den Festnetz-Telefonanschlüssen der Deutschen Telekom des Jahres 2005 ergeben sich nach Abzug der Bindestrich-Doppelnamen rund 850.000 unterschiedliche Familiennamen. Das aktuellste Wörterbuch (Duden Familiennamen 2005; Datenbasis von 1997) umfasst 20.000 Familiennamen, was gerade einmal rund 2,4 \% des Gesamtinventars entspricht. Das umfangreichste Nachschlagewerk (Gottschald 2006; letzte Neubearbeitung von 1932) verzeichnet rund 70.000 Familiennamen, das sind etwa $8,2 \%$ des Gesamtinventars. Besonders prekär stellt sich die Lage bei den niedrigfrequenten, meist regionalen wie auch den fremdsprachigen Namen insgesamt dar. Wenn überhaupt, werden sie nur in einigen wenigen Wörterbüchern aufgeführt. Ihre Etymologien muss man dann mühsam aus verschiedenen Einzelstudien heraussuchen und die sich oftmals widersprechenden Erklärungen gegeneinander abwägen. Konkret zieht dies folgende Herausforderungen bei der Namendeutung nach sich:

- Für eine einzelne Deutung muss man mindestens sechs Standardwerke konsultieren, nämlich Bahlow 2005, Brechenmacher 1957-63, Duden Familiennamen 2005, Gottschald 2006, Naumann 2007 und Zoder 1968.

- Dabei sind manche der genannten Namenbücher veraltet, widersprüchlich, regional begrenzt (Zoder 1968 etwa beschränkt sich auf Ostfalen) und oft wenig benutzerfreundlich (den mit 914 Telefonanschlüssen nicht gerade seltenen Familiennamen Robert findet man etwa bei Gottschald 2006 unter dem Eintrag HROD, der insgesamt 758 einzelne Namen umfasst, darunter so verschiedene wie Gruhl, Krupp, Rhode oder Riedel). 
- Die Deutungen berücksichtigen vielfach nicht den gegenwärtigen Stand der Forschung, etwa der Familiennamengeographie. Sie sind daher oft nicht mehr haltbar oder unzureichend gewichtet. So wird Schillo zu sorbisch šylo ,Pfriem' gestellt (Gottschald 2006, 433). Seine Verbreitung im Saarland und in der Südpfalz legt jedoch einen Zusammenhang mit dem französischen Patronym Gillot (zum Rufnamen Ägidius plus Diminutivendung -ot) nahe.

- Nicht immer sind alle Bedeutungskonkurrenzen mehrdeutiger Familiennamen in einem Namenbuch vollumfänglich erfasst.

Die Etymologisierung von Familiennamen gerät folglich zu einem sehr zeitaufwendigen und gerade bei seltenen Namen auch häufig erfolglosen Unterfangen. Stets muss man dafür die notwendige Literatur zur Hand haben und abweichende oder widersprüchliche Deutungen aus fachlicher Perspektive bewerten. Interessierte Laien können die genannten erforderlichen Schritte zu einer fundierten Namendeutung kaum selbst leisten. Nicht nur für sie fehlt ein umfassendes, gut zugängliches, einfach zu benutzendes Familiennamenwörterbuch. Daraus ergeben sich spezielle Anforderungen an das ,Digitale Familiennamenwörterbuch Deutschlands', die bei der Erarbeitung berücksichtigt werden müssen:

- Zunächst ist die Zusammenstellung und kritische Neubewertung der Deutungen in den Standardwerken zu leisten. Die Bedeutungskonkurrenzen müssen auf ihre Wahrscheinlichkeit hin überprüft und dementsprechend angeordnet, teilweise auch erweitert und genauer erläutert werden. Nicht zutreffende Etymologien kennzeichnen wir eigens als solche, sich neu anbietende prüfen wir eingehend und fügen sie ggf. hinzu.

- Notwendig ist auch die systematische Nutzung und Auswertung der anthroponomastischen Literatur, was zum Aufbau einer entsprechenden Bibliothek führt. Dabei berücksichtigen wir nicht nur die wichtigsten überregionalen Standardnachschlagewerke, sondern auch und vor allem die zahlreichen kleinräumigen, regionalen Untersuchungen, die oftmals nur unter großem Aufwand zugänglich sind.

- Da ein beachtlicher Teil der Familiennamen in Deutschland fremdsprachige Wurzeln besitzt, ziehen wir die Namenlexika der Nachbarländer sowie die im Internet verfügbaren Kartierungsprogramme (z.B. www.meertens.knaw.nl / für die Niederlande; lfa. uni.lu für Luxemburg; www.gen-evolu.de für die historische Verbreitung vor 1945) heran. Unabdingbar ist dies für die sichere Etymologisierung vieler regionaler Familiennamen in den Grenzregionen.

Auch in anderen europäischen Ländern hat man die Notwendigkeit moderner Nachschlagewerke für Familiennamen erkannt: In Österreich wurde ein digitales Familiennamenwörterbuch (,Familiennamen Österreichs (FamOS) ${ }^{`}$ ) begonnen, das sich anfänglich vor allem mit regionalen Namen beschäftigt. Eine Testversion ist bereits online. ${ }^{1}$ Das Projekt

1 hw.oeaw.ac.at/famos; die Buchstabenstrecke A bis J ist bereits gefüllt (21.06.2012). 
,Luxemburger Familiennamenatlas (LFA)‘ steht vor der Drucklegung und bietet nun auch die Möglichkeit, einen oder mehrere Namen im Großherzogtum sowie angrenzenden Nachbarländern zu kartieren. ${ }^{2}$ In Planung befindet sich ein ähnliches Projekt zu den Familiennamen Großbritanniens (,Family Names of the United Kingdom (FaNUK) ${ }^{6}$ ). Die Homepage des Meertens-Instituts stellt eine umfassende Datenbank der niederländischen Familiennamen mit Kartierungsmöglichkeit bereit. ${ }^{3}$

\section{Das ,Digitale Familiennamenwörterbuch Deutschlands ${ }^{6}$}

Im Herbst 2011 hat die Akademie der Wissenschaften und der Literatur Mainz das ,Digitale Familiennamenwörterbuch Deutschlands' als Langzeitprojekt angenommen. Während einer Laufzeit von 24 Jahren sollen mehr als 250.000 Namenartikel zuverlässig erarbeitet werden. Mit der Erfassung aller Familiennamen über 10 Tokens (= Telefonanschlüsse) und der Einbeziehung ihrer niedrigfrequenten Varianten auch unter 10 Tokens gelingt es, einen Großteil des rezenten Familiennamenbestandes in Deutschland abzudecken. Besonders bei Namen mittlerer bis niedriger Frequenz ist mit einer sehr hohen Zahl an Ersteinträgen, Neu- bzw. Erstdeutungen zu rechnen. Das im Internet kostenfrei zugängliche ,Digitale Familiennamenwörterbuch Deutschlands' soll benutzerfreundlich aufgebaut sein und sich am bekannten Aufbau eines Wikipedia-Artikels anlehnen. Die in Zusammenarbeit mit der Technischen Universität Darmstadt und der Digitalen Akademie entwickelte XML-Textstruktur gewährleistet dabei eine Langzeitarchivierung der Datensätze. Eine ideale Benutzeroberfläche kann allerdings erst nach einer kritischen Testphase entwickelt und online gestellt werden. Folgende Inhalte soll ein Namenartikel umfassen:

- Lemmaeintrag (= Familienname) + Tokenangabe (= Telefonanschlüsse), z.B. Meyer 83.586. Optional werden zusätzlich der Rang (hier: 6) unter den häufigsten Familiennamen Deutschlands angegeben sowie eine Audiodatei zur Aussprache eingebunden, sollte diese unklar sein.

- Angaben zu Sprache, Herkunftsland/-ländern bzw. -region(en).

- Die Bedeutung erörtern wir ausführlich bei der Hauptvariante, also der häufigsten bzw. typischsten Namenform. Bei Nebenvarianten steht ein Verweis auf diesen Haupteintrag. Bedeutungskonkurrenzen werden nach Wahrscheinlichkeit geordnet, ggf. auch als unsicher, nur in Einzelfällen zutreffend oder als weitgehend auszuschließen eingestuft.

- Die geographische Verteilung des einzelnen Familiennamens dokumentiert eine herunterladbare Namenkarte im PDF-Format. Aus Datenschutzgründen ist diese allerdings erst ab einer Frequenz von mindestens 10 Tokens möglich. Zur Stützung einer bestimmten Deutung können hier auch Kombinations- oder Ausschnittkarten und ein kurzer Kommentar beigefügt werden.

2 lfa.uni.lu (21.06.2012).

3 www.meertens.knaw.nl/nfb (21.06.2012). 
- Hinweise zu Vorkommen der Namen außerhalb der Bundesrepublik beziehen wir dann ein, wenn sie im Ausland eine höhere Tokenzahl als in Deutschland selbst aufweisen und/oder die Ergebnisse für die Namendeutung relevant sind. Bei ausschließlich in Grenzregionen verbreiteten Namen erfolgen diese Angaben grundsätzlich für die benachbarten Länder, um grenzüberschreitende Namenlandschaften aufzeigen zu können.

- Häufige Schreibvarianten werden, sofern vorhanden, aufgeführt, ebenso die Originalschreibung in den Herkunftsländern.

- Ausgewählte Literaturangaben beziehen sich auf die wichtigsten Nachschlagewerke und interessante weiterführende Titel.

- Weblinks verweisen auf im Internet zur Verfügung stehende Literatur, Nachschlagewerke und Kartierungsprogramme, im Idealfall auch auf weitere digitale Familiennamenwörterbücher (s.o.).

- Eine sinnvolle Erweiterung stellen die Angaben zu historischen Belegen und Schreibweisen dar, welche die Namendeutung stützen.

- Über die sprachliche Interpretation eines Namens hinaus werden Zusatzinformationen geboten, z.B. zu besonderen kulturgeschichtlichen Hintergründen, Namensystemen anderer Kulturen oder bestimmten Namentypen (Lallnamen etc.). Hierfür können wir sogenannte Infokästen mitgeben.

- Sämtlichen Namen sind Schlagworte zugeordnet, z.B. zu Motivgruppen (Herkunftsname, Patronym etc.), sprachlicher Herkunft (germanisch, slawisch etc.), morphologischer Struktur (Simplizia, Komposita etc.), die eine schnelle Suche mit großer Ergebnismenge ermöglichen.

Parallel zu den einzelnen Namenartikeln führen wir im Hintergrund ein datenbankgestütztes Redaktionssystem, in dem die jeweils angestellten Recherchen dokumentiert werden. Außerdem notieren wir hier, ob es sich um einen Ersteintrag bzw. eine Neu- oder Erstdeutung handelt. Mithilfe dieser Angaben kann die Zahl nicht oder falsch gedeuteter Namen erstmals statistisch erfasst und damit ein aufschlussreicher Beitrag zur Wissenschaftsgeschichte geleistet werden.

\section{Fehl- und Neudeutungen regionaler Familiennamen}

Bei regional verbreiteten, seltenen Namen ist die Beleglage in den vorhandenen Wörterbüchern besonders dürftig. Viele müssen nicht nur erstmalig in ein überregionales Namenlexikon aufgenommen, sondern auch zum ersten Mal überhaupt gedeutet werden. Als sehr bedeutsam für die Etymologisierung erweist sich die Auswertung der Namenverbreitung, ein Verfahren, das bisher nur unzureichend genutzt worden ist. Die folgenden Beispiele sollen dies verdeutlichen und zugleich die Probleme bei der Deutung an sich illustrieren. Alle ausgewählten Namen treten fast ausschließlich in der Grenzregion Niederlande - Belgien - Luxemburg - Frankreich - Deutschland auf. Ein weiteres Kriterium war, dass sie sich in keinem der oben genannten Standardnamenbücher finden 
bzw. dort nicht exakt gedeutet werden. Ihre Frequenz sollte im unteren bis mittleren Bereich liegen. ${ }^{4}$ Eine besondere Schwierigkeit im südlichen westmitteldeutschen Raum stellt das weitgehende Fehlen historischer Namenbücher für diese Region dar (vgl. DFA I, LXXV), eine Lücke, die erst in den kommenden Jahren mit Arbeiten des Instituts für Geschichtliche Landeskunde an der Universität Mainz ${ }^{5}$ zumindest teilweise geschlossen werden wird.

\subsection{Revision von Fehldeutungen für Namen deutscher Herkunft}

Einige etwas häufigere regionale Namen haben auch einen Eintrag in zumindest einem der sechs Standardwerke. Allerdings sind diese Deutungen nicht immer zuverlässig und müssen kritisch hinterfragt werden. Gerade in Grenzregionen bleibt der Blick in fremdsprachige Lexika unerlässlich; er führt oft zu einer Revision offensichtlich falscher oder wenig wahrscheinlicher Etymologien.

Bi(e)wer. Als Beispiel für eine unzutreffende Einordnung darf der Familienname Bi(e)wer (314 bzw. 152 Tokens) gelten. Gottschald 2006, 116 stellt Biwer zum Nagetier Biber - allerdings ohne nähere Angaben zum Benennungsmotiv. Zoder 1968 I, 244 führt darüber hinaus unter Biewer eine gleichnamige Wüstung bei Magdeburg an. Die anderen Standardwerke verzeichnen ihn gar nicht. Fündig wird man hingegen bei Debrabandere 2003, 131 und Germain/Herbillon 2007, 169. Sie geben die luxemburgische Gemeinde Biwer als Ursprungsort an und deuten die entsprechenden Familiennamen als Herkunftsnamen. Das Kartenbild bestätigt ihre Einschätzung: Der Name konzentriert sich an der Landesgrenze zwischen Trier und Saarbrücken. Auf deutscher Seite kommt damit auch der heutige Trierer Stadtteil Biewer in Frage; die von Zoder angeführte Wüstung in Sachsen-Anhalt jedoch auf keinen Fall. Bei einer derart konzentrierten Verbreitung um gleichnamige Ursprungsorte kann ein indirekter Berufsname für den Biberjäger, den Biberfellhändler oder den Hersteller und Verkäufer von Bibergeil, einem Sekret aus den Schwanzdrüsen des Tieres, ebenfalls weitgehend ausgeschlossen werden. In Luxemburg lauten 51 Telefonanschlüsse auf Biewer, 114 auf Biwer und 13 auf Biewers. Die in Deutschland mit 6 Tokens nur selten vertretene Variante Biver gibt es im Großherzogtum insgesamt 205 mal. $^{6}$

Kartentyp: absolut; Ausschnitt Moselraum; Kreise pro fünfstellige PLZ, Kreisgröße 5-50, entspricht 1-23 Tokens; Flächen pro zweistellige PLZ, Anzeigeschwelle 15 Tokens; Tokenangaben beziehen sich auf Gesamtdeutschland.

4 Die folgenden Karten sind mit dem Kartierungsprogramm des Deutschen Familiennamenatlasses (DFA) auf Basis der Telefon-Festnetzanschlüsse des Jahres 2005 erstellt worden. Näheres dazu in DFA I, XXXI-LXIII.

5 Jüngst veröffentlicht wurde der ,Familiennamenatlas: Rheinland-Pfalz, Hessen, Saarland“. Geplant ist zudem ein ,Historisches rheinfränkisches Personennamenbuch 14. bis 16. Jh.', beide von Rudolf Steffens.

6 Daten nach lfa.uni.lu. In Belgien ist Biver ebenfalls die frequenteste Variante (157 Einwohner 1998; www.familienaam.be; jeweils 21.06.2012). 


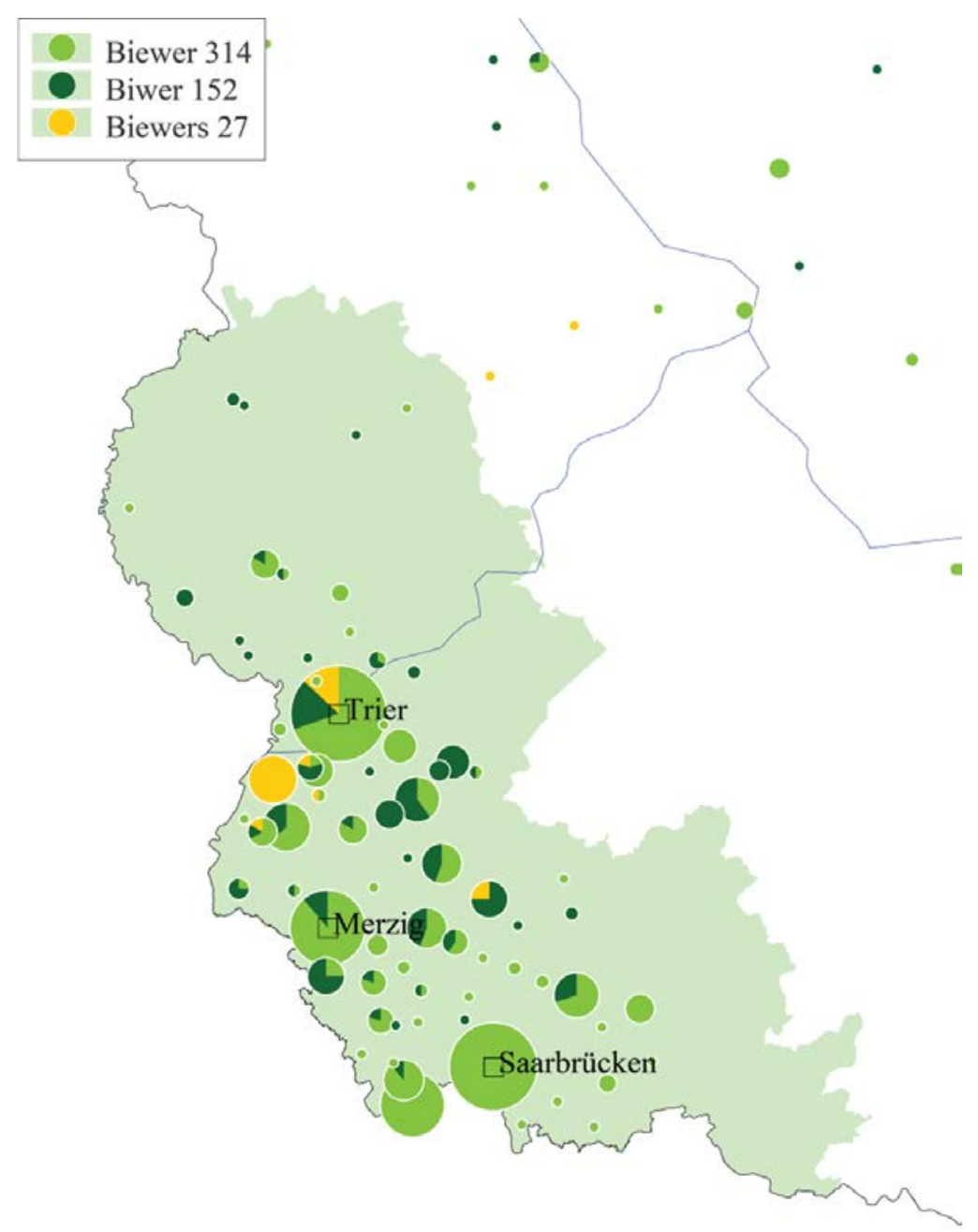

Karte 1: $\quad$ Verbreitung des Familiennamens Biewer mit Varianten

Frauenkron. Frauenkron wird einzig von Gottschald 2006, 189 verzeichnet, allerdings nur die nicht (mehr?) existierende Schreibvariante Frauenkrohn. Wiederum gibt er keine Bedeutung an; die Einordnung unter dem Stichwort Frau lässt aber auf einen Übernamen schließen. So sehen es auch Debrabandere 2003, 478 ("Frauenkron [...]: vrouwenkroon") und Germain/Herbillon 2007, 442 ("Frauenkrone [...]: couronne des dames"). In diesem Fall führen sie jedoch nicht zur richtigen Lösung, denn die konzentrierte Verbreitung des Namens in der Eifel weist ganz klar auf einen Herkunftsnamen hin. Und zwar zum gleichnamigen Ortsteil der Gemeinde Dahlem in der Nordeifel. Der Familienname (116 Tokens) bildet ein Nest im direkt benachbarten PLZ 53940 Hellenthal mit 28 Tokens. Auf der anderen Seite der Grenze ballen sich die 28 Namenträger im deutschsprachigen Teil Belgiens (www.familienaam.be; 21.06.2012). 


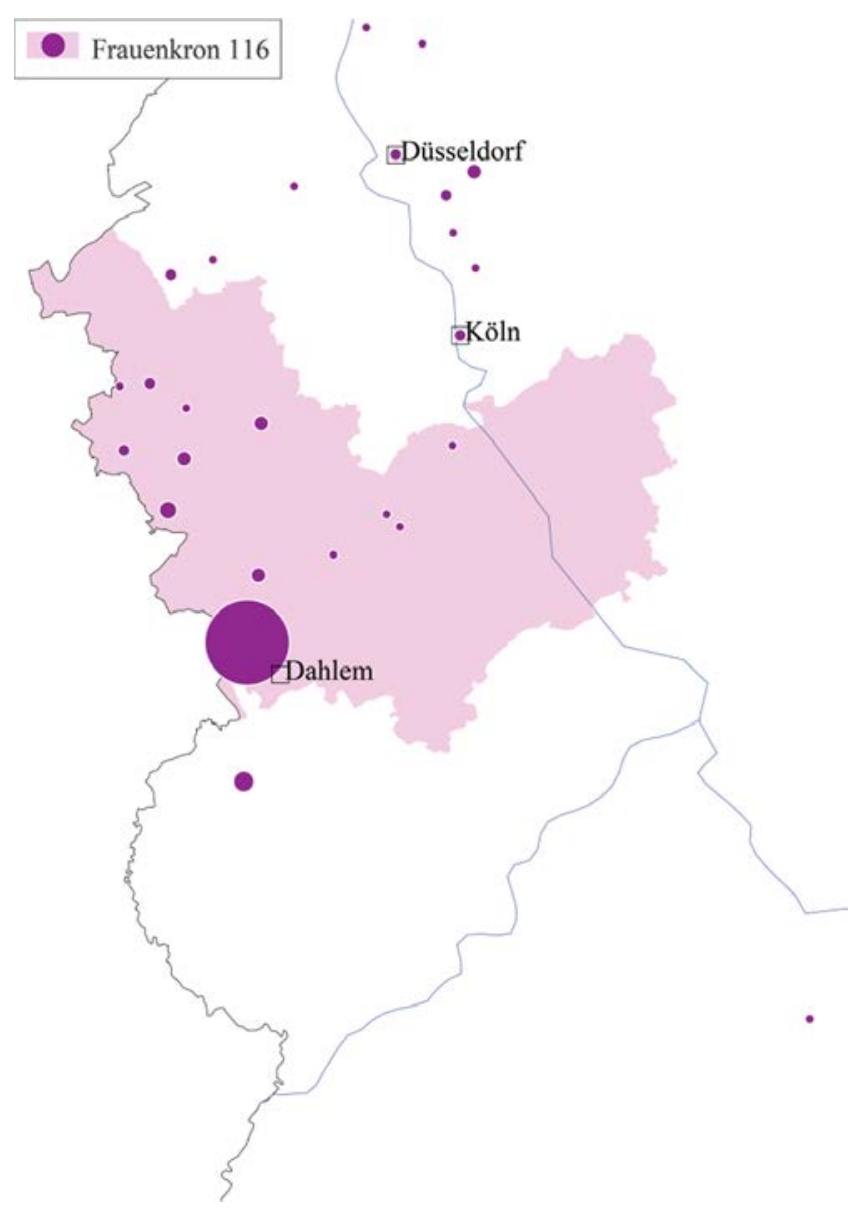

Karte 2: $\quad$ Verbreitung des Familiennamens Frauenkron

Kartentyp: absolut; Ausschnitt Rheinland; Kreise pro fünfstellige PLZ, Kreisgröße 5-50, entspricht 1-28 Tokens; Flächen pro zweistellige PLZ, Anzeigeschwelle 10 Tokens; Tokenangaben beziehen sich auf Gesamtdeutschland.

\subsection{Neudeutung von in Namenbüchern nicht belegten Namen deutscher Herkunft}

Viele deutsche Familiennamen, die nicht einmal äußerst selten sein müssen (das folgende Beispiel Fixemer etwa kommt hochgerechnet auf rund 314 Namenträger) ${ }^{7}$, tauchen in keinem der sechs Standardwerke auf. Daher rühren auch die vielen an die Mainzer Namenforschergruppe gerichteten privaten Namenanfragen. Meist handelt es sich um regionale Familiennamen oder Schreibvarianten häufigerer Namen, deren Deutung allzu oft einige Herausforderungen bereithält.

7 Statistisch gesehen teilen sich in Deutschland 2,8 Personen einen Festnetzanschluss. 
Fixemer. Dieser Familienname findet sich weder in den konsultierten deutschen noch in den einschlägigen fremdsprachigen Wörterbüchern.

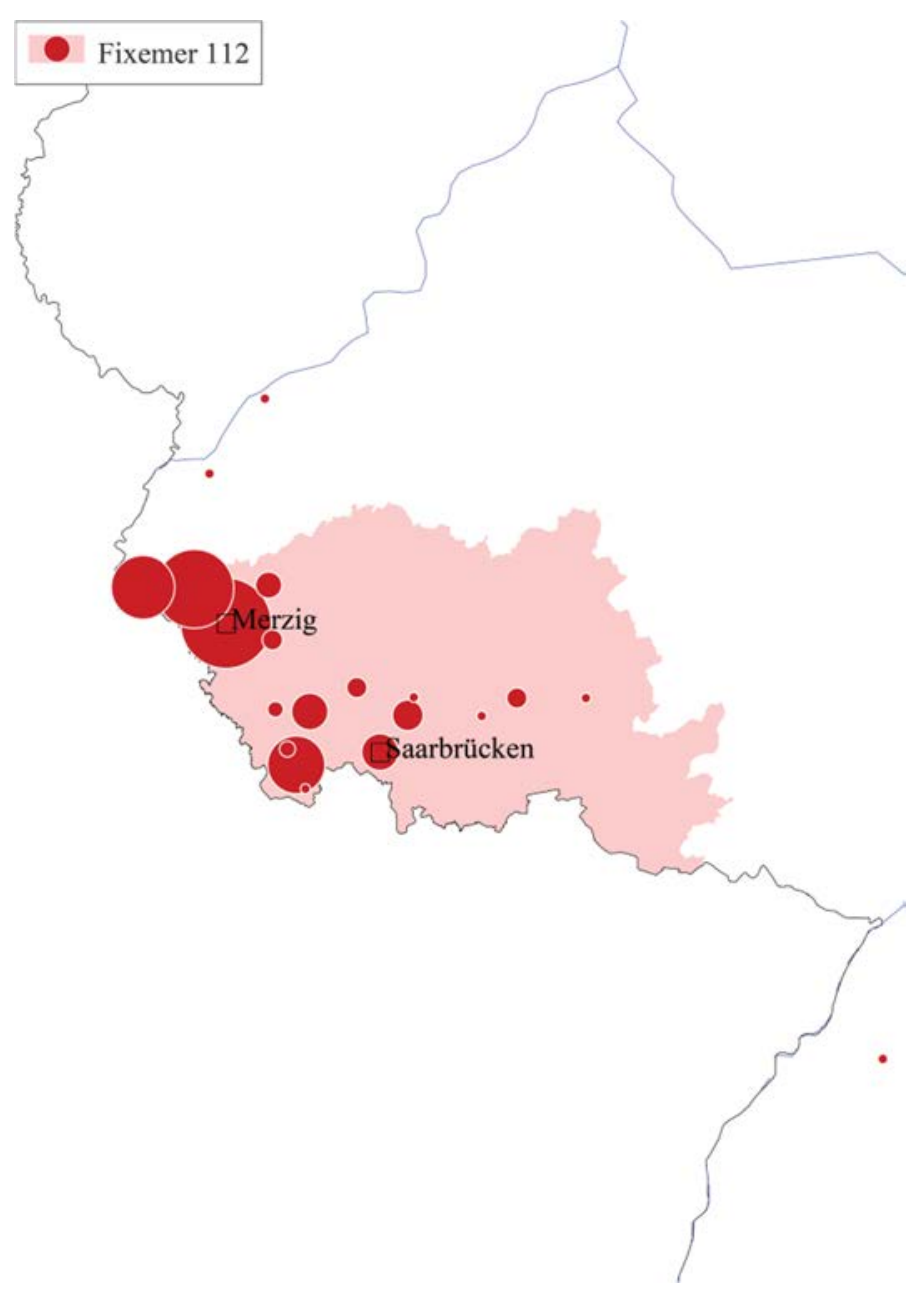

Karte 3: Verbreitung des Familiennamens Fixemer

Ein interessierter Laie käme an dieser Stelle bei der Suche nach der Bedeutung seines Namens nicht weiter. Dabei ist die Lösung eigentlich naheliegend. Die 112 Telefonanschlüsse treten gehäuft im Saarland auf (die 24 Tokens der synkopierten Variante Fixmer sind in Süddeutschland verstreut). Wie die kleinräumige Verbreitung bereits nahelegt, handelt es sich um einen Herkunftsnamen. Den Ursprungsort bildet die französische Gemeinde Fixem im Département Moselle, nordöstlich von Thionville. Gerade in Siedlungsnamen wird das Endglied -heim oft zu -em verschliffen. Die teilweise nur dialektal erfolgte Auflösung der Kompositionsfuge ist dann in die Familiennamen übernommen worden (vgl. DFA III, 626-629). 
Kartentyp: absolut; Ausschnitt Saarland; Kreise pro fünfstellige PLZ, Kreisgröße 5-50, entspricht 1-16 Tokens; Flächen pro zweistellige PLZ, Anzeigeschwelle 5 Tokens; Tokenangaben beziehen sich auf Gesamtdeutschland.

Erang. Auch bei Erang, mit lediglich 30 Telefonanschlüssen ungleich seltener als Fixemer, handelt es sich um einen Herkunftsnamen. Und zwar zu Ehrang, einem heutigen Stadtteil von Trier.

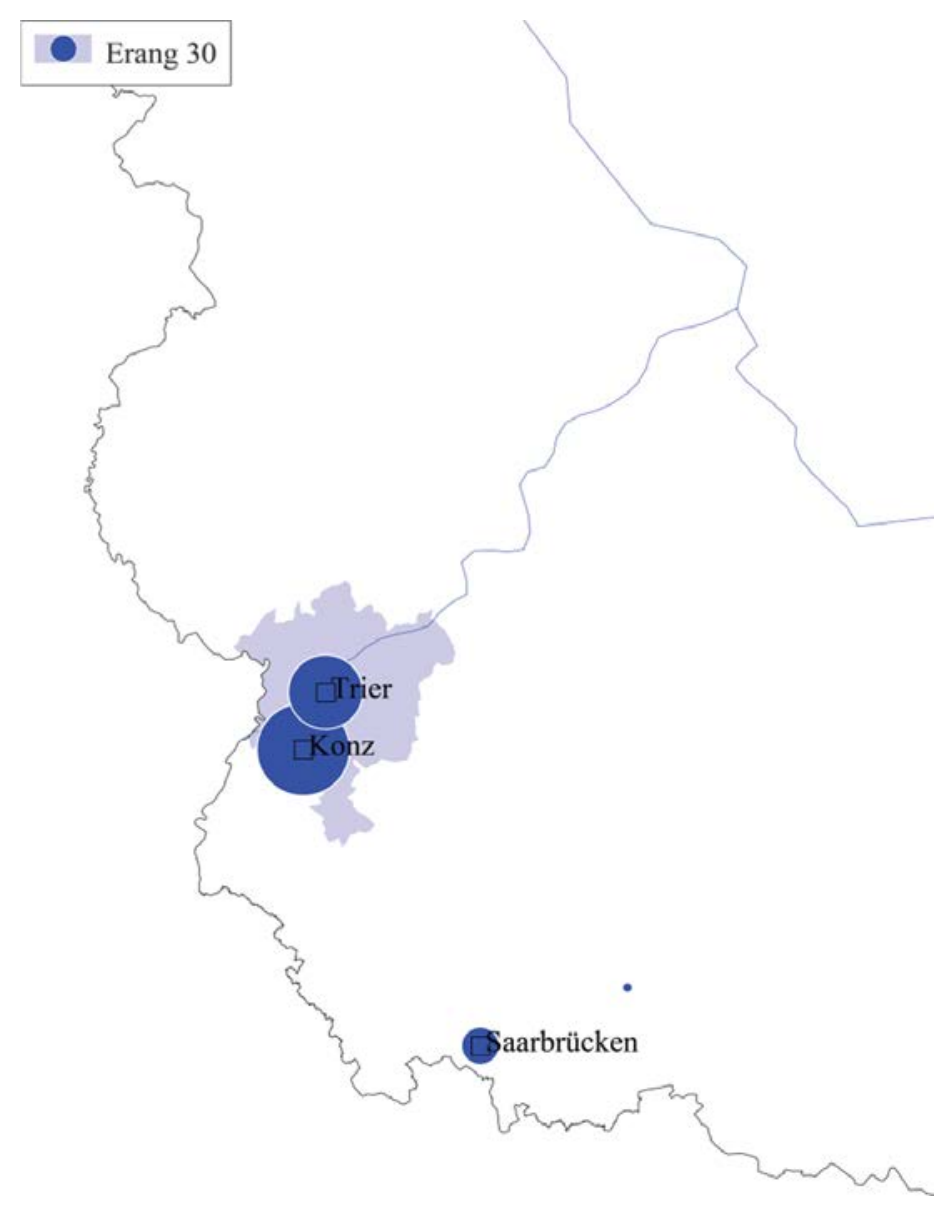

Karte 4: $\quad$ Verbreitung des Familiennamens Erang

Die Konzentration des Familiennamens im kleinen Radius um den Ursprungsort in Konz (10 Tokens) und Trier selbst (8 Tokens) lässt keinen anderen Schluss zu. Große Städte, wie z.B. Nürnberg, strahlen deutlich weiter. Entsprechende Familiennamen finden sich dann selbstverständlich auch in den Standardwerken, Erang hingegen nicht.

Kartentyp: absolut; Ausschnitt Moselraum; Kreise pro fünfstellige PLZ, Kreisgröße 5-50, entspricht 1-10 Tokens; Flächen pro dreistellige PLZ, Anzeigeschwelle 5 Tokens; Tokenangaben beziehen sich auf Gesamtdeutschland. 
Juchmes. Die Familiennamengeographie hilft oft beim Aufspüren niedrigfrequenter Varianten von häufigeren Familiennamen und trägt damit entscheidend zu deren Etymologisierung bei. Für das ,Digitale Familiennamenwörterbuch Deutschlands' erarbeiten wir stets ganze Namenstrecken, um bspw. alle Familiennamen zu ermitteln, die aus dem Rufnamen Joachim entstanden sind.

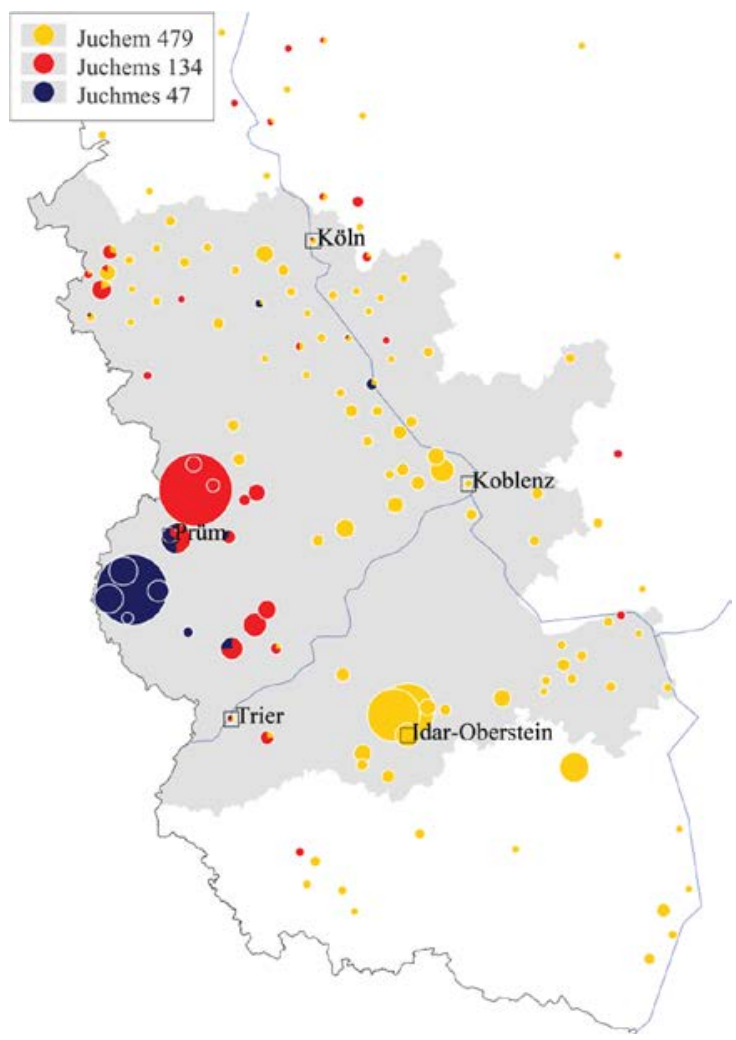

Karte 5: Verbreitung des Familiennamens Juchem mit Varianten

Eine solche, auf den ersten Blick nur schwer erkennbare verkürzte regionale Variante stellt auch Juchmes dar. Mit 47 Tokens vor allem in und um Prüm beheimatet, können wir sie zum starken patronymischen Genitiv Juchems oder zu Juchem selbst (134 bzw. 479 Tokens) stellen, die etwas weiträumiger verbreitet sind, und eine $m$-Metathese vermuten. Auch Debrabandere 2003, 657 vertritt diese Auffassung. Juchem und Juchems werden in einigen Standardwerken gelistet, z.B. bei Bahlow 2005, 267, und stützen so die Deutung von Juchmes. Da in Luxemburg auch die Variante Juchemes (10 Tokens) vorkommt, geht der LFA von einer erschlossenen Form Juchemus als ,partieller' Latinisierung von Juchem aus und sieht in Juchmes eine Synkope aus Juchemes.

Kartentyp: relativ; Ausschnitt Moselraum; Kreise pro fünfstellige PLZ, Kreisgröße 5-50, entspricht 0,01-10,72 \%; Flächen pro zweistellige PLZ, Anzeigeschwelle 0,05\%; Tokenangaben beziehen sich auf Gesamtdeutschland. 


\subsection{Neudeutung von in Namenbüchern nicht belegten Namen fremder Herkunft}

Obwohl Familiennamen nichtdeutscher Herkunft einen wesentlichen Bestandteil der Namenlandschaft in der Bundesrepublik bilden, fanden sie bislang kaum Aufnahme in Familiennamenwörterbücher. Dies betrifft sowohl die jüngst eingewanderten (türkisch, griechisch etc.) als auch die aufgrund historischer Migrationsbewegungen zu uns gekommenen und weitgehend eingedeutschten Familiennamen (Dumont, Schimanski u.a.). Im Folgenden wollen wir einige solcher Fälle betrachten, die in den Telefonbucheinträgen fast ausschließlich einheimische Rufnamen aufweisen und daher auch als "deutsche" Familiennamen gelten dürfen, unabhängig von ihren eigentlichen Ursprüngen.

Arimond. Die deutschen Standardnachschlagewerke helfen nun gar nicht mehr weiter. Beim Familiennamen Arimond, der sich in dieser Schreibweise nur in der Bundesrepublik findet (44 Tokens), liefern Debrabandere 2003, 302 und Germain/Herbillon 2007, 133 Anhaltspunkte für die Deutung als Herkunftsname. Genauer gesagt zum Siedlungsnamen Arimont bei Malmedy in der belgischen Provinz Lüttich, was sich durch die Kartierung erhärten lässt. Die Varianten Arimont und Darimont treten in Deutschland mit 33 bzw. 64 Telefonanschlüssen auf, ganze 161 bzw. 583 Namenträger gab es 1998 in Belgien (www.familienaam.be; 21.06.2012).

Kartentyp: absolut; Ausschnitt Rheinland; Kreise pro fünfstellige PLZ, Kreisgröße 5-50, entspricht 1-20 Tokens; Tokenangaben beziehen sich auf Gesamtdeutschland.

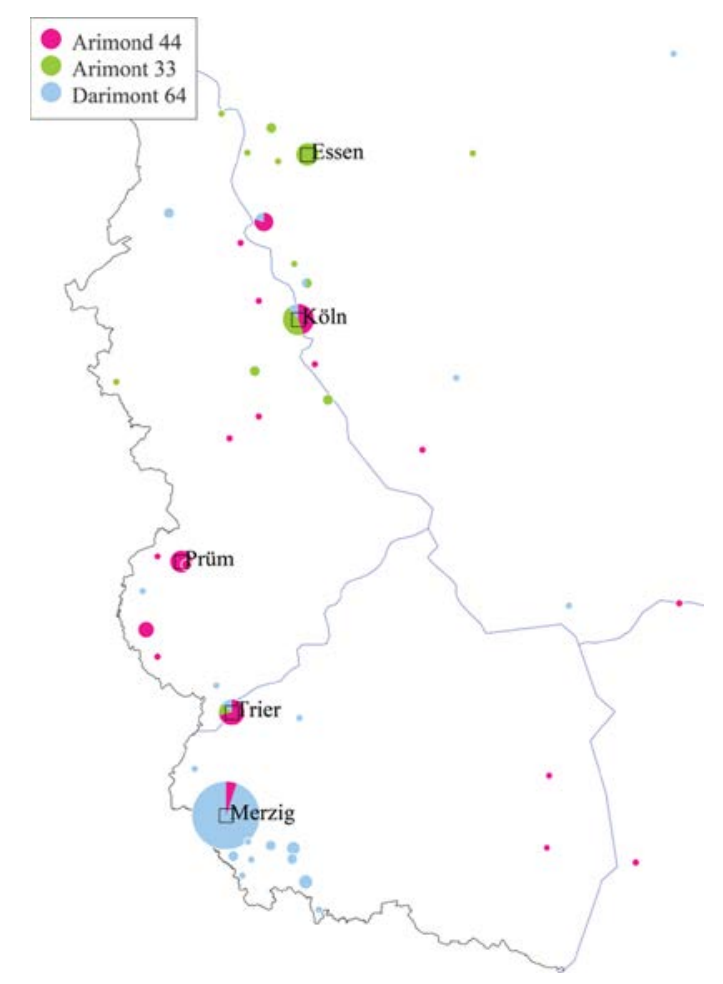

Karte 6: Verbreitung der Familiennamen Arimond/Arimont/Darimont 
Musti. Wiederum liegt ein Herkunftsname vor. Debrabandere 2003, 879 und Germain/ Herbillon 2007, 748 stellen ihn zu den häufigen Siedlungsnamen Moutier(s), Moustier(s), Mousty (zu französisch moutier ,Münster, Kirche') in Frankreich und Belgien. Dort sind daraus zahlreiche Familiennamen entstanden wie Moustier 111/104, Mousty 60/150, Moustie 32/92, Musty 9/45 (jeweils zuerst Anzahl der Geburten in Frankreich zwischen 1891 und 1915 nach www.geopatronyme.com, dann die Anzahl der Namenträger 1998 in Belgien nach www.familienaam.be; jeweils 21.06.2012). In Deutschland erscheint nur die Variante Musti mit 17 Tokens etwas häufiger. Konzentriert in und um Konz kommen als Ursprung der Siedlungsname Moutiers (Lothringen) oder entsprechende Örtlichkeitsnamen in der Wallonie (z.B. Moustî bei Malmedy) in Frage.

Kartentyp: absolut; Ausschnitt Moselraum; Kreise pro fünfstellige PLZ, Kreisgröße 5-50, entspricht 1-3 Tokens; Flächen pro dreistellige PLZ; Tokenangaben beziehen sich auf Gesamtdeutschland.

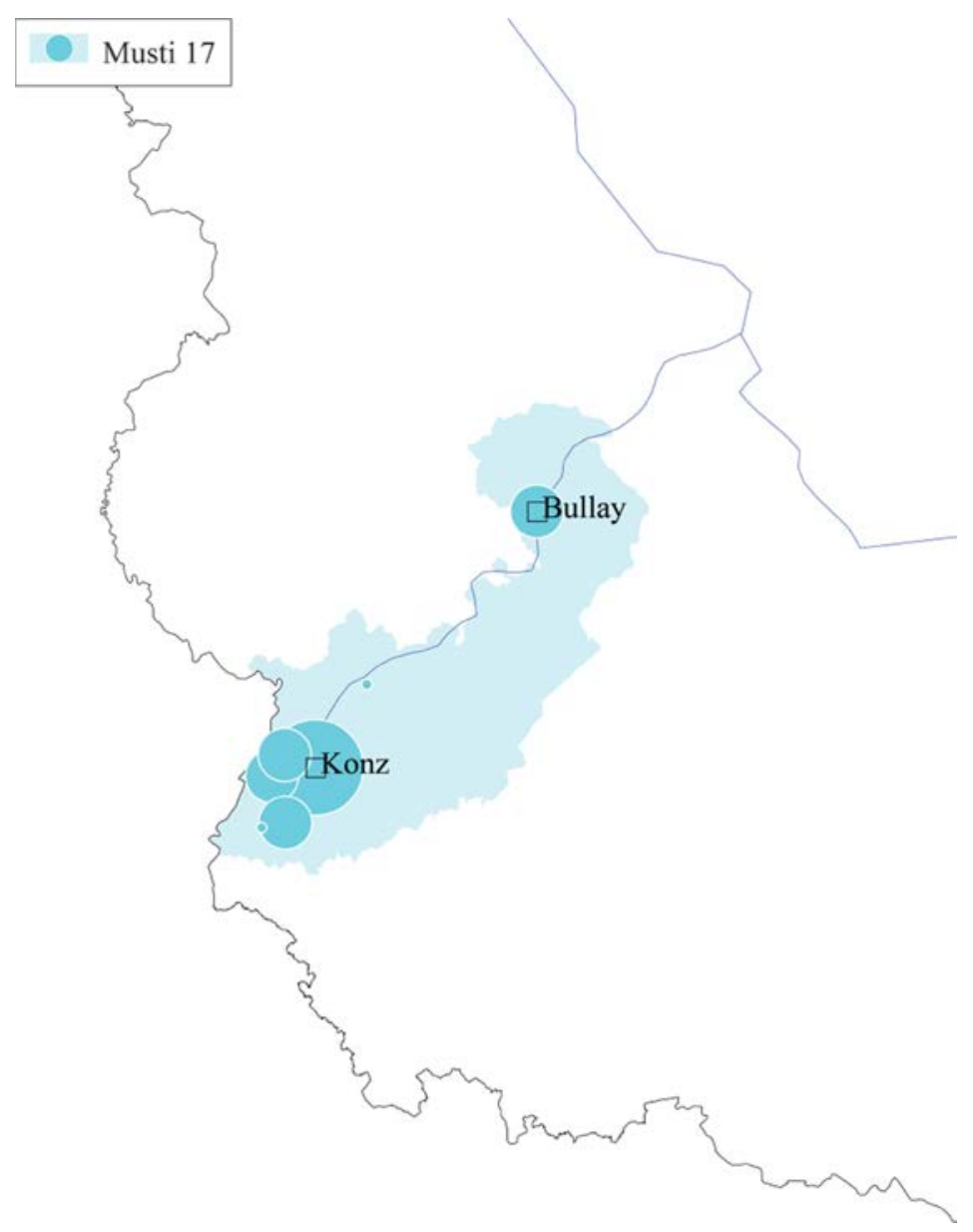

Karte 7: $\quad$ Verbreitung des Familiennamens Musti 
Permesang. Die folgenden drei Beispiele geben Rätsel auf, welche vorerst nicht endgültig gelöst werden können. Stattdessen wollen wir mit aller gebotenen Zurückhaltung einige Vermutungen aufstellen. Es zeigt sich, welche Schwierigkeiten mitunter die Ermittlung der Etymologie von niedrigfrequenten, regional begrenzt vorkommenden Familiennamen bereitet. Hier ist noch viel Forschungsarbeit zu leisten, auch und vor allem unter Beteiligung von Fachleuten anderer Disziplinen bzw. Sprachen. Gerade die Offenheit eines Online-Lexikons erlaubt zunächst die Veröffentlichung von Deutungshypothesen unter Vorbehalt sowie deren anschließende Bestätigung oder Korrektur durch gemeinsamen fächerübergreifenden Austausch mit Familienforschern, Historikern etc.

Bei Permesang (38 Tokens) gehen wir im Anschluss an Debrabandere 2003, 944 von einer eingedeutschten Variante des belgischen Herkunftsnamens Permesaen (7 Einwohner; www.familienaam.be; 21.06.2012) zur oberitalienischen Stadt Parma aus. Die Schreibung -ang zeigt eine für den Südwesten Deutschlands typische Anpassung nasalierter Vokale am Wortende, vgl. auch die aus dem Französischen eingedeutschten Namen Dussing ( $<$ Toussaint, Übername zum religiösen Fest ,Allerheiligen'), Lorang ( $<$ Laurent, Patronym zum gleichlautenden französischen Rufnamen) oder Wehrung $(<$ Véron, Herkunftsname zum gleichlautenden Siedlungsnamen im Burgund; vgl. Hartig/Schwanke 2009, 468 sowie Heuser 2011, 359f., 366 mit Karte 6).

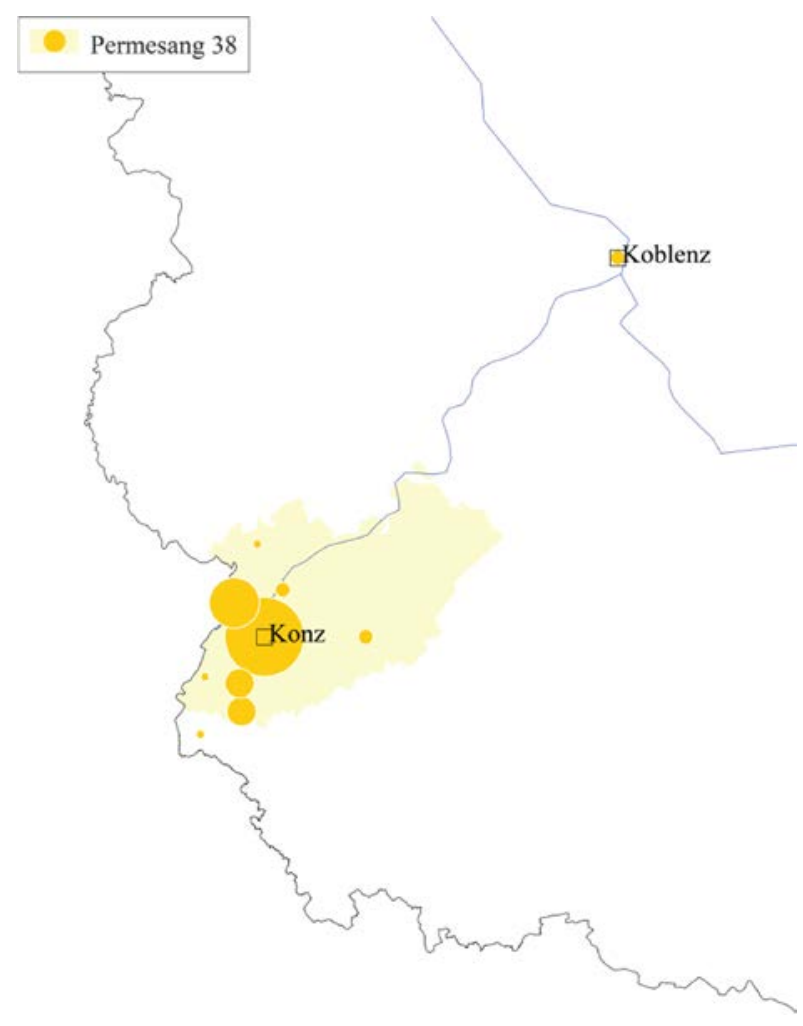

Karte 8: Verbreitung des Familiennamens Permesang 
Kartentyp: absolut; Ausschnitt Moselraum; Kreise pro fünfstellige PLZ, Kreisgröße 5-50, entspricht 1-11 Tokens; Flächen pro dreistellige PLZ, Anzeigeschwelle 3 Tokens; Tokenangaben beziehen sich auf Gesamtdeutschland.

Biwersi/Biniwersi. Mit Biwersi und Biniwersi ( 25 bzw. 8 Tokens) haben wir eventuell Herkunftsnamen zum Siedlungsnamen Bévercé vorliegen, einem Stadtteil von Malmedy in der belgischen Provinz Lüttich. Darauf deuten zumindest historische Schreibweisen dieser Familiennamen wie Biverze 1725, Biverzi 1780 oder Binversie 1813 hin: Ägidius Biverze wurde 1725 in der Eifelgemeinde Dudeldorf geboren. Sein Enkelsohn John schreibt sich dann zum ersten Mal Biwersi. ${ }^{8}$ Zur endgültigen Klärung der Etymologie sind aber noch weitere, vor allem frühere Namengleichungen notwendig.

Kartentyp: relativ; Ausschnitt Moselraum; Kreise pro fünfstellige PLZ, Kreisgröße 5-50, entspricht 0,03-6,16\% Tokens; Tokenangaben beziehen sich auf Gesamtdeutschland.

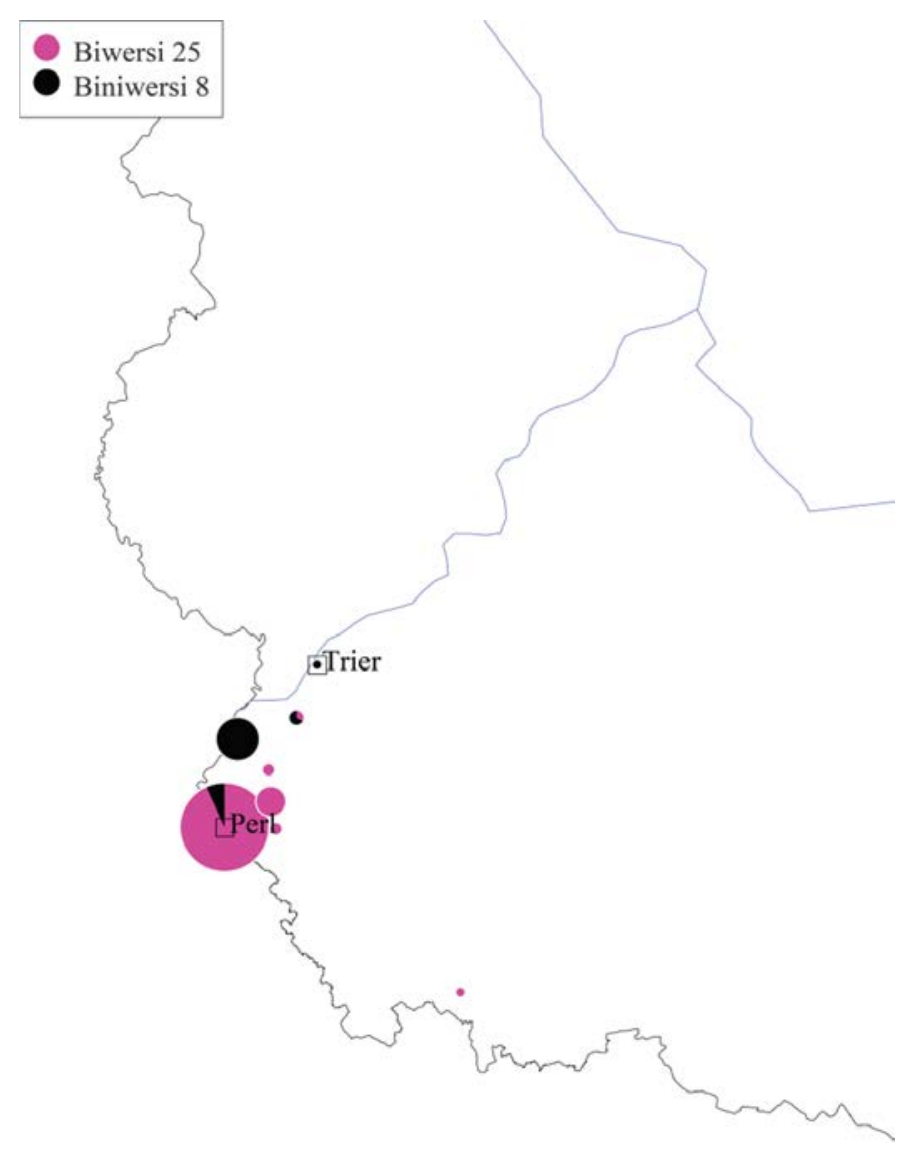

Karte 9: $\quad$ Verbreitung der Familiennamen Biwersi/Biniwersi

8 Gefunden in einem Stammbaum unter freepages.genealogy.rootsweb.ancestry.com/ kautzer/ biverze_a.html (21.06.2012). 
Bitzigeio. Beim Familiennamen Bitzigeio (24 Tokens) könnte es sich um eine eingedeutschte Form des italienischen Übernamens Pizzigallo (,Schmetterling') für einen flatterhaften Menschen handeln. Italienische Einwanderung in die Rheinlande und den Trierer Raum ist seit dem 17. Jh. belegt (vgl. Brendler 2009, 444f., 452f. sowie Dräger 2011, 333f.). Zur abschließenden Deutung des Namens dürfte die Aufarbeitung dieser historischen Migrationsbewegungen und genealogischer Hinweise mehr Aufschluss geben.

Kartentyp: absolut; Ausschnitt Moselraum; Kreise pro fünfstellige PLZ, Kreisgröße 5-50, entspricht 1-4 Tokens; Flächen pro zweistellige PLZ, Anzeigeschwelle 3 Tokens; Tokenangaben beziehen sich auf Gesamtdeutschland.

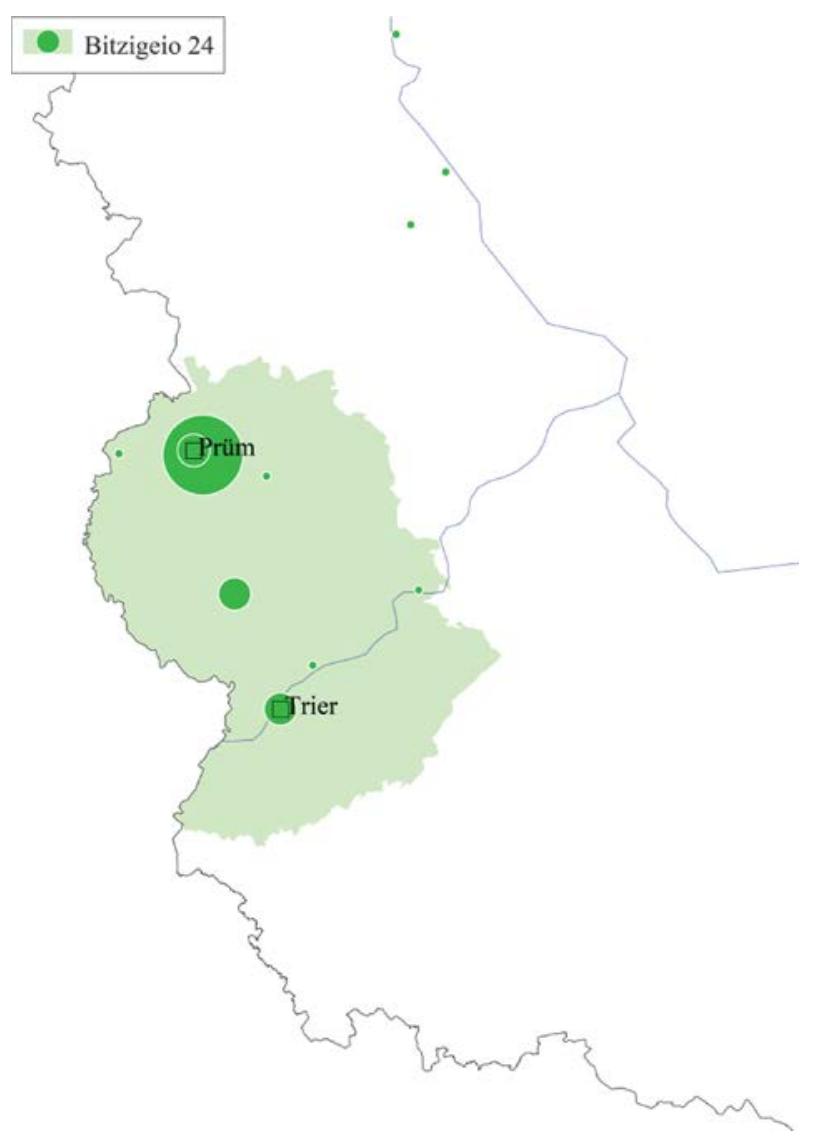

Karte 10: Verbreitung des Familiennamens Bitzigeio

\section{Auswertungsmöglichkeiten}

Durch das ,Digitale Familiennamenwörterbuch Deutschlands' können Familiennamen nicht nur für weitergehende oder regionale Fragestellungen der Onomastik, sondern auch als eine wertvolle Quelle für Nachbardisziplinen wie die Kulturanthropologie, die 
Mentalitäts- und Migrationsforschung nutzbar gemacht werden. Spezifische Suchmöglichkeiten sowie Schlagworte erlauben etwa, die Namen nach Motivgruppen (Berufsname, Herkunftsname etc.), Berufsfeldern (Bäcker, Schmied etc.) oder nach sprachlicher Herkunft (germanisch, slawisch etc.) zu gruppieren. Dies eröffnet die Möglichkeit, erstmals den genauen Anteil der fünf Motivgruppen (Patronyme, Berufs-, Herkunfts-, Wohnstätten- und Übernamen) am Gesamtinventar aller Familiennamen Deutschlands zu bestimmen oder sämtliche Patronyme, die sich aus einem einzelnen Rufnamen ableiten (z.B. aus Ägidius: Gilles, Gillot, Ilg, Schiltz etc.), zu ermitteln. Diese können folglich die Gesamtverbreitung eines Rufnamens im Mittelalter, aber auch die regionale Verteilung bestimmter Kurzformen und Varianten erhellen. Besonders Zentren von Heiligenverehrung lassen sich anhand regional typischer Patronyme lokalisieren. So zeigt die Karte der aus dem Heiligennamen Pirminius abgeleiteten Familiennamen Pfirrmann (459 Tokens), Pfirmann (91 Tokens) sowie Pirmann (76 Tokens) eine markante, auf die südliche Pfalz beschränkte Verbreitung. Dies erklärt sich vor allem mit dem Wirkungsgebiet und der Grablege des Heiligen im Kloster Hornbach bei Pirmasens, dessen Name ebenfalls auf Pirminius zurückgeführt wird (vgl. Duden Geographische Namen 1999, 228).

Kartentyp: absolut; Ausschnitt südliche Pfalz; Kreise pro fünfstellige PLZ, Kreisgröße 5-50, entspricht 1-113 Tokens; Tokenangaben beziehen sich auf Gesamtdeutschland.

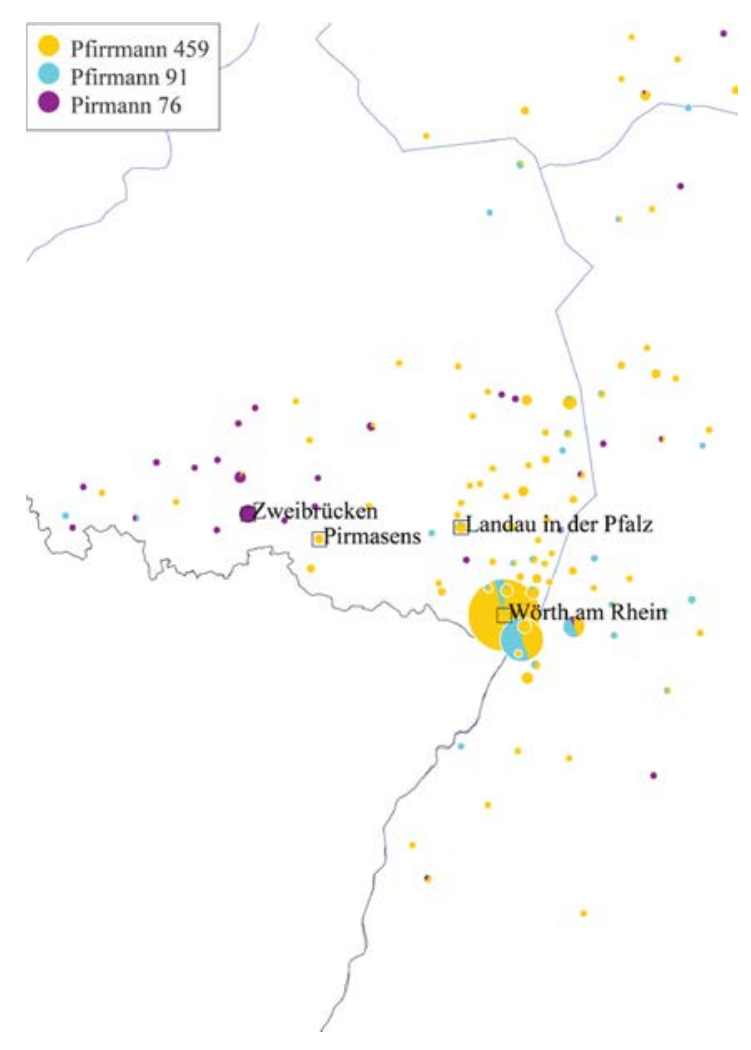

Karte 11: Verbreitung der Familiennamen Pfirrmann, Pfirmann, Pirmann 
Migrationsbewegungen spiegeln sich in der Verbreitung eingedeutschter Varianten wider, etwa in der Häufung der Namen Gillo, Gillot, Giloth, Schillo (alle zum französischen Patronym Gillot) in Südwestdeutschland. Konkrete Hinweise auf Einwanderung und Herkunftsgebiete geben vor allem die aus fremdsprachigen Siedlungsnamen (z.B. Arimont) abgeleiteten Familiennamen. Die Suchfunktionen unserer Datenbank erlauben hier, das Variantenspektrum eines Herkunftsnamens und dessen Verbreitungsraum vollständig zu erheben, etwa die auf den Stadt- bzw. Landesnamen Luxemburg zurückgehenden Familiennamen: Luxenburger (238 Tokens), Litzenburger (174 Tokens), Luxemburger (71 Tokens) u.v.a.m. Durch Einbeziehung der Daten aus den Nachbarländern lassen sich darüber hinaus grenzübergreifende Namenlandschaften nachvollziehen (z.B. die Verbreitung der Varianten Schilz/Schiltz im Raum Mosel - Eifel - Luxemburg).

\section{Ziele und Ergebnisse}

Mit dem ,Digitalen Familiennamenwörterbuch Deutschlands' entsteht erstmals ein umfassendes, modernes und jederzeit zugängliches Nachschlagewerk auf dem aktuellen Stand der Technik, das nicht nur den Ansprüchen der Fachleute, sondern auch dem großen Interesse des breiten Publikums an Familiennamen gerecht wird. Die systematische Erfassung und fundierte Etymologisierung des rezenten Familiennamenbestandes in Deutschland inklusive der fremdsprachigen Namen schließt zudem eine seit Jahrzehnten bestehende empfindliche Lücke in der Anthroponomastik. Besonders im Bereich der fremdsprachigen, regionalen und niedrigfrequenten Namen ist eine noch nicht abzuschätzende Vielzahl von Neueinträgen zu erwarten. Hier bietet sich in vielen Bereichen eine Zusammenarbeit auf der Ebene regionaler, historischer sowie auch internationaler Namenforschung an.

\section{Literatur}

Bahlow, Hans: Deutsches Namenlexikon, Familien- und Vornamen nach Ursprung und Sinn erklärt, 16. Auflage, Frankfurt/Main, 2005.

Brechenmacher, Josef Karlmann: Etymologisches Wörterbuch der Deutschen Familiennamen, 2 Bände, 2., von Grund auf neugearbeitete Auflage, Limburg/Lahn, 1957-63.

Brendler, Andrea: Italienische Familiennamen im Deutschen. In: Karlheinz Hengst/Dietlind Krüger: Familiennamen im Deutschen, Erforschung und Nachschlagewerke, Deutsche Familiennamen im deutschen Sprachraum, Leipzig, 2009, S. 439-458.

Debrabandere, Frans: Woordenboek van de familienamen in België en Noord-Frankrijk, Amsterdam/ Antwerpen, 2003.

Dräger, Kathrin: Italienische Familiennamen in Deutschland. In: Karlheinz Hengst/Dietlind Krüger: Familiennamen im Deutschen, Erforschung und Nachschlagewerke, Familiennamen aus fremden Sprachen im deutschen Sprachraum, Leipzig, 2011, S. 333-347.

Deutscher Familiennamenatlas (DFA). Herausgegeben von Konrad Kunze und Damaris Nübling, Band 1: Graphematik/Phonologie der Familiennamen I: Vokalismus, Band 2: Graphematik/Phonologie der Familiennamen II: Konsonantismus, Band 3: Morphologie der Familiennamen, Band 4: Familiennamen nach Herkunft und Wohnstätte, Berlin, New York, 2009, 2011, Berlin, Boston 2012, 2013. 
Duden Familiennamen, Herkunft und Bedeutung, Bearbeitet von Rosa Kohlheim und Volker Kohlheim, 2., völlig neu bearbeitete Auflage, Mannheim u.a., 2005.

Duden Geographische Namen in Deutschland, Bearbeitet von Dieter Berger, 2., überarbeitete Auflage, Mannheim u.a., 1999.

Germain, Jean/Herbillon, Jules: Dictionnaire des noms de famille en Wallonie et à Bruxelles, Brüssel, 2007.

Gottschald, Max: Deutsche Namenkunde, Mit einer Einführung in die Familiennamenkunde von Rudolf Schützeichel, 6. durchgesehene und bibliographisch aktualisierte Auflage, Berlin, New York, 2006.

Hartig, Margit/Schwanke, Judith: Französische Familiennamen im deutschen Sprachgebiet. In: Karlheinz Hengst/Dietlind Krüger: Familiennamen im Deutschen, Erforschung und Nachschlagewerke, Deutsche Familiennamen im deutschen Sprachraum, Leipzig, 2009, S. 459-473.

Heuser, Rita: Französische Familiennamen in Deutschland. In: Karlheinz Hengst/Dietlind Krüger: Familiennamen im Deutschen, Erforschung und Nachschlagewerke, Familiennamen aus fremden Sprachen im deutschen Sprachraum, Leipzig, 2011, S. 349-372.

Morlet, Marie-Thérèse: Dictionnaire étymologique des noms de famille, Paris, 1991.

Naumann, Horst: Das große Buch der Familiennamen, Alter, Herkunft, Bedeutung, München, 2007.

Steffens, Rudolf: Familiennamenatlas Rheinland-Pfalz, Hessen, Saarland. Ubstadt-Weiher, 2013.

Zoder, Rudolf: Familiennamen in Ostfalen, 2 Bände, Hildesheim, 1968. 


\section{Anschriften der Autorinnen und Autoren}

Fabian Fahlbusch

Dr. Rita Heuser

Deutscher Familiennamenatlas/Digitales

Familiennamenwörterbuch Deutschlands

Akademie der Wissenschaften und der Literatur Mainz,

Geschwister-Scholl-Str. 2, D-55131 Mainz

Frau Dräger hat die Freiburger Adresse, s. Konrad Kunze

Walter Amaru Flores Flores

Prof. Dr. Peter Gilles

Dr. Cristian Kollmann

Dr. Claire Muller

Université du Luxembourg

Laboratoire für luxemburgische Sprach- und Literaturwissenschaft

L-7201 Walferdange

Prof. Dr. Jean Germain

Université catholique de Louvain

Place de l'Université

B-1348 Louvain-la-Neuve

Dr. Kathrin Dräger

Prof. Dr. Konrad Kunze

Albert-Ludwigs-Universität Freiburg

Deutsches Seminar I D-79085 Freiburg

Prof. Dr. Ann Marynissen

Universität zu Köln

Philosophische Fakultät

Institut für Niederlandistik

D-50935 Köln

Dr. Jean-Claude Muller

12, allée des Tilleuls

L-8501 Redange-sur-Attert 
